\title{
BEHAVIOUR OF SANDS UNDER \\ GENERALIZED LOADING AND DRAINAGE CONDITIONS
}

\author{
A research thesis submitted to \\ the Faculty of Graduate Studies and Research \\ in a partial fulfillment of the requirements for the degree
}

Doctor of Philosophy

by

\section{Paramaguru Logeswaran}

M.A.Sc., Carleton University, Canada (2005)

B.Sc.Eng., University of Peradeniya, Sri Lanka (2001)

Department of Civil and Environmental Engineering

Carleton University

Ottawa-Carleton Institute of Civil and Environmental Engineering August 2010

CLogeswaran. P 


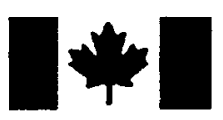

\author{
Library and Archives \\ Canada \\ Published Heritage \\ Branch \\ 395 Wellington Street \\ Ottawa ON K1A ON4 \\ Canada
}

Bibliotheque et

Archives Canada

Direction du

Patrimoine de l'édition

395 , rue Wellington

Ottawa ON K1A ON4

Canada
Your file Votre référence
ISBN: $978-0-494-70530-8$
Our file Notre référence
ISBN: $978-0-494-70530-8$
NOTICE:

The author has granted a nonexclusive license allowing Library and Archives Canada to reproduce, publish, archive, preserve, conserve, communicate to the public by telecommunication or on the Internet, loan, distribute and sell theses worldwide, for commercial or noncommercial purposes, in microform, paper, electronic and/or any other formats.

The author retains copyright ownership and moral rights in this thesis. Neither the thesis nor substantial extracts from it may be printed or otherwise reproduced without the author's permission.
AVIS:

L'auteur a accordé une licence non exclusive permettant à la Bibliothèque et Archives Canada de reproduire, publier, archiver, sauvegarder, conserver, transmettre au public par télécommunication ou par l'Internet, prêter, distribuer et vendre des thèses partout dans le monde, à des fins commerciales ou autres, sur support microforme, papier, électronique et/ou autres formats.

L'auteur conserve la propriété du droit d'auteur et des droits moraux qui protège cette thèse. $\mathrm{Ni}$ la thèse ni des extraits substantiels de celle-ci ne doivent être imprimés ou autrement reproduits sans son autorisation.
In compliance with the Canadian Privacy Act some supporting forms may have been removed from this thesis.

While these forms may be included in the document page count, their removal does not represent any loss of content from the thesis.
Conformément à la loi canadienne sur la protection de la vie privée, quelques formulaires secondaires ont été enlevés de cette thèse.

Bien que ces formulaires aient inclus dans la pagination, il n'y aura aucun contenu manquant.

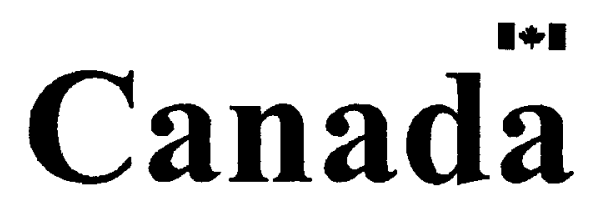




\begin{abstract}
Deformation characteristics of anisotropic materials depend on the principal stresses and their directions. Magnitude and direction of the principal stresses vary continuously in most of the in-situ loading. For a reliable assessment of soil response (and to predict the in-situ soil deformation characteristics), laboratory devices require a loading system to duplicate the in-situ loading very well.

Laboratory devices that are commonly used in soil testing are not capable of controlling all principal stresses and/or their directions independently in a soil specimen. Only a few devices (e.g. hollow cylinder torsional, directional shear cell) have the ability to control the principal stresses and their directions, but these are rarely used in soil testing practice. An automated Hollow Cylinder Torsional (HCT) shear device, capable of applying static and cyclic loading, was developed in the geotechnical laboratory of Carleton University. In addition, it is capable of simulating prescribed stress path or strain path controlled loading.
\end{abstract}

The undrained response of sands is dependent on the loading mode, and serious strength degradations occur as the inclination of major principal stress varies from $0^{\circ}$ to $45^{\circ}$ to vertical. The strength degradation is insignificant beyond $45^{\circ}$ (towards $90^{\circ}$ ). The effect of intermediate principal stress is not as critical as that of the direction of major principal stress in water deposited sands.

The undrained effective stress path has been shown to be unique regardless of total stress paths, even when the loading involves principal stress rotation, and changes in the intermediate principal stress parameter. 
Measured behaviour under volume-pore pressure coupled deformation demonstrates that undrained loading does not always represent the worst-case scenario. Pore pressure equalization following a short term loading (e.g. earthquake) might trigger strain softening deformation in sands that are considered dilative and stable under undrained conditions. This finding has serious practical implications since the current design practice generally assumes the undrained loading to be the worst-case scenario. field.

Cyclic loading tests to assess the effects of principal stress rotation on liquefaction susceptibility shows that the nature of principal stress rotation (i.e. jump vs. smooth) does not affect the cyclic resistance of sands when the cyclic loading causes limited rotation of the principal stresses (less than $60^{\circ}$ about the axis of symmetry). 


\section{ACKNOWLEDGEMENTS}

I am grateful to my supervisor Professor Siva Sivathayalan for his constant support, guidance, encouragement and enthusiastic interest during the course of study. I owe him a debt of gratitude for his commitment to this research.

Most importantly, I would like to express my deepest gratitude to my mother Sivagamiamma, brother Ramesh and sister Malini for their continuous support, patience and encouragement throughout my studies and life.

I would like to thank my wife Mohana for her encouragement and support during my study and my life. Her love, support and encouragement have been instrumental in guiding me to earn this doctoral degree.

Friends are always a huge part of any endeavor in life and I have been truly blessed throughout my seven years at Carleton University. Many hours has been spent on working, talking, playing and laughing with my friends and colleagues who got me through many rocky patches and truly made both life and studies much richer.

During the seven years of my graduate studies, I have earned knowledge and experience in the advanced geotechnical laboratory in Carleton University. I would like to acknowledge the laboratory technicians Stan, Pierre, Jason and Ken McMartin for their great support to do my lab experiments. 


\section{DEDICATION}

This research thesis is dedicated to my wonderful parents; my mother Sivagamiamma and late father Paramaguru who have motivated me to be the person I am today. You two have been with me in every step of my life, through good and bad times. The sacrifice and struggle of my mother in her life gave me some education. Thank you for all the unconditional love, guidance and support that you have always given me, helping me to succeed and instilling in me the confidence that I am capable of doing anything. Thank you for everything. I love you so much! 


\section{TABLE OF CONTENTS}

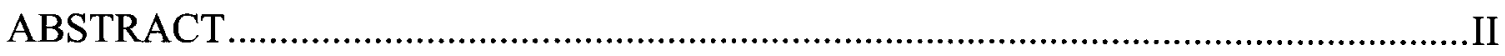

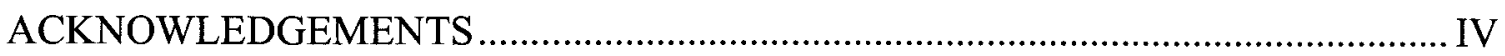

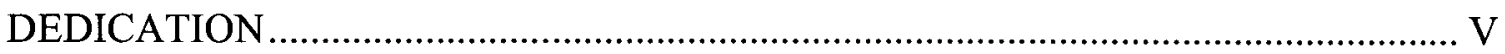

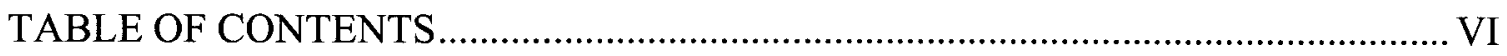

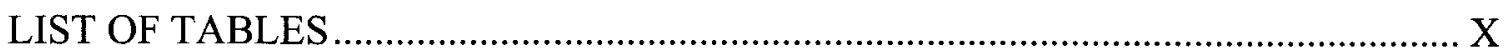

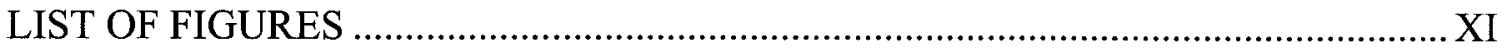

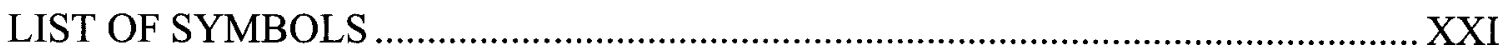

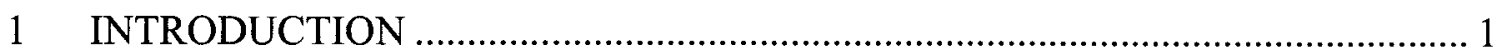

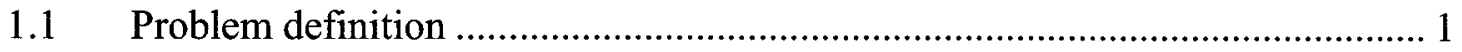

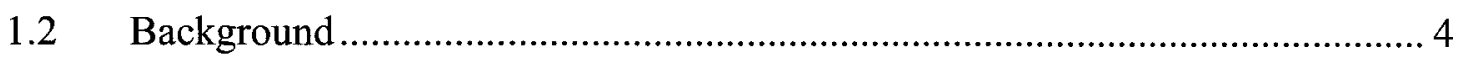

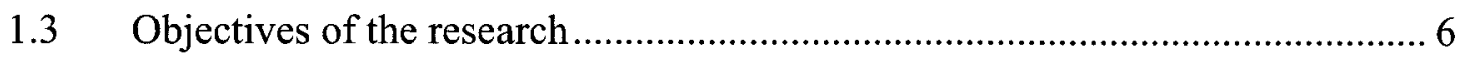

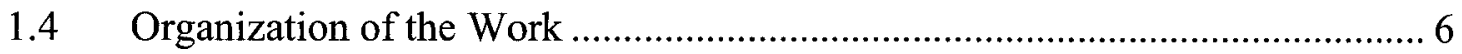

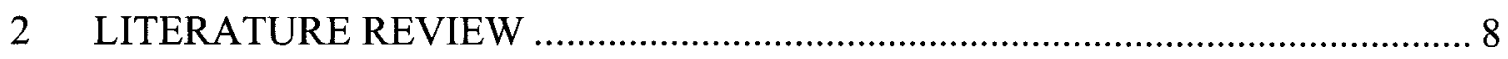

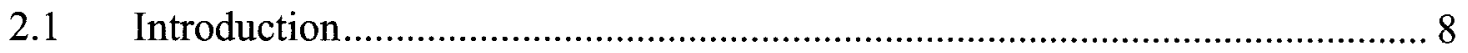

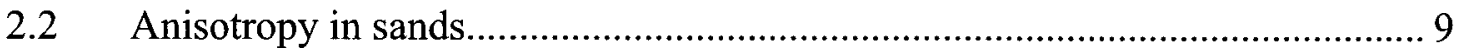

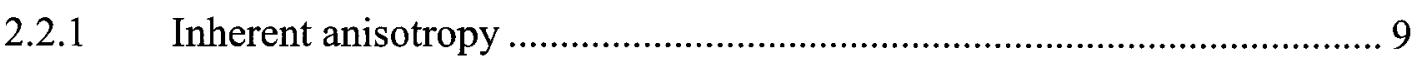

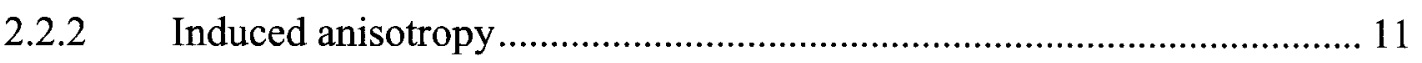

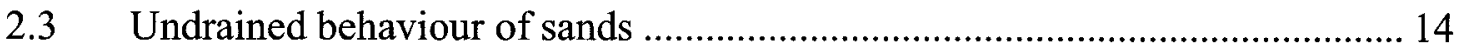

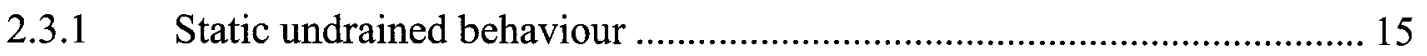

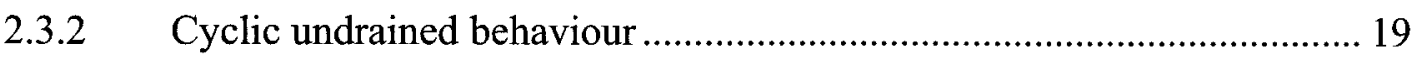

2.3.2.1 Mechanisms of strain development ……….................................... 19

2.3.3 Peak state and initiation of strain softening ............................................. 26

2.3.4 Phase Transformation (PT) and Steady State (SS) ................................... 27

$2.4 \quad$ Pore pressure generation in saturated soils ................................................... 28

2.5 Behaviour of sands under generalized stress path loading ............................. 31

2.5.1 Effect of principal stress direction ……………..................................... 32

2.5.2 Effect of intermediate principal stress .................................................. 36

2.5.3 Effect of mean normal stress and relative density ................................... 39 


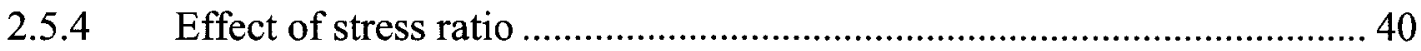

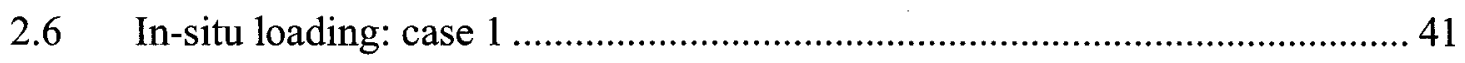

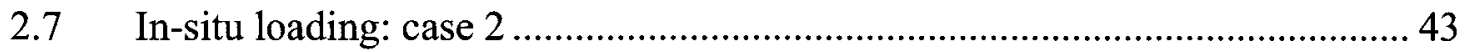

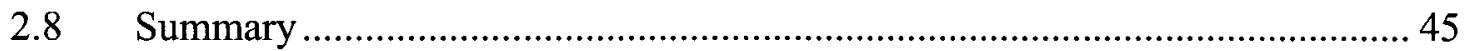

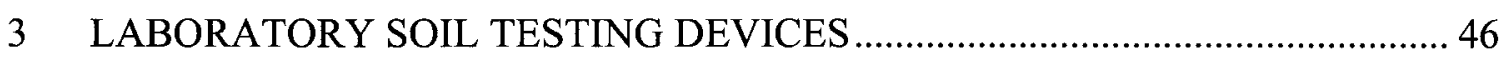

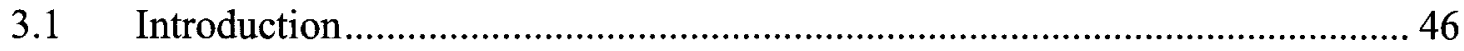

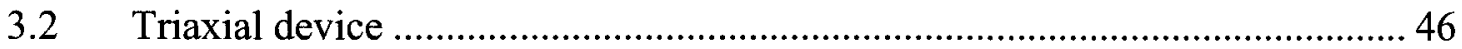

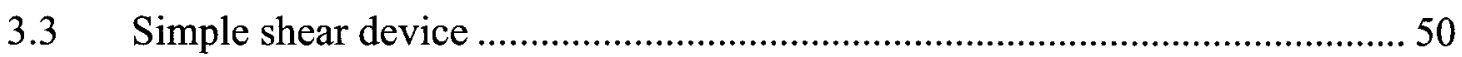

$3.4 \quad$ Hollow cylinder torsional shear device........................................................... 53

3.4.1 Description of the HCT device used.................................................... 53

3.4.2 Loading systems of the HCT device ...................................................... 55

3.4.2.1 Stress-controlled loading system ……………..................................... 56

3.4.2.2 Displacement-controlled loading system.............................................. 59

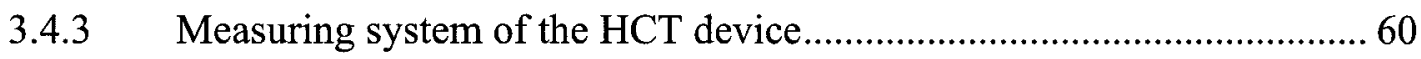

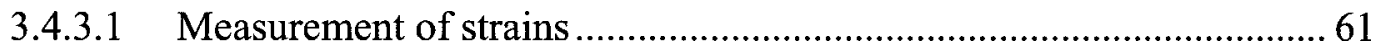

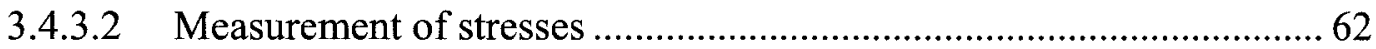

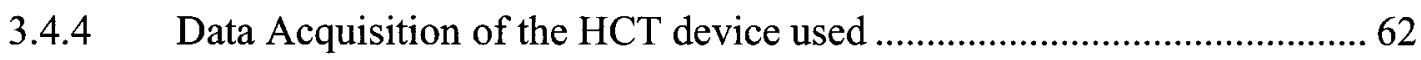

3.5 Definitions of stresses and strains in HCT specimens ……............................... 64

3.6 Stress nonuniformities in HCT specimens .....................................................6

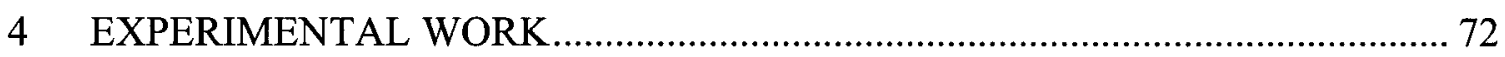

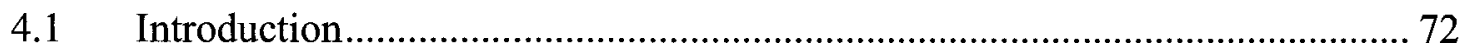

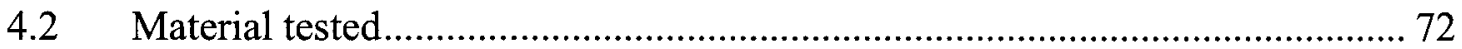

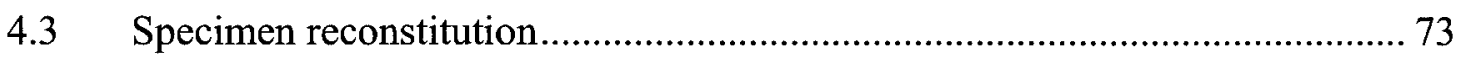

4.3.1 Preliminary steps of specimen reconstitution ........................................ 75

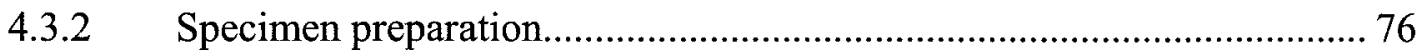

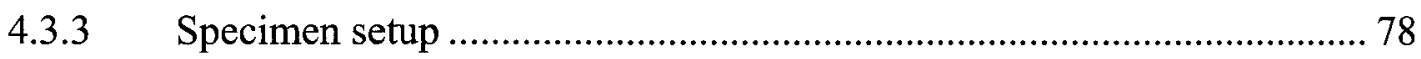

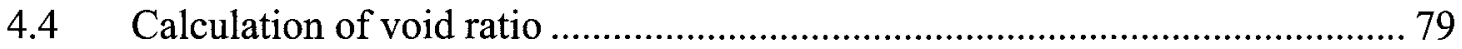

4.5 Performance and control of the HCT device .................................................. 79

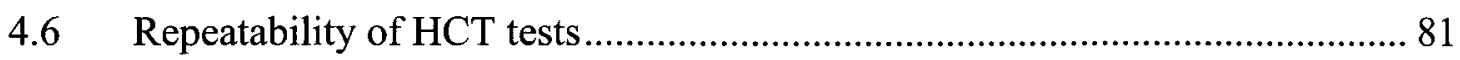

5 MONOTONIC RESPONSE UNDER GENERALIZED LOADING AND

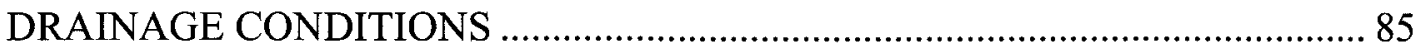




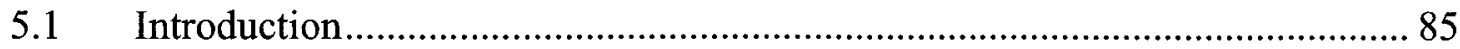

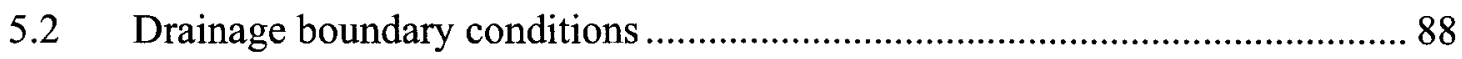

$5.3 \quad$ Undrained behaviour of sand ..................................................................... 91

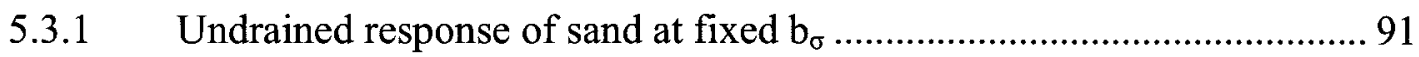

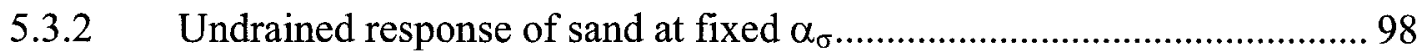

5.3.3 Undrained response of anisotropically consolidated sand ....................... 103

5.4 Strain path controlled behaviour of sand .................................................. 106

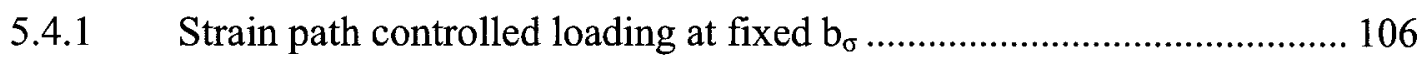

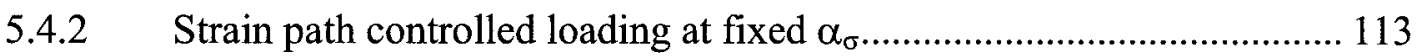

5.4.3 Strain path controlled loading on anisotropically consolidated sand...... 120

5.4.4 Influence of strain paths on sand behaviour .......................................... 126

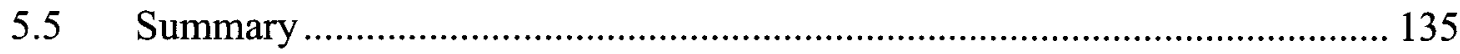

6 CYCLIC BEHAVIOUR OF SAND UNDER GENERALIZED LOADING.......... 139

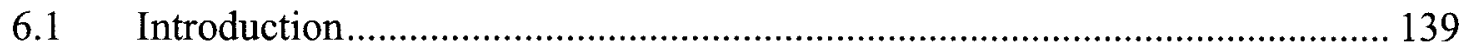

6.2 Simulation of triaxial mode of cyclic loading............................................... 143

6.3 Jump rotation of principal stress under plane strain condition ........................ 146

6.4 Nature of principal stress rotation and cyclic behaviour of sand.................... 149

6.4.1 Smooth rotation of principal stress direction at different stress ratios.... 149

6.4.2 Jump rotation of principal stress direction at different stress ratios ....... 155

6.4.3 Comparison of the nature of principal stress rotation on cyclic behaviour.. 158

6.5 Influence of $\alpha_{\sigma}$ on cyclic behaviour of sand ............................................... 160

6.5.1 Smooth rotation of $\alpha_{\sigma}$ at a fixed cyclic stress ratio................................. 160

6.5.2 Jump rotation of $\alpha_{\sigma}$ at a fixed cyclic stress ratio .................................... 166

6.6 Comparison of the nature of principal stress rotation.................................... 173

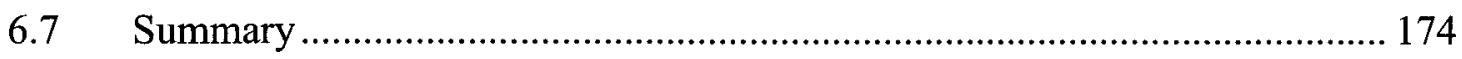

7 TOTAL STRESS PATHS AND PORE PRESSURE GENERATION ………….... 177

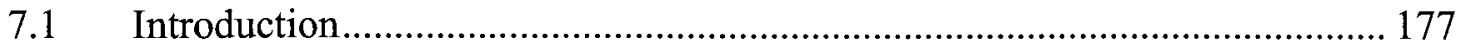

7.2 Total stress increment and pore pressure generation ………………............ 180

$7.3 \quad$ Pore pressure parameters ………................................................................ 185 
7.4 Effect of total stress path on pore pressure generation and sand behaviour... 192

7.5 Effect of $b_{\sigma}$ on pore pressure generation ........................................... 194

7.6 Effect of $\alpha_{\sigma}$ on pore pressure generation ................................................. 196

7.7 Effect of change of $b_{\sigma}$ on pore pressure generation and sand behaviour........ 201

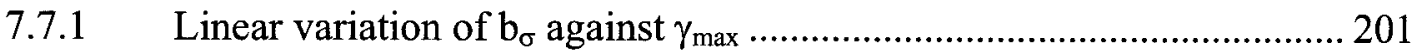

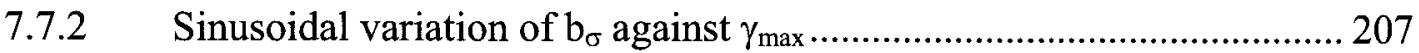

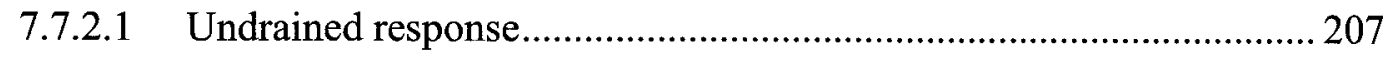

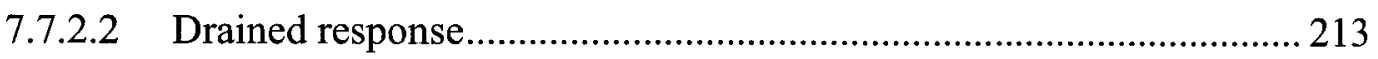

7.8 Effect of rotation of $\alpha_{\sigma}$ on pore pressure generation and sand behaviour ...... 216

7.9 Effect of simultaneous change of $\alpha_{\sigma}$ and $b_{\sigma}$ on pore pressure generation..... 222

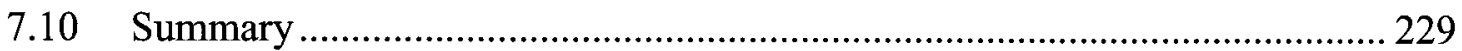

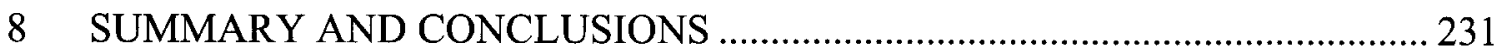

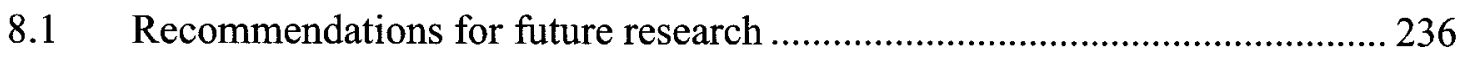

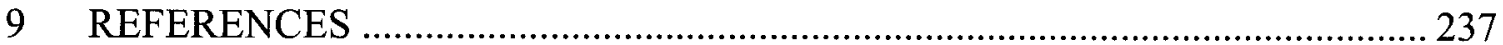




\section{LIST OF TABLES}

Table 5.1 Initial and loading states of undrained and strain path controlled tests ....... 86

Table 6.1 Initial and loading states of tests conducted under cyclic loading............. 142

Table 7.1 Initial and loading states of tests conducted under different total stress paths 


\section{LIST OF FIGURES}

Fig. 2.1 Induced anisotropy of sands (after Arthur et al., 1977) ............................ 13

Fig. 2.2 Influence of direction of loading and imposed strain level on induced anisotropy (Modified from Mould et al., 1982) ........................................ 14

Fig. 2.3 Characteristic behaviour of saturated sand under undrained monotonic loading (after Vaid and Chern, 1985) (a) stress-strain responses (b) stress paths.

Fig. 2.4 True liquefaction of sands during cyclic undrained shear (After Vaid and Chern, 1985)

Fig. 2.5 Limited liquefaction of sands during cyclic undrained shear (After Vaid and Chern, 1985)

Fig. 2.6 Cyclic mobility in sands during cyclic undrained shear with transient state of zero effective stress (After Vaid and Chern, 1985) 24

Fig. 2.7 Cyclic mobility in sands during cyclic undrained shear without transient state of zero effective stress (Vaid et al., 2001) 25

Fig. 2.8 Increment of principal stresses in axisymmetric soil element during rotation of principal stress (After Law and Holtz, 1978) 30

Fig. 2.9 Principal stress rotations underneath an offshore soil foundation (After Vaughan et al., 1976) 33

Fig. 2.10 (a) Contractant region of state boundary surface; (b) Complete state boundary surface. 35

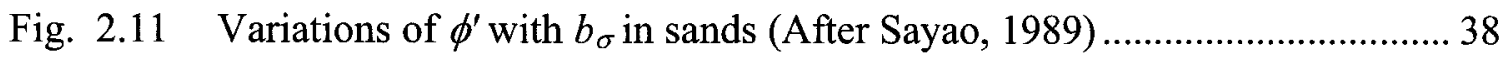

Fig. 2.12 Principal stress variation along potential failure surfaces. 42

Fig. 2.13 3-D representation of stresses in a soil element (a) at level ground before construction (b) after construction (c) during application of load... 43

Fig. 2.14 Schematic illustration of pore water migration due to piezometric head gradient in a heterogeneous earth structure (modified after Logeswaran, 2005)

Fig. 3.1 Mohr's circle representation of stress states during drained triaxial tests consolidated (1) isotropically (2) anisotropically . 48 
Fig. 3.2 Comparison of in-situ stress condition and simple shear loading (Modified

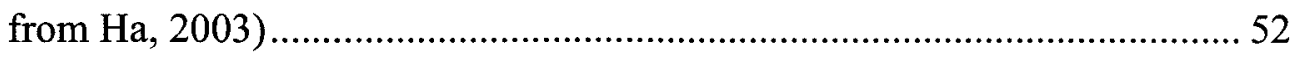

Fig. 3.3 Schematic diagram of the HCT apparatus ............................................... 54

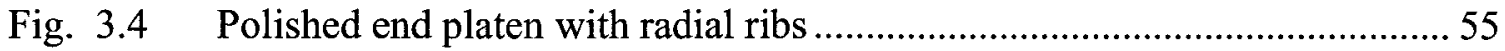

Fig. 3.5 Pressure and volume control of inner chamber of the HCT device............. 57

Fig. 3.6 Vertical stress and vertical displacement control of the HCT device........... 57

Fig. 3.7 Pressure control of external chamber of the HCT device ............................ 58

Fig. 3.8 Torque and torsional displacement control of the HCT device ................... 58

Fig. 3.9 Back pressure and specimen volume control of the HCT device ................ 59

Fig. 3.10 Torsional displacement measurement in the HCT device .......................... 61

Fig. 3.11 Schematic diagram of data acquisition process in the HCT device............. 63

Fig. 3.12 Surface tractions and stress state of a soil element in a HCT specimen ....... 65

Fig. 4.1 Grain size distribution of Fraser River sand used .................................... 73

Fig. 4.2 HCT specimen preparation by water pluviation technique (After Sayao,

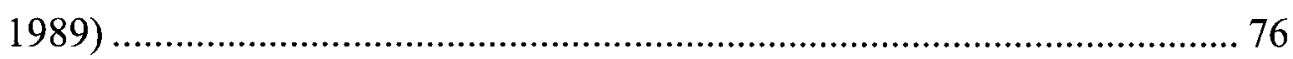

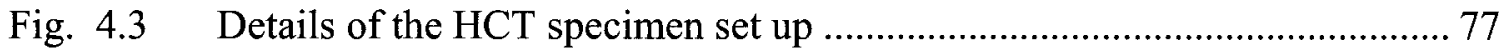

Fig. 4.4 Control of stresses and measured volumetric strain during anisotropic

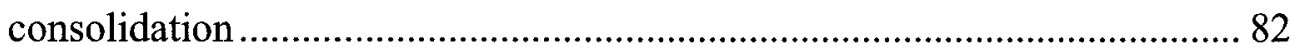

Fig. 4.5 Targeted and actual control parameters followed in typical HCT test ........ 83

Fig. 4.6 Repeatability of HCT undrained test at $b_{\sigma}=0.0$ and $\alpha_{\sigma}=90^{\circ} \ldots \ldots \ldots \ldots \ldots . . . . . . . . .84$

Fig. 5.1 Schematic illustration of conditions of drainage: strain paths and stress paths (After Logeswaran, 2005).

Fig. 5.2 Variation of principal strains with vertical strain during undrained shear tests at fixed $b_{\sigma}$ 92

Fig. 5.3 Undrained response of Fraser River sand at fixed $b_{\sigma}\left(\alpha_{\sigma}=0^{\circ}\right) \ldots \ldots \ldots \ldots \ldots \ldots . . . . . . .93$

Fig. 5.4 Variation of strength, excess pore pressure and stress state at PT state....... 95

Fig. 5.5 Contours of coefficient of nonuniformity at $R=3.0, \sigma_{m}^{\prime}=300 \mathrm{kPa}$ and $D_{r}=$ 30\% (After Wijewickreme, 1990) 96

Fig. 5.6 Effect of stress ratio on coefficient of nonuniformity at $\sigma_{m}^{\prime}=300 \mathrm{kPa}$ and $D_{r}$ $=30 \%($ After Wijewickreme, 1990) 
Fig. 5.7 Variation of principal strains with vertical strain during undrained shear tests at fixed $\alpha_{\sigma}$

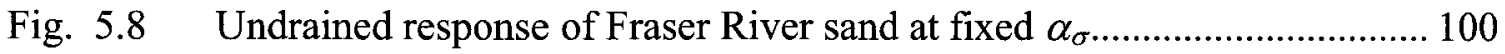

Fig. 5.9 Variation of strength, excess pore pressure and stress state at PT state...... 102

Fig. 5.10 Undrained response of anisotropically consolidated Fraser River sand ..... 104

Fig. 5.11 Variation of strength, excess pore pressure induced by deviatoric stress and stress state at PT state

Fig. 5.12 Variation of principal strains with vertical strain during strain path controlled tests at fixed $b_{\sigma}$ 108

Fig. 5.13 Response of Fraser River sand during strain path controlled tests at fixed $b_{\sigma}$ 109

Fig. 5.14 Peak strength, excess pore pressure and effective stress state at peak strength state during strain path controlled tests at fixed $b_{\sigma}$ 110

Fig. 5.15 Strength, excess pore pressure and stress state of peak pore pressure state during strain path controlled tests at fixed $b_{\sigma}$ 112

Fig. 5.16 Variation of principal strains during strain path controlled tests at fixed $\alpha_{\sigma}$ 114

Fig. 5.17 Response of Fraser River sand during strain path controlled tests at fixed $\alpha_{\sigma}$ 116

Fig. 5.18 Strength, excess pore pressure and effective stress state at peak strength state during strain path controlled tests at fixed $\alpha_{\sigma}$

Fig. 5.19 Strength, excess pore pressure and stress state of peak pore pressure state during strain path controlled tests at fixed $\alpha_{\sigma}$ 119

Fig. 5.20 Response of anisotropically consolidated Fraser River sand during strain path controlled loading 121

Fig. 5.21 Flow potential of anisotropically consolidated Fraser River sand during expansive volumetric strain

Fig. 5.22 Strength, excess pore pressure and effective stress state at peak strength state during strain path controlled loading..... 124 
Fig. 5.23 Strength, excess pore pressure and stress state at peak pore pressure state during strain path controlled loading ............................................... 125

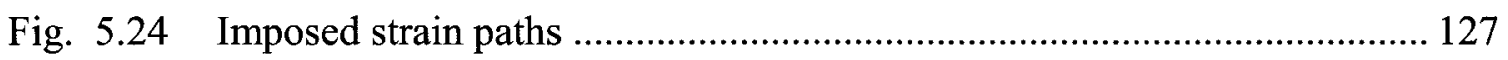

Fig. 5.25 Variation of principal strains with torsional strain at $b_{\sigma}=0.4$ and $\alpha_{\sigma}=45^{\circ}$ during strain path controlled tests 128

Fig. 5.26 Response of Fraser River sand at $b_{\sigma}=0.4$ and $\alpha_{\sigma}=45^{\circ}$ during strain path controlled tests 130

Fig. 5.27 Strength, excess pore pressure and mobilized friction angle at peak strength state during strain path controlled tests.

Fig. 5.28 Strength, excess pore pressure and effective stress state at peak pore pressure state during strain path controlled tests.

Fig. 6.1 Cyclic stress and nature of principal stress rotation imposed 140

Fig. 6.2 Response of Fraser River sand under triaxial mode of cyclic loading........ 145

Fig. 6.3 Cyclic response of Fraser River sand during jump rotation of $\alpha_{\sigma}$ between $45^{\circ}$ and $+45^{\circ}$ at $b_{\sigma}=0.40$ 148

Fig. 6.4 Imposed torsional shear stress during application of cyclic loading under smooth and jump rotations of $\alpha_{\sigma}$ 150

Fig. 6.5 Stress path of Fraser River sand during cyclic loading of $\sigma_{d} /\left(2 \sigma_{3 c}^{\prime}\right)=0.10$ with smooth rotation of $\alpha_{\sigma}$ between $-45^{\circ}$ and $+45^{\circ}$ 151

Fig. 6.6 Stress path of Fraser River sand during cyclic loading of $\sigma_{d} /\left(2 \sigma_{3 c}^{\prime}\right)=0.15$ with smooth rotation of $\alpha_{\sigma}$ between $-45^{\circ}$ and $+45^{\circ}$

Fig. 6.7 Stress path of Fraser River sand during cyclic loading of $\sigma_{d} /\left(2 \sigma_{3 c}^{\prime}\right)=0.20$ with smooth rotation of $\alpha_{\sigma}$ between $-45^{\circ}$ and $+45^{\circ}$ 152

Fig. 6.8 Stress path of Fraser River sand during cyclic loading of $\sigma_{d} /\left(2 \sigma_{3 c}^{\prime}\right)=0.25$ with smooth rotation of $\alpha_{\sigma}$ between $-45^{\circ}$ and $+45^{\circ}$ 152

Fig. 6.9 Excess pore pressure response of Fraser River sand during cyclic loading with smooth rotation of $\alpha_{\sigma}$ between $-45^{\circ}$ and $+45^{\circ}$ 154

Fig. 6.10 Induced maximum shear strain during cyclic loading with smooth rotation of $\alpha_{\sigma}$ between $-45^{\circ}$ and $+45^{\circ}$. 154 
Fig. 6.11 Stress path of Fraser River sand during cyclic loading of $\sigma_{d} /\left(2 \sigma_{3 c}^{\prime}\right)=0.15$ with jump rotation of $\alpha_{\sigma}$ between $-45^{\circ}$ and $+45^{\circ}$ 156

Fig. 6.12 Stress path of Fraser River sand during cyclic loading of $\sigma_{d} /\left(2 \sigma_{3 c}^{\prime}\right)=0.20$ with jump rotation of $\alpha_{\sigma}$ between $-45^{\circ}$ and $+45^{\circ}$ 156

Fig. 6.13 Stress path of Fraser River sand during cyclic loading of $\sigma_{d} /\left(2 \sigma_{3 c}^{\prime}\right)=0.25$ with jump rotation of $\alpha_{\sigma}$ between $-45^{\circ}$ and $+45^{\circ}$ 157

Fig. 6.14 Excess pore pressure response of Fraser River sand during cyclic loading with jump rotation of $\alpha_{\sigma}$ between $-45^{\circ}$ and $+45^{\circ}$

Fig. 6.15 Induced maximum shear strain during cyclic loading with jump rotation of $\alpha_{\sigma}$ between $-45^{\circ}$ and $+45^{\circ}$ 158

Fig. 6.16 Cyclic resistance curve of loose Fraser River sand during jump and smooth rotations of $\alpha_{\sigma}$ 159

Fig. 6.17 Torsional shear stress during smooth and jump rotations of $\alpha_{\sigma \ldots \ldots \ldots \ldots . . . . . . .161}$

Fig. 6.18 Stress path of Fraser River sand during cyclic loading of $\sigma_{d} /\left(2 \sigma^{\prime}{ }_{3 c}\right)=0.20$ with smooth rotation of $\alpha_{\sigma}$ between $-15^{\circ}$ and $+15^{\circ}$ 162

Fig. 6.19 Stress path of Fraser River sand during cyclic loading of $\sigma_{d} /\left(2 \sigma_{3 c}^{\prime}\right)=0.20$ with smooth rotation of $\alpha_{\sigma}$ between $-30^{\circ}$ and $+30^{\circ}$ 163

Fig. 6.20 Stress path of Fraser River sand during cyclic loading of $\sigma_{d} /\left(2 \sigma_{3 c}^{\prime}\right)=0.20$ with smooth rotation of $\alpha_{\sigma}$ between $-60^{\circ}$ and $+60^{\circ}$ 163

Fig. 6.21 Stress path of Fraser River sand during cyclic loading of $\sigma_{d} /\left(2 \sigma_{3 c}^{\prime}\right)=0.20$ with smooth rotation of $\alpha_{\sigma}$ between $-75^{\circ}$ and $+75^{\circ}$ 164

Fig. 6.22 Stress path of Fraser River sand during cyclic loading of $\sigma_{d} /\left(2 \sigma_{3 c}^{\prime}\right)=0.20$ with smooth rotation of $\alpha_{\sigma}$ between $-90^{\circ}$ and $+90^{\circ}$ 164

Fig. 6.23 Excess pore pressure response of Fraser River sand during cyclic loading with smooth rotation of $\alpha_{\sigma}$. 166

Fig. 6.24 Induced maximum shear strain during cyclic loading with smooth rotation of $\alpha_{\sigma}$ 167

Fig. 6.25 Stress path of Fraser River sand during cyclic loading of $\sigma_{d} /\left(2 \sigma_{3 c}^{\prime}\right)=0.20$ with jump rotation of $\alpha_{\sigma}$ between $-15^{\circ}$ and $+15^{\circ}$ 168 
Fig. 6.26 Stress path of Fraser River sand during cyclic loading of $\sigma_{d} /\left(2 \sigma_{3 c}^{\prime}\right)=0.20$ with jump rotation of $\alpha_{\sigma}$ between $-30^{\circ}$ and $+30^{\circ}$ 169

Fig. 6.27 Stress path of Fraser River sand during cyclic loading of $\sigma_{d} /\left(2 \sigma_{3 c}^{\prime}\right)=0.20$ with jump rotation of $\alpha_{\sigma}$ between $-60^{\circ}$ and $+60^{\circ}$ 169

Fig. 6.28 Stress path of Fraser River sand during cyclic loading of $\sigma_{d} /\left(2 \sigma_{3 c}^{\prime}\right)=0.20$ with jump rotation of $\alpha_{\sigma}$ between $-75^{\circ}$ and $+75^{\circ}$ 170

Fig. 6.29 Excess pore pressure response of Fraser River sand during cyclic loading with jump rotation of $\alpha_{\sigma}$. 171

Fig. 6.30 Imposed maximum shear strain during cyclic loading with jump rotation of $\alpha_{\sigma}$ 172

Fig. 6.31 Comparison of number of cycles required for liquefaction during smooth and jump rotations of $\alpha_{\sigma}$ 173

Fig. 6.32 Imposed torsional shear stress during jump and smooth rotations of $\alpha_{\sigma}$ between $-60^{\circ}$ and $+60^{\circ}$, and $-75^{\circ}$ and $+75^{\circ}$ 174

Fig. 7.1 Variation of $\Delta \sigma_{3}$ against $\Delta \sigma_{m}$ at fixed $b_{\sigma}=0.0$ and 0.4

Fig. 7.2 Change of $b_{\sigma}$ during application of a total stress path 184

Fig. 7.3 Variation of $\Delta \sigma_{3}$ against $\Delta \sigma_{m}$ during change of $b_{\sigma}$ corresponding to Fig. 7.2 184

Fig. 7.4 Excess pore pressure induced during isotropic increment of principal stresses 185

Fig. 7.5 Induced excess pore pressure during anisotropic principal stress increment (direction of $\sigma$ and direction of $\Delta \sigma$ coinciding)

Fig. 7.6 Variation of pore pressure parameters during anisotropic principal stress increment (direction of $\sigma$ and direction of $\Delta \sigma$ coinciding) ...................... 188

Fig. 7.7 Induced excess pore pressure during anisotropic principal stress increment (direction of $\sigma$ and direction of $\Delta \sigma$ not coincident)

Fig. 7.8 Variation of pore pressure parameters during anisotropic principal stress increment (direction of $\sigma$ and direction of $\Delta \sigma$ not coinciding) 191 
Fig. 7.9 Undrained response of Fraser River sand along different total stress paths $\left(b_{\sigma}\right.$ $=0$ and $\alpha_{\sigma}=0^{\circ}$ )

Fig. 7.10 Undrained response of Fraser River sand along different total stress paths $\left(b_{\sigma}\right.$ $=0$ and $\alpha_{\sigma}=90^{\circ}$ )

Fig. 7.11 Deviatoric stress induced excess pore pressure at $b_{\sigma}=0.0$ and 0.4 , and $\alpha_{\sigma}=$ $0^{\circ}$

Fig. 7.12 Variation of pore pressure parameters at fixed $b_{\sigma}=0.0$ and 0.4 , and $\alpha_{\sigma}=0^{\circ}$ 198

Fig. 7.13 Deviatoric stress induced excess pore pressure at $b_{\sigma}=0.4$ and $\alpha_{\sigma}=0^{\circ}$, and $b_{\sigma}=0.4$ and $\alpha_{\sigma}=45^{\circ}$

Fig. 7.14 Effect of $\alpha_{\sigma}$ on pore pressure parameters at fixed $b_{\sigma}=0.4 \ldots \ldots \ldots \ldots \ldots \ldots \ldots \ldots . . .200$

Fig. 7.15 Imposed total stress paths during undrained loading .................................. 202

Fig. 7.16 Undrained response of Fraser River sand during a systematic change of $b_{\sigma}$ with application of different total stress paths . 203

Fig. 7.17 Variation of principal strains during application of different total stress paths with change of $b_{\sigma}$ 205

Fig. 7.18 Excess pore pressure induced by deviatoric stress during application of different total stress paths with change of $b_{\sigma}$ 206

Fig. 7.19 Variation of pore pressure parameters during application of different total stress paths with change of $b_{\sigma}$ 208

Fig. 7.20 Variation of $b_{\sigma}$ during application of total stress paths 209

Fig. 7.21 Undrained response of Fraser River sand during application of different total stress paths with change of $b_{\sigma}$ 210

Fig. 7.22 Induced principal strains during application of different total stress paths with change of $b_{\sigma}$ 211

Fig. 7.23 Variation of excess pore pressure induced by deviatoric stress during the application of different total stress paths with change of $b_{\sigma}$. 212

Fig. 7.24 Variation of pore pressure parameters during change of $b_{\sigma}$ 214 
Fig. 7.25 Drained response of Fraser River sand during application of different total stress paths with change of $b_{\sigma}$

Fig. 7.26 Induced principal strains during application of different total stress paths with change of $b_{\sigma}$

Fig. 7.27 Undrained response of Fraser River sand during application of different total stress paths with rotation of $\alpha_{\sigma}$

Fig. 7.28 Induced principal strains during application of different total stress paths with rotation of $\alpha_{\sigma}$ 220

Fig. 7.29 Excess pore pressure induced by deviatoric stress during application of different total stress paths with rotation of $\alpha_{\sigma}$ 221

Fig. 7.30 Variation of pore pressure parameters during application of different total stress paths with rotation of $\alpha_{\sigma}$ 223

Fig. 7.31 Undrained response of Fraser River sand during change of $b_{\sigma}$ and rotation of $\alpha_{\sigma}$ 224

Fig. 7.32 Induced principal strains during change of $b_{\sigma}$ and rotation of $\alpha_{\sigma} \ldots \ldots \ldots \ldots . . .226$

Fig. 7.33 Excess pore pressure induced by deviatoric stress during change of $b_{\sigma}$ and rotation of $\alpha_{\sigma}$ 227

Fig. 7.34 Variation of pore pressure parameters during change of $b_{\sigma}$ and rotation of $\alpha_{\sigma}$

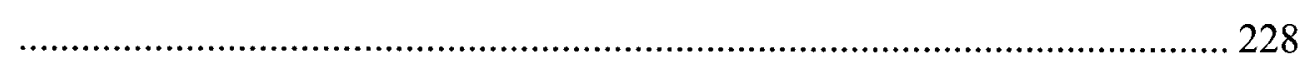

Figure 1 Modules of Data Acquisition program of the HCT device - 1 .................. 254

Figure 2 Modules of Data Acquisition program of the HCT device - 2 .................. 255

Figure 3 Modules of Data Acquisition program of the HCT device - 3 .................. 256

Figure 4 Modules of Data Acquisition program of the HCT device - 4 ................. 257

Figure 5 Fields, methods and properties of TAnalogInputModule class .................2258

Figure 6 Fields, methods and properties of classes (TThreadBaseClass, TBGDataSaveThreadManager and TAIScanThreadManager) in AnalogInputHelper module 259

Figure 7 Fields, methods and properties of TAnalogOutputModule class 259

Figure 8 Fields, methods and properties of class of TConsolidationPrefsForm class in ConsolidationOptions module 260 $\mathrm{xviii}$ 
Figure 9 Structures and enumerations in Constants module 261

Figure 10 Fields, methods and properties of TCyclicPrefsForm class in CyclicOptions module 261

Figure 11 Structures and enumerations of Defs module ………………………….... 262

Figure 12 Structures in TFileInfo class in FileInfo Module........................................ 262

Figure 13 Fields, methods and properties of TfrEPTControl class in FrameEPTControl module. 263

Figure 14 Fields, methods and properties of TFrSigma class in FrameSigma module263

Figure 15 Fields, methods and properties of TfrSMDControl class in FrameSMDControl module 264

Figure 16 Fields, methods and properties of TGainOffsetForm class in GainOffset module. 264

Figure 17 Fields, methods and properties of TDAQModule class in IOComponents module. 265

Figure 18 Fields, methods and properties of TMyStepperMotorControl class in UtilsThreadStepperMotor module .............................................................. 265

Figure 19 Fields, methods and properties of TMainForm class in Main module ....... 266

Figure 20 Fields, methods and properties of TMonotonicPrefsForm class in MonotonicOptions module

267

Figure 21 Fields, methods and properties of TStrainRatioForm class in StrainRatioUnit module. 267

Figure 22 Fields, methods and properties of TNoiseDisplayForm class in NoiseDisplay module. 268

Figure 23 Fields, methods and properties of TAIChannelScanner class in ThreadAIScanner module 269

Figure 24 Fields, methods and properties of TCyclicHCTFeedBackControl class in ThreadCyclicLoading module 270

Figure 25 Fields, methods and properties of classes (TSThreadDataCapture and TMsgWaitObject) in ThreadDataSaver module..... 270

Figure 26 Fields, methods and properties of THCTStepperMotorControl class in ThreadStepperMotor module 270 xix 
Figure 27 Fields, methods and properties of THCTFeedBackControl class in threadTargetPressure module............................................................ 271

Figure 28 Fields, methods and properties of classes (TSMDPulseClass, TEPTControllerClass, TSMDControllerClass and TSyncClass) in UtilsSync module. 271

Figure 29 Fields, methods and properties of TTimeHistoryViewerForm class in TimeHistoryViewer 272

Figure 30 Fields, methods, enumerations and properties of TGlobalVars class in UtilsObjectGlobal module 273

Figure 31 Fields, methods and properties of TThreadSafeTimerRecordStack class and structures of RTimerRecord in UtilsStack module ................................ 274

Figure 32 Fields, methods and properties of TStressStateFeedBackControl and TFeedBackControl classes in UtilsThreadFeedback module 274 


\section{LIST OF SYMBOLS}

\begin{tabular}{ll}
$A_{T}$ & Pore pressure parameter \\
$A / D$ & Analog to digital \\
$a$ & Pore pressure parameter \\
$C_{c}$ & Coefficient of curvature \\
$C_{u}$ & Uniformity coefficient \\
$C S R$ & Critical stress ratio \\
$D / A$ & Digital to analog \\
$D P T$ & Differential pressure transducer \\
$D P V C$ & Digital pressure/volume controller \\
$D S C$ & Directional shear cell \\
$D_{50}$ & Average particle size \\
$D_{r}$ & Relative density \\
$D_{r c}$ & Relative density at end of consolidation \\
$F_{z}$ & Vertical load \\
$H$ & Height of specimen \\
$H C T$ & Hollow cylinder torsional \\
$I_{B}$ & Brittleness index \\
$I_{B, m o d i f i e d}$ & Modified brittleness index \\
$K_{\mathrm{c}}$ & $=\sigma_{l c}^{\prime} / \sigma_{3 c}^{\prime}$, Effective stress ratio at the end of consolidation \\
$K_{0}$ & $=\sigma_{l}^{\prime} / \sigma_{3}^{\prime}$, Effective stress ratio \\
$L V D T$ & Linear variable displacement transducer \\
$M O$ & Maximum obliquity \\
$M S C$ & Multi-channel signal conditioner \\
$N$ & Number of cycles \\
$P_{e}$ & Outer chamber pressure \\
$P_{i}$ & Inner chamber pressure \\
$P S C$ & Plane strain compression \\
$P S E$ & Plane strain extension \\
$P T$ & Phase transformation \\
& \\
\hline &
\end{tabular}




\begin{tabular}{|c|c|}
\hline QSS & Quasi steady state \\
\hline$R$ & $=\sigma_{1}^{\prime} / \sigma_{3}^{\prime}$, stress ratio \\
\hline$R$ & Radius \\
\hline$R_{a v}$ & Average stress ratio \\
\hline$R_{e}$ & Outer radius of specimen \\
\hline$R_{i}$ & Inner radius of specimen \\
\hline$R_{\max }$ & Maximum stress ratio \\
\hline$R_{\min }$ & Minimum stress ratio \\
\hline$S B S$ & State boundary surface \\
\hline$S S$ & Steady state \\
\hline$T C$ & Triaxial compression \\
\hline$T E$ & Triaxial extension \\
\hline$T_{h}$ & Torque \\
\hline$b_{\sigma}$ & $=\left(\sigma_{2}-\sigma_{3}\right) /\left(\sigma_{1}-\sigma_{3}\right)$, Intermediate principal stress parameter \\
\hline$b_{\Delta \sigma}$ & $=\left(\Delta \sigma_{2}-\Delta \sigma_{3}\right) /\left(\Delta \sigma_{1}-\Delta \sigma_{3}\right)$, Intermediate principal stress parameter \\
\hline$b_{\infty c}$ & Intermediate principal stress parameter at the end of consolidation \\
\hline$d r$ & Change of radius \\
\hline$d \varepsilon_{v}$ & Change of volumetric strain \\
\hline$e_{\max }$ & Maximum void ratio \\
\hline$e_{\min }$ & Minimum void ratio \\
\hline$\alpha_{\sigma, \max }$ & Maximum inclination of principal stress with vertical axis \\
\hline$\alpha_{\sigma}$ & Inclination of major principal stress to the vertical axis \\
\hline$\alpha_{\sigma c}$ & $\begin{array}{l}\text { Inclination of major principal stress to vertical axis at the end of } \\
\text { consolidation }\end{array}$ \\
\hline$\beta$ & Constant \\
\hline$\beta_{3}$ & Nonuniformity parameter \\
\hline$\beta_{R}$ & Nonuniformity parameter \\
\hline$\phi^{\prime}$ & Effective internal friction angle \\
\hline$\phi_{C S R}$ & Friction angle mobilized at the trigger of strain softening \\
\hline$\phi_{P T}$ & Friction angle mobilized at PT state \\
\hline
\end{tabular}


$\tau_{\text {oct }}$

$\sigma_{l}$

$\sigma_{2}$

$\sigma_{3}$

$\sigma_{c}$

$\sigma_{d}$

$\sigma_{h}$

$\sigma_{m}$

$\sigma_{r}$

$\sigma_{v}$

$\sigma_{x}$

$\sigma_{y}$

$\sigma_{z}$

$\sigma_{\theta}$

$\sigma_{1}^{\prime}$

$\sigma_{2}^{\prime}$

$\sigma^{\prime}{ }^{\prime}$

$\sigma_{d, c y c}$

$\sigma_{m}^{\prime}$

$\sigma_{m c}^{\prime}$

$\Delta H$

$\Delta R_{e}$

$\Delta R_{i}$

$\Delta U$

$\Delta \theta$

$\Delta \tau_{\text {oct }}$
Friction angle mobilized at peak strength state

$=\frac{1}{3} \sqrt{\left(\sigma_{1}-\sigma_{2}\right)^{2}+\left(\sigma_{2}-\sigma_{3}\right)^{2}+\left(\sigma_{3}-\sigma_{1}\right)^{2}}$

Major principal stress

Intermediate principal stress

Minor principal stress

Confining stress

$=\sigma_{1}-\sigma_{3}$, deviatoric stress

Horizontal stress

Total mean normal stress

Radial stress

Vertical stress

Stress along the $x$ - axis

Stress along the $y$-axis

Vertical stress

Tangential stress

Effective major principal stress

Effective intermediate principal stress

Effective minor principal stress

Cyclic deviatoric stress

$=\left(\sigma_{1}^{\prime}+\sigma_{2}^{\prime}+\sigma_{3}^{\prime}\right) / 3$, Effective mean normal stress

$=\left(\sigma_{1 c}^{\prime}+\sigma_{2 c}^{\prime}+\sigma_{3 c}^{\prime}\right) / 3$, Effective mean normal stress at the end of

consolidation

Change of height of specimen

Change of outer radius of specimen

Change of inner radius of specimen

Excess pore pressure

Increment of angular rotation

$=\left(\tau_{o c t, 1}-\tau_{o c t, 2}\right)$ 


$\begin{array}{ll}\Delta \sigma_{1} & \text { Major component of stress increments } \\ \Delta \sigma_{2} & \text { Intermediate component of stress increments } \\ \Delta \sigma_{3} & \text { Minor component of stress increments } \\ \Delta \sigma_{d} & \text { Increment of deviatoric stress } \\ \Delta \sigma_{m} & \text { Increment of total mean normal stress } \\ \Delta \gamma_{\max } & \text { Change of maximum shear strain } \\ \Delta \varepsilon_{V} & \text { Change of volumetric strain } \\ \alpha_{\Delta \varepsilon} & \text { Major principal strain increment direction } \\ \alpha_{\Delta \sigma} & \text { Major principal stress increment direction } \\ \varepsilon_{1} & \text { Major principal strain } \\ \varepsilon_{2} & \text { Intermediate principal strain } \\ \varepsilon_{3} & \text { Minor principal strain } \\ \varepsilon_{a} & \text { Axial strain } \\ \varepsilon_{r} & \text { Radial strain } \\ \varepsilon_{v} & \text { Volumetric strain } \\ \varepsilon_{x} & \text { Strain along } x \text { - axis } \\ \varepsilon_{y} & \text { Strain along } y \text {-axis } \\ \varepsilon_{z} & \text { Axial strain } \\ \varepsilon_{\theta} & \text { Tangential strain } \\ \tau_{z \theta} & \text { Shear stress } \\ \tau_{z x} & \text { Shear stress along } \mathrm{z} \text { direction in x plane } \\ \tau_{z x} & \text { Shear stress along x direction in z plane } \\ \gamma_{m a x} & \text { Maximum shear strain } \\ \zeta & \text { Torsional shear strain } \\ & \text { Strain ratio } \\ & \end{array}$




\section{INTRODUCTION}

\subsection{Problem definition}

Laboratory assessment of soil behaviour is commonly carried out using conventional soil testing devices (e.g. triaxial, simple shear). These devices cannot represent the actual in-situ soil response except at certain loading scenarios because loading imposed by these devices commonly ignore the effects of intermediate principal stress, and principal stress direction/rotation. In addition, the nature of principal stress rotation in some of these devices may be completely different compared to that of in-situ. A confident assessment of soil response requires the laboratory soil-testing device to have the capability to control the magnitude and direction of principal stresses independently.

Behaviour of soils is dependent on the initial stress state that is characterized by stress parameters, such as three principal stresses (major, intermediate and minor) and their inclinations to a set of orthogonal coordinate axes, and the stress path during shearing. In geotechnical practice, the stress state parameters are commonly represented by

- Shear stress $\tau$ or deviatoric stress $\sigma_{d}=\sigma_{1}-\sigma_{3}$

- Effective confining pressure $\sigma_{3}^{\prime}$ or effective mean normal stress $\sigma_{m}^{\prime}=$ $\left(\sigma_{1}^{\prime}+\sigma_{2}^{\prime}+\sigma_{3}^{\prime}\right) / 3$

- Intermediate principal stress $\sigma_{2}$ or more conveniently, intermediate principal stress parameter $b_{\sigma}=\left(\sigma_{2}-\sigma_{3}\right) /\left(\sigma_{1}-\sigma_{3}\right)$

- Inclination of principal stresses to the deposition direction $\alpha_{\sigma}$ Triaxial device, which is one of the popular laboratory soil testing devices restricts intermediate principal stress parameter $b_{\sigma}$ and direction of principal stresses $\alpha_{\sigma}$ 
to certain values. Intermediate principal stress parameter $b_{\sigma}$ is fixed at 0 during triaxial compression loading and 1.0 during triaxial extension loading. The major principal stress acts along the vertical direction during compression and along the horizontal direction during extension loading. Therefore, a monotonic triaxial test is limited to either $b_{\sigma}=0$ and $\alpha_{\sigma}=0^{\circ}$ or $b_{\sigma}=1.0$ and $\alpha_{\sigma}=90^{\circ}$. In cyclic triaxial loading, $b_{\sigma}$ and $\alpha_{\sigma}$ may switch from $b_{\sigma}=0$ and $\alpha_{\sigma}=0^{\circ}$ to $b_{\sigma}=1.0$ and $\alpha_{\sigma}=90^{\circ}$ when cyclic shear stress amplitude exceeds static shear stress. The values of $b_{\sigma}$ and $\alpha_{\sigma}$ during triaxial cyclic loading change instantaneously. Such sudden jump rotation in the direction of principal stresses is not typical in the field loading. It is also possible in the cyclic triaxial tests that constant $b_{\sigma}=$ 0 and $\alpha_{\sigma}=0^{\circ}$ can be maintained when static shear stress is greater than cyclic shear stress.

Compared to the triaxial device, simple shear device is more versatile in controlling the principal stress directions. The simple shear device is capable of rotating principal stress direction smoothly during shearing, but the range of rotation is limited and the nature of principal stress rotation is uncontrolled. In addition, simple shear has no control on intermediate principal stress parameter. The intermediate principal stress in simple shear is derived from the enforced plane strain boundary conditions. However, the simple shear device simulates certain field loading scenarios fairly well (e.g vertically propagating shear waves).

Simulating actual in-situ loading conditions in the laboratory tests is not an easy task because it might involve six degrees of freedom and require twenty-one elastic constants to describe the problem (Love, 1927). Many field problems can be characterized by the four stress parameters noted above (three principal stresses and one 
direction) due to the cross anisotropic nature of most natural soils. The key objective of the research program is duplication of field loading as closely as possible. The closest simulation of field loading in laboratory tests would yield a reliable assessment of soil behaviour. The independent control of stress parameters of $b_{\sigma}$ and $\alpha_{\sigma}$ in laboratory testing may simulate some critical in-situ loading conditions fairly well.

Limited ability of controlling stress parameters using conventional laboratory devices has motivated researchers to develop a more flexible device in soil testing. True triaxial, directional shear and hollow cylinder torsional (HCT) shear devices attempt to improve the testing methodology. The HCT device was introduced in experimental practice many years ago, but it has been rarely used due to the complexities of configuration and testing method. A specimen in the HCT device can be loaded by controlling four independent tractions (vertical load, inner pressure, outer pressure and torsional shear stress). Independent control of these four surface tractions allows the soil testing under a generalized loading and permits control of three principal stresses and the direction of major and minor principal stresses in one plane.

Magnitudes of $b_{\sigma}$ and $\alpha_{\sigma}$ in an in-situ soil element may continuously change during the process of construction and performance (e.g. construction of dams and slopes). During repetitive loading (e.g. earthquakes and wave loading), the magnitudes of $b_{\sigma}$ and $\alpha_{\sigma}$ vary depending on the level of shear stress applied. This research study has established a better understanding of soil behaviour under the influence of $b_{\sigma}$ and $\alpha_{\sigma}$.

This research has also investigated the behaviour of soil during post loading deformation under generalized stress state. In the past, it has been pointed out that some earth structures (e.g. San Fernando Dam, Mochikoshi Dam) may have failed following 
the cessation of earthquakes. The term post loading deformation is used to represent the fact that failure of these soil structures has occurred following the cessation of the earthquake loading. Initiation of liquefaction, albeit delayed, due to void redistribution within soil deposits (Kokusho, 2003) has been the cause of such failures. Void redistribution occurs due to the existence of piezometric pressure gradients within soil deposit. Such liquefaction failure may be critical in slope structures due to the presence of initial static shear. The presence of static shear stress influences magnitude of principal stresses and its directions, so that the study on post loading deformation of soils under generalized loading may be useful.

Some studies reported in the literature (e.g. Vaid and Eliadorani, 1998 \& 2000; Gananathan, 2002; Sivathayalan and Logeswaran, 2007 \& 2008) have assessed such post loading deformation behaviour of soils. Those studies based on laboratory experiments were limited in the scope (to axisymmetric loading) and the effects of intermediate principal stress and direction of principal stress have not been assessed.

\subsection{Background}

Few soil-testing devices (e.g. True triaxial, Directional Shear Cell, DSC and Hollow Cylinder Torsional, HCT) permit soil testing under generalized loading. The true triaxial device (Kjellman, 1936; Ko and Scott, 1967; Pearce, 1971) has control on principal stresses and has limited control on principal stress directions $\left(\alpha_{\sigma}=0^{\circ}\right.$ or $\alpha_{\sigma}=$ $90^{\circ}$ ). The DSC (Arthur et al., 1977) was introduced to control the magnitudes of principal stresses and their directions, but it was limited to plane strain condition and a low confining stress level. The HCT device has further improved the control of principal stresses and their direction in one plane. 
In geotechnical design practice, in-situ soil behaviour is often predicted or modeled based on parameters obtained from laboratory soil testing. These laboratory tests should be reliable and applicable to the loading modes encountered in the field problems. Several researchers have studied soil behaviour under generalized loading conditions; however, soil behaviour under generalized loading is not completely understood and several gaps exist in the current state of knowledge.

Most of the previous studies under generalized loading (Symes et al., 1984 \& 1988; Miura et al., 1986; Sayao, 1989; Wijewickreme, 1990; Uthayakumar, 1995; Nakata et al., 1998; Sivathayalan, 2000) were conducted under fully drained or undrained conditions. The fully drained condition is applicable to long-term deformation and it cannot be expected in all in-situ soil problems. The undrained condition is commonly considered during short-term loading deformation. Previous studies have clearly shown that the undrained condition is more critical than drained in loose soil deposits. Deformation under undrained condition is coupled with excess pore pressure, instead of volumetric strain in drained deformation. Undrained deformation is scrutinized frequently in soils because of the excess pore pressure generation and its effect on the soil strength. Few researchers (Symes et al., 1985; Uthayakumar, 1995; Nakata, et al., 1998; Sivathayalan, 2000) have studied undrained behaviour of soils under generalized loading using HCT device. Nevertheless, this area needs further systematic studies to understand the undrained soil behaviour clearly. In addition, soil behaviour under simultaneous change of pore pressure and pore volume (partially drained condition) is commonly encountered in field problems, but has not been studied so far under generalized loading. 


\subsection{Objectives of the research}

This research studied the effects of intermediate principal stress and principal stress direction, and the impact of partial drainage on soil response. To the best of the knowledge of the author, the proposed research is the first to quantify partially drained response of soils under three-dimensional loading conditions. The experimental work was organized to study the behaviour of sands as follows.

- Undrained anisotropy of sand

- Effect of intermediate principal stress on undrained behaviour

- Effect of intermediate principal stress on sand behaviour under partial drainage

- Effect of principal stress direction on sand behaviour under partial drainage

- Effects of jump vs. smooth rotation of principal stress directions on cyclic behaviour of sand

- Effect of total stress paths on sand behaviour and pore pressure generation

\subsection{Organization of the Work}

The HCT device used in this research study has been newly built and modified to enable the identified research objectives. An in-house data acquisition program has been developed to control the application of surface tractions and measure the stresses and strains. A brief background of the research, technical information of the HCT device, details of experimental work and analysis of tests results are discussed in this thesis.

Chapter 2 presents a review of the literature to highlight the types of anisotropy in sands, the current understanding of undrained behaviour of sands under static and cyclic loading, recent findings about the sand behaviour under generalized loading and descriptions of stress states encountered in in-situ soil deposits. 
Chapter 3 discusses the typical laboratory shear devices and provides description of the HCT device used in this study.

Chapter 4 explicates the characteristics of material used, techniques of specimen preparation, test procedures, specimen setup and capability of the HCT device.

Chapter 5 studies the effect of intermediate principal stress parameter and direction of principal stress on undrained behaviour of sand. In addition, it describes the behaviour of sand under partial drainage.

Chapter 6 discusses the cyclic behaviour of sand under generalized stress state that closely simulates the in-situ loading.

Chapter 7 discusses the different approaches to quantify deviatoric stress induced excess pore pressure in saturated sands. In addition, it describes the pore pressure generation and sand behaviour along different total stress paths with change of intermediate principal stress parameter and rotation of principal stress direction.

Chapter 8 summarizes the findings and presents the conclusions. 


\section{LITERATURE REVIEW}

\subsection{Introduction}

Behaviour of soils is dependent on the direction of loading due to anisotropy. An understanding of the nature of the anisotropy and its effect on response is essential to characterize the behaviour of soils. Current understanding of the types of anisotropy in sand is briefly discussed at the first part of this chapter.

In the second part, behaviour of soils under static and cyclic undrained loading is discussed. The undrained condition is presumed when excess pore pressure cannot be released quickly from soils. Since positive excess pore pressure decreases the effective stresses, the undrained state is considered critical in geotechnical designs, and the shear strength at this state is generally assumed to be at the minimum. Undrained response of soils has been widely researched and a fundamental understanding of the undrained behaviour has been well established (e.g., Ishihara et al., 1975; Ishihara, 1993; Castro, 1969; Sladen et al., 1985; Mohamad and Dobry, 1986; Kramer and Seed, 1988; Vaid et al., 1989; Lade, 1992).

Thirdly, current understating of soil behaviour under generalized stress state is discussed. Generally, soil behaviour at a given density is studied only in terms of shear stress level (or deviatoric stress $\sigma_{d}=\sigma_{1^{-}} \sigma_{3}$ ) and effective confining stress (or effective mean normal stress $\left.\sigma_{m}^{\prime}=\left(\sigma_{1}^{\prime}+\sigma_{2}^{\prime}+\sigma_{3}^{\prime}\right) / 3\right)$. The influence of intermediate principal stress $\sigma_{2}$ and principal stress direction $\alpha_{\sigma}$ is often disregarded.

At the end of this chapter, common types of in-situ stress states along a potential failure surface and the concept of post loading deformations are explained. A clear 
understanding of magnitude and orientation of in situ stresses is essential to predict the response of soil during deformation.

\subsection{Anisotropy in sands}

Several studies have been carried out to study the anisotropy of soils over the decades. Kjellman (1936) and Parkin et al. (1968) have noted anisotropy effect of sands, in which strains along three principal directions were observed to be unequal during hydrostatic compression. Casagrande and Carrillo (1944) suggested that two different mechanisms are responsible for anisotropy, namely, inherent anisotropy and induced anisotropy.

\subsubsection{Inherent anisotropy}

Natural soil deposits exhibit an inherently anisotropic fabric due to the mode of deposition and the shape of individual grains. The existence of cross-anisotropy in deposited sands has been identified by Yamada and Ishihara (1979), and Lade and Wasif (1988) using a true triaxial device. Stokoe et al. (1985) have confirmed the in-situ cross anisotropy by measuring different shear wave velocities along horizontal and vertical planes. The laboratory experimental evidences presented by El-Sohby and Andrawes (1972), Ladd et al. (1977), and Yamada and Ishihara (1979) support the existence of inherent anisotropy in undisturbed sand specimens. The ratio between axial and radial strains during hydrostatic compression not being unity is an indicator of anisotropy.

Soils are commonly assumed isotropic in the interest of simplicity in practice. Alternatively, in some of the constitutive models, soils are considered inherently crossanisotropic materials. The inherent cross-anisotropy is considered in in-situ when formation of soils is established naturally due to vertical deposition process. In the 
inherent cross-anisotropic soil structure, axis of symmetry and the horizontal plane of isotropy are considered. Mechanical properties of soils along the vertical axis (deposition direction) differ from that along the horizontal plane.

Haruyama (1985) studied the directional dependency of the mechanical behaviour of sand under cubical triaxial tests, and reported that the reconstitution technique of water pluviation leads to inherent cross-anisotropic soil structure. Oda et al. (1978) have also noted cross-anisotropy in soils and indicated that reconstituted specimens by gravitational pluviation (air and water) closely duplicate the in-situ deposition process and fabric. Further, a higher stiffer response was obtained during the application of load along the direction of deposition compared to the application of load along the direction of bedding plane. Other reconstitution methods (e.g. moist tampering) may not exactly duplicate the natural inherent anisotropy due to random orientation of contact normals.

Phillips and May (1967), and Duncan and Dunlop (1969) used a specially constructed shear box to study inherent anisotropy by pouring sand in different directions with respect to the subsequent failure plane and showed that shear strength of sand is dependent on the inclination of bedding planes. Arthur and Menzies (1972) also studied inherent anisotropy of sand under a true triaxial stress system. It is clear from the literature that inherently anisotropic behaviour of sands is caused by the deposition process.

In order to investigate the directional dependency of mechanical properties of granular material, detailed studies of its morphological and physical properties are essential. Several researchers (e.g. Smith, 1932; Kallstenius and Bergau, 1961; Field, 1963; Oda, 1972a) have studied the configuration of particle assembly (fabric). Oda 
(1972a) has concluded that the shape of constituting particles and type of deposition process determine the characteristics of initial fabric of granular material. In addition, preferred alignment of non-spherical particles during the natural deposition process is often along the plane of deposition and a larger number of contact normals are perpendicular to that plane. Several researchers (Oda, 1981; Haruyama, 1981; Shibuya and Hight, 1987) have shown that anisotropy can be formed even in spherical particles. Oda (1981) has reported that anisotropy of spherical particles is established by uneven distribution of contact normals on account of gravitational stress along vertical plane.

\subsubsection{Induced anisotropy}

Cohesionless granular assemblies undergo continuous changes in the evolution of interparticle forces and internal geometry during deformation. As a result, spatial arrangement of solid particles and associated void of granular mass, termed fabric, progressively change (Oda et al., 1985). The changes in the fabric of soil may lead to a gradual increase in the degree of anisotropy. Such changes in anisotropy due to shearing have been termed induced anisotropy. Oda et al. (1985) have demonstrated that due to the application of shear stress, direction of contact normals tend to align with the direction of major principal stress and longitudinal axis of sand particles tends to align along the minor principal stress direction. Further, distribution of contact normals, shape of particles and shape of voids dictate the stress induced anisotropy of granular soil mass.

The term, induced anisotropy refers to the anisotropy due to straining of soils. The anisotropy induced during a stage of loading can be considered inherent to the next stage (Arthur and Menzies, 1972; Saada, 1981; Wong and Arthur, 1985). 
The study of stress-induced anisotropy is difficult because of limited control of principal stress rotation in soil testing devices. Oda (1972b) has studied stress-induced anisotropy in two special cases, in which directions of major and minor principal stress were interchangeable along deposition direction and bedding plane. It has been observed that no distinct changes in the process of fabric reconstruction occurred between the stages of pre-peak and peak stress states. Further, it has been noted that continuous reconstruction of initial fabric occurs during increasing axial strain by sliding particles and the sliding particles are aligned along a preferred orientation of long axes of particles that is perpendicular to major principal stress direction.

Arthur et al. (1977) developed a directional shear cell to study induced anisotropy through controlled rotation of principal stress direction. During specimen preparation for the direction shear cell, sand was deposited along the direction of major principal stress. The specimen was preloaded by increasing major principal stress $\sigma_{l}^{\prime}$ along the direction of deposition. After the preloading, the sample was unloaded to a hydrostatic stress state and reloaded ( $\sigma_{1}^{\prime}$ was applied) along an inclined direction $\alpha_{\sigma}$ with respect to the direction of deposition. It was noted that stiffness of the sand decreased with increasing $\alpha_{\sigma}$ as shown in Fig. 2.1. Further, cycles of unloading and reloading were carried out on same specimen. The observed stress-strain behaviour of second reloading was nearly similar to that of first reloading, even after a $90^{\circ}$ change occurred in the loading direction. This observation demonstrates that fabric changes during shearing process induce isotropic soil structure. 


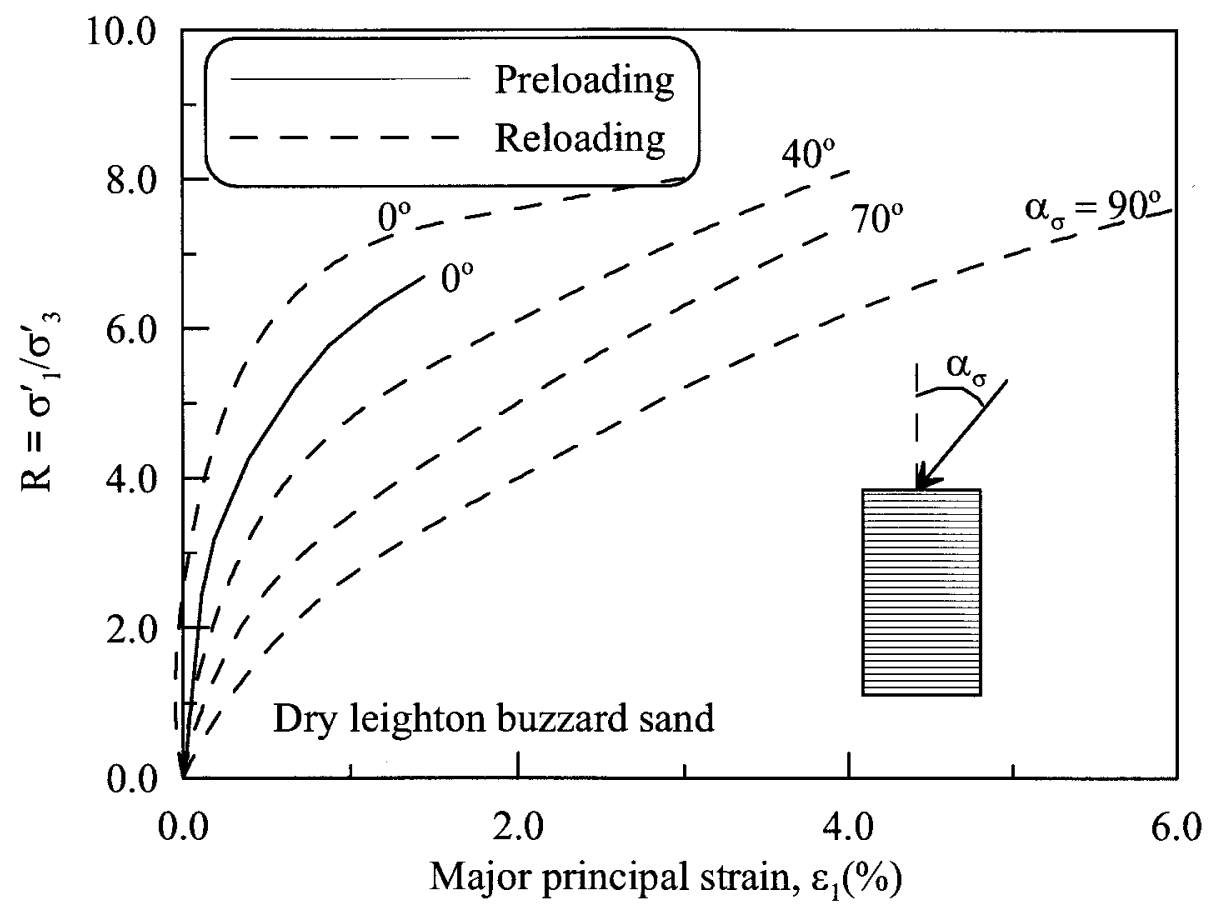

Fig. $2.1 \quad$ Induced anisotropy of sands (after Arthur et al., 1977)

Mould et al. (1982) carried out an experimental study to investigate stress-induced anisotropy in sands using a multi-axial cubical apparatus (similar to true triaxial) that allows the loading only along the deposition direction and the bedding plane. It was observed from the study that stress-induced anisotropy is more pronounced due to the rotation of principal stress as noted by Arthur et al. (1977). The stress-strain relationship during reloading was quite different from that during initial loading (Fig. 2.2). However, a similar stress-strain relationship was observed during reloading even the direction of loading direction differed by $90^{\circ}$. It was concluded that an isotropic process occurs during soil fabric dilation. In this loading, comparatively high shear strain was imposed during the successive reloading process (about $\gamma_{\max }=3 \%$ ) and may have developed a complex geometric fabric in the soil structure. The induced anisotropy would have been dependent on previous $\sigma_{l}^{\prime}$ and strain level. 


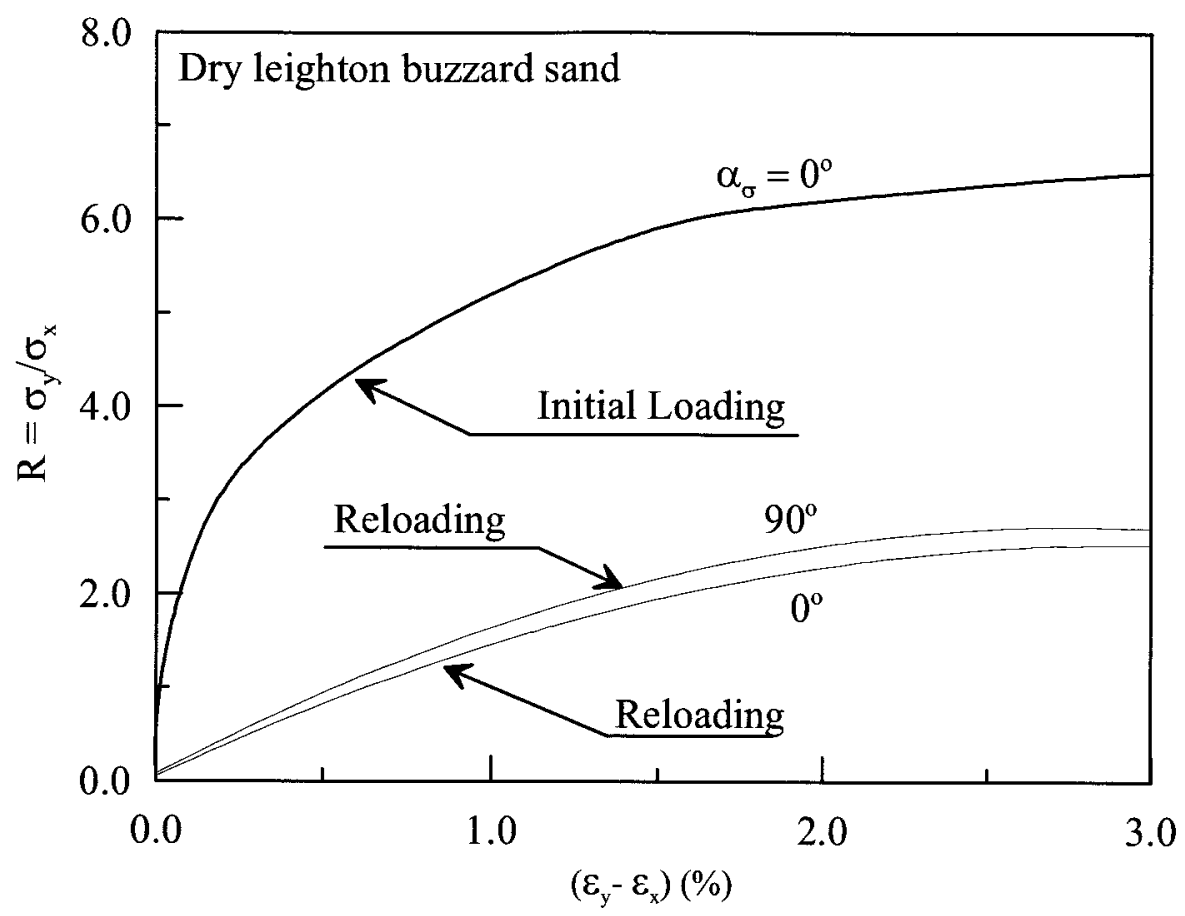

Fig. 2.2 Influence of direction of loading and imposed strain level on induced anisotropy (Modified from Mould et al., 1982)

\subsection{Undrained behaviour of sands}

Deformations under undrained conditions have caused several failures in soils. Several earthquakes (e.g. Alaska, USA, 1964; Niigata, Japan, 1964; Loma Prieta, USA. 1989; Kobe, Japan, 1995) have caused flow slides and consequently significant damages to property and lives.

Soil liquefaction is a phenomenon in which strength and stiffness of saturated soil are reduced by earthquake shaking or other rapid loading under undrained condition. The reduction of the strength of soil during liquefaction occurs due to significant level of excess pore pressure generation.

The phenomenon of liquefaction can be explained by the change of physical configuration of the soil structure. Rearrangement of the soil structure during rapid loading generates excess pore pressure. During rapid loading, the structure of saturated 
loose sand is broken down. Because of the broken soil structure, the loosely packed individual soil particles attempt to move into a denser configuration. In short-term loading scenarios, however, pore water cannot drain out. Instead, the water is trapped and prevents soil particles from moving closer together. The prevention of soil particle movement by the trapped water is accompanied by an increase of pore water pressure that reduces contact forces between individual soil particles, thereby softening and weakening the soil deposit.

Significant advances have been made in the understanding of soil liquefaction. Terzaghi and Peck (1948) originally referred to the sudden change in response of loose sands as "spontaneous liquefaction", in which soils behave in a manner similar to a viscous liquid. Several researchers (e.g. Castro, 1969; Lee and Seed, 1967; Vaid and Chern, 1985) have studied liquefaction of sands under cyclic and monotonic loading conditions mostly using conventional laboratory devices (triaxial and simple shear).

\subsubsection{Static undrained behaviour}

Static undrained behaviour of saturated sands has been widely studied under triaxial loading modes by several researchers (e.g. Castro, 1969; Lee and Seed, 1967; Chern, 1985; Thomas, 1992). In these studies, three different types of stress-strain behaviour have been identified depending on initial stress state and relative density of soil specimens as shown Fig. 2.3. The change in typical static responses from Type 1 to Type 3 is associated with increasing relative density at a constant confining pressure.

Loose sand at low confining stress responds as Type 1. Initially, the strength of the soil increases with deformation until reaching peak strength. After reaching the peak, the strength of the soil reduces towards a constant value. The unlimited deformation at 
the constant normal and shear stresses, and constant void ratio has been referred to as Steady State (SS) of deformation or flow deformation. Castro (1969) called this behaviour "liquefaction", but it is commonly referred to as flow liquefaction (Casagrande, 1975; Seed, 1979) or true liquefaction (Chern, 1985). In terms of pore pressure development, Type 1 deformation, in which no excess pore pressure reduction can be observed at any state of deformation, is always contractive (Castro, 1969).

Medium dense sand responds as Type 2 (Fig. 2.3). Initially, strength of sand increases until reaching peak state. During the deformation following the peak state, soil loses shear resistance until it reaches minimum strength. Subsequently, soil starts to regain shear resistance. This type of soil behaviour has been called partial liquefaction by Lee and Seed (1967) and limited liquefaction many others (Castro, 1969; Vaid and Chern, 1985). The minimum strength state can be considered as a transient steady state (similar to Type 1), and it has been termed Quasi Steady State (QSS) by Ishihara et al. (1975). QSS may be considered as a state during which sands may deform over a small range of strain at constant stress and void ratio as stated in steady state deformation. In terms of pore pressure development, behaviour of sand is contractive until it reaches QSS, and dilative afterwards. 

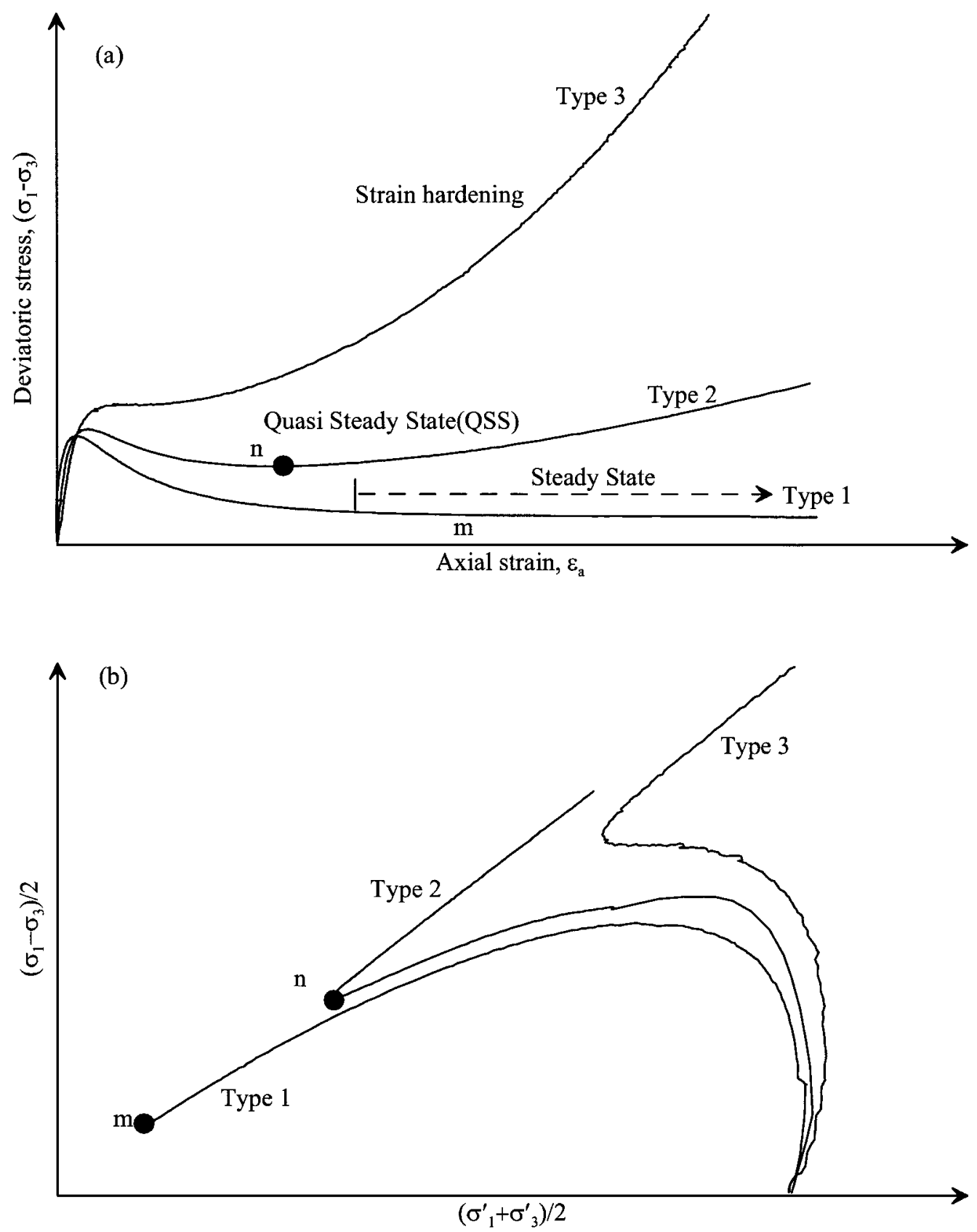

Fig. 2.3 Characteristic behaviour of saturated sand under undrained monotonic loading (after Vaid and Chern, 1985) (a) stress-strain responses (b) stress paths

Type 3 response (Fig. 2.3) illustrates either behaviour of dense sand or behaviour of loose sand at low confining stress. The sand does not exhibit any loss of strength during deformation. This sand initially develops positive excess pore pressure for a small extent of deformation and subsequently it develops large negative excess pore pressure. 
Dense sand may therefore show contractive behaviour initially and dilative behaviour at large strain level, but since the initial contractive region is often limited, and the dilative response corresponds to failure, such sands are often termed "dilative".

Three different types of sand responses discussed above are dependent on initial states of soil, such as soil relative density, confining stress, shear stress, fabric and loading mode. Increment of relative density may transform the undrained response of sand from Type 1 to Type 3 for a given loading mode (Been et al., 1991; Bishop, 1971; Vaid and Thomas, 1995; Vaid and Sivathayalan, 1996; Uthayakumar and Vaid, 1998). Increasing confining stress at other constant initial states (relative density, shear stress, fabric and loading mode) enhances the tendency for contractive behaviour (Castro, 1987; Been et al., 1991; Ishihara, 1993; Vaid and Thomas, 1995; Vaid and Sivathayalan, 1996; Riemer and Seed, 1997; Uthayakumar and Vaid, 1998).

Initial static shear stress level, reflected by $K_{c}$ in triaxial tests, slightly increases the contractiveness of sand (Vaid et al., 2001). The initial static shear significantly influences the response of sands under simple shear loading. The increase of initial static shear stress promotes more contractive behaviour despite a small increase in relative density. The undrained response of sand is highly dependent on fabric, and hence the specimen reconstitution method can significantly influence the type of response (Vaid et al. 1999).

The loading mode that is characterized by the direction of principal stress with respect to the bedding plane (or direction of deposition) also plays a key role in the undrained response. The sand behaviour may change from dilative to contractive when inclination of major principal stress changes from the deposition direction to along the 
bedding plane of the specimen (Hight et al., 1983; Symes et al., 1985; Shibuya and Hight, 1987; Uthayakumar, 1995). In addition, Vaid et al. (1989) have noted that water deposited sands exhibit contractive behaviour over a large range of void ratios in triaxial extension loading compared to triaxial compression, in which water pluviated sand is more dilative. The sand at the same initial state may be dilative and hence strain hardening in compression, but in extension, it could be contractive and hence strain softening. These observations are a clear indication of stress path dependency and a reflection of anisotropic nature of natural sands noted by Oda (1972a).

\subsubsection{Cyclic undrained behaviour}

Cyclic undrained loading may be imposed on in-situ soils due to earthquakes and waves or any other rapid loading. The potential for liquefaction under cyclic loading is generally higher than that under static loading, so that study of sand behaviour under cyclic loading becomes very important in the assessment of liquefaction susceptibility.

Vaid and Chern (1985) have reported different strain development mechanisms during cyclic loading depending on the initial state of sand. These strain development mechanisms consist of true liquefaction and limited liquefaction as introduced in static undrained loading, and cyclic mobility. Castro et al. (1982) related liquefaction and limited liquefaction of static loading with that of cyclic loading based on steady state line. No attention has been paid to the stress condition at the initiation of strain softening leading to liquefaction or limited liquefaction under either static or cyclic loading.

\subsubsection{Mechanisms of strain development}

Large deformation during cyclic loading may occur due to either strain softening or cyclic mobility (Castro, 1969; Vaid and Chern, 1985). The term cyclic mobility is used 
to characterize progressively larger development of deformation during cyclic loading with or without transient states of zero effective stress (Castro, 1969; Casagrande, 1975; Vaid et al., 2001). Unlike liquefaction, cyclic mobility causes accumulation of strains that are limited in magnitude. The initial development of strains is associated with stiffness degradation due to decrease of normal effective stress.

The mechanism of deformation (i.e. liquefaction or cyclic mobility) is dependent on relative density, grain structure or fabric, confining pressure and value of $K_{0}$ (Castro and Poulos, 1977; Vaid and Chern, 1985; Vaid and Sivathayalan, 2000), in addition to, prestrain history (Finn et al., 1971; Seed et al., 1977; Vaid and Sivathayalan, 2000). Fig. 2.4 shows typical true liquefaction response of loose sand under cyclic loading. True liquefaction under cyclic loading has the same characteristics as static loading (Fig. 2.3; Type 1). In the process of true liquefaction, a sharp increase of pore pressure is accompanied by sudden development of large axial strain. This phenomenon is associated with contractive flow deformation (Castro, 1969; Casagrande, 1975) that starts before stress state reaches failure line (Vaid and Chern, 1985). The triggering point of contractive flow deformation lies on a line, called Critical Stress Ratio (CSR) line or the flow line.

Induced axial deformation during cyclic loading is comparatively small until soil reaches triggering point of contractive flow deformation. However, large strain is induced during flow deformation at steady state. As shown in Fig. 2.4, after strain softening, strength of soil continues at a constant level and stress state remains constant. Theoretically, unlimited and unidirectional deformation can be induced during this steady state. 

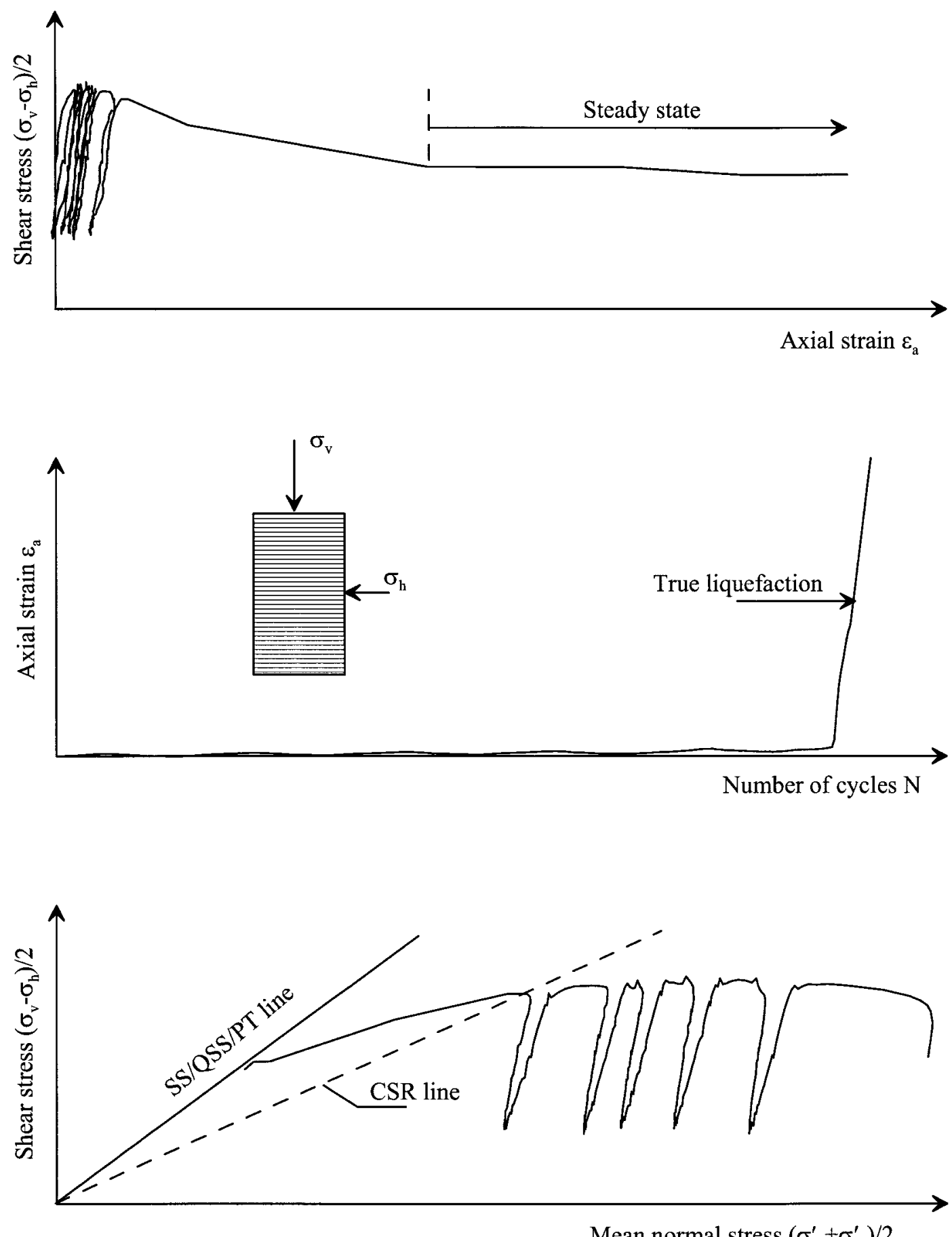

Fig. 2.4 True liquefaction of sands during cyclic undrained shear (After Vaid and Chern, 1985)

Fig. 2.5 shows undrained cyclic response of sands during limited liquefaction.

This response has the same characteristics as Type 2 (Fig 2.3) response of static loading. Deformation at a specified level may be due to either limited liquefaction or combination 
of limited liquefaction and cyclic mobility with continued cyclic loading (Vaid and Chern, 1983). Both true liquefaction and limited liquefaction can occur in cyclic loading, if the cyclic stress amplitude is larger than the minimum undrained strength of the material at the given state (Vaid and Chern, 1985). Transient states of zero effective stress may ensue following true or limited liquefaction depending on the number of loading cycles.

As noted earlier, two different types of cyclic responses can manifest during cyclic mobility. In the first type, soil experiences transient states of zero effective stress (Fig. 2.6). A state of zero effective stress is never realized the second type of cyclic mobility (Fig. 2.7). A transient state of zero effective stress is a necessary condition for the development of large deformation due to cyclic mobility, and it occurs depending on the relative magnitude of static and cyclic shear stress amplitudes. If the magnitude of static shear stress is higher than amplitude of cyclic shear stress then the effective stress never reaches a state of zero, and the strain development is gradual. 

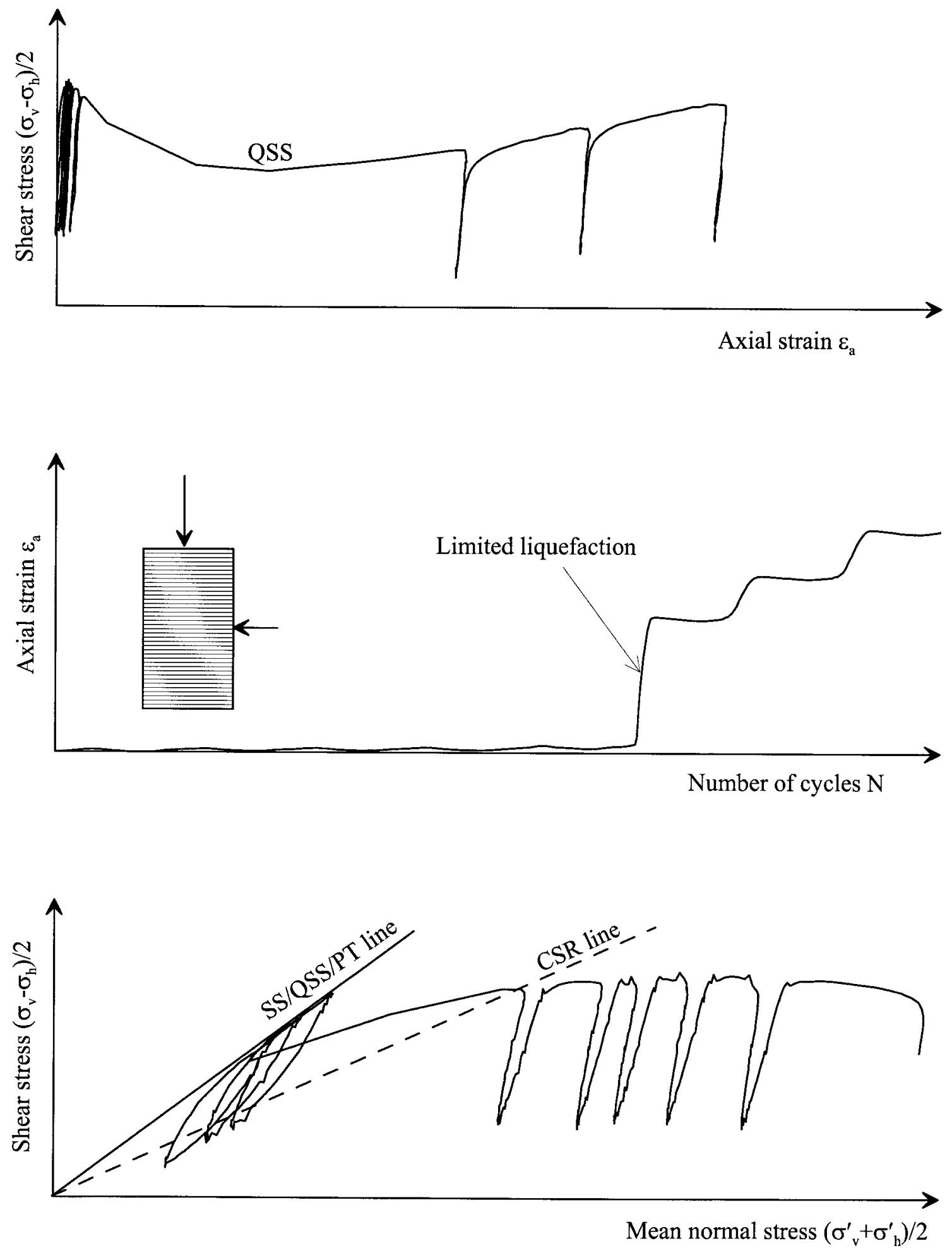

Fig. 2.5 Limited liquefaction of sands during cyclic undrained shear (After Vaid and Chern, 1985) 

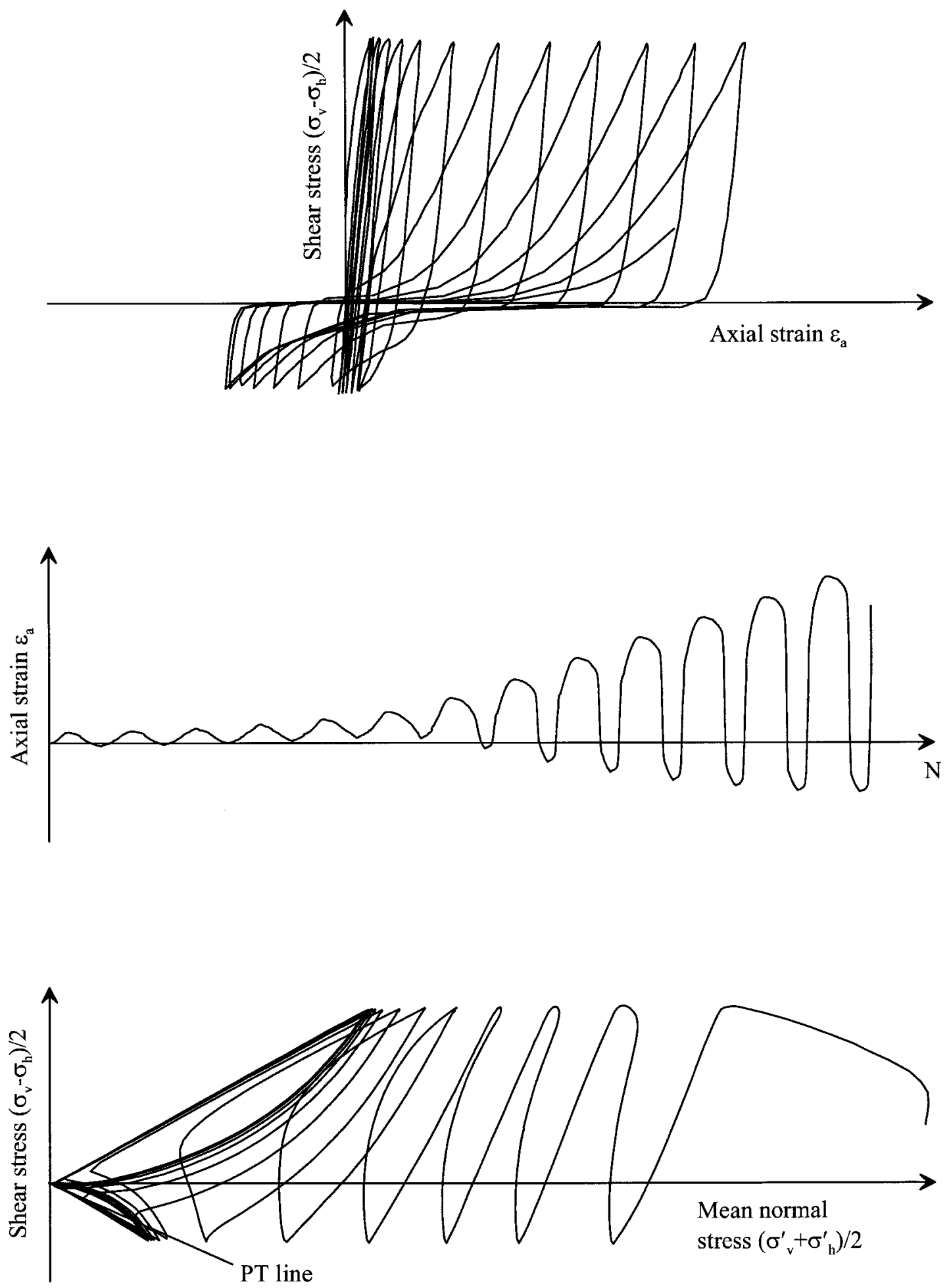

Fig. 2.6 Cyclic mobility in sands during cyclic undrained shear with transient state of zero effective stress (After Vaid and Chern, 1985) 

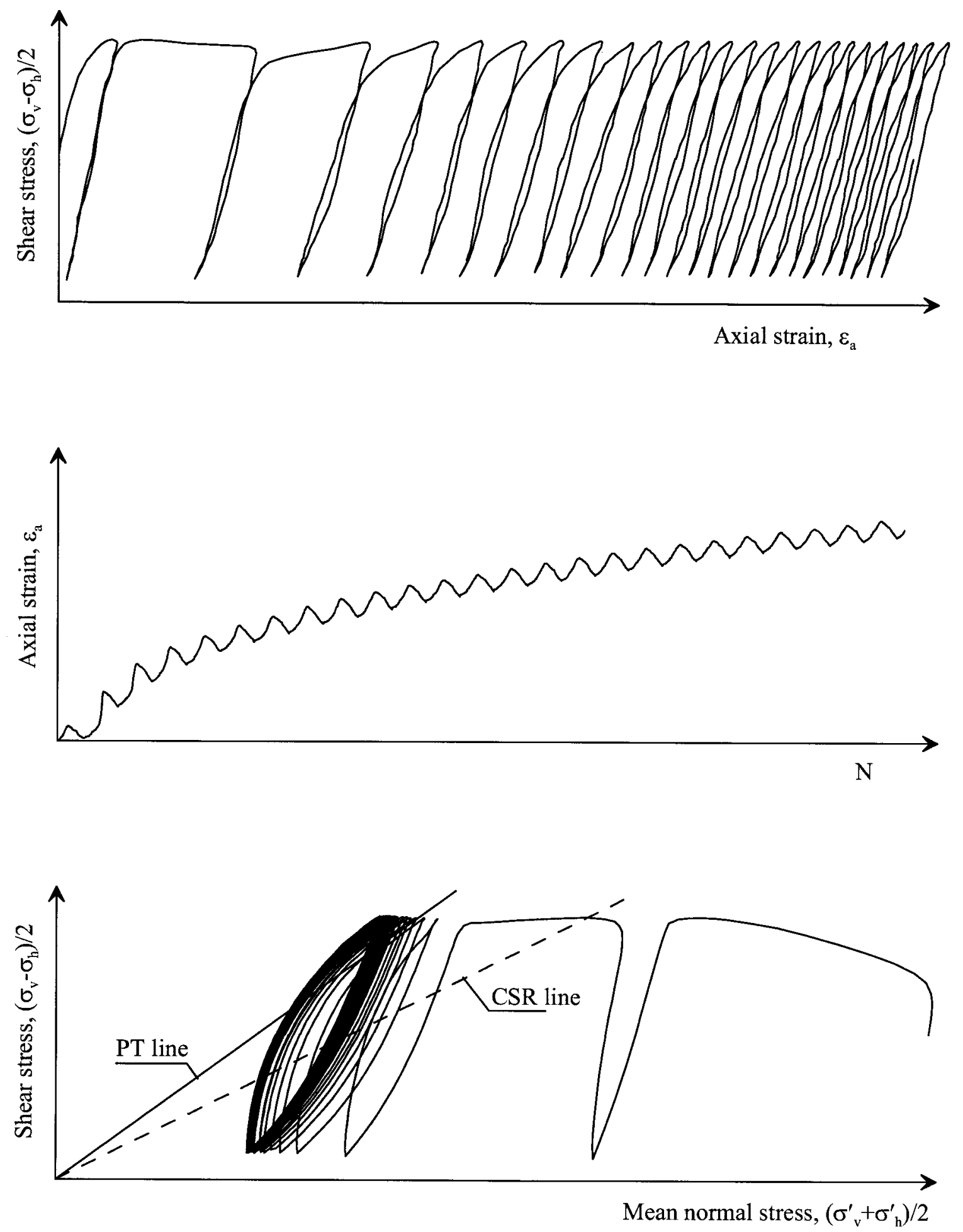

Fig. 2.7 Cyclic mobility in sands during cyclic undrained shear without transient state of zero effective stress (Vaid et al., 2001) 


\subsubsection{Peak state and initiation of strain softening}

Effective stress ratio $\sigma_{1}^{\prime} / \sigma_{3}^{\prime}$, at which strain softening is initiated has been called Critical Stress Ratio (CSR) by Vaid and Chern (1983). Based on triaxial compression tests, it was suggested that CSR is independent of the initial state of the sand (void ratio, effective confining stress and shear stress $/ K_{0}$ ) and amplitude of cyclic stress. However, recent studies have indicated that CSR is dependent on density, loading modes, confining stresses and fabric (Castro, 1969, Sladen et al., 1985, Vaid and Thomas, 1995; Vaid et al., 2001). In addition, CSR value in triaxial compression is considerably larger than that of triaxial extension and simple shear (Vaid et al., 2001). Both monotonic and cyclic loadings yield the same CSR.

Castro (1969) and Sladen et al. (1985) have also reported that CSR is not unique for moist tamped specimens at different void ratios and confining stress levels. The observation obtained from the moist temped specimens indicates that soil fabric influences CSR value. This observation is consistent with the findings of Vaid and his coresearchers in triaxial extension and simple shear loading modes. Further, Vaid et al. (1999) have noted that moist tamped specimens behave more contractively, leading to true liquefaction and a steady state compared to air/water pluviated specimens. Water pluviated specimens rarely strain soften during triaxial compression. In addition, Kuerbis (1989) also reported that CSR is dependent on reconstitution technique for a given loading mode.

Friction angle is one of the most frequently used soil parameters in geotechnical design practice. The understanding of mobilized friction angle at CSR $\phi_{C S R}$ is useful to assess liquefaction problem. $\phi_{C S R}$ is a reflection of CSR, so that the findings about CSR 
are applicable to $\phi_{C S R}$. A recent study on sand under three-dimensional HCT loading reveals that $\phi_{C S R}$ is dependent on both major principal stress direction $\alpha_{\sigma}$ and intermediate principal stress parameter $b_{\sigma}$, but not on $K_{c}$ (Uthayakumar and Vaid, 1998; Sivathayalan and Vaid, 2002).

\subsubsection{Phase Transformation (PT) and Steady State (SS)}

Ishihara et al. (1975) have introduced the term "Phase Transformation" (PT) to denote a state, at which the response of sand is transformed from contractive to dilative. The PT state has been clearly identified as the state of peak pore water pressure. Vaid and Thomas (1995) have suggested that QSS and PT are essentially coincident during conventional triaxial tests. However, Sukumaran et al. (1996) have pointed out that minimum undrained strength state may occur at or just prior to PT.

Steady State (SS) for any mass particles is defined as a state, in which continuous deformation occurs at constant volume, constant shear, constant effective normal stress and constant velocity of deformation (Poulos, $1981 \& 1985$ ). SS deformation occurs only when all particles have reached a statistically constant state and the structure of specimen has been destroyed.

PT occurs in both strain softening and strain hardening soils and SS occurs only in the case of true liquefaction, in which no state change from contractive to dilative occurs. Both PT and SS have different implications in geotechnical design. However, true liquefaction and limited liquefaction can be treated within the same framework of

effective stress space for a given loading mode, even though these characteristics are quite different in shear stress-void ratio space (Vaid and Chern, 1985). 
Mobilized friction angle at SS and PT is identical for particular sand at various initial states (Chern, 1985; Vaid and Thomas, 1994 \& 1995; Stedman, 1997). The combined treatment of SS and PT within a same framework has caused some confusion in the literature because of the contradiction between such an approach and the concepts of a unique steady state. Vaid and Thomas (1996) have noted that uniqueness of friction angle at PT of dilative material has no further implication other than it is equal to PT/SS of a contractive material.

The mobilized friction angle at PT state is an unique property of a given sand, and it is independent on void ratio $e$, initial stress state $\left(\sigma_{3 c}^{\prime}\right.$ and $\left.K_{c}\right)$, direction of principal stress $\alpha_{\sigma}$, intermediate principal stress level $b_{\sigma}$, type of response (contractive and dilative), mode of loading (cyclic or monotonic) and method of specimen reconstitution (Chern, 1985; Vaid and Chern, 1985; Vaid and Thomas, 1995; Stedman, 1997, Uthayakumar and Vaid, 1998; Vaid et al., 1999). Negussey et al., (1988) indicated that the friction angle at PT is only dependent on soil mineralogy and equal to constant volume friction angle under drained conditions.

\subsection{Pore pressure generation in saturated soils}

Amount of excess pore pressure generated during undrained loading is quantified in terms of increments of principal stresses. Skempton (1954) proposed a relationship to quantify the amount of excess pore pressure generated, in terms of major $\Delta \sigma_{l}$ and minor $\Delta \sigma_{3}$ principal stress increments as

$$
\Delta U=B\left[\Delta \sigma_{3}+A\left(\Delta \sigma_{1}-\Delta \sigma_{3}\right)\right]
$$

Here, $A$ and $B$ are defined as pore pressure coefficients. $B$ depends on the degree of saturation and $A$ accounts for the shear induced excess pore pressure. When degree of 
saturation is $100 \%$ (full saturation), $B$ is $1.0 . B$ decreases rapidly toward zero when degree of saturation decreases. The coefficient $A$ is determined experimentally.

Application of Skempton's pore pressure equation without regard to principal stress rotation and intermediate principal stress has led to some confusion in the literature. Originally, Skempton's equation was based only on axisymmetric triaxial compression. It was reviewed by Skempton (1961) and expressed in terms of absolute values of principal stress difference. However, Ladd and Lambe (1963) used a different approach for unloading in-situ conditions $\left(K_{0}<1\right)$. In this approach, $\Delta \sigma_{I}$ and $\Delta \sigma_{3}$ were defined as given in Eq. 2.3.

$\Delta \sigma_{1}=\Delta \sigma_{v}$ and $\Delta \sigma_{3}=\Delta \sigma_{h}$

Eq. 2.2

The same kind of unloading field problem was approached by Noorany and Seed (1965) in a different manner. $\Delta \sigma_{1}$ and $\Delta \sigma_{3}$ were defined as given in Eq. 2.3.

$\Delta \sigma_{1}=\Delta \sigma_{h}$ and $\Delta \sigma_{3}=\Delta \sigma_{v}$

These two definitions of $\Delta \sigma_{l}$ and $\Delta \sigma_{3}$ by Ladd and Lambe (1963), and Noorany and Seed (1965) have resulted in a completely different estimation of pore pressure parameter $A$. To unify the definition of $\Delta \sigma_{l}$ and $\Delta \sigma_{3}$, Law and Holtz (1978) have defined the stress increments as illustrated in Fig. 2.8. In that approach, the algebraically largest increment was considered as $\Delta \sigma_{1}$ (major component of stress increments) and the algebraically smallest increment was considered as $\Delta \sigma_{3}$ (minor component of stress increments) regardless of original major and minor principal stresses, so that $\Delta \sigma_{l}$ and $\Delta \sigma_{3}$ need not to be in the direction of $\sigma_{1}$ and $\sigma_{3}$ respectively. 

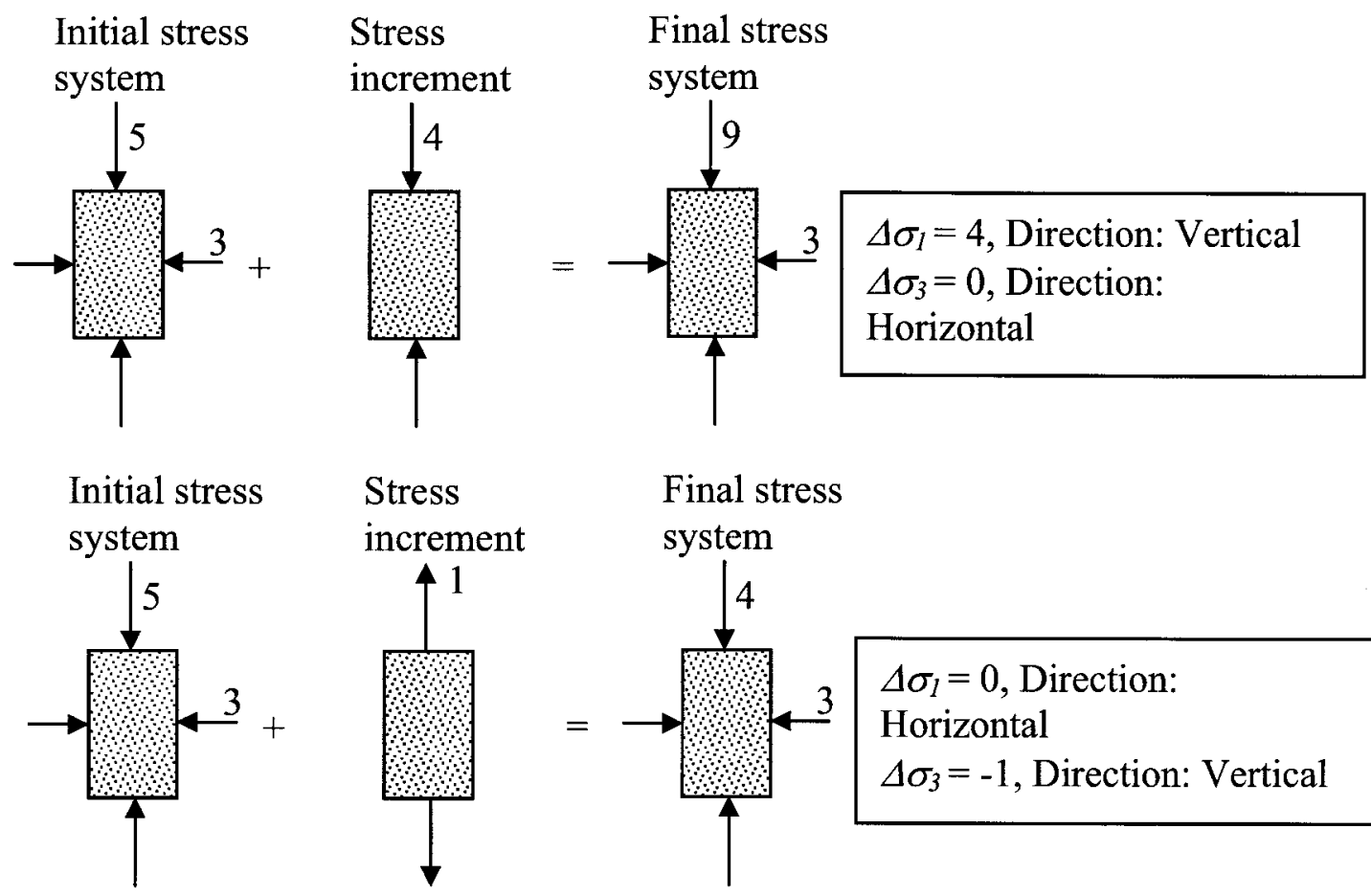

$\Delta \sigma_{l}=0$, Direction:

Horizontal

$\Delta \sigma_{3}=-1$, Direction: Vertical
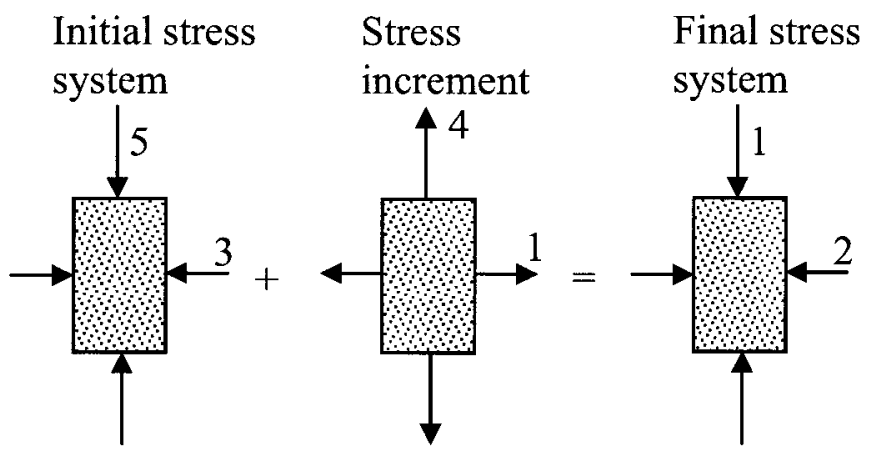

$\Delta \sigma_{1}=-1$

Direction: Horizontal

$\Delta \sigma_{3}=-4$,

Direction: Vertical

Fig. 2.8 Increment of principal stresses in axisymmetric soil element during rotation of principal stress (After Law and Holtz, 1978)

Henkel (1960) has suggested an equation in terms of principal stress increments to express the excess pore pressure generation in 3-D problems. The equation was based on the stress invariants expressed by the sum of square of the difference of principal stress increments and the sum of all principal stress increments.

$$
\Delta U=\frac{\Delta \sigma_{1}+\Delta \sigma_{2}+\Delta \sigma_{3}}{3}+\frac{a}{3} \sqrt{\left(\Delta \sigma_{1}-\Delta \sigma_{2}\right)^{2}+\left(\Delta \sigma_{2}-\Delta \sigma_{3}\right)^{2}+\left(\Delta \sigma_{3}-\Delta \sigma_{1}\right)^{2}}
$$


Here, $\Delta \sigma_{1}, \Delta \sigma_{2}$ and $\Delta \sigma_{3}$ are the changes of principal stresses and $a$ is pore pressure parameter depending on soil type, consolidation history and position of stress space. Later, Henkel and Wade (1966) have identified Eq. 2.4 as incorrect and modified it to

$$
\Delta U=\frac{\Delta \sigma_{1}+\Delta \sigma_{2}+\Delta \sigma_{3}}{3}+\frac{a}{3} \Delta \sqrt{\left(\sigma_{1}-\sigma_{2}\right)^{2}+\left(\sigma_{2}-\sigma_{3}\right)^{2}+\left(\sigma_{3}-\sigma_{1}\right)^{2}}
$$

The basic equation to estimate excess pore pressure in 2-D problem was slightly modified to include the effect of intermediate principal stress by Terzaghi et al. (1996) as shown in Eq. 2.6. The excess pore pressure equation was expressed in terms of principal changes of stress increments according to Law and Holtz (1978), rather than changes in preshear principal stress.

$$
\begin{aligned}
& \Delta U=\left[\Delta \sigma_{3}+A_{1}\left(\Delta \sigma_{1}-\Delta \sigma_{3}\right)+A_{2}\left(\Delta \sigma_{2}-\Delta \sigma_{3}\right)\right] \\
& \text { or } \\
& \Delta U=\left[\Delta \sigma_{3}+A_{T}\left(\Delta \sigma_{1}-\Delta \sigma_{3}\right)\right]
\end{aligned}
$$

Here, $A_{T}=A_{1}+A_{2} b_{\Delta \sigma}$

\subsection{Behaviour of sands under generalized stress path loading}

For a cross-anisotropic material, principal stresses $\left(\sigma_{1}^{\prime}, \sigma_{2}^{\prime}\right.$ and $\left.\sigma_{3}^{\prime}\right)$ and inclination of $\sigma_{1}^{\prime}$ with deposition direction $\alpha_{\sigma}$ can be used to characterize the response. For convenience, these four parameters are commonly substituted for effective mean normal stress $\sigma_{m}^{\prime}$, intermediate stress parameter $b_{\sigma}$, deviatoric stress $\sigma_{d}$ and direction of principal stress $\alpha_{\sigma .}$. Only a few studies have been carried out in the literature to assess the effect of these stress parameters on soil behaviour. 


\subsubsection{Effect of principal stress direction}

The effect of anisotropy on shear strength and stress-strain response is well established in the literature for both granular and cohesive soils (Arthur and Menzies, 1972; Arthur et al., 1977; Bishop, 1966; Duncan and Seed, 1966; Hansen and Gibson, 1948; Oda, 1972a). The deformation characteristics of inherently anisotropic material depend on the inclinations of principal stresses to the material axis (perpendicular to bedding plane) prior to stress changes for a given magnitude of principal stress increment. In most geotechnical problems, principal stress direction rotates gradually (Arthur et al., 1980), and it rotates continuously in cyclic loading. Fig. 2.9 shows an example of principal stress rotation obtained from finite element analysis conducted by Vaughan et al. (1976) beneath an offshore soil foundation.

Arthur et al. (1980) have conducted a study on sand that was subjected to jump and continuous rotations of principal stress direction. The jump rotation was imposed on specimens after an initial compressive strain $\left(\varepsilon_{I}=2.0 \%\right)$. No significant variation of strength of the specimen was noted during jump rotation. However, the reduction of stiffness was significant and dependent on the magnitude of allowed principal stress rotation. During continuous rotation, principal stress was allowed to rotate in a cyclic manner $\left(+20^{\circ}\right.$ to $\left.-20^{\circ}\right)$ at a constant effective stress ratio $\sigma_{l}^{\prime} / \sigma_{3}^{\prime}$. It was noted that major principal strain accumulated continuously even after a large number of cycles. No failure planes were observed in specimens as noted in the jump rotation. In addition, Arthur et al. (1980) had difficulties in independently controlling all other stress parameters $\left(\sigma_{m}^{\prime}, b_{\sigma}\right.$ and $\sigma_{d}$ ) during principal stress rotation, so that this study may not reflect absolute effect of rotation of principal stress direction. 


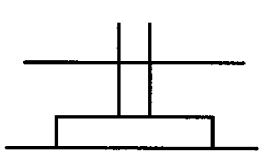

1
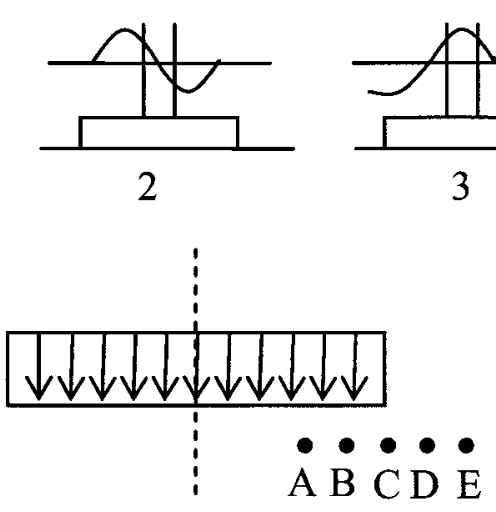

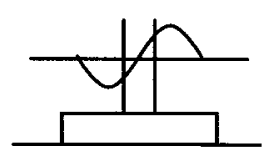

4

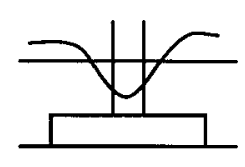

5
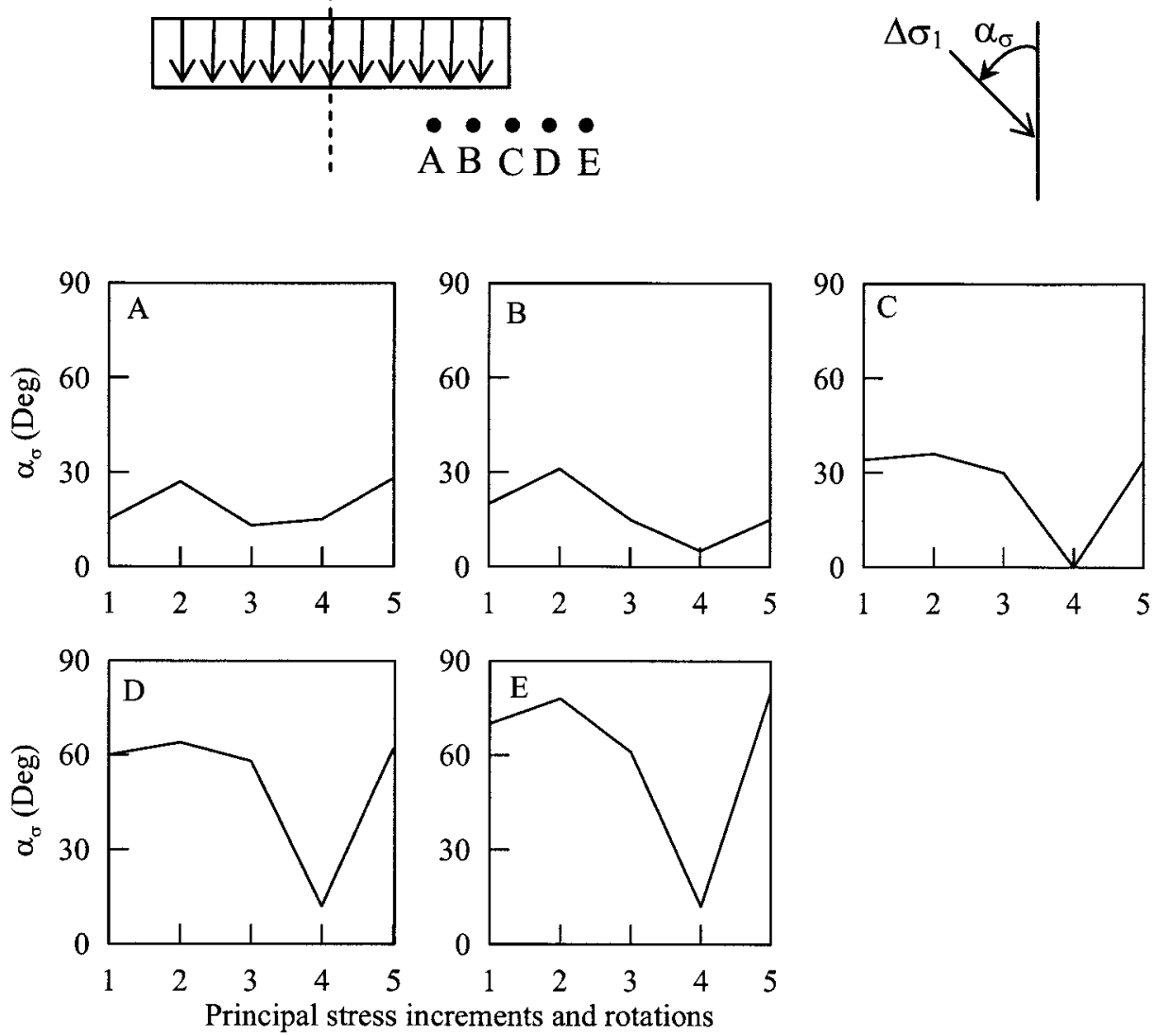

Fig. 2.9 Principal stress rotations underneath an offshore soil foundation (After Vaughan et al., 1976)

Broms and Casbarian (1965) conducted the first study of principal stress rotation on clay using hollow cylinder specimens under undrained condition. This study revealed that continuous principal stress rotation increased the pore water pressure and decreased the friction angle at failure, and consequently reduced the undrained strength of the soil.

Using HCT device, Symes et al. (1982) conducted a study to investigate the influence of principal stress rotation of sand behaviour at constant intermediate stress 
parameter $b_{\sigma}$. On approaching failure, the rotation of principal stress directions had a significant effect on strains. A large deviation was observed between stress increment and strain increment directions at the beginning of stress rotation. However, this deviation was minimized as rotation continued and failure approached. The friction angle at failure was affected less by the rotation of principal stress direction (Symes et al., 1982; Oda et al., 1978; Miura et al., 1986).

Symes et al. (1984) have interpreted the dependency of rotation of principal stress direction based on the concept of State Boundary Surface (SBS) introduced by Rendulic (1936) and Roscoe et al. (1958). SBS is originally defined in $q-p^{\prime}-e$ space. However, SBS may be connected to either $b_{\sigma}$ or $\alpha_{\sigma}$ if required. The explanation based on the SBS concept for undrained deformation at constant $b_{\sigma}$ is shown in Fig. 2.10, in which SBS is defined in $q-p^{\prime}-\alpha_{\sigma}$ space. The rotation of principal stress causes effective stress state on SBS or beneath SBS and pore pressure generation is inferred from the position of the effective stress state. If the effective stress state is on the SBS, a substantial amount of pore pressure is generated. The pore pressure generation is smaller for the case, in which the effective stress path is beneath SBS.

Also, Symes et al. (1988) studied the effect of principal stress rotation under drained condition. Specimens that were loaded along vertical direction (i.e major principal stress direction was along the deposition direction) exhibited higher shear strength and initial stiffness and it induced lower volumetric strain. The inclinations of principal stress direction reduced initial stiffness and shear strength, and increased induced volumetric strain. At constant shear stress level, rotation of principal stress direction generated volume contraction irrespective of the sense of rotation. However, the 
magnitude of volume contraction was dependent on the sense of rotation. These observations are analogous to the variation of pore pressure in undrained principal stress rotation, and suggest that excess pore pressure generation may be dependent on the principal stress direction and its rotation.

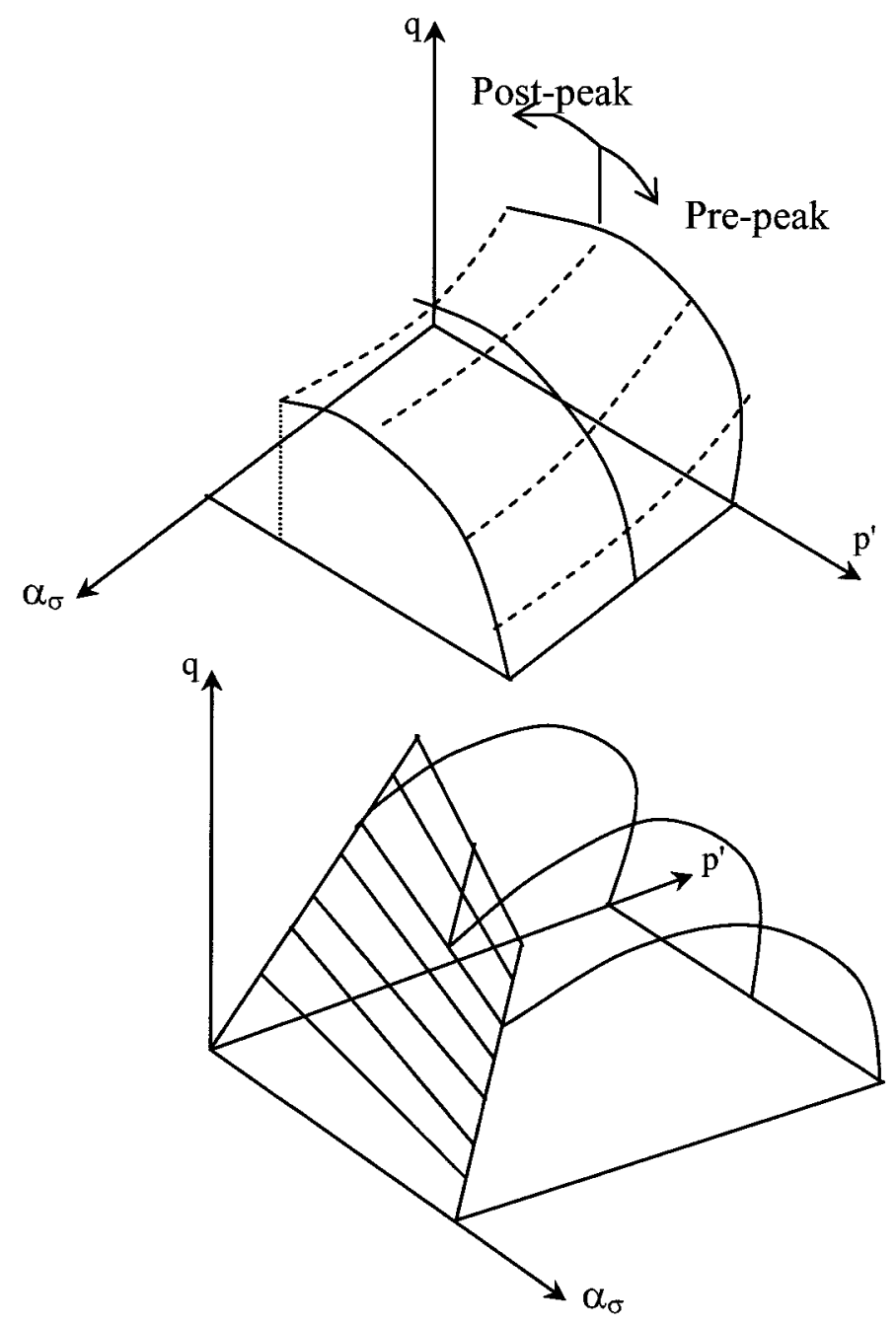

Fig. $2.10 \quad$ (a) Contractant region of state boundary surface; (b) Complete state boundary surface

Miura et al. (1986) also investigated the influence of principal stress direction and rotation on soil behaviour. The failure strength of soil at drained condition showed a strong dependence on the inclination of principal stress direction $\alpha_{\sigma}$ and it was reduced 
with increasing $\alpha_{\sigma}$. In addition, the principal stress rotation led to accumulated contraction, even though the three principal stresses were constant.

Sayao (1989) studied the drained behaviour of sands under continuous principal stress rotation at constant $\sigma_{m}^{\prime}, R$ and $b_{\sigma}$. During the rotation of principal stress direction, volume contraction and shear distortion progressively accumulated. In addition, the amount of volume contraction and distortion increased with decreasing relative density. In the rotation of principal stress in a cyclic manner, volume contraction and shear distortion were significant during the first cycle of rotation and strain accumulation was small in the subsequent cycles. Uthayakumar (1995) studied the static undrained behaviour of sand using a HCT device. The undrained response was dependent on principal stress direction. A stronger response was noted when principal stress direction was along the deposition direction and a weaker response was noted when principal stress direction was along the bedding plane.

\subsubsection{Effect of intermediate principal stress}

Effect of intermediate principal stress $\sigma_{2}^{\prime}$ on stress-strain characteristics of soils is frequently disregarded because of wide spread use of Mohr-Coulomb failure criteria which is formulated in terms of major and minor effective stresses only. In addition, general inability to handle $\sigma_{2}^{\prime}$ in standard laboratory testing and in-situ testing methods has contributed to this practice. Bishop (1966) indicated the importance of $\sigma_{2}^{\prime}$ in soil analysis. As noted earlier, intermediate principal stress parameter $b_{\sigma}=\frac{\sigma_{2}-\sigma_{3}}{\sigma_{1}-\sigma_{3}}$ is often used to represent the role of $\sigma_{2}^{\prime}$. (Habib, 1953; Saada and Bianchini, 1978; Saada, 1981; Saada and Puccini, 1985). A study about the effect of $\sigma_{2}^{\prime}$ will be useful to formulate a 
realistic constitutive model of soil behaviour, as well as to calibrate the existing constitutive models.

True triaxial device has been used frequently to study the effect of $\sigma_{2}^{\prime}$ in sand behaviour (Kjellman, 1936; Lade and Duncan, 1973 \&1975; Reades and Green, 1976; Yamada and Ishihara, 1979; Haruyama, 1981). Kjellman (1936) noted a significant difference in volumetric and shear responses of soil due to the variation of $b_{\sigma}$. Yamada and Ishihara (1979) have shown that the variation of $b_{\sigma}$ between 0 and 0.27 caused negative value of intermediate principal strain (extensional strain). In addition, $b_{\sigma}$ between 0.27 and 0.5 resulted in approximately zero intermediate principal strain, and thus plane strain condition would lie within that range of $b_{\sigma}$.

Several studies have indicated the dependency of friction angle $\phi^{\prime}$ at failure state on $b_{\sigma}$ as shown in Fig. 2.11. Lam and Tatsuoka (1988) have noted that $\phi^{\prime}$ is a function of $\alpha_{\sigma}, b_{\sigma}$ and failure mode (compression or extension) using triaxial tests. Symes et al. (1982) have noted that $\phi^{\prime}$ varies depending on $b_{\sigma}$ and $\phi^{\prime}$ at $b_{\sigma}=0.50$ was higher than $b_{\sigma}=$ 0.0. Hight et al. (1988) have also shown that $\phi^{\prime}$ is dependent on both $b_{\sigma}$ and $\alpha_{\sigma}$. The conflicting suggestions about the influence of $b_{\sigma}$ on $\phi^{\prime}$ may be due to the differences in anisotropic fabric, direction of principal stresses, variation in effective mean normal stress, testing techniques (loading or unloading) and experimental errors.

Sayao (1989) has conducted an experimental study to isolate the influence of $b_{\sigma}$ on sand behaviour using a HCT device. Tests on a medium dense sand showed that both volumetric strain and shear strain increase progressively regardless of $\alpha_{\sigma}$ as $b_{\sigma}$ increases from 0.0 to 1.0. In addition, the specimen sheared at $b_{\sigma}=0.3$ resulted in a stiffer response than the specimen at $b_{\sigma}=0.0$. 

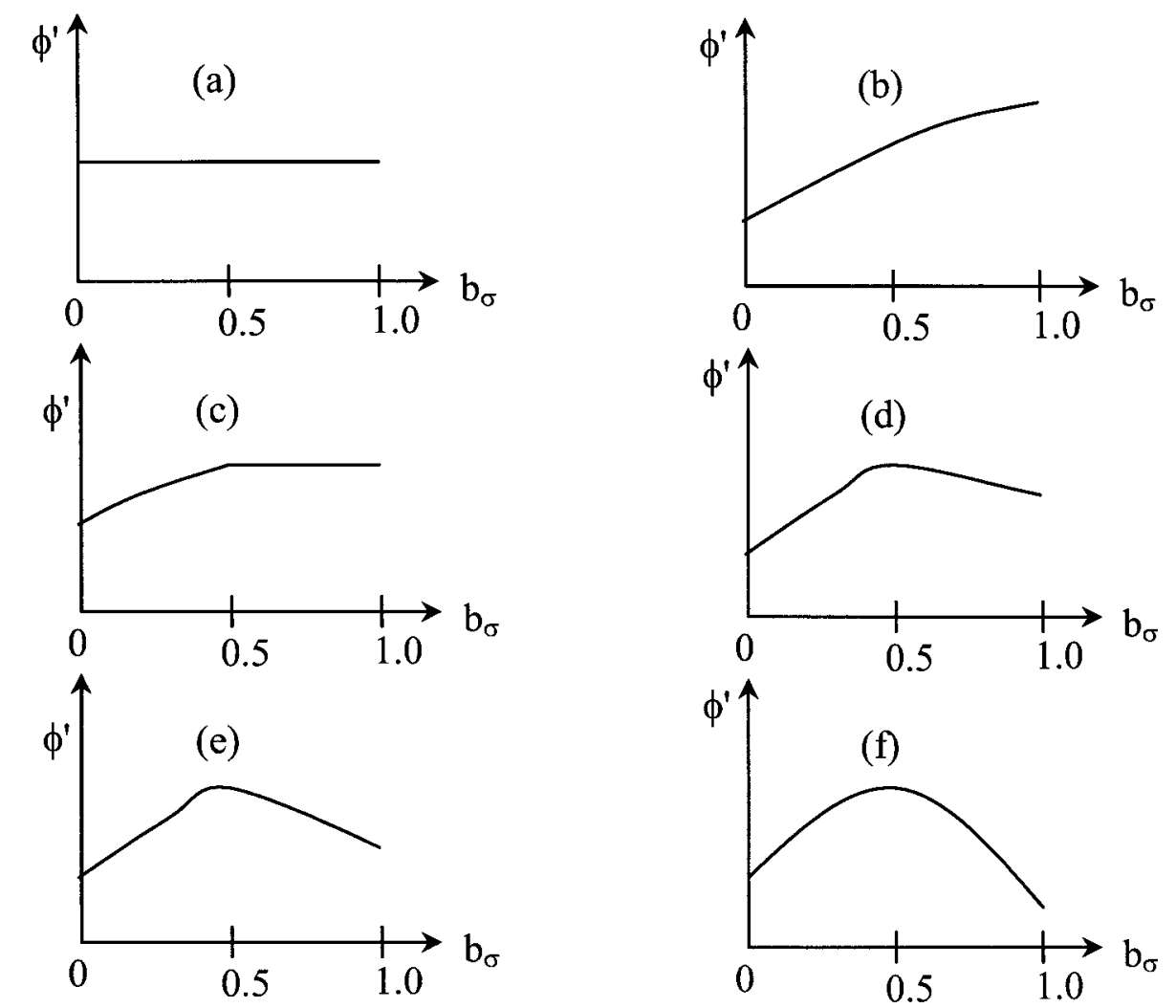

\begin{tabular}{|l|l|l|}
\hline Curve & \multicolumn{1}{|c|}{ Reference } & \multicolumn{1}{|c|}{ Relative Density } \\
\hline (a) & Bishop (1966) & Loose \\
\hline (b) & $\begin{array}{l}\text { Lade and Duncan (1973) } \\
\text { Reades and Green (1976) }\end{array}$ & $\begin{array}{l}\text { Loose } \\
\text { Loose to Dense }\end{array}$ \\
\hline (c) & $\begin{array}{l}\text { Green (1971) } \\
\text { Lade and Duncan (1973) }\end{array}$ & $\begin{array}{l}\text { Loose to Dense } \\
\text { Dense }\end{array}$ \\
\hline (d) & $\begin{array}{l}\text { Ergun (1981) } \\
\text { Haruyama (1981) }\end{array}$ & $\begin{array}{l}\text { Dense } \\
\text { Loose }\end{array}$ \\
\hline (e) & $\begin{array}{l}\text { Sutherland and Mesdary (1969) } \\
\text { Ramamurthy and Rowat (1973) }\end{array}$ & $\begin{array}{l}\text { Loose to Dense } \\
\text { Dense }\end{array}$ \\
\hline (f) & $\begin{array}{l}\text { Shankariah and Ramamurthy } \\
\text { (1980) }\end{array}$ & Medium to Dense \\
\hline
\end{tabular}

Fig. 2.11 Variations of $\phi^{\prime}$ with $b_{\sigma}$ in sands (After Sayao, 1989)

Further, Sayao (1989) conducted an experimental study on sand by proportionally increasing $\sigma_{m}^{\prime}(50$ to $300 \mathrm{kPa})$ and $b_{\sigma}(0$ to 0.5$)$ at constant $R$ and $\alpha_{\sigma}$. The study revealed that shear distortion in sand increased with simultaneous increase of $b_{\sigma}$ and $\sigma_{m}^{\prime}$. However, the induced volumetric strain increased with the increasing $\sigma_{m}^{\prime}$ and the influence of $b_{\sigma}$ 
was insignificant. The strain paths have remained essentially linear for the proportional increase of $\sigma_{m}^{\prime}$ and $b_{\sigma}$. This observation implies that initial cross anisotropy of sand remained unchanged during the proportional increase of $\sigma_{m}^{\prime}$ and $b_{\sigma}$.

\subsubsection{Effect of mean normal stress and relative density}

Effect of mean normal stress and relative density on soil behaviour is well understood in current practice. Increasing initial confining stress on sand at drained condition reduces the brittle characteristics of stress-strain curves and increases the strain to failure. In addition, it decreases the tendency for dilation (Lee and Seed, 1967). The initial confining stress does not influence the void ratio at failure. However, the friction angle at failure is dependent on the level of initial confining stress (Lee and Seed, 1967). The increase of relative density at a constant confining stress increases the strength of soil and tendency to dilate.

During undrained loading, the strain-hardening tendency of sand decreases with increasing initial effective confining stress. The sand exhibiting strain hardening behaviour at low effective confining stress may strain soften at high effective confining stress (i.e. increase of initial effective confining stress enhances the contractiveness of soil). A number of studies on undrained response at different confining stress and relative density have been reported in the literature (e.g. Vaid and Thomas 1994; Vaid and Sivathayalan 1996; Logeswaran 2005).

The above-mentioned stress-strain characteristics of sands under different confining stress and relative density states have been reported at $b_{\sigma}=0$ or 1.0 and $\alpha_{\sigma}=0^{\circ}$ or $90^{\circ}$. The influence of $\sigma_{m}^{\prime}$ and $D_{r}$ during the rotation of $\alpha_{\sigma}$ was studied by Sayao (1989) under generalized loading. The increase of $D_{r}$ during the rotation of $\alpha_{\sigma}$ at constant $b_{\sigma}, \sigma_{m}^{\prime}$ 
and $R$ significantly reduced both volumetric and shear strains and the increase of $\sigma_{m}$ at constant $b_{\sigma} D_{r}$ and $R$ increased both strains induced during rotation of $\alpha_{\sigma}$.

Sayao (1989) conducted another series of tests under proportional loading, in which $\sigma_{m}^{\prime}$ increased gradually during the loading at different $\alpha_{\sigma}$ and $D_{r}$. The volumetric strain and shear distortions increased with the increase of $\sigma_{m}$ at particular $\alpha_{\sigma}$. Both strains were more pronounced when major principal stress direction was more towards the bedding plane. In addition, contractiveness of sand increased with increasing $\sigma_{m}^{\prime}$ regardless of relative density at constant $b_{\sigma}, \alpha_{\sigma}$ and $R$.

\subsubsection{Effect of stress ratio}

Effective stress ratio $R$ could be alternatively substituted by deviatoric stress $q=$ $\sigma_{I^{-}} \sigma_{3}$ for a given initial stress state. Sayao (1989) conducted a few experiments to investigate the effect of $R$ at constant $\alpha_{\sigma}, \sigma_{m}^{\prime}$ and $b_{\sigma}$ on dense and loose sand. Dense sand experienced a higher amount of dilation than the loose sand and it started to dilate from the early stages of deformation. Higher values of $R$ at constant $\alpha_{\sigma}, D_{r}$, and $b_{\sigma}$ enhanced the tendency of contractiveness during gradual increase of $\sigma_{m}^{\prime}$.

Wijewickreme (1990) studied the behaviour of sands under simultaneous increase in stress parameters, $R$ and $\alpha_{\sigma}$. The obtained responses were compared with that of individual variation of stress parameter $R$. The strain response of the medium dense sand was path independent when the final stress state of the specimen maintained within $R \leq 2$ and $0^{\circ} \leq \alpha_{\sigma} \leq 45^{\circ}$. The strain responses became path dependent at higher $R$ and larger rotation of principal stress. As noted by Sayao (1989), sand subjected to principal stress rotation at higher $R$ developed large strains. When the stress state remained at $R \leq 2$ and $0 \leq \alpha_{\sigma} \leq 45^{\circ}$, the previous strain history did not influence the strain response of 
subsequent loading paths. During simultaneous increment of $R(0$ to 2$)$ and $\alpha_{\sigma}\left(0\right.$ to $\left.45^{\circ}\right)$, the influence of $b_{\sigma}$ and $\sigma_{m}^{\prime}$ on strain response of loose sand is significantly low. The increase of relative density retards the strain development.

\subsection{In-situ loading: case 1}

The stress state of a soil element is mainly dependent on the geometry of soil structure, the physical properties of soil (e.g $\left.K_{0}\right)$ and the nature of applied load. Fig. 2.12 shows the direction of principal stresses on the elements along a potential failure surface of an embankment and a gravity platform. The direction of principal stress changes from about $0^{\circ}$ at the top of the failure surface to about $90^{\circ}$ at the bottom of the failure surface.

Most of the field problems require three-dimensional analysis because of the three-dimensional stress states of soil elements. Fig. 2.13 shows 3-D stress states in a soil element at initial state, construction and cyclic loading. The soil element at initial state (Fig. 2.13a) has no shear stress, and hence load acting along axis $x, y$, and $z$ directions are the directions of principal stresses. During the construction of an earth structure, the soil element experiences shear stresses in addition to increases in normal stresses (Fig. 2.13b). The principal stresses act in different directions compared to initial principal stresses (i.e. principal stress rotates). The angle of rotation of principal stress depends on the magnitudes of shear stress and normal stresses. Fig. $2.13 \mathrm{c}$ shows the stress state in a soil element during a cyclic loading. The magnitude and direction of principal stresses change continuously depending on the magnitude of applied stresses. 


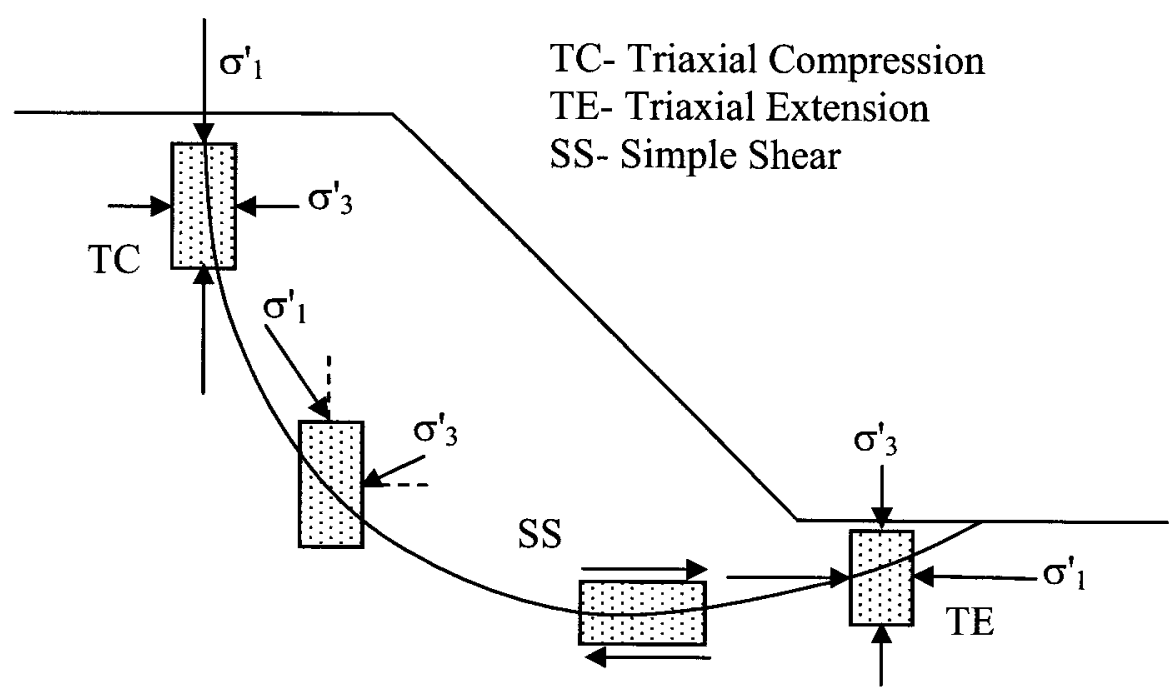

(a) Limiting equilibrium stress state beneath an embankment

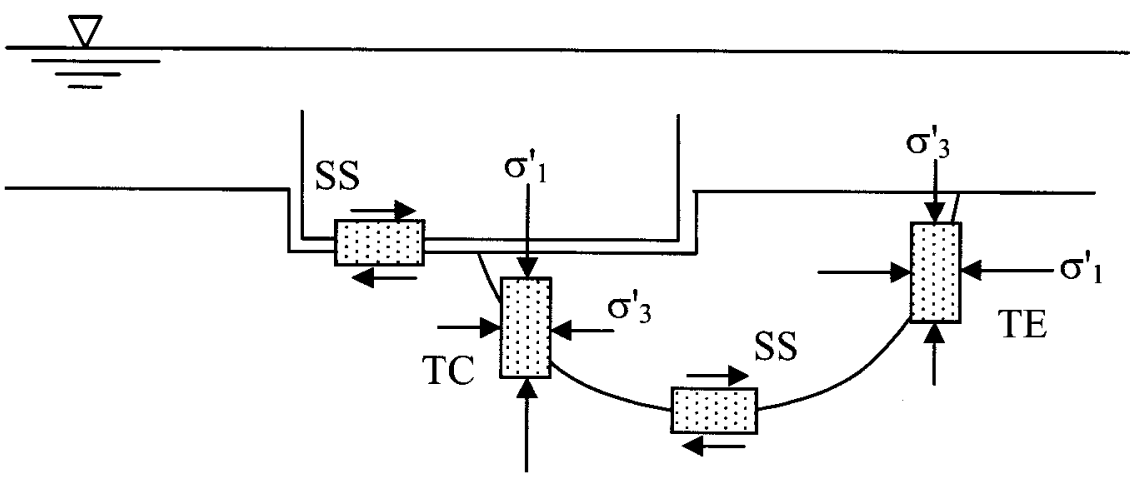

(b) Limiting equilibrium stress state beneath a gravity platform

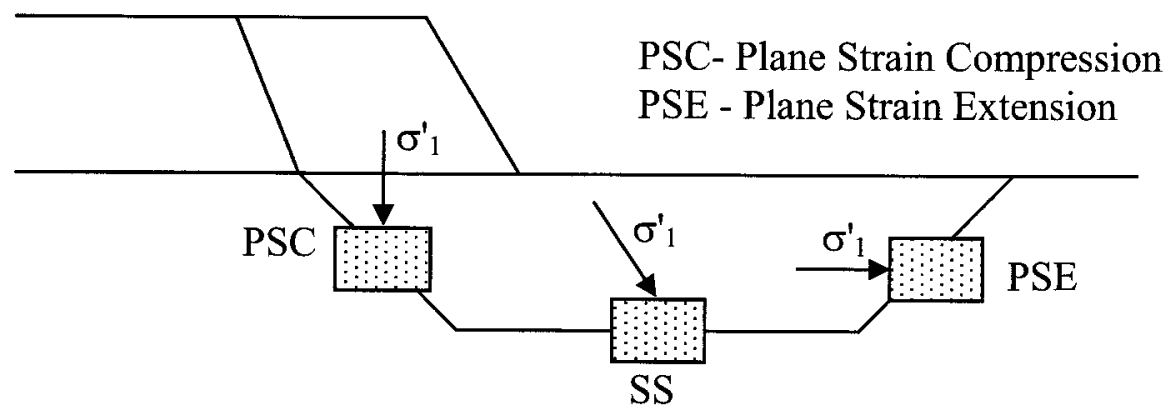

(c) Limiting equilibrium stress state in varved clay ground

Fig. 2.12 Principal stress variation along potential failure surfaces 


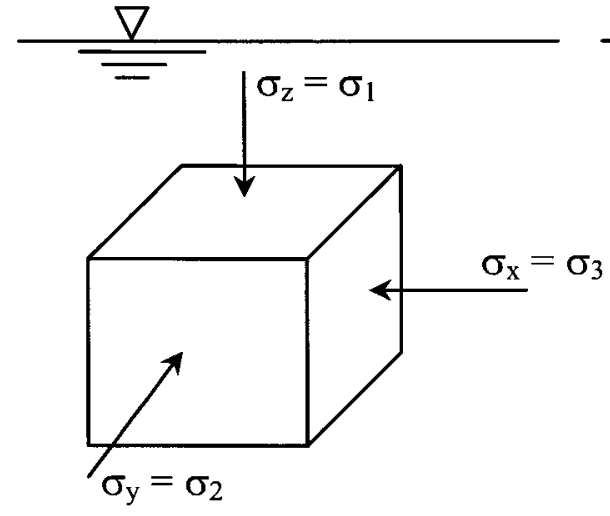

(a)

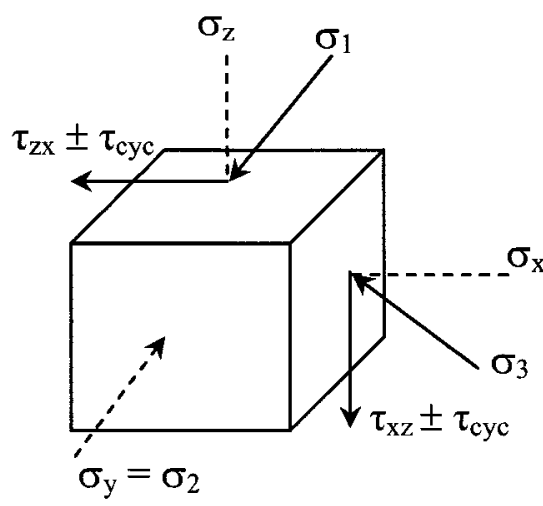

(c)

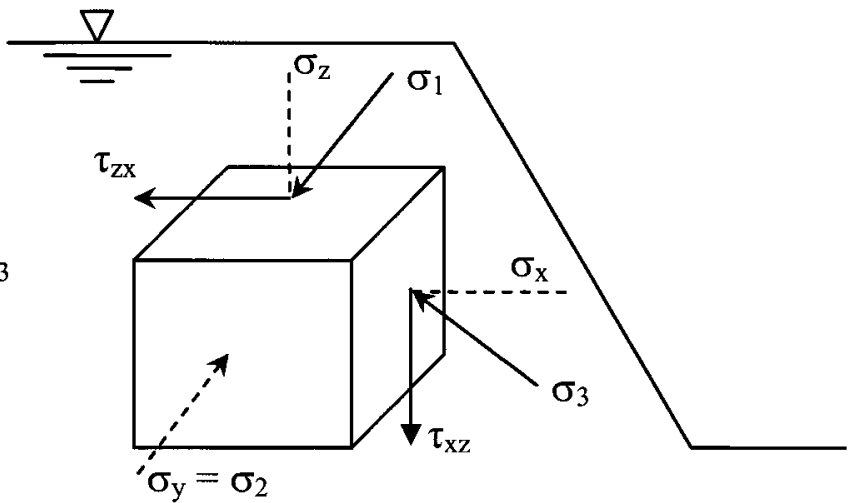

(b)

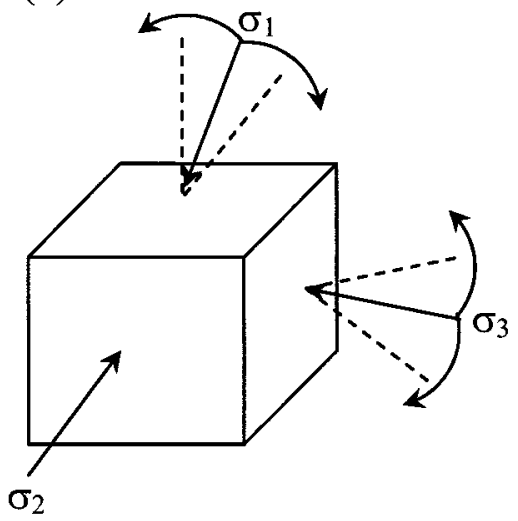

Fig. $2.13 \quad 3-D$ representation of stresses in a soil element (a) at level ground before construction (b) after construction (c) during application of load

\subsection{In-situ loading: case 2}

Generally, field deformation problems are approached as either undrained or drained. Even though these drainage conditions simplify the solution process, it is quite far from reality. In the undrained condition of deformation, volume of the soil remains constant $\left(\Delta \varepsilon_{v}=0\right)$ and pore pressure changes $(\Delta U \neq 0)$. In the drained condition, soil is allowed to full drain $\left(\Delta \varepsilon_{v} \neq 0\right)$, but the pore pressure remains constant $(\Delta U=0)$.

Saturated granular materials, such as sands and gravels are deemed to dissipate excess pore pressure immediately. The excess pore pressure dissipation is a time dependent process for clayey materials, even if free drainage is allowed. In most field 
situations, the soil element drains and experiences changes in volume and pore pressure with time $\left(\Delta U \neq 0, \Delta \varepsilon_{v} \neq 0\right)$.

When a saturated soil deposit is subjected to sudden loading, pore pressure may vary within the earth structure depending on the soil states and boundary conditions. The existence of piezometric pressure gradient in the soil structure may induce pore water migration that leads to volume inflow in some places and volume outflow in other places in earth structure as depicted in Fig. 2.16. In the above-mentioned scenario, pore pressure and volume changes occur simultaneously. Recent studies have revealed that small volume inflow could result in loss of strength (Eliadorani, 2000; Sivathayalan and Logeswaran, $2007 \& 2008)$. In addition, material that may be dilative under undrained loading may exhibit contractive behaviour due to volume inflow.

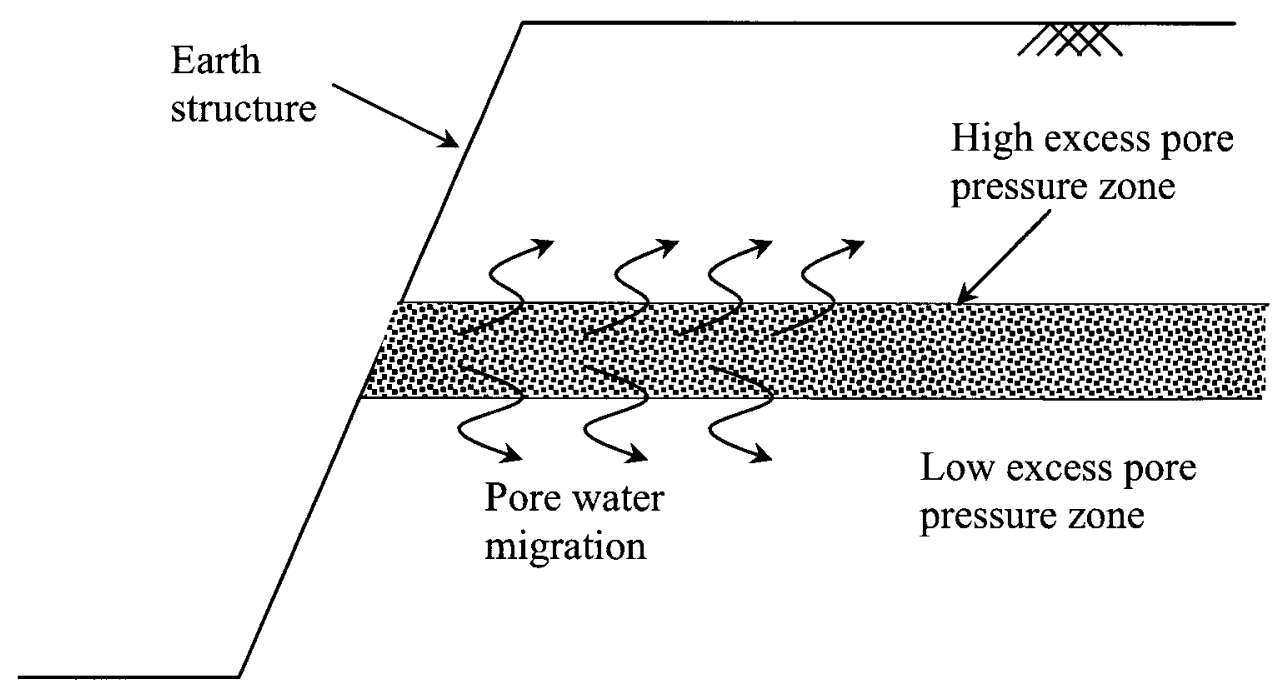

Fig. 2.14 Schematic illustration of pore water migration due to piezometric head gradient in a heterogeneous earth structure (modified after Logeswaran, 2005) 


\subsection{Summary}

It is clear from the review presented that the natural deposition process and laboratory reconstitution of specimens form anisotropic soil structure that results in directional dependency of soil behaviour. Soil behaviour has been often studied in the laboratory using conventional devices (e.g. triaxial and simple shear). These conventional laboratory devices mainly assess the soil behaviour only in the specific region of stress space because magnitudes of principal stresses and their directions are controlled in a restricted manner during the application of loading. The HCT device permits the investigation of soil behaviour in a generalized stress state, since it has the capability to control the magnitude of three principal stresses and rotation of direction of two principal stresses.

Only a few researchers have used the HCT device to understand the undrained behaviour of soils under generalized loading. An attempt is made to expand the knowledge of undrained soil behavior in this thesis. The main focus was on the effect of intermediate principal stress, direction of principal stresses and nature of principal stress rotation on undrained soil behaviour during both static and cyclic loading.

Another critical in-situ problem, volume-pore pressure coupled deformation was also studied in this research. A few earlier laboratory studies (Vaid and Eliadorani, 1998; Sivathayalan and Logeswaran, 2007 \& 2008) have investigated in this area and these studies have been performed using triaxial device that has a limited control on the principal stresses and directions. A research program was pioneered to investigate the soil behaviour controlling drainage under generalized loading using a newly built HCT device. 


\section{LABORATORY SOIL TESTING DEVICES}

\subsection{Introduction}

Different devices (e.g. simple shear, triaxial, true triaxial, torsional triaxial, true triaxial, directional shear cell, Hollow Cylinder Torsional (HCT) testing, etc.) are used in laboratory soil testing. Each device has merits and limitations depending on its capability and type of testing it is used for. The simple shear and triaxial devices are frequently used in laboratory soil testing and commonly used to assess the liquefaction susceptibility of soils. Soil testing using the HCT device is not common, even though this device is versatile and capable of representing field loading conditions very well.

In this chapter, merits and limitations of triaxial, simple shear and HCT testing devices are discussed. In addition, a detailed description of the HCT device used for this research study and nonuniformities found in HCT specimens are presented.

\subsection{Triaxial device}

The triaxial device is often used to examine the mechanical behaviour of soils because it is readily available and the testing procedure is simple and straightforward compared to other devices. Triaxial testing is performed on a tall cylindrical soil specimen surrounded by a non-reinforced latex membrane. The triaxial specimen is commonly sheared by the application of a vertical stress, while keeping lateral pressure constant.

Two stress components (major principal stress $\sigma_{l}$ and minor principal stress $\sigma_{3}$ ) can be controlled in this test. Depending on the nature of change in vertical stress (increase or decrease), a triaxial test may represent either compression or extension 
modes. In both compression and extension tests, vertical and horizontal stress directions coincide with major principal stress and minor principal stress directions or vice versa.

Drainage can be controlled quite well in a triaxial test and stress concentrations are significantly less compared to other conventional devices. The failure plane is not fixed during deformation as in the direct shear device, in which plane of failure is restricted along the horizontal plane. The failure plane in triaxial test develops based on material properties.

The triaxial stress path can be understood by using Mohr's circle as shown in Fig. 3.1. In both isotropic and anisotropic cases, principal stress direction rotates instantaneously by $90^{\circ}$ when axial deviatoric stress reverses the direction. The direction of major principal stress is generally vertical, but principal axis rotation occurs when major principal stress becomes horizontal (horizontal stress larger than vertical stress).

It is important to recognize that the loading condition of a triaxial test is axisymmetric and imposes a sudden principal stress rotation. These properties limit the nature of the stress paths that can be followed. The jump rotation of $90^{\circ}$ in principal stress directions cannot be expected in field problems where principal stress rotation is generally smooth and continuous.

Another limitation of the triaxial device is the incapability of control of intermediate principal stress $\sigma_{2}$. The influence of intermediate principal stress $\sigma_{2}$ cannot be evaluated in the triaxial test because of either $\sigma_{2}=\sigma_{1}$ or $\sigma_{2}=\sigma_{3}$. In field problems that are often approximated to the conditions of plane strain, $\sigma_{2}$ may be significantly higher than $\sigma_{3}$. Due to the negligence of $\sigma_{2}$ in triaxial tests, soil behaviour may not be a realistic representation of the field. 

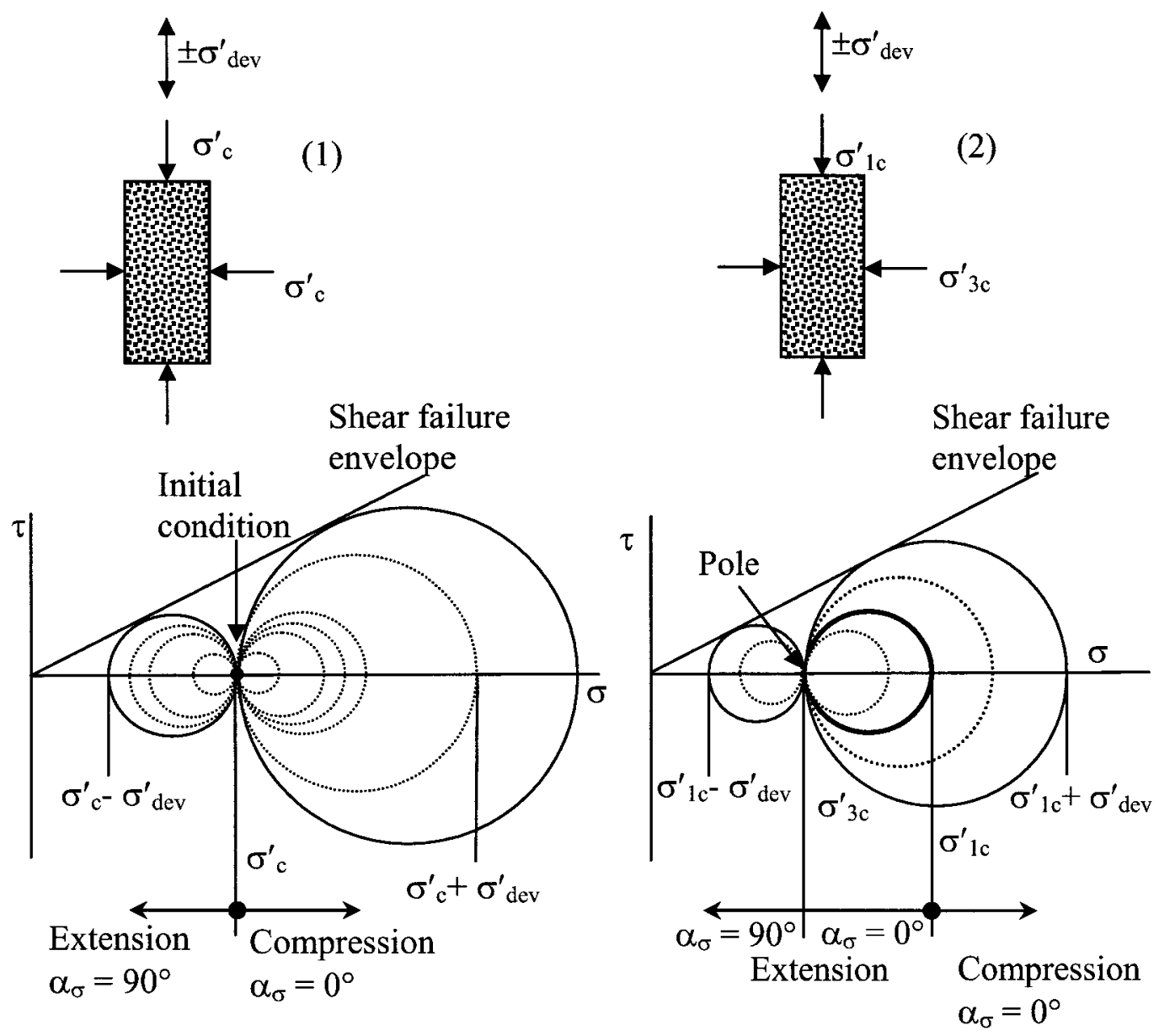

Fig. 3.1 Mohr's circle representation of stress states during drained triaxial tests consolidated (1) isotropically (2) anisotropically

The boundary conditions in the triaxial test are not entirely representative of actual field conditions. Lateral deformation is limited to one dimension in plane strain problems, but lateral deformation is axisymmetric in triaxial tests. The failure plane is inclined at an angle of $45+\phi / 2$ for Mohr-Coulomb materials, but failure may be limited to a particular plane during deformation in field.

The influence of end restraints (end caps) that provide the support to the specimen at the bottom and top cause non-uniform stresses, pore pressures and strains in the test specimens. This effect compromises the presumed equality of radial and tangential 
stresses and strains at larger strains (Casabarian and Jamal, 1963; Frydman et. al, 1971) and introduces uncertainty in the interpretation of tests results. However, the effect of end restraint on strength of soil can be reduced when a height to diameter ratio of two or larger is used (Taylor, 1948; Bishop and Green, 1965; Lade, 1982). The lubricated end platens (Rowe and Barden, 1964; Barden and Khayatt, 1966) reduce the end effect, but may introduce bedding errors (Sarsby et al., 1980).

The inability to control $\sigma_{2}$ in the typical triaxial device has been solved by the introduction of true triaxial devices, in which three the principal stresses are controlled independently. Using the true triaxial device, behaviour of the soil under the influence of intermediate principal stress may be assessed, but neither inclination of principal stresses nor rotation of principal stress directions is possible. Depending on the type of loading on the specimen, several types of true triaxial devices have been introduced in the literature as the following;

- True triaxial device with flexible boundaries: Uniform pressure is applied to all six prismatic specimen faces through pressured bags or membranes (Ko and Scott, 1967; Arthur and Menzies, 1968).

- True triaxial device with rigid boundaries: Uniform strains are applied to all six faces by rigid plates (Pearce, 1971; Hosseini and Cousens, 1988).

- True triaxial device with mixed boundaries: Rigid plates and flexible membranes are used to apply stresses and strains (Green, 1971; Lade, 1978).

The advantages and limitations of these devices have been described by Sture and Desai (1979). Commonly, stress nonuniformities are encountered around the adjacent plates or membranes in most of the true triaxial devices. 


\subsection{Simple shear device}

The simple shear device was used in the past by several researchers (e.g Roscoe, 1953; Bjerrum and Landva, 1966; Vaid and Finn, 1978; Franke et al., 1979; Ishihara and Yamazaki, 1980; Boulanger et al., 1993) to impose specific types of loading in soil testing. This device has been designed to impose loading as a combination of normal and shear stresses on anisotropically consolidated soil specimen. In practice, two types of simple shear devices are commonly used.

- NGI type device: The specimen is prepared like a circular disk and it is laterally confined by a steel reinforced rubber membrane.

- Cambridge type device: Specimen cross section is square and it is confined by six rigid faces.

The NGI type simple shear device was commonly used for liquefaction assessment by several researchers (e.g. Finn and Vaid, 1977; Finn et al., 1978; Kammerer, 2002). In the NGI type device, the wire-reinforced membrane imposes $K_{0}$ condition to specimen during application of vertical load. The lateral stresses, developed by a wire-reinforced membrane, maintain zero lateral deformation. Maintaining constant height of the specimen represents constant volume of the specimen during shear loading. As pore pressure of the specimen is always allowed to be atmospheric, the development of excess pore pressure is actually zero. The drop in vertical stress during shearing is equal to the excess pore pressure that would have been developed in a saturated undrained test (Dyvik et al., 1987). Maintaining constant volume during simple shear test ensures that no effort is required to maintain undrained state on specimens, and that the 
specimens do not suffer from system compliance effects which are encountered in other tests.

The combination of normal and shear loading allows for the smooth and continuous rotation of principal stress direction through $90^{\circ}$. Application of shear loading on the horizontal plane duplicates in-situ loading during propagation of SH wave as shown in locations (a) and (b) (Fig. 3.2). In addition, soil testing under simple shear loading closely simulates plane strain condition of in-situ loading.

Limitations of the simple shear testing have been discussed in the past (Saada and Townsend, 1981; Lacasse and Vucetic, 1981; Budhu, 1984). One of the major limitations is the absence of complementary shear stress on lateral boundaries of the specimen. Considerations of equilibrium and boundary conditions require non-uniform distribution of both shear stress and normal stress on the specimen's surfaces. In addition, radial expansion and pinching effects may enhance the non-uniformity of stress distributions. Therefore, use of a simple shear device to determine either stress-strain relations or absolute failure parameters would not be reliable (Saada and Townsend, 1981). The value of simple shear in the study of soil behaviour in terms of principal stress direction and rotation is limited, but this device is very useful in assessing the cyclic resistance of soils. 


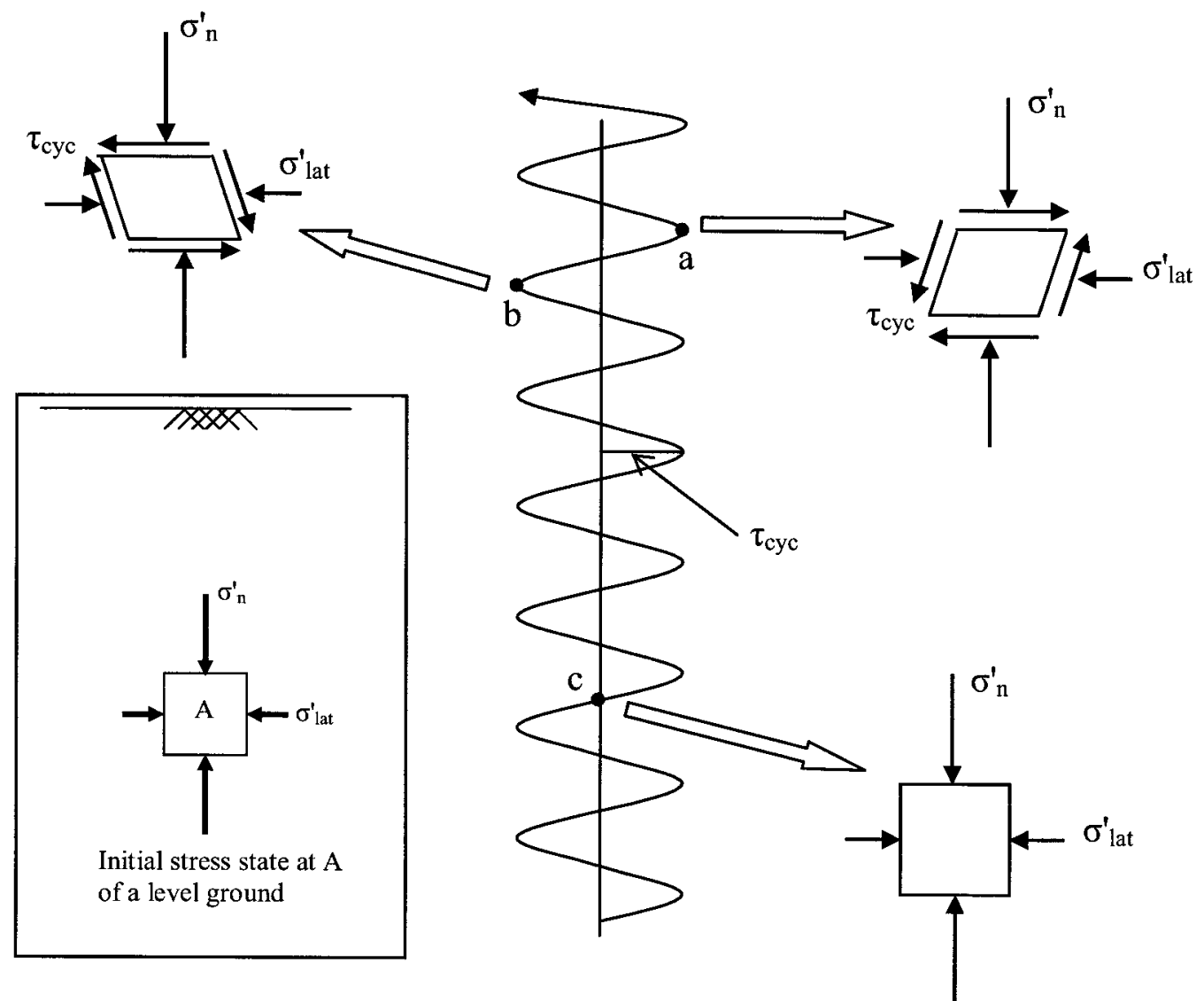

Stress state at A during the propagation of shear wave of a seismic event

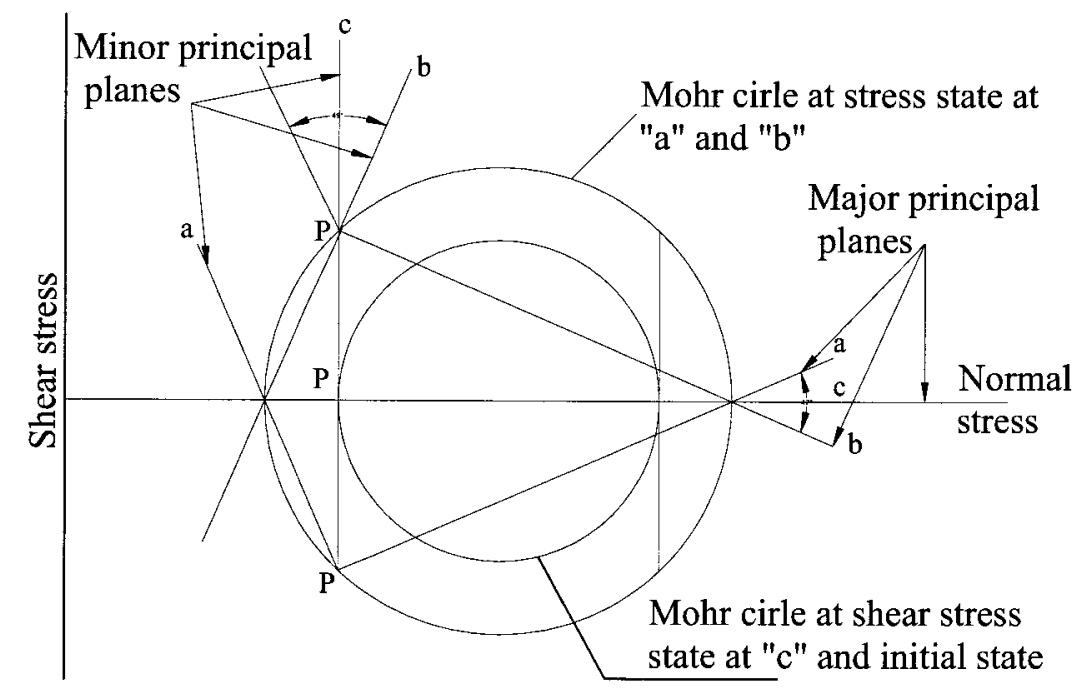

Fig. 3.2 Comparison of in-situ stress condition and simple shear loading (Modified from $\mathrm{Ha}, 2003$ ) 


\subsection{Hollow cylinder torsional shear device}

Using the hollow cylinder torsional (HCT) device, hollow soil specimens can be tested under the application of axial load, external confining pressure, internal confining pressure, and torque about vertical axis. The combination of these tractions allows the study of soil behaviour at any pre-failure stage in four-dimensional space $\left(\sigma_{l}, \sigma_{2}, \sigma_{3}, \alpha_{\sigma}\right)$. However, this device may not be suitable at certain stress states due to the high level of stress nonuniformities within the specimen (Sayao, 1989).

\subsubsection{Description of the HCT device used}

The HCT device in the advanced geotechnical laboratory at Carleton University is capable of performing controlled stress path testing on reconstituted sand specimens under monotonic and cyclic loading conditions. In addition, it permits strain path controlled tests by controlling inner volume, specimen volume, rotational displacement and vertical displacement.

A schematic layout of HCT device used is shown in Fig. 3.3. Dimensions of a specimen are approximately $300 \mathrm{~mm}$ of height, $150 \mathrm{~mm}$ of outer diameter and $25 \mathrm{~mm}$ of wall thickness. These selected dimensions result in a cross sectional area of about 100 $\mathrm{cm}^{2}$. The selection of comparatively larger dimensions of specimens (compared to other HCT devices in use) reduces the level of stress nonuniformities and improves the measurement resolutions. The internal and external pressures are applied laterally on flexible rubber membranes that have a thickness of about $0.3 \mathrm{~mm}$. Vertical and torsional loads are applied through the ribbed annular aluminum platens, which are located at the top and bottom of the specimens. To ensure complete drainage during deformation, six small porous stones are placed into the built-in cavities of each platen (Fig. 3.4). 


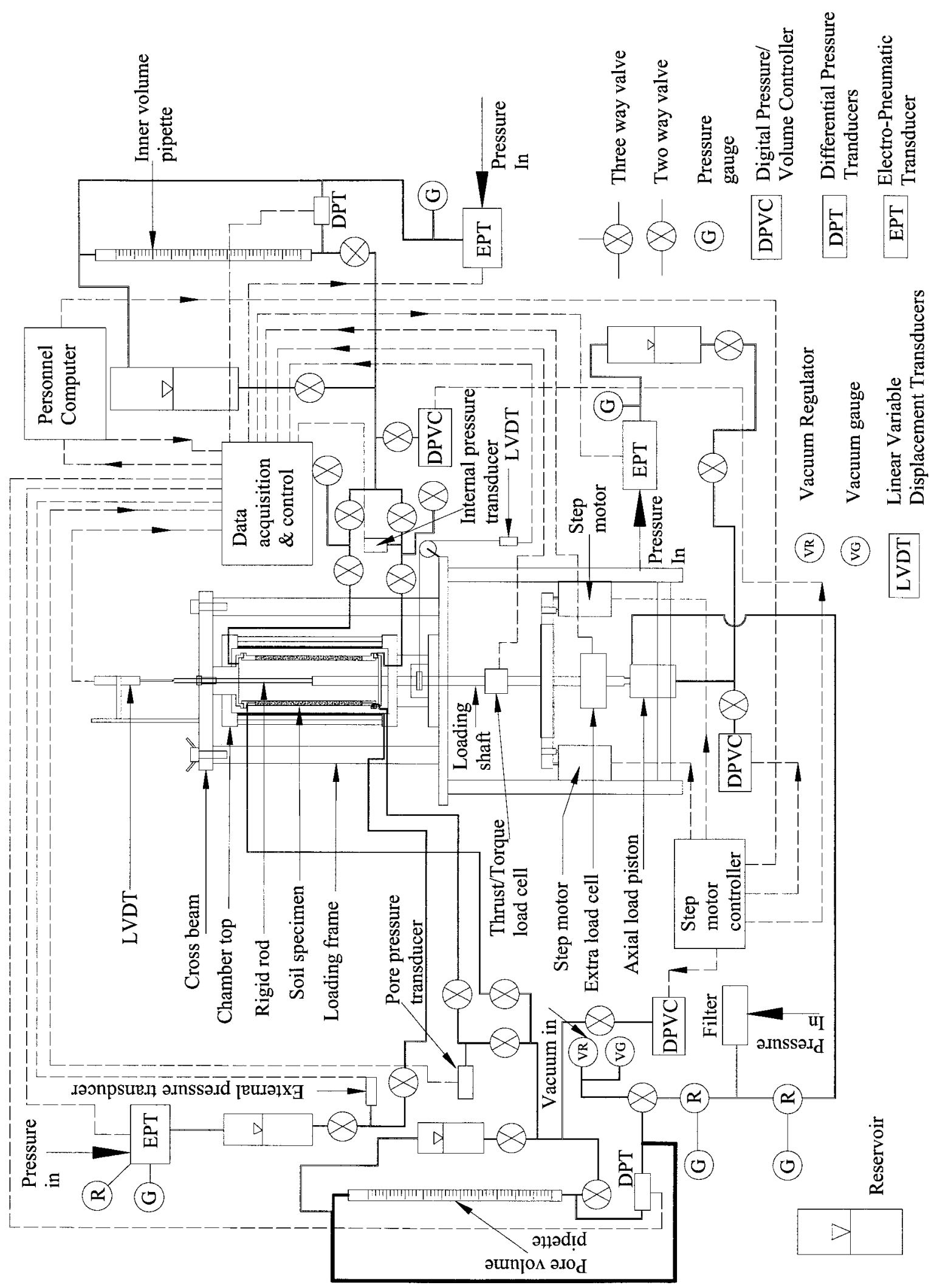

Fig. 3.3 Schematic diagram of the HCT apparatus 

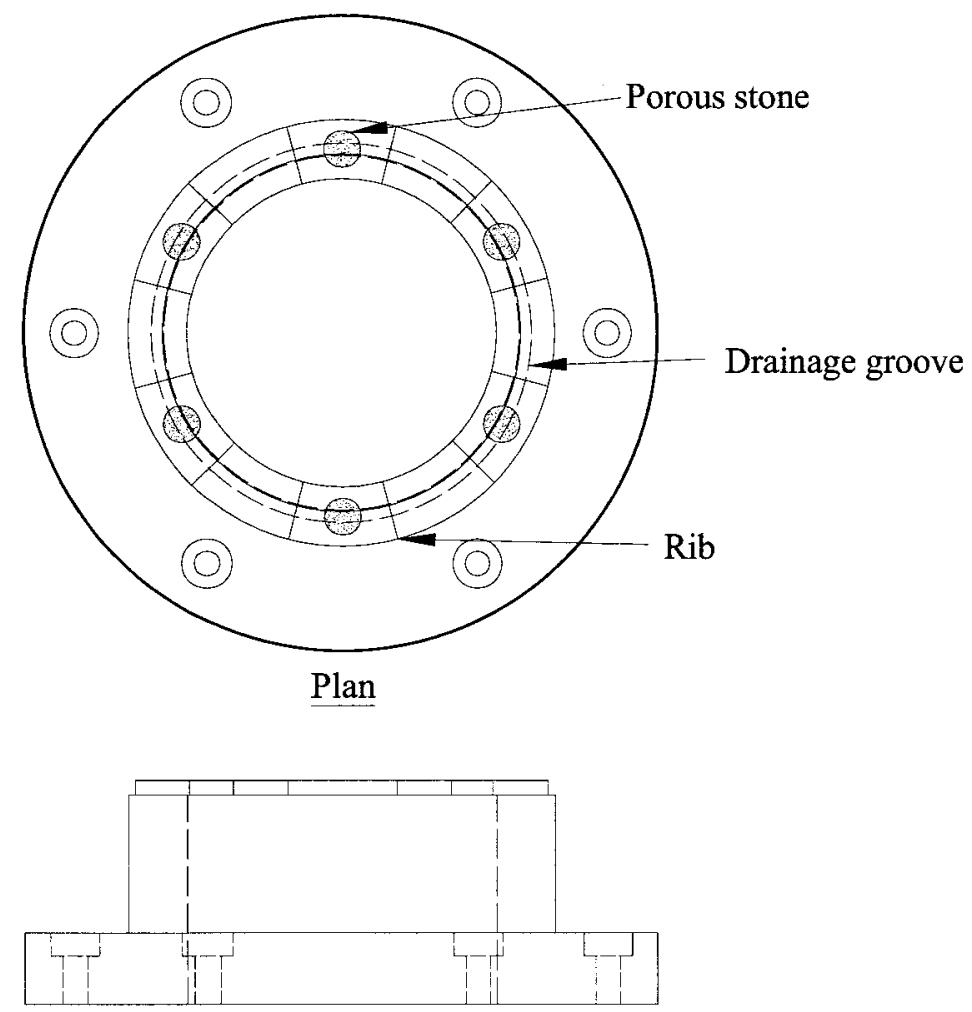

Front view

Fig. 3.4 Polished end platen with radial ribs

The HCT specimen is fixed at the top and loaded at the bottom. The application of load at the bottom makes specimen preparation easier and eliminates the requirement to correct the vertical stress for the weight of ram and load cell. A rigid steel frame attached at the bottom of specimen ensures the solid reaction of vertical and torsional loads.

Nine parameters, four stress components, four strain components and pore pressure are measured during the test. In most situations, interactive use of a computer is required for a proper stress/strain path control. Saturation of the testing system, noise level, and transducer gains are regularly monitored and checked.

\subsubsection{Loading systems of the HCT device}

Manual control of four surface tractions in the HCT specimen is a difficult task, so that several automatic systems were introduced. Saada and Baah (1967) introduced a 
pneumatic analog stress path control system. As a further advance in stress path testing, the servo controlled system was introduced (Hight et al., 1983; Sivakugan et al., 1988). Shibuya (1988) introduced a computer-controlled stepper motor system to replace the servo controlled system. However, the computer-controlled stepper motor system did not permit displacement controlled loading system. The stress-controlled loading system does not allow post peak behaviour because of specimen-device interaction (Chern, 1985) together with the inability of the device to follow a prescribed stress path. A displacement-controlled loading system is essential to assess the liquefaction potential of loose contractive sands that may strain soften during undrained loading.

\subsubsection{Stress-controlled loading system}

Stress-controlled loading is performed using three Electro-Pneumatic (E/P) transducers that are connected to inner chamber pressure (Fig. 3.5), axial load (Fig. 3.6) and external chamber pressure (Fig. 3.7). The E/P transducers have an output pressure range of 0 to $700 \mathrm{kPa}$. An E/P transducer converts an analog electrical signal input into a

directly proportional output pressure. Torque is applied by two precision step motors (Fig. 3.8) that apply pulses until reaching the target torque. The target torque is realized using a feedback loop of data acquisition program. 


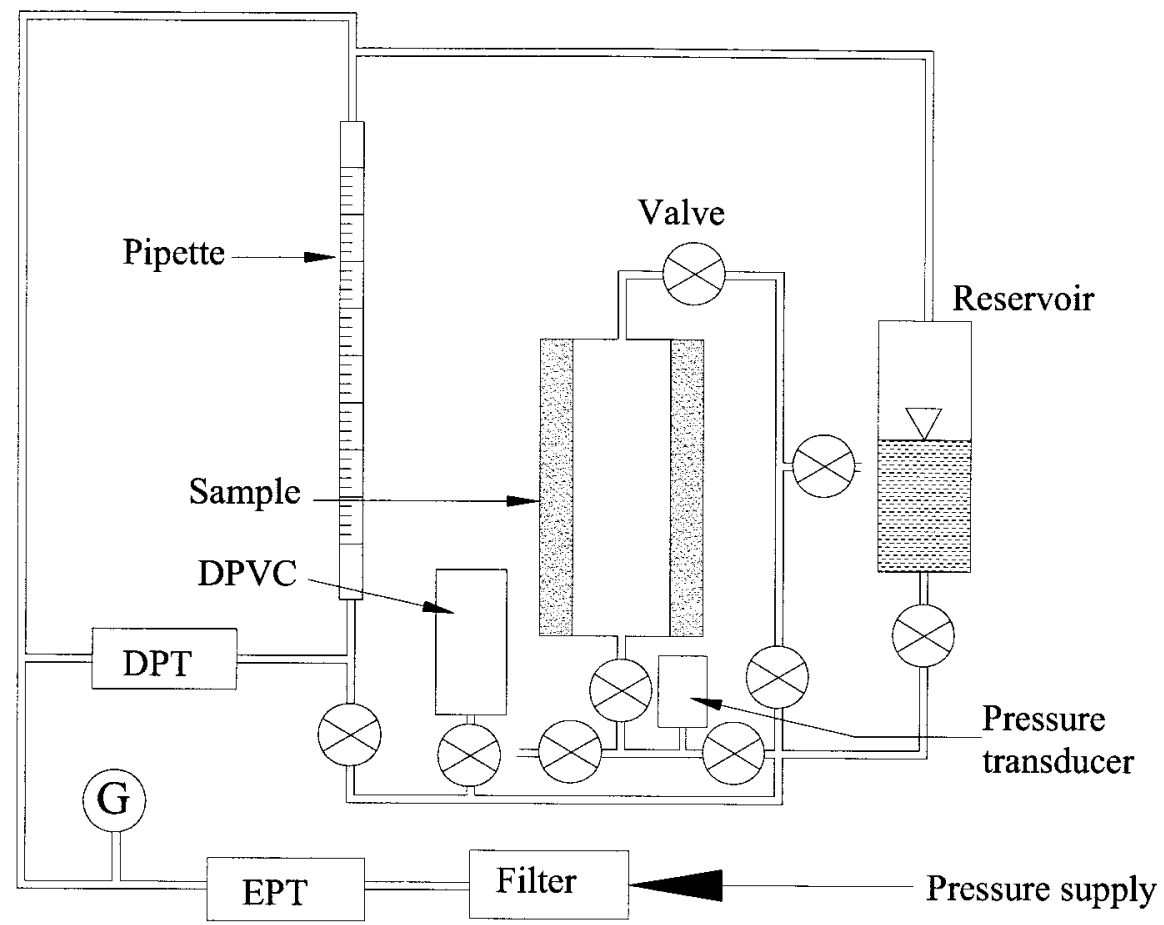

Fig. 3.5 Pressure and volume control of inner chamber of the HCT device

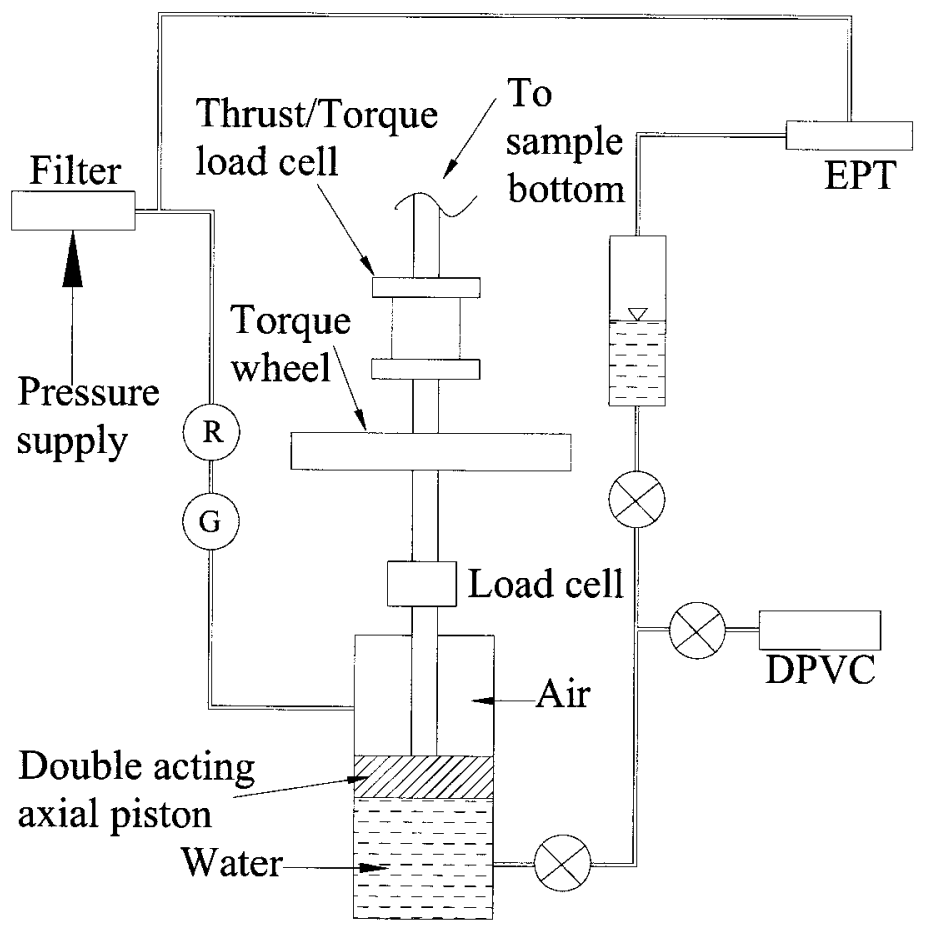

Fig. 3.6 Vertical stress and vertical displacement control of the HCT device 


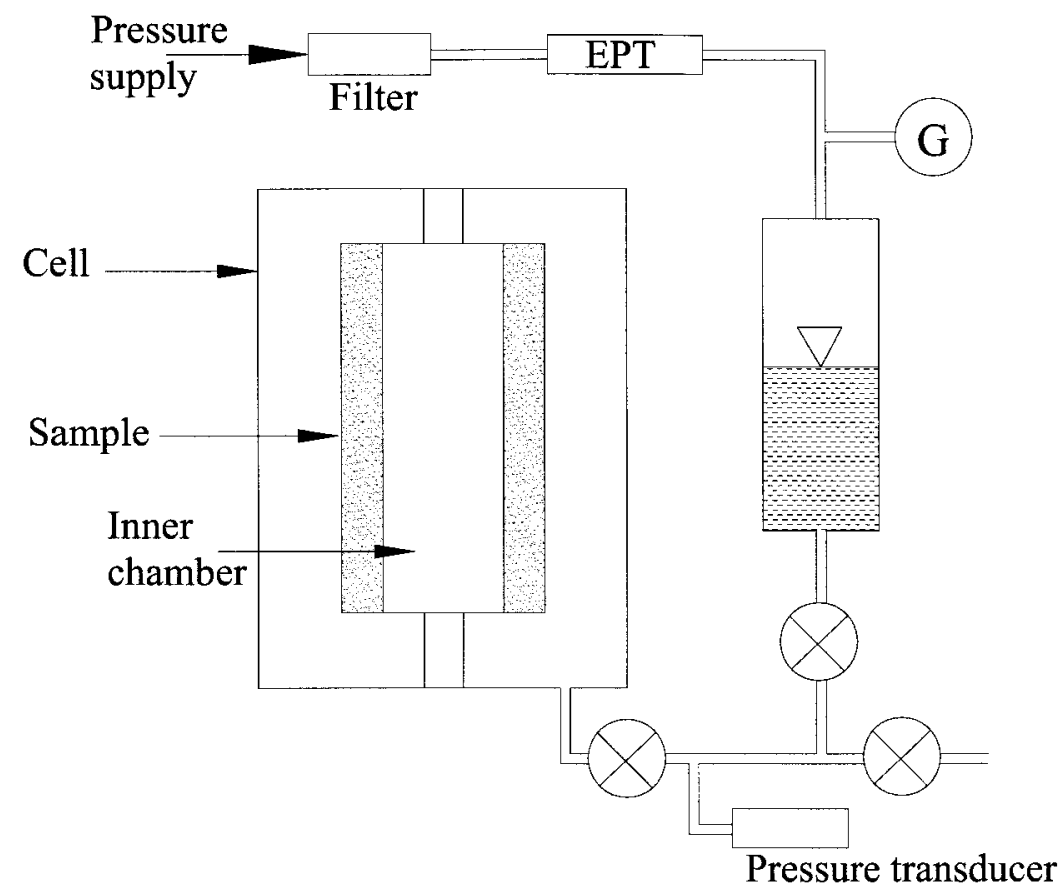

Fig. 3.7 Pressure control of external chamber of the HCT device

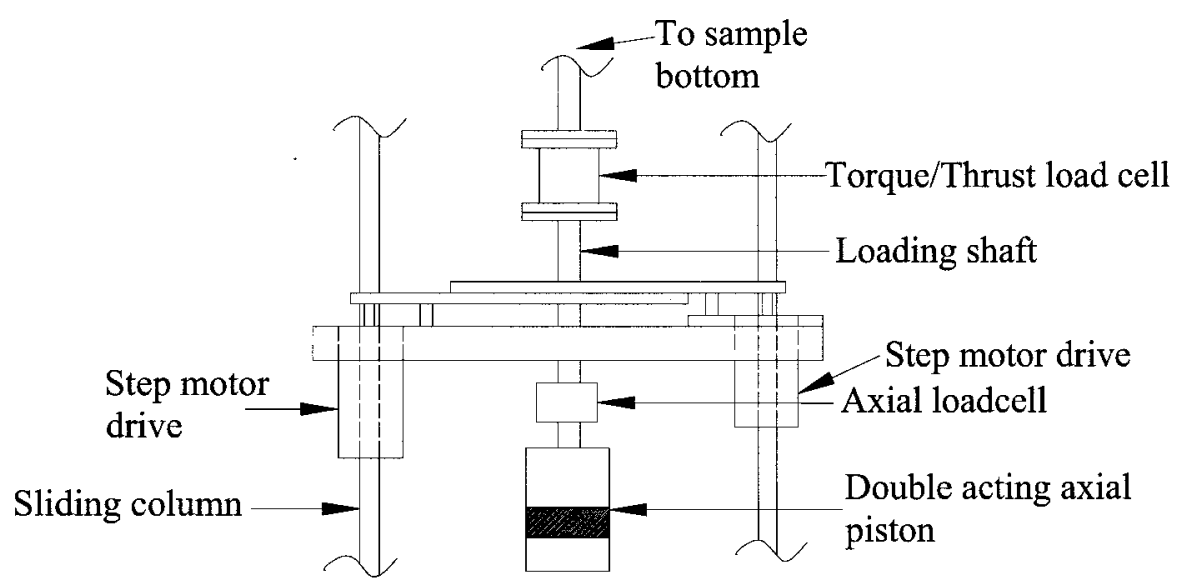

Front view

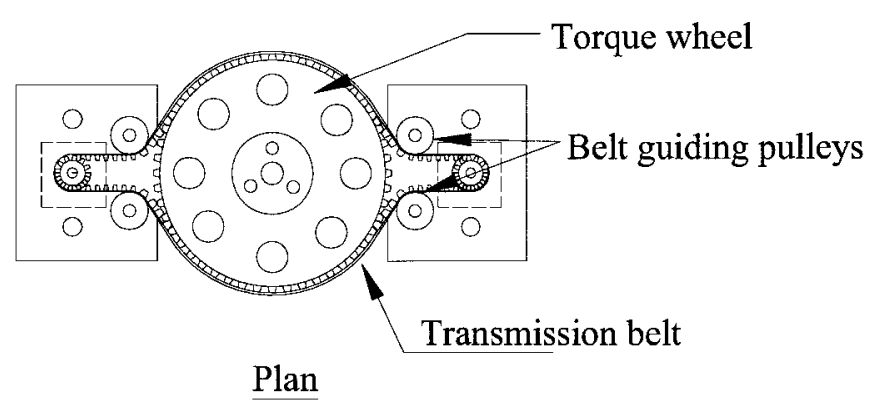

Fig. 3.8 Torque and torsional displacement control of the HCT device 


\subsubsection{Displacement-controlled loading system}

Displacement-controlled loading is achieved using three Digital Pressure/Volume Controllers (DPVC) connected to a vertical loading piston (Fig. 3.6), specimen volume (Fig. 3.9) and inner chamber (Fig. 3.5), and two torque motors attached at the bottom of the specimen to apply shear strain (Fig. 3.8). The DPVC connected to the vertical loading piston controls vertical displacement. The DPVCs connected to the inner chamber and specimen volume can manipulate the radial and tangential strains.

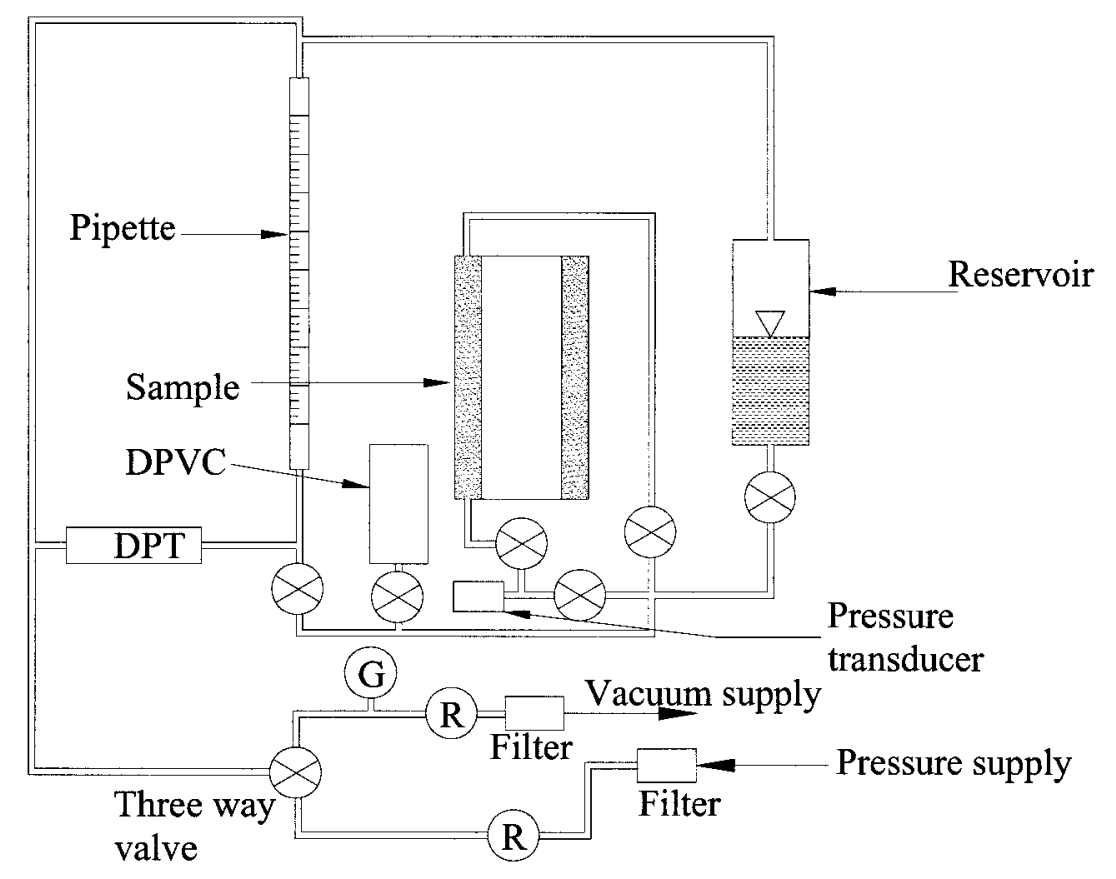

Fig. 3.9 Back pressure and specimen volume control of the HCT device

Menzies (1987) has outlined the principle of DPVC in soil testing. The DPVC consists of a water cylinder and a piston that is attached to a step motor by a ball screw. The DPVC has been designed to provide an accurate control of volume change depending on the amount of movement, diameter of the cylinder and pitch of the ball screw. The desired strain increment is applied by either extracting or injecting water. As strain increment is applied by volume control, the displacement loading system requires a 
fully saturated system. Incomplete saturation of the system would result in an inaccurate strain change due to compliance.

\subsubsection{Measuring system of the HCT device}

Loads, pressures and deformations are measured continuously by electronic transducers (Fig. 3.3). In the early development of HCT devices, internal measuring systems (Hight et al., 1983) were recommended. Such systems compounded the specimen preparation difficulties and are not commonly used in current practice. The recent evaluation of stress and strain measurements describes the advantages and disadvantages of both external and internal measuring systems in the laboratory instrumentation. Neither an internal nor an external measuring system is perfect (Atkinson and Evans, 1985; Jardine et al., 1985). The external measuring system enhances the amount of tilting and bedding/seating errors compared to the internal measuring system. However, the internal measuring system makes the specimen preparation and interpretation of the test results difficult. Negussey, (1984) indicated that errors occurring due to the external measuring system can be minimized by taking suitable measures and using improved techniques.

In an external vertical load measurement, friction between shaft and O-ring should be taken into account for an accurate measurement. The internal load cell (ElRuwayih, 1975; Hight, 1983) eliminates the problem due to the friction between shaft and O-ring. However, it introduces excessive deformability and effects the axial strain measurements of dense specimens (Costa Filho and Kupper, 1983). 


\subsubsection{Measurement of strains}

Four displacement components (vertical displacement, angular displacement, volume change of inner chamber and volume change of specimen) are used to estimate strains in the HCT specimen. The vertical and angular displacements are measured using Linear Variable Differential Transducers (LVDT) and translated to axial strain $\varepsilon_{z}$ and shear strain $\gamma_{z \theta}$.

The vertical LVDT is mounted at the top of the cross beam of loading frame (Fig. 3.3) and it reacts to the movement of a thin vertical rod that is attached to the centre of the bottom pedestal. The torsional LVDT measures the rotation of the specimen by changing the rotation as linear tangential displacement (Fig. 3.10). These two LVDTs are capable of measuring displacement in the order of $10^{-3} \mathrm{~mm}$. This resolution results in an accuracy level of axial and shear strains better than in the order of $5 \times 10^{-4}$.

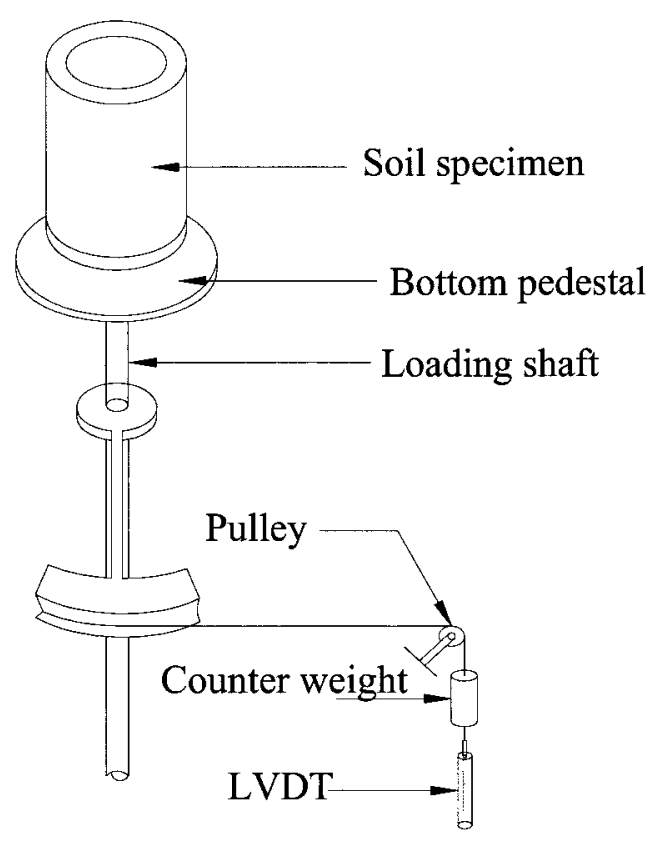

Fig. 3.10 Torsional displacement measurement in the HCT device 
Radial strain $\varepsilon_{r}$ and tangential normal strain $\varepsilon_{\theta}$ are estimated by measuring volume change of the inner chamber, and volume and height changes of the specimen. The volume changes are measured using Differential Pressure Transducers (DPT). From measured volume changes and height change, $\varepsilon_{r}$ and $\varepsilon_{\theta}$ are estimated with a resolution in the order of $10^{-4}$.

\subsubsection{Measurement of stresses}

Four surface tractions and pore water pressure are measured continuously during the HCT test. The pore pressure and chamber pressures are measured using precision pressure transducers, which have a resolution of about $\pm 0.1 \mathrm{kPa}$. The vertical load and torque are measured by a thrust/torque load cell. This load cell has a resolution of torque approximately $\pm 0.05 \mathrm{Nm}$ (shear stress in the order of $\pm 0.01 \mathrm{kPa}$ ) and axial load of approximately $\pm 4 \mathrm{~N}$ (vertical stress in the order of $\pm 0.5 \mathrm{kPa}$ ). The torque and thrust measurements have a negligible amount of crosstalk in this load cell. In addition, the vertical load is measured using an extra load cell attached at the bottom of the torque loading system (Fig. 3.3). This load cell has a resolution of approximately $\pm 1 \mathrm{~N}$ (vertical stress in the order of $\pm 0.1 \mathrm{kPa}$ ).

\subsubsection{Data Acquisition of the HCT device used}

The stress/strain measurement system, consisting of ten transducers is monitored through a multichannel signal conditioner (MSC-10) and an Analog-to-Digital (A/D) converter together with a personal computer as shown in Fig. 3.11. The MSC-10 is wired to a National Instruments PCI-6052E multifunctional A/D board and PCI-6703 Digitalto-Analog Converter (DAC). The PCI-6052E card is 16 bit resolution high speed (333 $\mathrm{kHz}$ ) with 16 analog inputs and 2 analog outputs. The PCI-6703 DAC is 16 bit analog 
output card with 16 channels. However, only four of those channels are wired in the HCT configuration.

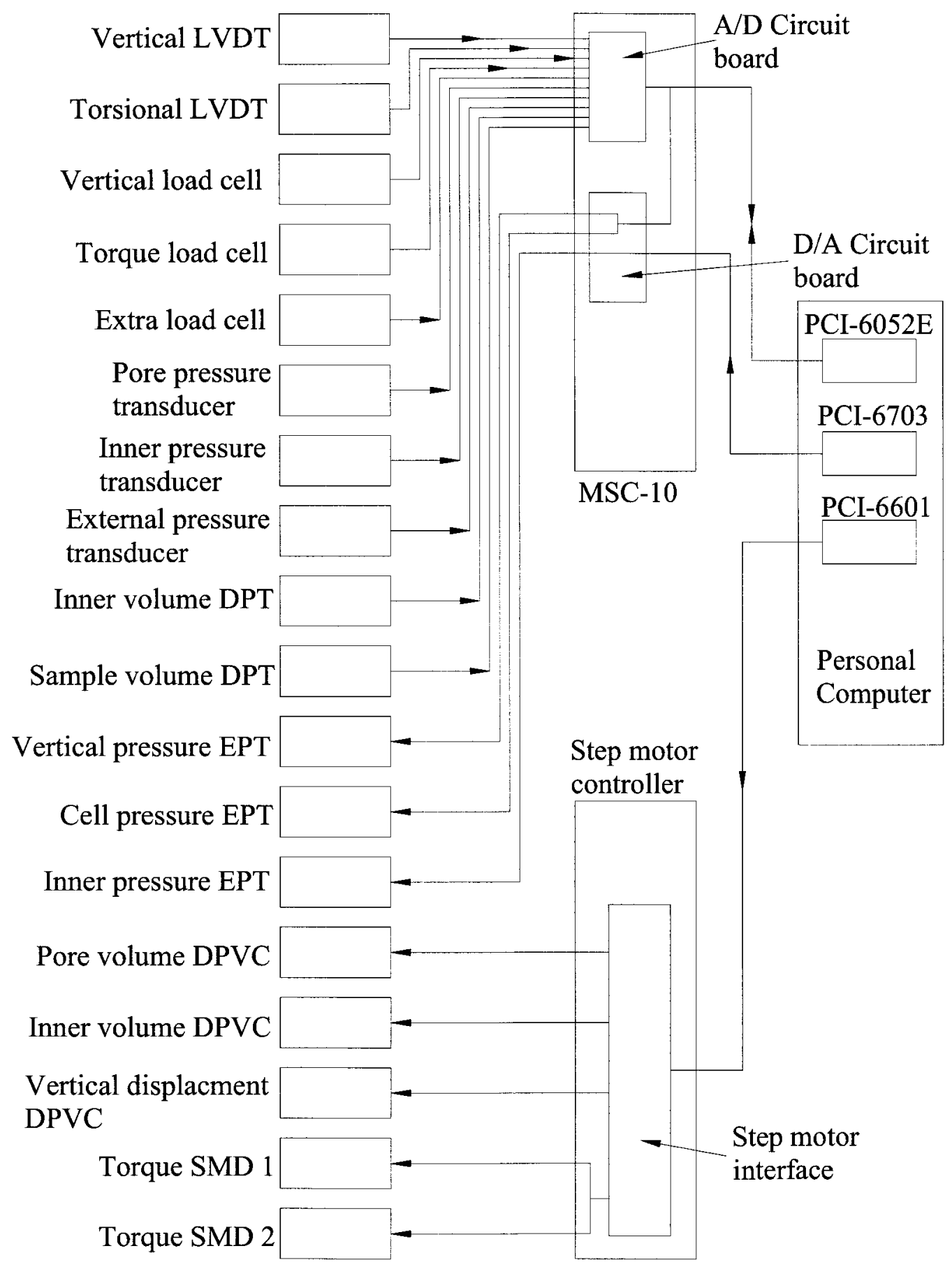

Fig. 3.11 Schematic diagram of data acquisition process in the HCT device 
Five step motor drivers of the HCT device are connected to step motor controller that is wired to PCI-6601 counter/timer. The PCI-6601 has four 32 bit counter channels. Two of five step motors that are connected to torque wheel is operated in the same manner by wiring to one channel of PCI-6601. Careful selection of this hardware of the HCT device ensures low noise level, and accurate measurements and application of tractions.

The MSC-10 provides regulated excitation, amplification and filtering for wide variety of transducers used. All transducers are read concurrently and analog voltage is collected for a certain period. From the collected readings, an average reading during that time interval is determined by an analog integrator. Then, the averaged value is converted to digital output by $\mathrm{A} / \mathrm{D}$ converters. The memory chips temporarily save the readings in the microcomputer for later retrieval.

A multithreaded data acquisition program, using object oriented programming techniques, was developed in-house to acquire the data and control the system. Multiple execution threads within a single process enable continuous and smooth operation of hardware and proper sampling of input channels without interruption or delay. Appendix A provides a summary of the modules used in the data acquisition programming together with the methods associated with different objects.

\subsection{Definitions of stresses and strains in HCT specimens}

Four surface tractions (vertical load $F_{z}$, torque $T_{h}$, outer chamber pressure $P_{e}$ and inner chamber pressure $P_{i}$ ) can be applied to impose a specified stress state in a HCT specimen (Fig. 3.12). Additionally, Fig. 3.12 shows the stress state and the direction of

principal stress in a soil element of a HCT specimen. The stress state in the soil element 
is expressed in terms of vertical stress $\sigma_{z}$, tangential stress $\sigma_{\theta}$, radial stress $\sigma_{r}$ and shear stress $\tau_{z \theta}$. The strains corresponding to the directional stresses are denoted by vertical strain $\varepsilon_{z}$, tangential strain $\varepsilon_{\theta}$, radial strain $\varepsilon_{r}$ and shear strain $\varepsilon_{z \theta}$. These directional stress/strain components are used to calculate principal stresses and strains, and inclination of major principal stress to vertical axis.

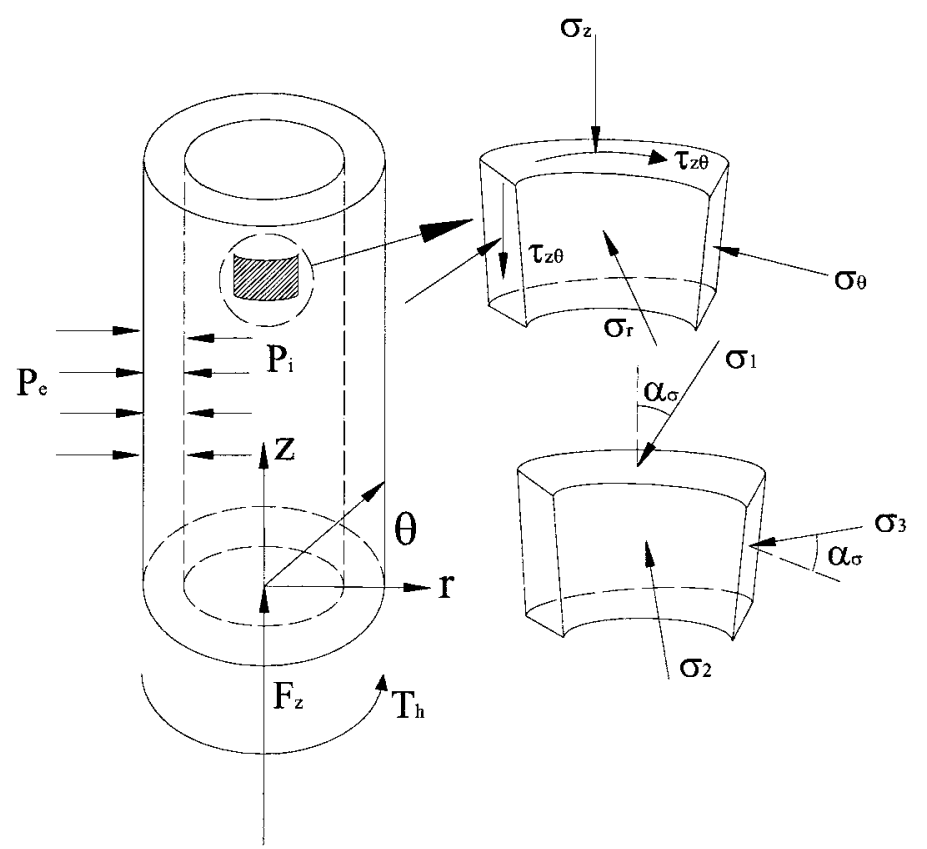

Fig. 3.12 Surface tractions and stress state of a soil element in a HCT specimen Normal stresses $\sigma_{\theta}$ and $\sigma_{r}$, and shear stress $\tau_{z \theta}$ vary along thickness of the specimen's wall. However, the average values of $\sigma_{\theta}, \sigma_{r}$ and $\tau_{z \theta}$ are calculated to determine the overall response of soil. Since a thick cylinder specimen is subjected to deformation, consideration of equilibrium alone cannot completely solve this 3-D problem. In addition, knowledge of the constitutive law of material is required to analyze this deformation problem.

$\sigma_{z}$ can be calculated directly from the vertical load and cross section of the specimen with the corrections for membrane stiffness, shaft friction and weight of the 
sand. The thick cylinder deformation problem has been solved for $\sigma_{\theta}, \sigma_{r}$, and $\tau_{z \theta}$ using an approximation of material behaviour. Symes et al. (1985) and Vaid et al. (1990b) calculated the directional stresses considering linearly elastic behaviour. $\sigma_{r}$ and $\sigma_{\theta}$ at a given radius $R$ were defined as given in Eqs. 3.1 and 3.2.

$$
\begin{aligned}
& \sigma_{r(a t r=R)}=\frac{P_{e} \cdot R_{e}^{2}-P_{i} \cdot R_{i}^{2}}{R_{e}^{2}-R_{i}^{2}}-\frac{\left(P_{e}-P_{i}\right) \cdot R_{e}^{2} \cdot R_{i}^{2}}{R_{e}^{2}-R_{i}^{2}} \cdot \frac{1}{R^{2}} \\
& \sigma_{\theta(a t r=R)}=\frac{P_{e} \cdot R_{e}^{2}-P_{i} \cdot R_{i}^{2}}{R_{e}^{2}-R_{i}^{2}}+\frac{\left(P_{e}-P_{i}\right) \cdot R_{e}^{2} \cdot R_{i}^{2}}{R_{e}^{2}-R_{i}^{2}} \cdot \frac{1}{R^{2}}
\end{aligned}
$$

Here, $R_{i}$ and $R_{e}$ are the internal and external radius of HCT specimen. $P_{i}$, and $P_{e}$ are the internal and external pressures.

Symes et al. (1985) have used the average of individual stresses as given in Eq. 3.3 and Vaid et al. (1990b) have considered the equilibrium of forces to derive the average $\sigma_{r}$ and $\sigma_{\theta}$ (Eq. 3.4). To calculate the average stresses, Miura et al. (1986) used a linear variation across the walls.

Symes et al. (1985): $\sigma_{r}=\frac{\int \sigma \cdot d r}{\int d r}$

Vaid et al. (1990b): $\sigma_{r}=\frac{\iint \sigma \cdot r d \theta \cdot d r}{\iint r d \theta \cdot d r}$

Eqs. 3.5, 3.6, 3.7 and 3.8 were used for this study to calculate directional stresses as proposed by Vaid et al. (1990b).

$$
\sigma_{z}=\frac{F_{z}+\pi \cdot\left(P_{e} \cdot R_{e}^{2}-P_{i} \cdot R_{i}^{2}\right)}{\pi\left(R_{e}^{2}-R_{i}^{2}\right)}
$$




$$
\begin{aligned}
& \sigma_{r}=\frac{\left(P_{e} \cdot R_{e}^{2}-P_{i} \cdot R_{i}^{2}\right)}{\left(R_{e}^{2}-R_{i}^{2}\right)}-\frac{2 \cdot\left(P_{e}-P_{i}\right) R_{e}^{2} R_{i}^{2} \ln \left(R_{e} / R_{i}\right)}{\left(R_{e}^{2}-R_{i}^{2}\right)^{2}} \\
& \sigma_{r}=\frac{\left(P_{e} \cdot R_{e}^{2}-P_{i} \cdot R_{i}^{2}\right)}{\left(R_{e}^{2}-R_{i}^{2}\right)}+\frac{2 \cdot\left(P_{e}-P_{i}\right) R_{e}^{2} R_{i}^{2} \ln \left(R_{e} / R_{i}\right)}{\left(R_{e}^{2}-R_{i}^{2}\right)^{2}} \\
& \tau_{z \theta}=\frac{4 \cdot T_{h} \cdot\left(R_{e}^{3}-R_{i}^{3}\right)}{3 \pi\left(R_{e}^{4}-R_{i}^{4}\right) \cdot\left(R_{e}^{2}-R_{i}^{2}\right)}
\end{aligned}
$$

Equations 3.9, 3.10, 3.11 and 3.12 were used for strain calculations assuming linear variation across the wall of specimen. The assumption of linear variation is consistent with the linear elastic response presumed in stress calculations.

$$
\begin{aligned}
& \varepsilon_{z}=-\frac{\Delta H}{H} \\
& \varepsilon_{r}=-\left(\frac{\Delta R_{e}-\Delta R_{i}}{R_{e}-R_{i}}\right) \\
& \varepsilon_{\theta}=-\left(\frac{\Delta R_{e}+\Delta R_{i}}{R_{e}+R_{i}}\right) \\
& \gamma_{z \theta}=\left(\frac{2 \Delta \theta\left(R_{e}^{3}-R_{i}^{3}\right)}{3 H\left(R_{e}^{2}-R_{i}^{2}\right)}\right)
\end{aligned}
$$

Here, $H$ and $\Delta H$ are height and change of height of the HCT specimen and $\Delta \theta$ is angular displacement. $\Delta R_{e}$ and $\Delta R_{i}$ are changes of external and internal radius, respectively. $\Delta R_{i}$ is calculated from measured values of $\Delta H$ and volume change of the inner chamber. $\Delta R_{e}$ is calculated from the measured value of $\Delta H$, volume change of specimen and $\Delta R_{i}$. 
In a soil element of a HCT specimen, shear stress along the direction of $r \theta$ and $r z$ is zero. Therefore, plane $r$ becomes one of the principal planes and normal stress acting on this plane is one of the principal stresses. The stress along direction $r$ is often identified as intermediate principal stress $\sigma_{2}$, and major $\sigma_{1}$ and minor $\sigma_{3}$ are calculated from $\sigma_{z}, \sigma_{\theta}$ and $\tau_{z \theta}$ using the principles of Mohr's circle. The principal strains are also calculated in a similar way.

\subsection{Stress nonuniformities in HCT specimens}

Use of a HCT device in soil testing is limited to an extent due to stress nonuniformities that are introduced in a HCT specimen during the application of torque or the different magnitudes of external and internal pressures. The degree of nonuniformity along the wall of a HCT specimen depends on stress state, specimen dimensions and constitutive law of soil.

Using finite element simulation, stress nonuniformities due to the curvature of HCT specimens have been studied by Hight et al. (1983), Sayao and Vaid (1991), and Wijewickreme and Vaid (1991). Hight et al. (1983) studied nonuniformities for individual stress components of $\sigma_{r}, \sigma_{\theta}$ and $\tau_{z} \theta \cdot \sigma_{z}$ that is independent of constitutive law and obtained by the equilibrium considerations was assumed uniform across specimen. $\sigma_{r}, \sigma_{\theta}$ and $\tau_{z} \theta$ were obtained by assuming soil as a linear elastic isotropic material. A coefficient $\beta_{3}$ was proposed by Hight et al. (1983) to estimate the stress nonuniformities and defined for each individual component as follows (Eq. 3.13).

$$
\beta_{3}=\frac{1}{R_{e}-R_{i}} \cdot \frac{1}{S_{L}} \int_{R_{i}}^{R_{e}}\left|S-S_{a v}\right| d r
$$


Here, $S$ is the stress component $\left(\sigma_{r}, \sigma_{\theta}\right.$ or $\left.\tau_{z}\right), S_{a v}$ is average stress component and $S_{L}$ is stress level, taken $\left(\tau_{z \theta}\right)_{a v}$ for $\tau_{z \theta}$ and $\frac{1}{2}\left[\left(\sigma_{r}\right)_{a v}+\left(\sigma_{\theta}\right)_{a v}\right]$ for $\sigma_{r}$ and $\sigma_{\theta}$. Hight et al. (1983) suggested that if $\beta_{3}<0.11$, level of stress nonuniformity can be considered acceptable.

In addition to the pressure difference and torque, rigid end restraint also contributes to the development of stress nonuniformities. The nonuniformities due to end restraint can be minimized by choosing appropriate dimensions and lubricated/polished end platens. The lubricated/polished end platens reduce radial shear at the end (Hight et al., 1983; Vaid et al., 1990b), nevertheless it may affect the transmission of torque load. To select the adequate dimensions of a specimen, Hight et al. (1983) have considered following criteria to choose $25.4 \mathrm{~mm}$ thickness of specimen's wall.

- Wall thickness should be sufficiently large relative to the maximum grain size of soils, so that the failure mechanism will not be constrained.

- Specimen volume change should be enough larger than the potential change of volume by membrane penetration.

- Uniform density is essential across the wall.

A ratio of 0.8 between inner radius and outer radius, and an outer radius of 127 $\mathrm{mm}$ were selected for a HCT specimen. No significant improvement in the stress nonuniformity was observed when the outer radius is larger than $127 \mathrm{~mm}$. In addition, height of HCT specimen $(254 \mathrm{~mm})$ was adopted to provide an adequate central gauge length for uniform stress and strain.

Sayao and Vaid (1991) adopted the inner and outer radii to be $51 \mathrm{~mm}$ and $76 \mathrm{~mm}$, respectively. For these selected dimensions, the stress nonuniformity coefficient of $\beta_{3}$ was 
acceptable level at certain stress state (less than 0.11). However, an unacceptable level of strain nonuniformities was introduced across the specimen's wall when a large variation of $R$ existed in the specimen's wall. In that sense, stress nonuniformity in terms of $R$ (Eq. 3.14) would be a more logical index than consideration of the individual stress component (Sayao and Vaid, 1991).

$\beta_{R}=\frac{R_{\max }-R_{\min }}{R_{a v}}$

Here, $R_{\max }$ and $R_{\min }$ are the highest and lowest level of stress ratios, respectively. $R_{a v}$ is average stress ratio. An acceptable level of nonuniformities could be considered when $\beta_{R} \leq 0.20 . \beta_{R} \leq 0.20$ corresponds to the maximum variation of $10 \%$ across the wall with respect to $R_{a v}$.

Sayao (1989) studied the influence of effective mean normal stress $\sigma_{m}^{\prime}$ and specimen geometry on stress nonuniformities at different $b_{\sigma}$ and $\alpha_{\sigma}$. In that study, soil was considered as linear elastic material. Analysis showed an unacceptable level of stress nonuniformities $\left(\beta_{R} \geq 0.20\right)$ when $R$ reached about 2.0 to 2.2 for the specimen geometry under consideration $\left(R_{e}=76 \mathrm{~mm}\right.$ and $\left.R_{i}=51 \mathrm{~mm}\right)$. This result revealed that most of the HCT devices having similar geometry in current soil testing are not suitable for generalized stress path loading if the nonuniformities are to be kept within the acceptable levels.

In addition, $\beta_{R}$ is not dependent on the level of $\sigma_{m}^{\prime}$ at a given stress state $\left(R, b_{\sigma}\right.$ and $\alpha_{\sigma}$ ), and the specimen geometry influences the nonuniformities at a given stress state. Based on experimental considerations and nonuniformities across the wall, it was suggested that specimen thickness within the range of 20 to $26 \mathrm{~mm}$, ratio between inner 
and outer radius $R_{i} / R_{e}$ within the range of 0.65 to 0.82 and ratio between height and outer diameter within the range of 1.8 to 2.2 would be suitable for a HCT specimen. Further, Sayao and Vaid (1991) have reported that nonuniformity analysis based on the linear elastic soil would be more severe than real nonlinear soil. However, errors from this analysis would be on the safe side in the selection of domain of stress space for the exploration of sand behaviour under generalized loading.

Wijewickreme and Vaid (1991) have studied stress nonuniformities in a HCT specimen based on incremental elastic hyperbolic stress-strain law, which represents the nonlinear soil behaviour well. In that study, effects of $\sigma_{m}^{\prime}, R$ and relative density on stress nonuniformities were examined. Based on the assumption of the nonlinear soil behaviour, $\beta_{R}$ did not increase continuously with increasing $R$ as noted in linear elastic assumption. $\beta_{R}$ reached a peak or even suffered a drop with a further increase of $R$. The influence of $\sigma_{m}^{\prime}$ on nonuniformity was small within the range of 100 to $900 \mathrm{kPa}$ that is mostly encountered in majority of the field problems. In addition, nonuniformities increased with the increase of relative density of the soil specimen. The loose sand specimens had a lesser degree of stress nonuniformity than that of dense specimens. The interpretation of HCT data of dense sand should be dealt with caution because of the high level of stress nonuniformities.

Based on linear elastic analysis, $\beta_{R}$ less than 0.2 was considered as an acceptable limit of nonuniformities to explore the soil using a HCT device. However, the realistic assumption of incremental elastic material yielded a much larger domain of general stress space even after the reduction of an acceptable $\beta_{R}$ of 0.1 . 


\section{EXPERIMENTAL WORK}

\subsection{Introduction}

This chapter details the experimental work carried out in pursuit of the objectives identified at the end of Chapter 1. A description of material tested, specimen reconstitution technique, specimen assembly, and performance and control of HCT are also provided.

\subsection{Material tested}

Fraser River sand dredged from the Fraser Delta in British Columbia was used for this research study. The sand was wet-sieved through a $0.075 \mathrm{~mm}$ sieve (\#200 sieve) to remove fine particles prior to testing. The removal of the fine particles was undertaken to enable the preparation of uniform specimens. The particle size distributions of the sand as received and after wet-sieving through $\# 200$ sieve are shown in Fig. 4.1. The amount of coarser particles larger than $0.850 \mathrm{~mm}$ (\#20 sieve) is negligible. The amount of fine particles less than $0.075 \mathrm{~mm}$ (\#200 sieve) is less than $2 \%$ in the original sand, so that the removal of fine particles is expected to not significantly alter the soil response.

Maximum and minimum void ratios of Fraser River sand, determined using ASTM D4253 (2001a) and D4254 (2001b) specifications are $e_{\max }=0.806$ and $e_{\min }=$ 0.509, respectively. The following information was obtained using ASTM standards for the test material: Average particle size $D_{50}=0.28 \mathrm{~mm}$, uniformity coefficient $C_{u}=2.92$, coefficient of curvature $C_{c}=1.27$ and specific gravity $G_{s}=2.67$. 


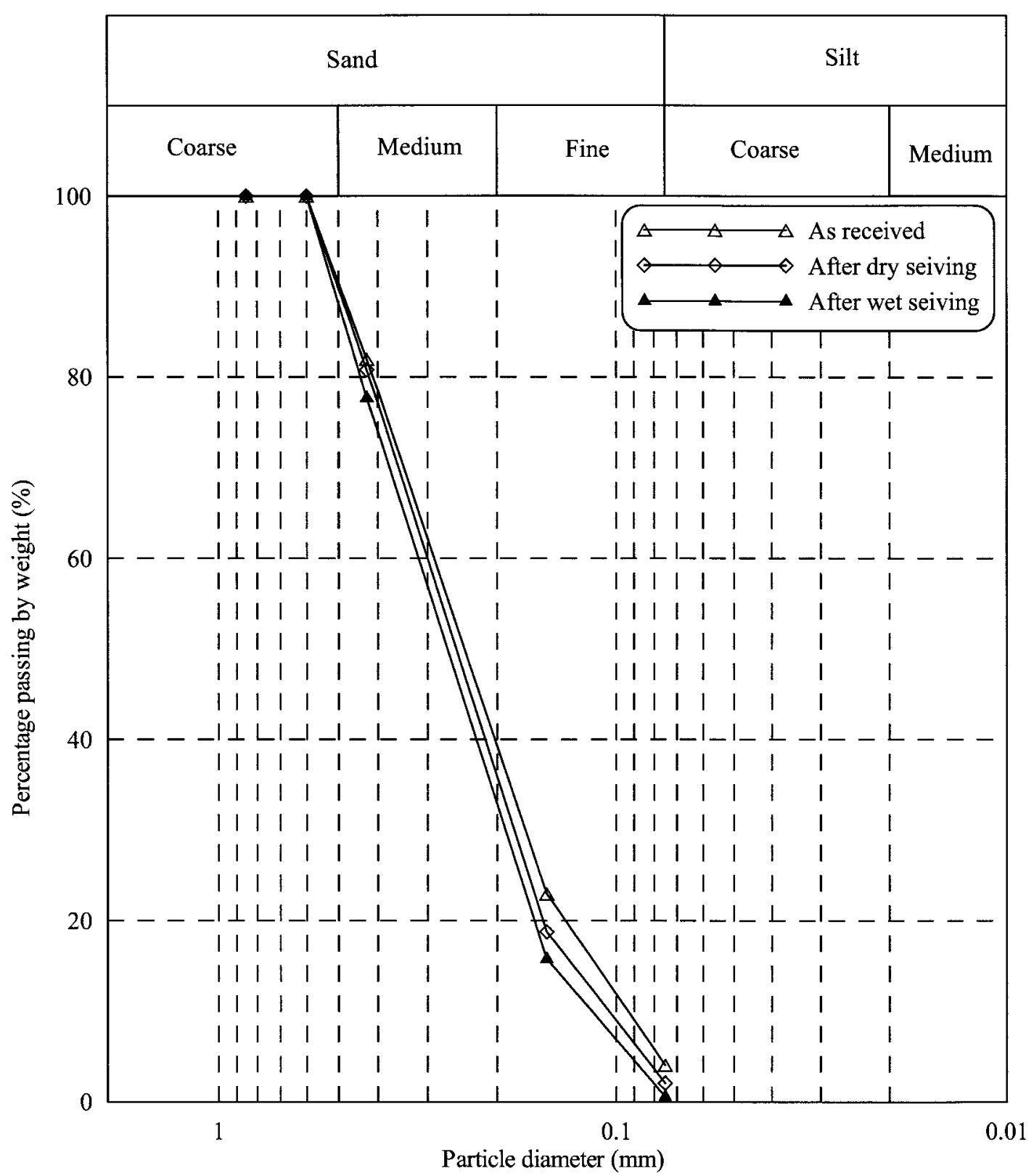

Fig. 4.1 Grain size distribution of Fraser River sand used

\subsection{Specimen reconstitution}

Undisturbed soil specimens would be expected to yield the true behaviour of insitu soils. However, obtaining undisturbed specimens from sandy deposits is a difficult task and conventional sampling techniques (e.g. borehole sampling) alter the mechanical properties of sandy soils (Seed et al., 1982). In-situ ground freezing technique is ideal (Yoshimi et al., 1977, 1978 \& 1989; Hatanaka et al., 1985) because it preserves the soil 
fabric during sampling. However, this method is extremely expensive and it is impossible to obtain several undisturbed specimens with identical characteristics for a systematic study.

Several reconstitution techniques have been used in laboratory studies to assess the fundamental behaviour of soils. The common techniques used for specimen reconstitution are pluviation (Kolbuszewski, 1948; Chaney and Mulilis, 1978; Miura and Toki, 1982) and moist tamping (Lambe, 1951; Ladd, 1978). The moist tamping technique compacts the sands at a lower water content, and as a result it produces looser void ratios on account of capillary forces. This technique may form a honeycomb like structure in the specimen (Casagrande, 1975).

Water pluviation is an excellent technique for specimen reconstitution as it yields uniform and repeatable specimens (Vaid and Negussey, 1988). Density of the water pluviated specimen is not generally affected by the height of particle drop (Vaid and Negussey, 1988), because particle deposition occurs at a constant velocity (terminal velocity) that is reached within a shorter drop height. Water pluviation simulates the natural deposition process in a hydraulic environment. Therefore, laboratory studies on water pluviated specimens represent the behaviour of natural soil deposits well (Oda et al., 1978; Miura and Toki, 1984).

Water pluviation technique yields fully saturated specimens, since soil particles are not exposed to air during the deposition process. However, this technique may result in particle segregation in well-graded soils. Sand used in this study is poorly graded and the percentage of fine particles is negligible. Therefore, this technique works well and produces homogenous specimens. 


\subsubsection{Preliminary steps of specimen reconstitution}

Sand was boiled in flasks to expel the air trapped between sand particles. After that, the flasks that contained the sand were allowed to cool at room temperature without air contact. Porous stones that are embedded into the end platens were also boiled to expel the air, and all drainage and pressure lines were saturated by flushing de-aired water.

A dial gauge was fixed to a movable stand and a reading was taken on the top of top platen during each step of specimen preparation. These readings were used to determine the initial specimen height after specimen preparation.

An inner membrane was positioned and sealed around the inner surface of the base platen. De-aired water was flushed through the base drainage line and the saturated porous stones were placed in the end platen. After that, the inner membrane was stretched around four pieces of an inner mould, which are positioned by two internal metallic discs and an annular base platen. An O-ring was positioned around the inner membrane at the top of the inner mould. An outer membrane was placed around the base platen and sealed by an O-ring. Next, two pieces of outer mould were placed. The outer membrane was flipped around the outer mould, which is made of porous plastic. Suction was applied around the inner surface of the outer mould to hold the membrane perfectly.

Figs. $4.2 \& 4.3$ show the details of the specimen deposition method and a schematic illustration of the specimen set up, respectively. Components of the specimen set up are labeled in both figures, and referred to in the descriptions of the specimen preparation and set up. 


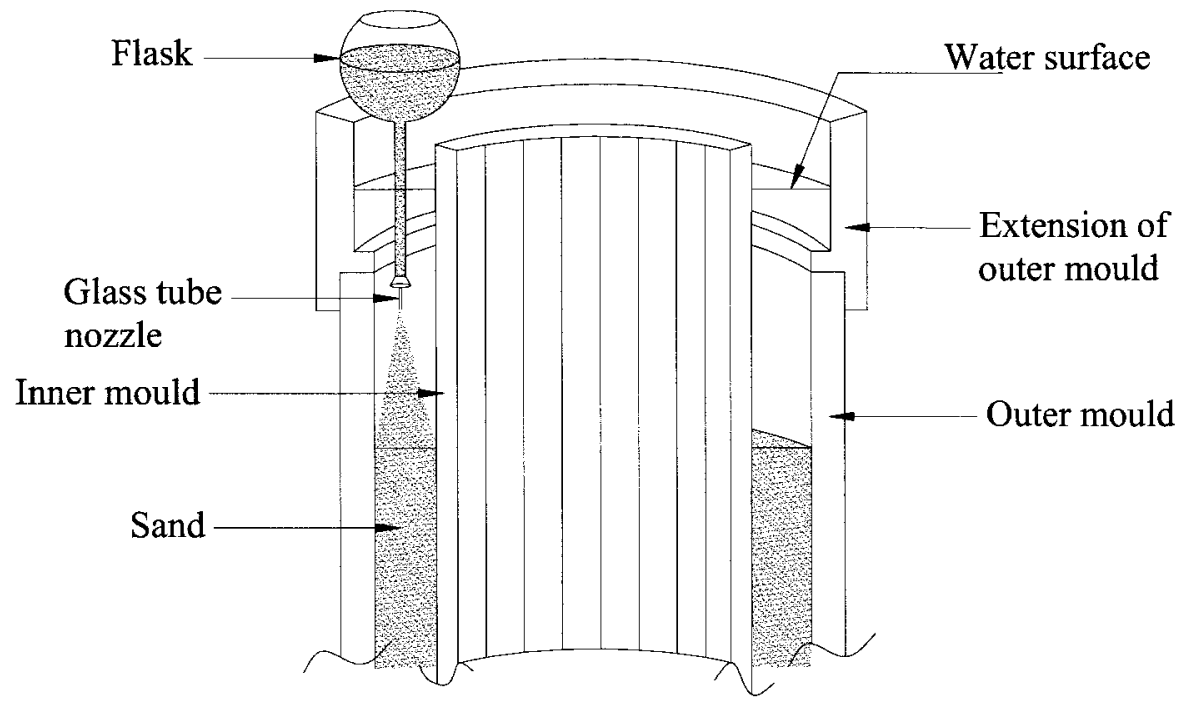

Fig. 4.2 HCT specimen preparation by water pluviation technique (After Sayao, 1989)

\subsubsection{Specimen preparation}

Circular cavity between inner and outer mould was completely filled with deaired water. The flasks that contained saturated sand were filled with de-aired water. Each flask was inverted into the circular cavity to drain the sand by gravity. During the deposition process, the flask was moved slowly along the annular cavity to maintain the approximately uniform level of deposited sand. The uniform level of sand ensures the horizontal plane of deposition. After filling the circular cavity with sand, excess sand was removed by siphoning and a leveled top surface was established. The siphoned sand was dried and weighed to determine the weight of sand used. The saturated porous stones were seated into the top platen and top platen was carefully placed on top of the specimen. De-aired water was percolated through with a small pressure gradient to remove entrapped air between the vertical wall of the top platen and the membranes. 


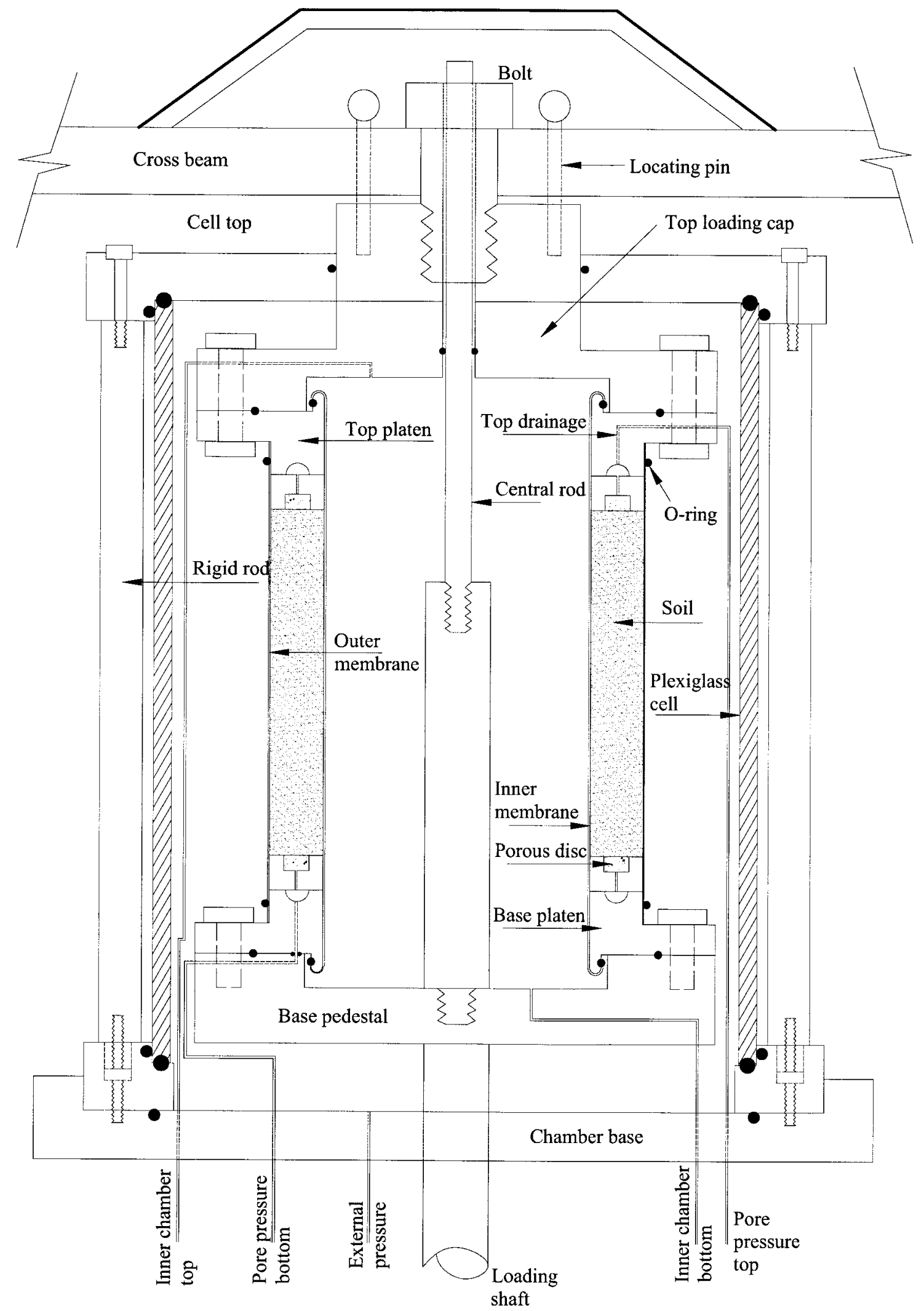

Fig. 4.3 Details of the HCT specimen set up 
The membranes were carefully flipped and o-rings were placed around the top platen. The top drainage valve was closed. After that, approximately $30 \mathrm{kPa}$ of suction was applied at the bottom of specimen to prevent the collapse of sand during removal of inner and outer moulds. From this stage to the end of the preparation, water removed from the specimen due to suction was recorded. The diameters of the specimen were corrected according to the membrane thickness. Finally, the top loading cap was carefully placed on the platen.

\subsubsection{Specimen setup}

The cell chamber was placed around the specimen and de-aired water was allowed to fill the inner and outer chambers simultaneously. After that, the cell top was placed on the cell chamber and the outer chamber was sealed. The top crossbeam was brought into position and fixed with the reaction frame. Installation of the central rod that is fixed at the loading shaft to measure the vertical displacement seals the inner chamber. The specimen was raised up slowly by increasing the pressure at the bottom of the double acting piston until a contact was made at the cross beam. Next, the top loading cap was fixed by a bolt to the cross beam to ensure vertical alignment of the specimen's axis and central axis of the frame. Two locating pins were inserted through the cross beam to avoid rotational movement of the loading cap during torque loading.

About $30 \mathrm{kPa}$ of confining pressure was applied initially under an undrained condition to increase pore pressure to a small positive value before starting the saturation test. Skempton's $B$-value was checked by several increments of confining pressure. Full saturation was confirmed by $B$ - value of about 0.99 in all tests. Subsequently, the specimen was consolidated and sheared according to the test requirements. 


\subsection{Calculation of void ratio}

Accurate calculation of the void ratio of a specimen is important in laboratory tests to enable valid comparison of test results. Specific gravity of soil particles, mass of dry soil and total volume of a specimen are required to calculate the void ratio. Traditionally, the volume of a specimen is calculated from measurements of height and diameters. The height of a specimen is measured accurately compared to the measurements of the diameters (inner and outer).

The diameters of the HCT specimen were measured as suggested by Vaid and Sivathayalan (1996). The outer diameter of the specimen was calculated by filling the membrane-lined cavity formed by the outer mould and measuring the height of the water. The outer diameter was accurately calculated from the mass of the water used to fill the cavity, the density of the water at room temperature and the height of water column. To calculate the inner diameter, the cavity formed by the inner and outer membranes was filled with water. The average inner diameter was determined from the mass and density of the water, and the height of the water column. During specimen preparation, volume change and height change of specimens were continuously measured, so that the void ratio was calculated confidently.

\subsection{Performance and control of the HCT device}

Simultaneous and independent control of four stress parameters is required to perform stress path control tests using a HCT device. The strain path tests with controlling stress parameter require not only the control of stress parameters but also the control of strain parameters. Depending on the test requirement, the following parameters were controlled in HCT tests. 
- Stress path controlled loading: Axial strain (independent control), torque, internal and external pressures OR torsional strain (independent control), axial stress, internal and external pressures

- Strain path controlled loading with control of stress parameters: Axial strain (independent control), volumetric strain, torque, internal and external pressures OR torsional strain (independent control), volumetric strain, axial stress, internal and external pressures

- Stress-controlled cyclic loading: Axial stress, torque, internal and external pressures

In order to follow prescribed stress/strain paths, smooth changes in pressures and loads should be applied either continuously or in small increments. Since the control system operates in a feedback loop, an appropriate loading rate was carefully chosen to get required adjustments in each stress parameter. To get proper response from analog input and output devices, a proper time delay was allowed for the hardware to reach target stress states. At the beginning of the study, a few tests were performed on the newly built HCT device to select an appropriate loading rate. When axial load was the independent control, a loading rate of $2 \%$ of axial strain per hour yielded satisfactory results. When torque was the independent control, a loading rate of $4 \%$ of torsional strain per hour was applied.

Most of the undrained and strain path controlled tests were conducted on isotropically consolidated specimens $\left(\sigma_{m c}^{\prime}=200 \mathrm{kPa}\right)$, and on a few anisotropically consolidated specimens. Fig. 4.4 shows the stages of an anisotropic consolidation process and the control of stresses. It can be noted that directional stresses $\left(\sigma_{z}, \sigma_{\theta}\right.$ and $\left.\sigma_{r}\right)$ were 
increased simultaneously to maintain the required stress state during the consolidation process. The consolidation stress was applied in increments of $25 \mathrm{kPa}$. A time interval of 3 minutes was allowed to complete the drainage before the next increment. Each $25 \mathrm{kPa}$ of increment was applied in 10 small increments of $2.5 \mathrm{kPa}$ continuously within the period of $15 \mathrm{sec}$. A $1.5 \mathrm{sec}$ of delay was provided during each small increment of stress $(2.5 \mathrm{kPa})$ to the analog input and output hardware (EPTs and step motors, transducers) to reach an equilibrium state.

Fig. 4.5 shows the capability of the HCT device to target required stress control parameters $\left(\Delta \sigma_{m}=456 \mathrm{kPa}, \alpha_{\sigma}=45^{\circ}\right.$ and $\left.b_{\sigma}=0.00\right)$. This plot indicates that prescribed stress paths are followed faithfully by the control system of the HCT device.

\subsection{Repeatability of HCT tests}

Reproduction of same relative density, replication of structure, measurement accuracy and exact duplication of test routine promote repeatability of tests. The presence of fines in soil leads to slower consolidation rate. To minimize the time dependent effect due to the presence of fines and to obtain a constant time dependent effect on Fraser River sand, a fixed time interval was allowed during consolidation for all specimens. Responses of two identical specimens of Fraser River sand under undrained loading are shown in Fig. 4.6. These specimens were consolidated to the same effective stress state and the same relative density prior to undrained compression loading. Identical responses confirm good repeatability of the specimen preparation technique and capabilities of the HCT device. 

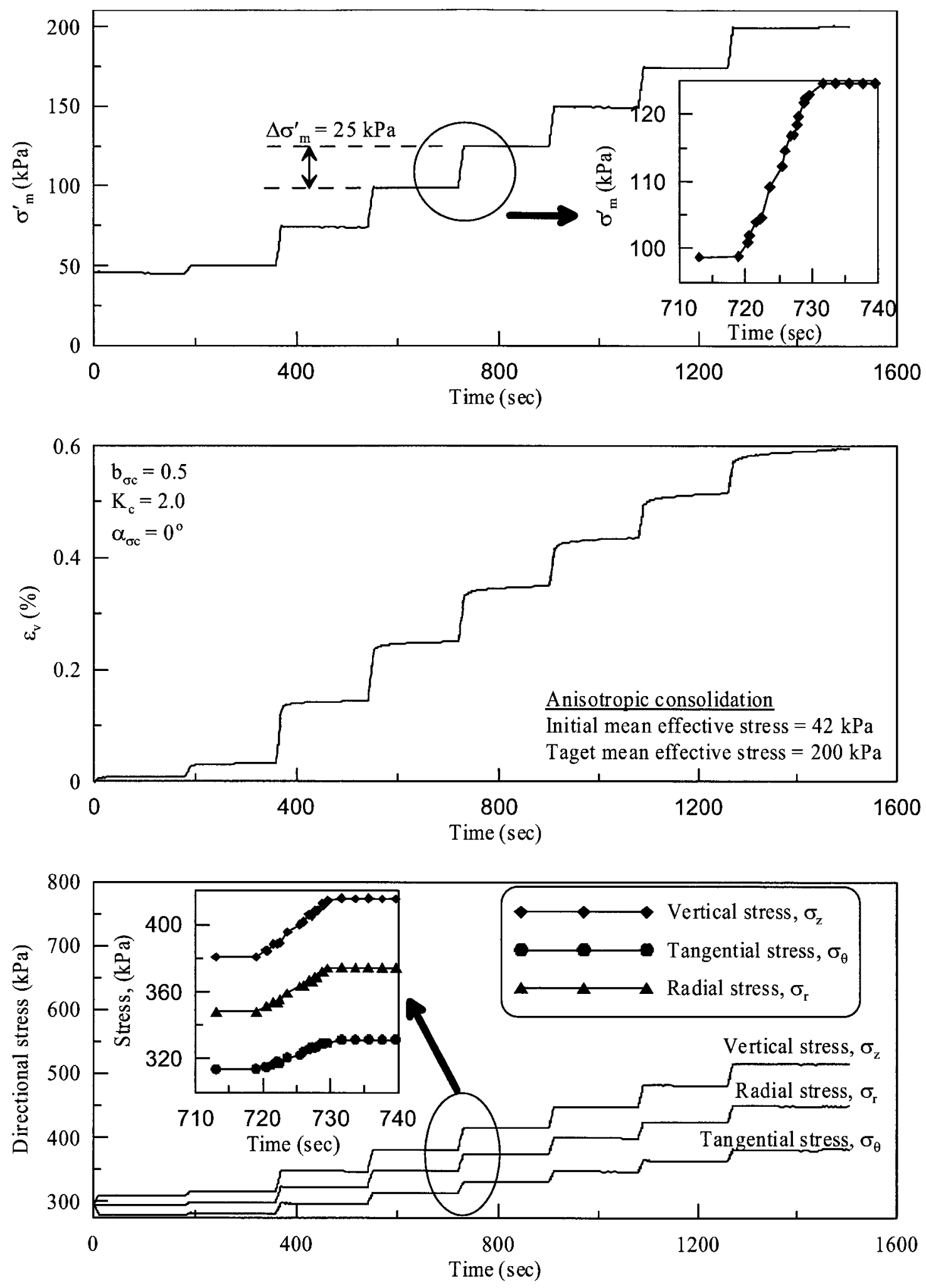

Fig. 4.4 Control of stresses and measured volumetric strain during anisotropic consolidation 

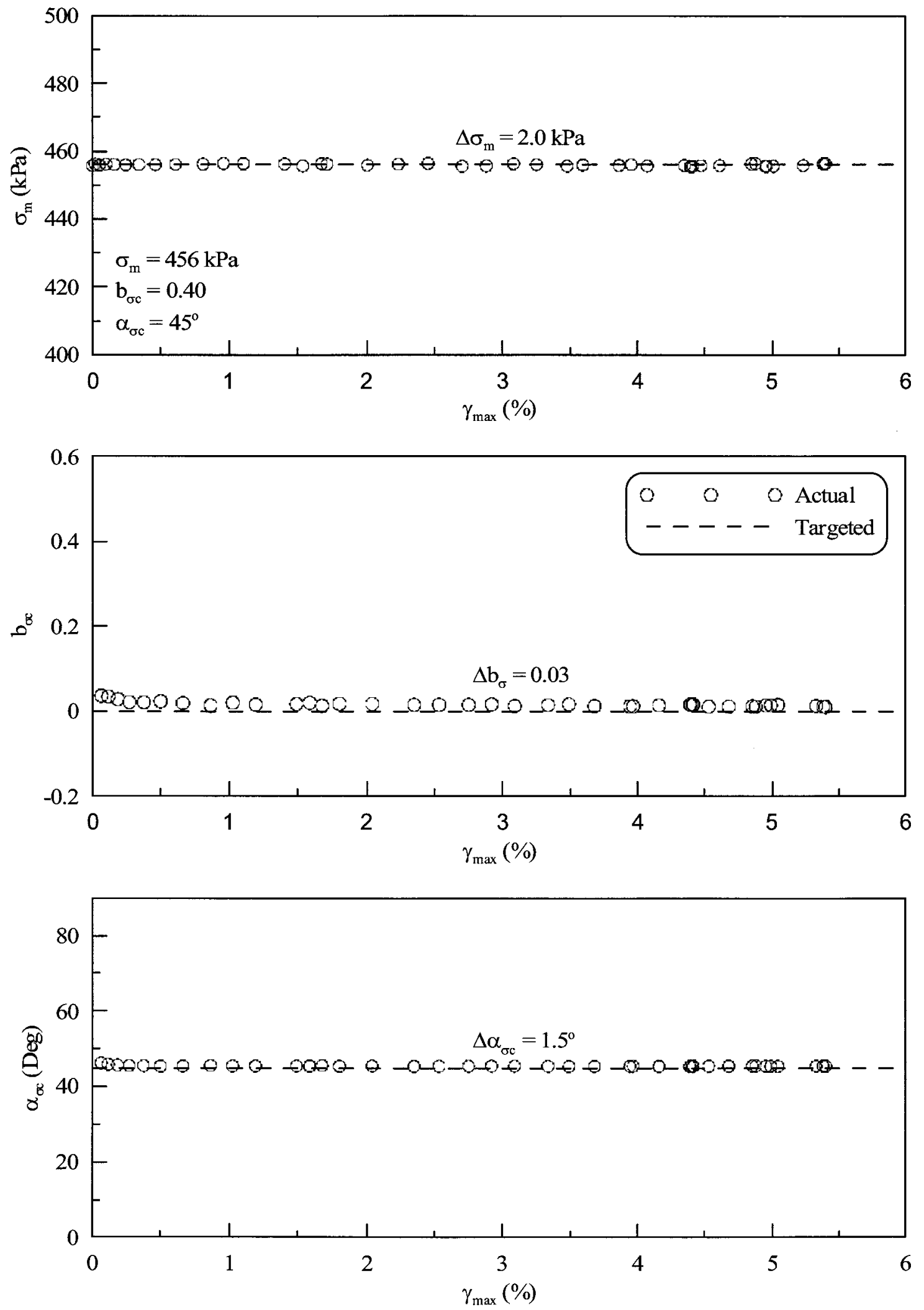

Fig. 4.5 Targeted and actual control parameters followed in typical HCT test 

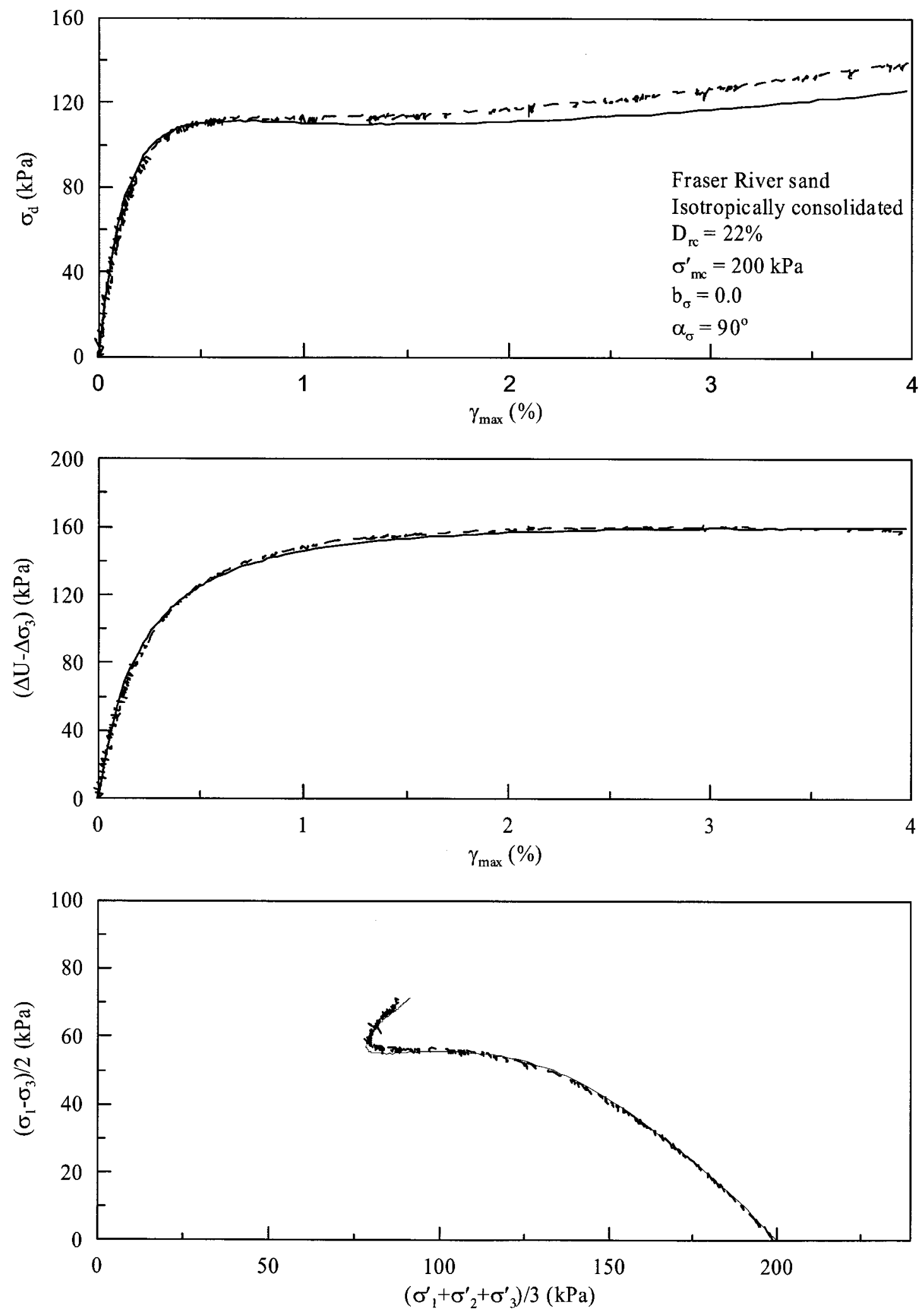

Fig. 4.6 Repeatability of HCT undrained test at $b_{\sigma}=0.0$ and $\alpha_{\sigma}=90^{\circ}$ 


\section{MONOTONIC RESPONSE UNDER GENERALIZED LOADING AND DRAINAGE CONDITIONS}

\subsection{Introduction}

This chapter presents the behaviour of Fraser River sand under undrained (no drainage) and strain path controlled loading that imposed simultaneous changes in pore pressure and volume during shearing. These tests were performed under threedimensional loading using the new HCT device that was built to perform both stress controlled and displacement controlled loading.

Specimens were reconstituted at loosest state, and the relative density at the end of consolidation ranged between 20.5 and $23 \%$. The effective stress state of the specimens at the end of consolidation was dependent on the test requirement. For convenience, stress state at the end of consolidation is represented by effective mean normal stress $\sigma_{m c}^{\prime}$, intermediate principal stress parameter $b_{\sigma c}$, effective stress ratio $K_{c}$ and principal stress direction $\alpha_{\sigma c}$.

In undrained tests, influence of stress parameters $\alpha_{\sigma}, b_{\sigma}$ and $K c$ were studied. In strain path controlled tests, influence of the same stress parameters were studied by imposing linear strain paths. During strain path controlled tests, drainage of the specimens was controlled by injecting or extracting an appropriate volume of water from soil's specimen. To make this process systematic, the imposed volumetric strain was linearly related to maximum shear strain induced, such that the ratio between volumetric strain and maximum shear strain was constant. Details of the monotonic test program and the test variables are given in Table 5.1. 
Table 5.1 Initial and loading states of undrained and strain path controlled tests

\begin{tabular}{|c|c|c|c|c|c|c|c|c|}
\hline \multirow{2}{*}{$\begin{array}{l}\text { Test } \\
\text { No }\end{array}$} & \multicolumn{5}{|c|}{ Consolidation state } & \multicolumn{2}{|c|}{$\begin{array}{l}\text { Loading } \\
\text { state }\end{array}$} & \multirow[t]{2}{*}{ Remarks } \\
\hline & $\begin{array}{l}D_{r c} \\
(\%) \\
\end{array}$ & $\begin{array}{c}\sigma_{m c}^{\prime} \\
(\mathrm{kPa})\end{array}$ & $b_{\sigma c}$ & $\begin{array}{c}\alpha_{\sigma c} \\
(\mathrm{Deg})\end{array}$ & $K_{c}$ & $b_{\sigma}$ & $\begin{array}{c}\alpha_{\sigma} \\
(\mathrm{Deg})\end{array}$ & \\
\hline M1 & 21.3 & 200 & - & - & 1.0 & 0.0 & 0 & \multirow{5}{*}{$\sigma_{\mathrm{d}} \uparrow$} \\
\hline M2 & 22.2 & 200 & - & - & 1.0 & 0.2 & 0 & \\
\hline M3 & 21.9 & 200 & - & - & 1.0 & 0.4 & 0 & \\
\hline M4 & 23.0 & 200 & - & - & 1.0 & 0.7 & 0 & \\
\hline M5 & 22.5 & 200 & - & - & 1.0 & 1.0 & 0 & \\
\hline M6 & 21.3 & 200 & - & - & 1.0 & 0 & 30 & \multirow{4}{*}{$\sigma_{\mathrm{d}}$ Undrained } \\
\hline M7 & 22.8 & 200 & - & - & 1.0 & 0 & 45 & \\
\hline M8 & 21.0 & 200 & - & - & 1.0 & 0 & 60 & \\
\hline M9 & 22.0 & 200 & - & - & 1.0 & 0 & 90 & \\
\hline M10 & 21.2 & 200 & - & - & 1.0 & 0 & 0 & \multirow{4}{*}{ Undrained } \\
\hline M11 & 22.7 & 200 & 0.0 & 0 & 1.5 & 0 & 0 & \\
\hline M12 & 21.9 & 200 & 0.0 & 0 & 2.0 & 0 & 0 & \\
\hline M13 & 22.4 & 200 & 0.0 & 0 & 2.5 & 0 & 0 & \\
\hline
\end{tabular}




\begin{tabular}{|c|c|c|c|c|c|c|c|c|}
\hline M14 & 20.5 & 200 & - & - & 1.0 & 0.0 & 0 & \multirow{6}{*}{$\underbrace{\uparrow \uparrow \alpha_{\sigma}}_{0.2}$} \\
\hline M15 & 22.9 & 200 & - & - & 1.0 & 0.2 & 0 & \\
\hline M16 & 21.0 & 200 & - & - & 1.0 & 0.4 & 0 & \\
\hline M17 & 20.6 & 200 & - & - & 1.0 & 0.6 & 0 & \\
\hline M18 & 20.5 & 200 & - & - & 1.0 & 0.8 & 0 & \\
\hline M19 & 20.8 & 200 & - & - & 1.0 & 1.0 & 0 & \\
\hline M20 & 20.5 & 200 & - & - & 1.0 & 0 & 0 & \multirow{3}{*}{ 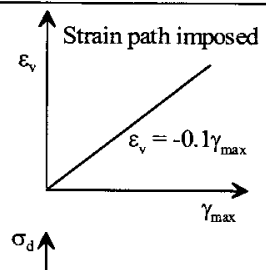 } \\
\hline M21 & 22.2 & 200 & - & - & 1.0 & 0 & 30 & \\
\hline M22 & 20.8 & 200 & - & - & 1.0 & 0 & 45 & \\
\hline M23 & 21.7 & 200 & - & - & 1.0 & 0 & 60 & \\
\hline M24 & 22.2 & 200 & - & - & 1.0 & 0 & 90 & \\
\hline M25 & 20.2 & 200 & - & - & 1.0 & 0 & 0 & $\varepsilon_{\mathrm{v}}$ \\
\hline M26 & 20.8 & 200 & 0.0 & 0 & 1.5 & 0 & 0 & \\
\hline M27 & 21.1 & 200 & 0.0 & 0 & 2.0 & 0 & 0 & \\
\hline M28 & 22.2 & 200 & 0.0 & 0 & 2.5 & 0 & 0 & \\
\hline M29 & 21.9 & 200 & - & - & 1.0 & 0.4 & 45 & $\varepsilon_{v} \uparrow$ \\
\hline M30 & 21.0 & 200 & - & - & 1.0 & 0.4 & 45 & \\
\hline M31 & 22.1 & 200 & - & - & 1.0 & 0.4 & 45 & \\
\hline M32 & 22.2 & 200 & - & - & 1.0 & 0.4 & 45 & \\
\hline
\end{tabular}




\subsection{Drainage boundary conditions}

Fig. 5.1 shows an illustration of possible drainage and stress paths that a soil element might undergo during deformation. The paths shown in this figure correspond to different drainage conditions under typical triaxial compression loading in loose sand (Sivathayalan and Logeswaran, 2007). The drainage boundary conditions dictate the effective stress paths under a given loading scenario. In drained conditions, the effective stress increment is equal to the total stress increment and pore pressure remains constant. Type 2 response represents a typical drained response of sand under triaxial compression. Type 1 response corresponds to undrained condition, which refers to deformation at constant volume (no drainage is allowed). Type 3 response is termed as partial drainage, in which complete drainage is not allowed. Only a portion of drainage compared to the drained test (free draining) is allowed. This scenario may occur in the field when loading rate is slow in a fine-grained soil, or when coarse-grained soils are subject to rapidly changing loads. The volumetric strain of soil is bounded by drained and undrained volumetric responses, and hence the stress path is expected to be bound by the drained and undrained stress paths.

Types 4 and 5 may occur in the field when pore pressure boundary condition is different from that of the pore pressure of the soil element in consideration (Fig. 2.14). The existence of a piezometric gradient between adjacent or surrounding soil elements may lead to such volumetric change. The soil element subjected to a lower piezometric head would experience pore water inflow causing expansive volume change as the soil element undergoes shear deformation (Type 4). The soil element having a higher piezometric gradient would undergo pore volume outflow and may experience 
contractive volume change (Type 5). Due to the loss of pore volume, the soil element may experience the drainage more than the full drainage.
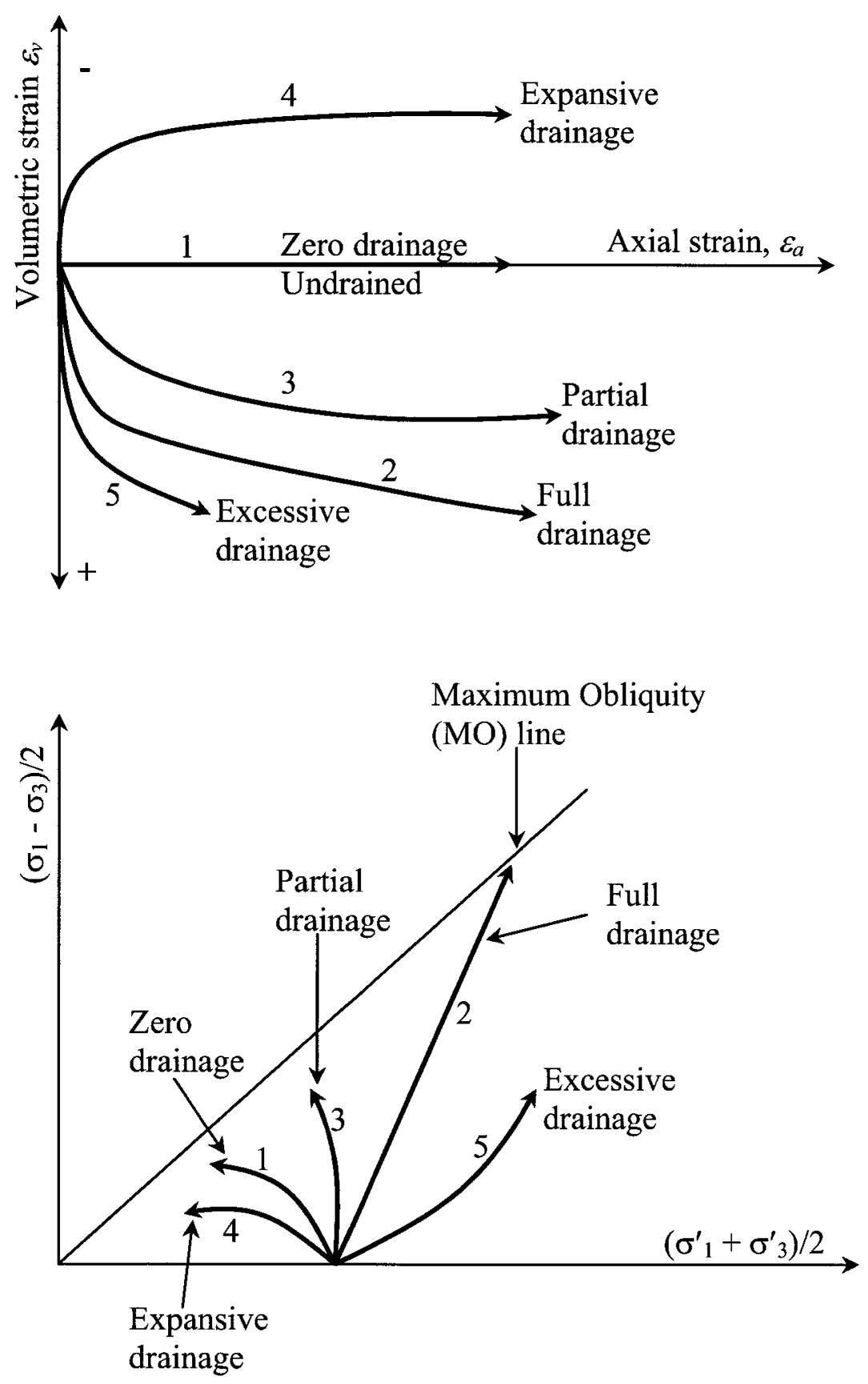

Fig. 5.1 Schematic illustration of conditions of drainage: strain paths and stress paths (After Logeswaran, 2005) 
The volume inflow contributes to the development of higher excess pore pressure in the soil element. The induced pore pressure in the soil element in this case would be due to both the volume transfer and shearing. In the case of volume inflow, stress paths are expected to be below the undrained stress path. The adjacent soil element subjected to volume outflow experiences loss of pore pressure, such that the pore volume of the soil element reduces. The stress paths are expected to be on the right side of the drained response. The behaviour of soils along the strain paths described by paths 4 and 5 have also been called 'partially drained' in the literature (Vaid and Eliadorani, 1998). However, the nature of boundary conditions leading to such strain paths is quite different from those characterized by Type 3 that is bounded by drained and undrained paths. To distinguish the responses, Type 4 has been named expansive drainage and Type 5 has been named excessive drainage by Sivathayalan and Logeswaran (2007).

The effect of stress path on the undrained and drained behaviour of soils has been discussed in the literature (e.g., Vaid et al., 1990a; Smith et al., 1992), but the paths explored have been limited to typical loading modes and were not representative of all possible drainage boundary conditions. Lade et al. (1988) and Chu et al. (1992) have attempted to understand the effects of drainage boundary conditions on the potential of instability in granular soils. Vaid and Eliadorani (1998 \& 2000), and Sivathayalan and Logeswaran (2007) have assessed the potential of strain softening and the effect of different stress increment vectors on secant shear modulus at different drainage conditions. These previous studies were based solely on triaxial type loadings. In this research program, an attempt was made to explore the soil behaviour by imposing drainage conditions under generalized loading. 


\subsection{Undrained behaviour of sand}

The undrained condition is created when the excess pore pressure cannot be released during the increment of stress. The soil that has the tendency to compress (contractive) generates positive excess pore pressure, thus experiences a reduction of effective stress between soil particles. The soil that has a tendency to expand (dilative) the volume generates negative excess pore pressure. The negative excess pore pressure increases the effective stress between soil particles. Most soils exhibit contractive tendencies (and hence positive excess pore water pressure) during early stages of loading, therefore, the undrained condition is often considered critical in geotechnical practice.

\subsubsection{Undrained response of sand at fixed $b_{\sigma}$}

Five tests were performed on Fraser River sand at $\alpha_{\sigma}=0^{\circ}$ and $b_{\sigma}=0.0,0.2,0.4$, 0.7 and 1.0 during shearing. Initially, the specimens were isotropically consolidated to $\sigma_{m c}^{\prime}=200 \mathrm{kPa}$ and $D_{r c}$ was in a narrow range between 21 and $23 \%$.

Induced principal strains during undrained loading are illustrated in Fig. 5.2. Intermediate principal strain $\varepsilon_{2}$ varies from positive to negative when $b_{\sigma}$ changes from 0 to 1.0. $\varepsilon_{2}$ is close to zero at $b_{\sigma}=0.4$ that corresponds to the plane strain deformation. This observation is consistent with the findings of previous researchers (Uthayakumar and Vaid, 1998; Yoshimine et al., 1998; Sivathayalan, 2000). The strain paths referred to a fixed coordinate system are essentially linear. This observation implies that the degree of initial anisotropy in sand specimens preserved during shearing and induced anisotropy is not profoundly developed. 

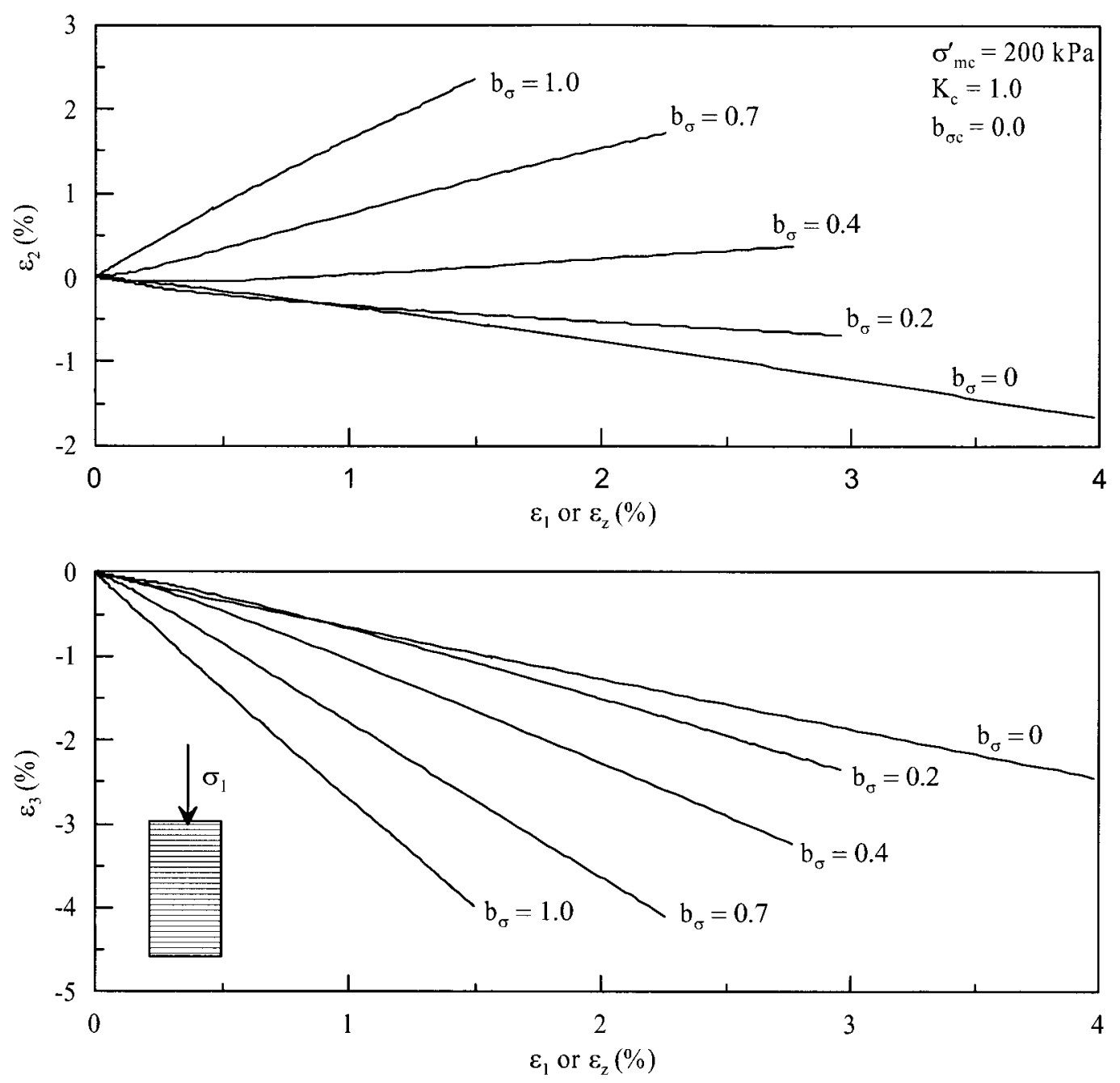

Fig. 5.2 Variation of principal strains with vertical strain during undrained shear tests at fixed $b_{\sigma}$

The stress-strain, pore pressure responses and the stress paths followed during these tests are shown in Fig. 5.3. The sand experiences strain hardening, regardless of the $b_{\sigma}$ value. Increase of $b_{\sigma}$ from 0.0 to 1.0 makes sand relatively softer. This observation is consistent with the data reported in the literature (Uthayakumar and Vaid, 1998; Yoshimine et al., 1998). Higher excess pore pressure is generated at $b_{\sigma}=1.0$ and lower excess pore pressure is generated at $b_{\sigma}=0.0$. A systematic variation of excess pore pressure generation is expected when $b_{\sigma}$ increases from 0.0 to 1.0 . 

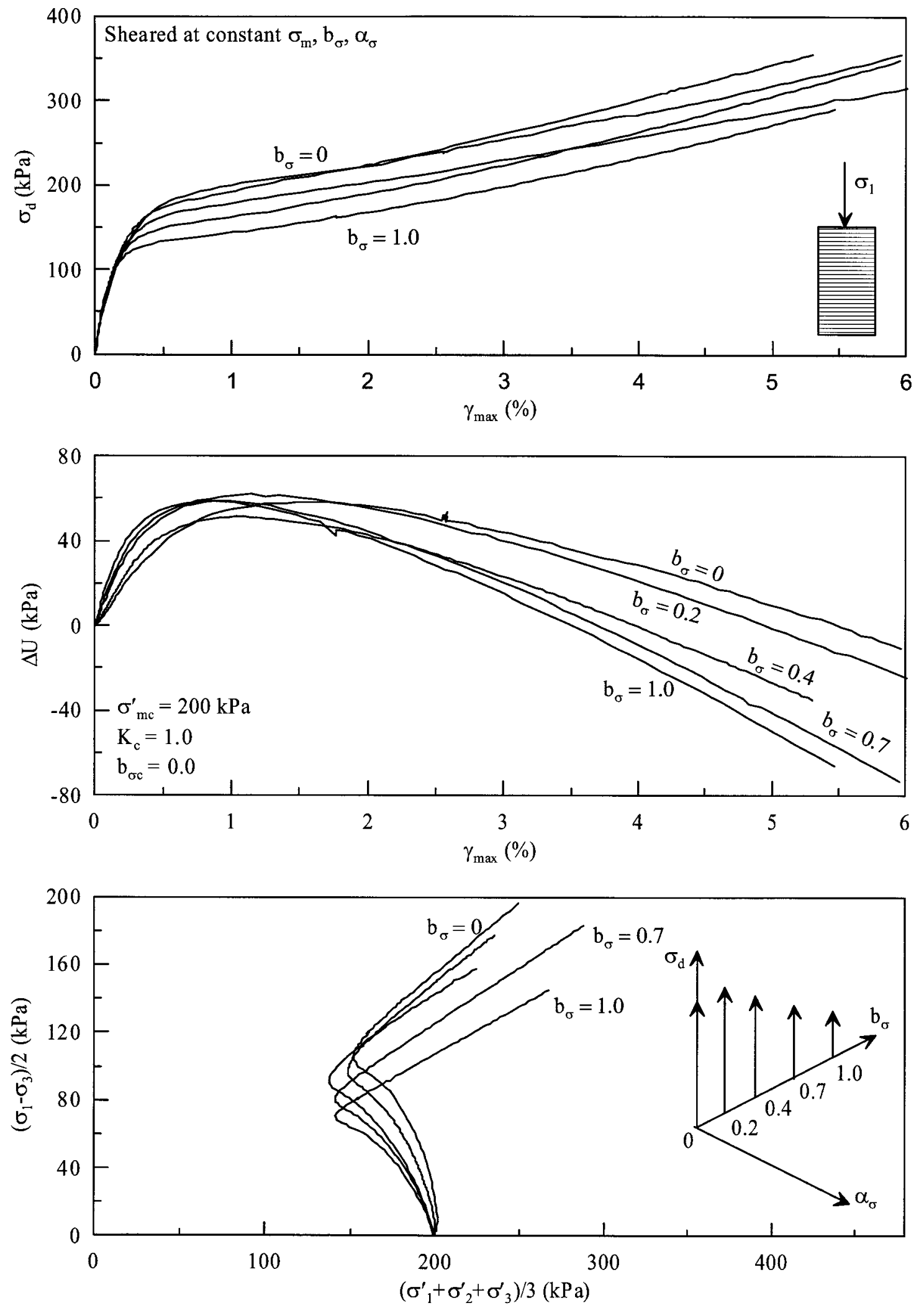

Fig. 5.3 Undrained response of Fraser River sand at fixed $b_{\sigma}\left(\alpha_{\sigma}=0^{\circ}\right)$ 
Shear strength $S_{P T}$, induced excess pore pressure $\Delta U_{P T}$, and stress state at PT are shown in Fig. 5.4. $S_{P T}$ systematically reduces with increasing $b_{\sigma}$. About $33 \%$ of strength reduction at PT state is noted as $b_{\sigma}$ changes from 0.0 to $1.0\left(S_{P T}\right.$ at $b_{\sigma}=0.0$ is $105 \mathrm{kPa}$ and $S_{P T}$ at $b_{\sigma}=1.0$ is $\left.70 \mathrm{kPa}\right) . \Delta U_{P T}$ increases noticeably from $b_{\sigma}=0.0$ to $0.4(45$ to $60 \mathrm{kPa})$. However, when $b_{\sigma}$ is greater than 0.4 , changes in $\Delta U_{P T}$ are insignificant. The stress states at PT lie along a straight line passing through the origin and result the friction angle at PT $\phi_{P T}$ of $34.2^{\circ}$. The observed $\phi_{P T}$ is consistent with previous studies (Uthayakumar, 1995; Sivathayalan, 2000; Logeswaran, 2005) for the same batch of Fraser River sand. It has also been noted in previous research (Uthayakumar, 1995) that $\phi_{P T}$ is a unique soil property and it is independent of $b_{\sigma}$.

Previous studies on this sand (Logeswaran, 2005), reported the friction angle at Maximum Obliquity (MO) $\phi_{M O}$ to be $37^{\circ}$ under triaxial compression. The test conducted at $b_{\sigma}=0$ and $\alpha_{\sigma}=0^{\circ}$ (triaxial mode of compression) results in $\phi_{M O}$ of $38^{\circ}$. It is noted that $\phi_{M O}$ slightly increases with increasing $b_{\sigma}$. Estimated $\phi_{M O}$ at $b_{\sigma}=1.0$ is $42^{\circ}$. This deviation of $\phi_{M O}$ could be due to stress nonuniformity induced across the specimen's wall. To justify the noted deviation in $\phi_{M O}$, Fig. 5.5 shows the variation of the coefficient of stress nonuniformity in terms of $b_{\sigma}$ and $\alpha_{\sigma}$ as illustrated by Wijewickreme (1990). In this figure, the stress nonuniformity in a hollow cylinder test was assessed by finite element modeling assuming a constitutive theory. Even though assumption of isotropic linear elasticity and hardening elasto-plasticity theories suggested different levels of stress nonuniformity in the HCT specimen at a given stress state, high stress nonuniformity was realized at $b_{\sigma}=0$ and $\alpha_{\sigma}=90^{\circ}$. Further, these model studies have assumed that stress nonuniformity is zero at $b_{\sigma}=0$ and $\alpha_{\sigma}=0^{\circ}$ and its closer values. 

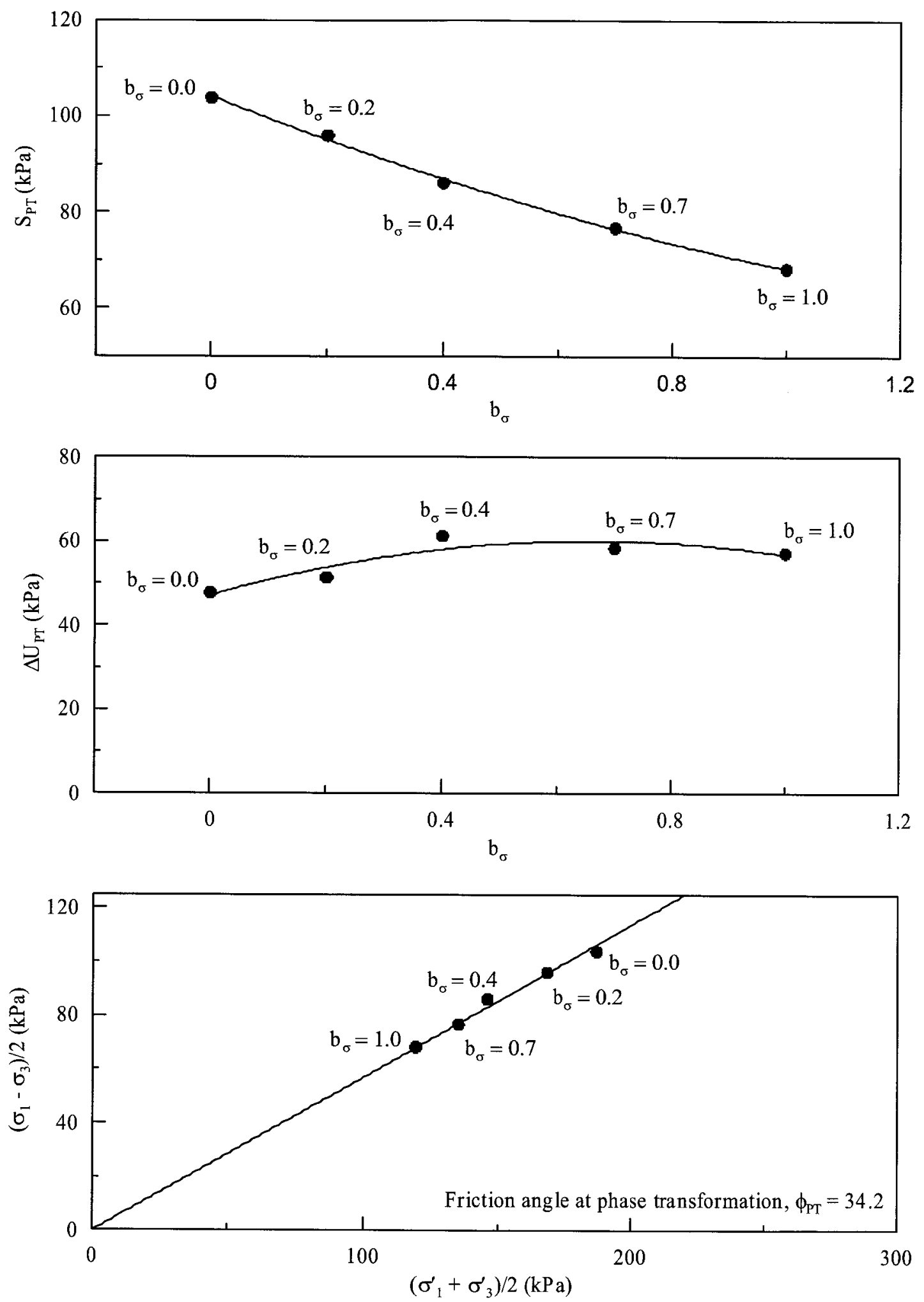

Fig. 5.4 Variation of strength, excess pore pressure and stress state at PT state 


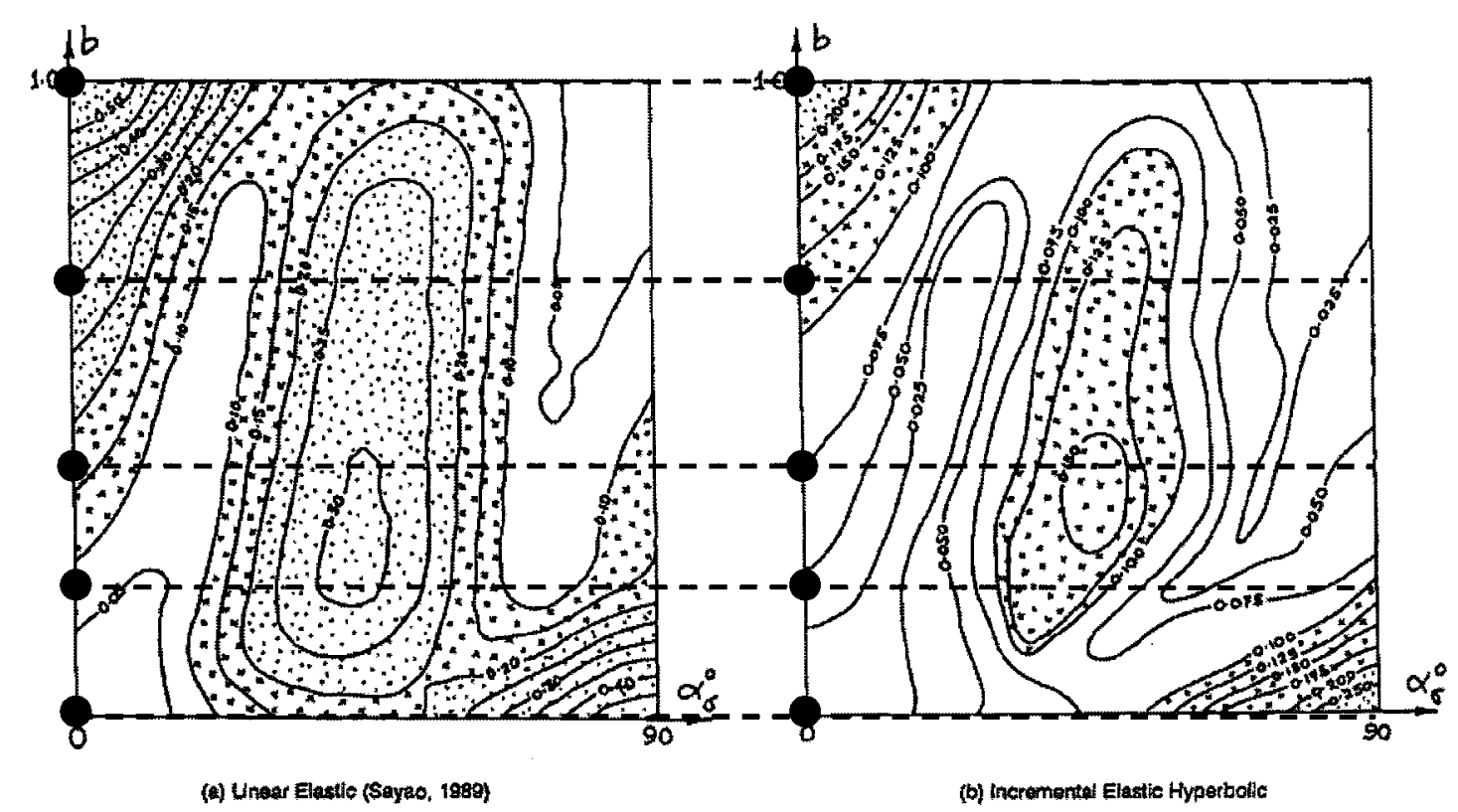

Fig. 5.5 Contours of coefficient of nonuniformity at $R=3.0, \sigma_{m}^{\prime}=300 \mathrm{kPa}$ and $D_{r}$ $=30 \%$ (After Wijewickreme, 1990)

Stress nonuniformity is also dependent on mobilized effective stress ratio $R$. Fig. 5.6 shows the variation of stress nonuniformity at different levels of $R$ with $\alpha_{\sigma}$ as reported by Wijewickreme (1990). Increase of mobilized $R$ essentially increases stress nonuniformity at $b_{\sigma}=0$ with increasing $\alpha_{\sigma}$.

As shown in Fig. 5.3, $\phi_{M O}$ increases with increasing $b_{\sigma}$. At $b_{\sigma}=1.0, R$ at $\mathrm{MO}$ is comparatively high (around $R=4.5$ ) and the difference between the inner and outer pressure reaches as high as $120 \mathrm{kPa}$, which implies relatively large stress non-uniformity. Thus, the higher $\phi_{M O}$ at higher $b_{\sigma}$ values might be reflection of the higher level of stress nonuniformity induced at such states. $\phi_{P T}$ at $b_{\sigma}=1.0$ is approximately equal to $\phi_{P T}$ at $b_{\sigma}=$ 0 . In PT state of the test at $b_{\sigma}=1.0$, the specimen experiences a comparatively lower $R$ of 3.0 and the difference between inner and outer pressure is comparatively low (around 60 $\mathrm{kPa}$ ). The stress nonuniformities at high stress ratios make the tests at higher $b_{\sigma}$ and lower $\alpha_{\sigma}\left(\right.$ or lower $b_{\sigma}$ and higher $\alpha_{\sigma}$ ) less reliable. 

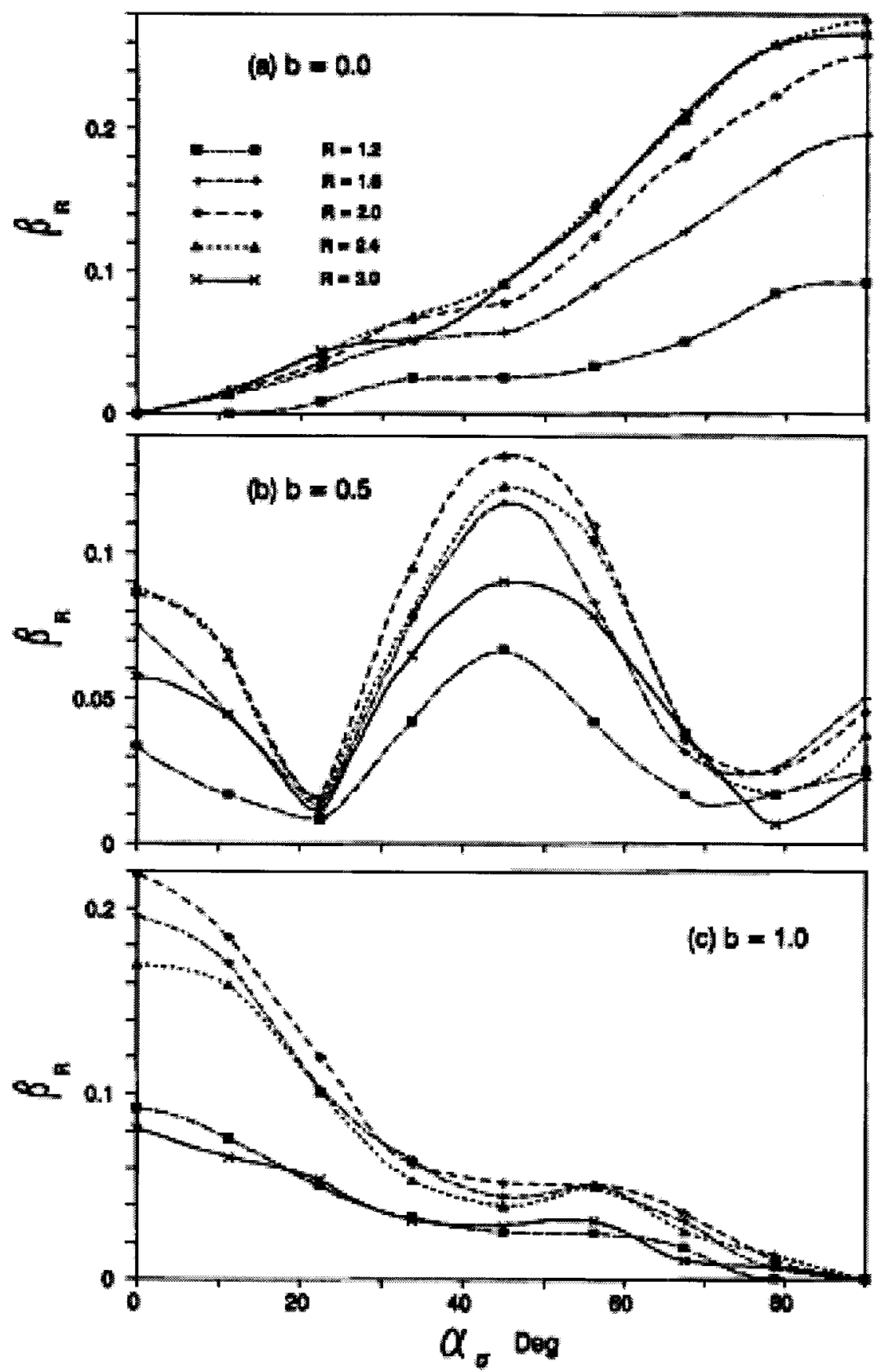

Fig. 5.6 Effect of stress ratio on coefficient of nonuniformity at $\sigma_{m}^{\prime}=300 \mathrm{kPa}$ and $D_{r}=30 \%($ After Wijewickreme, 1990) 


\subsubsection{Undrained response of sand at fixed $\alpha_{\sigma}$}

A series of tests were conducted to investigate the influence of principal stress direction $\alpha_{\sigma}$ on the undrained behaviour of Fraser River sand $\left(\alpha_{\sigma}=0^{\circ}, 30^{\circ}, 45^{\circ}, 60^{\circ}\right.$ and $90^{\circ}$ ). The intermediate principal stress parameter $b_{\sigma}$ was maintained at zero; thus, intermediate principal stress was equal to minor principal stress. The specimens were isotropically consolidated to $\sigma_{m c}^{\prime}=200 \mathrm{kPa}$ and relative density of the specimens was approximately $22 \%$.

The strain paths (Fig. 5.7) are essentially linear for all tests at the small strain level, but progressively become nonlinear at large strains. This observation implies that the degree of initial anisotropy is preserved during the early stages of shearing, and that strain induced anisotropy progressively increases during shearing and plays a role in strain development.

Fig. 5.8 shows stress-strain and pore pressure responses and effective stress paths of Fraser River sand at different $\alpha_{\sigma}$ values. The degree of anisotropy was found to be profound during undrained shear. When the direction of major principal stress changes from $\alpha_{\sigma}=0^{\circ}$ (sand deposition direction) to $\alpha_{\sigma}=90^{\circ}$ (bedding plane), the response of the sand changes from dilative to contractive. The sand exhibits highest strength at $\alpha_{\sigma}=0^{\circ}$ and lowest strength at $\alpha_{\sigma}=90^{\circ}$. Similar trends in anisotropic behaviour were also noted in previous studies (Uthayakumar, 1995, Yoshimine et al., 1998). The observation of the lowest strength at $\alpha_{\sigma}=90^{\circ}$ supports that higher deformability along bedding plane than that of deposition direction. Sayao (1989) and Wijewickreme (1990) have made a consistent observation in drained response, in which volumetric deformation for a given $\sigma_{d}$ increased with increasing $\alpha_{\sigma}$. 

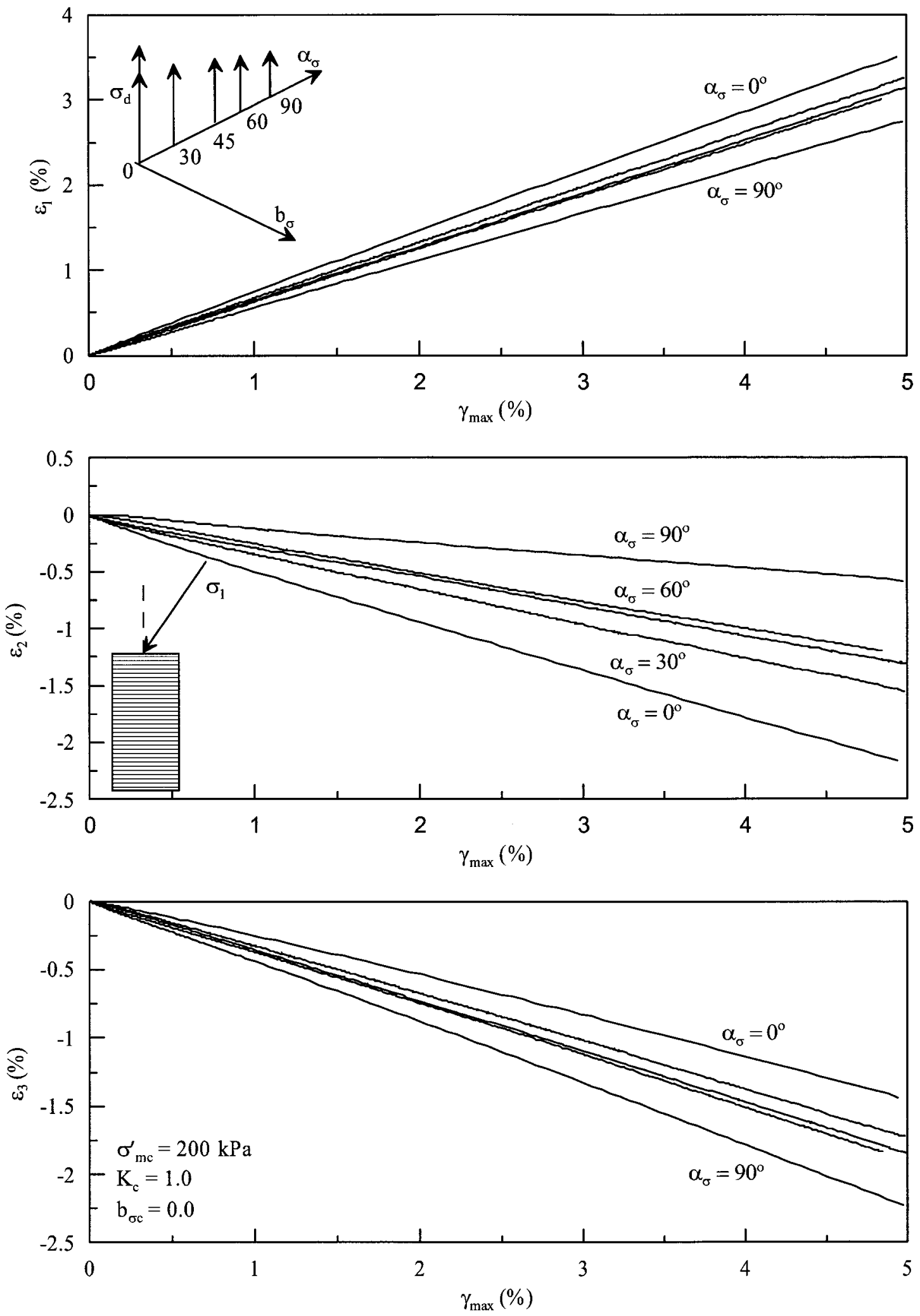

Fig. 5.7 Variation of principal strains with vertical strain during undrained shear tests at fixed $\alpha_{\sigma}$ 

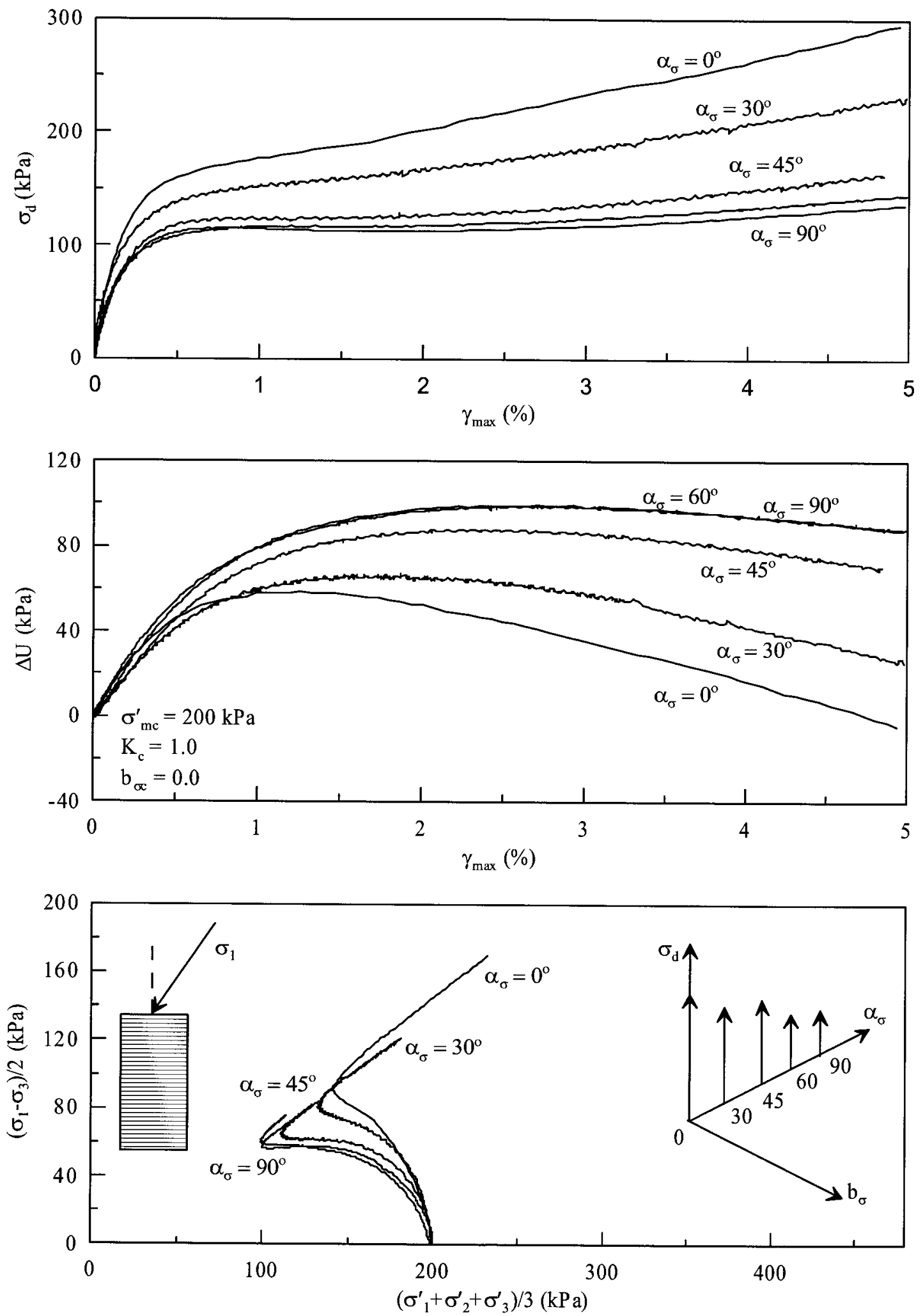

Fig. 5.8 Undrained response of Fraser River sand at fixed $\alpha_{\sigma}$ 
The strength of the sand significantly reduces when $\alpha_{\sigma}$ increases from $0^{\circ}$ to $45^{\circ}$. When $\alpha_{\sigma}$ is beyond $45^{\circ}$, the strength reduction is insignificant. At $\alpha_{\sigma}$ of $0^{\circ}$ and $30^{\circ}$, the sand response is completely strain hardening. However, sand undergoes marginally limited liquefaction at $\alpha_{\sigma}$ of $45^{\circ}, 60^{\circ}$ and $90^{\circ}$. A systematic increment of excess pore pressure is noted with increasing $\alpha_{\sigma}$ (Fig. 5.8). The sand generates higher excess pore pressure at $\alpha_{\sigma}=90^{\circ}$ and consequently the most contractive response corresponds to this $\alpha_{\sigma}$ value. Lower excess pore pressure is generated at $\alpha_{\sigma}=0^{\circ}$ and sand response is more dilative. The variation of excess pore pressure generation is significant between $\alpha_{\sigma}$ of $0^{\circ}$ and $45^{\circ}$ and it is only marginal beyond $\alpha_{\sigma}$ of $45^{\circ}$.

Fig. 5.9 illustrates strength $S_{P T}$, excess pore pressure $\Delta U_{P T}$ and stress state at PT state against $\alpha_{\sigma}$. Overall, $S_{P T}$ reduces when the principal stress direction moves from the direction of soil deposition to bedding plane. This observation demonstrates that $S_{P T}$ at constant initial void ratio and confining stress level depends on $\alpha_{\sigma}$ and supports the findings of Uthyakumar (1996). However, it was also reported in the literature (Castro, 1969; Ishihara, 1993) that $S_{P T}$ is only dependent on initial void ratio and initial confining stress.

Effective stress states at PT seem to be on a straight line passing through the origin and result in friction angle mobilized at $\mathrm{PT} \phi_{P T}$ of $34.2^{\circ}$, so that $\phi_{P T}$ is independent of $\alpha_{\sigma}$. This observation is also consistent with previous research by Uthayakumar (1995). In the literature, it was pointed out that $\phi_{P T}$ is also independent of stress path, relative density, consolidation history and mode of loading (Bishop, 1966 \& 1971; Vaid and Chern, 1985; Kuerbis et al., 1988; Vaid et al., 1990a). 

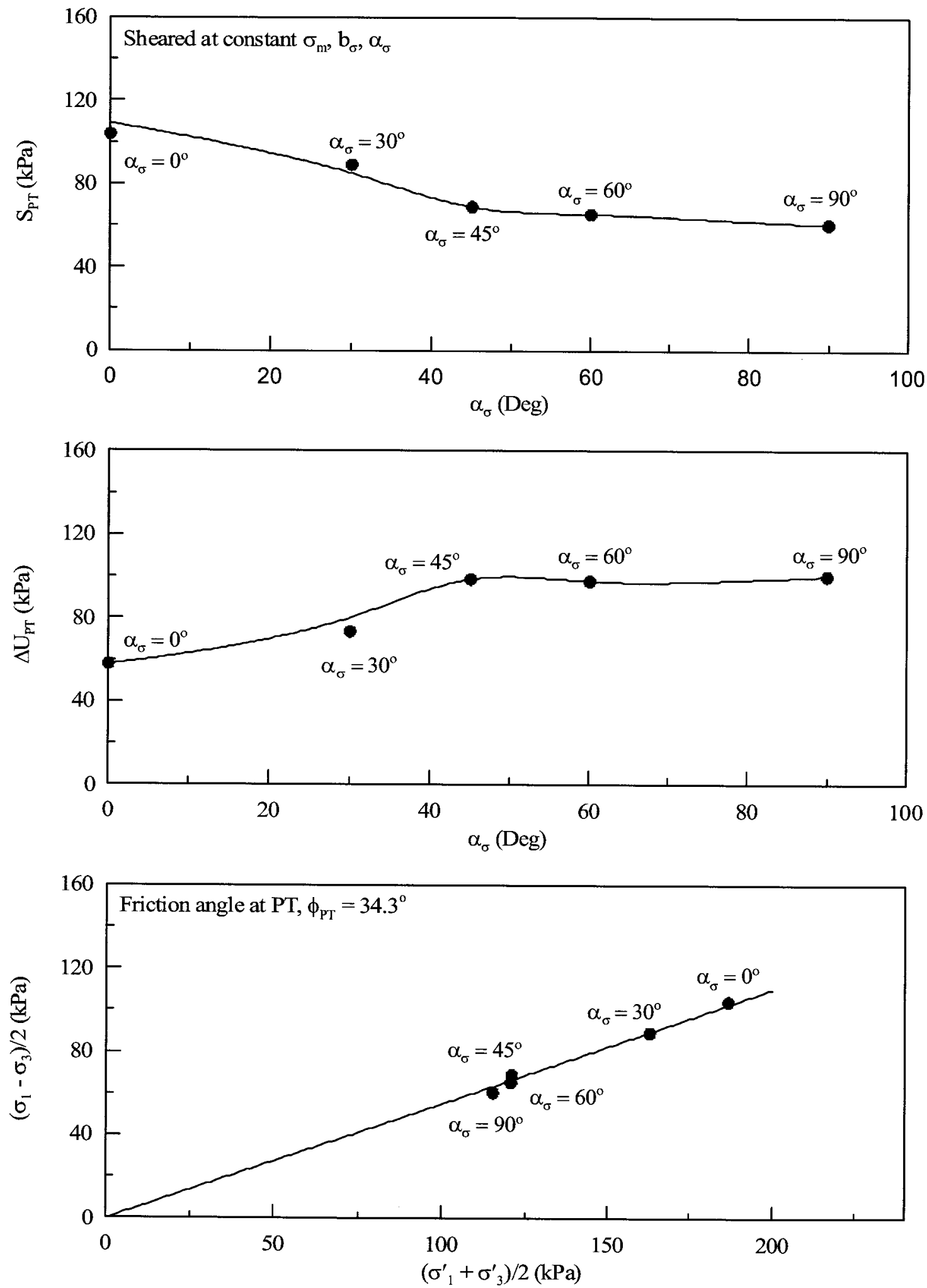

Fig. $5.9 \quad$ Variation of strength, excess pore pressure and stress state at PT state 
Effective stress ratio mobilized in these tests are comparatively in lower range (less than 3.2) until the end of tests. The stress nonuniformity induced is expected to be lower at this level of effective stress ratio. The noted induced friction angle at MO is $36.5^{\circ}$ and it is consistent with the friction angle reported in the literature for same batch of Fraser River sand.

\subsubsection{Undrained response of anisotropically consolidated sand}

The comparative undrained responses of Fraser River sand at different initial effective stress level $K_{c}$ are illustrated in Fig. 5.10. In these tests, specimens were consolidated at $K_{c}=1.0,1.5,2.0$, and $2.5, \sigma_{m c}^{\prime}=200 \mathrm{kPa}, b_{\sigma c}=0.0$ and $\alpha_{\sigma c}=0^{\circ}$. During shearing, lateral stresses were maintained constant and the specimens were axially loaded $\left(\alpha_{\sigma}=0^{\circ}\right)$.

The sand exhibits strain hardening regardless of $K_{c}$ and no tendency for strain softening due to changing $K_{c}$ levels is noted. As reported by Vaid et al. (2001), $K_{c}$ does promote strain softening, but its influence is relatively minor at $\alpha_{\sigma}=0^{\circ}$. The amount of excess pore pressure induced decreases with increasing $K_{c}$ because increment of deviatoric stress experienced by the sand reduces with increasing $K_{c}$. The sand reached the PT state after a $190 \mathrm{kPa}$ increment of deviatoric stress in the test with $K_{c}=1.0$, but only $45 \mathrm{kPa}$ increment in deviatoric stress was required to reach the PT state when $K_{c}=$ 2.5.

Fig. 5.11 shows variation of strength $S_{P T}$, excess pore pressure induced by deviatoric stress $\Delta U_{P T}$ and effective stress state at PT state. $S_{P T}$ increases and $\Delta U_{P T}$ decreases with increasing $K c$. The effective stress states at PT are along a unique straight line passing through origin and result in the friction angle $\phi_{P T}$ of $34.2^{\circ}$. 

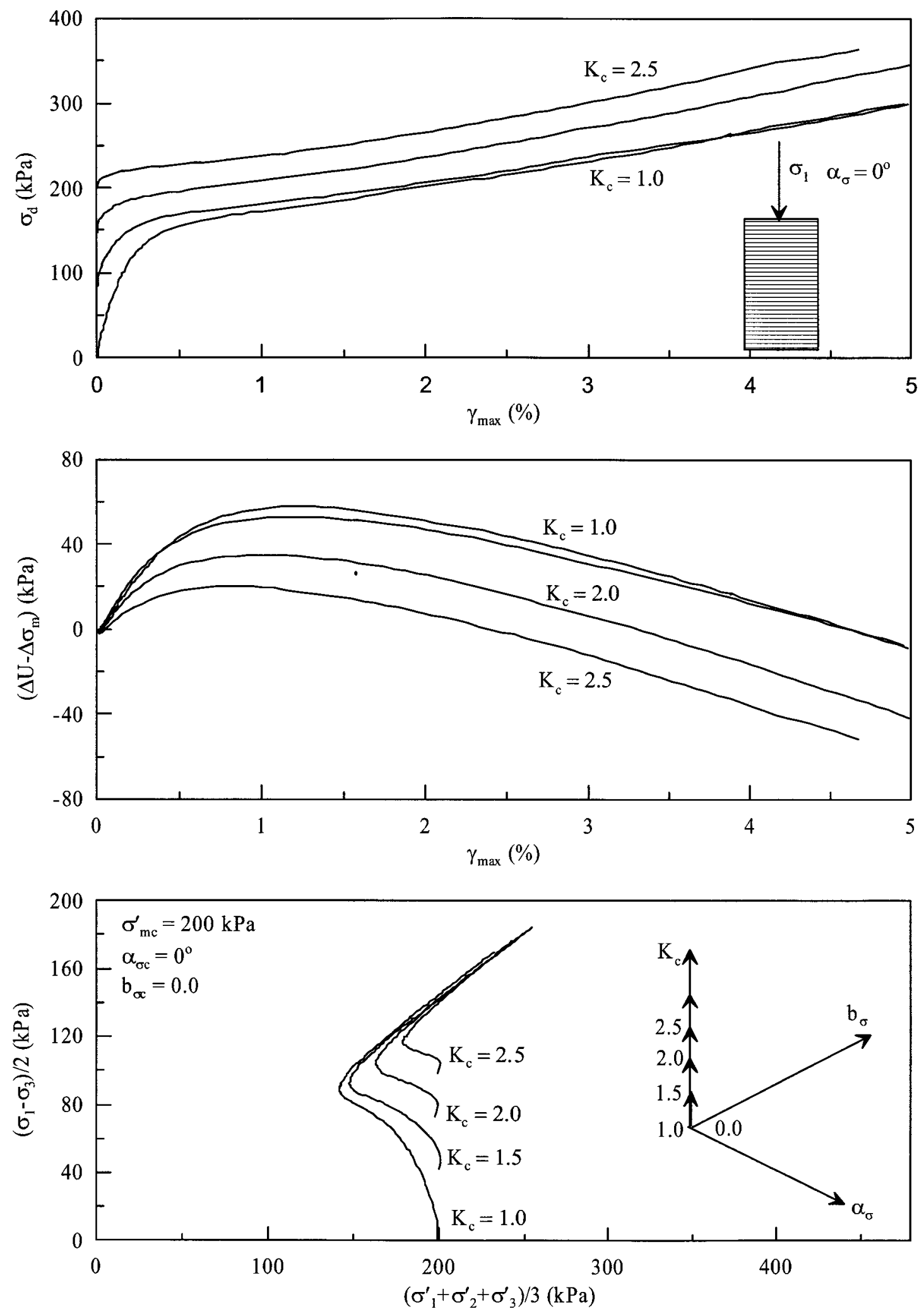

Fig. 5.10 Undrained response of anisotropically consolidated Fraser River sand 

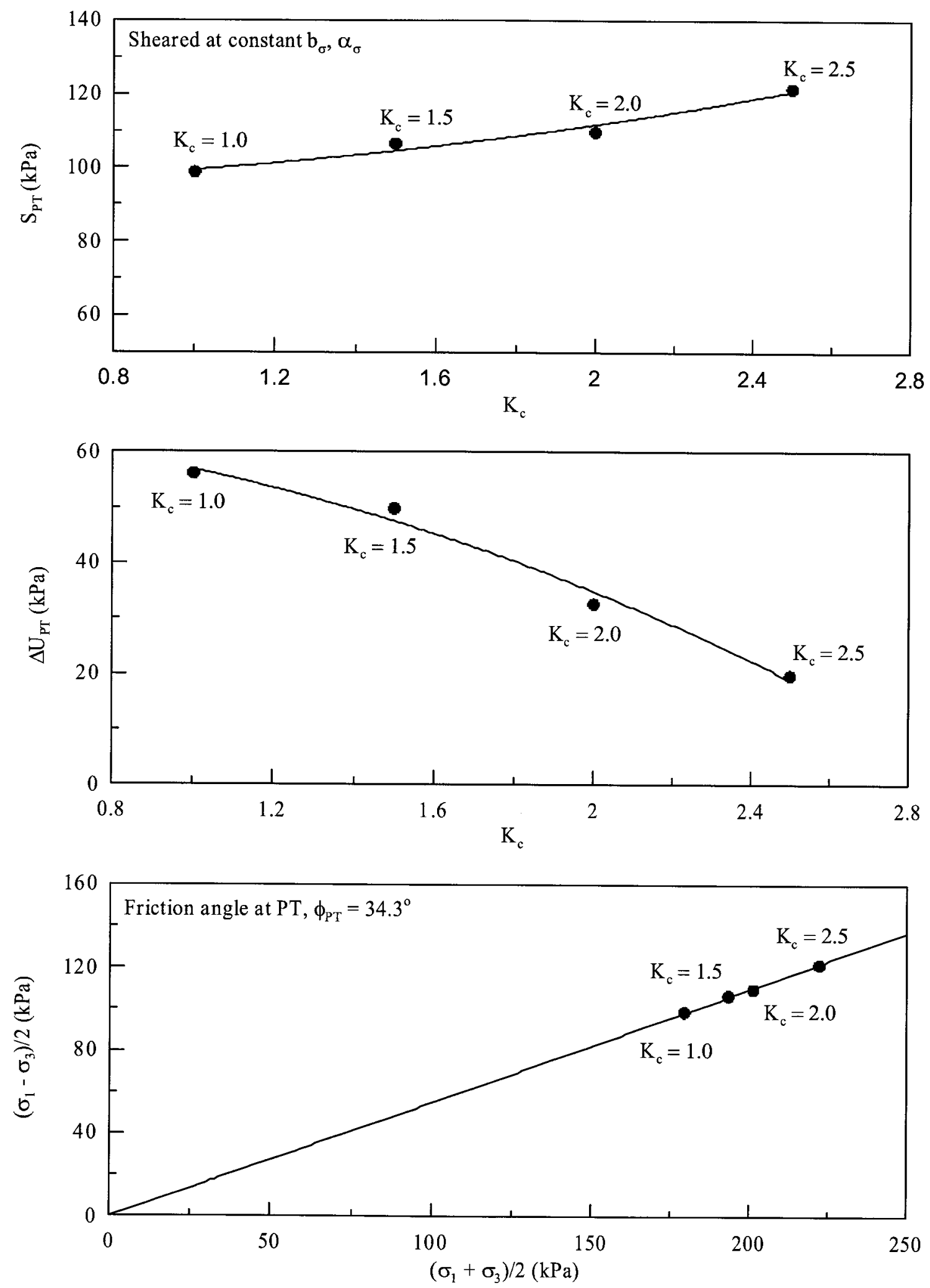

Fig. 5.11 Variation of strength, excess pore pressure induced by deviatoric stress and stress state at PT state 


\subsection{Strain path controlled behaviour of sand}

Loosest deposited Fraser River sand was subjected to simultaneous changes of pore pressure and pore volume to assess the effects of such coupled deformation under generalized loading conditions. Linear strain paths were imposed by maintaining a constant ratio between imposed volumetric strain $\Delta \varepsilon_{v}$ and maximum shear strain $\Delta \gamma_{\max }$ experienced by specimen $\left(\zeta=\Delta \varepsilon_{v} / \Delta \gamma_{\max }=\right.$ constant $)$. A negative sign of the constant implies volume injection into the specimen and the positive sign denotes volume extraction from the specimen.

The undrained tests that studied the influence of stress parameters $b_{\sigma}, \alpha_{\sigma}$ and $K_{c}$ are necessary to make a comparison of responses under undrained and strain path controlled loadings. A constant total mean normal stress was applied to specimens during loading with accurate control of $\alpha_{\sigma}$ and $b_{\sigma}$. The details of the strain paths controlled tests conducted are tabulated in Table 5.1.

\subsubsection{Strain path controlled loading at fixed $b_{\sigma}$}

A set of tests at fixed $b_{\sigma}=0.0,0.2,0.4,0.6,0.8$ and 1.0 were carried out to investigate the influence of $b_{\sigma}$ during simultaneous changes in pore pressure and pore volume. The specimens were isotropically consolidated to $\sigma_{m c}^{\prime}=200 \mathrm{kPa}$ and $D_{r c}=22 \%$. During shearing, principal stress direction was kept along the deposition direction $\left(\alpha_{\sigma}=\right.$ $0^{\circ}$ ). A linear strain path of $\Delta \varepsilon_{v} / \Delta \gamma_{\max }=-0.10$ was imposed on each specimen.

Induced principal strains $\left(\varepsilon_{1}, \varepsilon_{2}, \varepsilon_{3}\right)$ are illustrated in Fig. 5.12. A systematic variation of strains is noted when $b_{\sigma}$ changes from 0 to 1.0 . Intermediate principal strain $\varepsilon_{2}$ changes from positive to negative when $b_{\sigma}$ changes from 1.0 to 0 . The magnitude of minor principal strain $\varepsilon_{3}$ increases with increasing $b_{\sigma} . \varepsilon_{2}$ is much closer to zero at $b_{\sigma}=$ 
0.40 implying that $b_{\sigma}=0.40$ still imposes plane strain deformation as in the undrained loading. This observation demonstrates that the plane strain condition is preserved during intrusion of a small amount of volume into the sand $(0.4 \%$ of expansive volumetric strain at $\gamma_{\max }=4 \%$ ). The strain paths referred to fixed coordinate system is linear. This observation implies that the degree of initial anisotropy in soil preserved during shearing and contribution of induced anisotropy in strains is at low level.

Fig. 5.13 shows the stress-strain response of Fraser River sand under strain path controlled loading at fixed $b_{\sigma}$. A systematic variation in stress-strain response is noted as $b_{\sigma}$ changes from 0 to 1.0 . At a given $\gamma_{\max }$, water inflow causes the sand to exhibit lower strength than undrained sand. Comparison of the strength at undrained and strain controlled loading is discussed at the end of this subsection.

The expansive volumetric strain (volume intrusion) of the sand promotes strain softening. However, at $b_{\sigma}=0.0$, the sand still exhibits strain hardening, but at other $b_{\sigma}$ values the sand exhibits marginal strain softening. In comparison, it exhibited strain hardening behaviour for all $b_{\sigma}$ during undrained loading (Fig. 5.3). This observation demonstrates that a small amount of volume inflow into the sand at higher $b_{\sigma}$ is more vulnerable to liquefaction than soil at lower $b_{\sigma}$ due to the higher tendency of strain softening at higher $b_{\sigma}$.

The excess pore pressure generated includes both a deviatoric stress induced component and a volume change induced component, and both components are treated together in this discussion. Higher excess pore pressure is generated at $b_{\sigma}=1.0$ and lower excess pore pressure is generated at $b_{\sigma}=0.0$. During undrained loading at fixed $b_{\sigma}$, the sand experienced contractive type of deformation at the initial stage and dilative type of 
deformation after reaching peak excess pore pressure (Fig. 5.3). However, in the strain path controlled loading (volume intrusion), the sand does not generate excess pore pressure resulting dilative deformation (except $b_{\sigma}=0.0$ test). Fig. 5.14 shows the variation of peak strength $S_{\text {peak }}$ and corresponding excess pore pressure $\Delta U_{\text {peak }}$ as a function of $b_{\sigma}$ and stress states. A systematic reduction of $S_{\text {peak }}$ is noted with increasing $b_{\sigma} . S_{\text {peak }}$ at $b_{\sigma}=0.20$ is $84 \mathrm{kPa}$ and at $b_{\sigma}=1.0$, it is $51 \mathrm{kPa}$. Comparison of $S_{\text {peak }}$ with peak strength of undrained loading is not possible because no peak state was realized during undrained loading (Fig. 5.3).
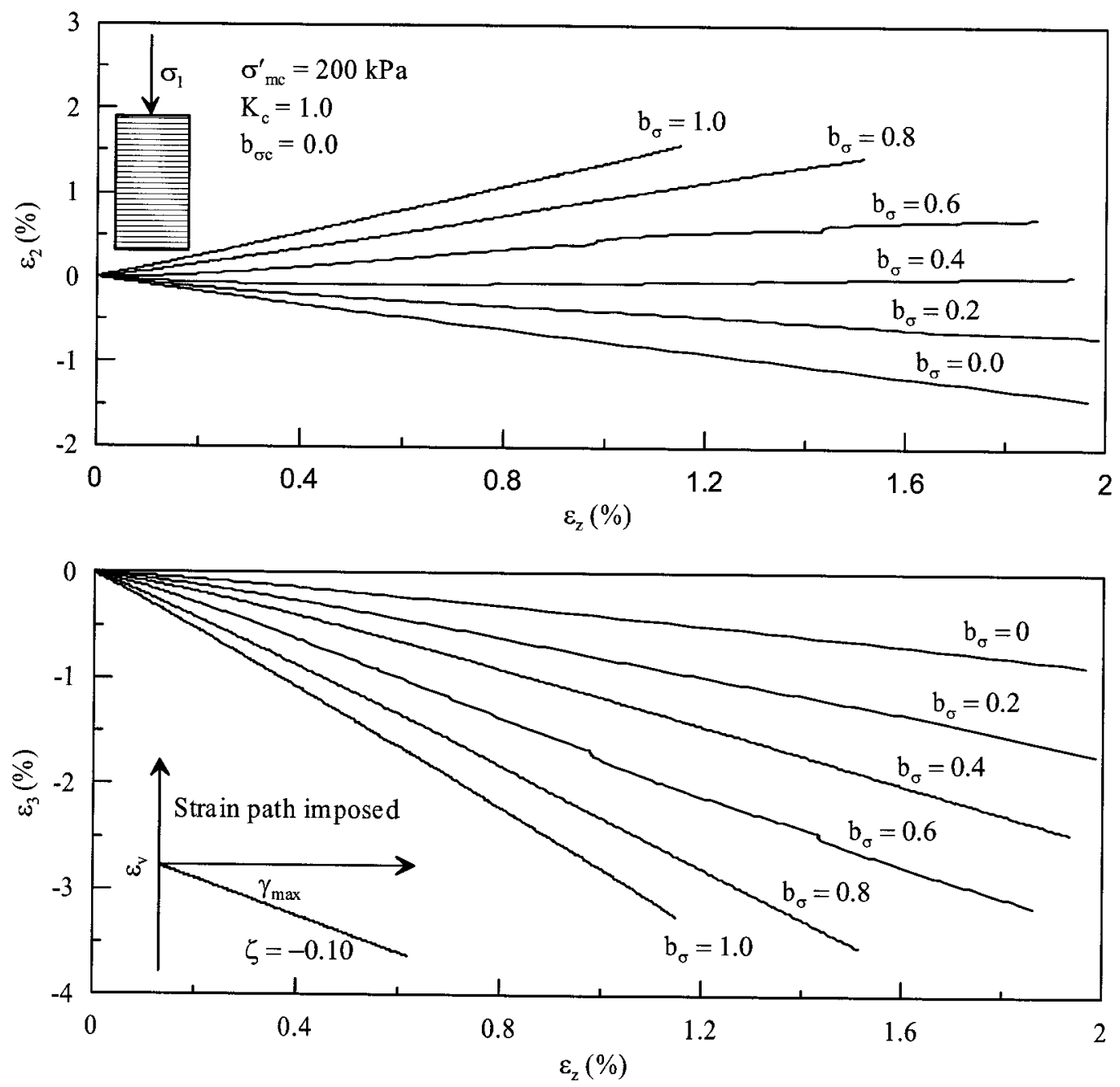

Fig. 5.12 Variation of principal strains with vertical strain during strain path controlled tests at fixed $b_{\sigma}$ 

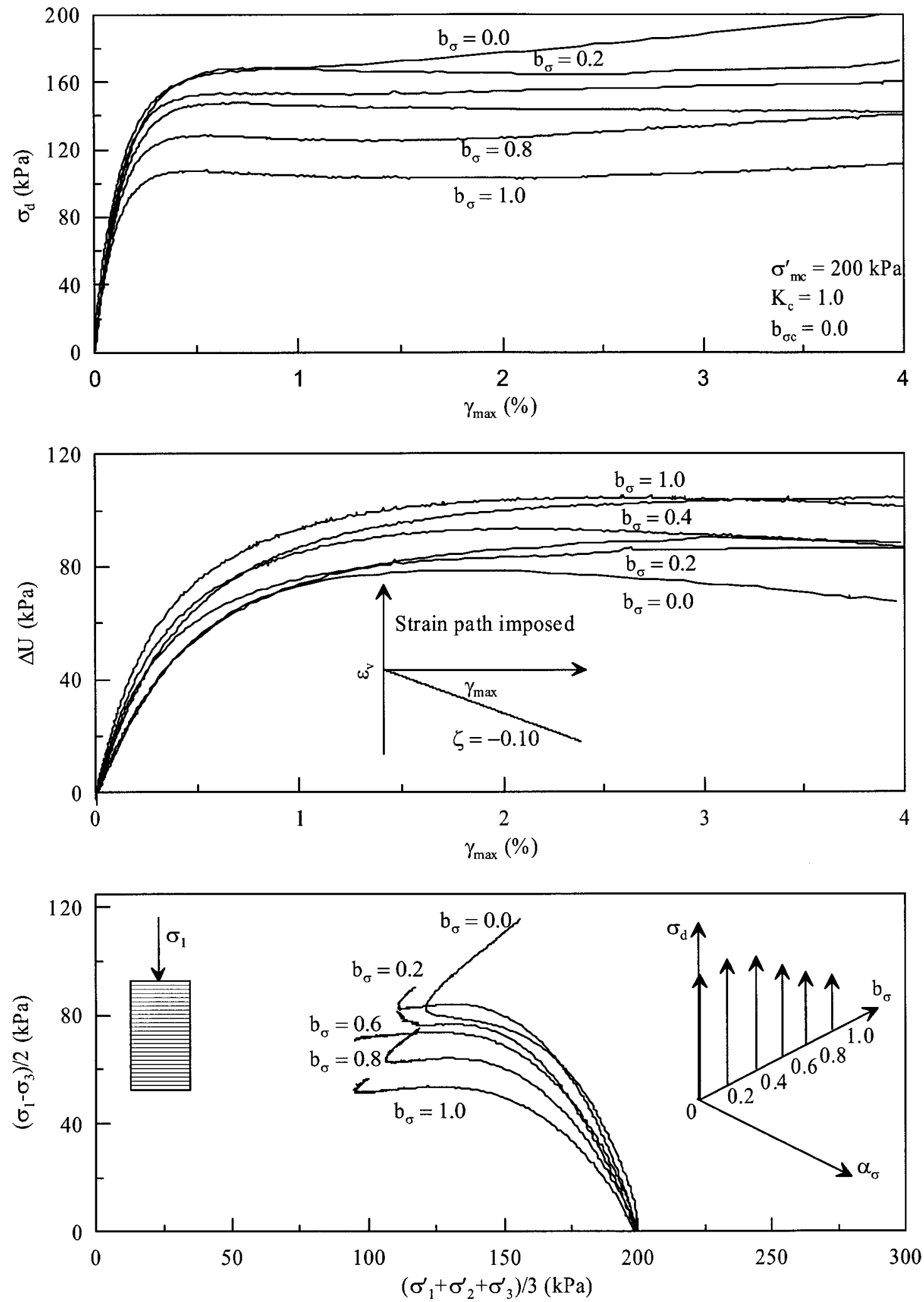

Fig. 5.13 Response of Fraser River sand during strain path controlled tests at fixed $b_{\sigma}$ 

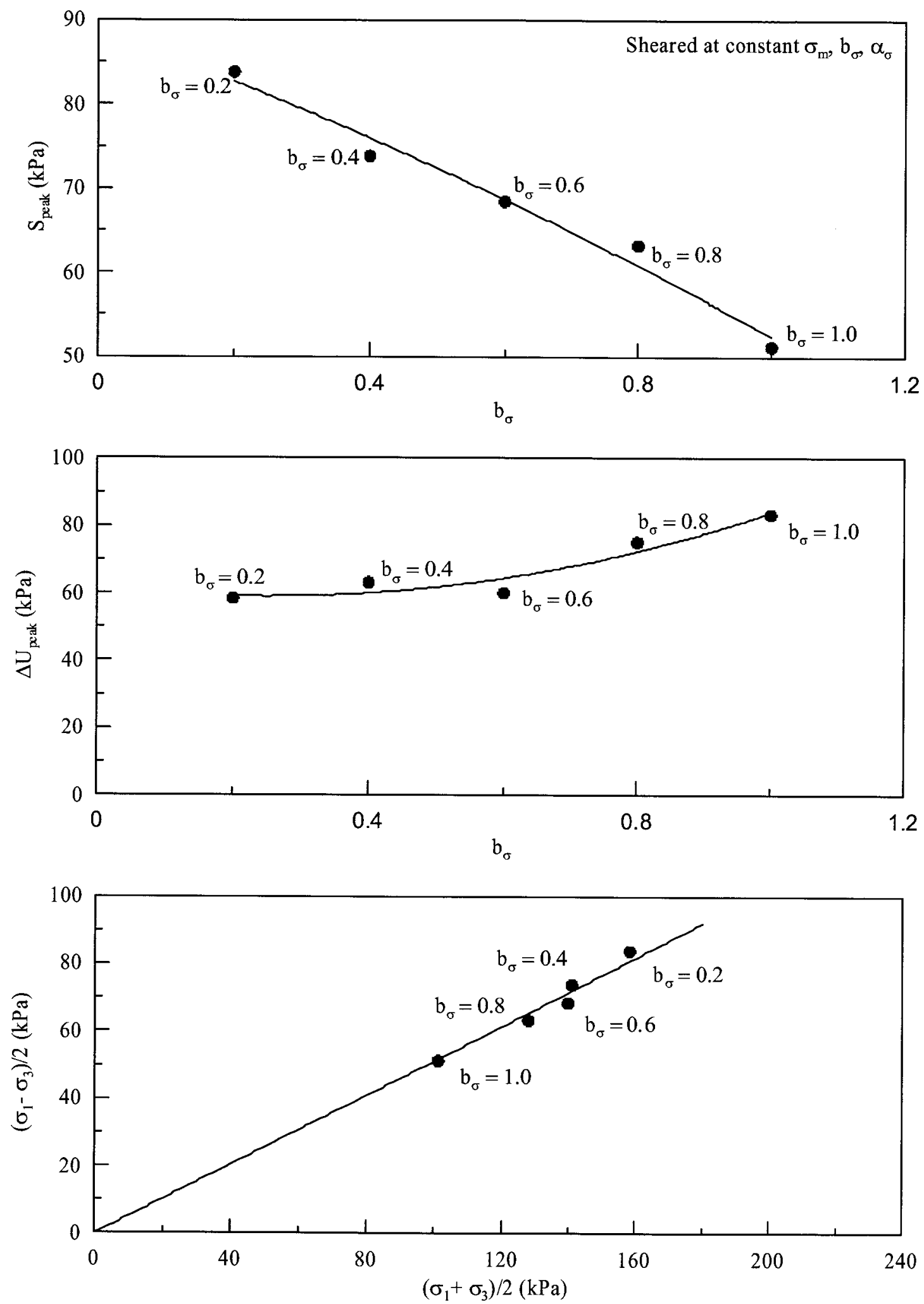

Fig. 5.14 Peak strength, excess pore pressure and effective stress state at peak strength state during strain path controlled tests at fixed $b_{\sigma}$ 
A systematic increase of $\Delta U_{\text {peak }}$ is noted at peak strength state when $b_{\sigma}$ increases from 0.2 to 1.0 . The friction angle mobilized at peak strength state $\phi_{\text {peak }}$ is in narrow range between $30^{\circ}$ and $32^{\circ}$. It has been noted in the literature (Uthayakumar, 1995) that friction angle induced at peak strength during undrained loading $\phi_{C S R}$ is dependent on $b_{\sigma}$ and $\alpha_{\sigma}$. Even though 'peak' and 'CSR' refer to the peak strength state of strain path controlled loading and undrained loading, these states are differentiated in this discussion because peak strength state of strain path controlled loading reflects the influences of shearing and volume transfer, and peak strength state of undrained loading reflects the influence of shearing only.

Fig. 5.15 illustrates the variation of strength $S_{\text {peakPP }}$, excess pore pressure $\Delta U_{P e a k P P}$ and effective stress state at peak pore pressure state. As referred in undrained tests, peak pressure state is not termed as PT in strain path controlled tests. The excess pore pressure induced in the strain path controlled tests includes not only a shear induced component but also a volume induced component. To distinguish undrained and strain controlled loading, this state is termed as peak pore pressure state and denoted as 'PeakPP'.

The sand exhibits a systematic reduction of $S_{\text {peakPP }}$ with increasing $b_{\sigma} . S_{\text {peakPP }}$ is less than $S_{P T}$ for all $b_{\sigma} . S_{p e a k P P}$ is $86 \mathrm{kPa}$ and $S_{P T}$ is $104 \mathrm{kPa}$ at $b_{\sigma}=0.0(17 \%$ of reduction of strength). $S_{\text {peakPP }}$ is $50 \mathrm{kPa}$ and $S_{P T}$ is $68 \mathrm{kPa}$ at $b_{\sigma}=1.0$ (26\% of reduction of strength). However, the reduction of strength during strain path controlled loading at intermediate $b_{\sigma}$ values $(0.4,0.6)$ appears low. Overall, $\Delta U_{P e a k P P}$ is higher than $\Delta U_{P T}$. However, differences between the maximum excess pore pressure during undrained and strain path controlled loading is smaller at intermediate $b_{\sigma}$ values $(0.4,0.6)$ as noted in the strength 
variation above. The available data is limited to draw a firm conclusion about whether this is an experimental artifact, or actual characteristics of soil response.
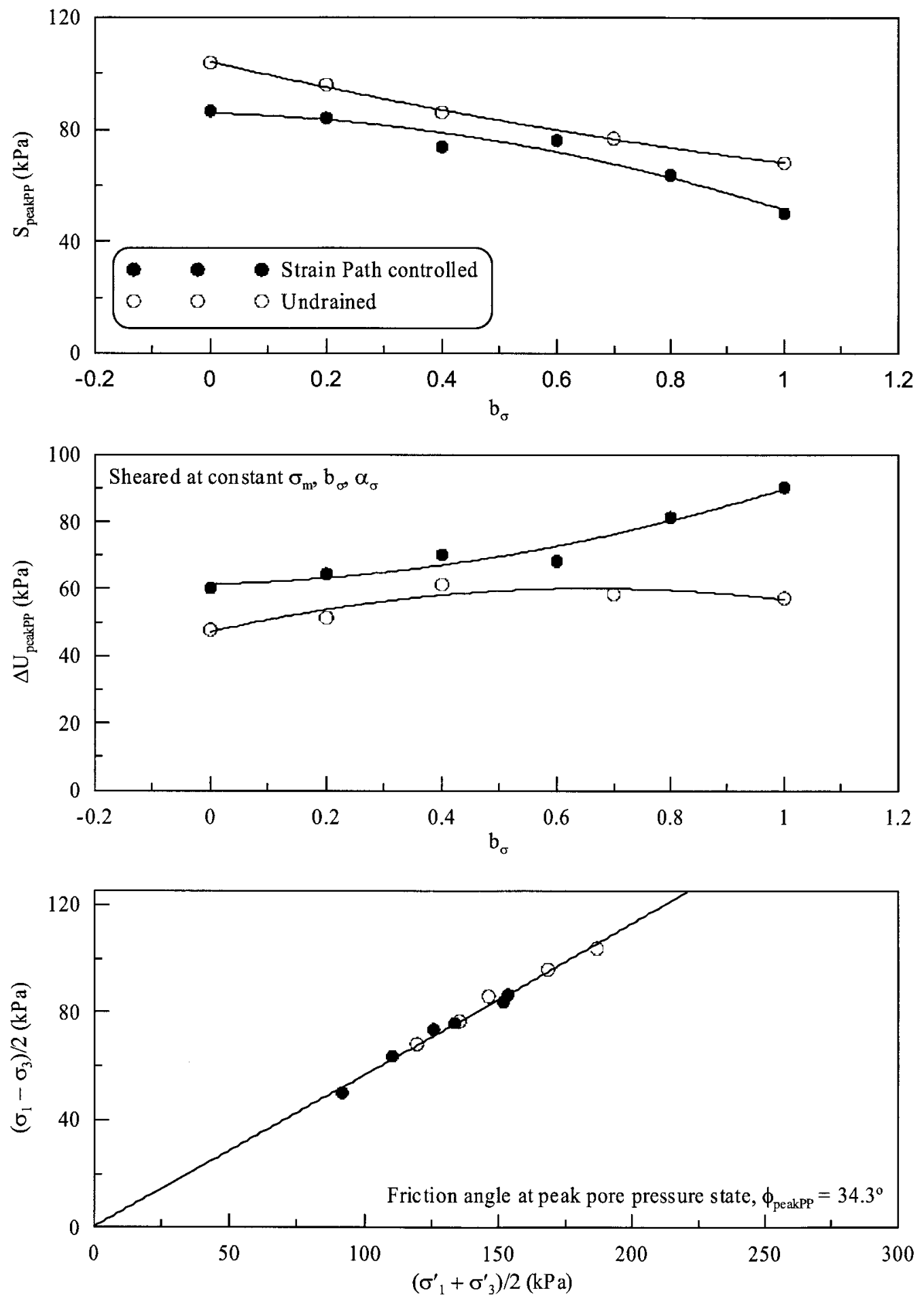

Fig. 5.15 Strength, excess pore pressure and stress state of peak pore pressure state during strain path controlled tests at fixed $b_{\sigma}$ 
Effective stress states at peak pore pressure of strain path controlled tests lie along a unique straight line passing through the origin and result in a friction angle $\phi_{\text {peakPP }}$ of $34.3^{\circ} . \phi_{\text {peakPP }}$ is equal to the friction angle noted at PT $\phi_{P T}$ during undrained shear. This observation is consistent with previous studies on this topic under axisymmetric triaxial loading (Sivathayalan and Logeswaran, 2007). It has been pointed out that $\phi_{\text {peakPP }}$ is unique during simultaneous changes in pore pressure and volume and it is independent of strain paths imposed.

\subsubsection{Strain path controlled loading at fixed $\alpha_{\sigma}$}

A set of tests at fixed $\alpha_{\sigma}=0^{\circ}, 30^{\circ}, 45^{\circ}, 60^{\circ}$ and $90^{\circ}$ were carried out to investigate the influence of principal stress direction $\alpha_{\sigma}$ during simultaneous changes in pore pressure and pore volume. Specimens were isotropically consolidated to $\sigma_{m c}^{\prime}=200$ $\mathrm{kPa}$ and $D_{r c}=22 \%$ prior to shearing. Total mean normal stress was maintained constant and intermediate and minor principal stresses were equal throughout the testing (i.e., $b_{\sigma}=$ 0.0). A linear strain path of $\zeta=-0.10$ was imposed on the specimens.

Induced principal strains $\left(\varepsilon_{1}, \varepsilon_{2}, \varepsilon_{3}\right)$ during strain path controlled loading are illustrated in Fig. 5.16. A systematic variation of strains is noted when $\alpha_{\sigma}$ changes from $0^{\circ}$ to $90^{\circ}$. Magnitudes of $\varepsilon_{1}$ and $\varepsilon_{2}$ decrease with increasing $\alpha_{\sigma}$ at a given level of $\gamma_{\max }$; however, magnitude of $\varepsilon_{3}$ increases. The strain paths referred to a fixed coordinate system is linear at small strains and this observation implies that the degree of initial anisotropy in the specimen is preserved during the shearing. However, at comparatively large strains, the strain paths become progressively nonlinear with increasing stress ratio. This observation demonstrates the gradual influence of induced anisotropy. 

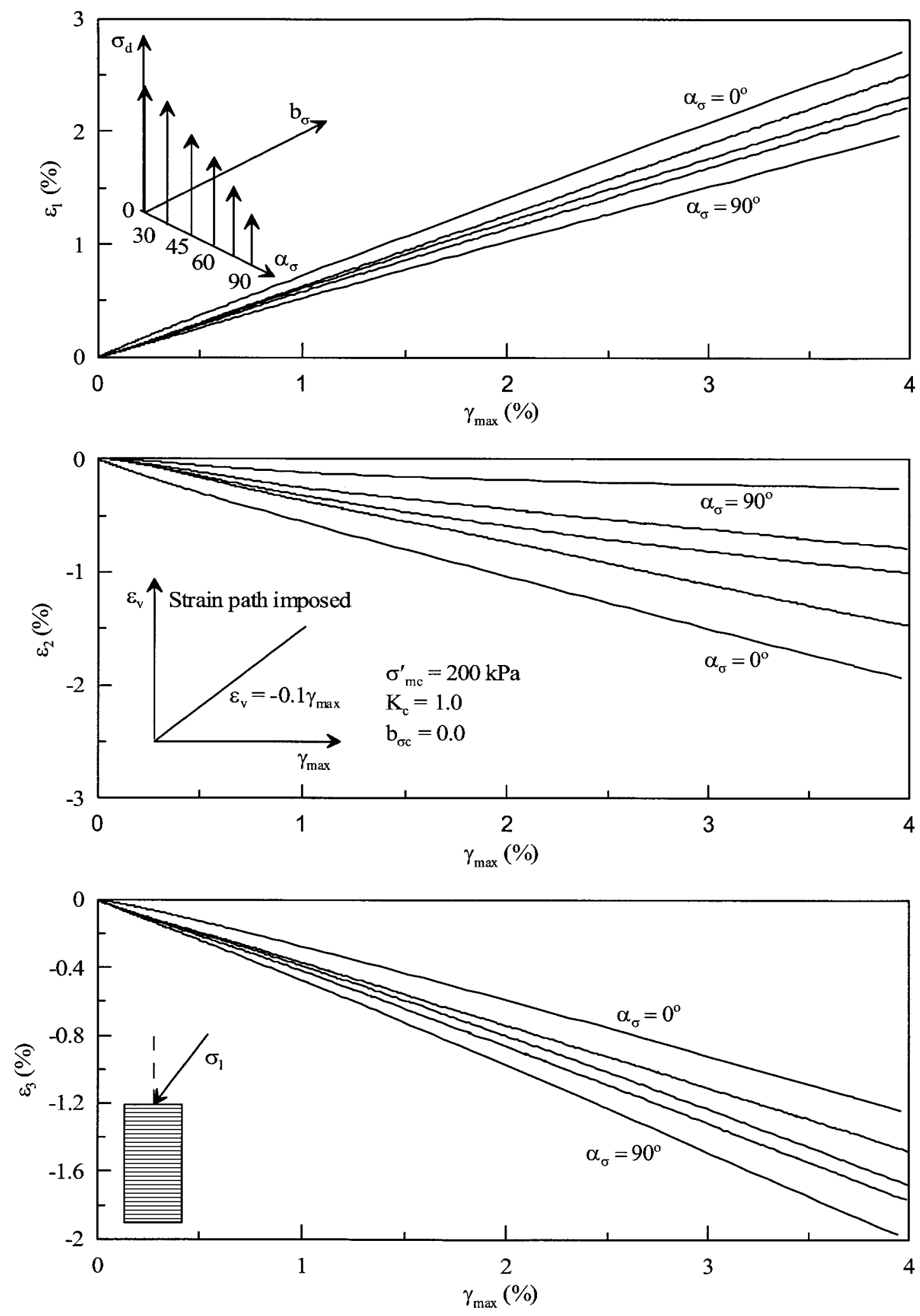

Fig. 5.16 Variation of principal strains during strain path controlled tests at fixed $\alpha_{\sigma}$ 
Fig. 5.17 shows stress-strain responses, excess pore pressure responses and stress paths of Fraser River sand under strain-controlled loading at fixed $\alpha_{\sigma}$. A systematic variation of stress-strain response is noted as $\alpha_{\sigma}$ changes from $0^{\circ}$ to $90^{\circ}$, and the strength of the sand decreases when $\alpha_{\sigma}$ changes from $0^{\circ}$ to $90^{\circ}$. The rate of strength reduction and the pore pressure generation is significant only within the range of $\alpha_{\sigma}$ between $0^{\circ}$ and $45^{\circ}$ as noted in undrained responses. The effect of $\alpha_{\sigma}$ is relatively minor beyond $\alpha_{\sigma}=45^{\circ}$. The sand exhibits strain hardening behaviour at $\alpha_{\sigma}=0^{\circ}$ and $30^{\circ}$ and strain softening behaviour at $\alpha_{\sigma}=45^{\circ}, 60^{\circ}$ and $90^{\circ}$. At a given $\alpha_{\sigma}$, water inflow causes the sand to exhibit lower strength than undrained. At $\alpha_{\sigma}$ of $45^{\circ}, 60^{\circ}$ and $90^{\circ}$, the sand exhibited partially strain-softening behaviour under undrained condition (Fig. 5.8). However, when the sand is subjected to comparatively small expansive volumetric strain at $\alpha_{\sigma}$ of $45^{\circ}, 60^{\circ}$ and $90^{\circ}$, it behaves completely strain softening and deforms at constant strength after reaching maximum excess pore pressure. The excess pore pressure responses at $\alpha_{\sigma}=0^{\circ}$ and $30^{\circ}$ show marginally dilative response at larger strains following contractive response during the initial stages of the loading. However, the response of the sand is always contractive when $\alpha_{\sigma}=45^{\circ}, 60^{\circ}$ or $90^{\circ}$.

Fig. 5.18 shows the variation of strength $S_{\text {peak }}$, excess pore pressure $\Delta U_{\text {peak }}$, and stress states at peak strength state. The peak strength state is only noted in the tests conducted at $\alpha_{\sigma}=45^{\circ}, 60^{\circ}$ and $90^{\circ}$. $S_{\text {peak }}$ systematically reduces with increasing $\alpha_{\sigma} . S_{\text {peak }}$ is $56 \mathrm{kPa}$ at $\alpha_{\sigma}=45^{\circ}$ and $47 \mathrm{kPa}$ at $\alpha_{\sigma}=90^{\circ}$ (16\% strength reduction). $\Delta U_{\text {peak }}$ systematically increases with increasing $\alpha_{\sigma}$. 

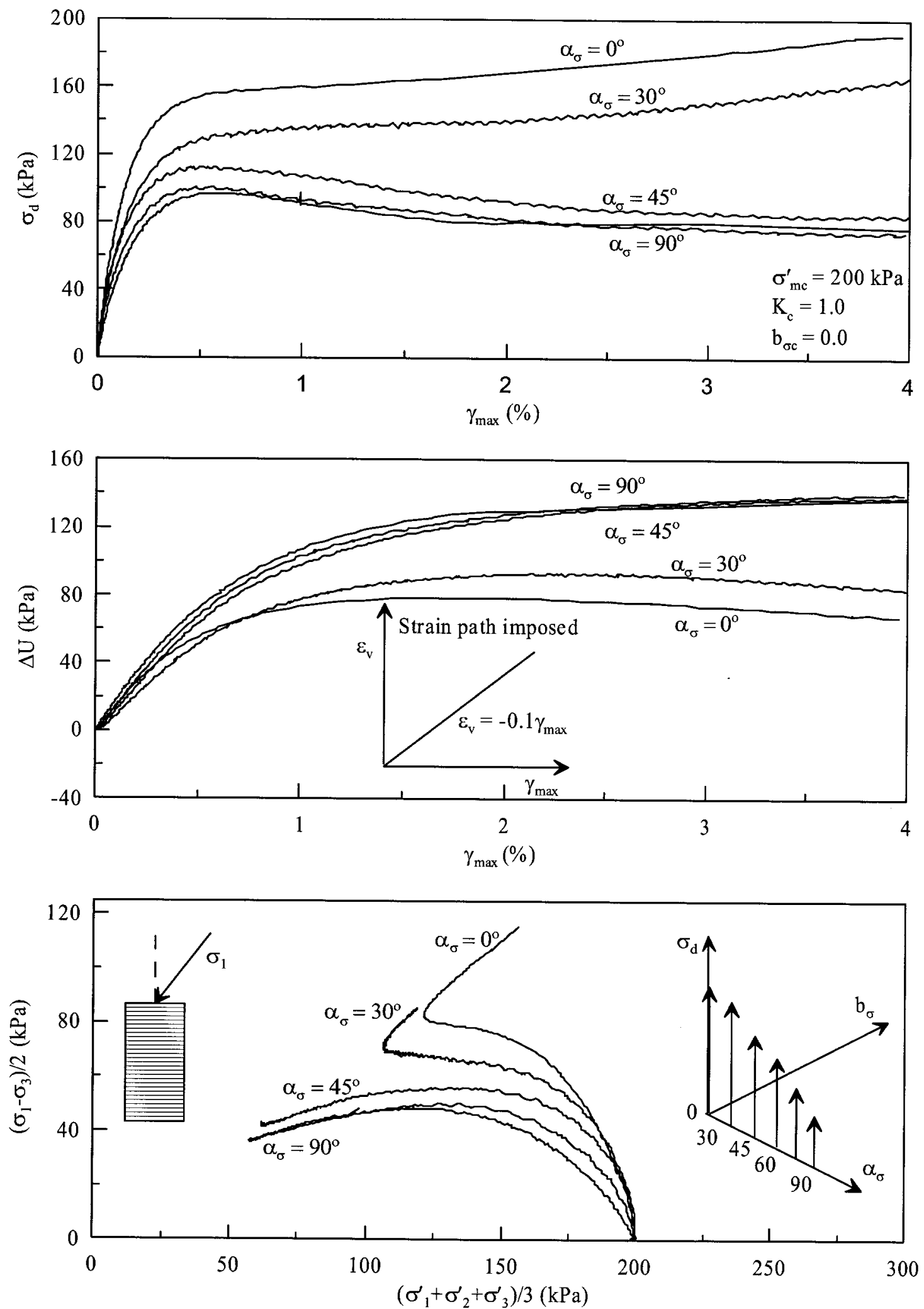

Fig. 5.17 Response of Fraser River sand during strain path controlled tests at fixed $\alpha_{\sigma}$ 

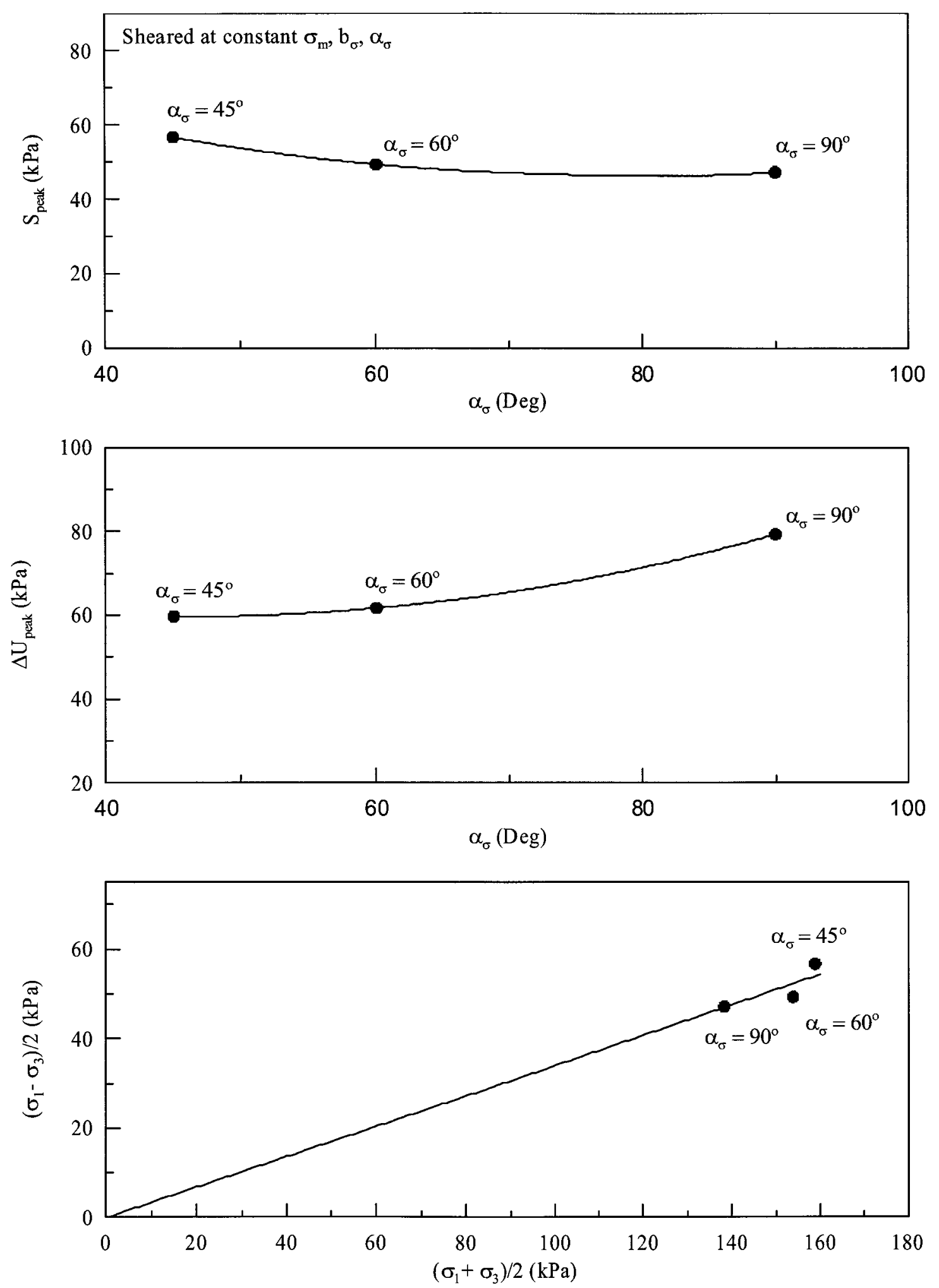

Fig. 5.18 Strength, excess pore pressure and effective stress state at peak strength state during strain path controlled tests at fixed $\alpha_{\sigma}$ 
Variation $\phi_{p e a k}$ is within a small range $\left(20^{\circ}-22^{\circ}\right)$ with change of $\alpha_{\sigma}$. It is suggested in the literature (Uthayakumar, 1995) that friction angle at peak strength under undrained condition $\phi_{C S R}$ is unique for a given $\alpha_{\sigma}$ and reduces with increasing $\alpha_{\sigma}$.

Fig. 5.19 shows strength $S_{\text {peakPP }}$, excess pore pressure $\Delta U_{\text {peakPP }}$ and effective stress state at peak pore pressure state during undrained and strain path controlled tests. $S_{\text {peakPP }}$ systematically reduces with increasing $\alpha_{\sigma}$. The reduction of $S_{p e a k P P}$ is significant when $\alpha_{\sigma}$ changes from $0^{\circ}$ to $45^{\circ} . S_{\text {peakPP }}$ is $81 \mathrm{kPa}$ at $\alpha_{\sigma}=0^{\circ}$ and $42 \mathrm{kPa}$ at $\alpha_{\sigma}=45^{\circ}$ (around $50 \%$ strength reduction). The strength reduction is insignificant when $\alpha_{\sigma}$ is beyond $45^{\circ} . S_{\text {peakPP }}$ is $36 \mathrm{kPa}$ at $\alpha_{\sigma}=90^{\circ}$ (only $14 \%$ of strength reduction compared to $S_{\text {peakPP }}$ at $\alpha_{\sigma}=45^{\circ}$ ). Overall, excess pore pressure at peak pore pressure state increases with increasing $\alpha_{\sigma}$. The increment of $\triangle U_{\text {peakPP }}$ is significant between $\alpha_{\sigma}$ of $0^{\circ}$ and $45^{\circ}$ and is minor beyond $\alpha_{\sigma}=45^{\circ}$.

Effective stress states at the instance of peak pore pressure lie along a straight line, passing through the origin and result in a $\phi_{\text {peakPP }}$ of $34.3^{\circ}$. The $\phi_{p e a k P P}$ is equal to the friction angle mobilized at PT state $\phi_{P T}$ under undrained condition. This observation also demonstrates that $\phi_{p e a k P P}$ can be treated as a unique material property regardless of $\alpha_{\sigma}$ and drainage conditions. 

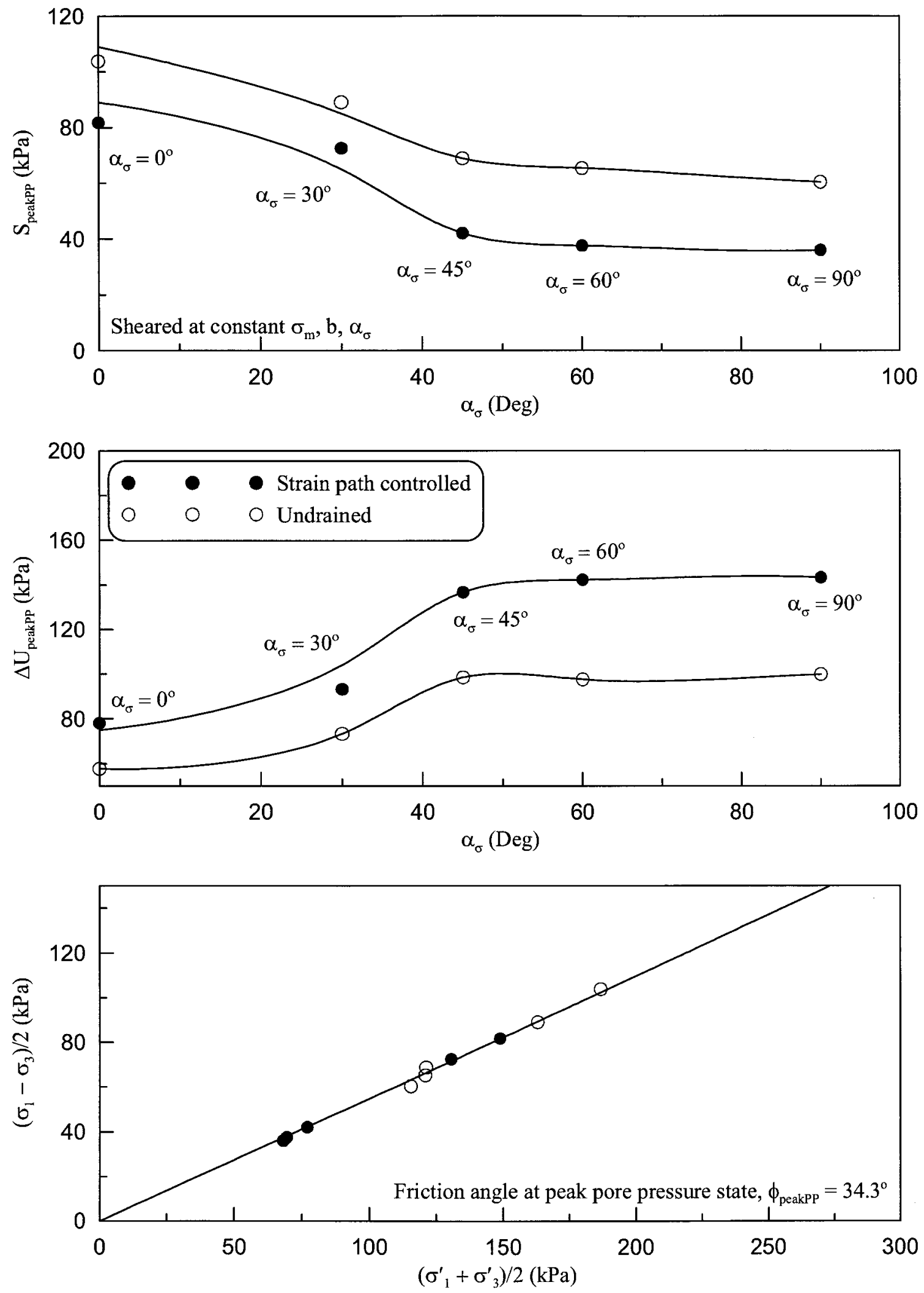

Fig. 5.19 Strength, excess pore pressure and stress state of peak pore pressure state during strain path controlled tests at fixed $\alpha_{\sigma}$ 


\subsubsection{Strain path controlled loading on anisotropically consolidated sand}

To investigate the influence of initial effective stress ratio $K c$ on simultaneous changes in pore pressure and pore volume, a set of tests at $K_{c}=1.0,2.0,2.0$ and 2.5 were carried out by imposing a linear strain path with the same rate of volumetric deformation as earlier $\left(\varepsilon_{v} / \gamma_{\max }=-0.10\right)$. In these tests, $b_{\sigma}$ was kept at zero and principal stress direction was along the specimen deposition direction $\left(\alpha_{\sigma}=0^{\circ}\right)$ throughout the deformation. The initial effective mean normal stress $\sigma_{m c}^{\prime}$ was set to $200 \mathrm{kPa}$. During shearing, inner and outer pressures of the sample were held constant.

The comparative behaviour of sand at identical $b_{\sigma}$ and $\alpha_{\sigma}$, but at a different level of $K_{c}$ under strain path controlled loading is illustrated in Fig. 5.20. No strain softening behaviour was noted during undrained tests regardless of $K_{c}$ (Fig. 5.10). However, expansive volumetric strain has promoted strain softening. The sand marginally strain softened at all $K_{c}$ values. Induced excess pore pressure systematically changes with $K_{c}$ as excess pore pressure decreases from about $120 \mathrm{kPa}$ at $K_{c}=1.0$ to about $60 \mathrm{kPa}$ at $K_{c}=$ 2.5 .

The degree of strain softening is often characterized by brittleness index $I_{B}$ to describe the flow potential of contractive sand (Bishop, 1971).

$$
I_{B}=\frac{S_{\text {peak }}-S_{\text {min }}}{S_{\text {peak }}}
$$

Here, $S_{\text {peak }}$ and $S_{\min }$ are the peak and minimum undrained strengths. However, this definition is unable to describe the flow potential for anisotropically consolidated sands and a modified brittleness index $I_{B, \text { modified }}$ is defined as below. 

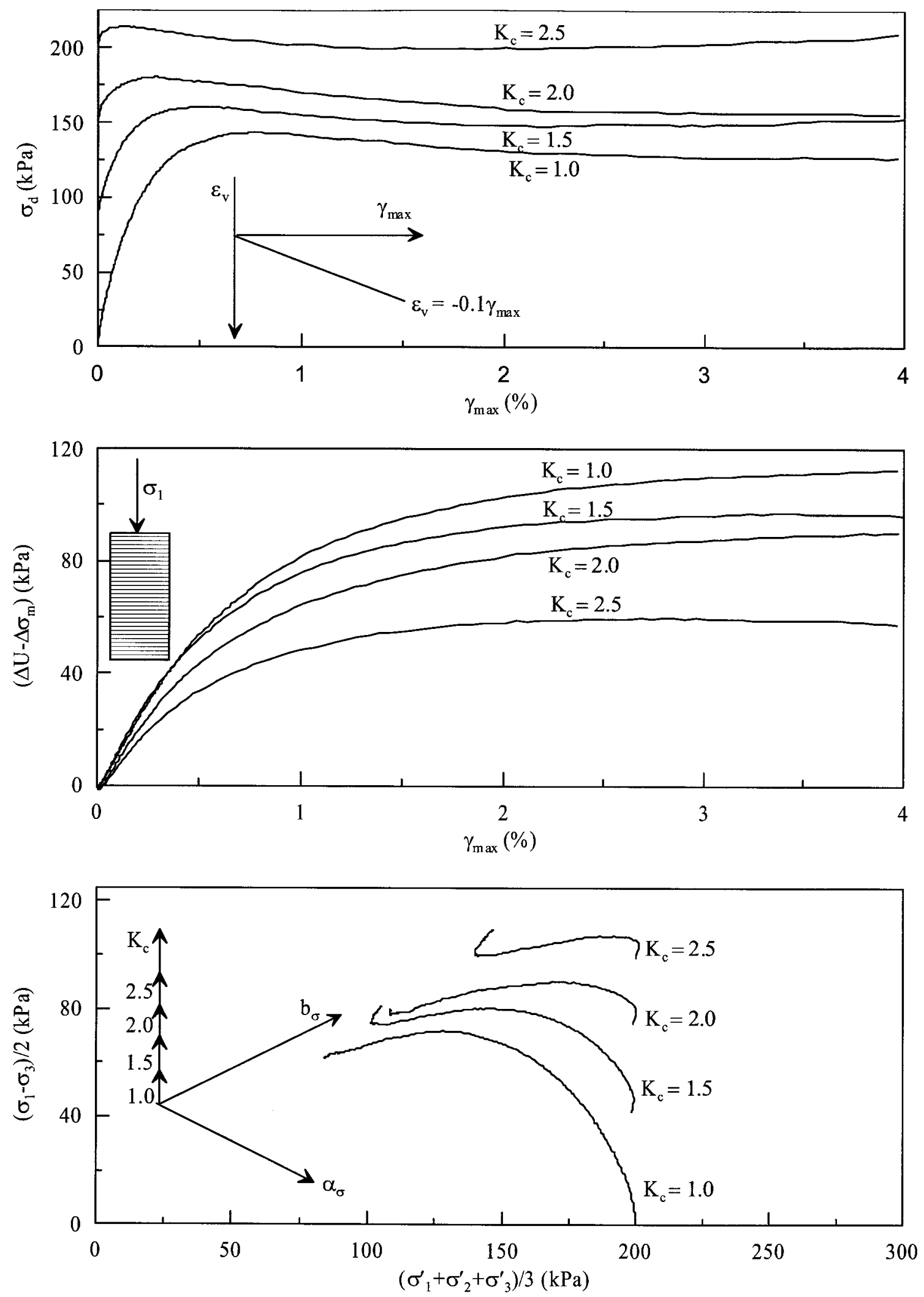

Fig. 5.20 Response of anisotropically consolidated Fraser River sand during strain path controlled loading 


$$
I_{B, \text { modified }}=\frac{S_{\text {peak }}-S_{\text {min }}}{S_{\text {peak }}-S_{\text {static }}}
$$

The value of $I_{B, \text { modified }}=0$ represents no strain softening and $I_{B, \text { modified }}=1.0 \mathrm{implies}$

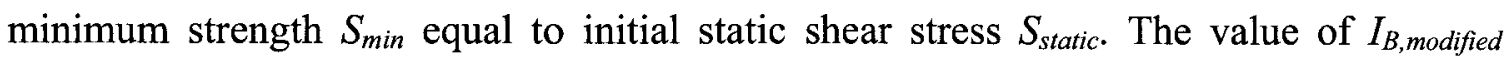
larger than 1.0 corresponds to minimum strength lower than $S_{\text {static }}$ and it would imply a catastrophic collapse, if the equilibrium is disturbed by a small perturbation.

During the strain path controlled loading, $I_{B, \text { modiffed }}$ increases with increasing $K_{c}$ (Fig. 5.21). $I_{B, \text { modified }}$ value is less than 1.0 for all $K_{c}$ value except $K_{c}=2.5$. This observation clearly demonstrates that higher initial static shear $S_{\text {static }}$ increases the flow potential when the sand is subjected to expansive volumetric strain.

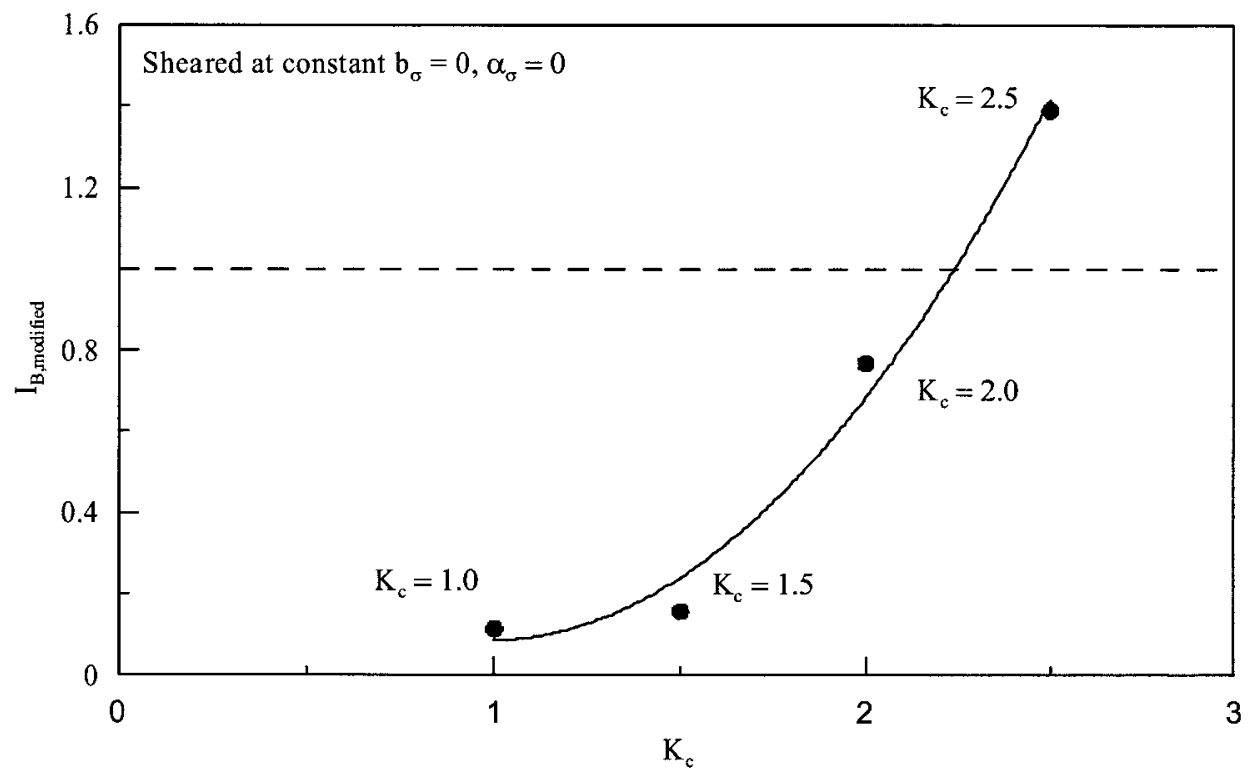

Fig. 5.21 Flow potential of anisotropically consolidated Fraser River sand during expansive volumetric strain

Fig. 5.22 shows strength $S_{\text {peak }}$ and excess pore pressure $\left(\Delta U-\Delta \sigma_{m}\right)_{p e a k}$ and stress states at peak strength state. A systematic variation of $S_{\text {peak }}$ and $\left(\Delta U-\Delta \sigma_{m}\right)_{\text {peak }}$ is observed during strain path controlled loading. However, it can be noted that the incremental shear stress needed to trigger strain softening deformation $\left(S_{\text {peak }}-S_{\text {static }}\right)$ decreases with 
increasing $K_{c}$. The excess pore pressure induced by both volume induced and shear induced components is expressed as a difference between the total induced excess pore pressure and hydrostatic excess pore pressure $\left(\Delta U-\Delta \sigma_{m}\right) . \phi_{\text {peak }}$ is essentially constant (approximately $28^{\circ}$ ) and can be treated as a constant regardless of $K_{c}$. A similar observation was made in undrained tests by Sivathayalan and Vaid (2002) that $\phi_{C S R}$ is essentially constant regardless of $K_{c}$ at fixed $b_{\sigma}$ and $\alpha_{\sigma}$.

Fig. 5.23 shows the variation of strength $S_{\text {peakPP}}$, excess pore pressure $(\Delta U$ $\left.\Delta \sigma_{m}\right)_{\text {peakPP }}$ and effective stress state at peak pore pressure state during undrained and strain path imposed loading. A systematic variation of strength is noted and $S_{\text {peakPP }}$ increases with increasing $K_{c}$. This figure also shows that a substantial amount of strength at maximum pore pressure state is lost during strain path controlled loading, compared to the undrained loading.

At $K_{c}=1.0$, strength at maximum pore pressure state $S_{P T}$ is $98 \mathrm{kPa}$ under undrained condition and $S_{\text {peakPP }}$ is $64 \mathrm{kPa}$ during strain path controlled loading $(34 \%$ of strength reduction). At $K_{c}=2.5, S_{P T}$ is $128 \mathrm{kPa}$ and $S_{\text {peakPP }}$ during strain path controlled loading is $97 \mathrm{kPa}$ ( $27 \%$ of strength reduction). Fig. 5.23 shows that strength reduction occurs due to expansive volumetric strain, but it is not affected much by $K_{c}$ (essentially the same amount of strength reduction is noted at each $K_{c}$ ).

$\left(\Delta U-\Delta \sigma_{m}\right)_{\text {peakPP }}$ reduces with increasing $K_{c}$. The effective stress states of peak pore pressure state lie on unique straight line, passing through the origin. The mobilized friction angle at peak pore pressure state $\phi_{p e a k P P}$ is $34.3^{\circ}$ and identical to $\phi_{P T}$ of undrained loading. This observation demonstrates that $\phi_{p e a k P P}$ can be treated as a unique material property of the sand during strain path controlled loading regardless of $K_{c}$. 

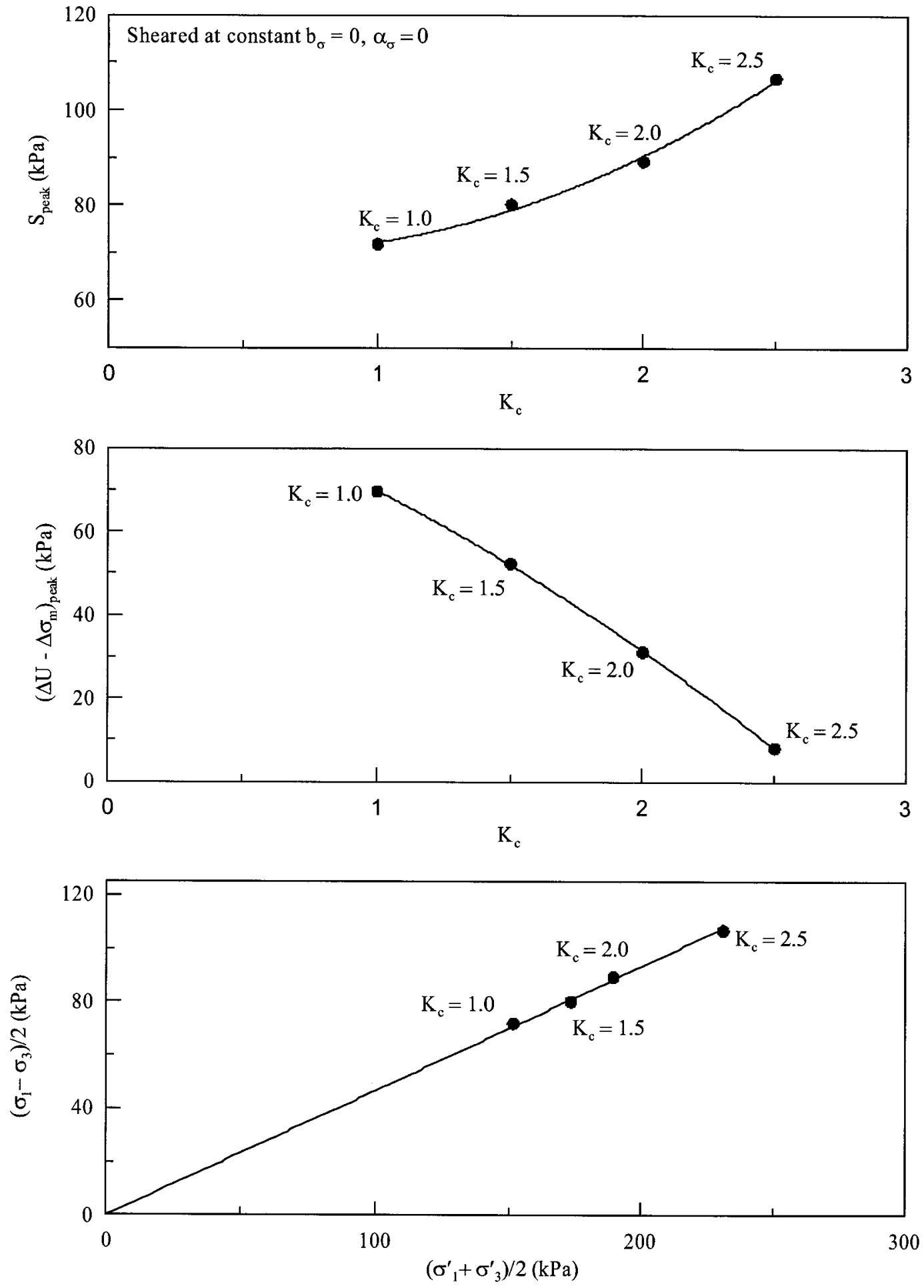

Fig. 5.22 Strength, excess pore pressure and effective stress state at peak strength state during strain path controlled loading 

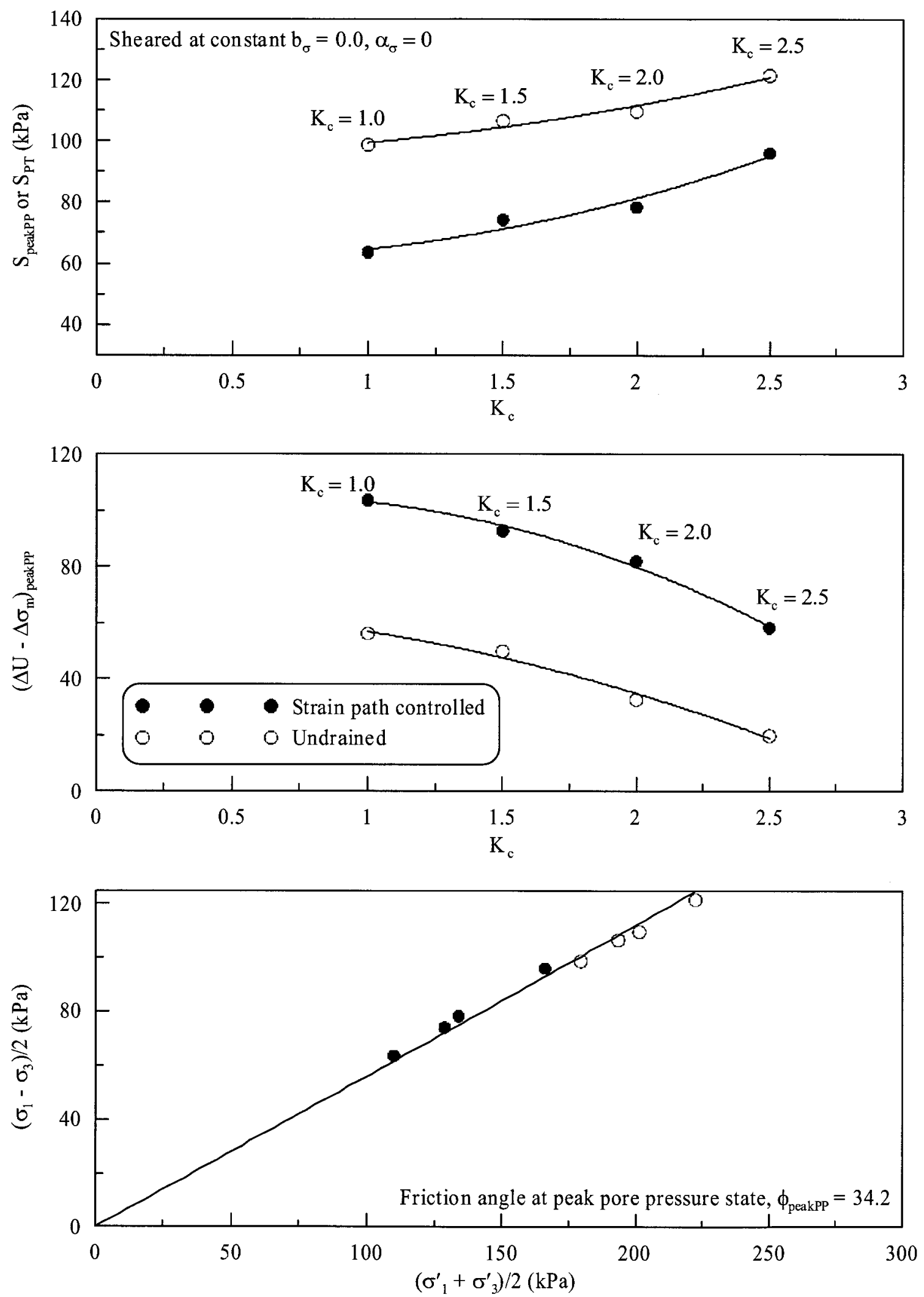

Fig. 5.23 Strength, excess pore pressure and stress state at peak pore pressure state during strain path controlled loading 


\subsubsection{Influence of strain paths on sand behaviour}

The volume inflow or outflow of an in-situ soil element occurs depending on the peizometric head gradient between the adjacent soil elements. The rate of pore volume distribution decreases with time due to reduction of the peizometric head gradient following pore pressure equalization. Generally, soil elements in-situ will not be subjected to linear strain paths (constant rate of volume transfer between soil elements) as studied in this research. However, the prediction of actual strain paths in in-situ soil during pore volume transfer is more complex and unknown. An understanding of the response of soils under such linear strain paths will help to comprehend the nature of the full spectrum of responses and is considered as a required initial step in this area of research.

A set of tests was carried out in the laboratory to provide an understanding of the response of in-situ soil elements that are subjected to various pore pressure boundary conditions under three-dimensional loading. Shearing characteristics of loosest deposited Fraser River sand subjected to expansive and contractive volumetric strains as shown in Fig. 5.24 were used to understand the effects of drainage conditions under generalized loading.

The specimens were hydrostatically consolidated to $\sigma_{m c}^{\prime}=200 \mathrm{kPa}$ and $D_{r c}=22 \%$ prior to shearing. Total mean normal stress was maintained constant throughout the shearing $\left(\sigma_{m}=\sigma_{m c}\right)$. During shear loading, principal stress was kept along $45^{\circ}$ to the deposition direction $\left(\alpha_{\sigma}=45^{\circ}\right)$. The intermediate principal stress parameter $b_{\sigma}$ was kept at 0.4 in order to simulate plane strain deformation. An undrained test was also conducted at this initial stress state to compare soil responses with strain path controlled loading. 


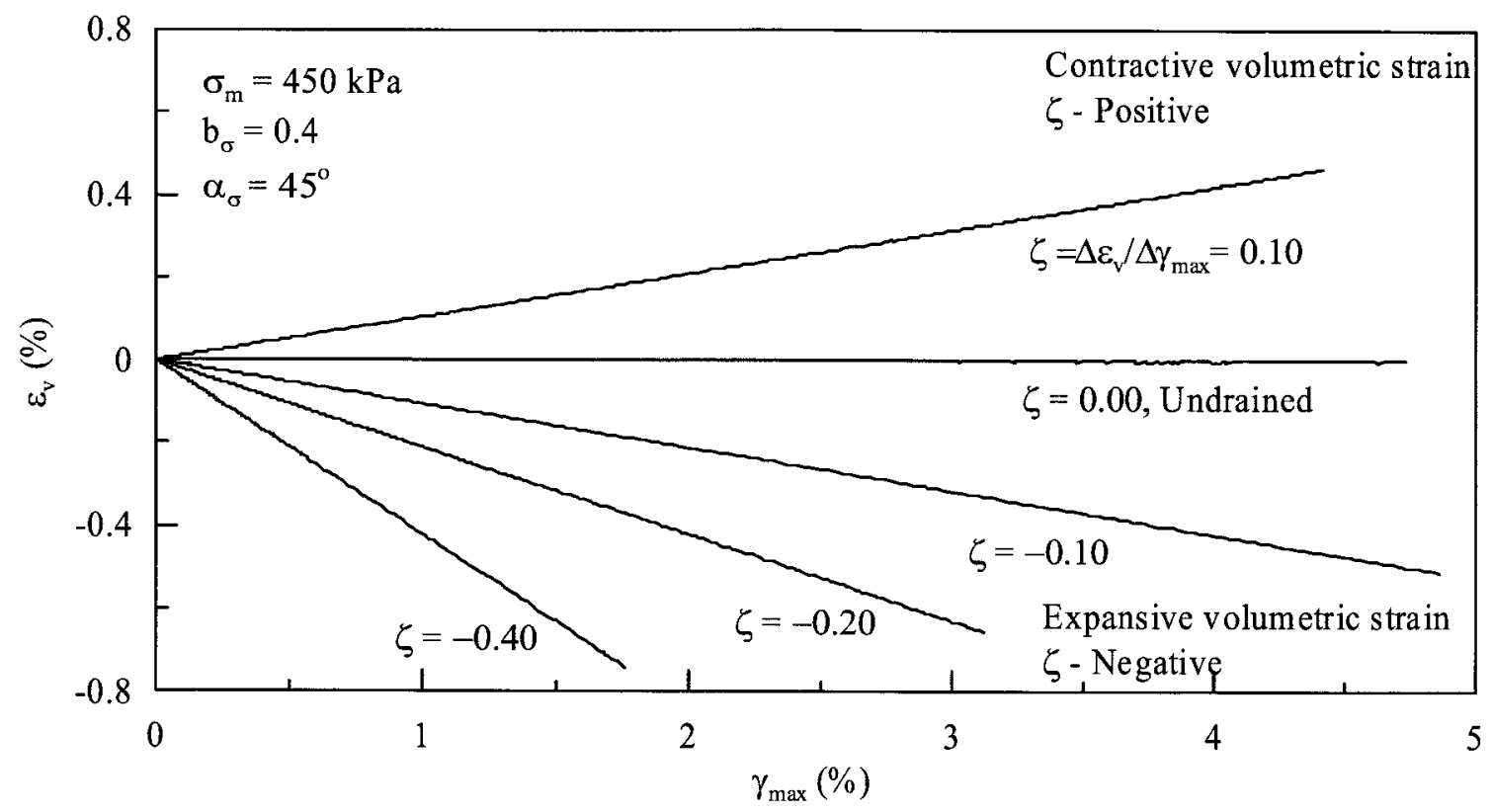

Fig. $5.24 \quad$ Imposed strain paths

Induced principal strains $\left(\varepsilon_{1}, \varepsilon_{2}\right.$ and $\left.\varepsilon_{3}\right)$ are shown in Fig. 5.25 against torsional strain $\gamma_{z \theta}$. A systematic variation of strains is noted against the imposed strain paths. The strains are linear at a small strain level. This observation demonstrates that initial anisotropy of the sand is preserved and contribution of induced anisotropy is relatively low at a small strain level. However, the strains progressively become nonlinear at larger strain levels. The induced anisotropy becomes significant at the large strain level, in which sand experiences higher effective stress ratio $R$.

It can be further noted that strains are more nonlinear at $\zeta$ of -0.2 and -0.4 . In the tests at $\zeta=-0.20$ and -0.40 , excess pore pressure development is very large, and thus the sand experiences very low effective stress. The effective stress ratio $R$ reaches a very high or an undefined level and may not be an accurate indicator to predict the induced anisotropy in sand. 

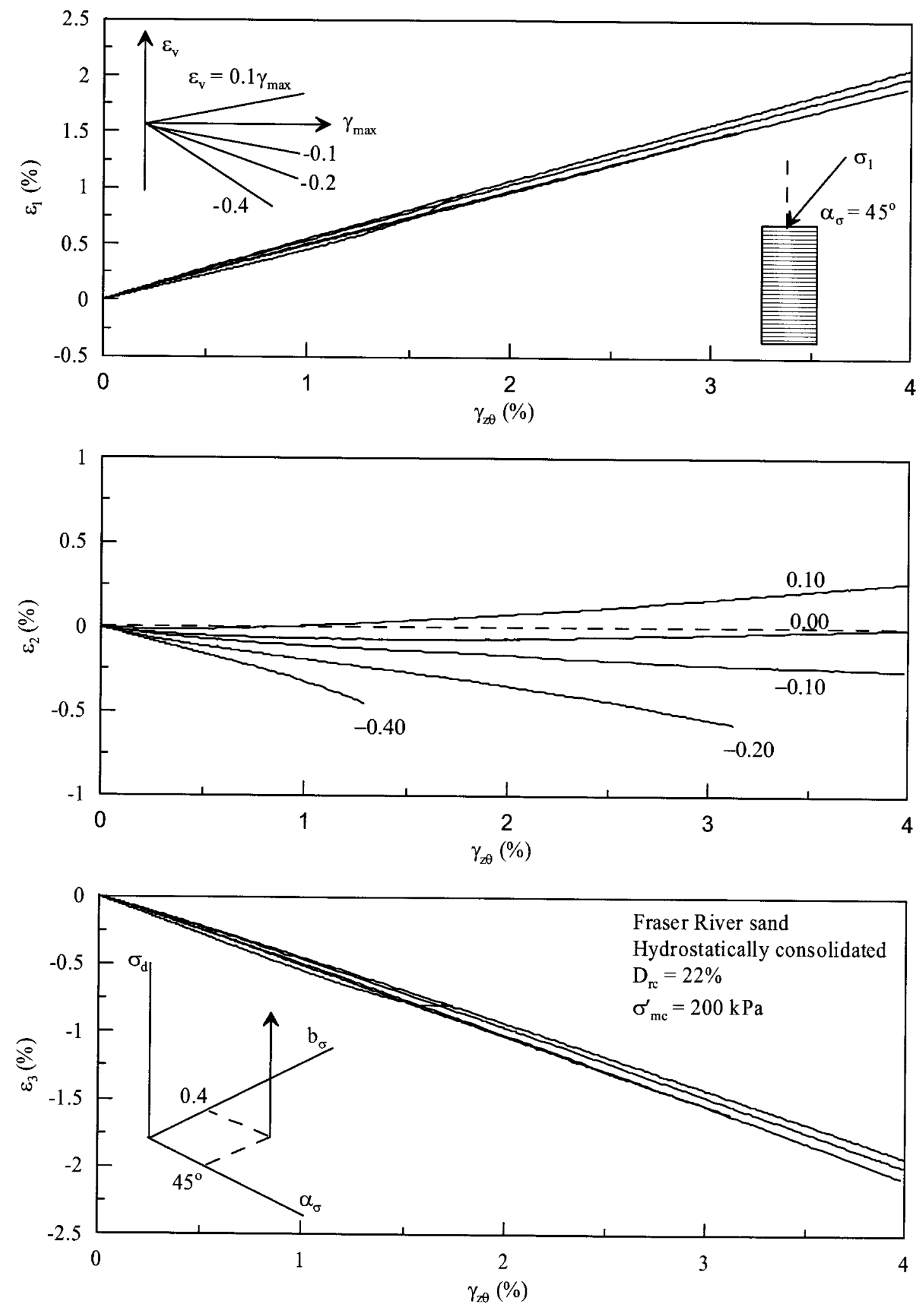

Fig. 5.25 Variation of principal strains with torsional strain at $b_{\sigma}=0.4$ and $\alpha_{\sigma}=45^{\circ}$ during strain path controlled tests 
As shown in Fig. 5.25, intermediate principal strain $\varepsilon_{2}$ at $b_{\sigma}=0.4$ and $\alpha_{\sigma}=45^{\circ}$ under undrained condition is much closer to zero. However, magnitude of $\varepsilon_{2}$ significantly deviates from zero during the application of both expansive and contractive volumetric strain. The plane strain condition ( $b_{\sigma}$ closer to 0.4 in undrained condition) no longer exists at this $b_{\sigma}$ value when sand deforms under strain path controlled loading. $\varepsilon_{2}$ is negative and its magnitude increases with increasing rate of expansive volumetric strain. $\varepsilon_{2}$ is positive during the contractive volumetric strain. The major principal strain $\varepsilon_{1}$ is positive and its variation is small as drainage changes from contractive to expansive. The minor principal strain, $\varepsilon_{3}$ is negative and the same trend of variation is noted as $\varepsilon_{1}$.

Stress-strain response of Fraser River sand subjected to the linear strain paths, and corresponding effective stress paths and excess pore pressure responses are shown in Fig. 5.26. The sand responses systematically change from strain hardening to strain softening when the imposed drainage conditions change from contractive to expansive volumetric strain. $\zeta=0.10$ (volume outflow from specimen) results in complete strain hardening behaviour and the strength of the sand is much higher than undrained strength. At the same stress condition, the undrained response $(\zeta=0.00)$ is marginally strain softening with almost negligible reduction in strength following the peak state. During expansive volumetric strain, $\zeta$ of $-0.10,-0.20$ and -0.40 results in complete strain softening behaviour and a much weaker response than undrained. 

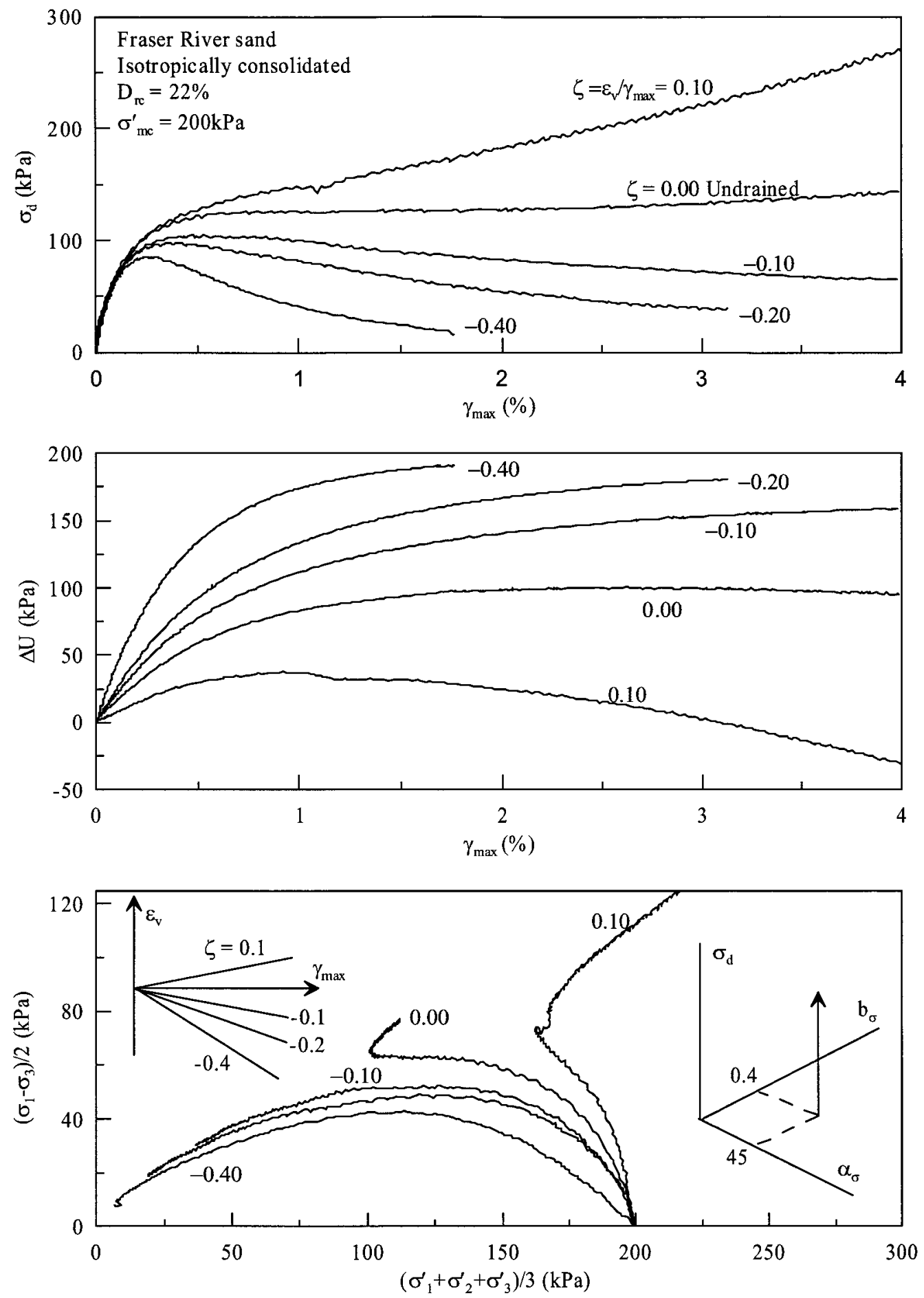

Fig. 5.26 Response of Fraser River sand at $b_{\sigma}=0.4$ and $\alpha_{\sigma}=45^{\circ}$ during strain path controlled tests

Expansive volumetric strains cause significant damage to the strength of the soil by drastically increasing the tendency for strain softening. The minimum strength 
realized during the application of expansive volumetric strain is smaller than the undrained strength of the soil. As the rate of volume inflow increases, the minimum strength realized become smaller and the excess pore pressure generated becomes high (excess pore pressure induced is almost $90 \%$ of $\sigma_{3 c}^{\prime}$ when $\zeta=-0.4$ ), and thus the sand may even reach a state of zero effective stress under monotonic loading. This results in the deformation of sand at essentially zero shear strength.

Variation of strength $S_{\text {peak }}$, excess pore pressure $\Delta U_{\text {peak }}$ and friction angle $\phi_{\text {peak }}$ at peak strength state is shown in Fig. 5.27 against the strain increment ratio $\zeta$. The peak strength state is only realized in strain softening materials, and thus limited to tests between $\zeta=0.0$ and $\zeta=-0.4$. $S_{\text {peak }}$ systematically reduces with an increasing rate of imposed expansive volumetric strain. The peak strength state of the test at $\zeta=-0.4$ was realized at $\gamma_{\max }=0.3 \%$ and sand experienced only $0.12 \%$ of expansive volumetric strain at that shear strain level. In terms of relative density, this corresponds to about $0.2 \%$ increment, a very minor change. This small amount of expansive volumetric strain caused about $31 \%$ reduction in $S_{\text {peak }}$ compared to $S_{\text {peak }}$ of the undrained test.

A systematic variation of $\Delta U_{p e a k}$ is noted (Fig. 5.27) as $\zeta$ varying from 0.0 to 0.40 . About $23 \%$ of increment in $\Delta U_{\text {peak }}$ is noted when $\zeta$ varies from 0.0 (undrained) to 0.4. $\phi_{\text {peak }}$ reduces with the increasing rate of expansive volumetric strain. The friction angle mobilized at peak strength state $\phi_{C S R}$ under undrained condition is approximately $27^{\circ}$ and $\phi_{p e a k}$ reduces to $21^{\circ}$ at $\zeta$ of -0.40 . This observation is consistent with a previous study by Sivathayalan and Logeswaran (2008). It was reported that $\phi_{\text {peak }}$ decreases with an increasing rate of expansive volumetric strain under triaxial mode of loading. 

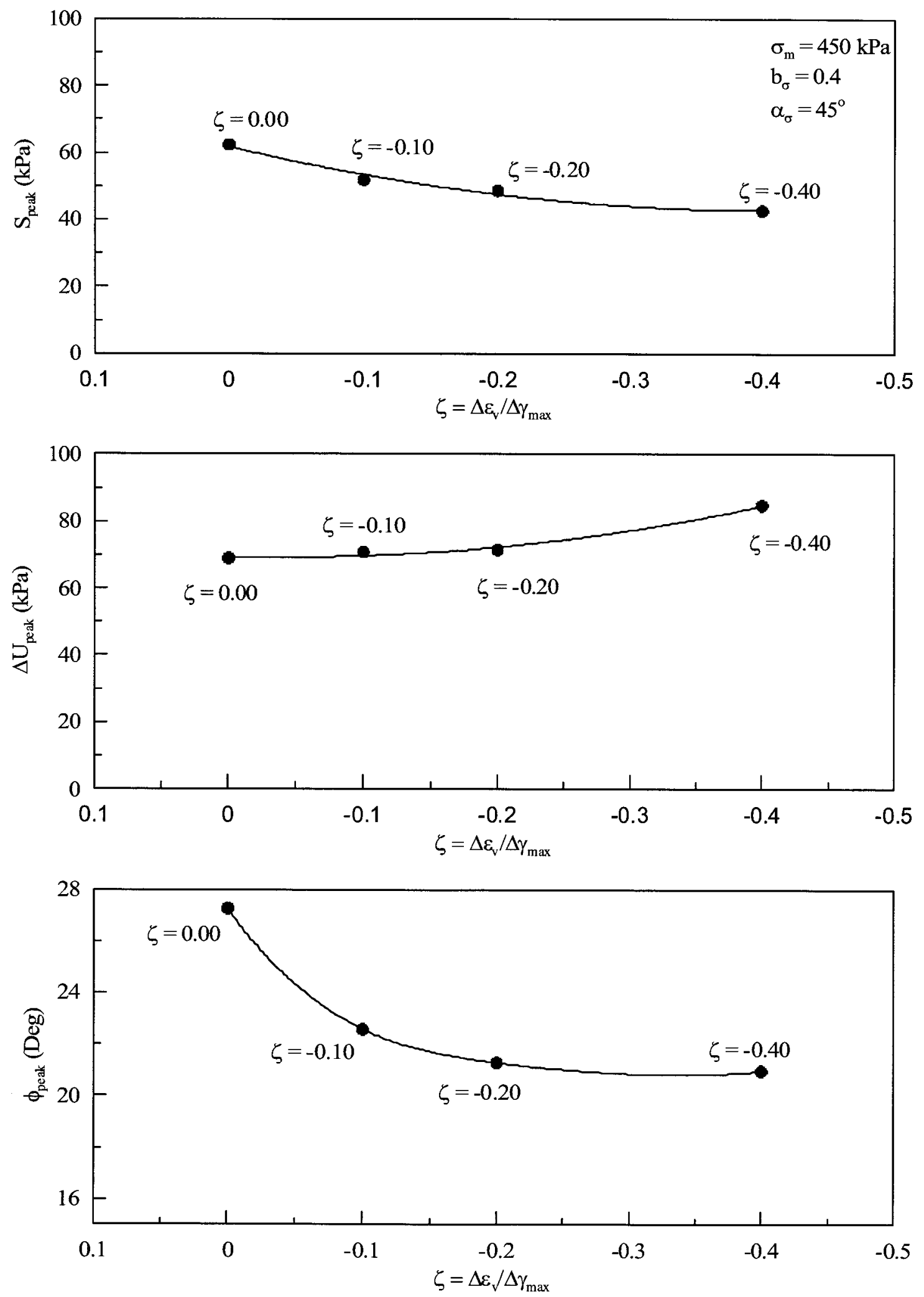

Fig. 5.27 Strength, excess pore pressure and mobilized friction angle at peak strength state during strain path controlled tests 
Fig. 5.28 shows the variation of strength $S_{\text {peakPP }}$, excess pore pressure $\Delta U_{\text {peakPP }}$ and the effective stress state at the instant of peak pore pressure. $S_{\text {peakPP }}$ corresponds to the minimum strength in strain softening sands. A minimum strength cannot be defined for fully strain hardening sands, which was the case when the sand was sheared with positive $\zeta$. A systematic reduction of strength is noted as $\zeta$ varies from positive (contractive volumetric strain) to negative (expansive volumetric strain). Strength at peak pore pressure state under undrained condition $S_{P T}$ (phase transformation) is $63 \mathrm{kPa}$ at $b_{\sigma}=$ 0.4 and $\alpha_{\sigma}=45^{\circ}$. About $60 \%$ reduction in $S_{\text {peakPP }}$ can be noted in Fraser River sand during the application of $\zeta=-0.4\left(S_{\text {peakPP }}\right.$ at $\zeta=-0.40$ is $\left.25 \mathrm{kPa}\right)$.

As shown in the excess pore pressure variation at peak pore pressure state (Fig. 5.28), a systematic increment of excess pore pressure is noted as $\zeta$ varies from positive to negative. Peak excess pore pressure induced under undrained condition $\Delta U_{P T}$ is around $100 \mathrm{kPa}$. About $72 \%$ increment in $\triangle U_{\text {peakPP }}$ can be noted during the application of $\zeta=-$ 0.4 and about $70 \%$ reduction in $\Delta U_{\text {peakPP }}$ is observed during contractive volumetric strain $\zeta=0.10$ compared to $\Delta U_{P T}$.

Effective stress states at the peak pore pressure state essentially lie on a straight line passing through the origin and result in a friction angle at the peak pore pressure state $\phi_{\text {peakPP }}$ of $34.3^{\circ}$. $\phi_{\text {peakPP }}$ can be treated as constant and is equal to the friction angle mobilized at PT state of undrained tests $\phi_{P T}$. In previous studies by Sivathayalan and Logeswaran (2007 \& 2008) using triaxial tests, it was noted that effective stress states at the instant of peak excess pore pressure in various linear and nonlinear strain path tests resulted in $\phi_{\text {peakPP }}$ equal to $\phi_{P T}$. 

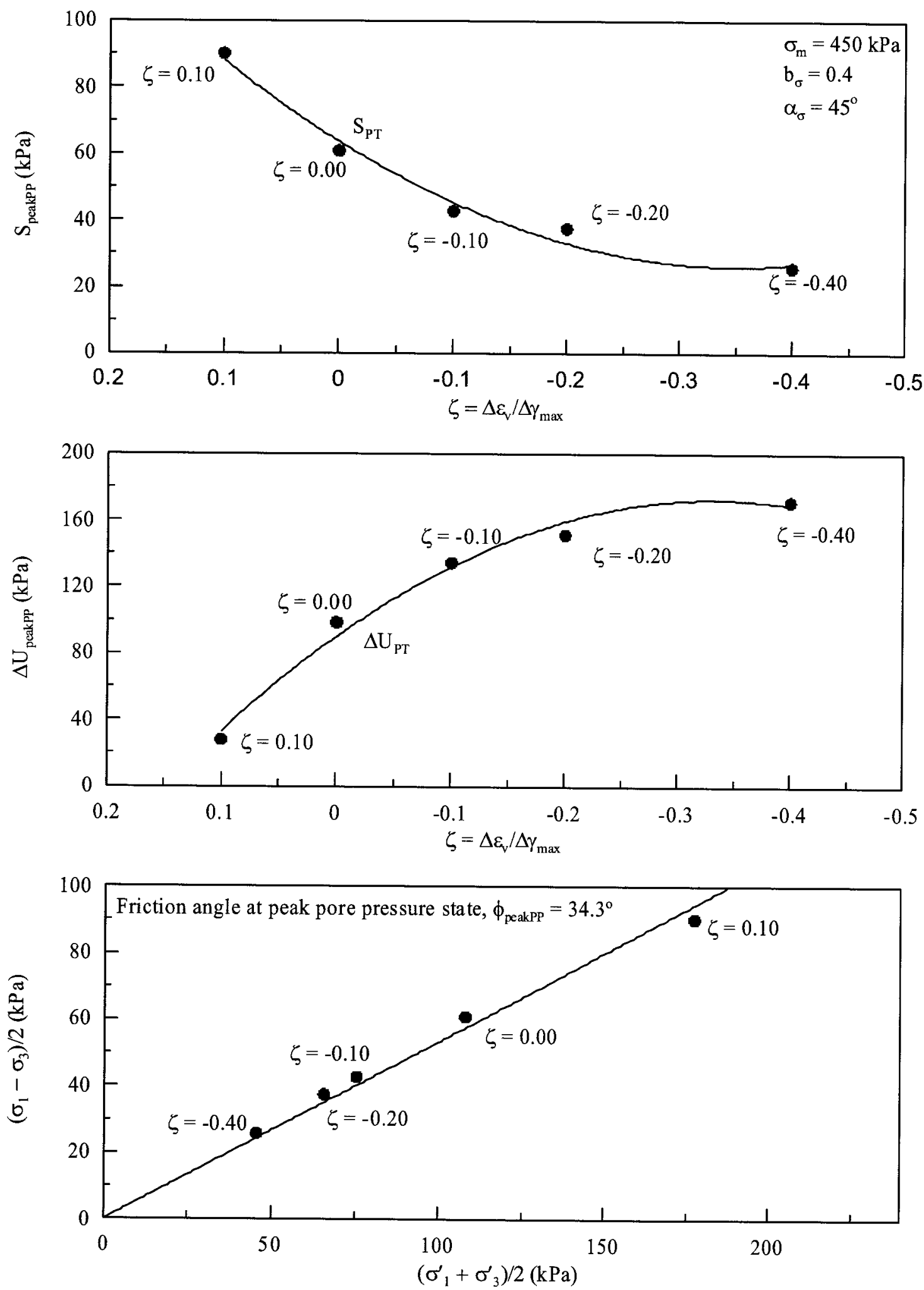

Fig. 5.28 Strength, excess pore pressure and effective stress state at peak pore pressure state during strain path controlled tests 
These test results clearly demonstrate that the undrained state does not always represent the most damaging scenario in the field, and the static liquefaction potential of a soil deposit may be significantly influenced by the drainage boundary conditions. A small amount of pore water intrusion may change the stronger behaviour of soil (dilative) to softer behaviour (contractive). It was noted in undrained tests that sand behaviour became softer when the intermediate principal stress parameter is high and principal stress direction is closer to the bedding plane. Soils at these states (i.e. high $b_{\sigma}$ and $\alpha_{\sigma}$ ), may be further weakened due to intrusion of pore water depending on the pore pressure boundary conditions.

\subsection{Summary}

Undrained effective stress-strain behaviour of Fraser River sand is dependent on the intermediate principal stress parameter $b_{\sigma}$ when principal stress direction $\alpha_{\sigma}$ and total mean normal stress are held constant. Fraser River sand exhibits strain hardening behaviour at different levels of $b_{\sigma}$ at fixed $\alpha_{\sigma}=0^{\circ}$. The strength of the sand systematically reduces with increasing $b_{\sigma}$. The friction angle mobilized at Phase transformation (PT) is constant regardless of $b_{\sigma}$. The undrained response of Fraser River sand is highly dependent on $\alpha_{\sigma}$. Fraser River sand exhibits strain hardening behaviour and stronger at $b_{\sigma}=0.0$ and $\alpha_{\sigma}=0^{\circ}$ and it exhibits strain softening behaviour at $b_{\sigma}=0.0$ and $\alpha_{\sigma}=90^{\circ}$. This observation confirms the existence of anisotropy that is formed during the deposition process and results in high deformability along the bedding plane. Also, it confirms that the friction angle mobilized at the peak pore pressure (phase transformation) state is independent of principal stress direction and strength at PT is dependent of $b_{\sigma}$ and $\alpha_{\sigma}$. Dependence of undrained response on $b_{\sigma}$ and $\alpha_{\sigma}$ has been well 
established in the literature. Nevertheless, it was studied again to provide the confidence required in using a new $\mathrm{HCT}$ device, and to provide a baseline for proper comparison with shear-volume couple deformation (i.e., strain path controlled tests). Experiments conducted to study the behaviour of Fraser River sand under simultaneous changes in pore pressure and pore volume show that strain paths imposing expansive volumetric strains can trigger liquefaction during static loading in sands that are dilative and stable under undrained conditions.

Fraser River sand exhibits strain hardening behaviour at $b_{\sigma}=0.0$ and $\alpha_{\sigma}=0^{\circ}$ during the application of strain path $\Delta \varepsilon_{v} / \Delta \varepsilon_{a}=-0.10$ and undrained loading. The sand strain softens at $b_{\sigma}=1.0$ and $\alpha_{\sigma}=0^{\circ}$ during the application of strain path $\Delta \varepsilon_{v} / \Delta \varepsilon_{a}=-0.10$ and it exhibits strain hardening under undrained condition. The distinguishing observations on sand response demonstrate that the expansive volumetric strain may be more critical at high $b_{\sigma}$. The strength at the peak strength state decreases with increasing $b_{\sigma}$ and the friction angle mobilized at this state is essentially constant at a strain increment ratio of $\zeta=-0.10$. The strength at the peak pore pressure state systematically reduces with increasing $b_{\sigma}$. The friction angle mobilized at the peak pore pressure state is constant regardless of $b_{\sigma}$.

Fraser River sand strain hardens at $\alpha_{\sigma}=0^{\circ}$ and $30^{\circ}$ during the application of strain path $\left(\Delta \varepsilon_{v} / \Delta \varepsilon_{a}=-0.10\right)$ when $b_{\sigma}$ is held at zero. The sand strain softens and shows a weaker response when principal stress direction moves further towards the bedding plane $\left(\alpha_{\sigma}=45^{\circ}, 60^{\circ}\right.$ and $90^{\circ}$ ). The strength mobilized at the peak strength state during the application of the strain path reduces systematically with increasing $\alpha_{\sigma}$. Friction angle mobilized at this state has a minor variation. 
The mobilized strength at the peak pore pressure state during strain path controlled loading $\left(\Delta \varepsilon_{v} / \Delta \varepsilon_{a}=-0.10\right)$ decreases with increasing $\alpha_{\sigma}$. Decrement of strength is significant when $\alpha_{\sigma}$ changes from $0^{\circ}$ to $45^{\circ}$. However, the decrement is minor when the principal stress direction is more towards the bedding plane (beyond $\alpha_{\sigma}=45^{\circ}$ ). The same trend of decrement of strength at the peak excess pore pressure (PT) state has been observed in undrained loading as well. Regardless of $\alpha_{\sigma}$, the strength reduction at the state of peak pore pressure due to expansive volumetric strain $\left(\Delta \varepsilon_{v} / \Delta \varepsilon_{a}=-0.10\right)$ is approximately equal to that at PT state in undrained loading. The friction angle mobilized at the state of peak excess pore pressure is unique regardless of $\alpha_{\sigma}$.

As shown from the tests conducted along different strain paths (application of contractive and expansive volumetric strain), the sand becomes weaker when it is subjected to volume intrusion (expansive volumetric strain). As a result, it liquefies at an early stage of its deformation. A small amount of pore volume extrusion (contractive volumetric strain) results in a stronger soil response than in undrained. A significant decrement is noted in strength and friction angle at the peak strength state with increasing rate of volume inflow (expansive volumetric strain).

The friction angle mobilized at the state of peak pore pressure can be treated as a unique material property even if the sand experiences different drainage conditions than undrained. The friction angle mobilized at the state of peak pore pressure is independent of the principal stress direction $\alpha_{\sigma}$, intermediate principal stress parameter $b_{\sigma}$, initial effective stress ratio, $K_{c}$ and strain paths. Even though the data herein is limited to one density, data in the literature suggests that this conclusion applies regardless of the density state. Sivathayalan and Logeswaran (2008) also noted that the friction angle at the 
minimum strength state, which corresponds to the maximum pore pressure state, is constant regardless of the drainage condition (strain paths).

Current design practice only considers drained and undrained states, and it generally assumes that undrained condition corresponds to the lowest shear strength. The experimental results presented herein clearly demonstrate that undrained strength may not be the lowest, if pore pressure redistribution occurs in the in-situ soil elements that are subjected to expansive volumetric strain. The principal stress along the bedding plane at a fixed $b_{\sigma}$ and a higher intermediate principal stress parameter at fixed $\alpha_{\sigma}$ in soil loading result in a softer response and a lower strength under undrained condition. During application of expansive volumetric strain, the soil will further experience strength degradation. It could be suggested that consideration of strength, based on the stress states and the drainage boundary conditions may further improve the confidence in the design practice. 


\section{CYCLIC BEHAVIOUR OF SAND UNDER GENERALIZED LOADING}

\subsection{Introduction}

Undrained cyclic behaviour of Fraser River sand under generalized stress state is presented in this Chapter. Triaxial and simple shear devices that are commonly used to assess the liquefaction potential of soils in laboratory tests cannot properly simulate the stress conditions in-situ. The nature of rotation of principal stress direction during cyclic loading in these laboratory tests and in-situ are quite different. Jump rotation is imposed in the triaxial cyclic loading and uncontrolled smooth rotation is applied in the simple shear. However, in the field, the smooth and continuous rotation of principal stress direction is very common. Investigation of cyclic soil behaviour under jump and smooth rotation of principal stress direction may be useful to improve the current liquefaction assessment.

Fig. 6.1 shows the nature of the rotation of principal stress directions imposed on Fraser River sand under three-dimensional loading. In the jump rotation tests, direction of principal stress is instantaneously switched from positive to negative value when cyclic shear stress changes its direction. In the smooth rotation test, direction of principal stress rotates smoothly during application of cyclic stress. The smooth rotation of principal stress was applied as a sinusoidal function of time. The signs of the direction of principal stress are defined based on direction of the torsional shear stress $\tau_{z} \theta$. The positive sign of direction of principal stress denotes that $\tau_{z \theta}$ is counter clockwise and the negative sign of principal stress direction denotes that $\tau_{z}$ is clockwise. 


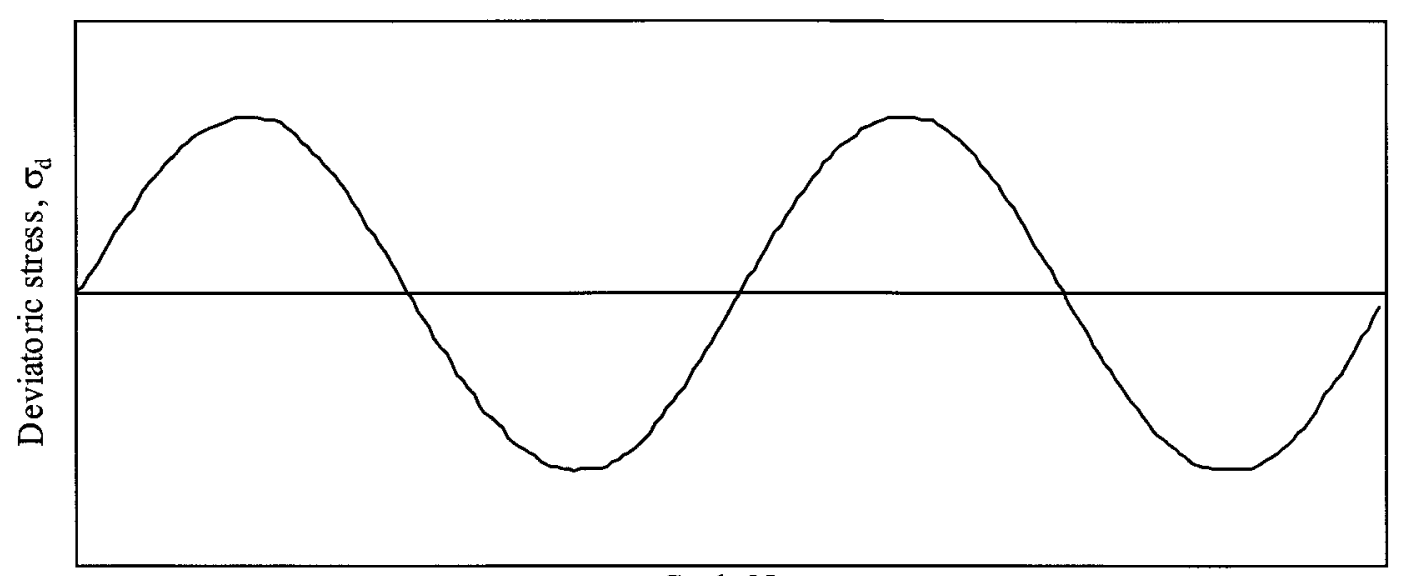

Cycle No.

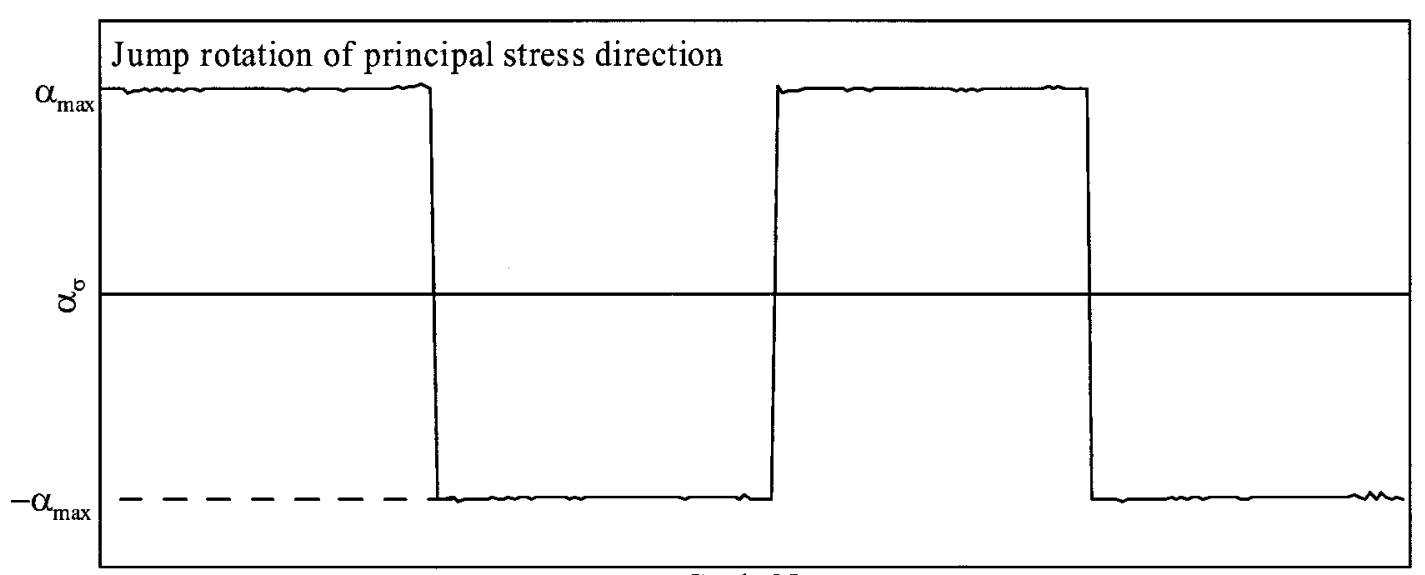

Cycle No.

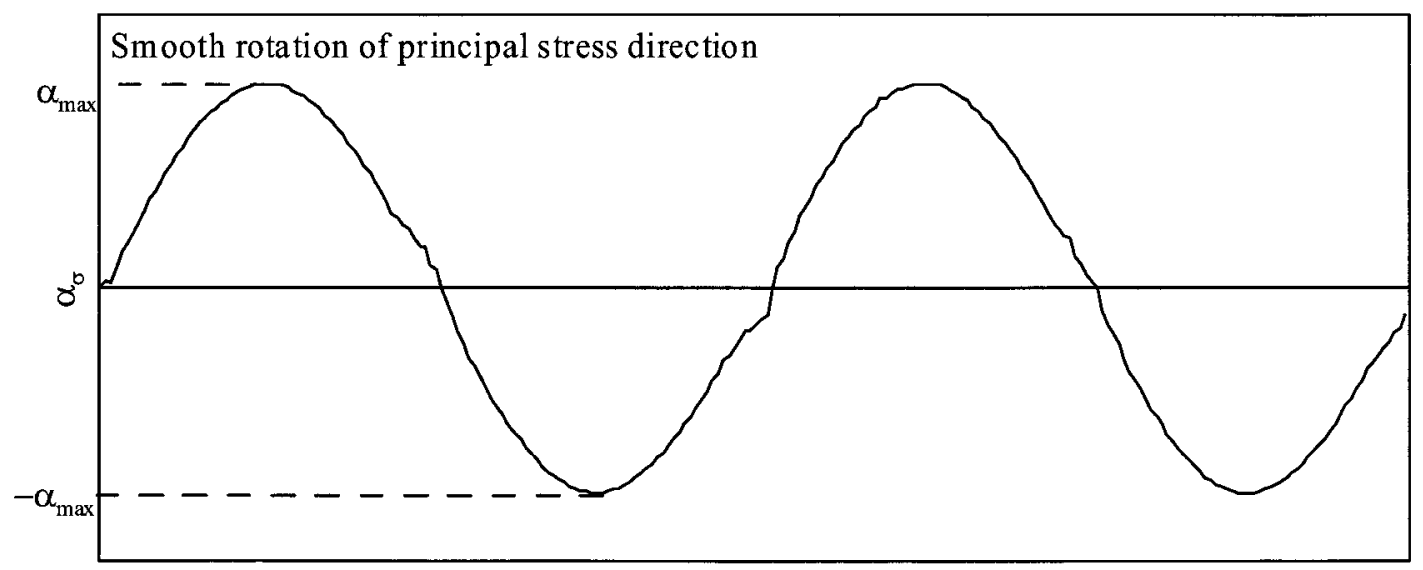

Cycle No.

Fig. 6.1 Cyclic stress and nature of principal stress rotation imposed 
The cyclic shear stress was applied to the hollow cylindrical specimen according to Eq. 6.1 as given below.

$$
\frac{\sigma_{d}}{2 \sigma_{m c}^{\prime}}=\frac{\sigma_{d}}{2 \sigma_{3 c}^{\prime}}=\beta_{0}+\beta_{\max } \sin (\omega t)
$$

$\sigma_{d}$ is deviatoric stress, $\sigma_{3 c}^{\prime}$ is effective minor principal stress at the end of consolidation. $\sigma_{m c}^{\prime}$ is effective total mean normal stress at the end of consolidation. As specimens are isotropically consolidated, $\sigma_{3 c}^{\prime}$ is equal to $\sigma_{m c}^{\prime} . \beta_{0}$ is initial stress ratio and $\beta_{\max }$ is the amplitude of cyclic stress ratio. $\beta_{0}$ is zero in the tests because of isotropic consolidation. In order to rotate the principal stress direction during the application of cyclic stress smoothly, $\alpha_{\sigma}$ was changed in sinusoidal manner as given in Eq. 6.2.

$$
\begin{aligned}
\alpha_{\sigma}= & \alpha_{\sigma, 0}+\alpha_{\sigma, \max } \sin (\omega t) \\
& \alpha_{\sigma} \text { is principal stress direction with specimen deposition axis, } \alpha_{\sigma, 0} \text { is initial }
\end{aligned}
$$
principal stress direction at the end of consolidation. $\alpha_{\sigma, \max }$ is allowed maximum principal stress rotation.

From Mohr's circle, torsional shear stress applied $\tau_{z \theta}$ on the specimens can be expressed with respect to $\sigma_{d}$ and $\alpha_{\sigma}$ as given in Eq. 6.3. During the smooth rotation of principal stress, shear stress is calculated by multiplying the two sine functions.

$$
\begin{aligned}
& \tau_{z \theta}=\left(\frac{\sigma_{d} \sin \left(2 \alpha_{\sigma}\right)}{2}\right) \\
& \text { or } \\
& \frac{\tau_{z \theta}}{\sigma_{3 c}^{\prime}}=\left(\beta_{0}+\beta_{\max } \sin (\omega t)\right)\left[\sin \left(2 \alpha_{\sigma}\right)\right]
\end{aligned}
$$


The loosest deposited sand specimens by water pluviation were hydrostatically consolidated to $\sigma_{m c}^{\prime}=200 \mathrm{kPa}$ prior to cyclic shearing. The relative density of the specimens at the end of consolidation was about $22 \%$. During cyclic loading, total mean normal stress $\sigma_{m c}$ was held constant except during a test that was conducted to simulate the triaxial mode of cyclic loading. The period of cyclic loading was maintained at 240 seconds to enable proper feedback control. The details of the tests conducted are given in Table 6.1.

The term liquefaction as discussed in Chapter 2 refers herein to all phenomena involving excessive deformation. The soil is deemed to have liquefied when maximum shear strain $\gamma_{\max }$ exceeds $3.75 \%$ (NRC, 1985). This strain may have accumulated with or without the occurrence of contractive deformation during cyclic loading.

Table 6.1 Initial and loading states of tests conducted under cyclic loading

\begin{tabular}{|c|c|c|c|c|c|c|c|}
\hline \multirow{2}{*}{ Test No } & \multicolumn{4}{|c|}{ Consolidation state } & \multicolumn{2}{|c|}{$\begin{array}{l}\text { Loading } \\
\text { state }\end{array}$} & \multirow{2}{*}{ Remarks } \\
\hline & $\begin{array}{l}D_{r c} \\
(\%) \\
\end{array}$ & $\begin{array}{c}\sigma_{m c}^{\prime} \\
(\mathrm{kPa})\end{array}$ & $b_{\sigma c}$ & $\begin{array}{c}\alpha_{\sigma c} \\
(\text { Deg) }\end{array}$ & $\begin{array}{c}\alpha_{\sigma} \\
(\mathrm{Deg})\end{array}$ & $\sigma_{d} / 2 \sigma_{3 c}^{\prime}$ & \\
\hline CY01 & 21.3 & 200 & - & - & $0-+90$ & 0.20 & $\begin{array}{c}\text { Triaxial cyclic } \\
\text { loading }\end{array}$ \\
\hline CY02 & 22.1 & 200 & - & - & $-45-+45$ & 0.20 & $\begin{array}{c}\text { Jump rotation } \\
b_{\sigma}=0.40\end{array}$ \\
\hline CY03 & 22.2 & 200 & - & - & $-45-+45$ & 0.10 & \\
\hline CY04 & 21.4 & 200 & - & - & $-45-+45$ & 0.15 & \\
\hline CY05 & 20.2 & 200 & - & - & $-45-+45$ & 0.20 & \\
\hline CY06 & 20.8 & 200 & - & - & $-45-+45$ & 0.25 & \\
\hline
\end{tabular}




\begin{tabular}{|c|c|c|c|c|c|c|c|}
\hline CY07 & 20.8 & 200 & - & - & $-45-+45$ & 0.15 & \multirow{3}{*}{ 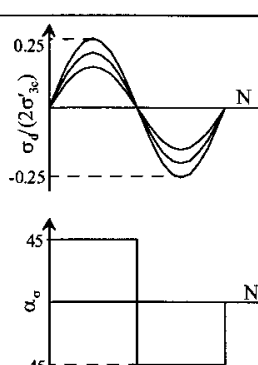 } \\
\hline CY08 & 23.2 & 200 & - & - & $-45-+45$ & 0.20 & \\
\hline CY09 & 22.0 & 200 & - & - & $-45-+45$ & 0.25 & \\
\hline CY10 & 20.3 & 200 & - & - & $-15-+15$ & 0.20 & \multirow{6}{*}{$\begin{array}{l}\text { Smooth } \\
\text { rotation } \\
b_{\sigma}=0.0\end{array}$} \\
\hline CY11 & 20.8 & 200 & - & - & $-30-+30$ & 0.20 & \\
\hline CY12 & 20.2 & 200 & - & - & $-45-+45$ & 0.20 & \\
\hline CY13 & 20.5 & 200 & - & - & $-60-+60$ & 0.20 & \\
\hline CY14 & 22.7 & 200 & - & - & $-75-+75$ & 0.20 & \\
\hline CY15 & 22.7 & 200 & - & - & $-90-+90$ & 0.20 & \\
\hline CY16 & 20.2 & 200 & - & - & $-15-+15$ & 0.20 & \multirow{5}{*}{ Jump rotation } \\
\hline CY17 & 23.0 & 200 & - & - & $-30-+30$ & 0.20 & \\
\hline CY18 & 23.2 & 200 & - & - & $-45-+45$ & 0.20 & \\
\hline CY19 & 22.4 & 200 & - & - & $-60-+60$ & 0.20 & \\
\hline CY20 & 22.4 & 200 & - & - & $-75-+75$ & 0.20 & \\
\hline
\end{tabular}

\subsection{Simulation of triaxial mode of cyclic loading}

The HCT device was used to simulate a cyclic triaxial test for comparison purposes. Major principal stress $\sigma_{l}$ was kept along deposition direction $\left(\alpha_{\sigma}=0^{\circ}\right)$ during positive half cycle and along the bedding plane $\left(\alpha_{\sigma}=90^{\circ}\right)$ during negative half cycle, and 
thus $90^{\circ}$ jump rotation was imposed to the principal stresses. The intermediate principal stress parameter $b_{\sigma}$ was allowed to change from 0 to 1.0 . In this test, the inner and outer pressures of the specimen were maintained constant. The cyclic stress ratio $\sigma_{d} /\left(2 \sigma_{m c}^{\prime}\right)=$ 0.20 was imposed.

Fig. 6.2 shows the response of Fraser River sand under typical triaxial mode of cyclic loading. Effective stress paths are not symmetrical about the effective mean normal stress $\sigma_{m}^{\prime}$ axis. The inclination of the effective stress path to the right demonstrates that larger effective stress ratio is mobilized in extension compared to compression. No strain softening behaviour is noted and the soil is deemed to have liquefied based on the maximum shear strain criterion. Only a small level of strain was induced until the $34^{\text {th }}$ cycle, in which the stress path reached the Phase Transformation (PT) line and induced large shear strain. Both excess pore pressure (75\% of $\left.\sigma_{m c}^{\prime}\right)$ and shear strain $\left(\gamma_{\max }=4.0 \%\right)$ reached their maximum values during this cycle.

The excess pore pressure response shows peaks and troughs during application of cyclic loading. Positive increments of excess pore pressure are noted in the first and fourth quarters of each cycle. In the second and third quarters of each cycle, negative increments of excess pore pressure are noted. The increment of excess pore pressure corresponds to the increment of mean normal stress $\Delta \sigma_{m}$ during the first and fourth quarters. The decrement of excess pore pressure relates to the decrement of $\Delta \sigma_{m}$ during the second and third quarters. Nevertheless, cumulative excess pore pressure is positive and increases with an increasing number of applied cycles. 

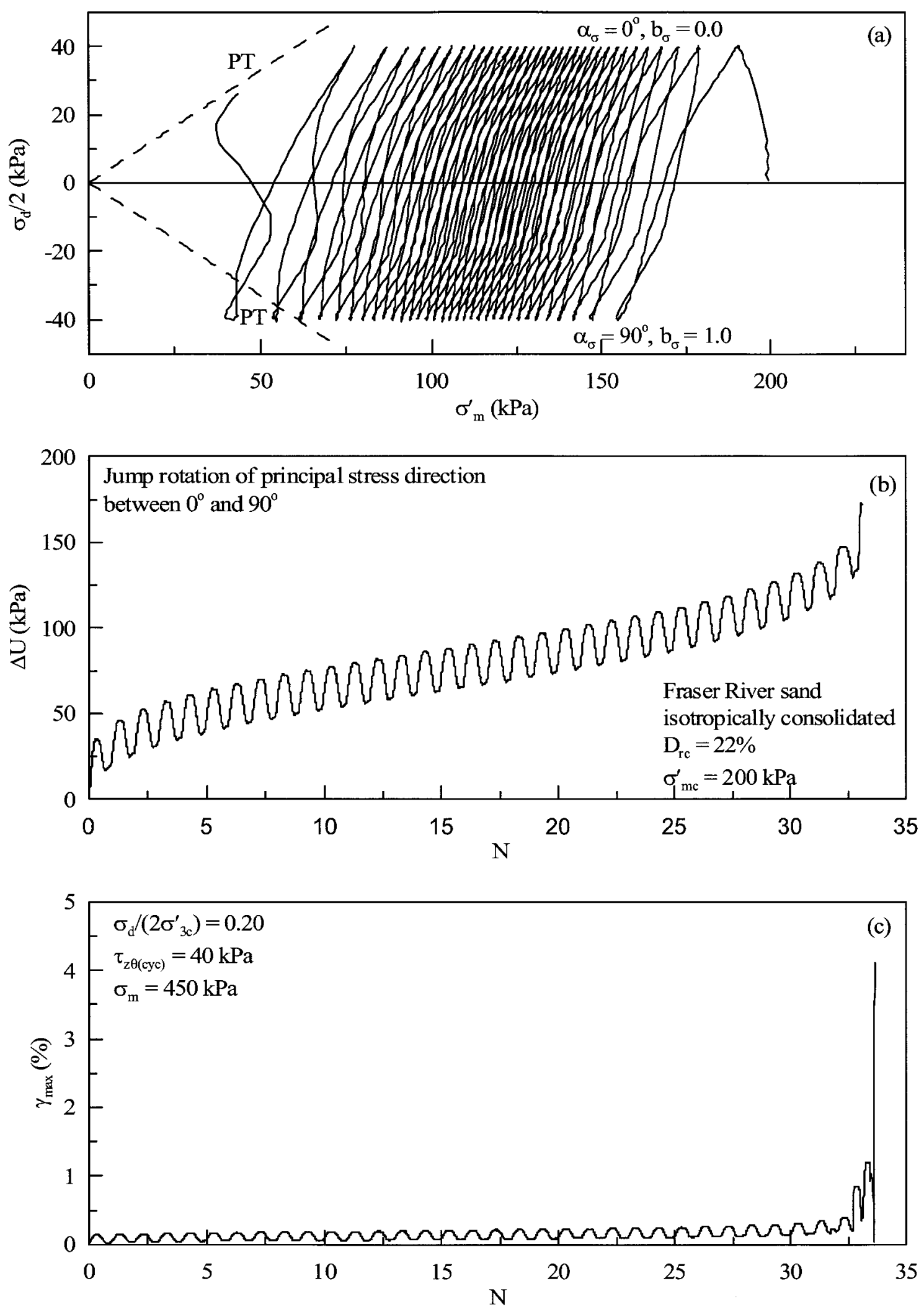

Fig. 6.2 Response of Fraser River sand under triaxial mode of cyclic loading. 


\subsection{Jump rotation of principal stress under plane strain condition}

Cyclic behaviour of Fraser River sand was studied under plane strain condition that corresponds to $b_{\sigma}=0.40$. The direction of principal stress was rotated in a jump manner on either side of the deposition direction (between $-45^{\circ}$ and $+45^{\circ}$ ). The specimen was hydrostatically consolidated to $\sigma_{m c}^{\prime}=200 \mathrm{kPa}$ and cyclic stress ratio of 0.20 was imposed to specimen.

Fig. 6.3 shows stress path, excess pore pressure response and induced strain during cyclic loading. The stress path is symmetric about $\sigma_{m}^{\prime}$ axis when direction of principal stress is rotated symmetrically about the deposition direction because the rotation of major principal stress is symmetrical about the axis of cross anisotropy and both $\sigma_{m}$ and $b_{\sigma}$ are held constant during cycles. The sand liquefies at the $6^{\text {th }}$ cycles, at which the stress path has reached the PT line. Only a small level of shear strain is induced in the sand $\left(\gamma_{\max }\right.$ is less than 0.5$)$ until the PT line is reached. The induced excess pore pressure at the end of the test is about $90 \%$ of $\sigma_{m c}^{\prime}$. A large strain was mobilized when the state reached the PT state. Cumulative excess pore pressure increases with the number of cycles and excess pore pressure response rarely shows peaks and troughs compared to triaxial mode of cyclic loading.

The jump rotation between $-45^{\circ}$ and $+45^{\circ}$ at the cyclic stress ratio of 0.20 liquefies the loosest deposited Fraser River sand at the $6^{\text {th }}$ cycle. However, the number of cycles required to liquefy the sand at the same cyclic stress ratio, but under cyclic triaxial mode is quite higher (34 cycles). This observation clearly demonstrates that the laboratory devices may misinterpret the true cyclic resistance of soils under in-situ 
loading. These findings are consistent with the data reported in the literature about the effects of principal stress direction and rotation (Uthayakumar, 1995).

In both cases (Fig. 6.2 and 6.3), the principal stresses were rotated by $90^{\circ}$, but the nature of the rotation is different. In the positive half cycle, sand experiences the stronger mode of loading at $\alpha_{\sigma}=0^{\circ}$ and $b_{\sigma}=0$ (triaxial compression) than that of the jump rotation to $\alpha_{\sigma}=+45^{\circ}$ at a constant $b_{\sigma}=0.40$. It was also confirmed in static loading that the soil at $\alpha_{\sigma}=0^{\circ}$ and $b_{\sigma}=0$ resulted in higher strength than at $\alpha_{\sigma}=0^{\circ}$ and $b_{\sigma}=0.40$ (Fig. 5.3) and soil experienced lower strength when principal stress direction was more close to the bedding plane (Fig. 5.8). In the negative half cycle, sand experienced the weaker mode of loading at $\alpha_{\sigma}=90^{\circ}$ and $b_{\sigma}=1.0$ than that of the jump rotation to $\alpha_{\sigma}=$ $45^{\circ}$ at $b_{\sigma}=0.40$. It was shown in the static loading (Fig. 5.8) that $\alpha_{\sigma}$ beyond $45^{\circ}$ did not influence much on strength reduction. Over all, in the triaxial cyclic test, sand experiences the stronger mode of loading and liquefies at a higher number of cycles. The triaxial cyclic tests, in which the rotation of principal stress direction is allowed in a jump manner between $0^{\circ}$ and $90^{\circ}$ may overestimate the cyclic resistance of soils. The nature of principal stress rotation plays a key role in the estimation of cyclic resistance. In the literature, it has been also pointed out that liquefaction can be induced by mere rotation of principal stress alone (Symes et al., 1984). 

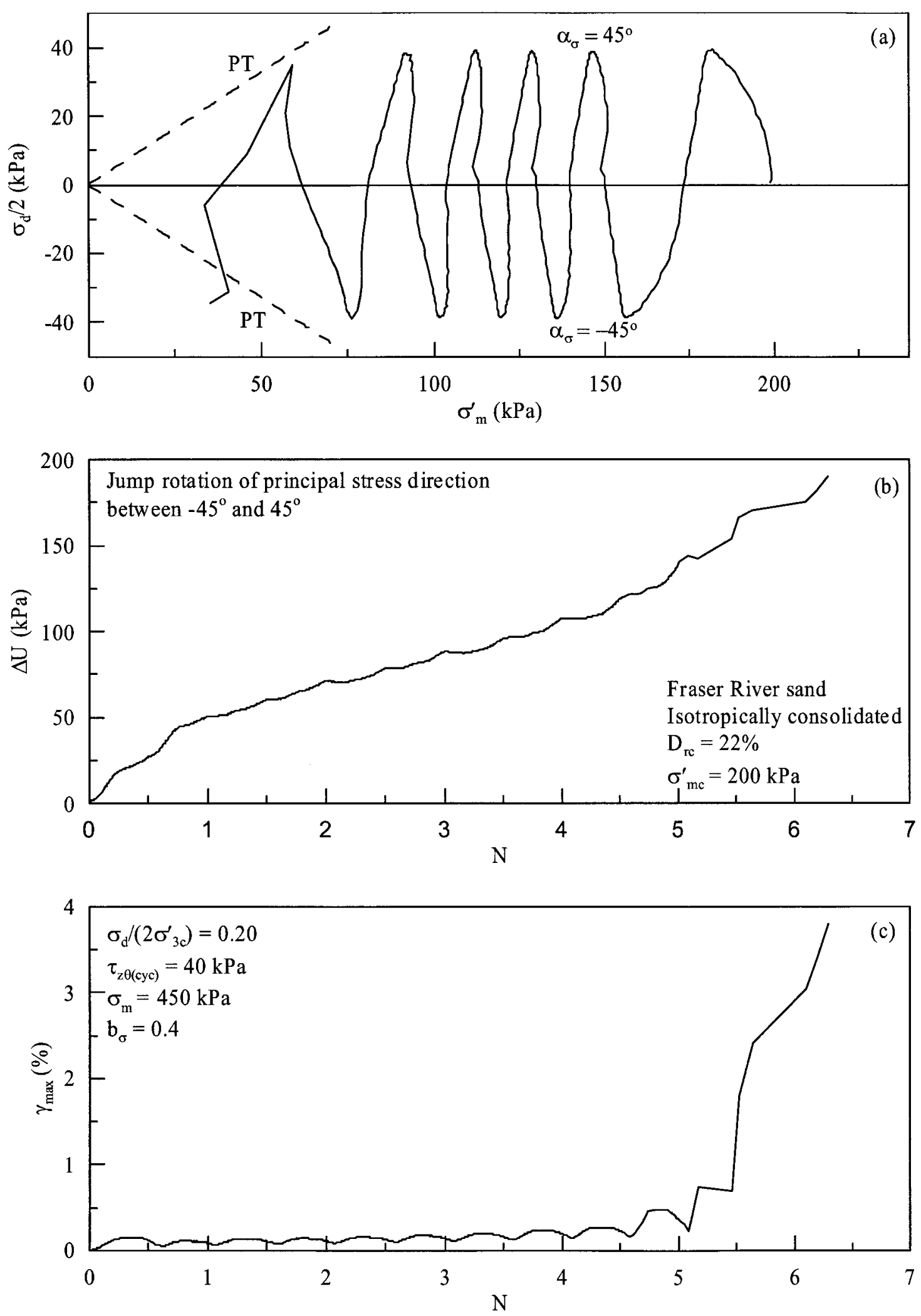

Fig. 6.3 Cyclic response of Fraser River sand during jump rotation of $\alpha_{\sigma}$ between $45^{\circ}$ and $+45^{\circ}$ at $b_{\sigma}=0.40$ 


\subsection{Nature of principal stress rotation and cyclic behaviour of sand}

Cyclic stress ratios of $0.10,0.15,0.20$ and 0.25 were imposed on loosest deposited Fraser River sand specimens. The direction of principal stress was rotated in smooth and jump manner between $+45^{\circ}$ and $-45^{\circ}$ (both sides of the deposition direction). During these tests, total mean normal stress $\sigma_{m}$ was kept at $450 \mathrm{kPa}$ and the intermediate principal stress parameter $b_{\sigma}$ was held at zero. Fig. 6.4 shows the variation of torsional shear stress $\tau_{z \theta}$ during the application of cyclic loading with both a smooth and a jump rotation of principal stress direction. Even though the peak amplitude of $\tau_{z} \theta$ is the same at a given cyclic stress ratio, $\tau_{z \theta}$ experienced by the specimen during the loading cycle is different.

\subsubsection{Smooth rotation of principal stress direction at different stress ratios}

Figs. 6.5 to 6.8 illustrate the cyclic behaviour of Fraser River sand under smooth rotation of principal stress direction between $+45^{\circ}$ and $-45^{\circ}$. The stress paths are symmetrical about $\sigma_{m}^{\prime}$ axis because the direction of major principal stress was rotated symmetrically about the deposition direction, when both $\sigma_{m}$ and $b_{\sigma}$ were held constant. This observation also suggests that cross anisotropy exists along the vertical axis of specimen.

Fig. 6.5 shows the stress path during the application of a cyclic stress ratio of 0.10 . The sand does not liquefy even after a large number of loading cycles (more than 40 cycles) because it experiences a relatively small cyclic stress ratio. When the sand experiences cyclic stress ratio of 0.15 (Fig. 6.6), liquefaction is observed in the $28^{\text {th }}$ cycle by exceeding $\gamma_{\max }=3.75 \%$. Fig. 6.7 shows the cyclic response of the sand at cyclic stress ratio of 0.20 and liquefaction is noted at the $8^{\text {th }}$ cycle. At a cyclic stress ratio of 0.25 , the 
sand liquefied in just 2 cycles (Fig. 6.8) and experienced a very large amount of deformation upon reaching the PT line.
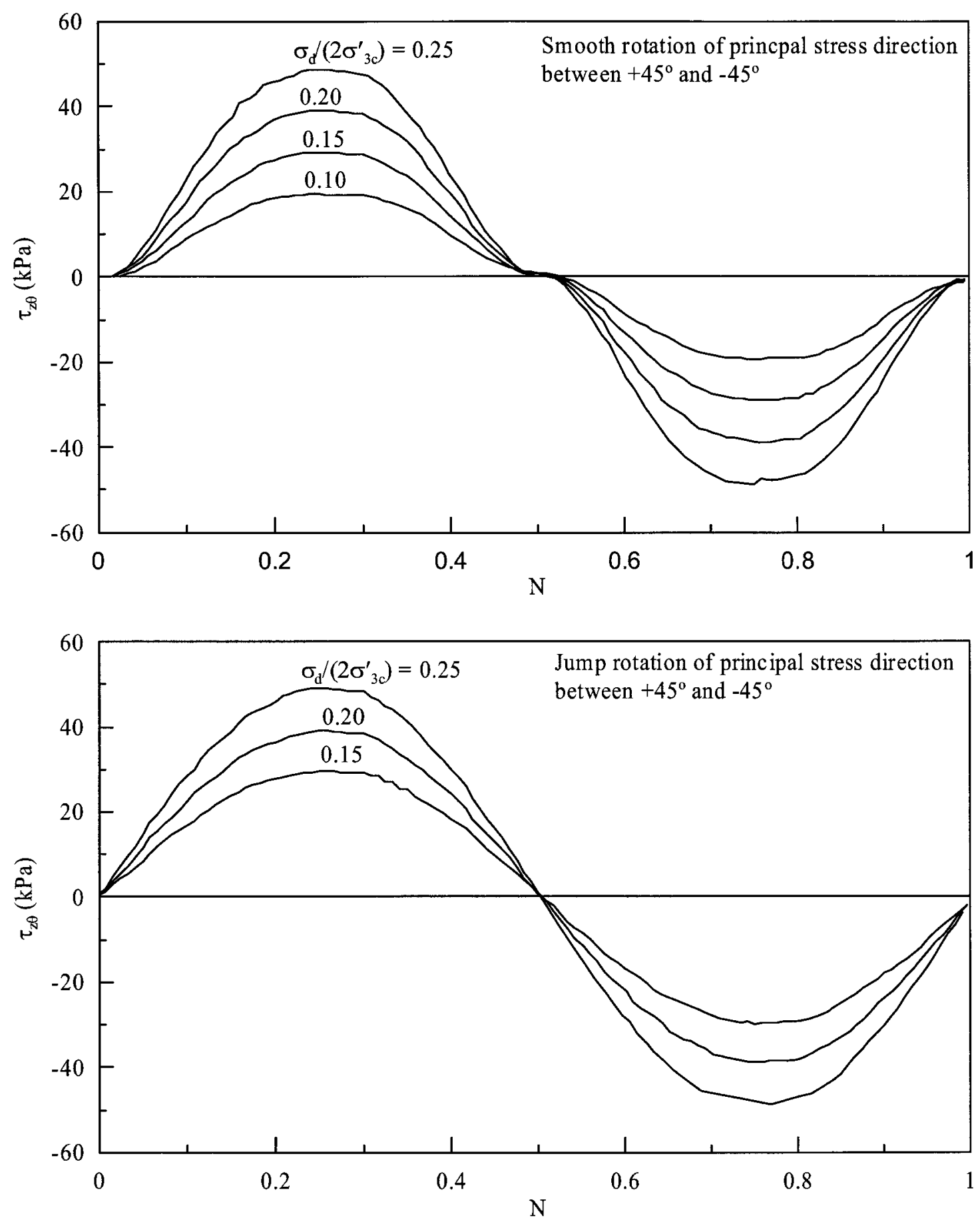

Fig. 6.4 Imposed torsional shear stress during application of cyclic loading under smooth and jump rotations of $\alpha_{\sigma}$ 


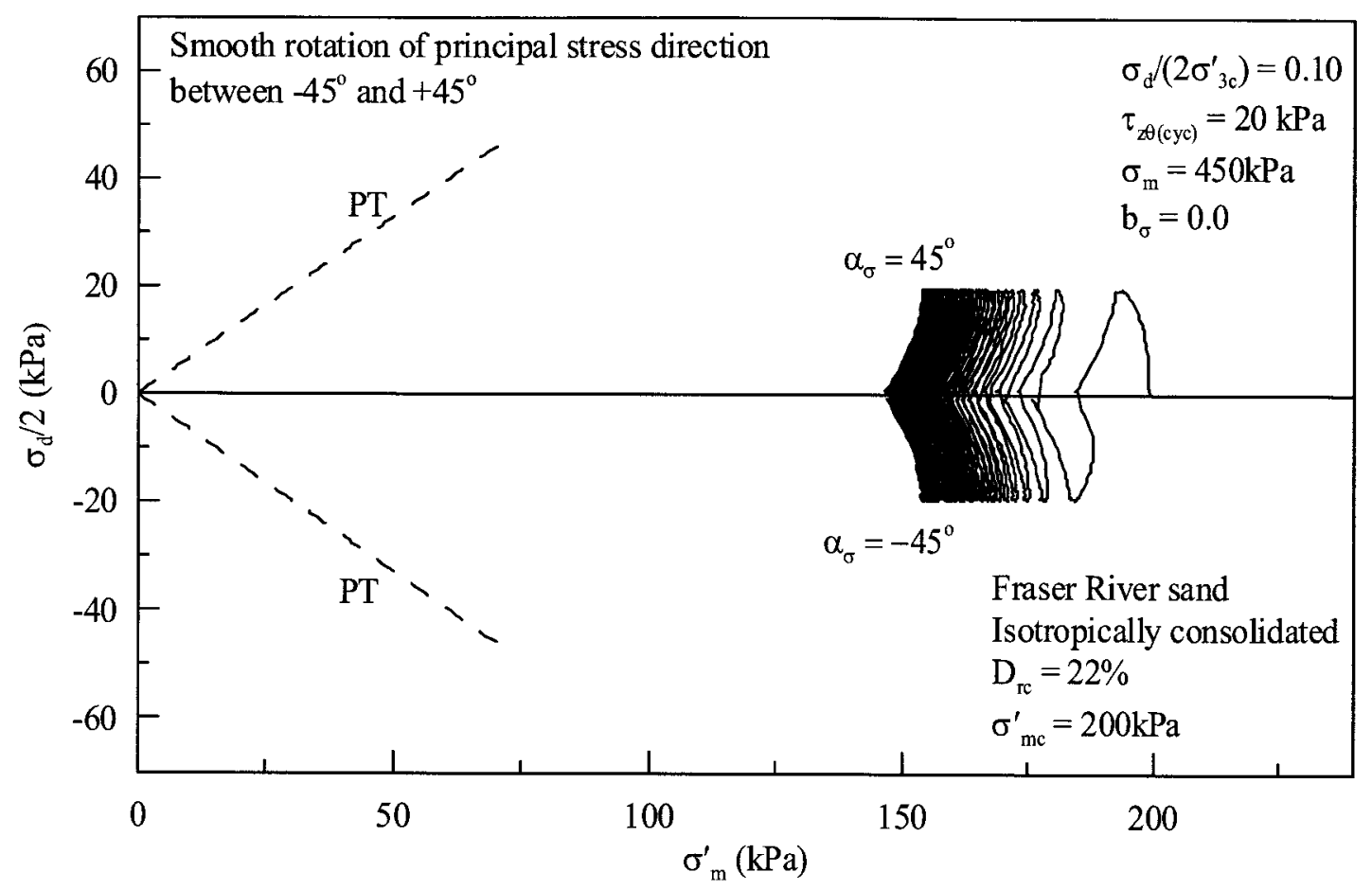

Fig. 6.5 Stress path of Fraser River sand during cyclic loading of $\sigma_{d} /\left(2 \sigma_{3 c}^{\prime}\right)=0.10$ with smooth rotation of $\alpha_{\sigma}$ between $-45^{\circ}$ and $+45^{\circ}$

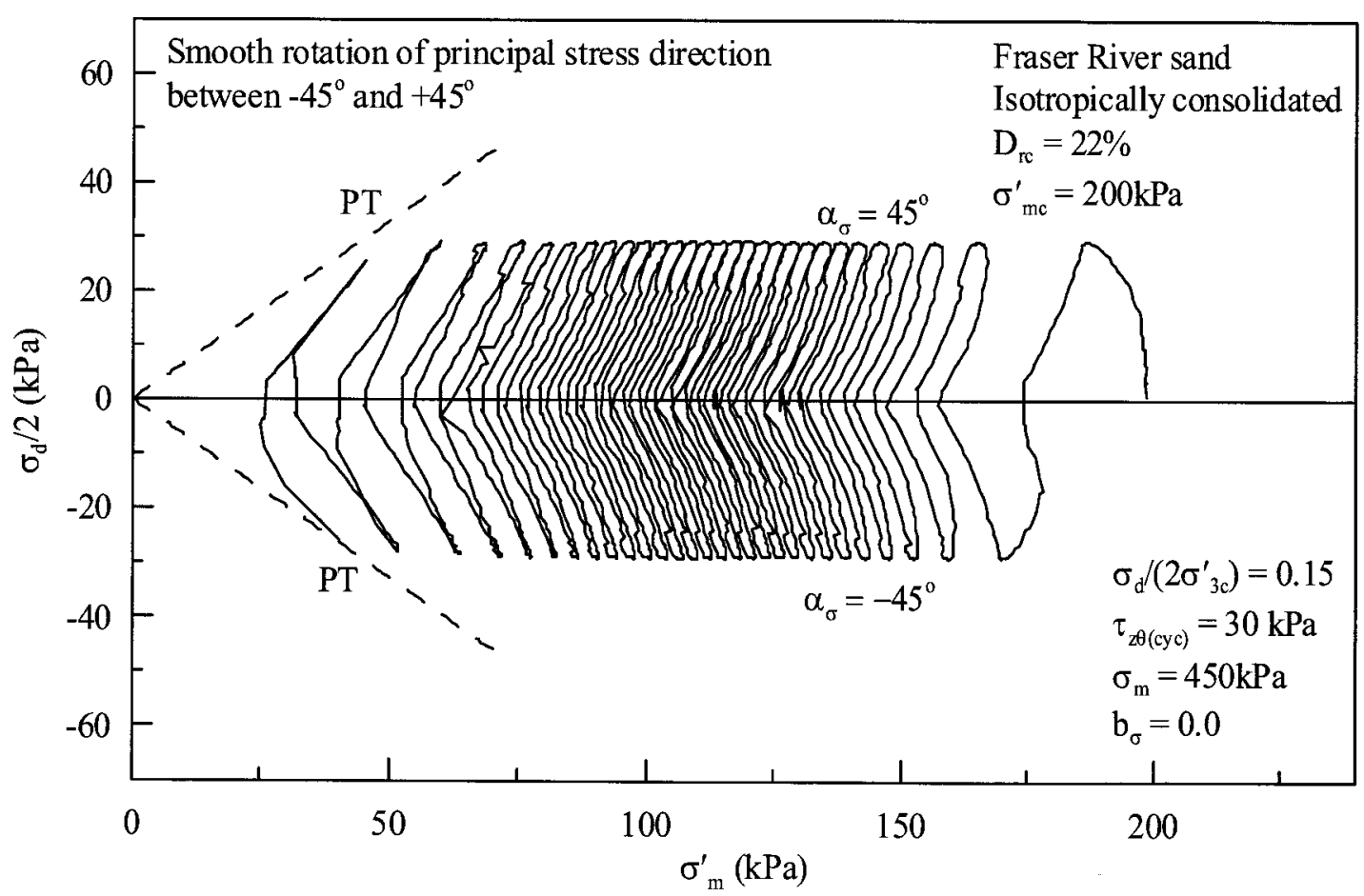

Fig. 6.6 Stress path of Fraser River sand during cyclic loading of $\sigma_{d} /\left(2 \sigma_{3 c}^{\prime}\right)=0.15$ with smooth rotation of $\alpha_{\sigma}$ between $-45^{\circ}$ and $+45^{\circ}$ 


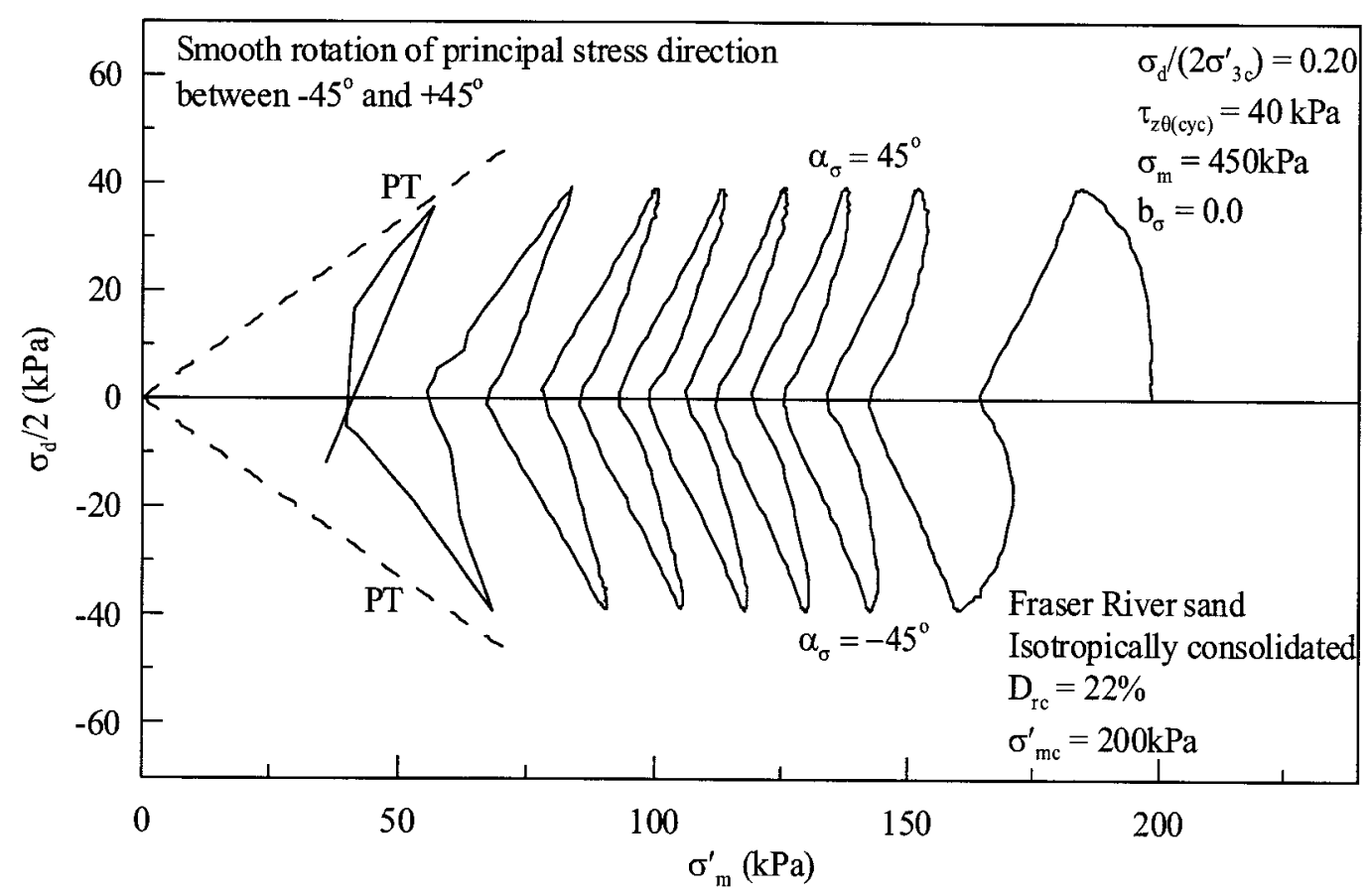

Fig. 6.7 Stress path of Fraser River sand during cyclic loading of $\sigma_{d} /\left(2 \sigma_{3 c}^{\prime}\right)=0.20$ with smooth rotation of $\alpha_{\sigma}$ between $-45^{\circ}$ and $+45^{\circ}$

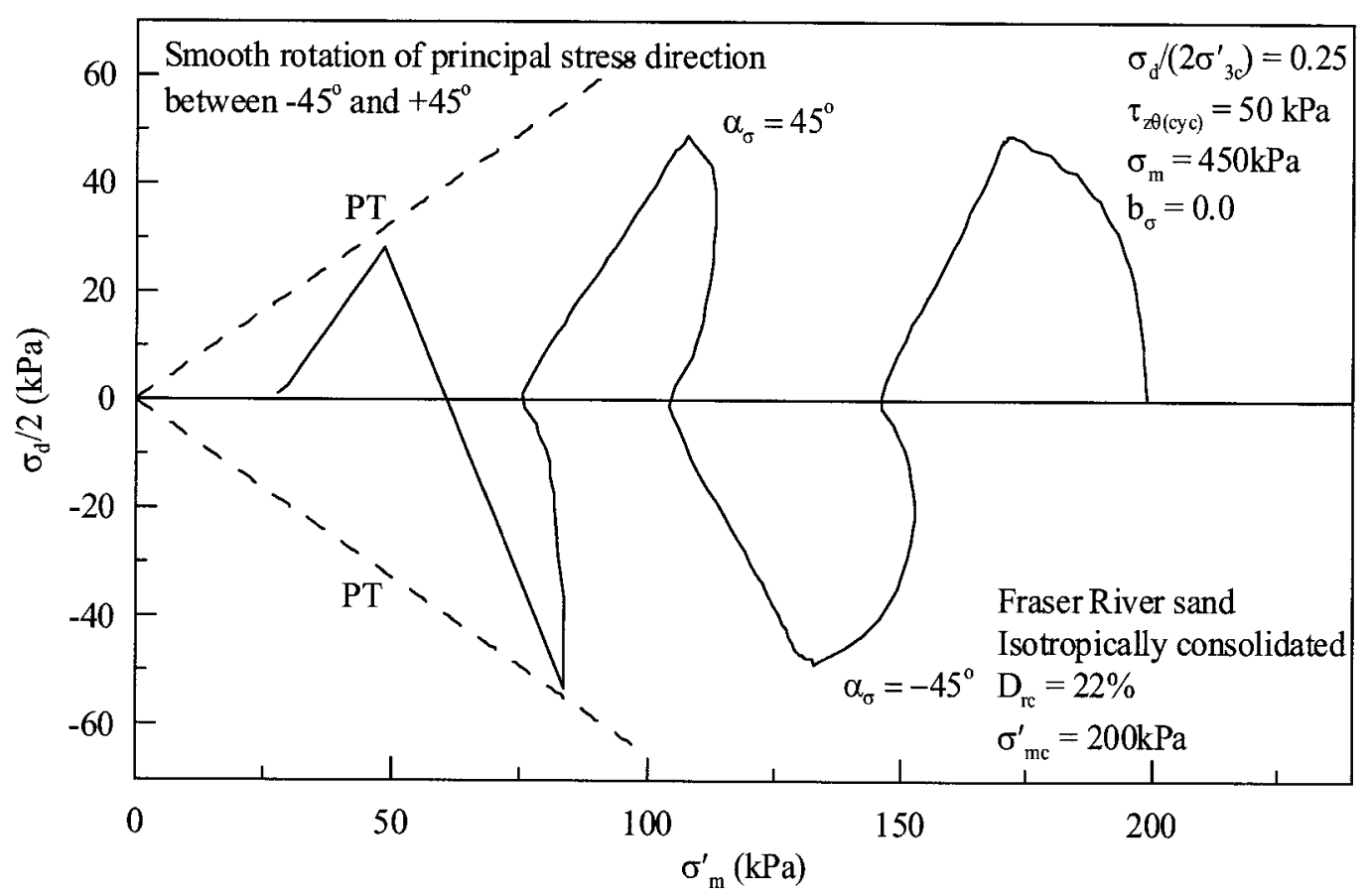

Fig. 6.8 Stress path of Fraser River sand during cyclic loading of $\sigma_{d} /\left(2 \sigma_{3 c}^{\prime}\right)=0.25$ with smooth rotation of $\alpha_{\sigma}$ between $-45^{\circ}$ and $+45^{\circ}$

The number of cycles required to liquefy the sand reduces with increasing cyclic stress ratio. As noted in the triaxial cyclic loading at a cyclic stress ratio of 0.20 in Fig. 
6.2 , liquefaction was observed after 34 cycles. However, during smooth rotation between $-45^{\circ}$ and $+45^{\circ}$ with cyclic stress ratio of 0.20 , the liquefaction is noted after 8 cycles (Fig. $6.7)$.

Fig. 6.9 shows the variation of excess pore pressure induced during cyclic loading with the smooth rotation of principal stress direction at different levels of cyclic stress ratios. The excess pore pressure responses show peaks and troughs in each cycle. However, the cumulative excess pore pressure induced is positive regardless of the sign of the principal stress direction. In each cycle, negative increments of excess pore pressure in the first and third quarters and positive increments in second and fourth quarters may be observed. The magnitude of excess pore pressure systematically increases with increasing cyclic stress ratio at a given cycle. The test performed at cyclic stress ratio of 0.10 develops the least excess pore pressure and exhibits approximately 40 $\mathrm{kPa}$ of excess pore pressure ( $20 \%$ of initial effective confining stress) after 40 cycles. A higher cyclic stress ratio of 0.25 generates $180 \mathrm{kPa}$ ( $90 \%$ of initial effective confining stress) excess pore pressure within two cycles.

Fig. 6.10 shows the variation of induced maximum shear strain $\gamma_{\max }$ during application of cyclic loading under smooth rotation of the principal stress direction. A systematic accumulation of $\gamma_{\max }$ is noted with increasing cyclic stress ratio as noted in excess pore pressure generation. A lower rate of accumulation of $\gamma_{\max }$ is noted at a cyclic stress ratio of 0.10 and higher rate is noted at a cyclic stress ratio of 0.25 . Induced $\gamma_{\max }$ is relatively low until the stress paths reaches PT line, and the rate of strain development rapidly increases afterwards. 


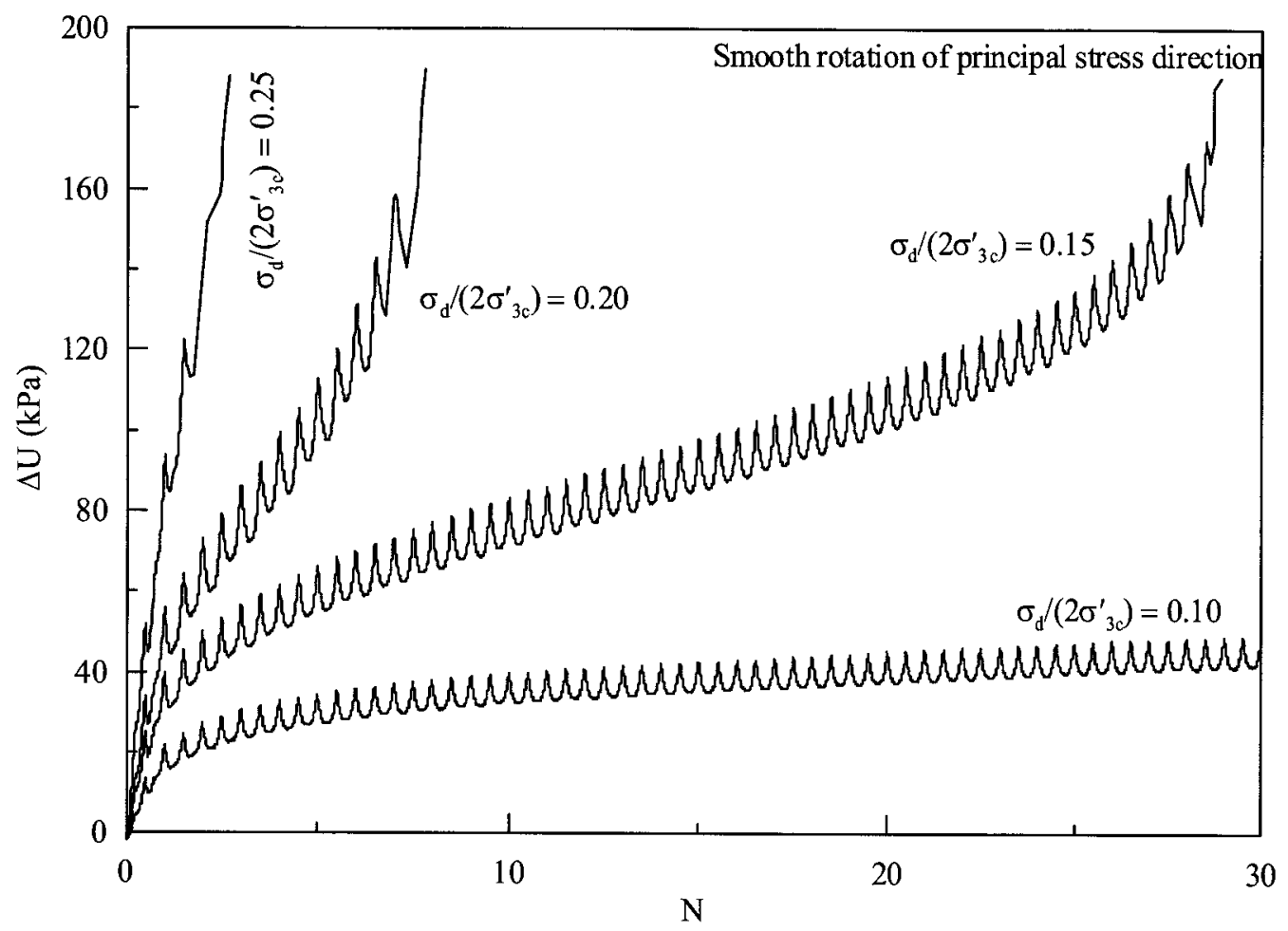

Fig. 6.9 Excess pore pressure response of Fraser River sand during cyclic loading with smooth rotation of $\alpha_{\sigma}$ between $-45^{\circ}$ and $+45^{\circ}$

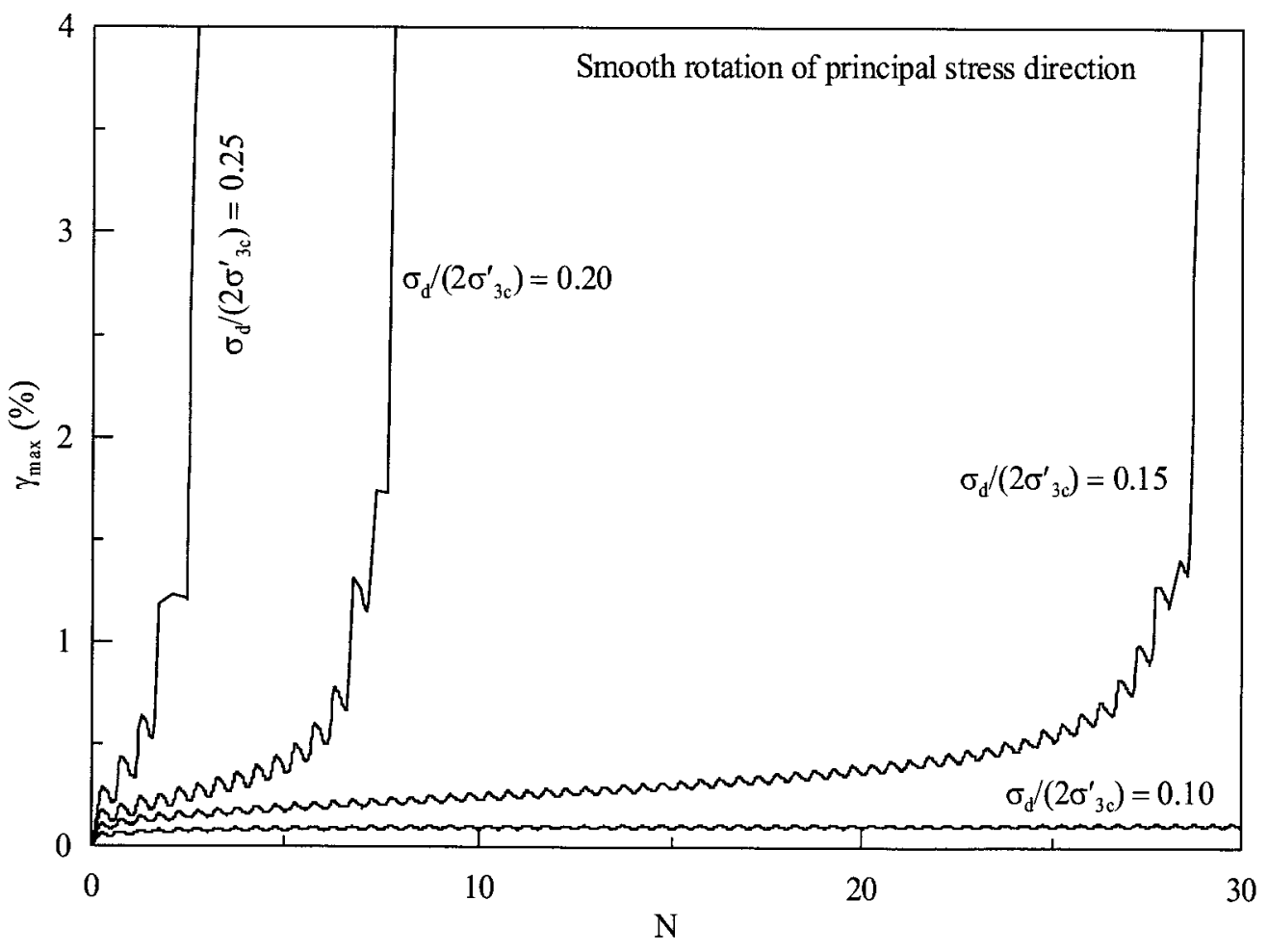

Fig. $6.10 \quad$ Induced maximum shear strain during cyclic loading with smooth rotation of $\alpha_{\sigma}$ between $-45^{\circ}$ and $+45^{\circ}$ 


\subsubsection{Jump rotation of principal stress direction at different stress ratios}

A series of tests with jump rotation of principal stress directions was carried out on identical Fraser River sand specimens. Fig. 6.4 shows the variation of torsional shear stress $\tau_{z \theta}$ during the application of cyclic stress ratios of $0.15,0.20$ and 0.25 . The specimens were isotropically consolidated to $\sigma_{m c}^{\prime}=200 \mathrm{kPa}$ prior to shearing. During loading, $b_{\sigma}$ was held at zero and $\sigma_{m}$ was constant.

Figs. 6.11, 6.12 and 6.13 illustrate the stress paths during jump rotation of the principal stress direction between $+45^{\circ}$ and $-45^{\circ}$. The stress paths are symmetrical about the $\sigma_{m}^{\prime}$ axis as the principal stress direction rotates symmetrically about the axis of cross anisotropy (direction of sand deposition). The soils liquefied (by exceeding the threshold strain level) in 23,8 and 3 cycles at cyclic stress ratios of $0.15,0.20$ and 0.25 respectively. The decrease in required cycles to trigger liquefaction with increasing CSR is similar to that observed in the tests with smooth rotation of principal stress direction.

Fig. 6.14 shows the variation of excess pore pressure induced during cyclic loading with jump rotation of principal stress direction. Even though peaks and troughs are noted in each cycle of loading, the excess pore pressure continuously accumulates. In each cycle, negative increments of excess pore pressure in the first and third quarters and positive increments in second and fourth quarters may be observed. The magnitude of excess pore pressure systematically increases with the increasing cyclic stress ratio at a given cycle. In the test conducted at a cyclic stress ratio of 0.25 , the peaks and troughs are rarely noted and the rate of excess pore pressure generation is high. In this test, excess pore pressure reaches approximately $90 \%$ of initial effective confining stress in 3 cycles. 


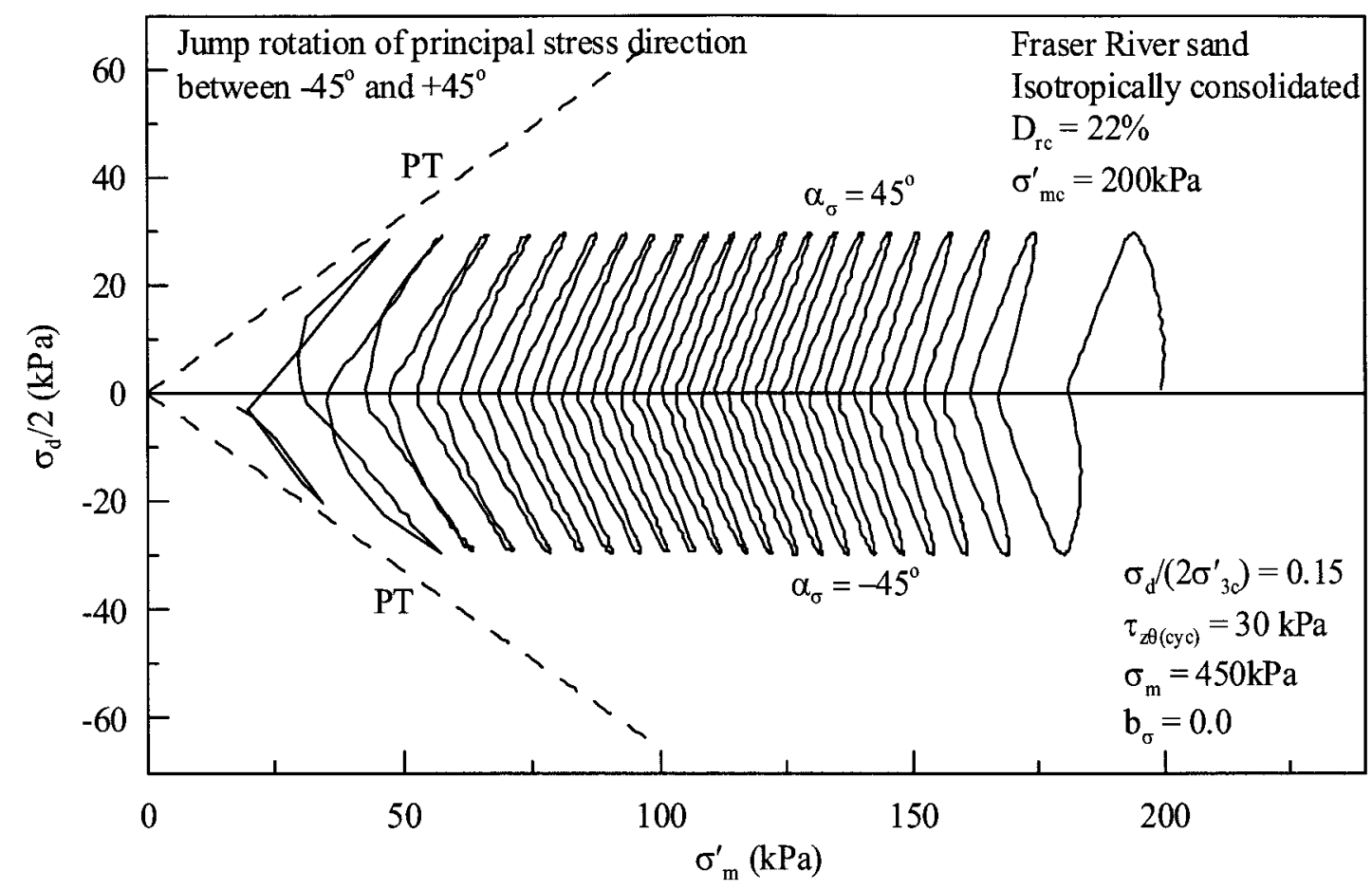

Fig. 6.11 Stress path of Fraser River sand during cyclic loading of $\sigma_{d} /\left(2 \sigma^{\prime}{ }^{\prime} c\right)=0.15$ with jump rotation of $\alpha_{\sigma}$ between $-45^{\circ}$ and $+45^{\circ}$

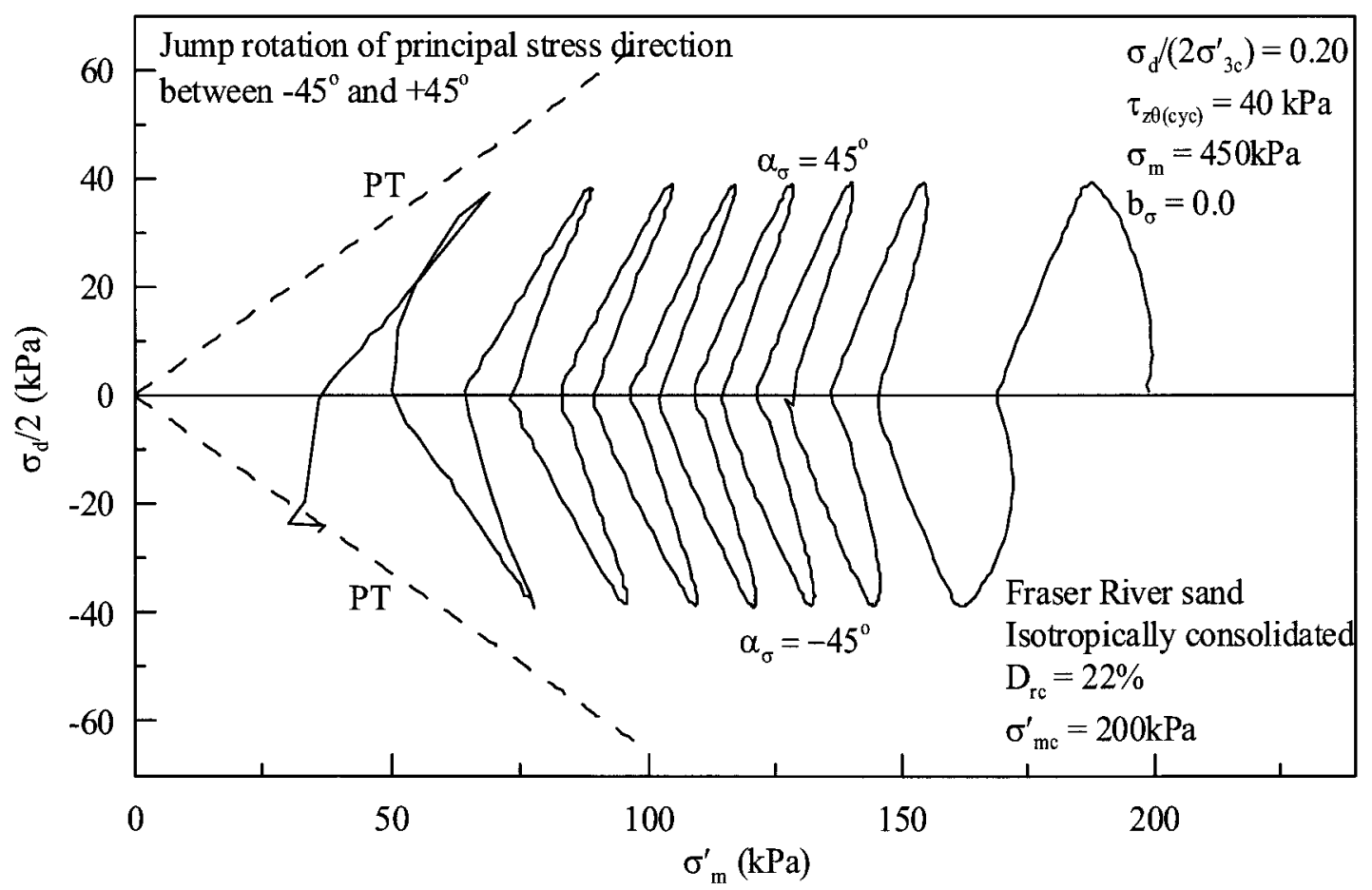

Fig. 6.12 Stress path of Fraser River sand during cyclic loading of $\sigma_{d} /\left(2 \sigma_{3 c}^{\prime}\right)=0.20$ with jump rotation of $\alpha_{\sigma}$ between $-45^{\circ}$ and $+45^{\circ}$ 


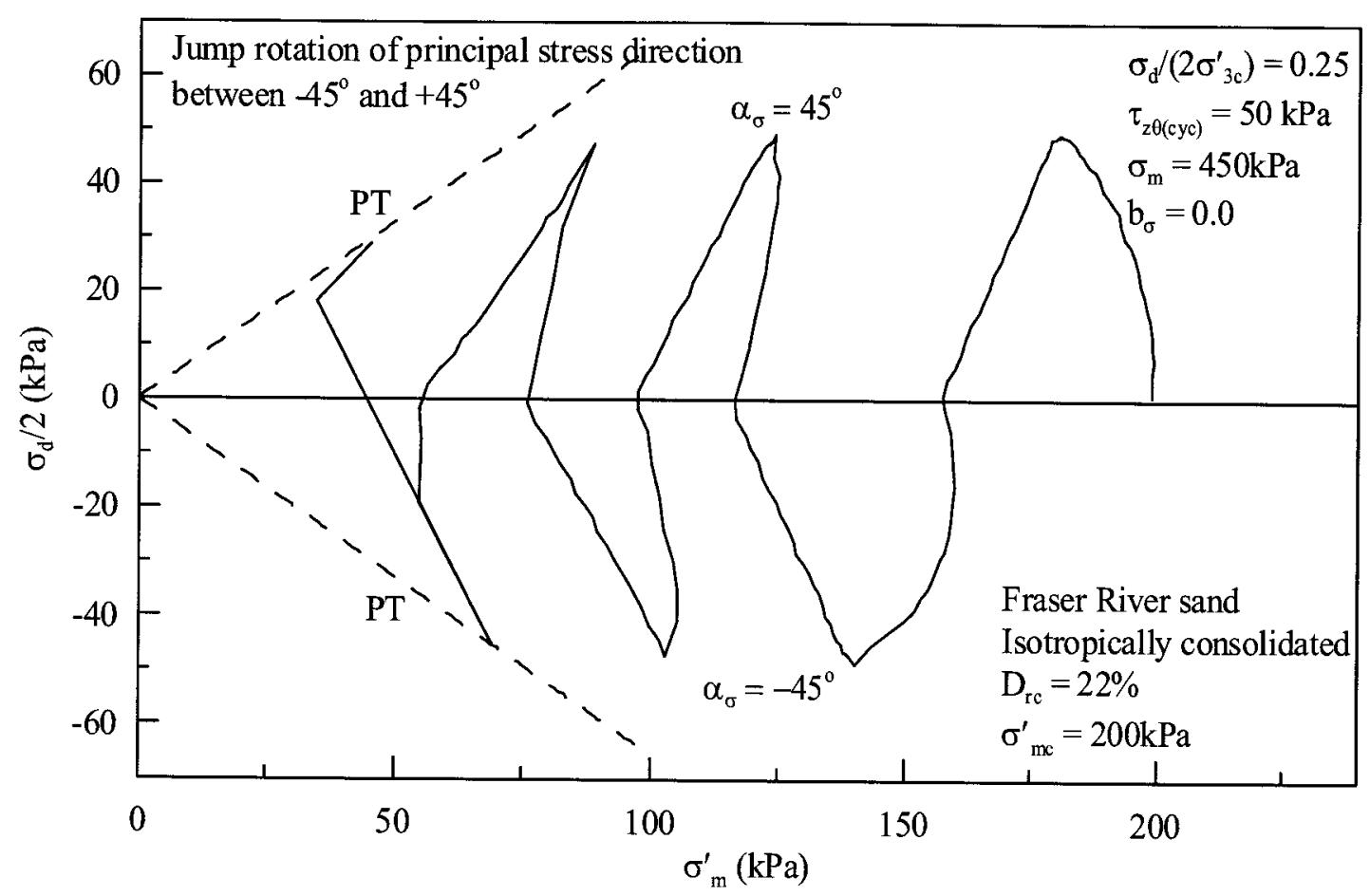

Fig. 6.13 Stress path of Fraser River sand during cyclic loading of $\sigma_{d} /\left(2 \sigma_{3 c}^{\prime}\right)=0.25$ with jump rotation of $\alpha_{\sigma}$ between $-45^{\circ}$ and $+45^{\circ}$

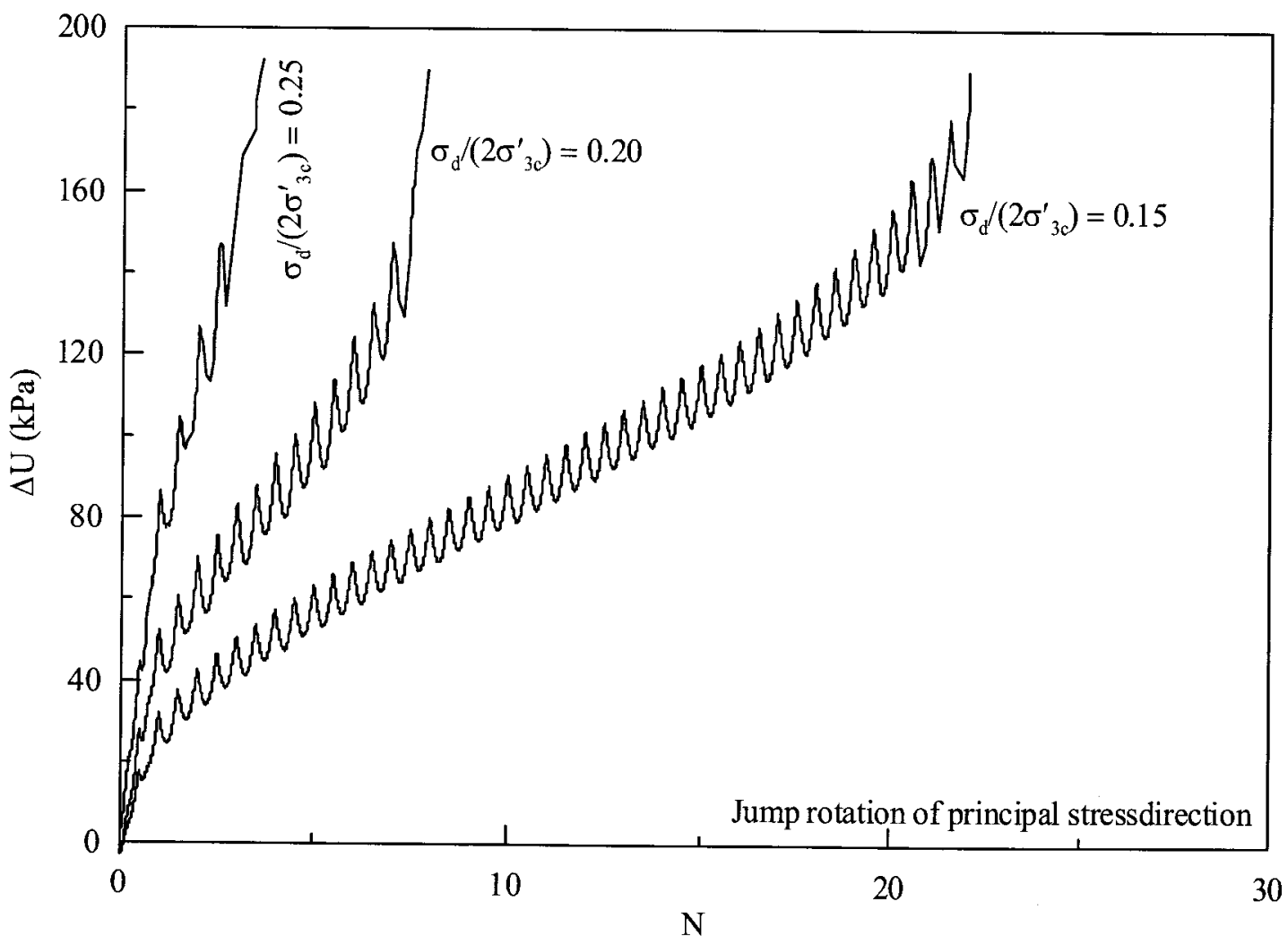

Fig. 6.14 Excess pore pressure response of Fraser River sand during cyclic loading with jump rotation of $\alpha_{\sigma}$ between $-45^{\circ}$ and $+45^{\circ}$ 
Fig. 6.15 shows the variation of induced maximum shear strain $\gamma_{\max }$ during the application of cyclic loading under jump rotation of principal stress direction. A systematic increment of $\gamma_{\max }$ at a given load cycle is noted with increasing cyclic stress ratio. Comparatively, a small level of $\gamma_{\max }$ is induced for cyclic shear stress ratios of 0.15 and 0.20 until stress paths reach the PT line. After that, sand undergoes large deformation.

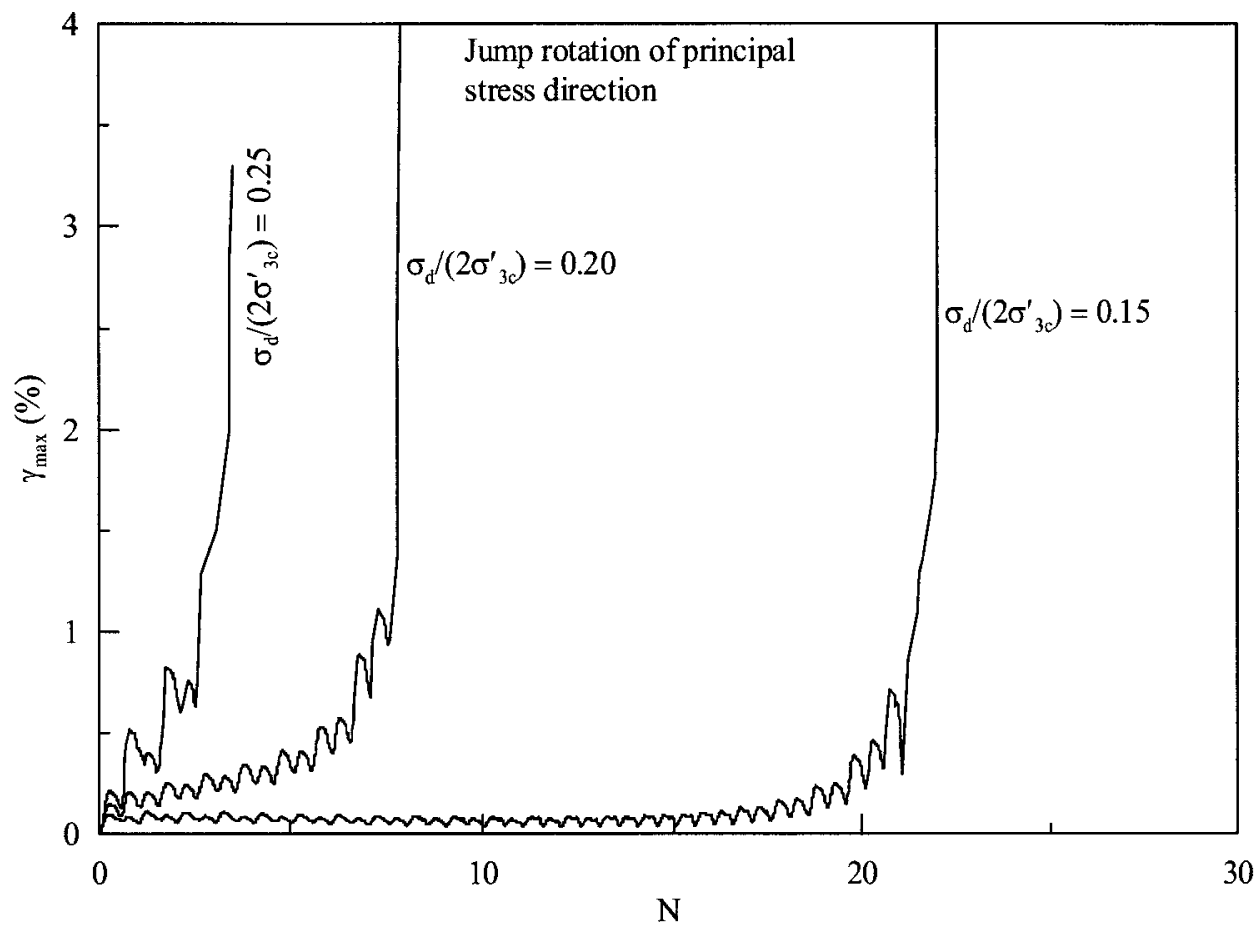

Fig. 6.15 Induced maximum shear strain during cyclic loading with jump rotation of $\alpha_{\sigma}$ between $-45^{\circ}$ and $+45^{\circ}$

\subsubsection{Comparison of the nature of principal stress rotation on cyclic behaviour}

Liquefaction resistance of Fraser River sand is compared under jump and smooth rotation of principal stress direction. The principal stress direction was rotated between $45^{\circ}$ and $+45^{\circ}$ smoothly in one series of tests, and suddenly in the other. Fig. 6.16 shows the variation of cyclic stress ratio and number of cycles required to liquefy. It is noted that the number of cycles required for liquefaction increase with decreasing cyclic stress 
ratio for both natures of rotation of the principal stress direction. At lower cyclic stress ratio, the sand does not liquefy even after reaching 40 cycles. No significant differences were noted in the number of cycles required for liquefaction. This indicates that the nature of rotation (jump vs smooth) does not influence the cyclic resistance. This conclusion is subject to the condition that the rotation is symmetric about the axis of deposition (i.e. principal stress direction between $+45^{\circ}$ and $-45^{\circ}$ ).

These test results are consistent with the data presented by Uthayakumar (1995) on loosest deposited Fraser River sand under jump rotation of principal stress direction between $-45^{\circ}$ and $+45^{\circ}$. It was observed that the sand liquefied at 8 cycles during the application of a cyclic stress ratio of 0.175 at $\sigma_{m c}^{\prime}=200 \mathrm{kPa}$ and fixed $b_{\sigma}=0.5$. The test according to this study (Fig. 6.16) results that the sand requires approximately 10 cycles to liquefy during the application of a cyclic stress ratio of 0.175 at $\sigma_{m c}^{\prime}=200 \mathrm{kPa}$ and fixed $b_{\sigma}=0.0$.

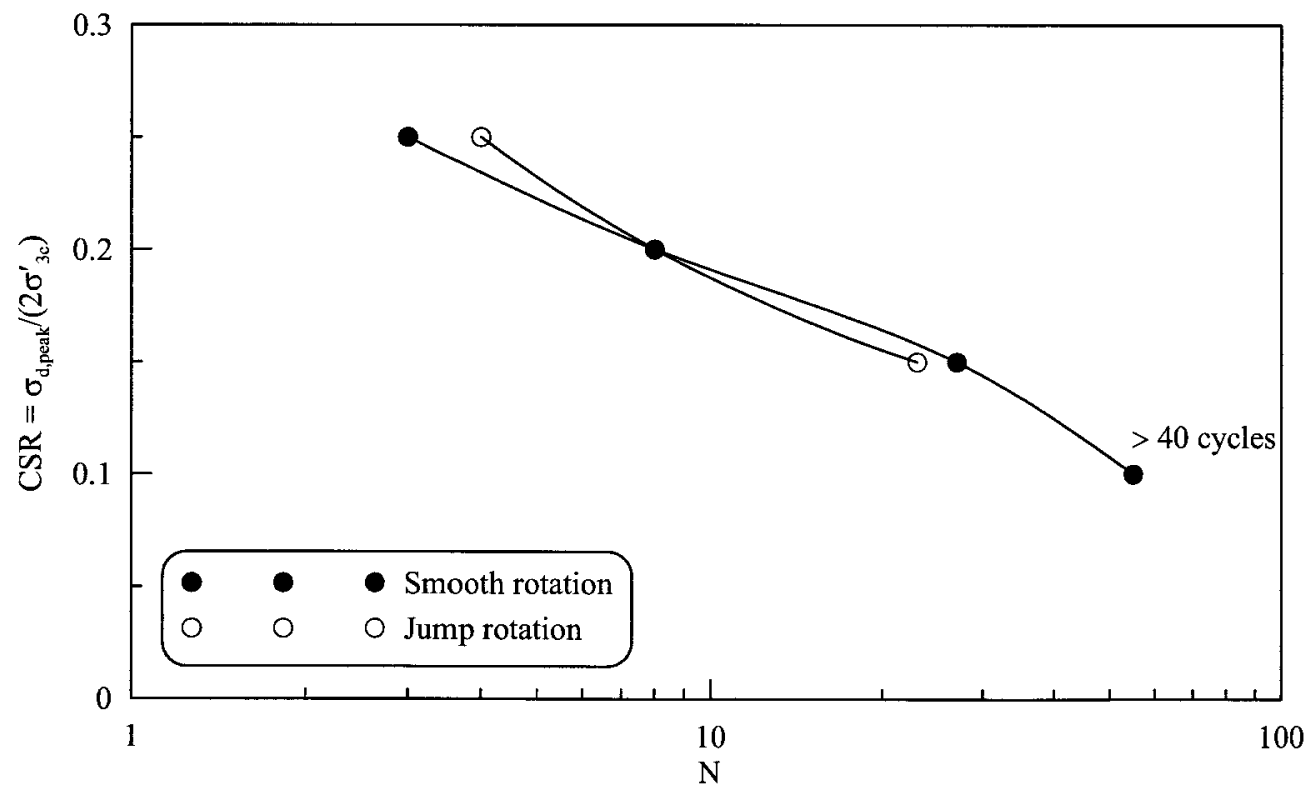

Fig. 6.16 Cyclic resistance curve of loose Fraser River sand during jump and smooth rotations of $\alpha_{\sigma}$ 


\subsection{Influence of $\alpha_{\sigma}$ on cyclic behaviour of sand}

This series of cyclic tests were performed at a fixed cyclic stress ratio $\frac{\sigma_{d}}{2 \sigma_{3 c}^{\prime}}=$ 0.20 , but with different amount of principal stress rotation. The principal stress direction was rotated in smooth and jump manner about the vertical axis of the specimen (axis of cross anisotropy). During these tests, total mean normal stress $\sigma_{m}$ was held at $450 \mathrm{kPa}$ and the intermediate principal stress parameter $b_{\sigma}$ was held at zero.

\subsubsection{Smooth rotation of $\alpha_{\sigma}$ at a fixed cyclic stress ratio}

Fig. 6.17a shows the variation of $\tau_{z \theta}$ during different levels of smooth rotation of the principal stress direction at a fixed cyclic stress ratio of 0.20 . When maximum rotation of the principal stress direction $\alpha_{\sigma, \max }$ is $45^{\circ}$ or less $\left(-45^{\circ} \leq \alpha_{\sigma, \max } \leq+45^{\circ}\right), \tau_{z} \theta$ reaches the peak at the instant of the peak cyclic stress ratio. When $\alpha_{\sigma, \max }$ is greater than $45^{\circ}$, the maximum value of $\tau_{z \theta}$ is not reached at the instant of peak cyclic stress ratio.

Figs. $6.18,6.19,6.20,6.21$, and 6.22 show the cyclic response of Fraser River sand when the principal direction is smoothly rotated for a range of $\alpha_{\sigma, \max }$ from $15^{\circ}$ to $90^{\circ}$. The symmetry of the stress paths with respect to $\sigma_{m}^{\prime}$ is noted for all tests.

No liquefaction is noted until the $40^{\text {th }}$ cycle, when $\alpha_{\sigma}$ is rotated by a relatively small angle between $-15^{\circ}$ and $15^{\circ}$ about the axis of cross anisotropy. The application of more cycles of loading may move the stress path gradually towards the PT line. When $\alpha_{\sigma}$ is rotated between $-30^{\circ}$ and $+30^{\circ}$, liquefaction is induced at the $14^{\text {th }}$ cycle. The same trend of stress path movement is noted when $\alpha_{\sigma}$ is rotated smoothly between $-45^{\circ}$ and $+45^{\circ}$ (Fig. 6.7), However, the liquefaction is induced in 8 cycles. 

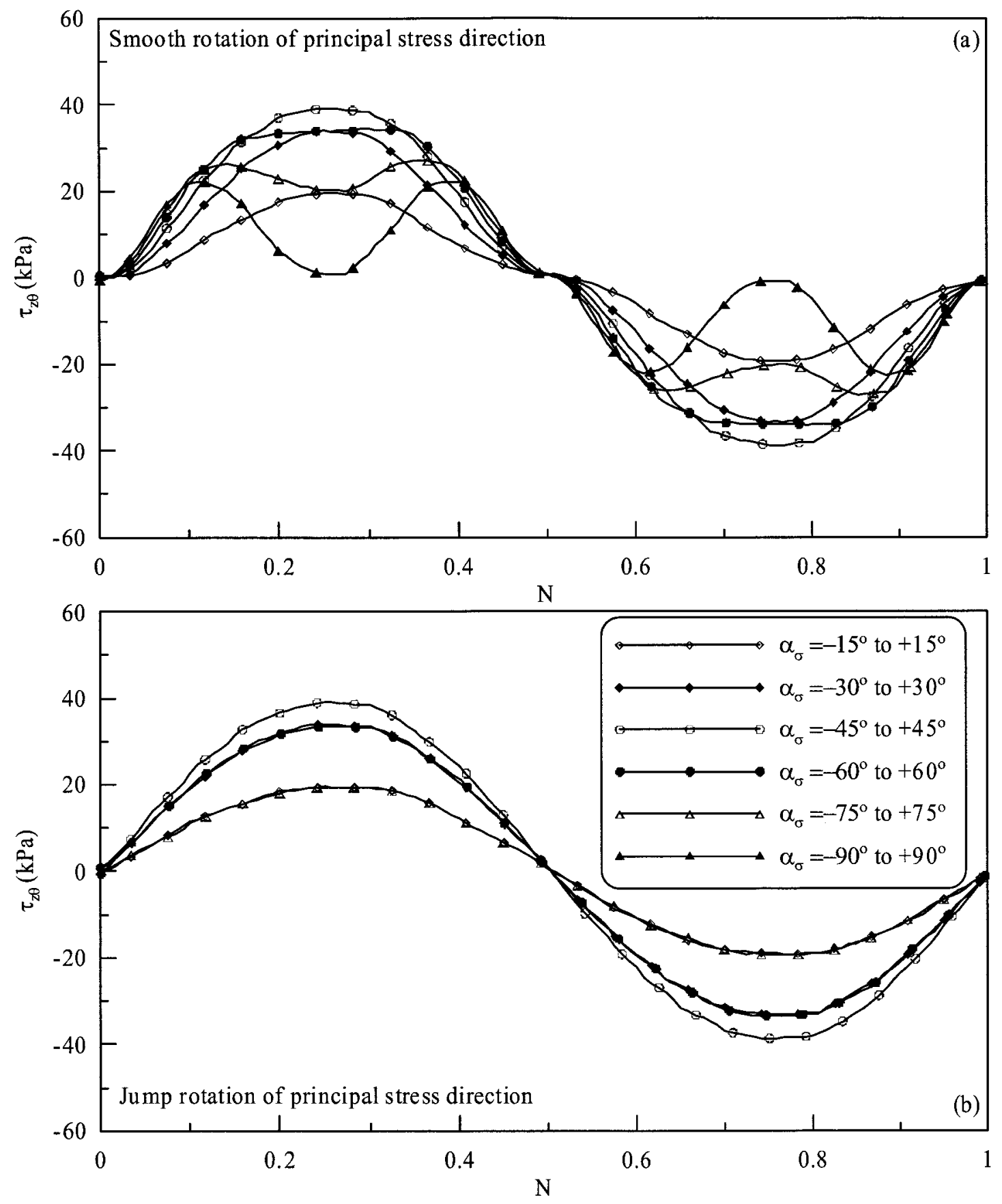

Fig. 6.17 Torsional shear stress during smooth and jump rotations of $\alpha_{\sigma}$

For the rotation of $\alpha_{\sigma}$ between $-60^{\circ}$ and $+60^{\circ}$, liquefaction is observed again in the $8^{\text {th }}$ cycle (Fig. 6.20). However, liquefaction is noted during the rotation of $\alpha_{\sigma}$ through $-75^{\circ}$ to $+75^{\circ}$ in the $14^{\text {th }}$ cycle (Fig. 6.21 ). It could be noted that the number of cycles required to liquefy the sand are the same ( 7 to 8 cycles) when $\alpha_{\sigma}$ is smoothly rotated 
between $-45^{\circ}$ and $+45^{\circ}$, and $-60^{\circ}$ and $+60^{\circ}$. This observation is consistent with static undrained loading at a fixed $\alpha_{\sigma}$ (Fig. 5.8). The static undrained strength of the sand did not change much when $\alpha_{\sigma}$ was fixed along $45^{\circ}$ and $60^{\circ}$. As $\alpha_{\sigma}$ is rotated between $-90^{\circ}$ and $+90^{\circ}$, liquefaction is noted at the $19^{\text {th }}$ cycle (Fig. 6.22). Even though $\alpha_{\sigma}$ is rotated through a large angle $\left(\alpha_{\sigma, \max }=75^{\circ}\right.$ and $\left.90^{\circ}\right)$, a larger number of cycles were needed to trigger liquefaction. This deviation may be due to the imposed $\tau_{z}$ that is comparatively lower than $\tau_{z \theta}$ of $\alpha_{\sigma, \max }=75^{\circ}$ and $90^{\circ}$. In addition, $\tau_{z \theta}$ reaches a lower value than its peak at the instance of peak cyclic stress for $\alpha_{\sigma, \max }=75^{\circ}$ and $90^{\circ}$ (Fig. 6.17).

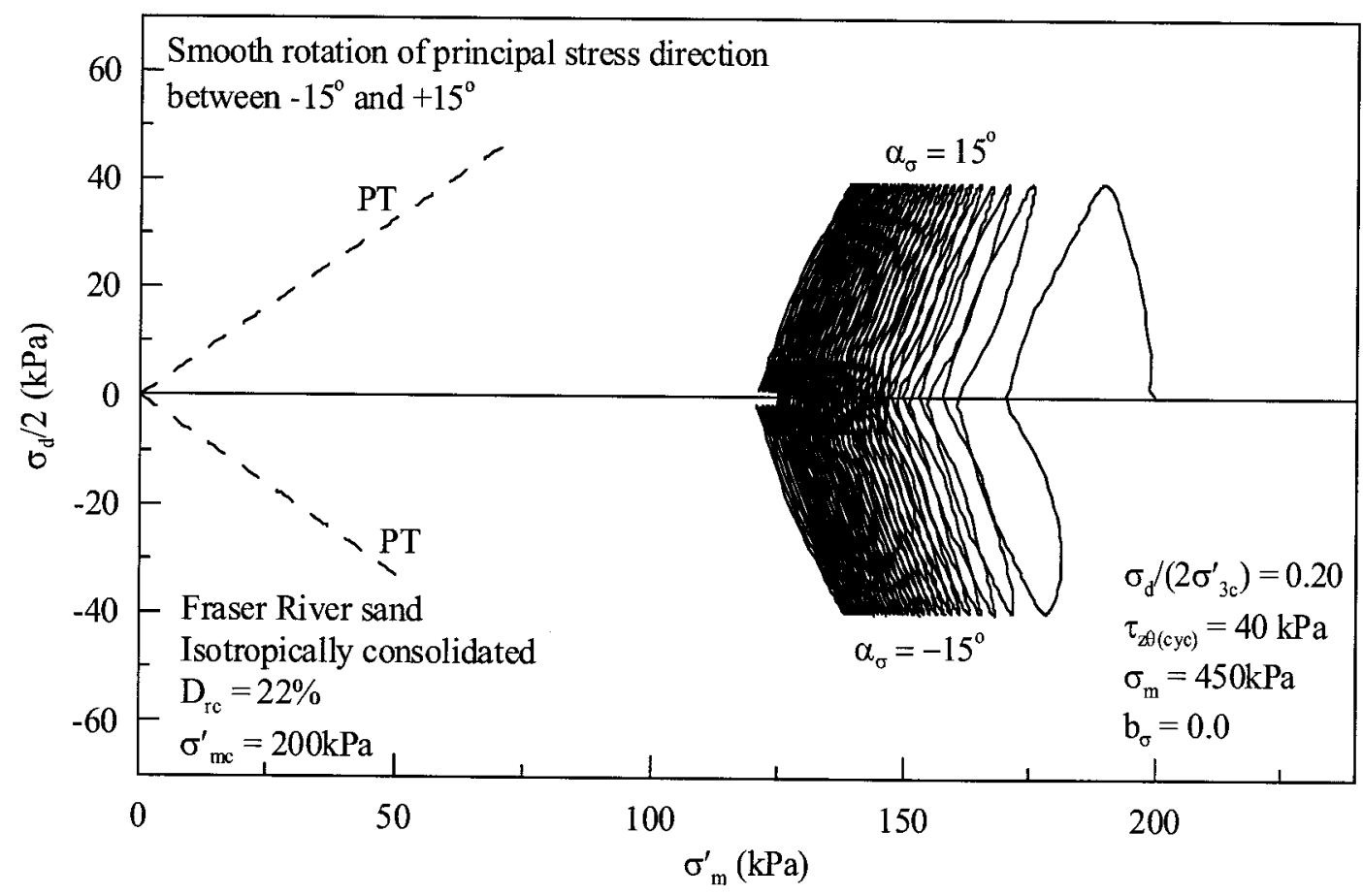

Fig. 6.18 Stress path of Fraser River sand during cyclic loading of $\sigma_{d} /\left(2 \sigma_{3 c}^{\prime}\right)=0.20$ with smooth rotation of $\alpha_{\sigma}$ between $-15^{\circ}$ and $+15^{\circ}$ 


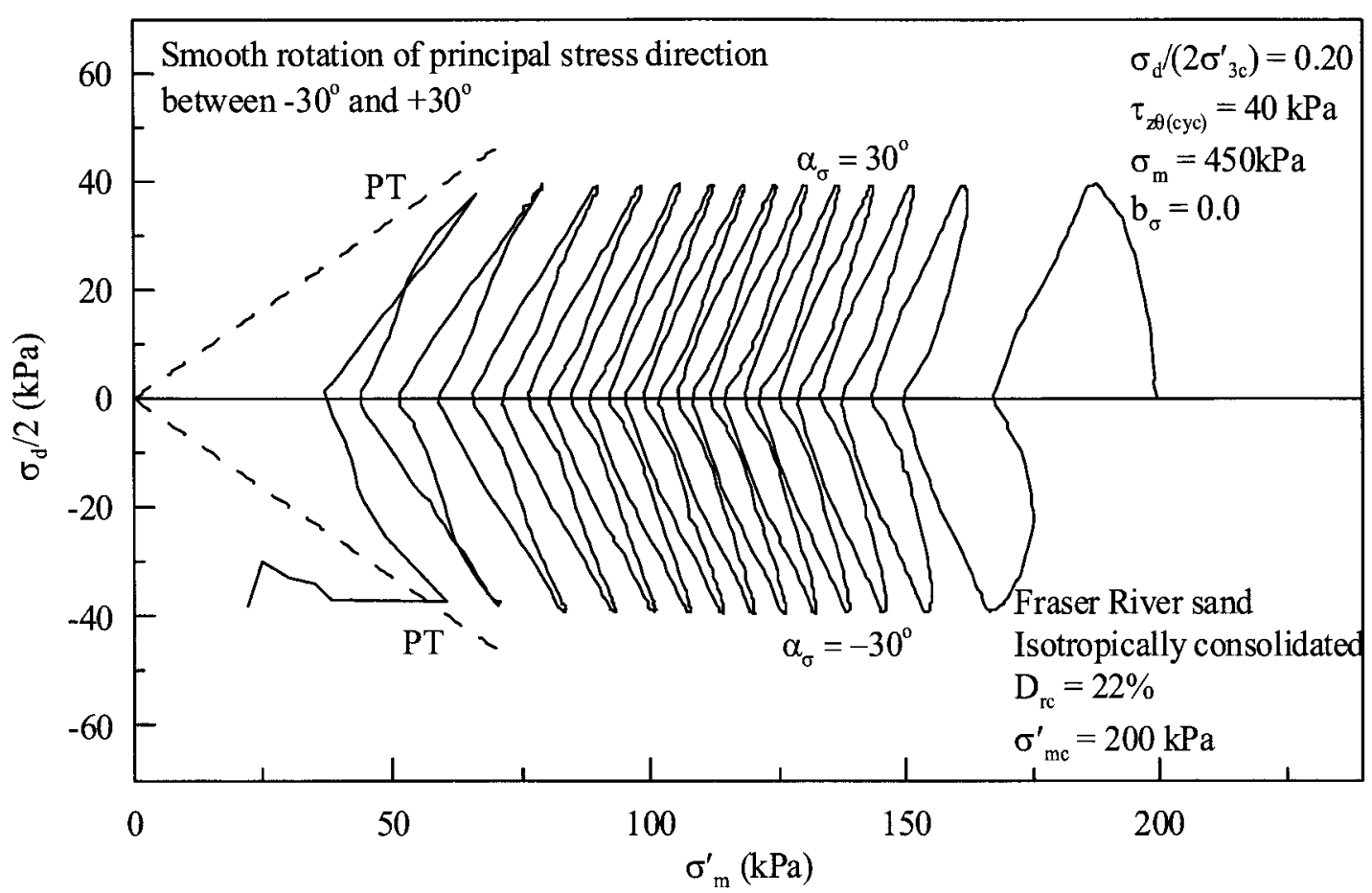

Fig. 6.19 Stress path of Fraser River sand during cyclic loading of $\sigma_{d} /\left(2 \sigma^{\prime}{ }_{3 c}\right)=0.20$ with smooth rotation of $\alpha_{\sigma}$ between $-30^{\circ}$ and $+30^{\circ}$

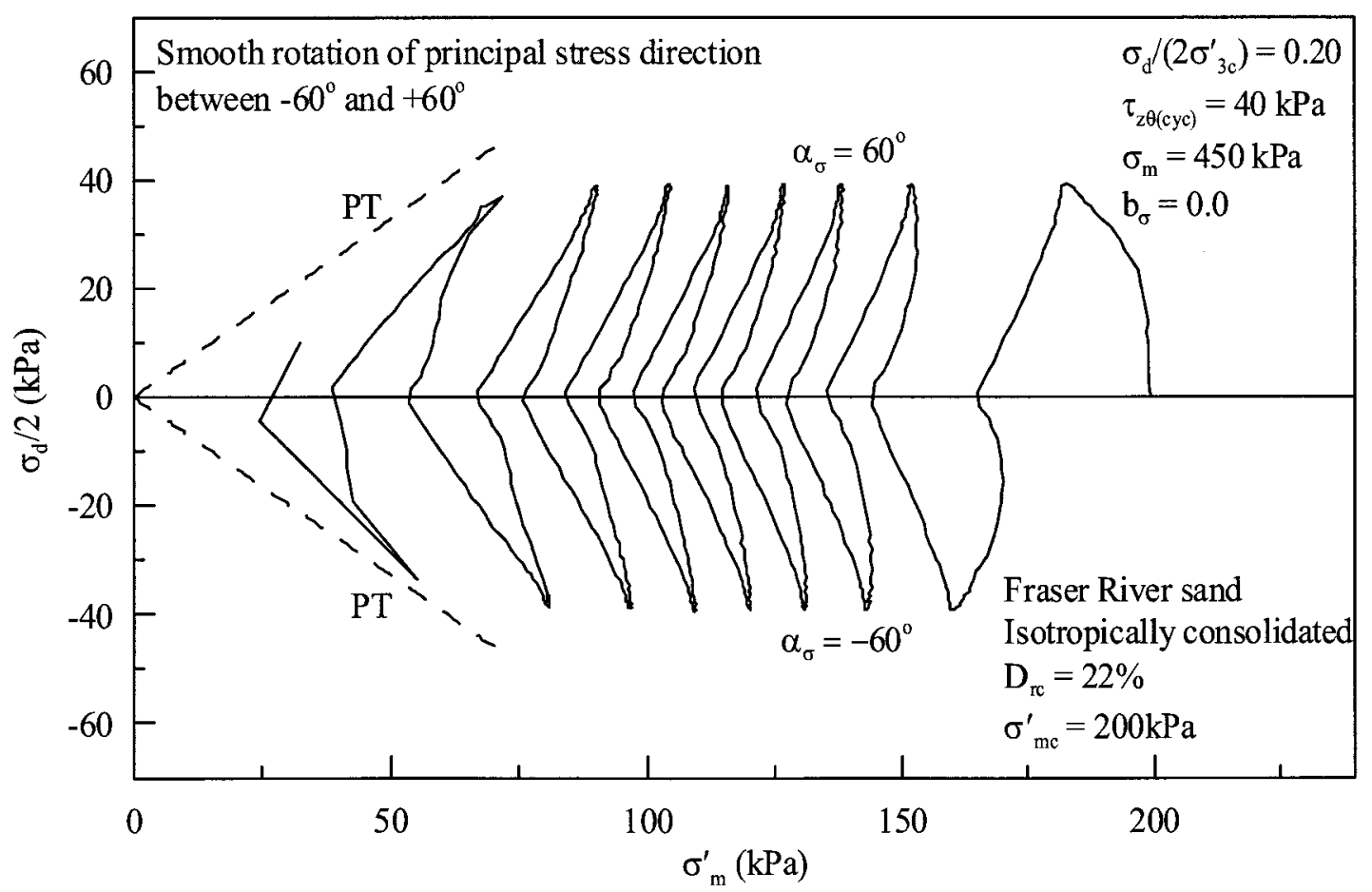

Fig. 6.20 Stress path of Fraser River sand during cyclic loading of $\sigma_{d} /\left(2 \sigma^{\prime}{ }_{3 c}\right)=0.20$ with smooth rotation of $\alpha_{\sigma}$ between $-60^{\circ}$ and $+60^{\circ}$ 


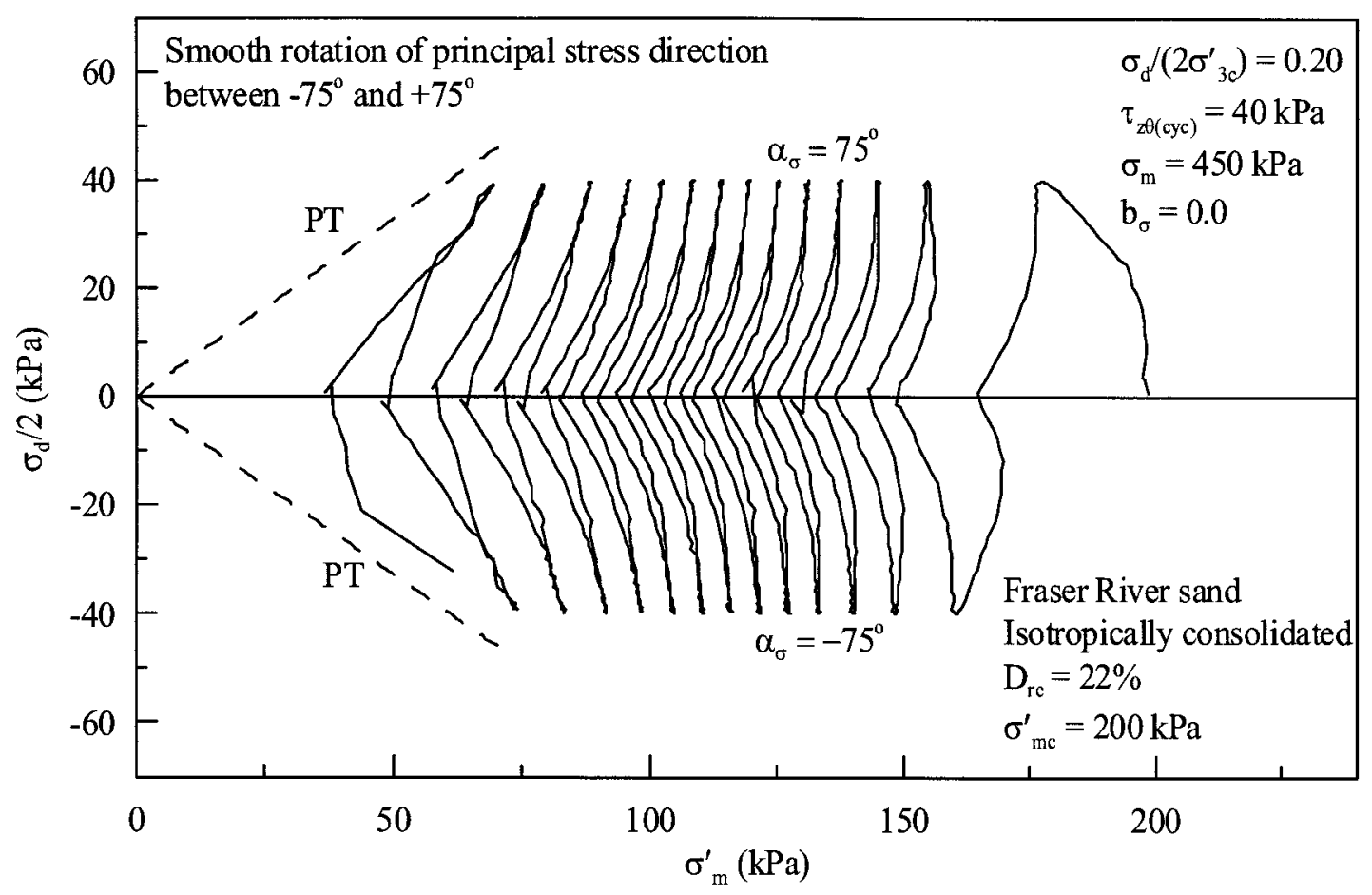

Fig. 6.21 Stress path of Fraser River sand during cyclic loading of $\sigma_{d} /\left(2 \sigma_{3 c}^{\prime}\right)=0.20$ with smooth rotation of $\alpha_{\sigma}$ between $-75^{\circ}$ and $+75^{\circ}$

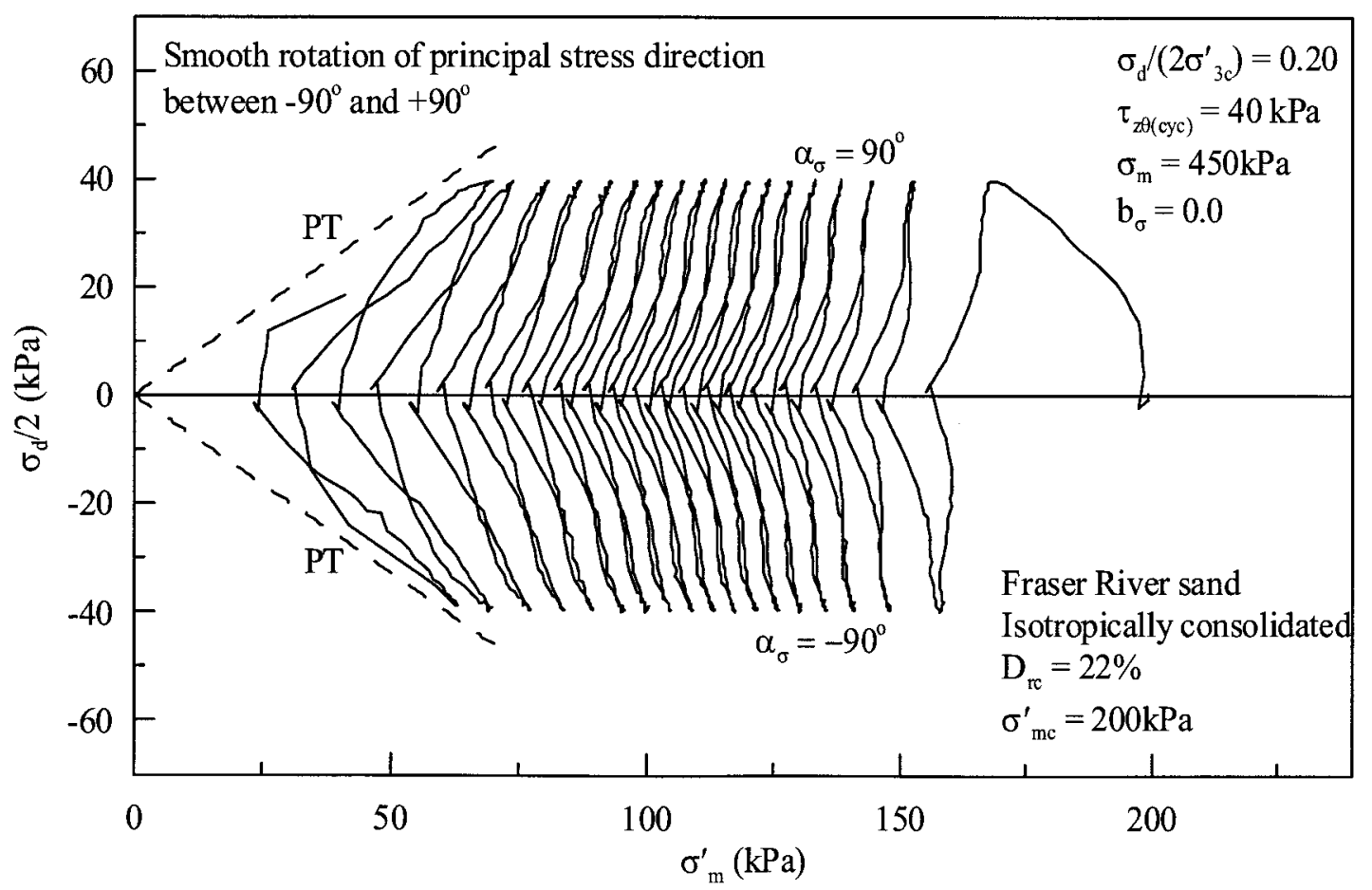

Fig. 6.22 Stress path of Fraser River sand during cyclic loading of $\sigma_{d} /\left(2 \sigma_{3 c}^{\prime}\right)=0.20$ with smooth rotation of $\alpha_{\sigma}$ between $-90^{\circ}$ and $+90^{\circ}$ 
Fig. 6.23 illustrates the induced excess pore pressure response during cyclic loading with smooth rotation of $\alpha_{\sigma}$. Positive excess pore pressure accumulates with increasing number of cycles. Depending on the range of $\alpha_{\sigma}$, the rate of excess pore pressure accumulation differs. The least amount of excess pore pressure is generated at the cyclic test with the smooth rotation of $\alpha_{\sigma}$ between $-15^{\circ}$ and $+15^{\circ}$, and the sand experiences approximately $60 \mathrm{kPa}$ of excess pore pressure $\left(30 \%\right.$ of $\left.\sigma_{m c}^{\prime}\right)$ after the $42^{\text {nd }}$ cycle. When $\alpha_{\sigma}$ is smoothly rotated between $-30^{\circ}$ to $+30^{\circ}$, the sand experiences the excess pore pressure of approximately $80 \%$ of $\sigma_{m c}^{\prime}$ at liquefaction state. During the smooth rotation of $\alpha_{\sigma}$ between $-45^{\circ}$ and $+45^{\circ}$, and $-60^{\circ}$ and $+60^{\circ}$, the sand experiences the same trend of excess pore pressure generation regardless of $\alpha_{\sigma, \max }$ and the excess pore pressure reaches approximately $90 \%$ of $\sigma_{m c}^{\prime}$ at liquefaction. This observation is consistent with the excess pore pressure response of static undrained loading that resulted in less deviation in the excess pore pressure at fixed $\alpha_{\sigma}=45^{\circ}$ and $60^{\circ}$ (Fig. 5.8). During smooth rotation of $\alpha_{\sigma}$ between $-75^{\circ}$ and $+75^{\circ}$, the sand exhibits relatively the same trend of excess pore pressure response as smooth rotation of $\alpha_{\sigma}$ between $-30^{\circ}$ and $+30^{\circ}$. During smooth rotation of $\alpha_{\sigma}$ between $-90^{\circ}$ and $+90^{\circ}$, the sand generates relatively less excess pore pressure than the smooth rotation of $\alpha_{\sigma}$ between $-30^{\circ}$ and $+30^{\circ}$. This data suggests that the use of CSR, defined based on peak deviator stress and effective mean normal stress as the only consideration in the assessment of cyclic resistance may not be fully satisfactory.

Fig. 6.24 shows the variation of induced maximum shear strain $\gamma_{\max }$ during cyclic loading with smooth rotation of $\alpha_{\sigma}$. The developed $\gamma_{\max }$ is small until the stress state 
reaches the PT line. After that, the specimen experiences a large increment of $\gamma_{\max }$. The test conducted at smooth rotation of $\alpha_{\sigma}$ between $-15^{\circ}$ and $+15^{\circ}$ generates only $0.25 \%$ of $\gamma_{\max }$ at the end of the $42^{\text {nd }}$ cycle, and the tests corresponding to $\alpha_{\sigma}$ rotation between $\pm 45^{\circ}$ and $\pm 60^{\circ}$ represent the fastest strain accumulation.

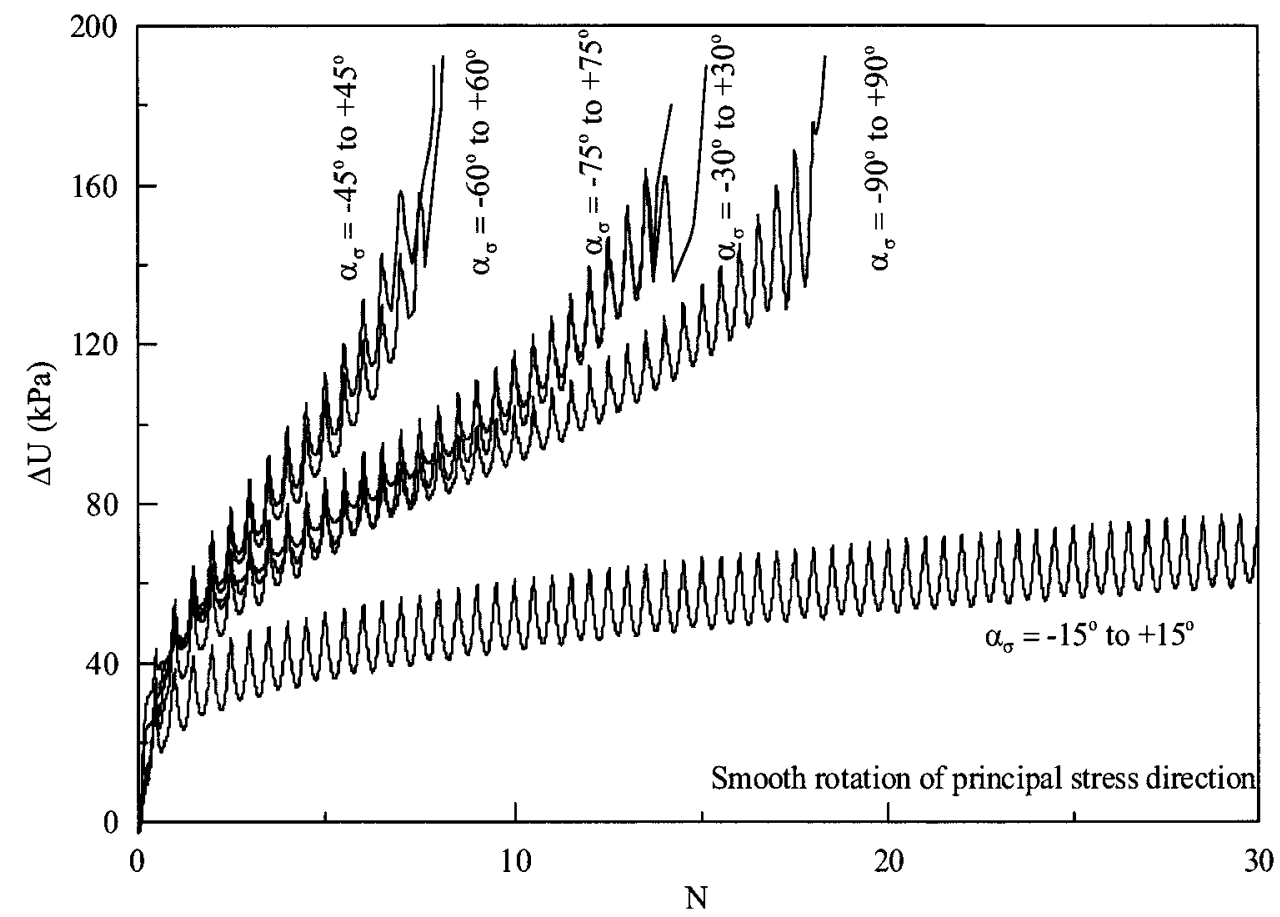

Fig. 6.23 Excess pore pressure response of Fraser River sand during cyclic loading with smooth rotation of $\alpha_{\sigma}$

\subsubsection{Jump rotation of $\alpha_{\sigma}$ at a fixed cyclic stress ratio}

A set of cyclic tests were carried out rotating $\alpha_{\sigma}$ in jump manner at a constant cyclic stress ratio $\sigma_{d} /\left(2 \sigma_{3 c}^{\prime}\right)=0.20$. The specimens were isotropically consolidated to $\sigma_{m c}^{\prime}$ $200 \mathrm{kPa}$ prior to cyclic loading. $\alpha_{\sigma}$ was rotated symmetrically on either side of the deposition direction. $\alpha_{\sigma}$ remained a fixed positive value during the positive half cycle and jumped to a negative value during the negative half cycle. Torsional shear stress $\tau_{z} \theta$ varied in a sinusoidal manner (Fig. 6.17b). The amplitude of $\tau_{z \theta}$ increased with increasing $\alpha_{\sigma, \max }$ for the tests that were conducted with jump rotation of $\alpha_{\sigma}$ between $-15^{\circ}$ and $+15^{\circ}$, - 
$30^{\circ}$ and $+30^{\circ}$, and $-45^{\circ}$ and $+45^{\circ}$. The $\tau_{z}$ of tests, rotating $\alpha_{\sigma}$ between $-60^{\circ}$ and $+60^{\circ}$, and $-75^{\circ}$ and $+75^{\circ}$ is equal to the $\tau_{z \theta}$ of tests rotating $\alpha_{\sigma}$ between $-30^{\circ}$ and $+30^{\circ}$, and $-15^{\circ}$ and $+15^{\circ}$, respectively.

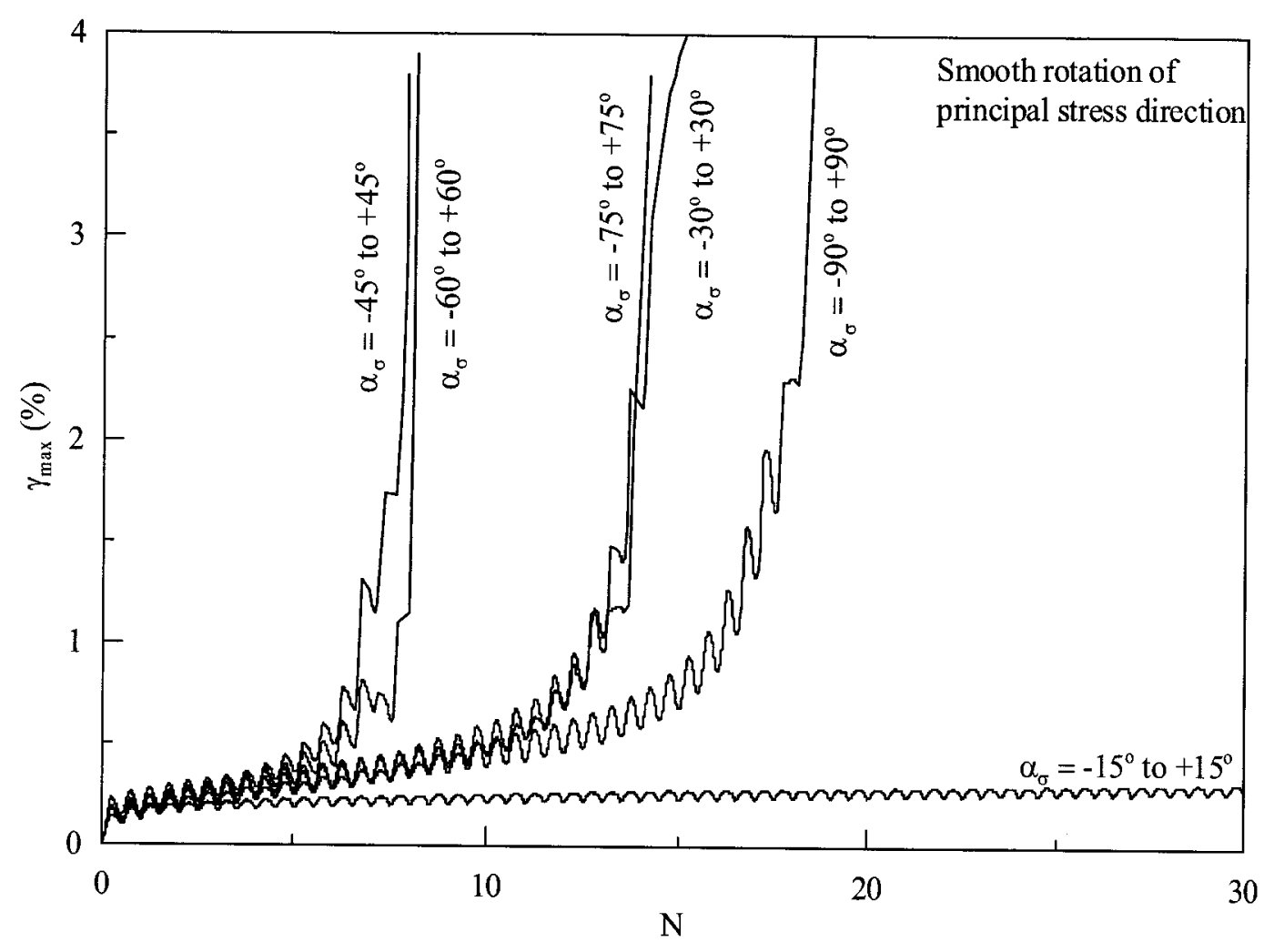

Fig. 6.24 Induced maximum shear strain during cyclic loading with smooth rotation of $\alpha_{\sigma}$

Figs. $6.25,6.26,6.27$ and 6.28 show the cyclic response of Fraser River sand during jump rotation of $\alpha_{\sigma}$ between $-15^{\circ}$ and $+15^{\circ},-30^{\circ}$ and $+30^{\circ},-60^{\circ}$ and $+60^{\circ}$, and $75^{\circ}$ and $+75^{\circ}$, respectively. In all tests, symmetry of the stress paths about the axis of $\sigma_{m}^{\prime}$ is noted. When $\alpha_{\sigma}$ is rotated between $-15^{\circ}$ and $15^{\circ}$, no liquefaction is noted until the $40^{\text {th }}$ stress cycle as noted in the smooth rotation of $\alpha_{\sigma}$ at the same cyclic stress ratio of 0.20 . When $\alpha_{\sigma}$ is rotated between $-30^{\circ}$ and $+30^{\circ}$, liquefaction is noted at the $18^{\text {th }}$ cycle. The number of cycles required to liquefy the sand during jump rotation of $\alpha_{\sigma}$ between $-45^{\circ}$ to $+45^{\circ}$ is further reduced (Fig. 6.12) and liquefaction is observed in 8 cycles. During jump 
rotation of $\alpha_{\sigma}$ between $-60^{\circ}$ and $+60^{\circ}$, liquefaction is observed at the $9^{\text {th }}$ cycle. The sand requires 25 stress cycles to liquefy during jump rotation of $\alpha_{\sigma}$ between $-75^{\circ}$ and $+75^{\circ}$. The tests with jump rotation, in which $\alpha_{\sigma}$ is more closer to the bedding plane experience liquefaction in less number of cycles, even though the same magnitude of $\tau_{z \theta}$ is imposed (imposed $\tau_{z \theta}$ is same for the tests at $\alpha_{\sigma}=-15^{\circ}-+15^{\circ}$ and $\alpha_{\sigma}=-75^{\circ}-+75^{\circ}, \tau_{z \theta}$ is same for the tests at $\alpha_{\sigma}=-30^{\circ}-+30^{\circ}$ and $\alpha_{\sigma}=-60^{\circ}-+60^{\circ}$ ). This observation also confirms the formation of anisotropy in soil structure with weaker plane along the bedding plane.

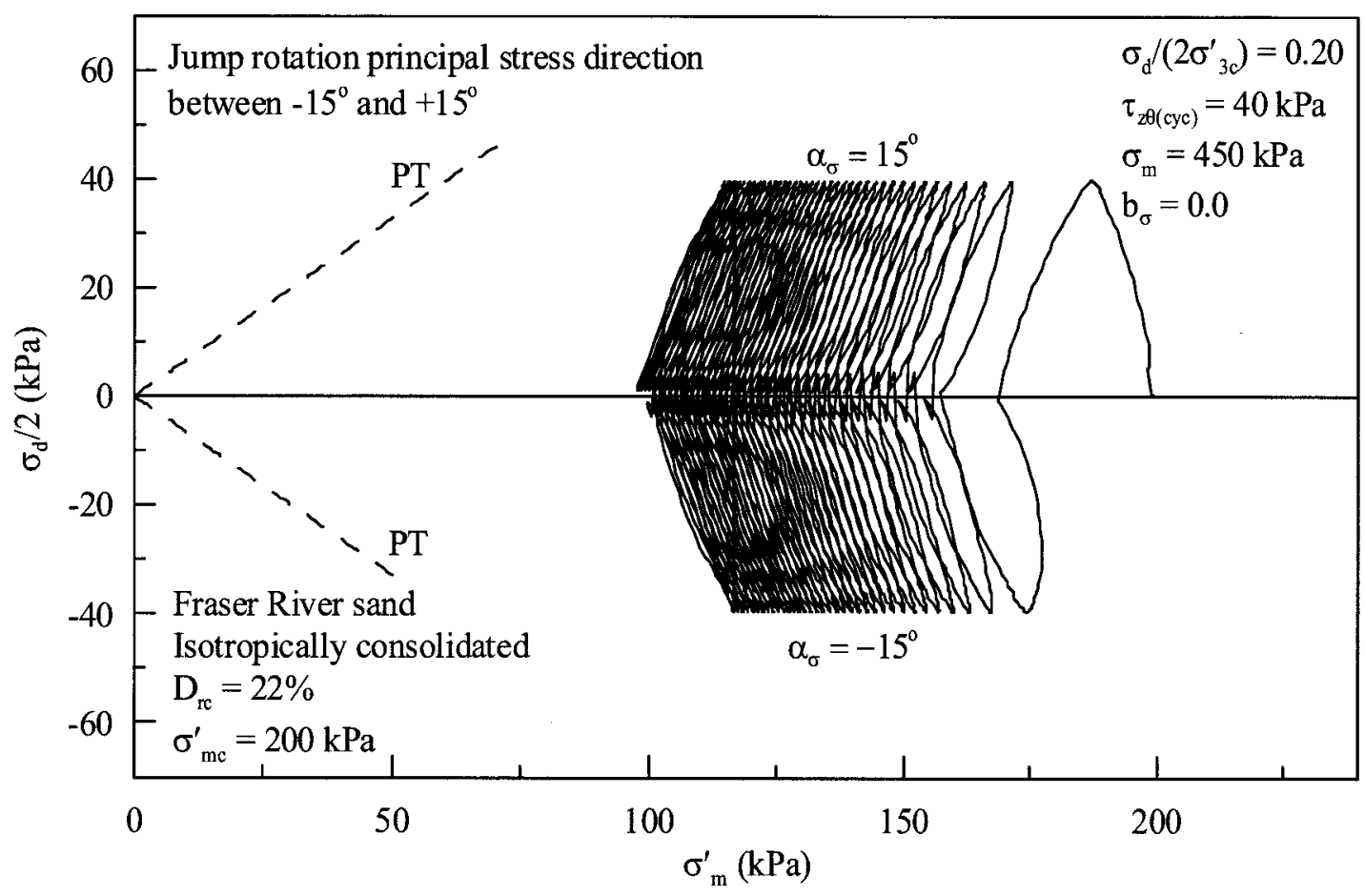

Fig. 6.25 Stress path of Fraser River sand during cyclic loading of $\sigma_{d} /\left(2 \sigma_{3 c}^{\prime}\right)=0.20$ with jump rotation of $\alpha_{\sigma}$ between $-15^{\circ}$ and $+15^{\circ}$ 


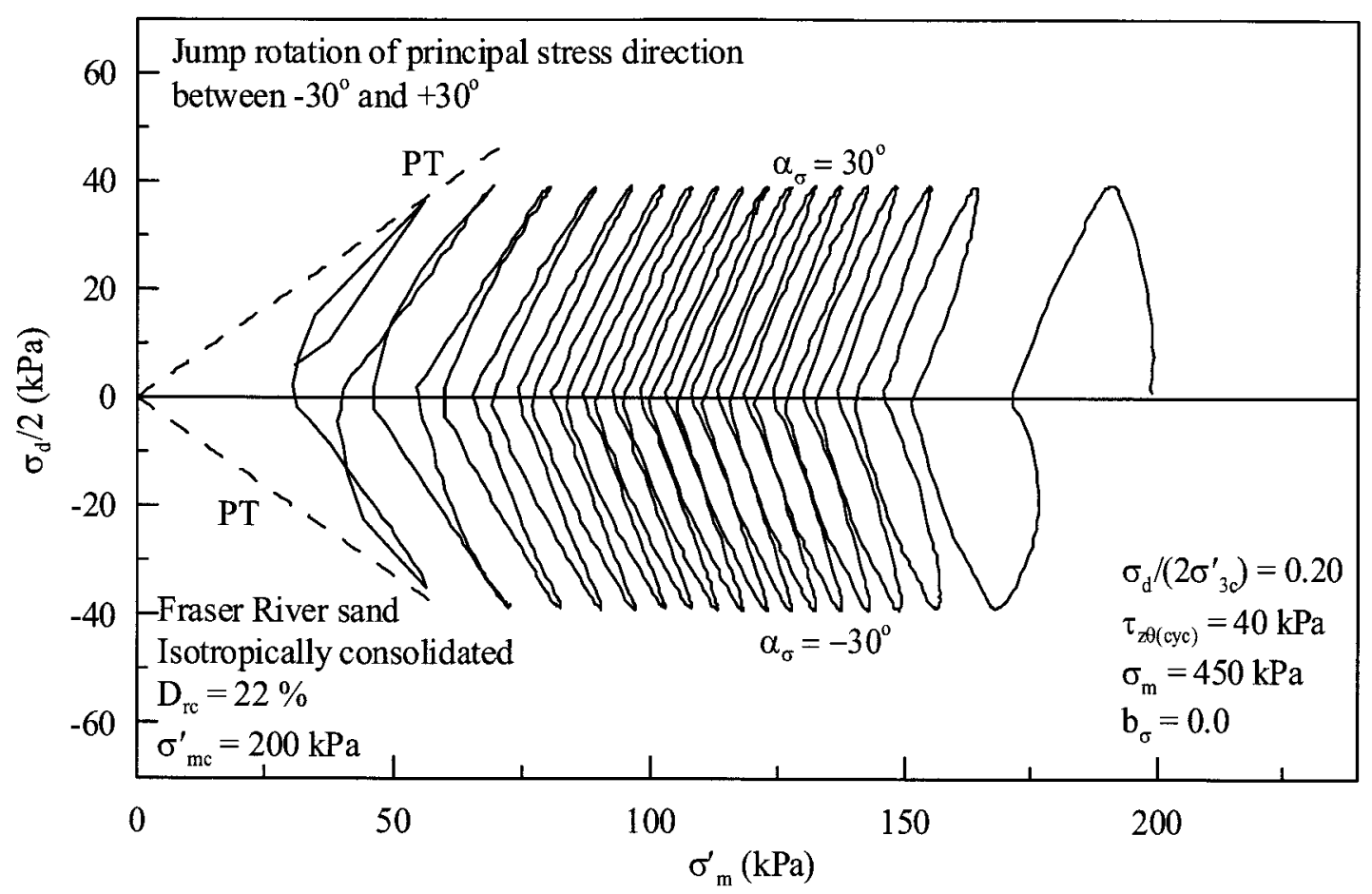

Fig. 6.26 Stress path of Fraser River sand during cyclic loading of $\sigma_{d} /\left(2 \sigma_{3 c}^{\prime}\right)=0.20$ with jump rotation of $\alpha_{\sigma}$ between $-30^{\circ}$ and $+30^{\circ}$

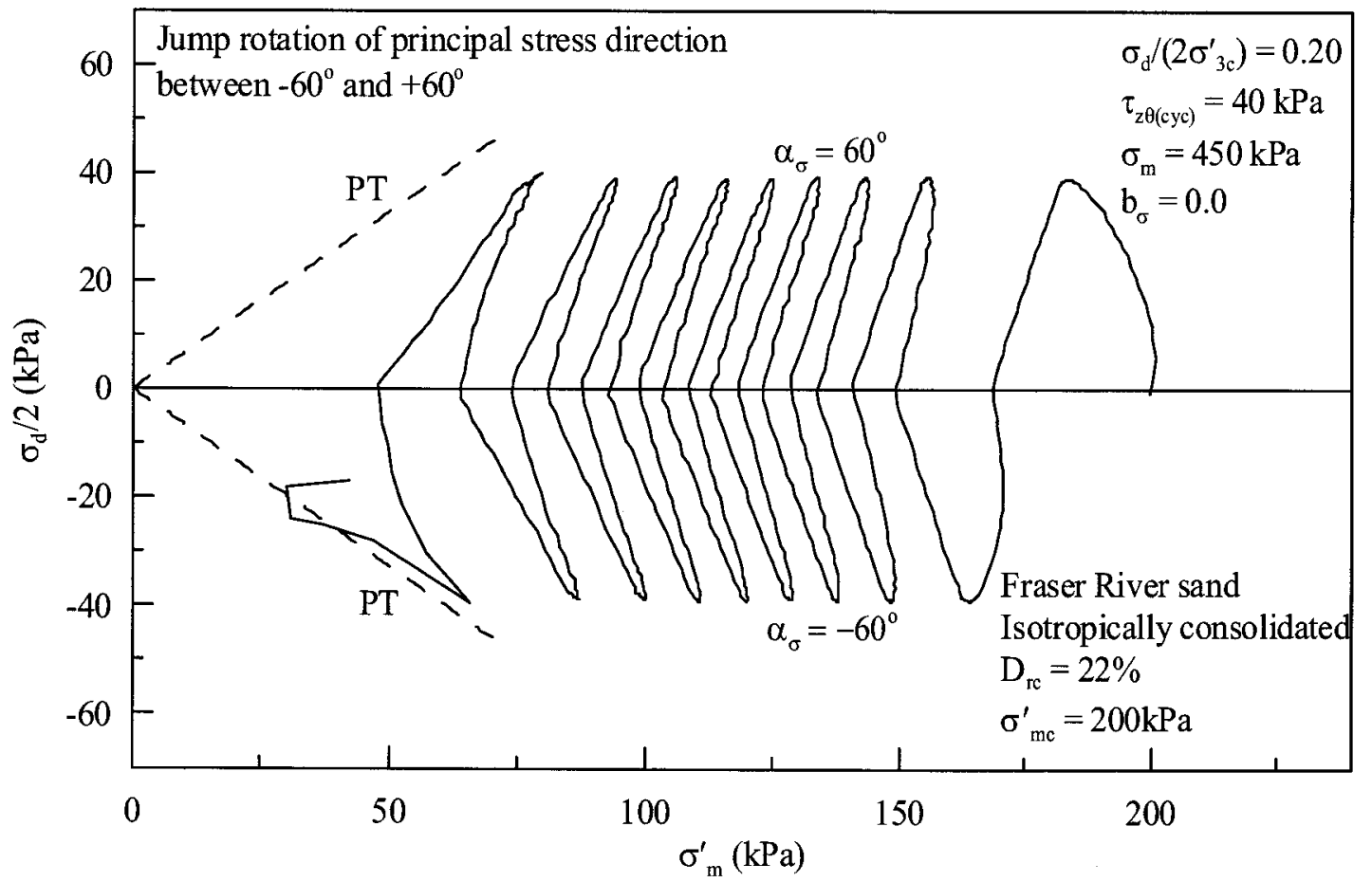

Fig. 6.27 Stress path of Fraser River sand during cyclic loading of $\sigma_{d} /\left(2 \sigma_{3 c}^{\prime}\right)=0.20$ with jump rotation of $\alpha_{\sigma}$ between $-60^{\circ}$ and $+60^{\circ}$ 


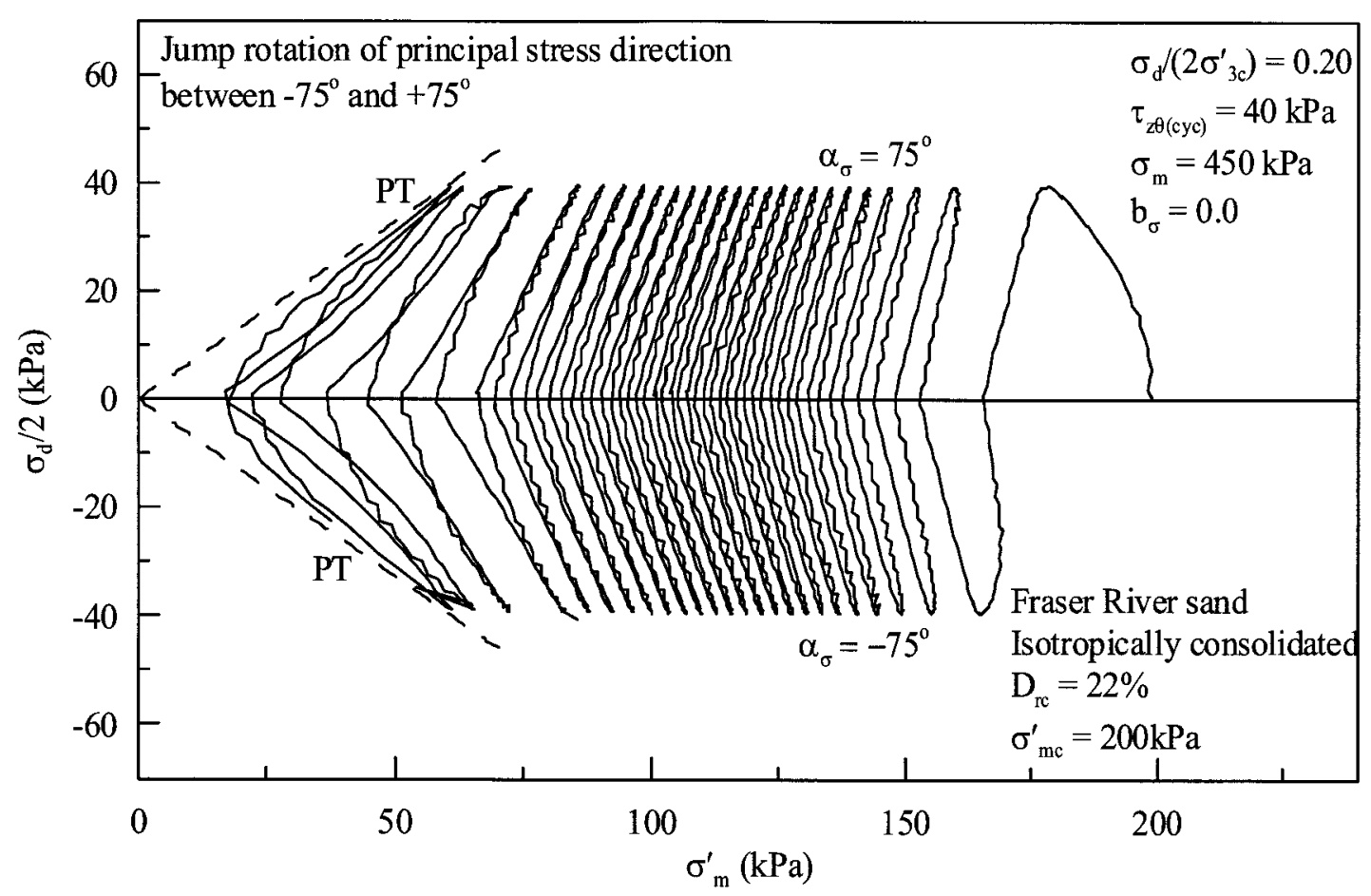

Fig. 6.28 Stress path of Fraser River sand during cyclic loading of $\sigma_{d} /\left(2 \sigma_{3 c}^{\prime}\right)=0.20$ with jump rotation of $\alpha_{\sigma}$ between $-75^{\circ}$ and $+75^{\circ}$

Fig. 6.29 illustrates the excess pore pressure response of Fraser River sand during cyclic loading with jump rotation of $\alpha_{\sigma}$. In all tests, the excess pore pressure accumulates with increasing number of cycles. Depending on $\alpha_{\sigma, \max }$, the rate of excess pore pressure accumulation differs. The test rotating $\alpha_{\sigma}$ between $-15^{\circ}$ and $+15^{\circ}$ experiences the lowest rate of excess pore pressure accumulation and the sand experiences approximately $80 \mathrm{kPa}$ of excess pore pressure at the end of 40 cycles $\left(40 \%\right.$ of $\left.\sigma_{m c}^{\prime}\right)$. At a given cycle, the rate of excess pore pressure accumulation increases with increasing $\alpha_{\sigma, \max }$ (until $\alpha_{\sigma, \max }=45^{\circ}$ ). Beyond $\alpha_{\sigma, \max }=45^{\circ}$, the rate of excess pore pressure accumulation reduces.

The rate of accumulation of excess pore pressure during jump rotation of $\alpha_{\sigma}$ between $-60^{\circ}$ and $+60^{\circ}$ is slightly less than that of accumulation during jump rotation of $\alpha_{\sigma}$ between $-45^{\circ}$ and $+45^{\circ}$. This observation may be explained by the level of $\tau_{z \theta}$ experienced in these two tests (Fig. 6.17b). The cyclic test conducted with jump rotation 
of $\alpha_{\sigma}$ between $-45^{\circ}$ and $+45^{\circ}$ experiences higher $\tau_{z \theta}$ than the cyclic test conducted with jump rotation of $\alpha_{\sigma}$ between $-60^{\circ}$ and $+60^{\circ}$. However, the rate of excess pore pressure accumulation during jump rotation of $\alpha_{\sigma}$ between $-60^{\circ}$ and $+60^{\circ}$ is higher than the rate of accumulation during jump rotation of $\alpha_{\sigma}$ between $-30^{\circ}$ and $+30^{\circ}$. In these two tests, imposed $\tau_{z \theta}$ is equal. The difference in the excess pore pressure accumulation can be attributed to the higher strength of the sand along the deposition direction and the lower strength along the bedding plane. The same trend of deviation in excess pore pressure accumulation is noted in the tests conducted with jump rotation of $\alpha_{\sigma}$ between $-15^{\circ}$ and $+15^{\circ}$, and $-75^{\circ}$ and $+75^{\circ}$.

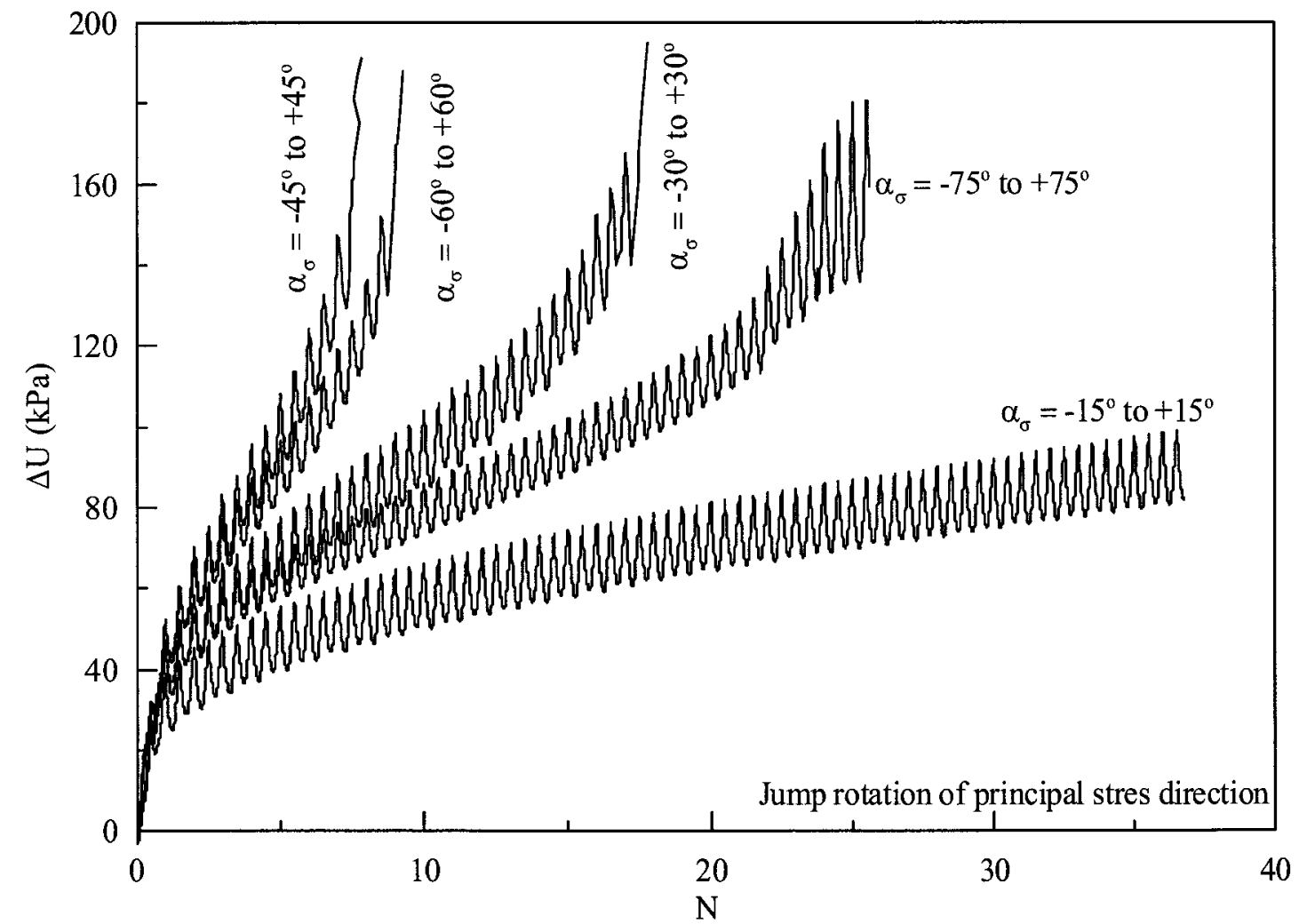

Fig. 6.29 Excess pore pressure response of Fraser River sand during cyclic loading with jump rotation of $\alpha_{\sigma}$ 
Fig. 6.30 shows the variation of induced maximum shear strain $\gamma_{\max }$ during cyclic loading with jump rotation of $\alpha_{\sigma}$. The developed $\gamma_{\max }$ is comparatively small, until the stress path reaches the PT line. After that, the specimen experiences a large increment of $\gamma_{\max }$. The test conducted at jump rotation of $\gamma_{\max }$ between $-15^{\circ}$ and $+15^{\circ}$ induces only $0.35 \%$ of $\gamma_{\max }$ at the end of 40 cycles of loading. In all other tests, $\gamma_{\max }$ reaches more than $3.75 \%$ of maximum shear strain due to liquefaction.

The friction angle of $33.4^{\circ}$ is mobilized at PT during both smooth and jump rotations of $\alpha_{\sigma}$. It is equal to the friction angle mobilized at PT state during the static undrained loading.

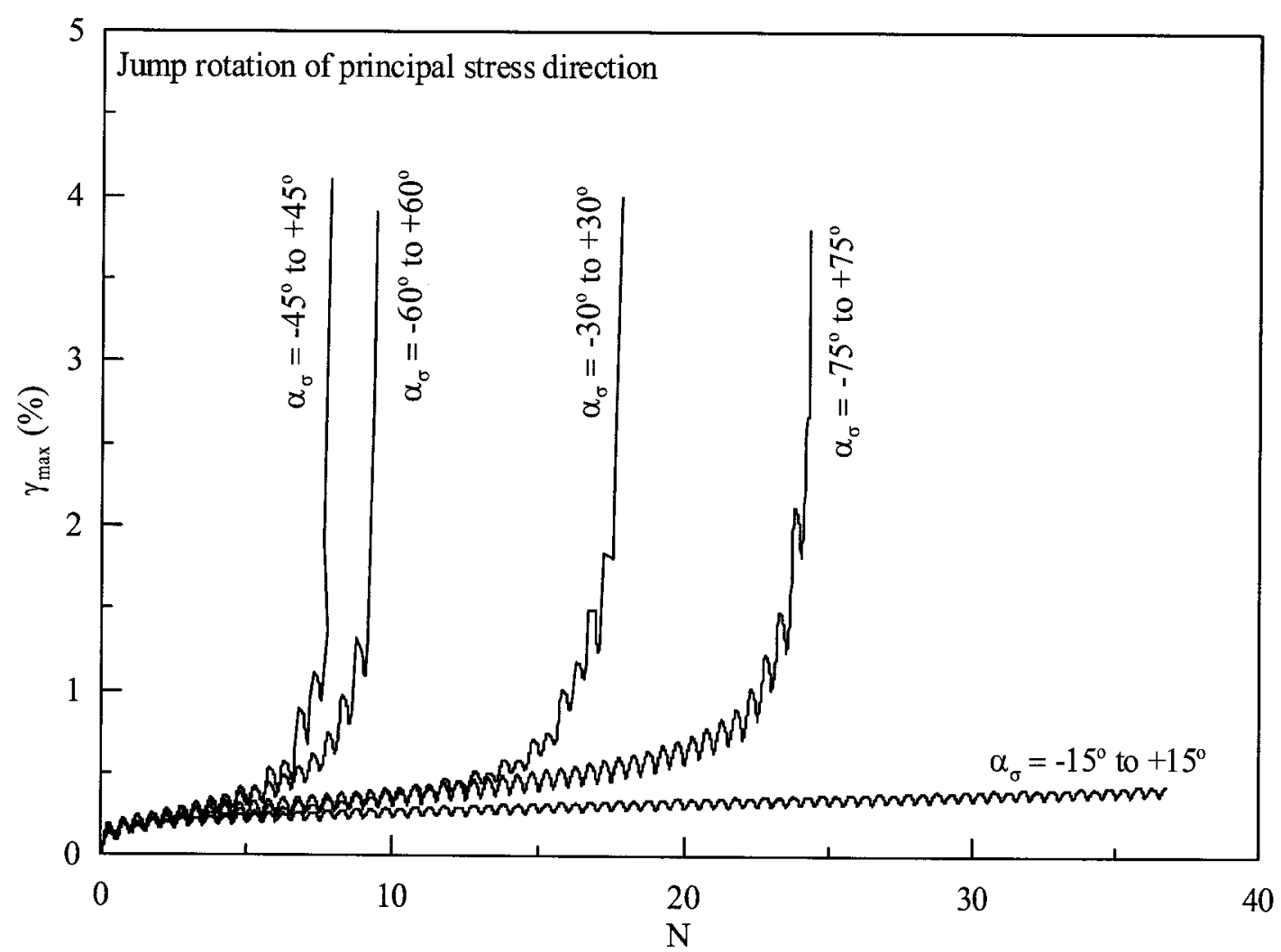

Fig. 6.30 Imposed maximum shear strain during cyclic loading with jump rotation of $\alpha_{\sigma}$ 


\subsection{Comparison of the nature of principal stress rotation}

The effect of smooth and jump rotations of $\alpha_{\sigma}$ during cyclic loading at a fixed cyclic stress ratio of 0.20 is compared. Fig. 6.31 shows the variation of the number of cycles required to liquefy the sand against $\alpha_{\sigma, \max }$. It is noted that the number of cycles required to liquefy the sand during both types of rotations is decreased when $\alpha_{\sigma, \max }$ increases to $\alpha_{\sigma, \max }=45^{\circ}$. In addition, the influence of the nature of the rotations on the cyclic resistance of the sand appears insignificant. Nearly the same trend of variation is noted for both types of stress rotations.

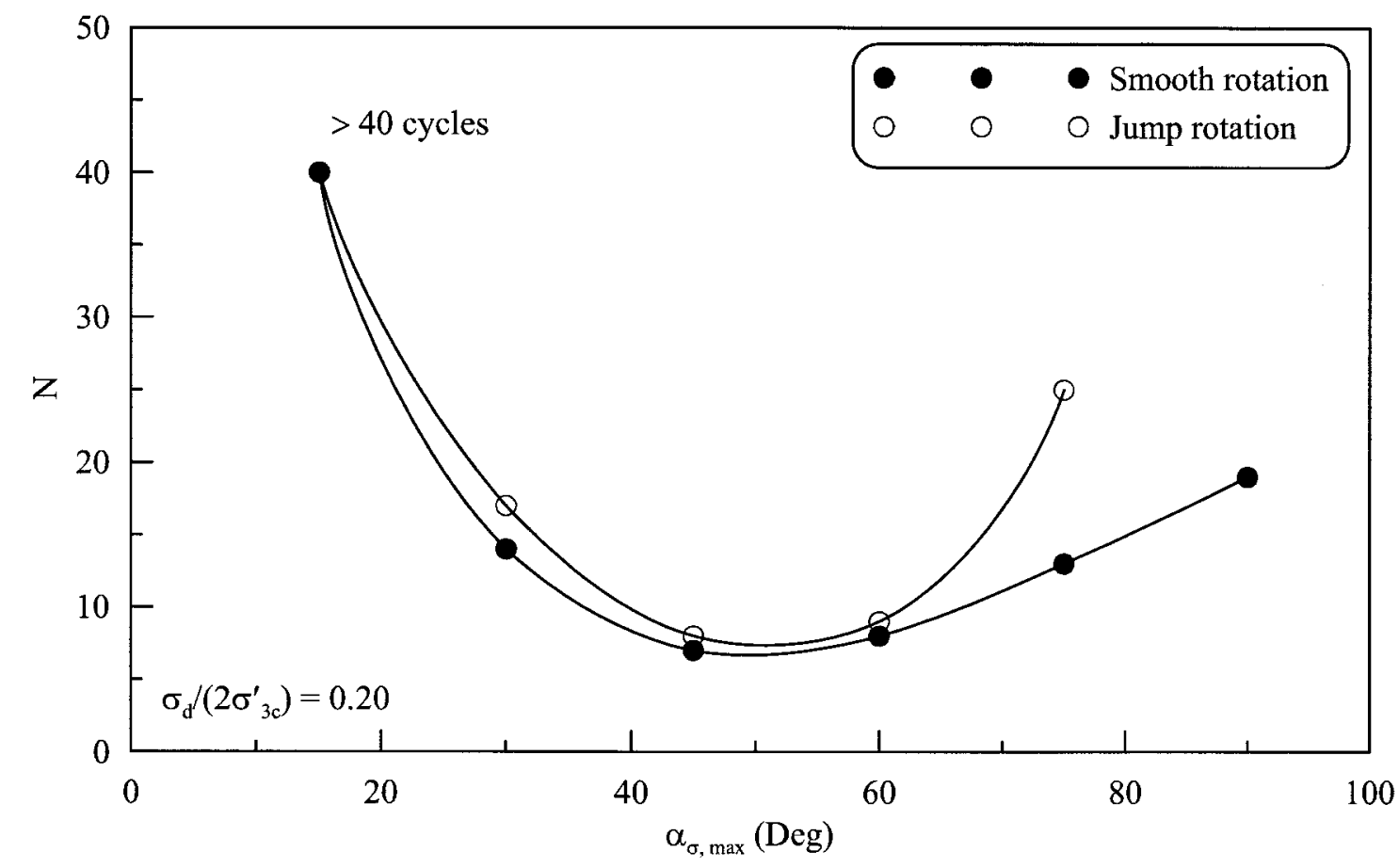

Fig. 6.31 Comparison of number of cycles required for liquefaction during smooth and jump rotations of $\alpha_{\sigma}$

The limited data available suggests that the nature of the rotation might significantly affect the cyclic resistance of the sand when $\alpha_{\sigma, \max }$ is beyond $60^{\circ}$. Liquefaction resistance of the sand increases with increasing $\alpha_{\sigma, \max }$. However, the trend is quite different from one to the other. Smooth rotation of $\alpha_{\sigma}$ shows a lower cyclic 
resistance of the sand compared to the jump rotation of $\alpha_{\sigma}$. This might be a reflection of the imposed $\tau_{z \theta}$ as shown in Fig. 6.32. This figure shows the comparison of $\tau_{z \theta}$ during jump and smooth rotations of $\alpha_{\sigma}$ between $-60^{\circ}$ and $+60^{\circ}$, and $-75^{\circ}$ and $+75^{\circ}$. During smooth rotation, the sand experiences a higher amount of $\tau_{z \theta}$ compared to the corresponding jump rotation tests. In addition, the specimen experiences higher $\tau_{z \theta}$ during smooth rotation of $\alpha_{\sigma}$ prior to reaching peak deviatoric stress.

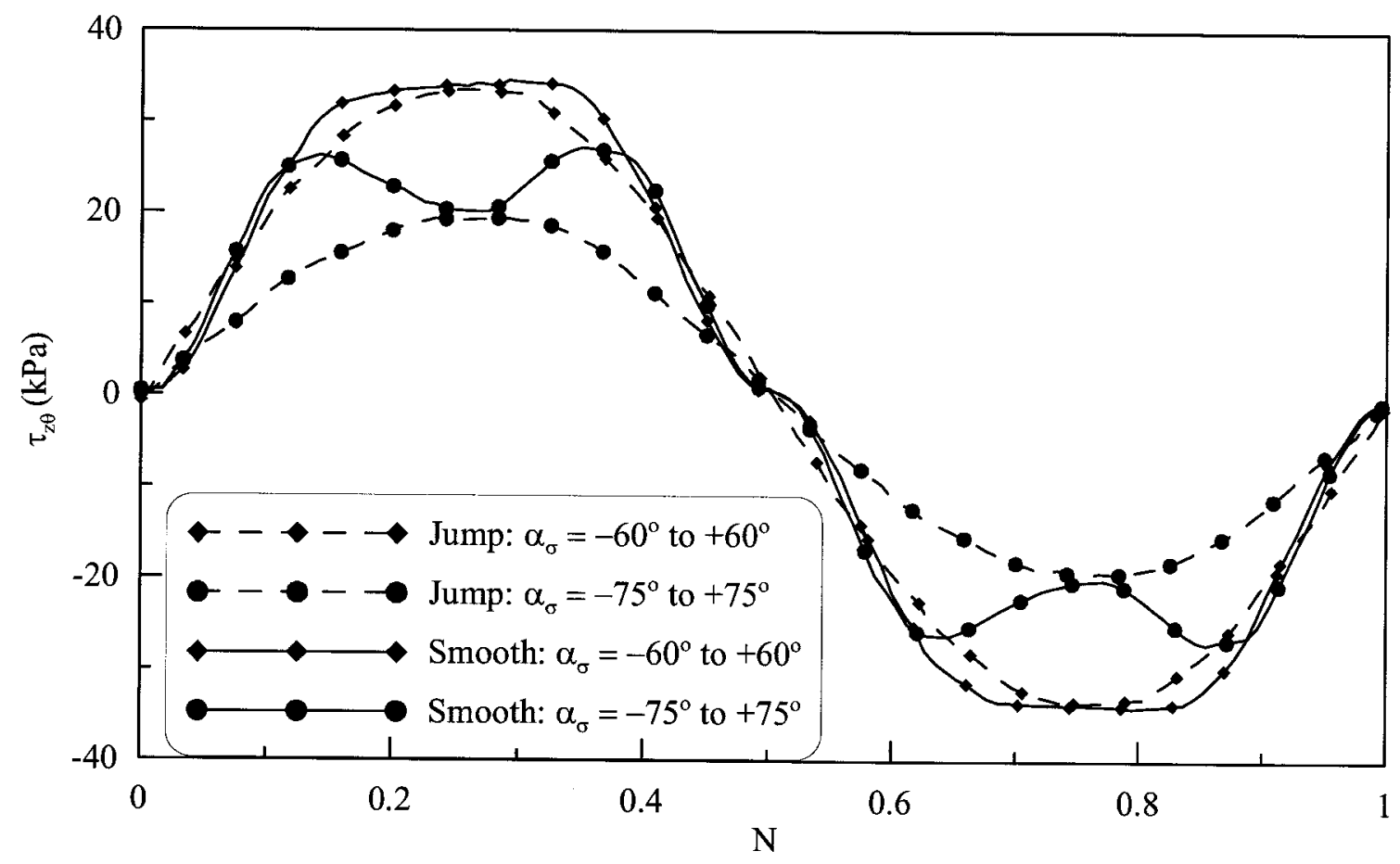

Fig. 6.32 Imposed torsional shear stress during jump and smooth rotations of $\alpha_{\sigma}$ between $-60^{\circ}$ and $+60^{\circ}$, and $-75^{\circ}$ and $+75^{\circ}$

\subsection{Summary}

This chapter studied the cyclic behaviour of Fraser River sand under smooth and jump rotation using an automated HCT device. The experiments carried out to study the nature of rotation of principal stress direction lead to following conclusions.

The stress path during triaxial mode of cyclic loading is not symmetrical because rotation of the principal stress direction is not symmetrical about the axis of cross 
anisotropy of the specimen. The triaxial cyclic loading induces the stronger mode during compression $\left(\alpha_{\sigma}=0^{\circ}\right)$ and the weaker mode during extension $\left(\alpha_{\sigma}=90^{\circ}\right)$. The liquefaction assessment based on triaxial cyclic loading may misinterpret the cyclic resistance of soils. Fraser River sand liquefies in 34 stress cycles at a cyclic stress ratio of 0.20 . However, the sand under plane strain condition $\left(b_{\sigma}=0.40\right)$ at a cyclic stress ratio of 0.20 liquefies in 6 cycles.

The tests carried out under smooth and jump rotation of the principal stress direction at $\alpha_{\sigma, \max }=45^{\circ}$ indicate that the nature of the rotation has minimal influence on liquefaction resistance. Nearly, same trend of variation in cyclic resistance is noted in both jump and smooth rotations (Fig. 6.16). The number of cycles required for liquefaction decreases with increasing cyclic stress ratio as noted in the literature.

The cyclic resistance of the sand is almost the same at a given cyclic stress ratio and a given $\alpha_{\sigma, \max }$ for both types of rotations when $\alpha_{\sigma, \max }$ is less $60^{\circ}$. However, $\alpha_{\sigma, \max }$ affects the cyclic resistance of the sand depending on the nature of the rotation when it is beyond $60^{\circ}$. The sand exhibits higher cyclic resistance when the rotation of principal stress is closer to the deposition direction (i.e. $\alpha_{\sigma}$ between $-15^{\circ}$ and $+15^{\circ}$ ). When the rotation of principal stress is further towards the bedding plane, the cyclic resistance reduces drastically (i.e. $\alpha_{\sigma}$ between $-45^{\circ}$ and $+45^{\circ}$ ). This observation may be due to the fact that the soil becomes weaker as the major principal stress aligns towards the bedding plane. The cyclic resistance of the sand during smooth rotation is quite lower than that of jump rotation when the principal stress is rotated beyond $60^{\circ}$. This observation may be explained by the differences in the torsional shear stress imposed. 
Excess pore pressure and strain developments are relatively small prior to the triggering of liquefaction. The rates of excess pore pressure and strain developments increase when the principal stress direction is rotated more towards the bedding plane $\left(\alpha_{\sigma, \max } \leq 45^{\circ}\right)$. The friction angle mobilized at the phase transformation state during cyclic loading is essentially identical to that noted during static loading. 


\section{TOTAL STRESS PATHS AND PORE PRESSURE GENERATION}

\subsection{Introduction}

Pore pressure generation in a saturated soil during undrained loading significantly influences the strength of the soil. Pore pressure generation under undrained condition in soils is generally related to tendency for volume change during the application of shear stress. The shear stress is reflected by unequal increments of principal stresses. An isotropic increment of principal stresses in a saturated soil generates pore pressure equal to the magnitude of the stress increment. Generally, the amount of induced excess pore pressure due to the changes of total stresses has been expressed as a sum of two components (isotropic increment of total stress and deviatoric stress increment). Different approaches are used in practice to quantify the excess pore pressure in terms of principal stress increments. This chapter studies the applicability of these approaches under threedimensional loading. In addition, it investigates the influence of the intermediate principal stress and the direction of principal stress on excess pore pressure generation and stress-strain response during increments of total stress.

Total stress paths applied in these tests were linear in stress space of deviatoric stress $\sigma_{d}$ and total mean normal stress $\sigma_{m}$. During shearing along different total stress paths, change of intermediate principal stress parameter $b_{\sigma}$ and rotation of principal stress direction were imposed in a systematic manner, in which $b_{\sigma}$ and $\alpha_{\sigma}$ were changed against either maximum shear strain $\gamma_{\max }$ or deviatoric stress $\sigma_{d}$. Previous studies have indicated that the undrained behaviour of soils is independent of applied total stress paths at constant $b_{\sigma}$ and $\alpha_{\sigma}$. This study further expands the state of knowledge by assessing pore 
pressure generation under undrained loading with changing $b_{\sigma}$ and $\alpha_{\sigma}$ during the application of different total stress paths. The details of the tests performed are given in Table 7.1.

Table 7.1 Initial and loading states of tests conducted under different total stress paths

\begin{tabular}{|c|c|c|c|c|c|c|c|c|}
\hline \multirow{2}{*}{$\begin{array}{l}\text { Test } \\
\text { No }\end{array}$} & \multicolumn{5}{|c|}{ Consolidation state } & \multicolumn{2}{|c|}{$\begin{array}{l}\text { Loading } \\
\text { state }\end{array}$} & \multirow{2}{*}{ Remarks } \\
\hline & $\begin{array}{l}D_{r c} \\
(\%)\end{array}$ & $\begin{array}{c}\sigma_{m c}^{\prime} \\
(\mathrm{kPa})\end{array}$ & $b_{o c}$ & $\begin{array}{c}\alpha_{\sigma c} \\
(\mathrm{Deg})\end{array}$ & $K_{c}$ & $b_{\sigma}$ & $\begin{array}{c}\alpha_{\sigma} \\
(\mathrm{Deg})\end{array}$ & \\
\hline P01 & 22.7 & 200 & 0.5 & 0 & 1.7 & 0.5 & 0 & $\Delta \sigma_{m}=25 \mathrm{kPa}$ \\
\hline P02 & 22.1 & 200 & - & - & 1.0 & - & 0 & $\Delta \sigma_{m}=0$ \\
\hline P03 & 21.2 & 200 & 1.0 & 0 & 1.5 & - & 0 & $\begin{array}{c}\Delta \sigma_{1}=\Delta \sigma_{2}= \\
25, \Delta \sigma_{3}=0 \\
\mathrm{kPa}\end{array}$ \\
\hline P04 & 21.7 & 200 & - & - & 1.0 & 0.0 & 0 & \multirow{2}{*}{ 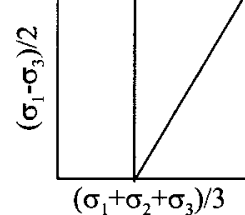 } \\
\hline P05 & 21.4 & 200 & - & - & 1.0 & 0.0 & 0 & \\
\hline P06 & 22.7 & 200 & - & - & 1.0 & 1.0 & 90 & \multirow{2}{*}{ 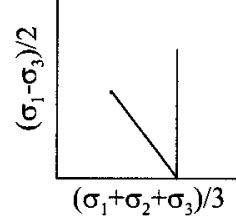 } \\
\hline P07 & 23.0 & 200 & - & - & 1.0 & 1.0 & 90 & \\
\hline P08 & 22.8 & 200 & - & - & 1.0 & 0.0 & 0 & \multirow{2}{*}{ 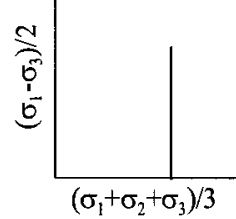 } \\
\hline P09 & 22.2 & 200 & - & - & 1.0 & 0.4 & 0 & \\
\hline P10 & 21.9 & 200 & - & - & 1.0 & 0.4 & 0 & \multirow{2}{*}{ 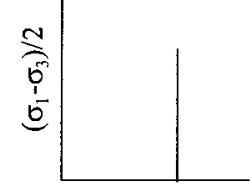 } \\
\hline P11 & 21.6 & 200 & - & - & 1.0 & 0.4 & 45 & \\
\hline
\end{tabular}




\begin{tabular}{|c|c|c|c|c|c|c|c|c|}
\hline $\mathrm{P} 12$ & 21.6 & 200 & - & - & 1.0 & $\begin{array}{c}0.0- \\
0.5\end{array}$ & 0 & \multirow{5}{*}{ 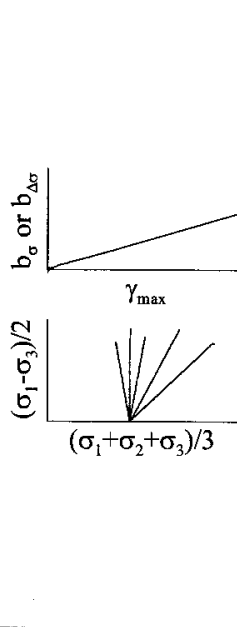 } \\
\hline P13 & 22.4 & 200 & - & - & 1.0 & $\begin{array}{c}0.0- \\
0.5\end{array}$ & 0 & \\
\hline P14 & 21.1 & 200 & - & - & 1.0 & $\begin{array}{c}0.0- \\
0.5\end{array}$ & 0 & \\
\hline P15 & 22.5 & 200 & - & - & 1.0 & $\begin{array}{c}0.0- \\
0.5\end{array}$ & 0 & \\
\hline P16 & 21.6 & 200 & - & - & 1.0 & $\begin{array}{c}0.0- \\
0.5\end{array}$ & 0 & \\
\hline P17 & 23.0 & 200 & - & - & 1.0 & Sine & 0 & \multirow{6}{*}{ 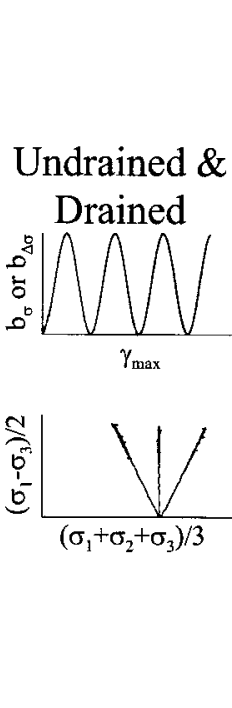 } \\
\hline P18 & 21.1 & 200 & - & - & 1.0 & Sine & 0 & \\
\hline P19 & 22.1 & 200 & - & - & 1.0 & Sine & 0 & \\
\hline P20 & 22.3 & 200 & - & - & 1.0 & Sine & 0 & \\
\hline P21 & 22.7 & 200 & - & - & 1.0 & Sine & 0 & \\
\hline P22 & 21.4 & 200 & - & - & 1.0 & Sine & 0 & \\
\hline P23 & 22.1 & 200 & - & - & 1.0 & 0 & $0-45$ & \\
\hline P24 & 23.0 & 200 & - & - & 1.0 & 0 & $0-45$ & \\
\hline P25 & 22.5 & 200 & - & - & 1.0 & 0 & $0-45$ & $\left(\sigma_{1}+c\right.$ \\
\hline P26 & 22.2 & 200 & - & - & 1.0 & $\begin{array}{c}0.0- \\
1.0\end{array}$ & $15-75$ & \\
\hline P27 & 22.9 & 200 & - & - & 1.0 & $\begin{array}{l}0.0- \\
1.0\end{array}$ & $15-75$ & \\
\hline P28 & 21.3 & 200 & - & - & 1.0 & $\begin{array}{l}0.0- \\
1.0\end{array}$ & $15-75$ & $+\sigma_{3} / 3$ \\
\hline
\end{tabular}




\subsection{Total stress increment and pore pressure generation}

Total stress increment in soil elements can be divided into a hydrostatic stress component and a shear (deviatoric) stress component. The induced excess pore pressure during the application of a total stress increment will be due to both of these components. In practice, two different methods are used to express the increment of total stress tensor in terms of hydrostatic stress tensor and deviatoric stress tensor as shown in Eqs. 7.1 and 7.2. As it is known, hydrostatic total stress increment implies that induced excess pore pressure in saturated soils due to the hydrostatic component is equal to its magnitude.

In the first formulation (Eq. 7.1), the hydrostatic stress tensor is formed by the minor component of stress increments $\Delta \sigma_{3}$ that is selected without considering the magnitude of preshear principal stresses as suggested by Law and Holtz (1978). In the second formulation (Eq. 7.2) that is commonly used in mechanics the hydrostatic stress tensor is formed in terms of increment of mean normal stress $\Delta \sigma_{m}$. The deviatoric stress tensors in both formulations are given in terms of intermediate principal stress parameter $b_{\Delta \sigma}=\frac{\Delta \sigma_{2}-\Delta \sigma_{3}}{\Delta \sigma_{1}-\Delta \sigma_{3}}$ and incremental deviatoric stress $\Delta \sigma_{d}$ that are estimated based on principal components of stress increments.

As defined earlier (Chapter 2), $b_{\sigma}=\frac{\sigma_{2}-\sigma_{3}}{\sigma_{1}-\sigma_{3}}$ is defined based on principal stresses. Parameter $b_{\Delta \sigma}$ becomes equal to $b_{\sigma}$ and takes the value between 0 and 1.0 when the direction of principal stresses and direction of principal components of stress increments coincide. It is also possible in a loading, in which both the direction of principal stresses and the direction of principal components of stress increments are different. In that case, $b_{\Delta \sigma}$ may not be equal to $b_{\sigma}$. In the tests conducted for this research 
program (with the exception of one test), the direction of principal stresses coincided with the direction of the principal components of stress increments throughout shearing.

$$
\begin{aligned}
& {\left[\begin{array}{ccc}
\Delta \sigma_{1} & 0 & 0 \\
0 & \Delta \sigma_{2} & 0 \\
0 & 0 & \Delta \sigma_{3}
\end{array}\right]=\left[\begin{array}{ccc}
\Delta \sigma_{3} & 0 & 0 \\
0 & \Delta \sigma_{3} & 0 \\
0 & 0 & \Delta \sigma_{3}
\end{array}\right]+\left[\begin{array}{ccc}
\Delta \sigma_{1}-\Delta \sigma_{3} & 0 & 0 \\
0 & b_{\Delta \sigma}\left(\Delta \sigma_{1}-\Delta \sigma_{3}\right) & 0 \\
0 & 0 & 0
\end{array}\right] \text { Eq. } 7.1}
\end{aligned}
$$

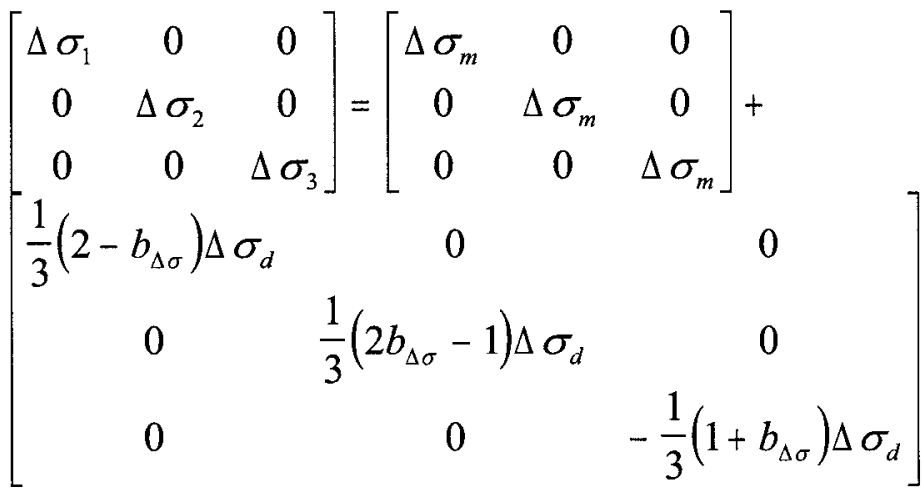

Here, $\Delta \sigma_{1}, \Delta \sigma_{2}$ and $\Delta \sigma_{3}$ are principal components of stress increments. $\Delta \sigma_{m}$ is an increment of total mean normal stress. $\Delta \sigma_{d}$ is incremental deviatoric stress with respect to the principal components of stress increments.

The hydrostatic stress increment tensors $\left[\Delta \sigma_{3}\right]$ and $\left[\Delta \sigma_{m}\right]$ are considered in completely different formats that lead to a different manifestation of excess pore pressure induced by the hydrostatic stress increment. According to the first formulation, the hydrostatic excess pore pressure generated for a particular increment of total stress is equal to the minor component of stress increments. However, the second approach suggests that the hydrostatic excess pore pressure is equal to the increment of mean normal total stress. During undrained shearing of saturated soils, excess pore pressure induced by shear stress is estimated by deducting the hydrostatic total stress increment 
from the total excess pore pressure measured, so that the estimation of excess pore pressure induced by shear stress (deviatoric stress) differs from each other.

For a particular total stress increment, the hydrostatic stress increment $\Delta \sigma_{m}$ is only dependent on the increment of mean normal stress (i.e total stress path in $\sigma_{m}$ - $\sigma_{d}$ space). The hydrostatic stress component $\Delta \sigma_{3}$ can be divided as a hydrostatic component of total mean normal stress increment and a hydrostatic component that is a function of the incremental deviatoric stress and intermediate principal stress parameter $b_{\Delta \sigma}$ (Eq. 7.3). The manipulation of the hydrostatic stress increment clearly shows that $\Delta \sigma_{3}$ is dependent not only on the total stress increment (total stress path) but also on stress state $\left(b_{\Delta \sigma}\right)$.

$\Delta \sigma_{3}=\sigma_{m}-\frac{1}{3}\left(1+b_{\Delta \sigma}\right) \Delta \sigma_{d}$

The total stress paths imposed is linear in the stress space of $\sigma_{m}$ and $\sigma_{d}$ and it can be formulated as given in Eq. 7.4.

$\sigma_{m}=\sigma_{m c}+\beta \sigma_{d}$ or $\Delta \sigma_{m}=\beta \Delta \sigma_{d}$

Here, $\sigma_{m}$ is the total mean normal stress. $\sigma_{m c}$ is the total mean normal stress at the end of consolidation. $\sigma_{d}$ is deviatoric stress. $\Delta \sigma_{m}$ is the increment of mean normal stress. $\Delta \sigma_{d}$ is the increment of deviatoric stress and $\beta$ is constant. Subsequently, the substitution of Eq. 7.4 into Eq. 7.3 results in Eq. 7.5 as a function of $b_{\Delta \sigma}$ and $\Delta \sigma_{m}$.

$\left[\Delta \sigma_{3}\right]=\left[f\left(b_{\Delta \sigma}\right)\right]\left[\Delta \sigma_{m}\right]$

If $b_{\Delta \sigma}$ is a constant throughout the shearing, $\left[f\left(b_{\Delta \sigma}\right)\right]$ would be a scalar, so that the hydrostatic component $\Delta \sigma_{3}$ will be a fold of the hydrostatic component $\Delta \sigma_{m}$. Even though both approaches manifest the different magnitude of excess pore pressure due to the 
deviatoric stress component at a fixed $b_{\Delta \sigma}$, these approaches do not at least change the trend of excess pore pressure generated by deviatoric stress.

Fig. 7.1 shows the variations of $\Delta \sigma_{3}$ against $\Delta \sigma_{m}$ during the application of total stress paths at a fixed $b_{\sigma}$ of 0.0 and 0.4 . In this stress condition of tests, the direction of principal stresses and the direction of principal components of the stress increments coincide, such that $b_{\sigma}$ is equal to $b_{\Delta \sigma}$. The linear relationship exists for both $b_{\sigma}$ in the space of $\Delta \sigma_{m}$ and $\Delta \sigma_{3}$. However, it is different depending on $b_{\sigma}$. As noted earlier, the hydrostatic stress increment formed by $\Delta \sigma_{3}$ differs based on $b_{\sigma}$, so that the comparison of excess pore pressure generated due to deviatoric stress at a given total stress path may not lead to a consistent approach.

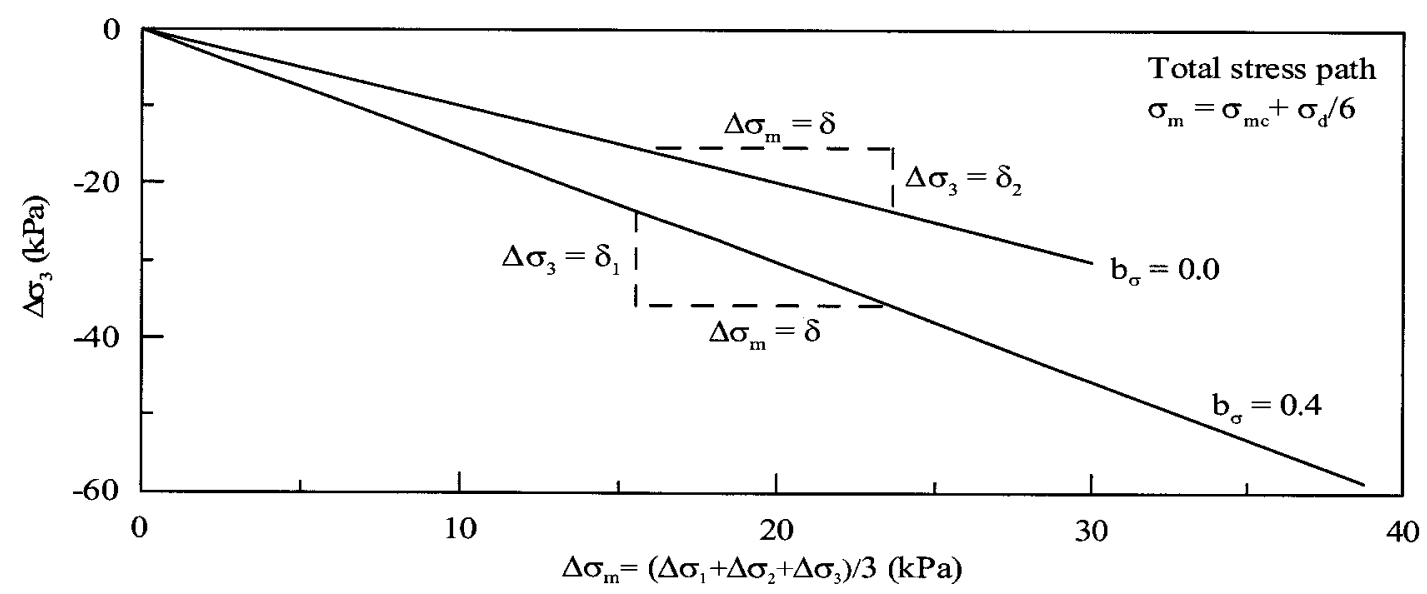

Fig. 7.1 Variation of $\Delta \sigma_{3}$ against $\Delta \sigma_{m}$ at fixed $b_{\sigma}=0.0$ and 0.4

It is also possible that $b_{\sigma}$ can change for a given total stress path. Fig. 7.2 shows the variation of $b_{\sigma}$ against the maximum shear strain $\gamma_{\max }$ during application of the total stress path $\left(\sigma_{m}=\sigma_{m c}+\sigma_{d} / 6\right)$. The variation of $b_{\sigma}$ is linear and it increases from 0 to 0.5 until $5 \%$ of $\gamma_{\max }$. Fig. 7.3 shows the variation of $\Delta \sigma_{3}$ against $\Delta \sigma_{m}$ and the variation represents a nonlinear relationship between $\Delta \sigma_{3}$ and $\Delta \sigma_{m}$. For a particular increment of $\Delta \sigma_{m}, \Delta \sigma_{3}$ takes a value depending on current $b_{\sigma}$. As shown in Fig. 7.3, for the first 
increment of $\Delta \sigma_{m}=\delta$ and the increment of $\Delta \sigma_{3}$ is $\delta_{1}$ and for the second increment of $\Delta \sigma_{m}$ $=\delta, \Delta \sigma_{3}$ is $\delta_{2}$. The hydrostatic stress component of the first increment differs from that of the second increment and thus, hydrostatic excess pore pressure in both increments is different.

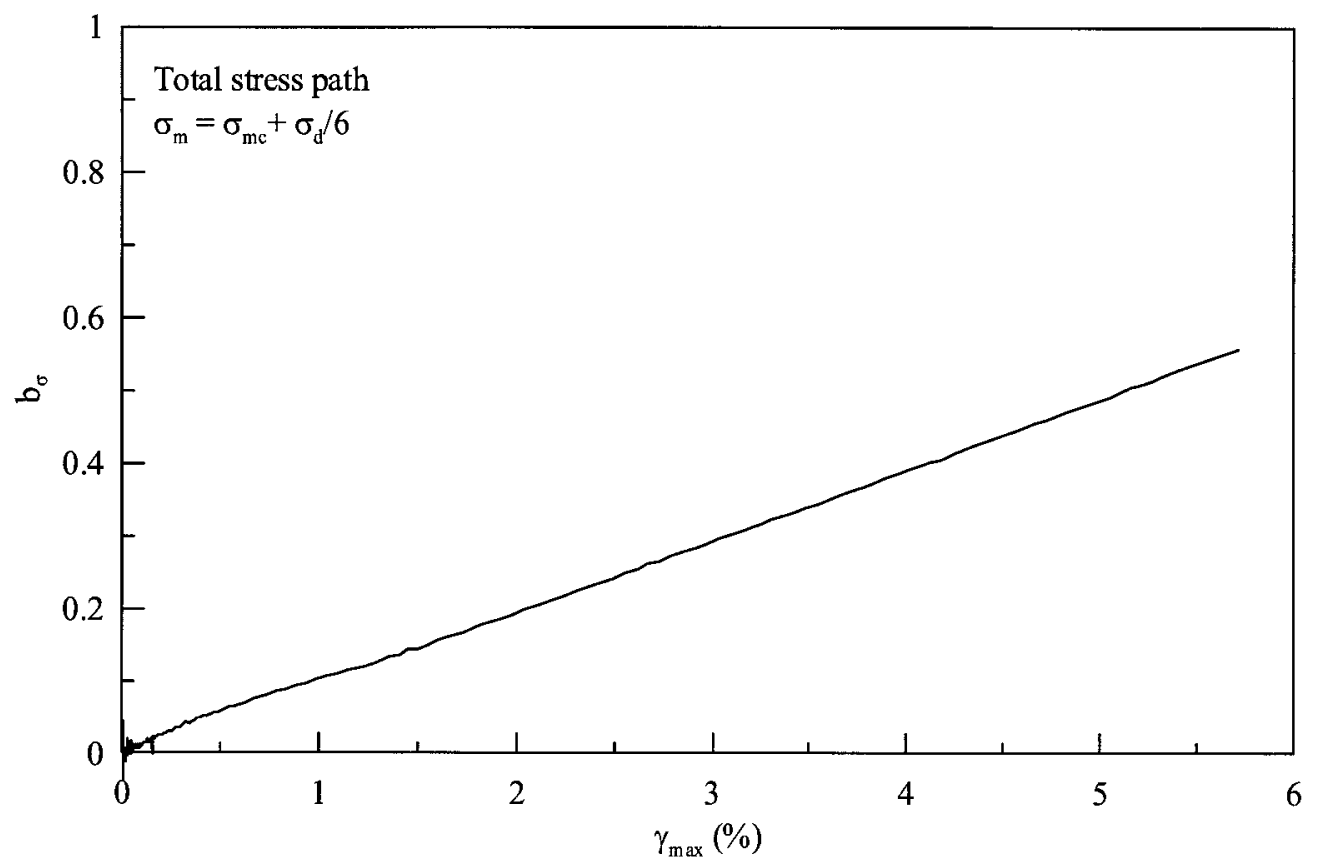

Fig. 7.2 Change of $b_{\sigma}$ during application of a total stress path

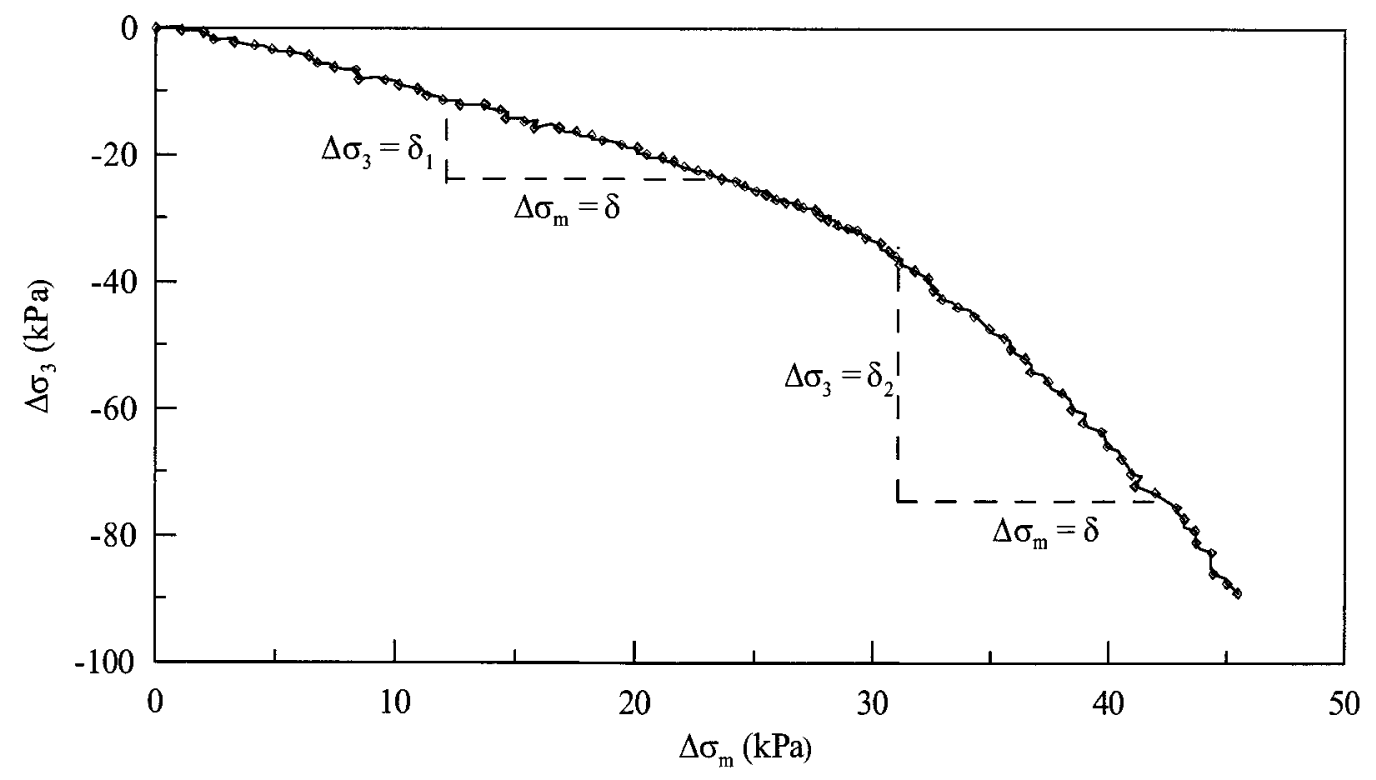

Fig. 7.3 Variation of $\Delta \sigma_{3}$ against $\Delta \sigma_{m}$ during change of $b_{\sigma}$ corresponding to Fig. 7.2 


\subsection{Pore pressure parameters}

The pore pressure equations (Eqs. 2.4, 2.5 and 2.6) have not found wide application in practice because generalization of the pore pressure parameters is not possible. The pore pressure parameters ( $A_{T}$ and $a$ ) that are used to conveniently quantify the shear induced excess pore water pressures depend on the factors, such as loading mode, consolidation history and stress state. In this section, some test results are presented to understand the nature of pore pressure parameters $\left(A_{T}\right.$ and $\left.a\right)$ during change of total stresses in fully saturated sand.

In the first test, the specimen was consolidated to $b_{\sigma c}=0.5, \sigma_{m c}^{\prime}=200 \mathrm{kPa}$ and $K_{c}$ $=1.7$ and $\alpha_{\sigma c}=0^{\circ}$. Each component of principal stresses in the soil element was increased by $25 \mathrm{kPa}$ simultaneously, such that $\Delta \sigma_{I}=\Delta \sigma_{2}=\Delta \sigma_{3}=25 \mathrm{kPa}$. It was noted from Fig. 7.4 that developed excess pore pressure was equal to $\Delta \sigma_{3}$. In other words, it was equal to $\Delta \sigma_{m}$. The deviatoric stress increment was zero, so that the pore pressure parameters $\left(A_{T}\right.$ and $\left.a\right)$ need not to be defined.

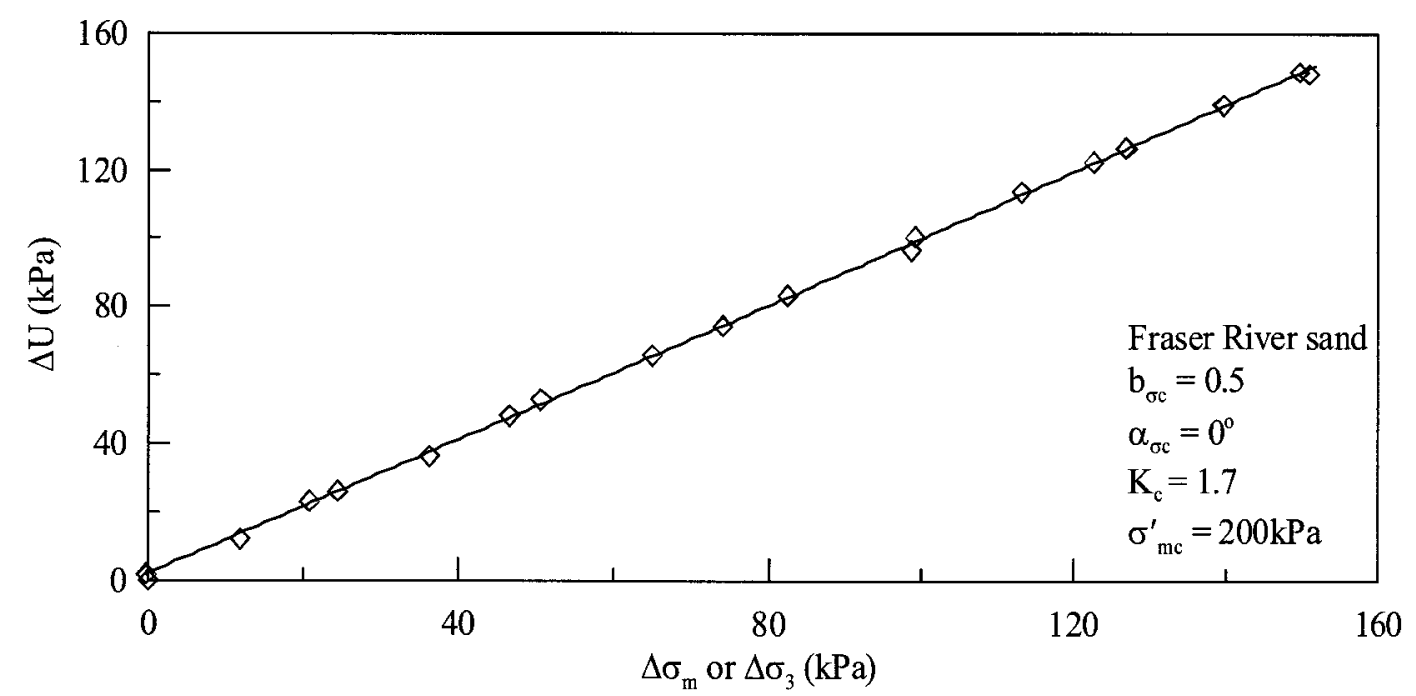

Fig. 7.4 Excess pore pressure induced during isotropic increment of principal stresses 
In the second test, a saturated sand specimen was isotropically consolidated to $\sigma_{m c}^{\prime}=200 \mathrm{kPa}$. The major principal stress was increased by $25 \mathrm{kPa}$ in each step and the intermediate and minor principal stresses were reduced by $2.5 \mathrm{kPa}$ and $22.5 \mathrm{kPa}$, respectively $\left(\Delta \sigma_{1}=25 \mathrm{kPa}, \Delta \sigma_{2}=-2.5 \mathrm{kPa}, \Delta \sigma_{3}=-22.5 \mathrm{kPa}\right)$. The total mean normal stress was thus maintained constant and the direction of principal stress and the direction of principal components of stress increments were coincident.

Fig. 7.5 shows the variation of excess pore pressure induced by a hydrostatic stress increment and a deviatoric stress increment. The induced excess pore pressure due to incremental deviatoric stress is shaded. The hydrostatic stress increment of $\Delta \sigma_{3}$ is negative ( $\sigma_{3}$ were reduced in each increment) and thus, excess pore pressure induced by $\Delta \sigma_{3}$ is negative. The excess pore pressure due to the hydrostatic stress increment of $\Delta \sigma_{m}$ is zero because of the constant total mean normal stress $\left(\Delta \sigma_{m}=0\right)$. These two approaches clearly quantify different amounts of excess pore pressure induced by the deviatoric stress increment. As the excess pore pressure induced by deviatoric stress is positive, pore pressure parameter $A_{T}$ continuously increases (Fig. 7.6). The pore pressure parameters $a$ defined by Henkel (1960), and Henkel and Wade (1966) also increase and the variations are identical. The pore pressure parameters $\left(A_{T}\right.$ and $\left.a\right)$ have different magnitudes at a particular stress state. However, trend of the variations is the same.

In the third test, a specimen was consolidated to $b_{c}=1.0, K_{c}=1.5, \sigma_{m c}^{\prime}=200 \mathrm{kPa}$ and $\alpha_{\sigma c}=0^{\circ}$. Major and minor principal stresses were increased by $25 \mathrm{kPa}$ in each step until intermediate and minor principal stresses become equal, such that the stress increments $\Delta \sigma_{1}=25 \mathrm{kPa}, \Delta \sigma_{2}=25 \mathrm{kPa}$ and $\Delta \sigma_{3}=0 \mathrm{kPa}$. The direction of the principal stress and direction of the principal components of the stress increments were not 
coinciding. The deviatoric stress $\left(\sigma_{1}-\sigma_{3}\right)$ was maintained constant. However, the incremental deviatoric stress with respect to the principal components of stress increments $\left(\Delta \sigma_{1}-\Delta \sigma_{3}\right)$ was increased by $25 \mathrm{kPa}$ in each step. The hydrostatic stress increment of $\Delta \sigma_{3}$ does not exist. However, the hydrostatic stress increment of $\Delta \sigma_{m}$ and the deviatoric stress increment exist.
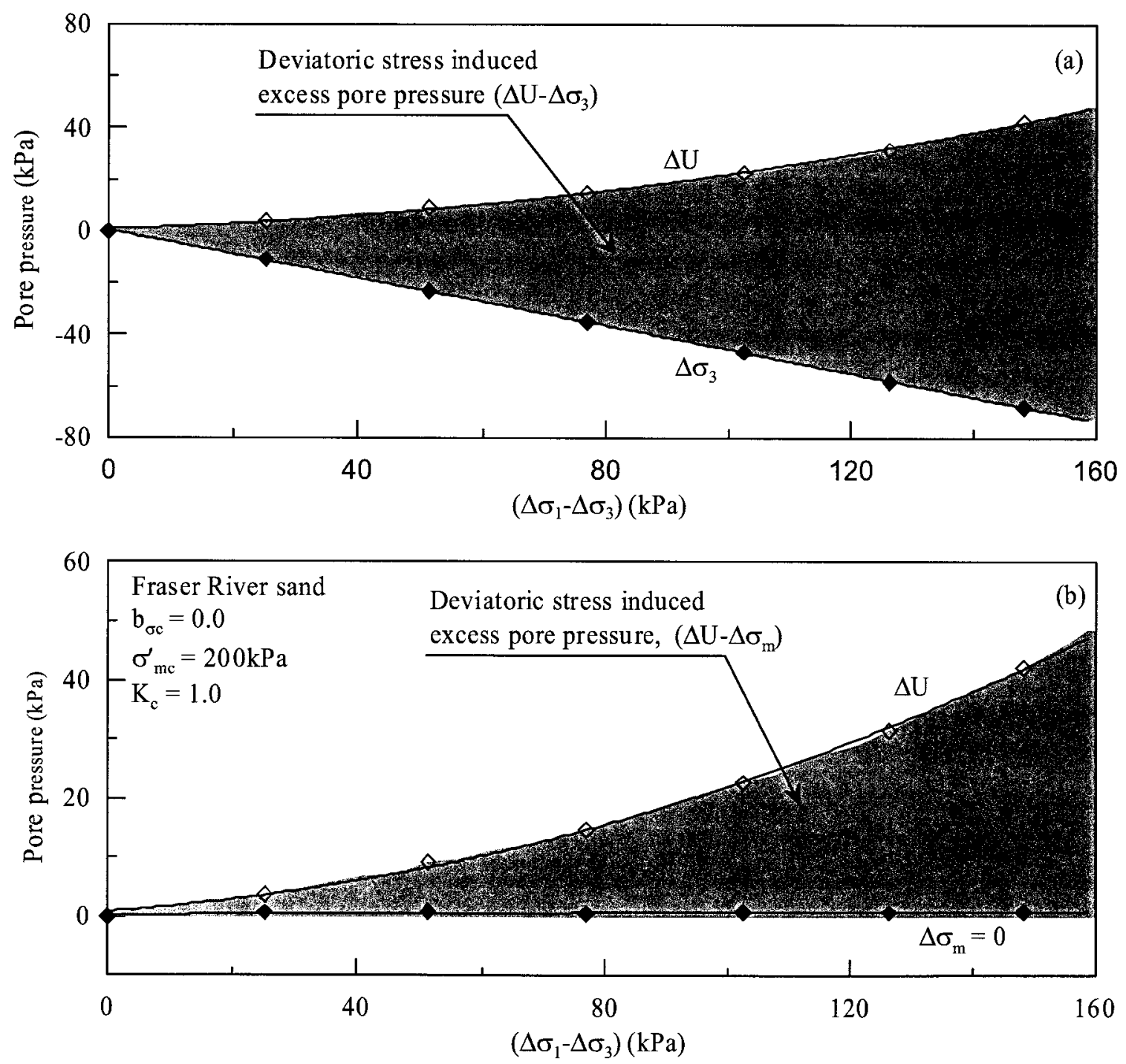

Fig. 7.5 Induced excess pore pressure during anisotropic principal stress increment (direction of $\sigma$ and direction of $\Delta \sigma$ coinciding) 

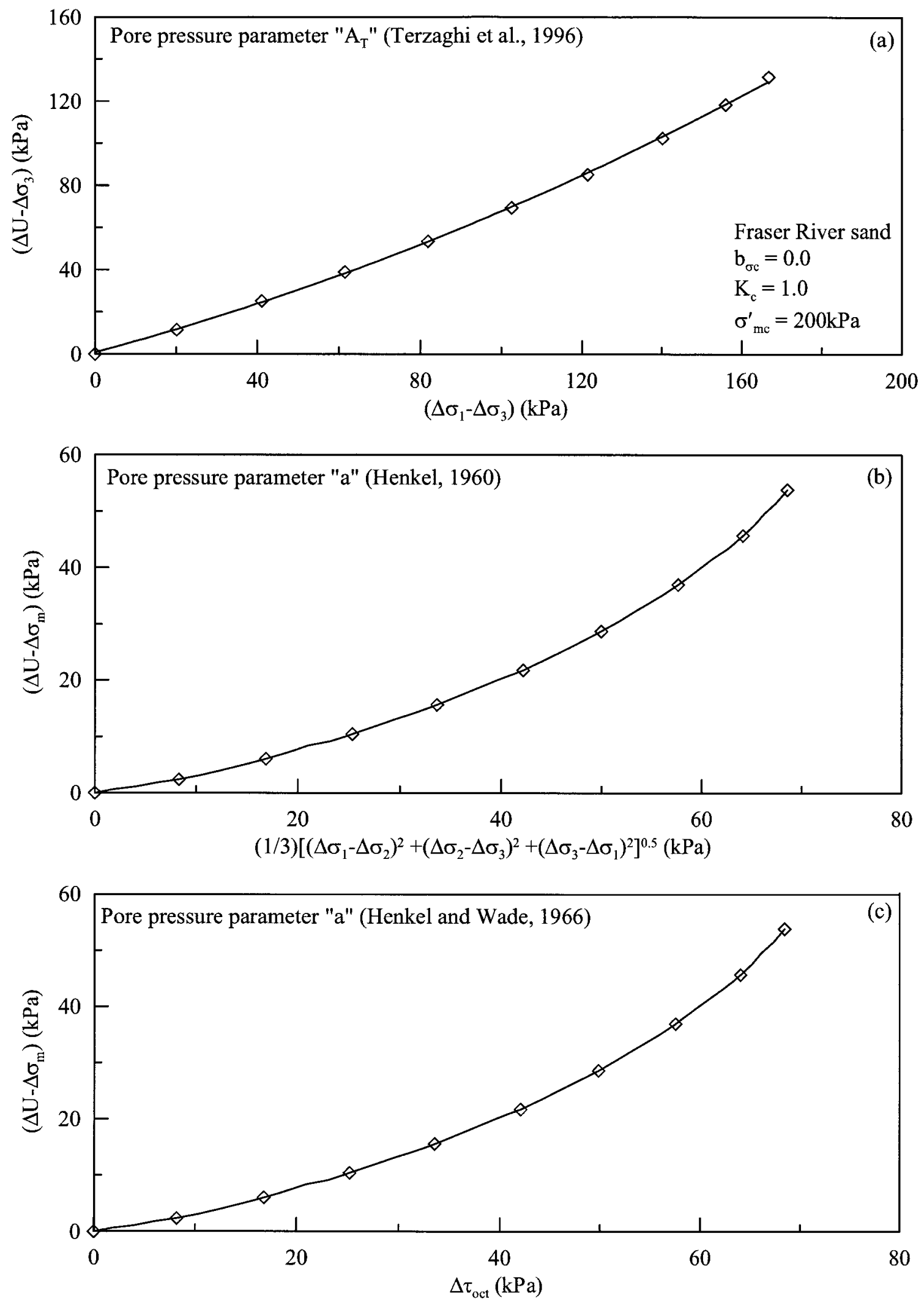

Fig. 7.6 Variation of pore pressure parameters during anisotropic principal stress increment (direction of $\sigma$ and direction of $\Delta \sigma$ coinciding) 
Fig. 7.7 shows the excess pore pressure induced by the hydrostatic stress and deviatoric stress components. The induced excess pore pressure by deviatoric stress components is shaded. These two approaches clearly quantify the different amount of excess pore pressure induced by incremental deviatoric stress.
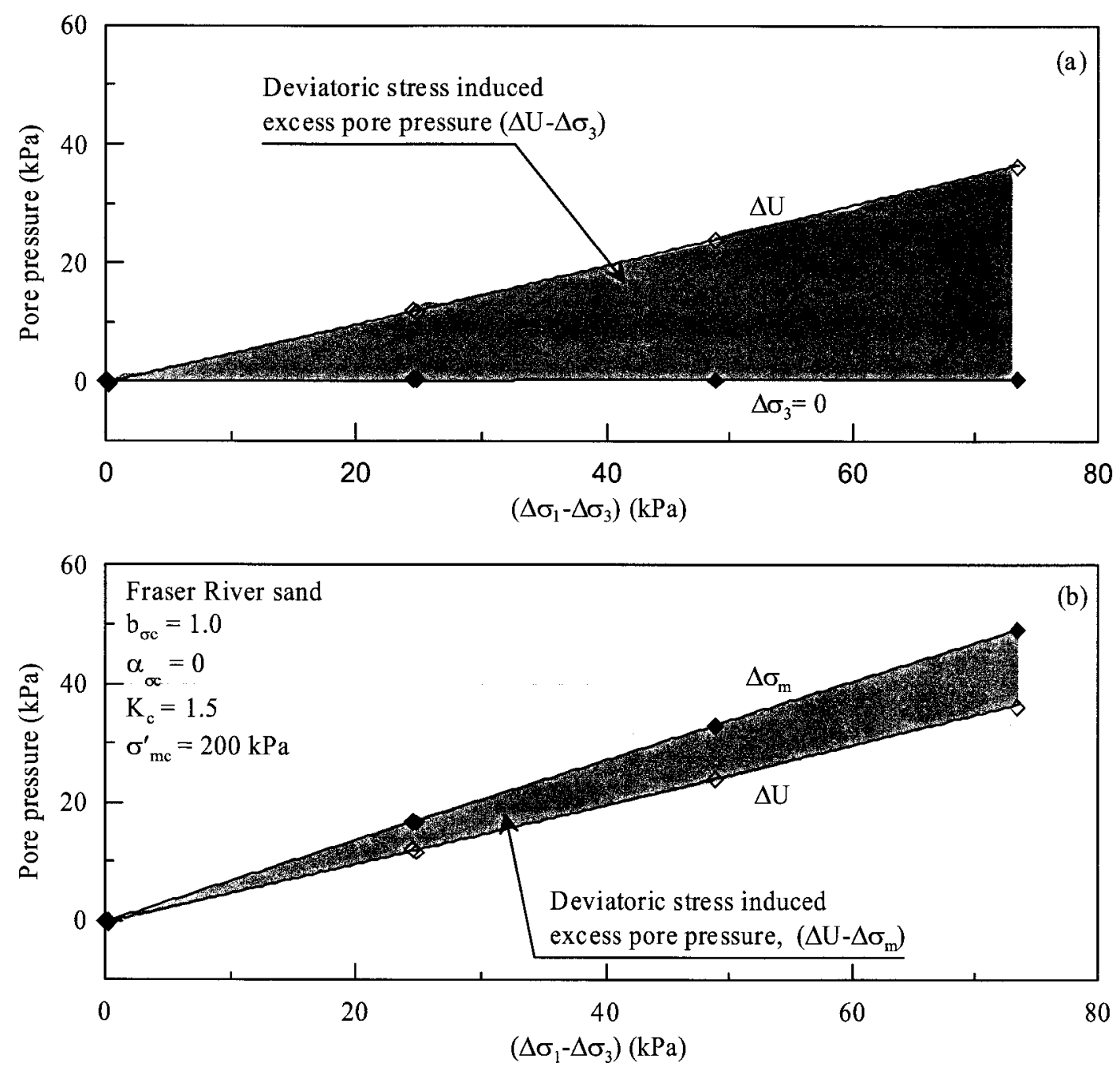

Fig. 7.7 Induced excess pore pressure during anisotropic principal stress increment (direction of $\sigma$ and direction of $\Delta \sigma$ not coincident)

Fig. 7.8a shows the variation of excess pore pressure induced by deviatoric stress against incremental deviatoric stress. The pore pressure parameter $A_{T}$ is positive and slightly increases with increasing $\left(\Delta \sigma_{1}-\Delta \sigma_{3}\right)$. Fig. $7.8 \mathrm{~b}$ shows the variation of the pore pressure parameter $a$ (Eq. 2.4). The parameter $a$ is negative and its magnitude slightly 
decreases with increasing $\left(\Delta \sigma_{1}-\Delta \sigma_{3}\right)$. The parameter plotted along the $\mathrm{x}$-axis (Eq. 7.6) is dependent on $b_{\Delta \sigma}$ and $\left(\Delta \sigma_{1}-\Delta \sigma_{3}\right) . b_{\Delta \sigma}$ remains at 1.0 for each increment $\left(\Delta \sigma_{1}=25 \mathrm{kPa}\right.$, $\Delta \sigma_{2}=25 \mathrm{kPa}$ and $\left.\Delta \sigma_{3}=0 \mathrm{kPa}\right)$.

$\frac{1}{3} \sqrt{\left(\Delta \sigma_{1}-\Delta \sigma_{2}\right)^{2}+\left(\Delta \sigma_{2}-\Delta \sigma_{3}\right)^{2}+\left(\Delta \sigma_{3}-\Delta \sigma_{1}\right)^{2}}=\frac{1}{3} \sqrt{2\left(1-b_{\Delta \sigma}+b_{\Delta \sigma}{ }^{2}\right)\left(\Delta \sigma_{1}-\Delta \sigma_{3}\right)^{2}}$

Eq. 7.6

Fig. $7.8 \mathrm{c}$ shows the variation of the pore pressure parameter $a$ according to Henkel and Wade (1966) definition. As stated in Eq. 2.5, the parameter plotted in $\mathrm{x}$ - axis $\Delta \tau_{o c t}\left(\Delta \tau_{o c t}=\tau_{\text {oct, current }}-\tau_{\text {oct, } \text {,initial }}\right)$ is dependent on $b_{\sigma}$ and deviatoric stress $\left(\sigma_{1}-\sigma_{3}\right)$ that are estimated from principal stresses (Eq. 7.7). In the test, $b_{\sigma}$ started from 1.0 and gradually decreased to zero $\left(\sigma_{3}\right.$ was increased towards the magnitude of $\left.\sigma_{2}\right)$ and the deviatoric stress was maintained constant. $\tau_{o c t}$ reaches peak value at $b_{\sigma}=0.5$. However, $\Delta \tau_{\text {oct }}$ becomes zero when $b_{\sigma}=0.0$ and $1.0\left(\tau_{o c t, \text { current }}=\tau_{o c t, \text { initial }}\right)$. In these cases $\left(b_{\sigma}=0.0\right.$ and 1.0), pore pressure parameter $a$ reaches an infinite value. The variation of Henkel's (1966) parameter $a$ is completely different from Henkel's (1960) parameter $a$ when the direction of principal stress and the direction of principal components of the stress increments are not coinciding.

$$
\tau_{\text {oct }}=\frac{1}{3} \sqrt{\left(\sigma_{1}-\sigma_{2}\right)^{2}+\left(\sigma_{2}-\sigma_{3}\right)^{2}+\left(\sigma_{3}-\sigma_{1}\right)^{2}}=\frac{1}{3} \sqrt{2\left(1-b+b^{2}\right)\left(\sigma_{1}-\sigma_{3}\right)^{2}}
$$

The excess pore pressure equation suggested by Terzaghi et al. (1996) requires the principal components of the stress increments to evaluate the hydrostatic and deviatoric stress components. However, Henkel's excess pore pressure equations do not necessarily require the principal components of stress increments. 

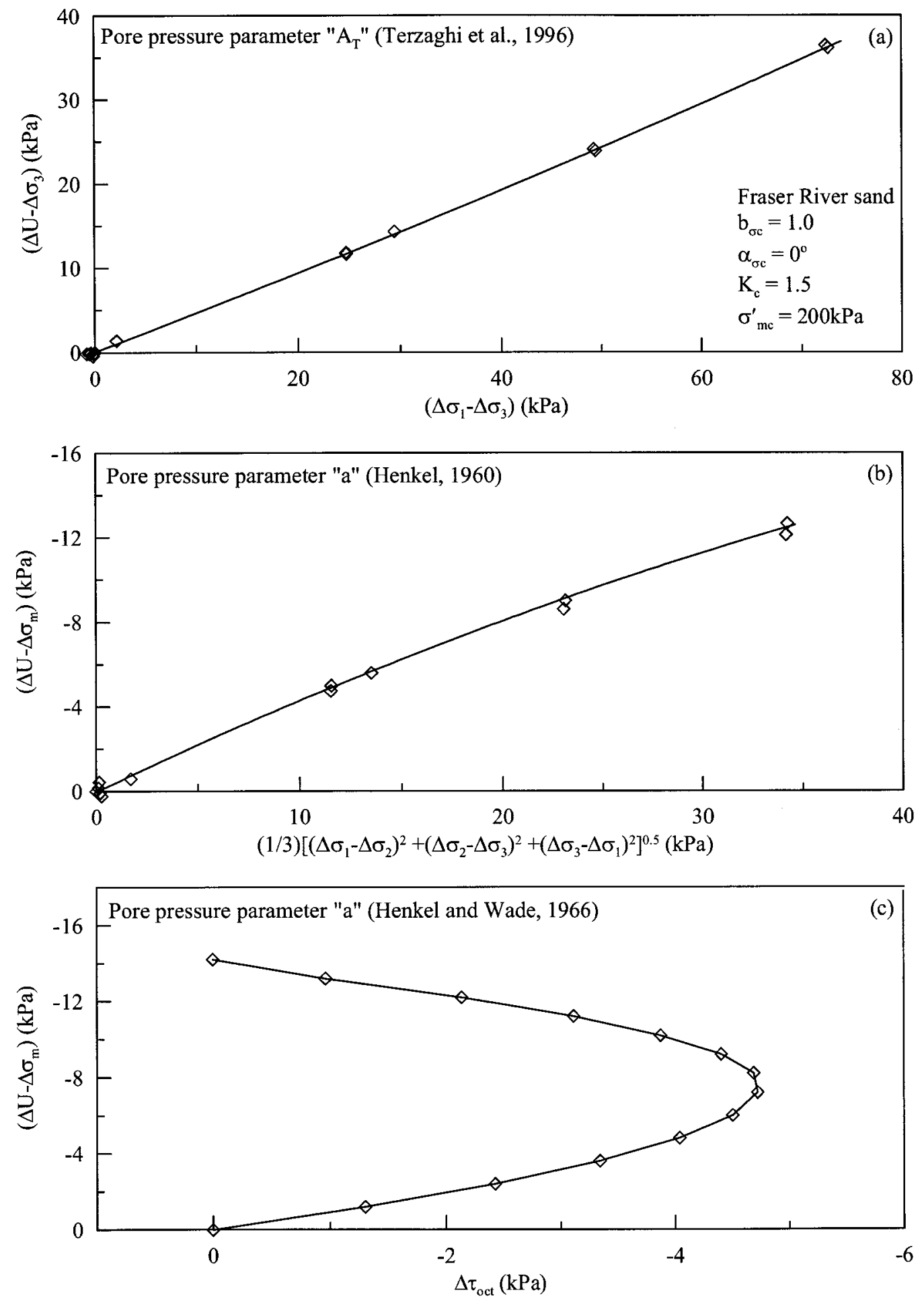

Fig. 7.8 Variation of pore pressure parameters during anisotropic principal stress increment (direction of $\sigma$ and direction of $\Delta \sigma$ not coinciding)

In Henkel's equations, the deviatoric stress component is established by either invariants of the principal stress tensor (square terms of differences in principal stresses) 
or invariants of the incremental principal stress tensor (square terms of differences in incremental principal stresses). In addition, the hydrostatic stress increment is established by adding all of the principal stress increments (first invariant of incremental principal stress tensor).

\subsection{Effect of total stress path on pore pressure generation and sand behaviour}

Two different total stress paths were imposed on loosest deposited Fraser River sand specimens. Total stress path 1 corresponds to typical triaxial compression loading that is represented by $\sigma_{m}=\sigma_{m c}+\left(\sigma_{l}-\sigma_{3}\right) / 3$, in which $\sigma_{m c}$ is the total mean normal stress at the end of consolidation. During the application of total stress path 2 , the total mean normal stress was maintained constant throughout the shearing $\left(\sigma_{m}=\sigma_{m c}\right)$. In these two tests, $b_{\sigma}$ was maintained at zero and the major principal stress was kept in the vertical direction $\left(\alpha_{\sigma}=0^{\circ}\right)$.

As shown in Fig. 7.9, effective stress paths, responses of excess pore pressure in terms of $\left(\Delta U-\Delta \sigma_{3}\right)$ and $\left(\Delta U-\Delta \sigma_{m}\right)$, and stress-strain responses are identical regardless of total stress paths as noted in several earlier studies (Henkel, 1960; Vaid and Campanella, 1974; Bishop and Wesley, 1975; Vaid et al., 1989; Kuerbis, 1989; Uthayakumar, 1995). The excess pore pressure due to the deviatoric stress increment is quite different for hydrostatic stress components of $\Delta \sigma_{3}$ and $\Delta \sigma_{m}$. However, the trends of excess pore pressure generation are exactly same. Both excess pore pressure responses display contractive and dilative pore pressure generation and the maximum pore pressure state (phase transformation) at $1.6 \%$ of $\gamma_{\max }$. The obtained identical responses along different total stress paths confirm that the undrained behaviour of soil is independent of total stress paths at constant $\alpha_{\sigma}$ and $b_{\sigma}$. 

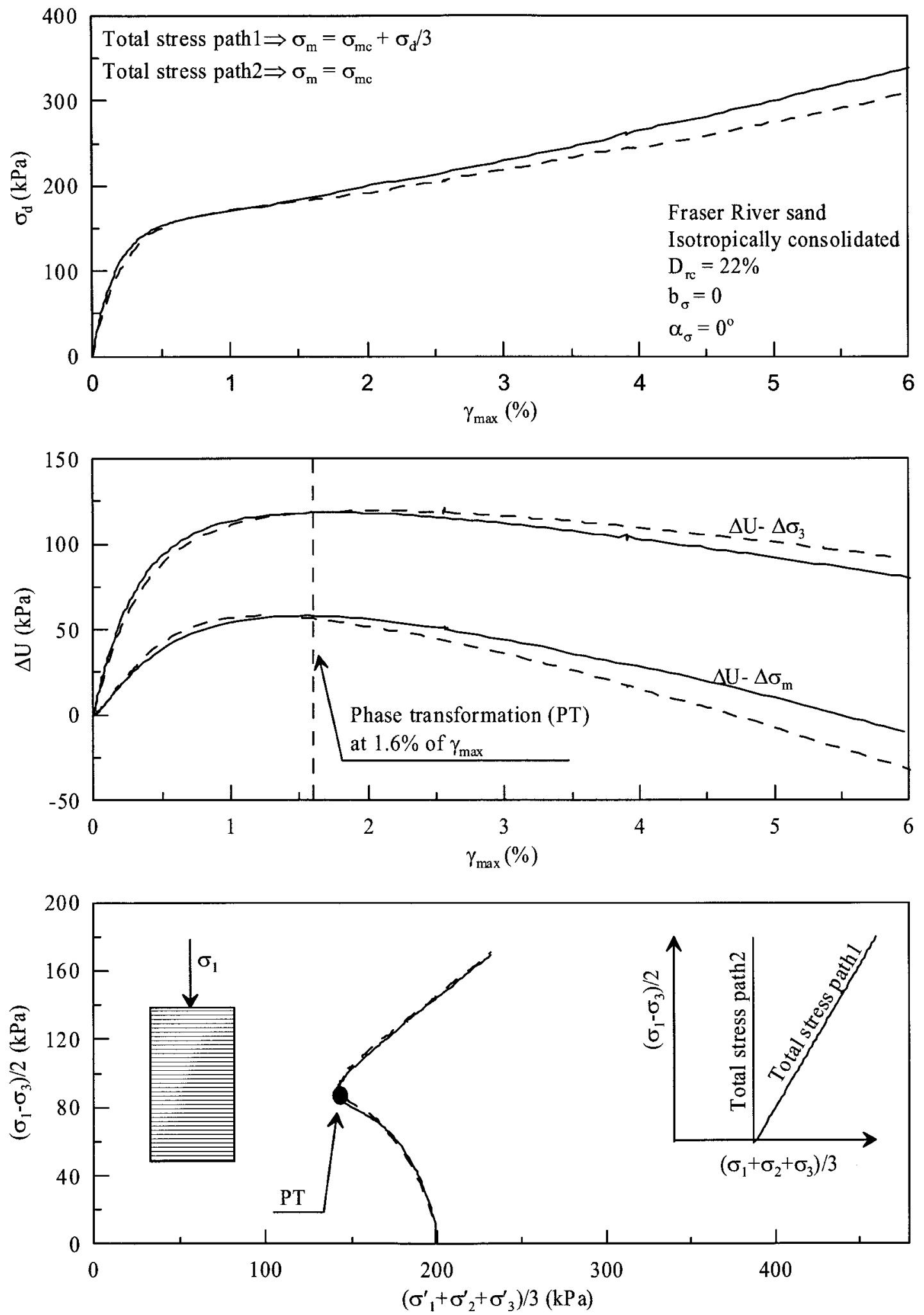

Fig. 7.9 Undrained response of Fraser River sand along different total stress paths $\left(b_{\sigma}=0\right.$ and $\left.\alpha_{\sigma}=0^{\circ}\right)$ 
Fig. 7.10 illustrates the undrained behaviour of Fraser River sand at fixed $\alpha_{\sigma}=$ $90^{\circ}$ and $b_{\sigma}=0.0$. In this set of tests, total stress path 1 was represented by $\sigma_{m}=\sigma_{m c}-\left(\sigma_{I}-\right.$ $\left.\sigma_{3}\right) / 3$ and total stress path 2 was represented by $\sigma_{m}=\sigma_{m c}$. Even though the principal stress direction is not along soil deposition direction as in triaxial compression, the undrained behaviour of sand is independent of total stress paths.

The magnitude of excess pore pressure due to deviatoric stress is quite different for the hydrostatic stress components of $\Delta \sigma_{3}$ and $\Delta \sigma_{m}$. The trend of excess pore pressure generation (contractive and dilative) is exactly same for both approaches. The sand response is initially contractive and slightly dilates after reaching peak pore pressure state (PT). In addition, both approaches display peak pore pressure state at $\gamma_{\max }=2.0 \%$.

\subsection{Effect of $b_{\sigma}$ on pore pressure generation}

Two different tests were performed on Fraser River sand along a total stress path $\left(\sigma_{m}=\sigma_{m c}\right)$. Specimens were isotropically consolidated to $\sigma_{m c}^{\prime}=200 \mathrm{kPa}$ and $D_{r c}=22 \%$. During the shearing, $b_{\sigma}$ was maintained 0.0 and 0.4 and $\alpha_{\sigma}$ was kept along deposition direction $\left(\alpha_{\sigma}=0^{\circ}\right)$. The test at $b_{\sigma}=0.0$ and $\alpha_{\sigma}=0^{\circ}$ imposes the same conditions as typical triaxial compression test except total stress path.

According to Eqs. 7.1 and 7.2, the deviatoric stress tensor is a function of $\Delta \sigma_{d}$ and $b_{\Delta \sigma}$ that is equal to $b_{\sigma}$ for these tests. Previous studies in the literature (Yoshimine et al., 1998; Uthayakumar and Vaid, 1998) and Chapter 5 have clearly shown that stress-strain response is dependent on $b_{\sigma}$. 

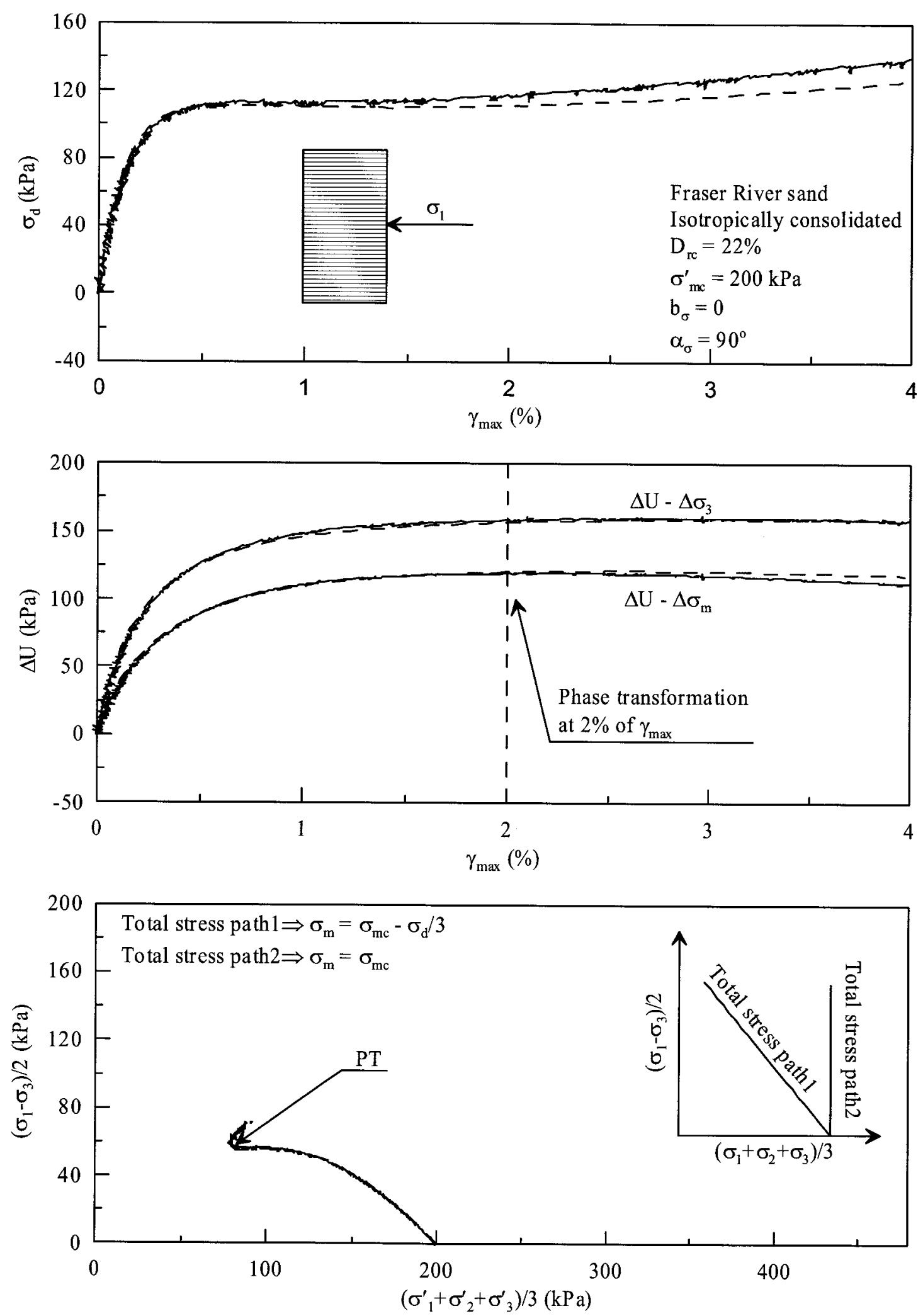

Fig. 7.10 Undrained response of Fraser River sand along different total stress paths ( $b_{\sigma}=0$ and $\alpha_{\sigma}=90^{\circ}$ ) 
Fig. 7.11 shows the variation of the excess pore pressure induced by the deviatoric stress increment. The amount of excess pore pressure generated due to the deviatoric stress increment is different for both approaches (hydrostatic stress components of $\Delta \sigma_{3}$ and $\Delta \sigma_{m}$ ). However, the trend of excess pore pressure responses (portion of contractive and dilative) is consistent. Peak excess pore pressure states can be clearly located from excess pore pressure response and stress states corresponding to that state coincide with stress state identified in stress paths as phase transformation. Regardless of hydrostatic stress components, deviatoric stress induced excess pore pressure is dependent on $b_{\sigma}$ at fixed $\alpha_{\sigma}$.

Fig. 7.12 shows the variation of deviatoric stress induced excess pore pressure against deviatoric component at different $b_{\sigma}$ of 0.0 and 0.4 . This plot clearly suggests that the pore pressure parameters are dependent on $b_{\sigma}$. The pore pressure parameters take a high value at phase transformation. As suggested by Henkel (1960) and Henkel and Wade (1966), the formulations to quantify the excess pore pressure are quite different. However, the variations of parameter $a$ are the same at given $b_{\sigma}$ for this set of tests because deviatoric components of those pore pressure equations (Eqs. 2.4 and 2.5) have the same variation.

\subsection{Effect of $\alpha_{\sigma}$ on pore pressure generation}

Two tests were performed on Fraser River sand along a same total stress path $\left(\sigma_{m}\right.$ $\left.=\sigma_{m c}\right)$. The specimens were isotropically consolidated to $\sigma_{m c}^{\prime}=200$ and $D_{r c}=22 \%$ and were sheared at $b_{\sigma}=0.4$ and $\alpha_{\sigma}=0^{\circ}$ and $45^{\circ}$. 

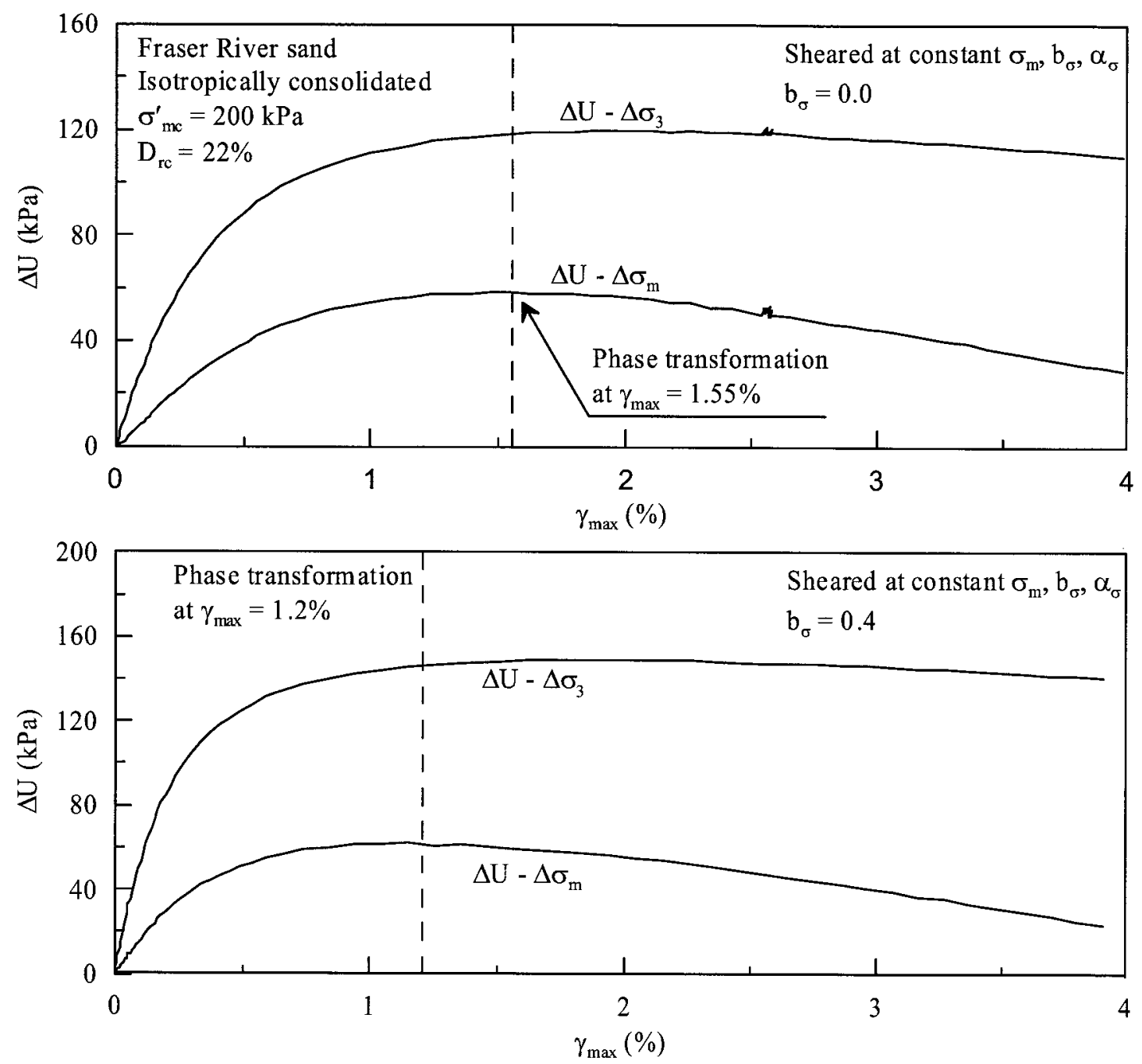

Fig. 7.11 Deviatoric stress induced excess pore pressure at $b_{\sigma}=0.0$ and 0.4 , and $\alpha_{\sigma}$ $=0^{\circ}$

Fig. 7.13 shows the variation of excess pore pressure induced by deviatoric stress.

The amount of excess pore pressure predicted by both approaches is different. However, the trend of the excess pore pressure responses is consistent. The excess pore pressure responses demonstrate that the sand initially deforms contractive and subsequently dilative after reaching peak pore pressure. The peak excess pore pressure states can be clearly located from the excess pore pressure responses. The stress states corresponding to the peak pore pressure state coincide with the stress state identified in the stress paths as phase transformation. The difference in deviatoric stress induced excess pore pressure 
responses clearly shows that excess pore pressure generation is dependent on principal stress direction.
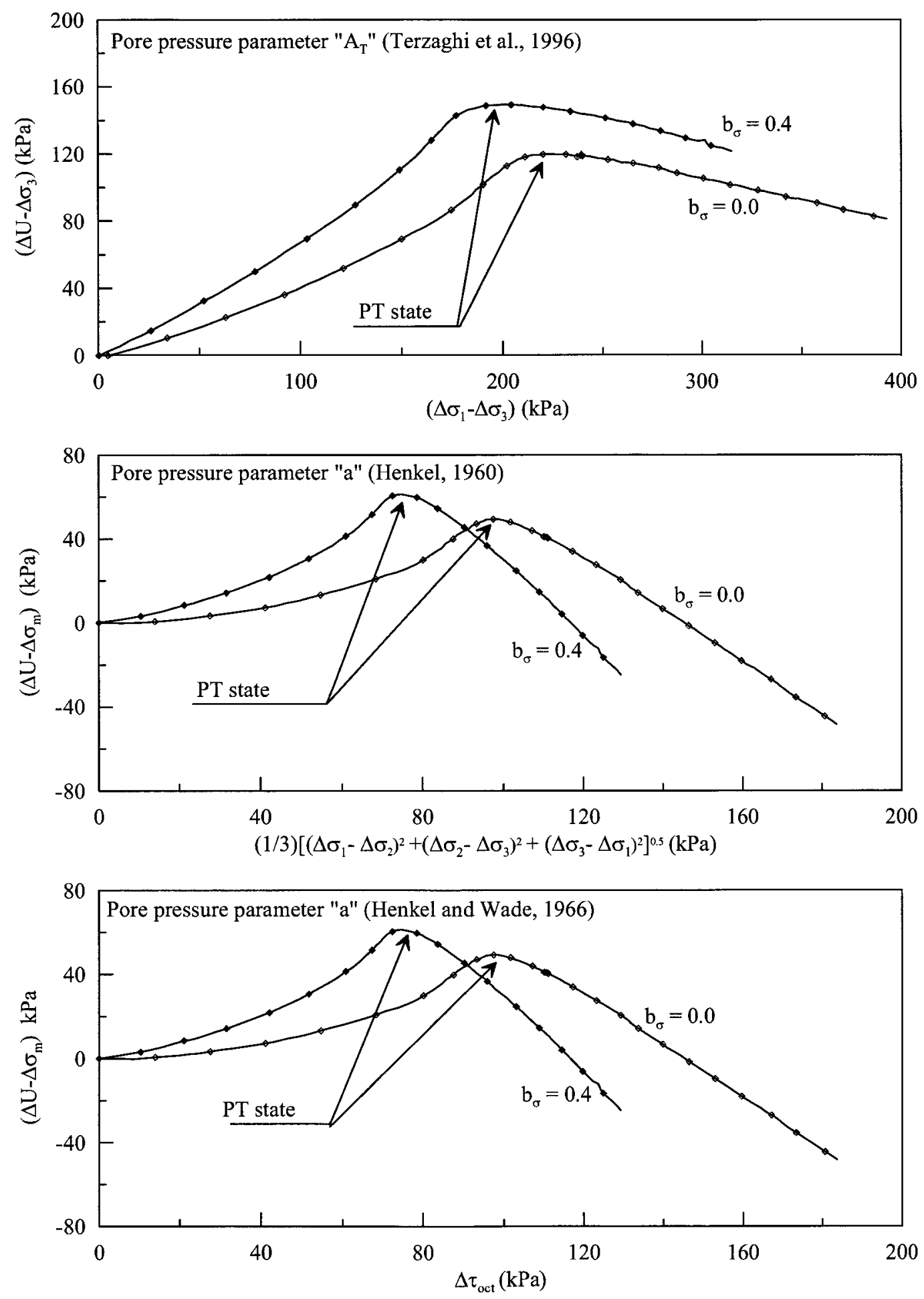

Fig. 7.12 Variation of pore pressure parameters at fixed $b_{\sigma}=0.0$ and 0.4 , and $\alpha_{\sigma}=$ $0^{\circ}$ 
Fig. 7.14 shows the variation of deviatoric stress induced excess pore pressure against deviatoric component at different $\alpha_{\sigma}$. The pore pressure parameters reach high value at phase transformation. After that, it reduces during reduction of excess pore pressure. As noted in fixed $b_{\sigma}$ tests, the variation of Henkel's pore pressure parameters $a$ as suggested in 1960 and 1966 are identical at a given $\alpha_{\sigma}$ due to the same variation of the deviatoric components of those equations.
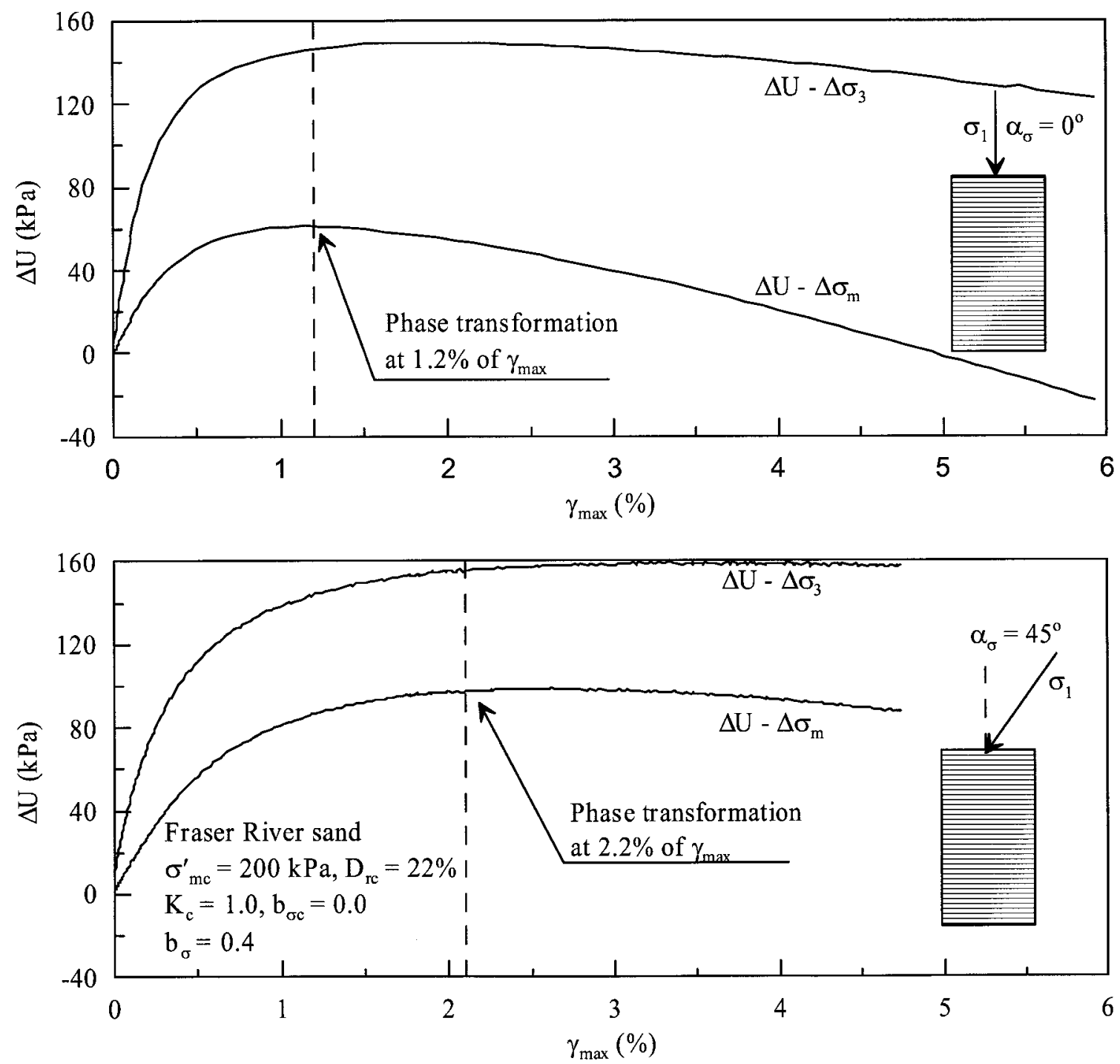

Fig. 7.13 Deviatoric stress induced excess pore pressure at $b_{\sigma}=0.4$ and $\alpha_{\sigma}=0^{\circ}$, and $b_{\sigma}=0.4$ and $\alpha_{\sigma}=45^{\circ}$ 

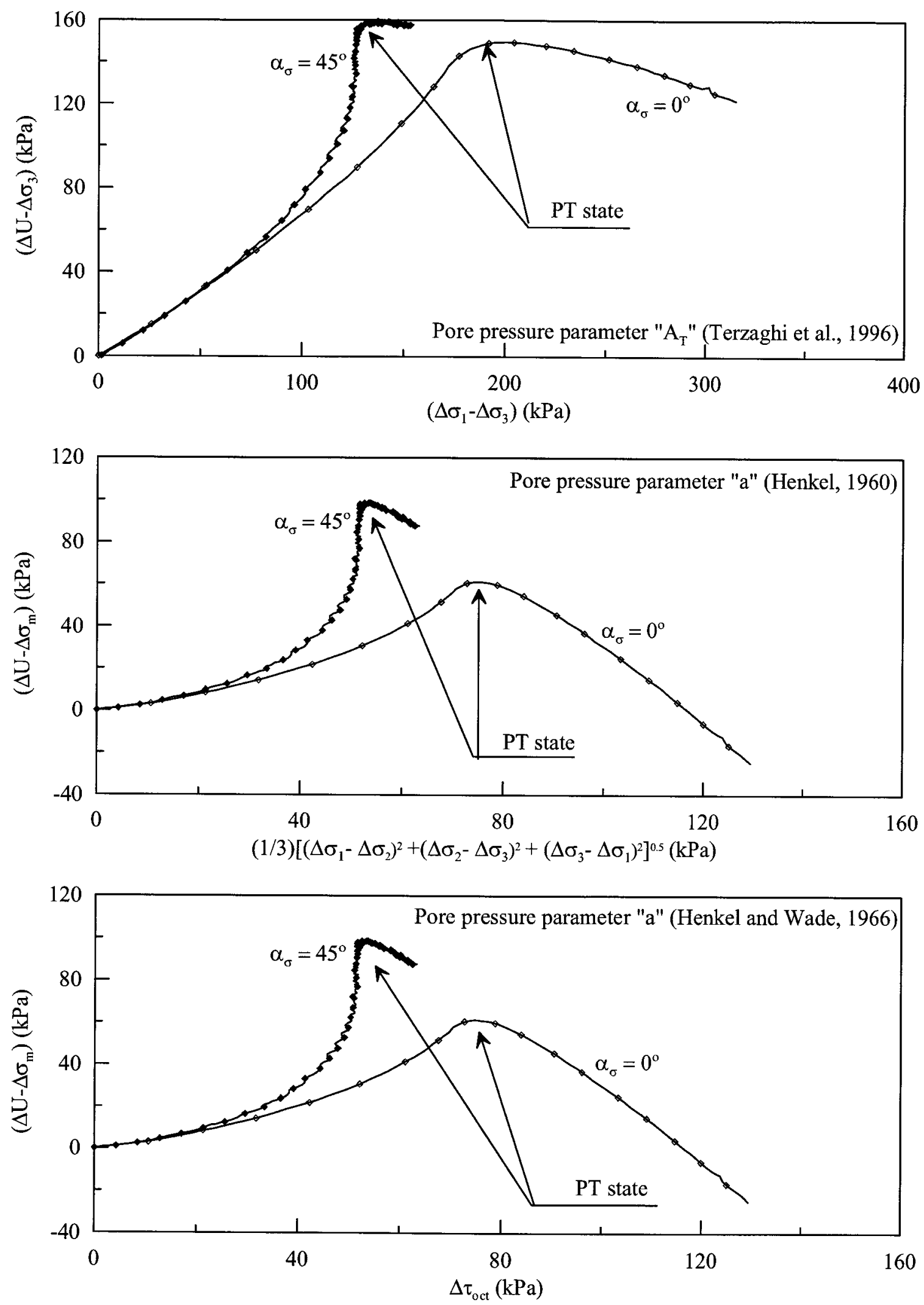

Fig. 7.14 Effect of $\alpha_{\sigma}$ on pore pressure parameters at fixed $b_{\sigma}=0.4$ 


\subsection{Effect of change of $b_{\sigma}$ on pore pressure generation and sand behaviour}

Literature and the tests reported in previous sections indicate that the undrained response of soil is independent of total stress paths. These finding were based on tests at constant $b_{\sigma}$ and $\alpha_{\sigma}$ and thus are limited to such simple loading paths. A series of tests was conducted to assess the effect of total stress path on the undrained behavior under generalized loading conditions. $b_{\sigma}$ was changed in a systematic manner in each total stress path to study the effect of the change of $b_{\sigma}$ on sand behaviour at fixed $\alpha_{\sigma}$. In the

first series of tests, $b_{\sigma}$ was changed linearly with maximum shear strain $\gamma_{\max }$ and a more drastic change was imposed in the second series of tests.

\subsubsection{Linear variation of $b_{\sigma}$ against $\gamma_{\max }$}

$b_{\sigma}$ was changed linearly from 0.0 at the beginning of the test, to 0.5 at $\gamma_{\max }=5 \%$. The specimen was isotropically consolidated to $\sigma_{m c}^{\prime}=200 \mathrm{kPa}$ and $D_{r c}=22 \%$. The total mean normal stress at the end of consolidation was $450 \mathrm{kPa}$ and a set of linear total stress paths was imposed during shearing (Fig. 7.15). The principal stress directions remained fixed during shearing $\left(\alpha_{\sigma}=0^{\circ}\right)$.

Fig. 7.16 shows the stress-strain response, excess pore pressure response and the effective stress paths of Fraser River sand during the application of the total stress paths. The sand experiences strain-hardening behaviour. It can be noted that stress-strain responses and effective stress paths are essentially identical regardless of the change in $b_{\sigma}$ and the total stress paths.

A significant difference in the induced excess pore pressure is noted because of the different total stress paths. The induced excess pore pressure includes both hydrostatic and deviatoric stress induced components and changes according to the 
magnitude of the total stress increment. The application of the total stress path of $\sigma_{m}=$ $\sigma_{m c}+\sigma_{d}$ that imposes a highest increment of total mean normal stress for a particular increment of deviatoric stress, and results in highest excess pore pressure. The total stress path of $\sigma_{m}=\sigma_{m c}-\sigma_{d} / 6$ that applies a decrement of total mean normal stress for a particular increment of deviatoric stress results in lowest excess pore pressure. Excess pore pressure response is not a good indicator of whether the sand is dilative or contractive, since it is hard to determine the location of maximum pore pressure state (PT) is impossible to detect in some total stress paths. However, the effective stress paths clearly display PT state as a knee shaped bend closer to the maximum obliquity line.

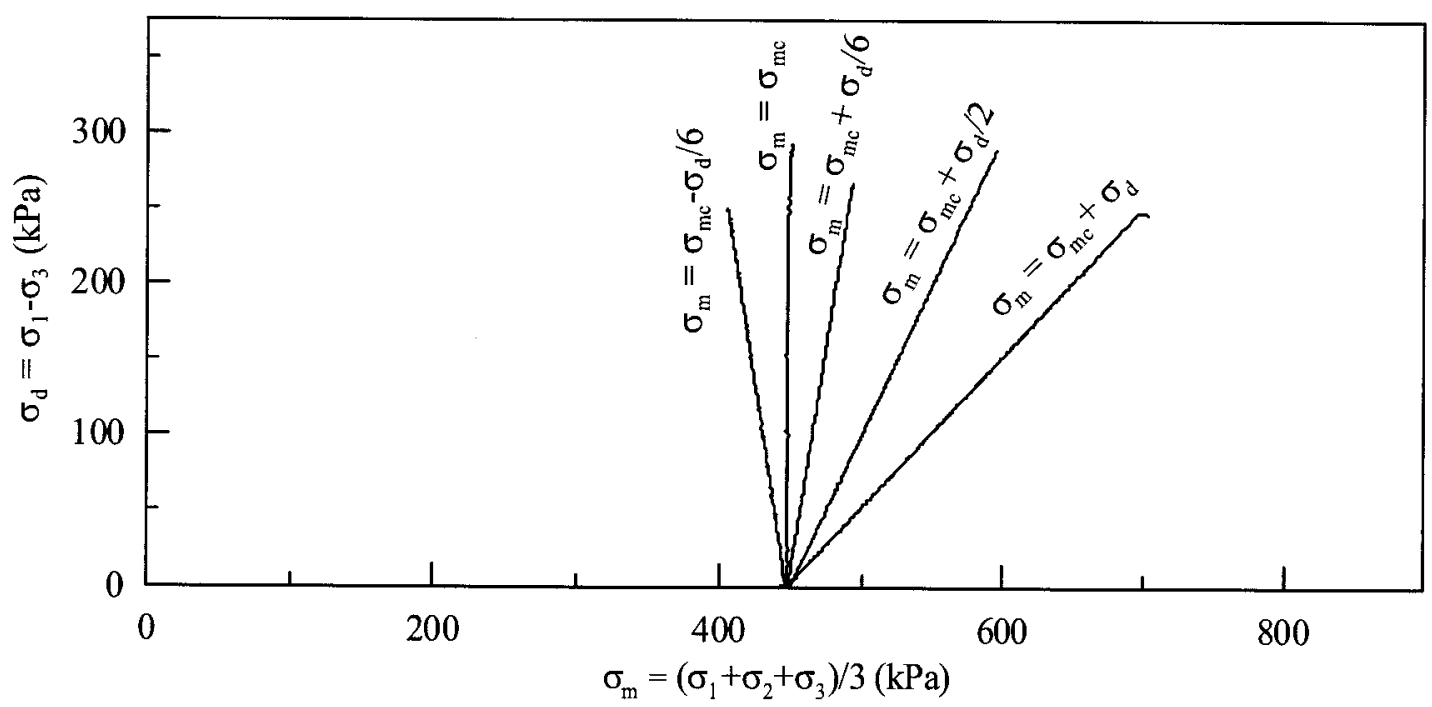

Fig. 7.15 Imposed total stress paths during undrained loading

The effective stress paths in $\sigma_{m}^{\prime}$ and $\sigma_{d}$ space are identical regardless of the total stress paths imposed. The mobilized friction angle at PT is $34.2^{\circ}$ and it narrowly deviates by about $0.4^{\circ}$ from previous studies (Logeswaran, 2005) on the same batch of Fraser River sand under triaxial loading. 

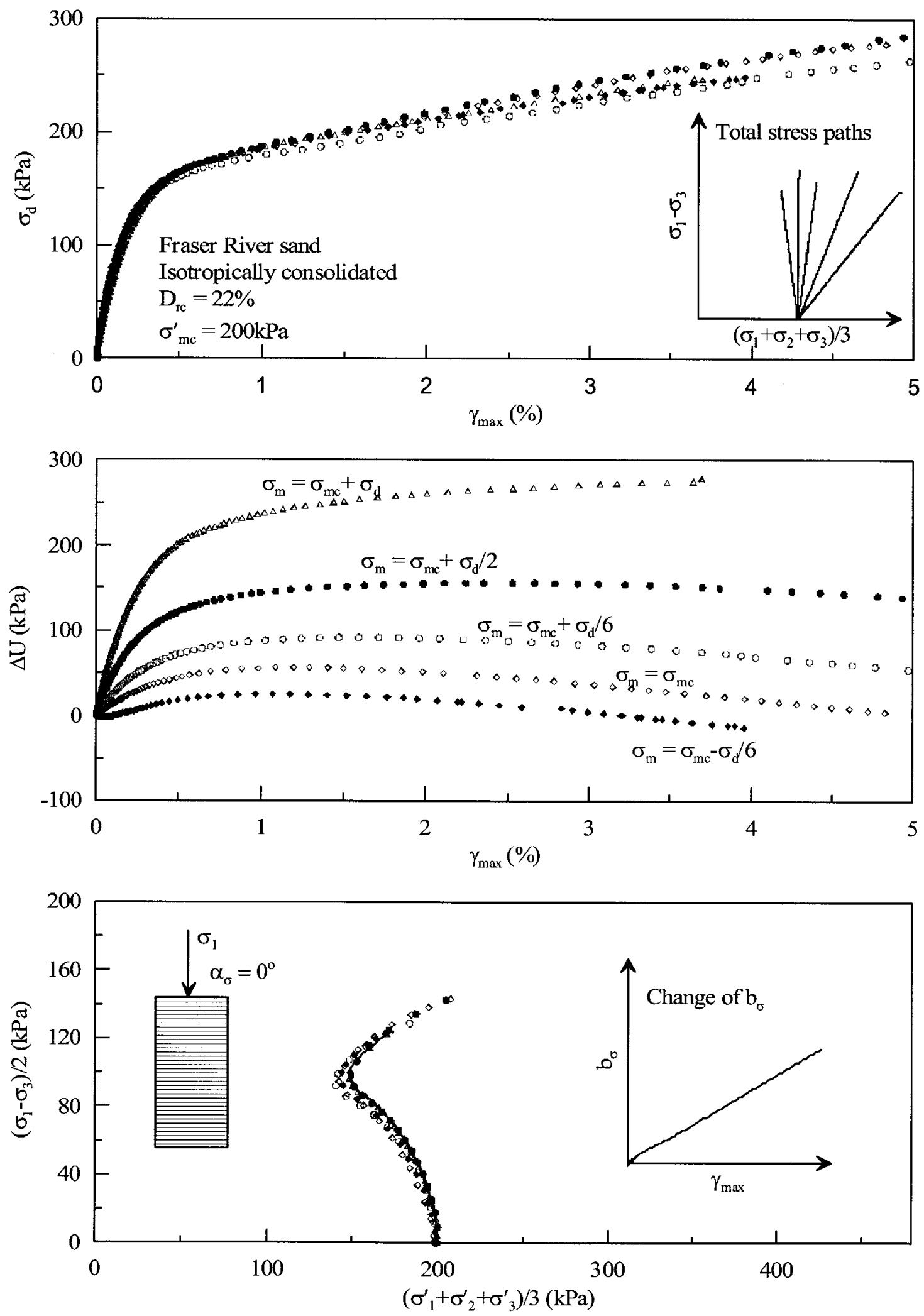

Fig. 7.16 Undrained response of Fraser River sand during a systematic change of $b_{\sigma}$ with application of different total stress paths 
Fig. 7.17 shows the variation of principal strains induced against maximum shear strain $\gamma_{\max }$ along each total stress path. The variations of the three principal strains $\left(\varepsilon_{1}, \varepsilon_{2}\right.$ and $\varepsilon_{3}$ ) are identical for each total stress path regardless of the change in $b_{\sigma}$. The major principal strain $\varepsilon_{l}$, which is along the deposition direction and independently controlled, increases monotonically with increasing $\gamma_{z}$. The minor principal strain $\varepsilon_{3}$ continuously reduces with increasing $\gamma_{z}$. However, the intermediate principal strain $\varepsilon_{2}$ initially decreases until $b_{\sigma}$ of about $0.25\left(\gamma_{\max }\right.$ of $\left.2.5 \%\right)$. After this stage, $\varepsilon_{2}$ gradually increases and becomes approximately zero when $b_{\sigma}$ is 0.42 and $\gamma_{\max }=4.2 \%$. Subsequently, $\varepsilon_{2}$ becomes positive. The observations on $\varepsilon_{2}$ are consistent with undrained tests performed on Fraser River sand at fixed $b_{\sigma}$ (Fig. 5.2). The sand exhibits a negative value of $\varepsilon_{2}$ when $b_{\sigma}$ is below 0.4. The sand experiences a positive value of $\varepsilon_{2}$ when $b_{\sigma}$ is above 0.4 . In addition, undrained test at a fixed $b_{\sigma}$ of 0.4 exhibits plane strain deformation $\left(\varepsilon_{2} \approx 0\right)$.

Fig. 7.18 shows the variation of induced excess pore pressure due to deviatoric stress during the application of total stress paths. A unique response of excess pore pressure induced by the deviatoric stress increment $\Delta U-\Delta \sigma_{3}$ (Fig. 7.18a) exists for all total stress paths regardless of change in $b_{\sigma}$. According to that response, $\Delta U-\Delta \sigma_{3}$ continuously increases throughout shearing, and reaches an asymptotic maximum value at about $140 \mathrm{kPa}$. There is no reduction in shear induced pore pressure However, effective stress paths shown in Fig. 7.16 clearly display PT state that occurred at the effective stress state of $\sigma_{m}^{\prime}=142.5 \mathrm{kPa}$ and $\sigma_{d}=187 \mathrm{kPa}$, and at $\gamma_{\max }=1.34 \%$. 

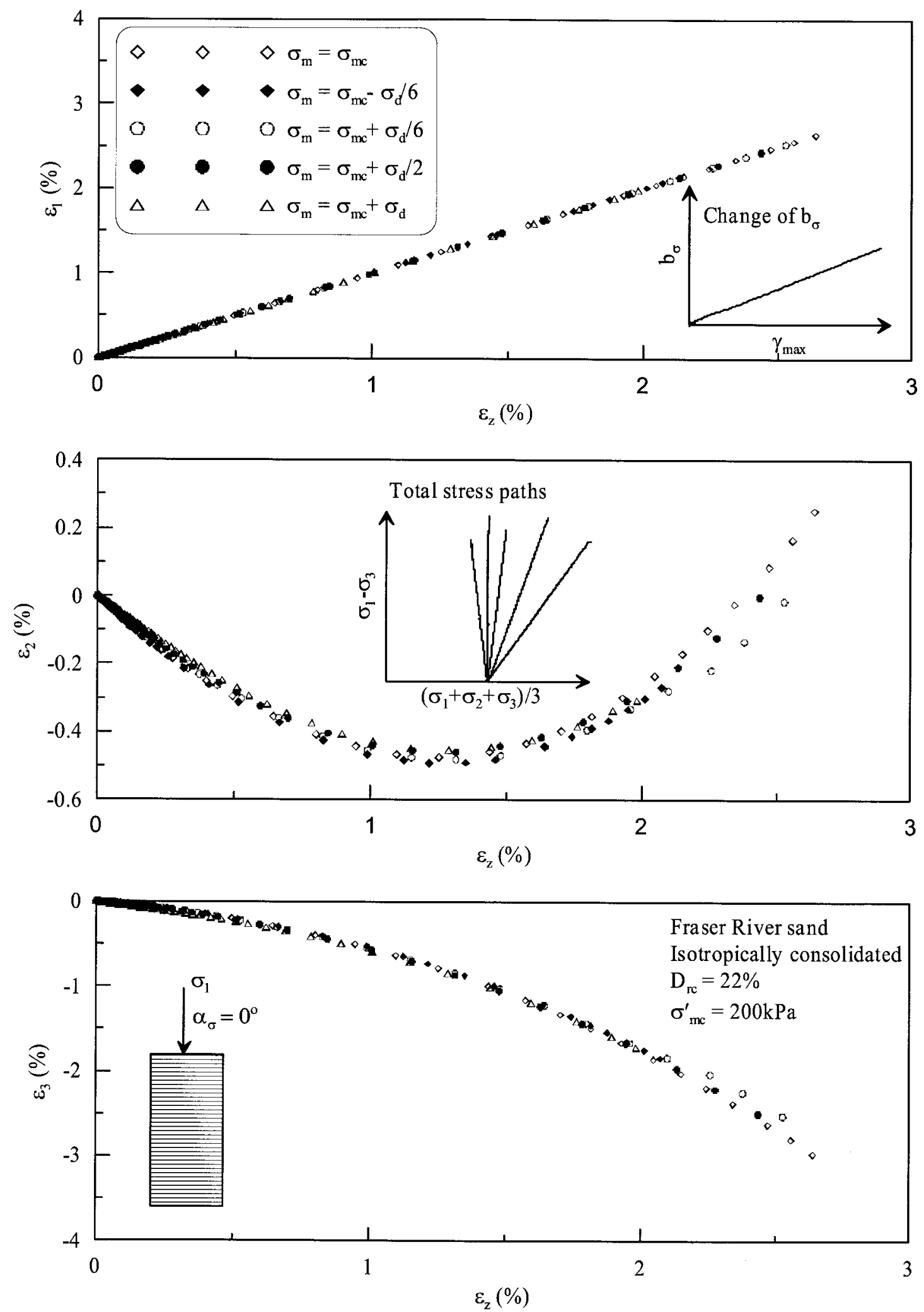

Fig. 7.17 Variation of principal strains during application of different total stress paths with change of $b_{\sigma}$ 

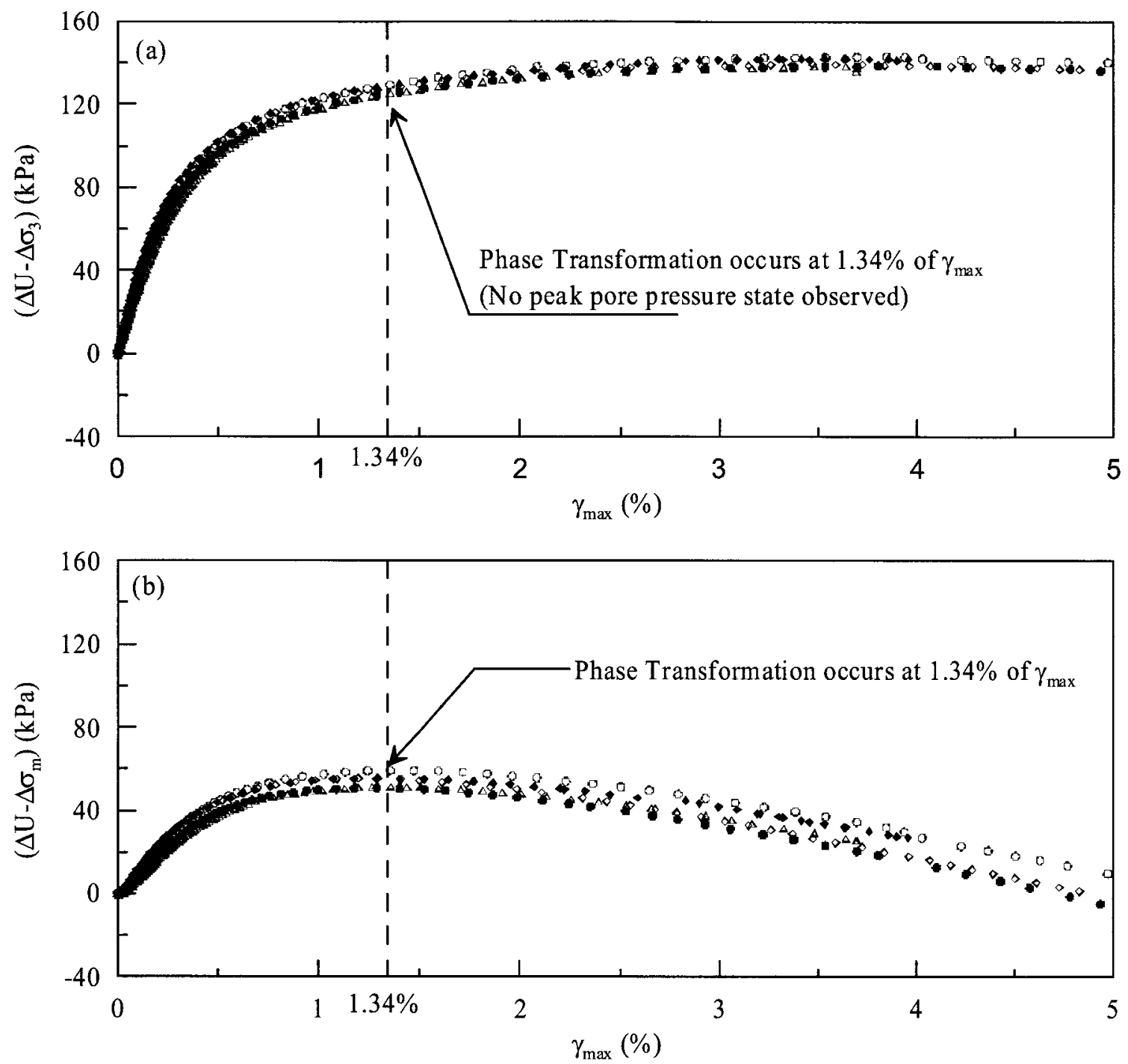

Fig. 7.18 Excess pore pressure induced by deviatoric stress during application of different total stress paths with change of $b_{\sigma}$

The response of excess pore pressure induced by deviatoric stress $\Delta U-\Delta \sigma_{m}$ (Fig. 7.18b) clearly displays contractive and dilative behaviour of Fraser River sand. The maximum value of $\Delta U-\Delta \sigma_{m}$ is realized at $1.34 \%$ of $\gamma_{\max }$ and the stress state at maximum $\Delta U-\Delta \sigma_{m}$ coincides with the effective stress state of PT as noted effective stress paths (Fig. 7.16).

Even though both approaches result in different responses of excess pore pressure induced by deviatoric stress component, responses are independent of total stress paths 
and change in $b_{\sigma}$. The excess pore pressure $\left(\Delta U-\Delta \sigma_{3}\right)$ does not capture the dilation, and in that sense, it misinterprets the actual behaviour of the sand. However, the excess pore pressure $\left(\Delta U-\Delta \sigma_{m}\right)$ correctly displays the trend of the sand deformation according to the effective stress paths (dilative, contractive and PT state).

Fig. 7.19 shows the variation of the pore pressure parameters during change in $b_{\sigma}$ along different total stress paths. It can be noted that the pore pressure parameters are independent of total stress paths, even though $b_{\sigma}$ is changed. In each case, the pore pressure parameters continuously increase until reaching PT state. Subsequently, it reduces. However, Henkel's pore pressure parameters $a$ reduces drastically beyond PT state. The variations of both Henkel's pore pressure parameters $a$ are same as noted earlier.

\subsubsection{Sinusoidal variation of $b_{\sigma}$ against $\gamma_{\max }$}

In this set of tests, $b_{\sigma}$ was changed as a sinusoidal function of $\gamma_{\max }$ (Fig. 7.20). Within $1 \%$ of $\gamma_{\max }, b_{\sigma}$ was increased from 0 to 1.0 and it was decreased to zero. The rate of change of $b_{\sigma}$ is drastic compared to the previous series, and may clearly reflect the effects of $b_{\sigma}$ change during application of total stress paths.

\subsubsection{Undrained response}

Fraser River sand specimens were isotropically consolidated to $\sigma_{m c}^{\prime}=200 \mathrm{kPa}$ and $D_{r c}=22 \%$. During shearing, $\alpha_{\sigma}$ was kept at $60^{\circ}$ to the deposition direction $\left(\alpha_{\sigma}=\right.$ $60^{\circ}$ ). Fig. 7.21 shows response of the sand during the application of total stress paths with change in $b_{\sigma}$. The effective stress-strain responses are essentially identical. This observation again demonstrates that effective stress-strain responses are independent of total stress paths regardless of changes in $b_{\sigma}$. 

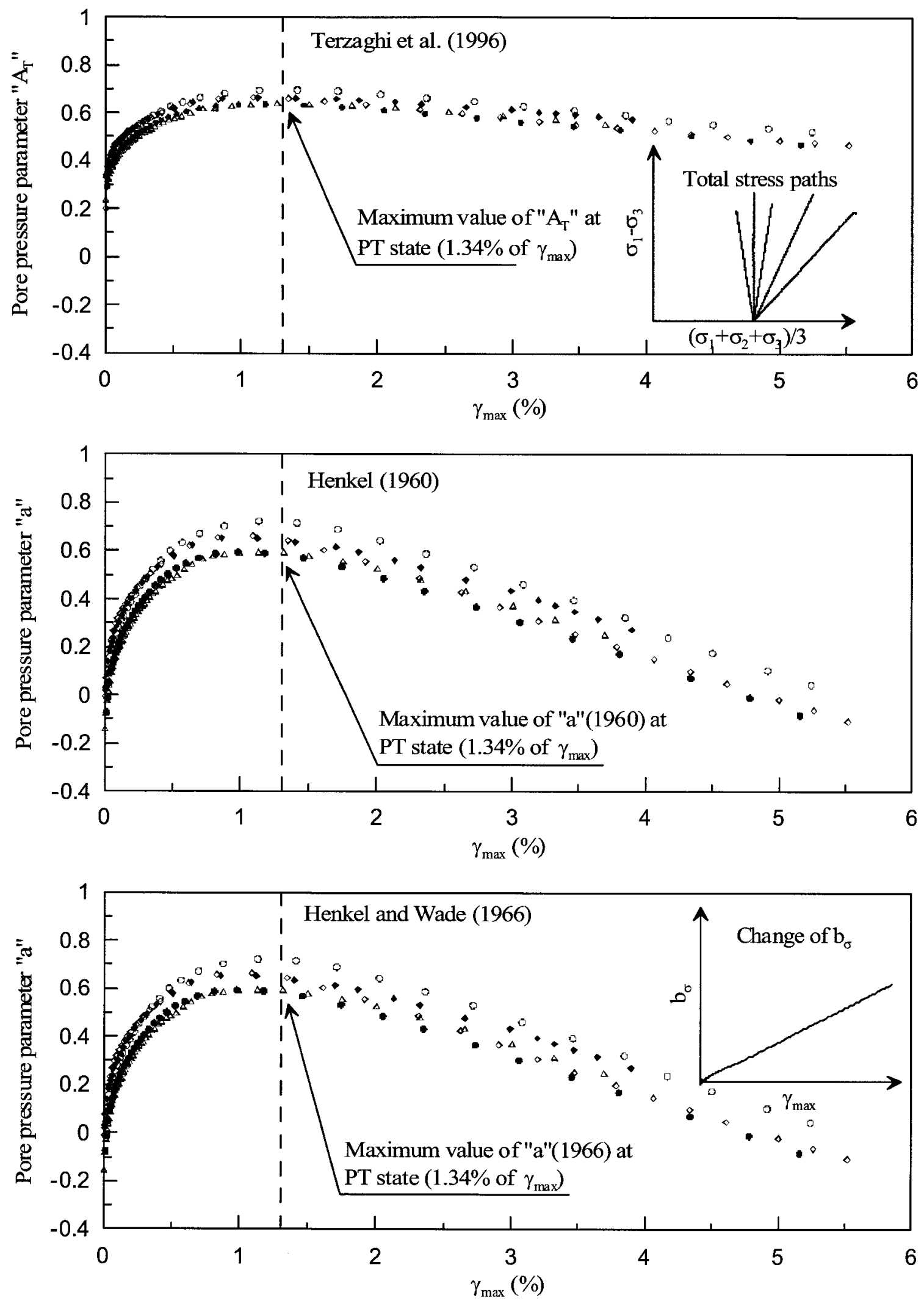

Fig. 7.19 Variation of pore pressure parameters during application of different total stress paths with change of $b_{\sigma}$ 


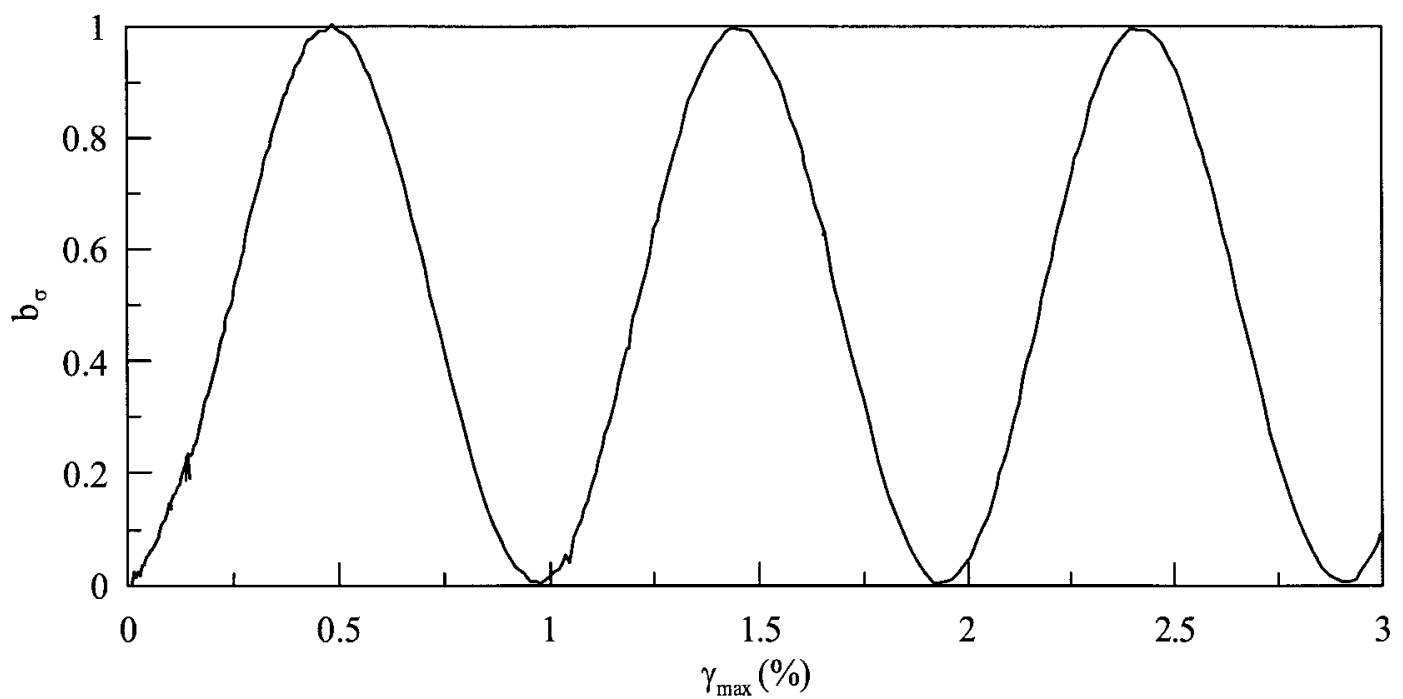

Fig. 7.20 Variation of $b_{\sigma}$ during application of total stress paths

The excess pore pressure changes according to the total stress paths imposed. The application of total stress path $\sigma_{m}=\sigma_{m c}+\sigma_{d} / 6$ results in higher excess pore pressure and $\sigma_{m}=\sigma_{m c}-\sigma_{d} / 6$ results in lower excess pore pressure. The effective stress paths are essentially identical regardless of the total stress paths imposed.

Fig. 7.22 shows the variation of principal strains during change of $b_{\sigma}$ along different total stress paths. The responses of principal strains are identical regardless of the total stress paths. The major principal strain $\varepsilon_{l}$ increases and the minor principal strain $\varepsilon_{3}$ decreases continuously with increasing $\gamma_{z \theta}$. However, the intermediate principal strain $\varepsilon_{2}$ shows peaks and troughs depending on $b_{\sigma} . \varepsilon_{2}$ at the peaks increases and $\varepsilon_{2}$ at the troughs decreases with the increasing number of rotations of $b_{\sigma}$. This observation may be due to the strain softening behaviour during cyclic change of $b_{\sigma}$. The positive value of $\varepsilon_{2}$ is observed when $b_{\sigma}$ is approximately above 0.45 and the negative value of $\varepsilon_{2}$ is noted when $b_{\sigma}$ is below 0.45 . This observation is consistent with the observation made on the static undrained test at a fixed $b_{\sigma}$ (Fig. 5.2). 

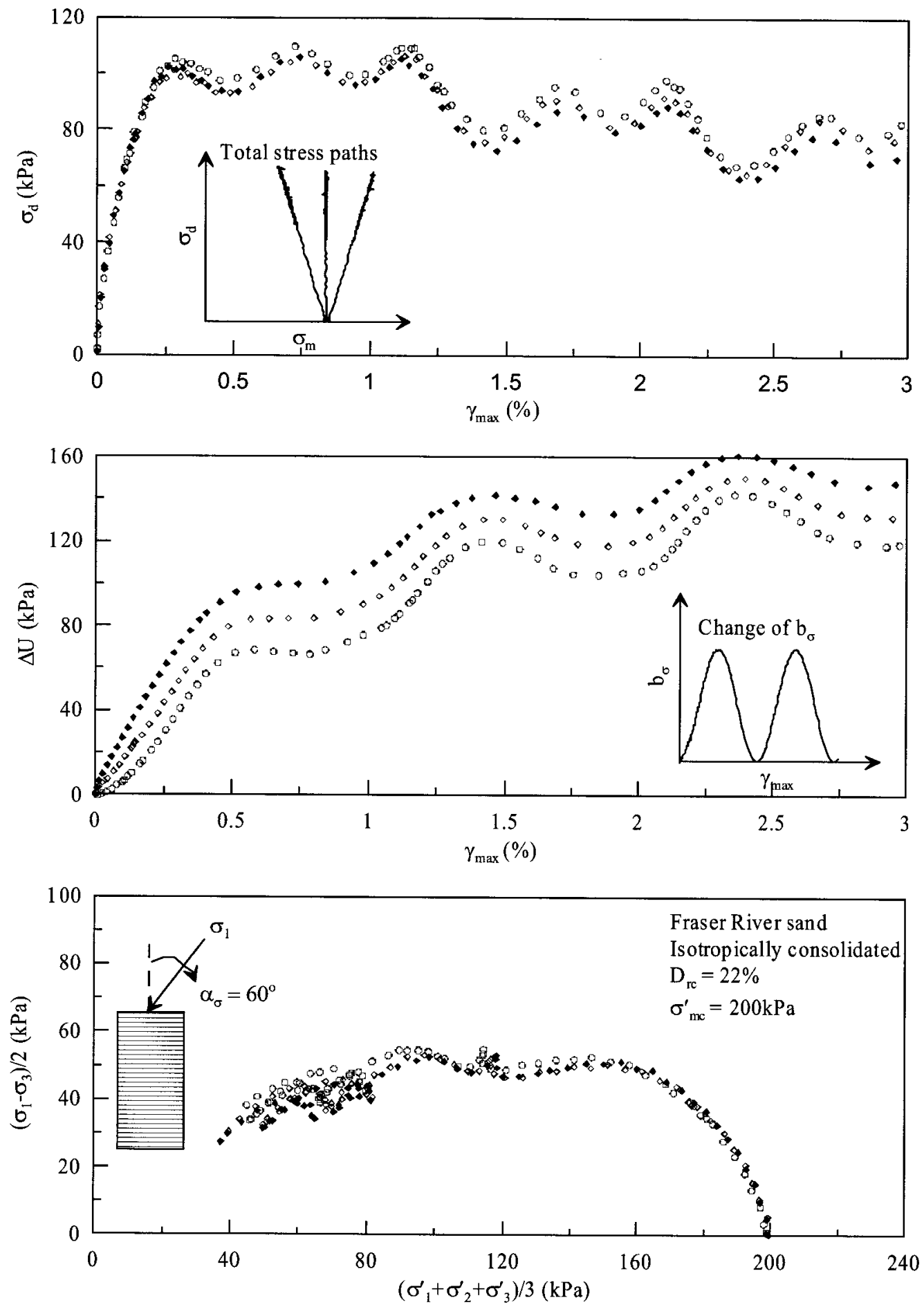

Fig. 7.21 Undrained response of Fraser River sand during application of different total stress paths with change of $b_{\sigma}$ 

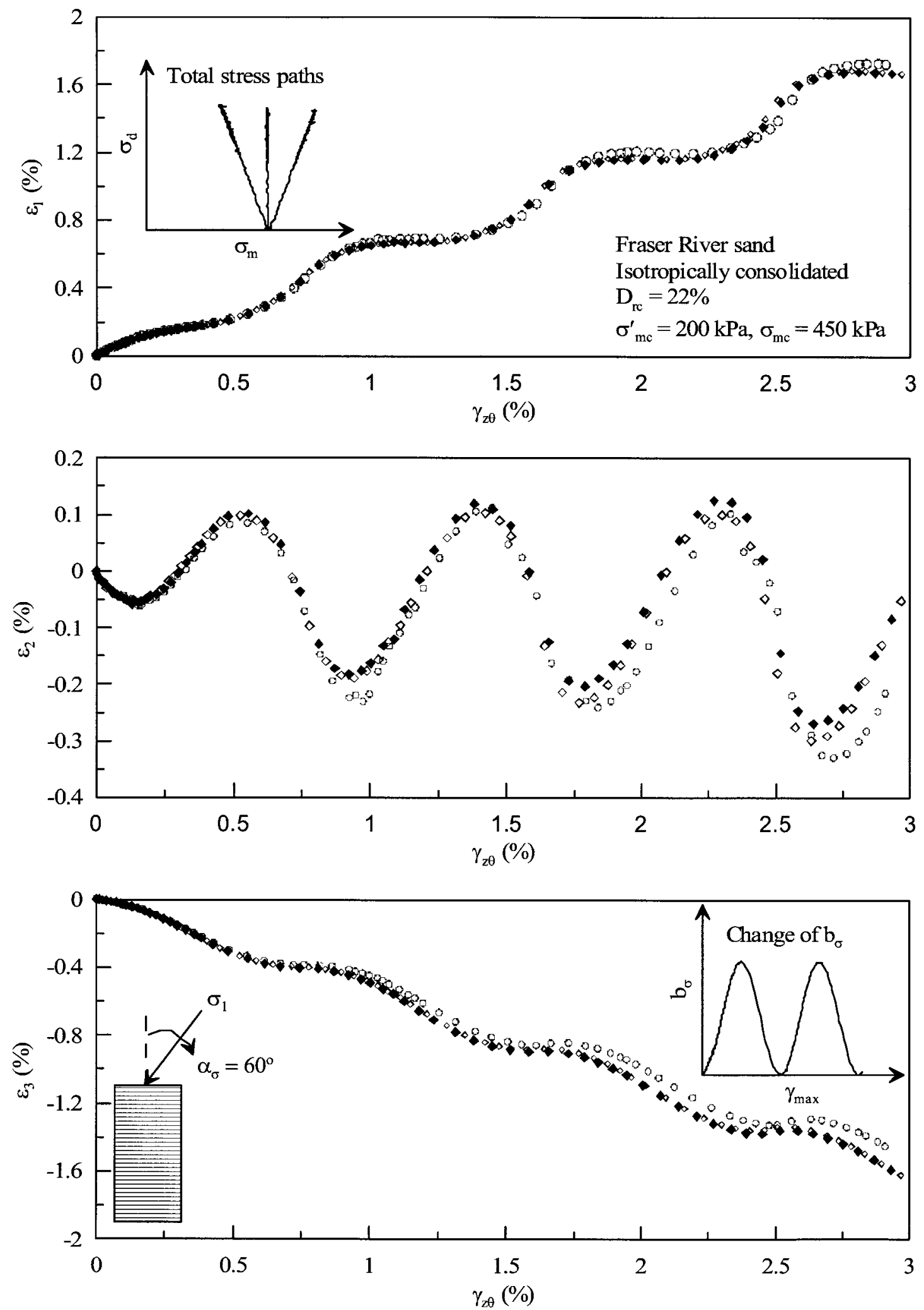

Fig. 7.22 Induced principal strains during application of different total stress paths with change of $b_{\sigma}$ 
Fig. 7.23 shows the variation of excess pore pressure induced by deviatoric stress for both consideration of hydrostatic stress. Both approaches result in a different amount of excess pore pressure at a particular level of $\gamma_{\max }$. The excess pore pressure responses are independent of total stress paths regardless of formulation. Peaks and troughs are noted during the changes of $b_{\sigma}$. The peaks are realized when $b_{\sigma}$ becomes $1.0\left(\gamma_{\max }=0.5\right.$, 1.5 and $2.5 \%)$. The troughs are noted when $b_{\sigma}$ becomes $0.0\left(\gamma_{\max }=1.0,2.0\right.$ and $\left.3.0 \%\right)$. However, cumulative excess pore pressure continuously increases with the number of rotations of $b_{\sigma}$.
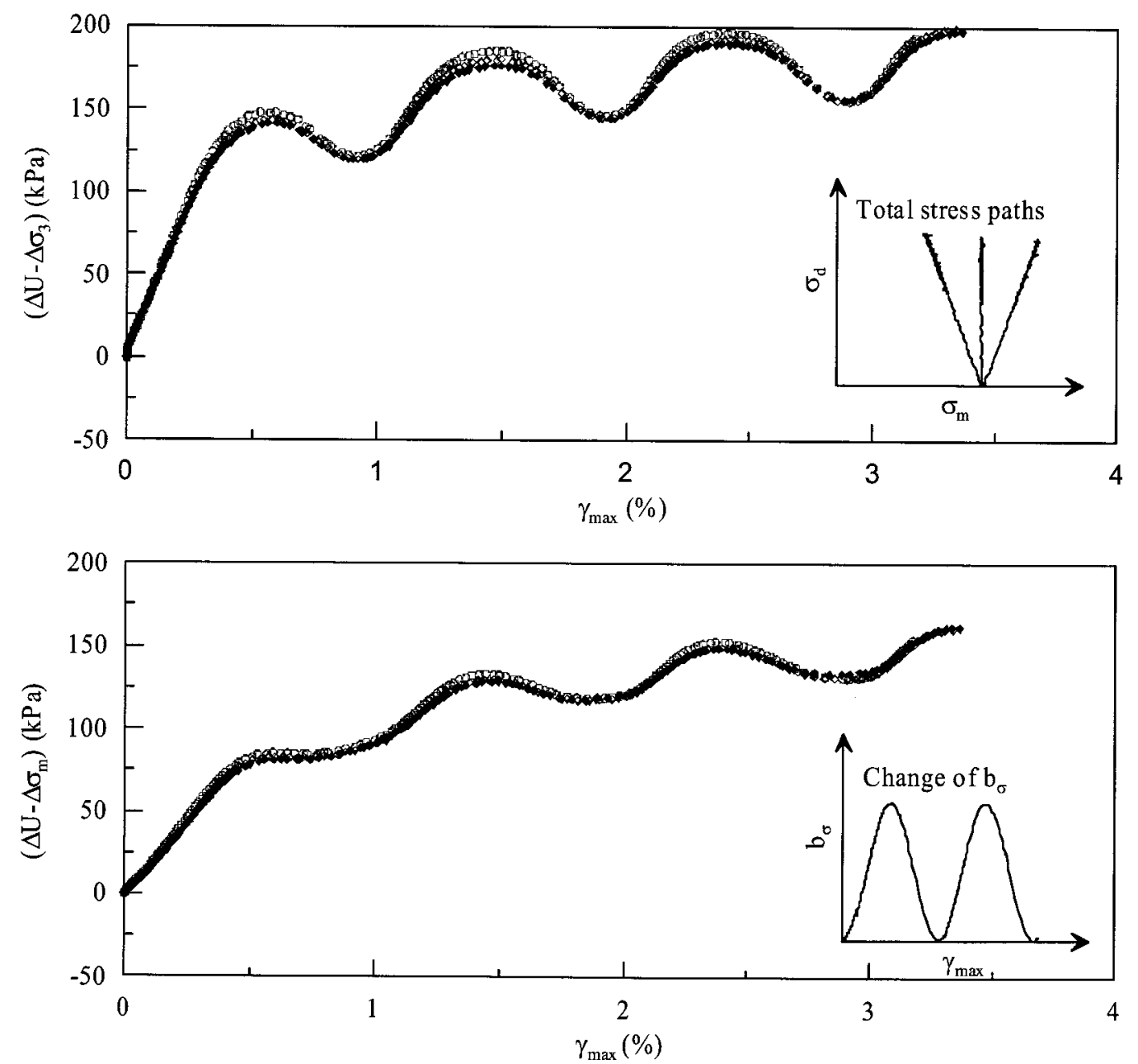

Fig. 7.23 Variation of excess pore pressure induced by deviatoric stress during the application of different total stress paths with change of $b_{\sigma}$ 
Fig. 7.24 shows the variation of excess pore pressure parameters. These plots show the unique variations of pore pressure parameters regardless of total stress paths, change of $b_{\sigma}$ and method of formulations. The variation of the pore pressure parameter also shows some peaks and troughs depending on $b_{\sigma}$ as noted in the excess pore pressure responses (Fig. 7.23).

\subsubsection{Drained response}

In this series of tests, $b_{\sigma}$ was changed in a drastic manner (Fig. 7.20) to study the effect of change of $b_{\sigma}$ on drained behaviour during the application of total stress paths. Specimens were isotropically consolidated to $\sigma_{m c}^{\prime}=200 \mathrm{kPa}$ and $D_{r c}=22 \%$.

The stress-strain responses are shown in Fig. 7.25. The strength of the sand increases depending on the total stress paths imposed. The total stress path $\sigma_{m}=\sigma_{m c}+$ $\sigma_{d} / 6$ results in higher strength and the total stress path $\sigma_{m}=\sigma_{m c}-\sigma_{d} / 6$ results in lower strength. The strength responses show peaks and troughs depending on $b_{\sigma}$. The peaks are realized at $b_{\sigma}$ closer to $0.0\left(\gamma_{\max }=1.0,2.0\right.$ and $\left.3.0 \%\right)$ and troughs are realized at $b_{\sigma}$ closer to $1.0\left(\gamma_{\max }=1.5 \%\right.$ and $\left.2.5 \%\right)$.

Induced volumetric strain increases systematically with increasing total mean normal stress. The increase of volumetric strain increases the relative density of the sand and thus, higher total mean normal stress leads to stronger response. This observation is analogous to the undrained tests (Fig. 7.21) that exhibits higher excess pore pressure during the application of higher mean normal stress and lower excess pore pressure during the application of lower mean normal stress. 

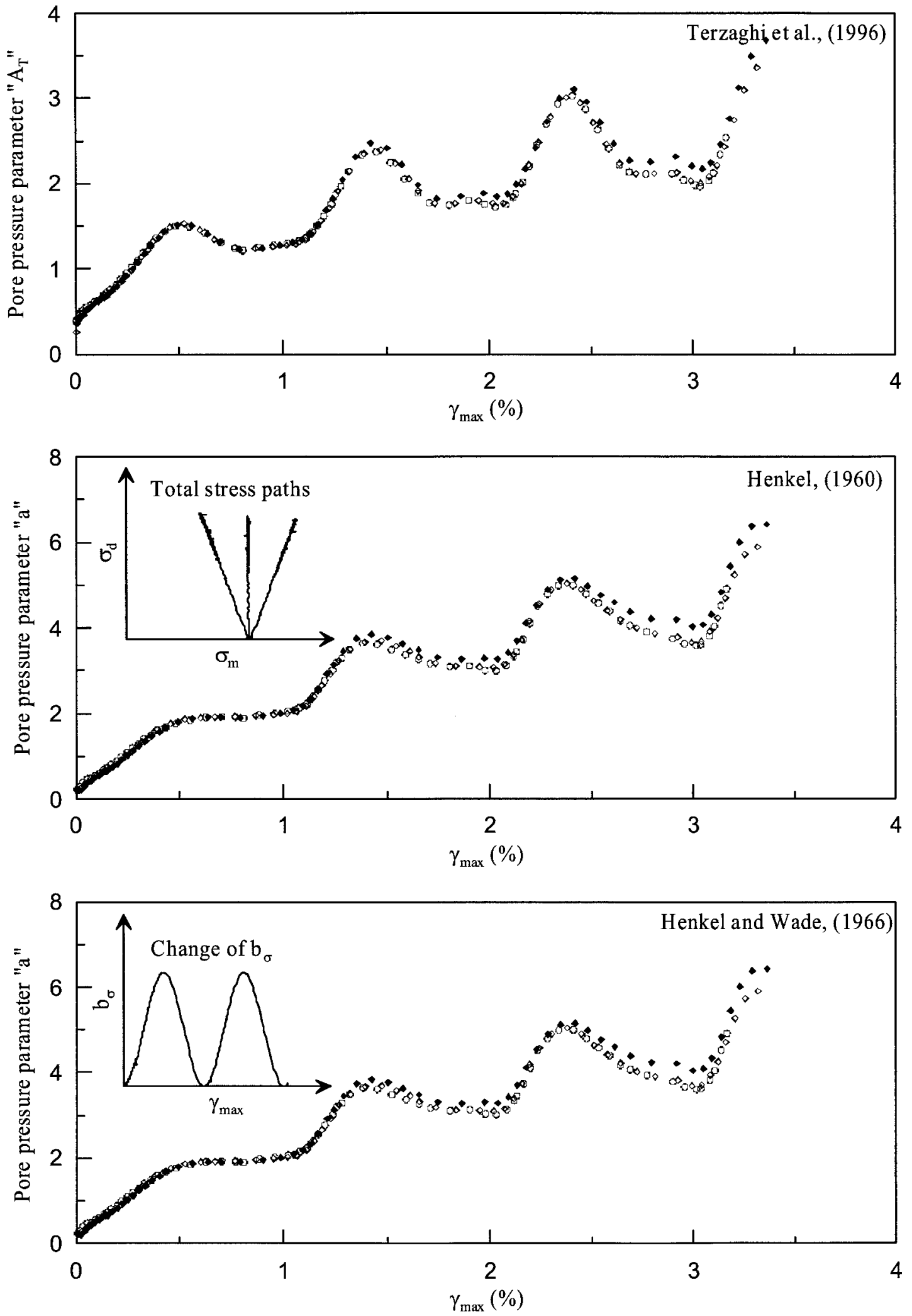

Fig. 7.24 Variation of pore pressure parameters during change of $b_{\sigma}$ 

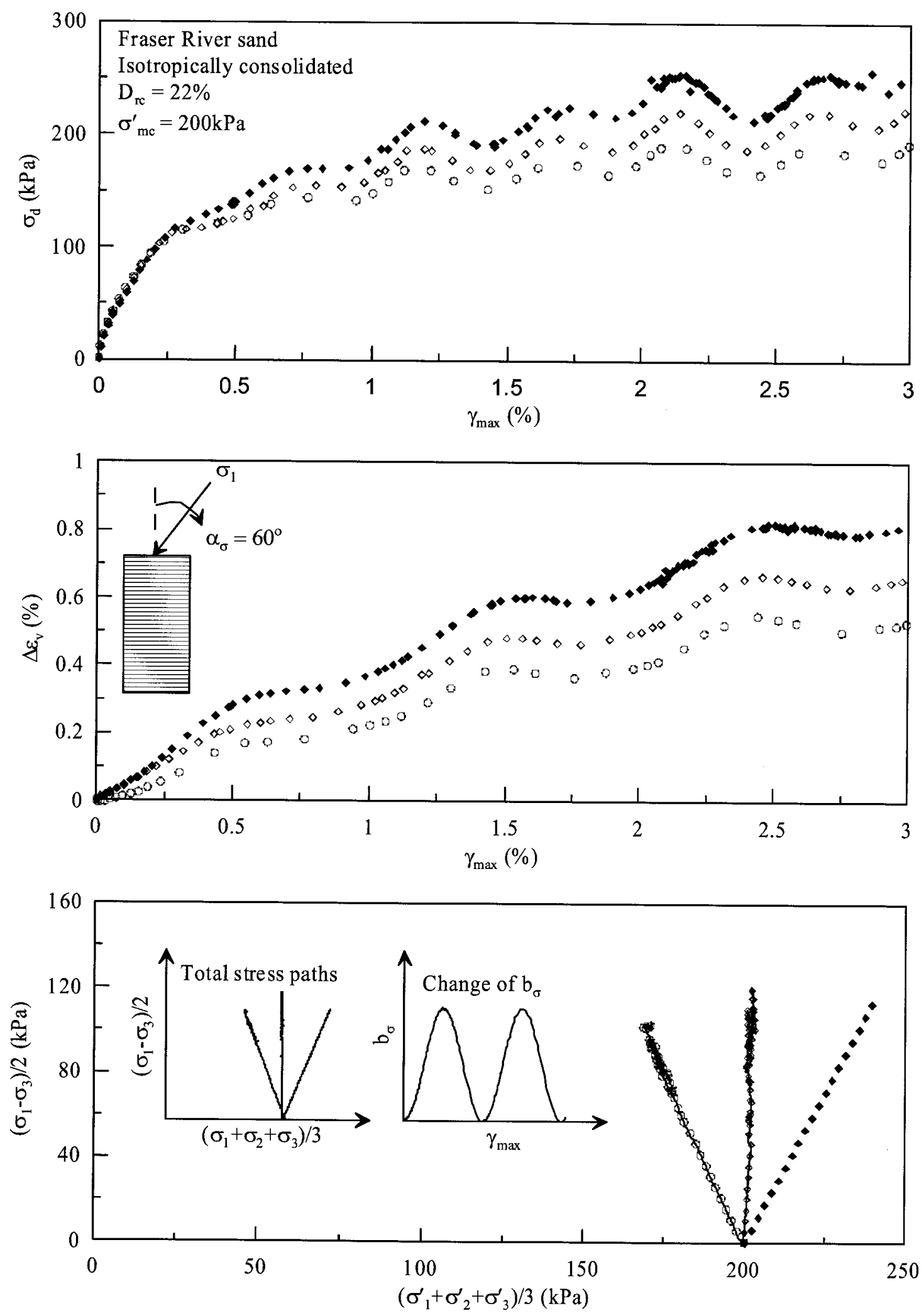

Fig. 7.25 Drained response of Fraser River sand during application of different total stress paths with change of $b_{\sigma}$ 
The induced volumetric strain is contractive and progressively increased with $\gamma_{\max }$, even though the peaks and troughs are noted in the volumetric response. The peaks are realized at $b_{\sigma}$ closer to 1.0 and the troughs are realized at $b_{\sigma}$ closer to 0.0 . This observation confirms that higher deformability exists along the bedding plane and lower deformability exists along the deposition direction of the sand (Sayao, 1989).

Fig. 7.26 shows the variation of the principal strains against $\gamma_{z \theta}$. The major principal strain $\varepsilon_{l}$ progressively increases and the minor principal strain $\varepsilon_{3}$ decreases with the increasing number of cycles of $b_{\sigma}$ rotation. As noted in undrained loading (Fig. 7.22), the same trends of variations in $\varepsilon_{1}$ and $\varepsilon_{3}$ are noted during drained loading with the increasing number of cycles of $b_{\sigma}$ rotation. The induced $\varepsilon_{I}$ and $\varepsilon_{3}$ are independent of total stress paths, however the intermediate principal strain $\varepsilon_{2}$ is dependent on the total stress paths. The deviation of $\varepsilon_{2}$ at a given level of $\gamma_{\max }$ in each total stress path would be equal to the deviation of volumetric strain noted in Fig. 7.25.

\subsection{Effect of rotation of $\alpha_{\sigma}$ on pore pressure generation and sand behaviour}

In this series of tests, principal stress direction $\alpha_{\sigma}$ was rotated in a systematic manner to study the effect of change of $\alpha_{\sigma}$ on sand behaviour along different total stress paths. $\alpha_{\sigma}$ was changed linearly increased from $0^{\circ}$ to $45^{\circ}$ as deviatoric stress increased from 0 to $90 \mathrm{kPa}$. The intermediate principal stress parameter $b_{\sigma}$ was maintained at zero throughout shearing. The specimen was isotropically consolidated to $\sigma_{m c}^{\prime}=200 \mathrm{kPa}$ and $D_{r c}=22 \%$. 

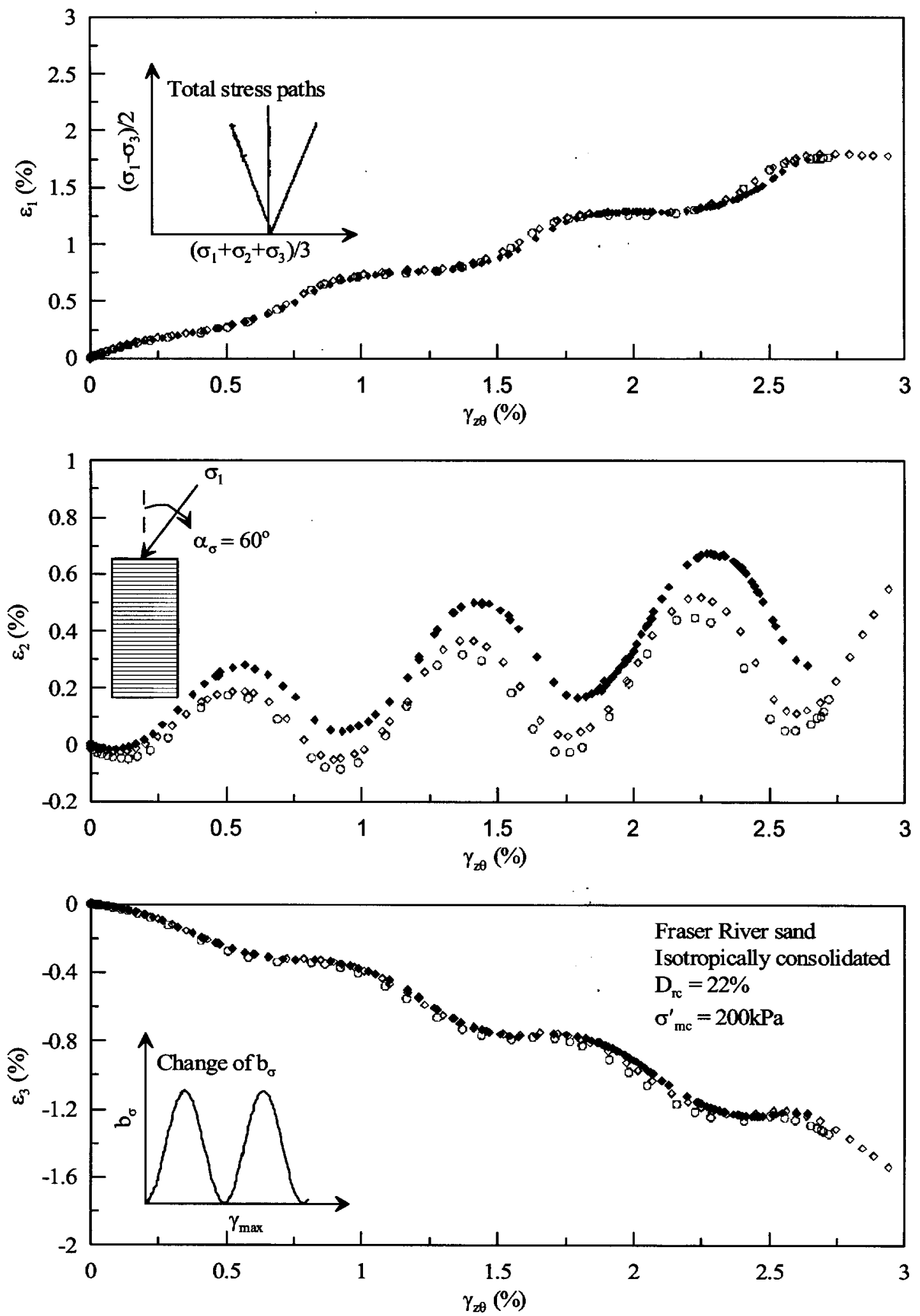

Fig. 7.26 Induced principal strains during application of different total stress paths with change of $b_{\sigma}$ 
Fig. 7.27 shows the response of Fraser River sand during the application of different total stress paths with rotation of $\alpha_{\sigma}$. The sand exhibits partially strain softening behaviour, in which strength of the sand decreases from peak strength state until reaching the phase transformation state, and subsequently it the sand strain hardens. Identical effective stress-strain responses are noted during rotation of $\alpha_{\sigma}$ for each total stress path.

Responses of induced excess pore pressure change systematically with the total mean normal stress imposed. During the application of a higher total mean normal stress increment $\left(\sigma_{m}=\sigma_{m c}+\sigma_{d} / 6\right)$, the sand generates highest excess pore pressure and it experiences lowest excess pore pressure during the application of a decrement of total mean normal stress $\left(\sigma_{m}=\sigma_{m c}-\sigma_{d} / 0\right)$. The effective stress paths are also identical regardless of the total stress paths. This observation clearly demonstrates that effective stress-strain response is independent of total stress paths, even $\alpha_{\sigma}$ is rotated at fixed $b_{\sigma}$. This finding further expands that undrained responses (stress-strain behavior, and effective stress path) for different total stress paths are unique, even though $\alpha_{\sigma}$ is rotated at a fixed $b_{\sigma}$.

Fig. 7.28 shows the induced principal strains $\left(\varepsilon_{1}, \varepsilon_{2}\right.$, and $\left.\varepsilon_{3}\right)$ during rotation of $\alpha_{\sigma}$ along different total stress paths. It is observed that strain responses are independent of total stress paths. The major principal strain $\varepsilon_{l}$ progressively increases, and the minor $\varepsilon_{3}$ and intermediate $\varepsilon_{2}$ principal strains decrease with rotation of $\alpha_{\sigma}$ and increasing $\gamma_{\max }$. 

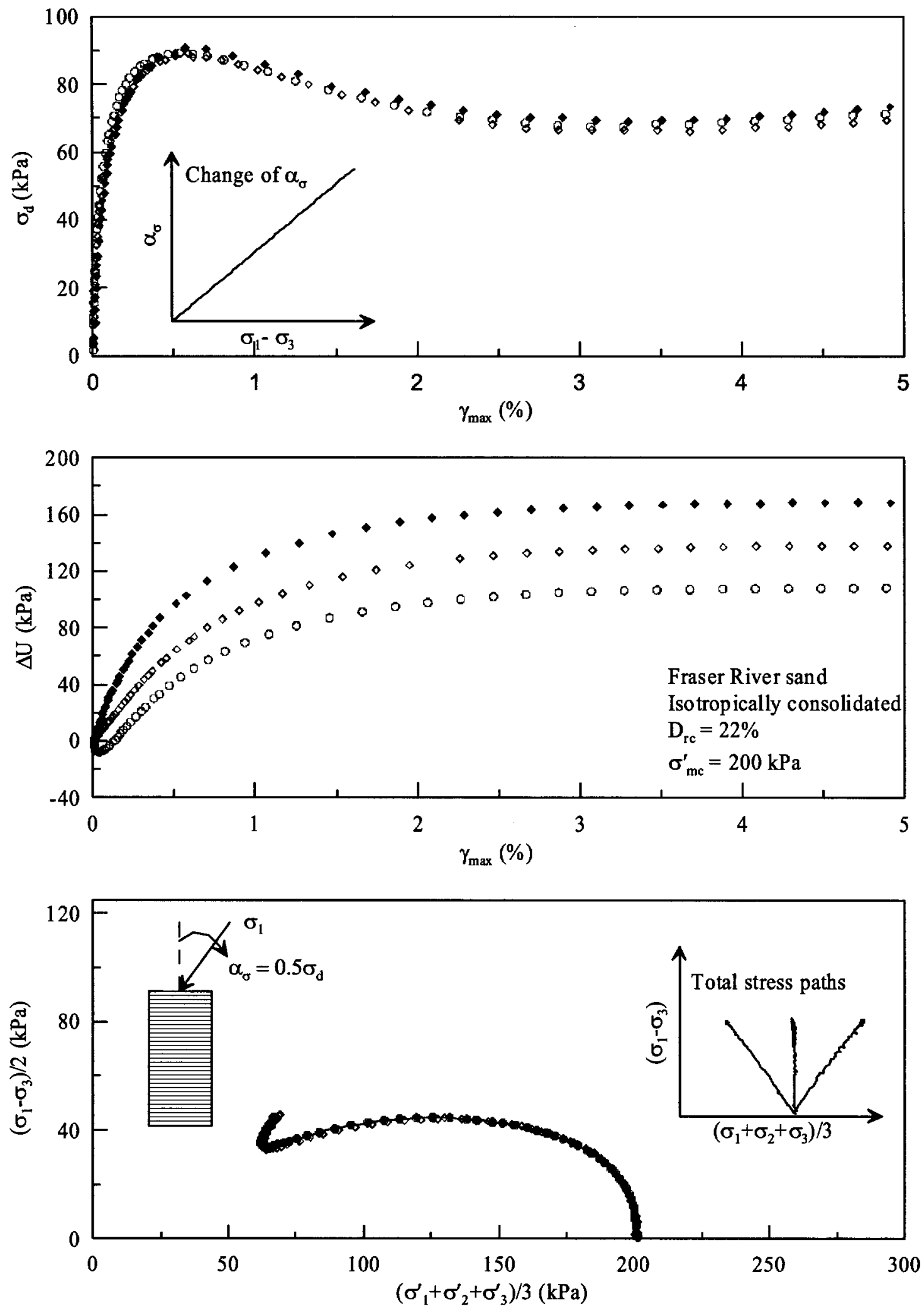

Fig. 7.27 Undrained response of Fraser River sand during application of different total stress paths with rotation of $\alpha_{\sigma}$ 

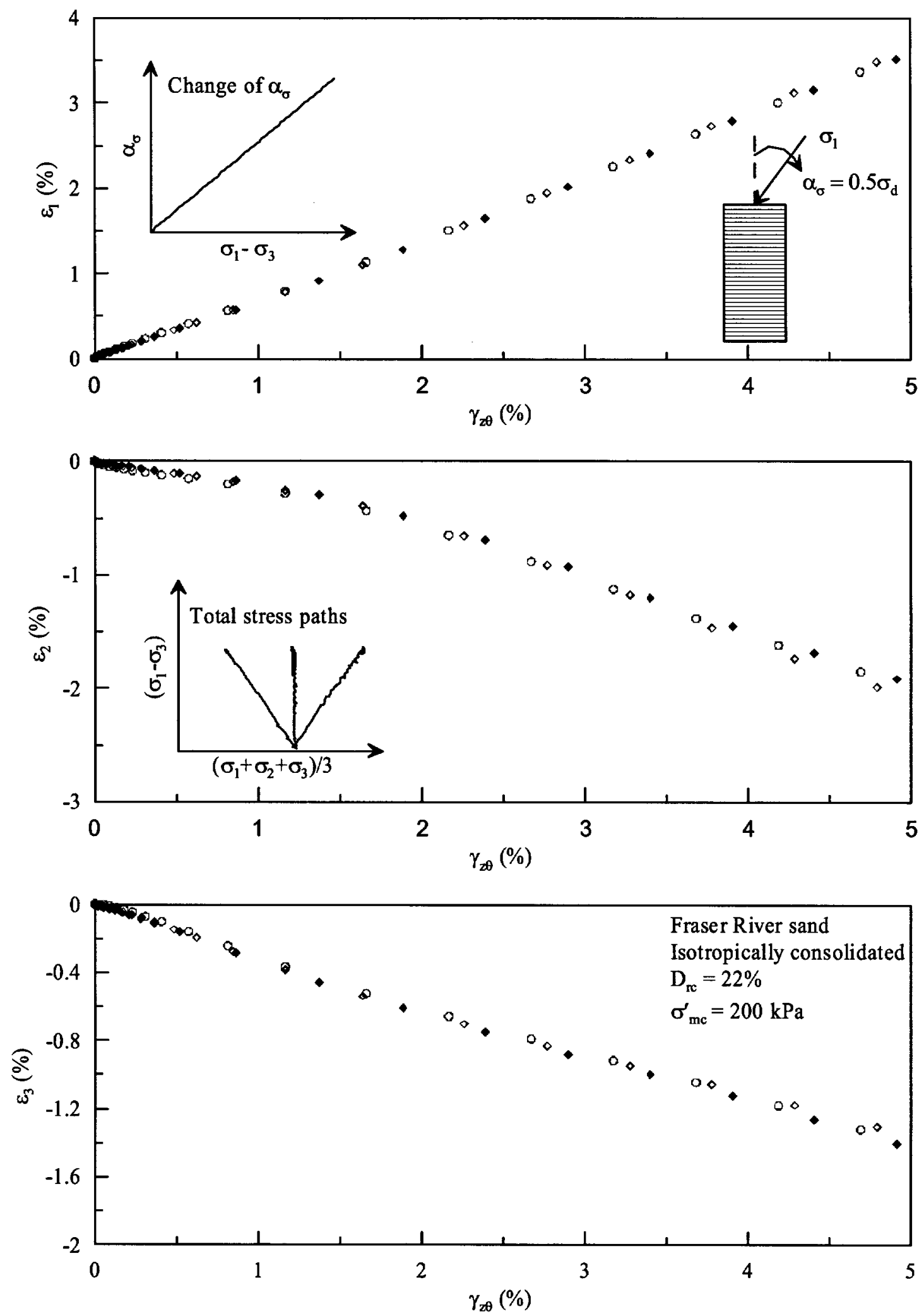

Fig. 7.28 Induced principal strains during application of different total stress paths with rotation of $\alpha_{\sigma}$ 
As shown in Fig. 7.29, the response of excess pore pressure induced by deviatoric stress is unique for all total stress paths regardless of the hydrostatic stress increments $\left(\Delta \sigma_{3}\right.$ and $\left.\Delta \sigma_{m}\right)$. The trend of excess pore pressure response is same; however, different magnitude of excess pore pressure at a given $\gamma_{\max }$ is noted. PT state is realized at $\sigma_{d}=68$ $\mathrm{kPa}$ and $\sigma_{m}^{\prime}=63 \mathrm{kPa}$ (Fig. 7.27) and it corresponds to $\gamma_{\max }=4.0 \%$. In that stress state, excess pore pressure $\left(\Delta U-\Delta \sigma_{3}\right)$ is $162 \mathrm{kPa}$ and excess pore pressure $\left(\Delta U-\Delta \sigma_{m}\right)$ is $138 \mathrm{kPa}$.
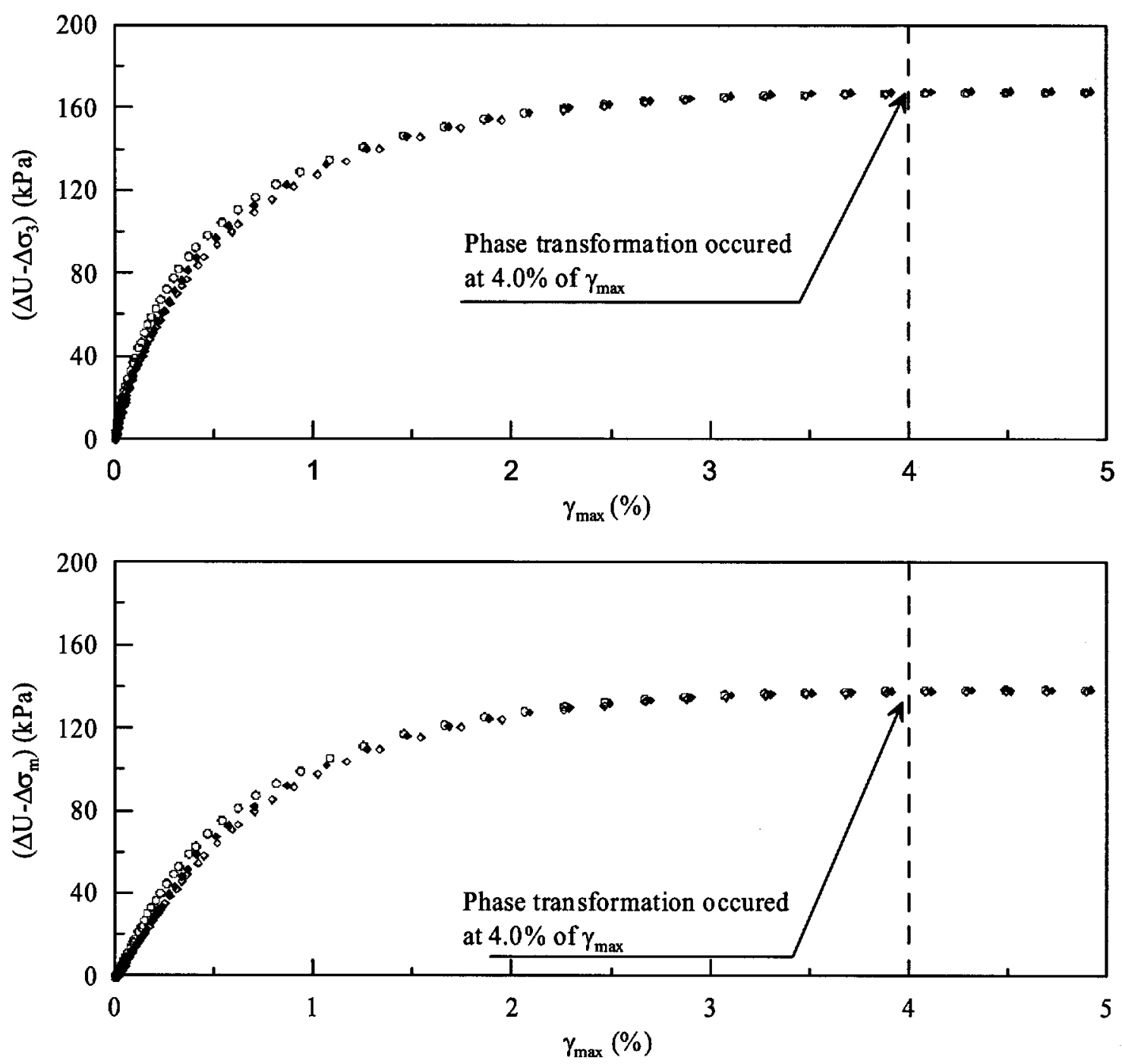

Fig. 7.29 Excess pore pressure induced by deviatoric stress during application of different total stress paths with rotation of $\alpha_{\sigma}$ 
Fig. 5.30 shows the variation of pore pressure parameters during rotation of $\alpha_{\sigma}$ along different total stress paths. Identical variations for each formulation are noted regardless of total stress paths and rotation of $\alpha_{\sigma}$. Even though the magnitudes of pore pressure parameters vary according to the formulations, a similar trend of variation is noted. The pore pressure parameters increase until PT state and subsequently decrease as noted in the excess pore pressure responses (Fig. 7.29).

\subsection{Effect of simultaneous change of $\alpha_{\sigma}$ and $b_{\sigma}$ on pore pressure generation}

A series of tests were performed to study the effect of simultaneous changes in $\alpha_{\sigma}$ and $b_{\sigma}$ along different total stress paths. $\alpha_{\sigma}$ was increased linearly against $\gamma_{\max }$ from $15^{\circ}$ to $75^{\circ} . b_{\sigma}$ was increased from 0 to 1.0 until reaching $\gamma_{\max }=2 \%$ and continuously reduced to 0.0 until reaching $\gamma_{\max }=4 \%$ (as a sine function). The specimens were isotropically consolidated to $\sigma_{m c}^{\prime}=200 \mathrm{kPa}$ and $D_{r c}=22 \%$.

Fig. 7.31 shows the response of Fraser River sand during simultaneous change in $\alpha_{\sigma}$ and $b_{\sigma}$ along different total stress paths. The sand experiences strain softening and peak strength of $150 \mathrm{kPa}$ is reached at $\gamma_{\max }=0.5 \%$. After the peak, the strength of the sand reduces and subsequently, the sand deforms at a constant strength. The effective stress-strain responses are identical for all total stress paths.

The excess pore pressure changes depending on the total stress path. The application of total stress path $\sigma_{m}=\sigma_{m c}+\sigma_{d} / 6$ results in higher excess pore pressure and the application of total stress path $\sigma_{m}=\sigma_{m c}-\sigma_{d} / 6$ results in lower excess pore pressure. The induced excess pore pressure continuously increases with increasing $\gamma_{\max }$. 

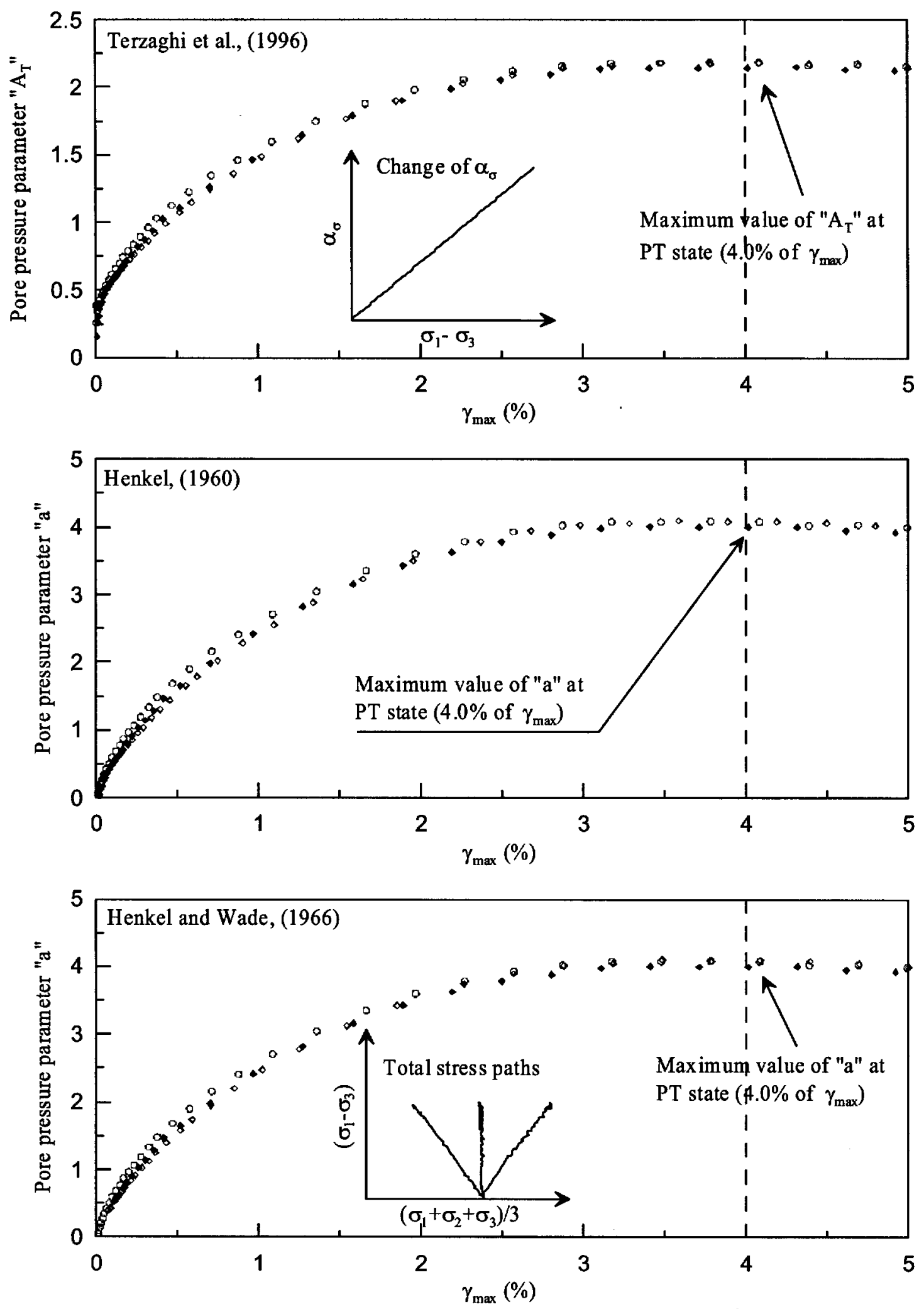

Fig. 7.30 Variation of pore pressure parameters during application of different total stress paths with rotation of $\alpha_{\sigma}$ 

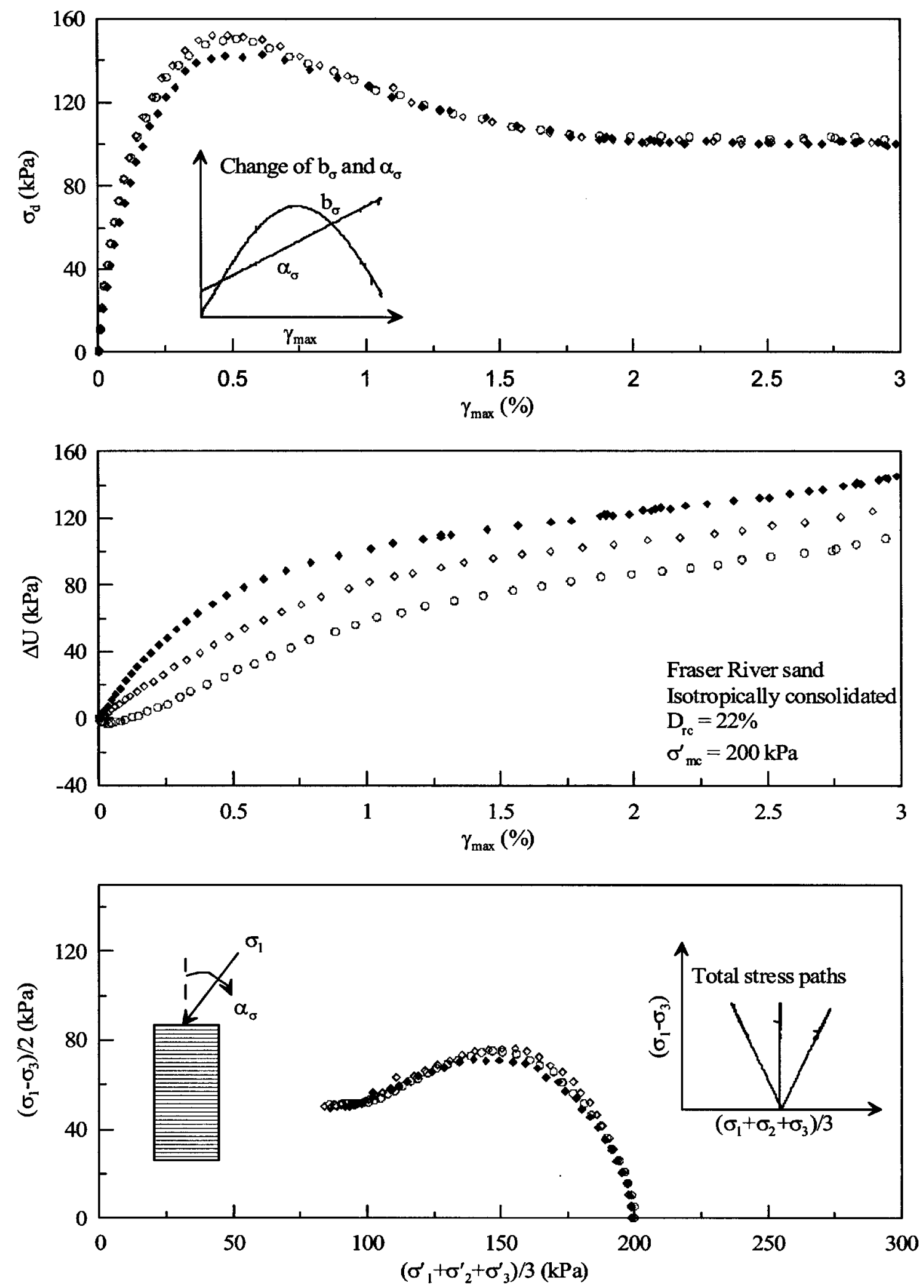

Fig. 7.31 Undrained response of Fraser River sand during change of $b_{\sigma}$ and rotation of $\alpha_{\sigma}$ 
The effective stress paths are identical, and it is clear that effective stress-strain response is independent of total stress paths, even though $\alpha_{\sigma}$ and $b_{\sigma}$ are simultaneously changed.

Fig. 7.32 shows the variation of principal strains during simultaneous changes in $\alpha_{\sigma}$ and $b_{\sigma}$ along different total stress paths. The responses of strains are essentially identical and independent of total stress paths. The major principal strain $\varepsilon_{l}$ increases and the minor principal strain $\varepsilon_{3}$ decreases continuously with increasing $\gamma_{z}$. At the initial stage of deformation, intermediate principal strain $\varepsilon_{2}$ is negative until reaching about $\gamma_{z}=$ $0.60 \%$, in which $b_{\sigma}$ reaches 0.45 . At $b_{\sigma}=0.45, \varepsilon_{2}$ is approximately zero. Subsequently, $\varepsilon_{2}$ becomes positive and increases continuously. The observation of $\varepsilon_{2}=0$ at $b_{\sigma}=0.45$ is consistent with the principal strain responses noted in static undrained tests at a fixed $b_{\sigma}$ (Fig. 5.2).

Fig. 7.33 shows the response of excess pore pressure induced by deviatoric stress. The magnitude of excess pore pressure at a certain level of $\gamma_{\max }$ differs from each approach. Both responses display the contractive type of deformation during shearing. However, the rate of contractiveness is displayed differently by both approaches. At the initial stage of deformation, Consideration of $\Delta \sigma_{3}$ shows higher contractiveness than that of $\Delta \sigma_{m}$. As $b_{\sigma}$ was changed with respect to $\gamma_{\max }$, consideration of $\Delta \sigma_{3}$ as hydrostatic stress increment may misinterpret the response of excess pore pressure induced by deviatoric stress because of dependency of $\Delta \sigma_{3}$ on $b_{\sigma}$.

Fig. 7.34 shows the variation of pore pressure parameters. Identical variations are noted for all total stress paths. Each pore pressure parameter increases with $\gamma_{\max }$ as noted 
in Fig. 7.33. The pore pressure parameters are independent regardless of total stress paths that may allow change of $b_{\sigma}$ and rotation of $\alpha_{\sigma}$.
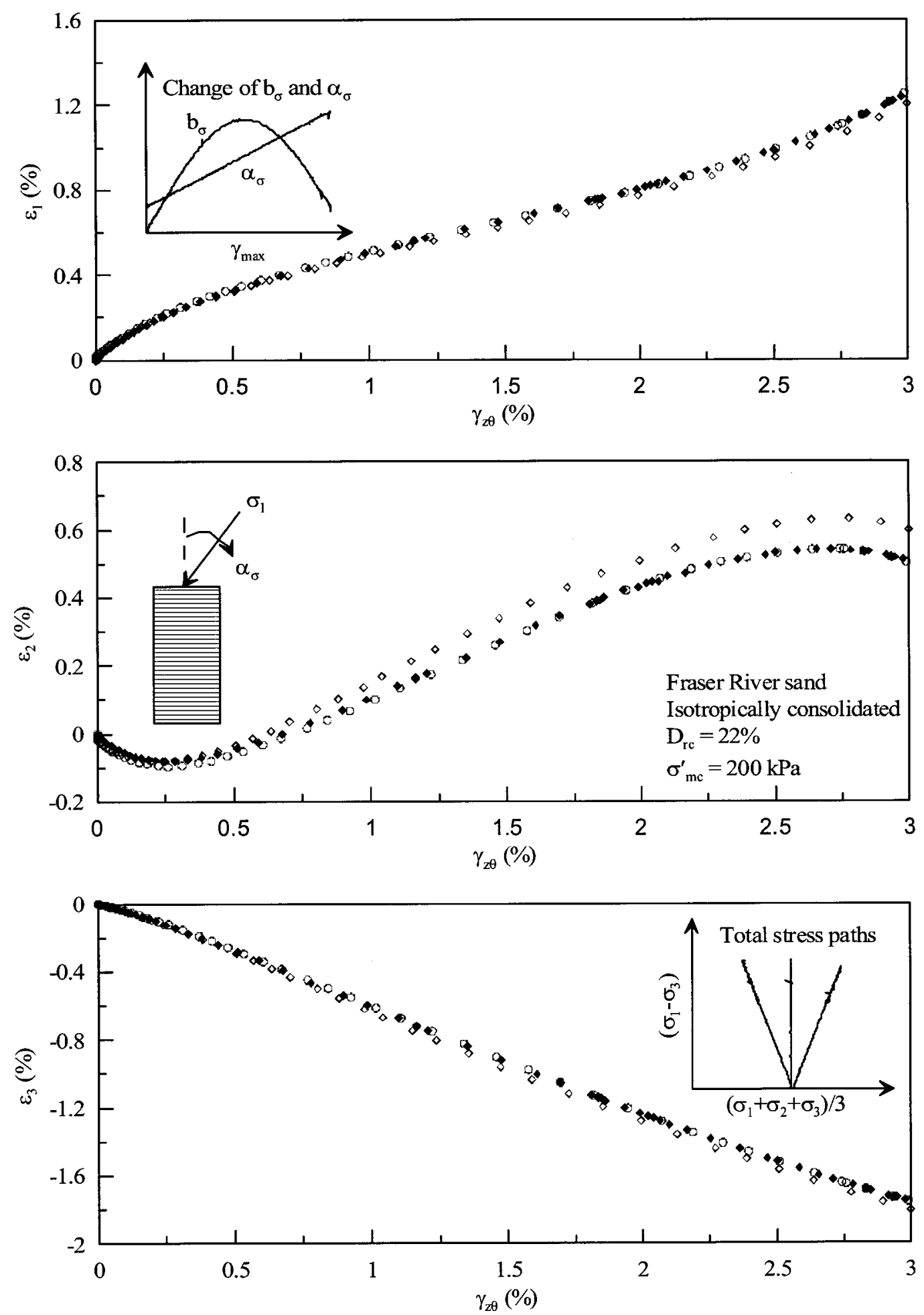

Fig. 7.32 Induced principal strains during change of $b_{\sigma}$ and rotation of $\alpha_{\sigma}$ 

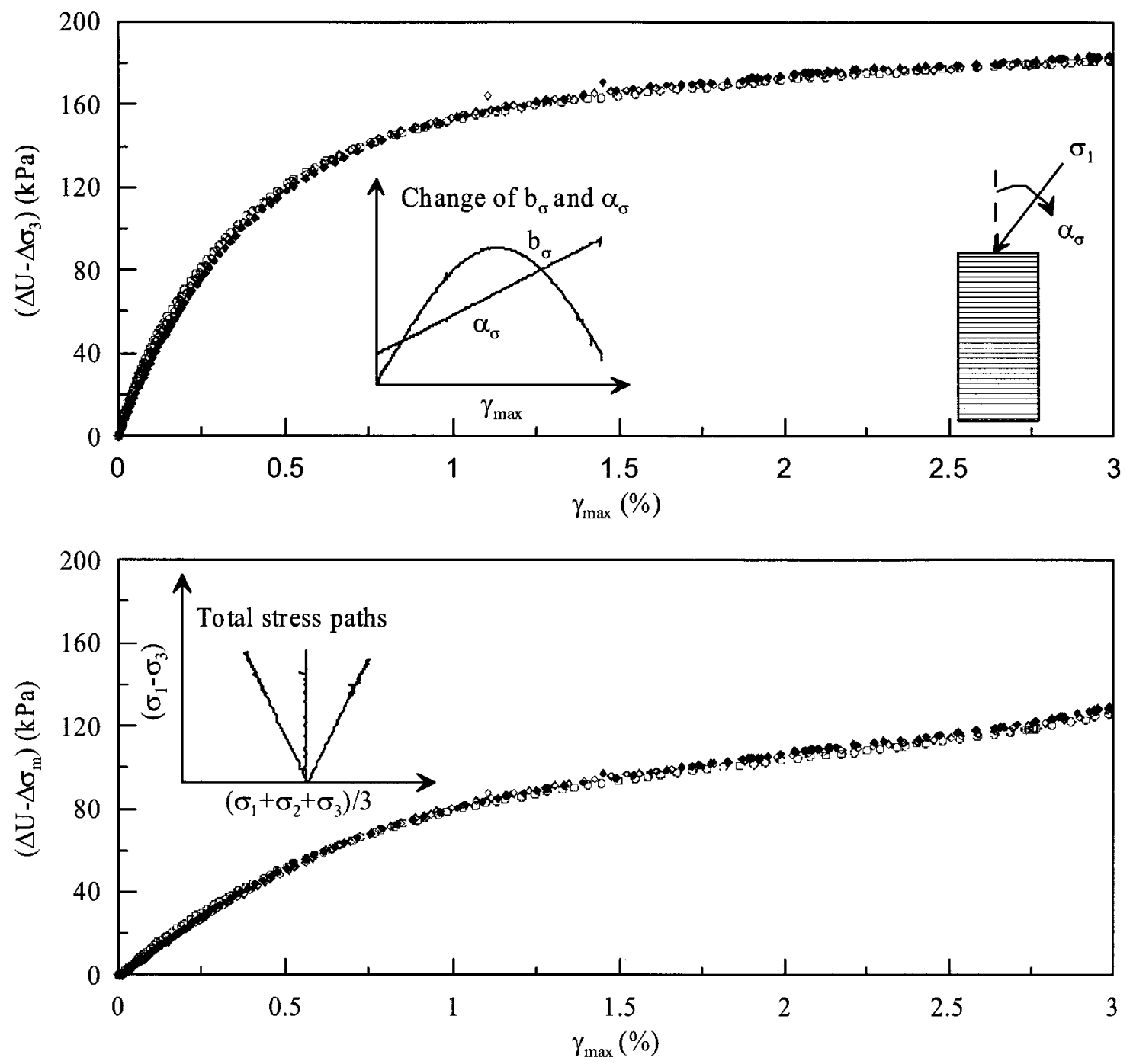

Fig. 7.33 Excess pore pressure induced by deviatoric stress during change of $b_{\sigma}$ and rotation of $\alpha_{\sigma}$ 

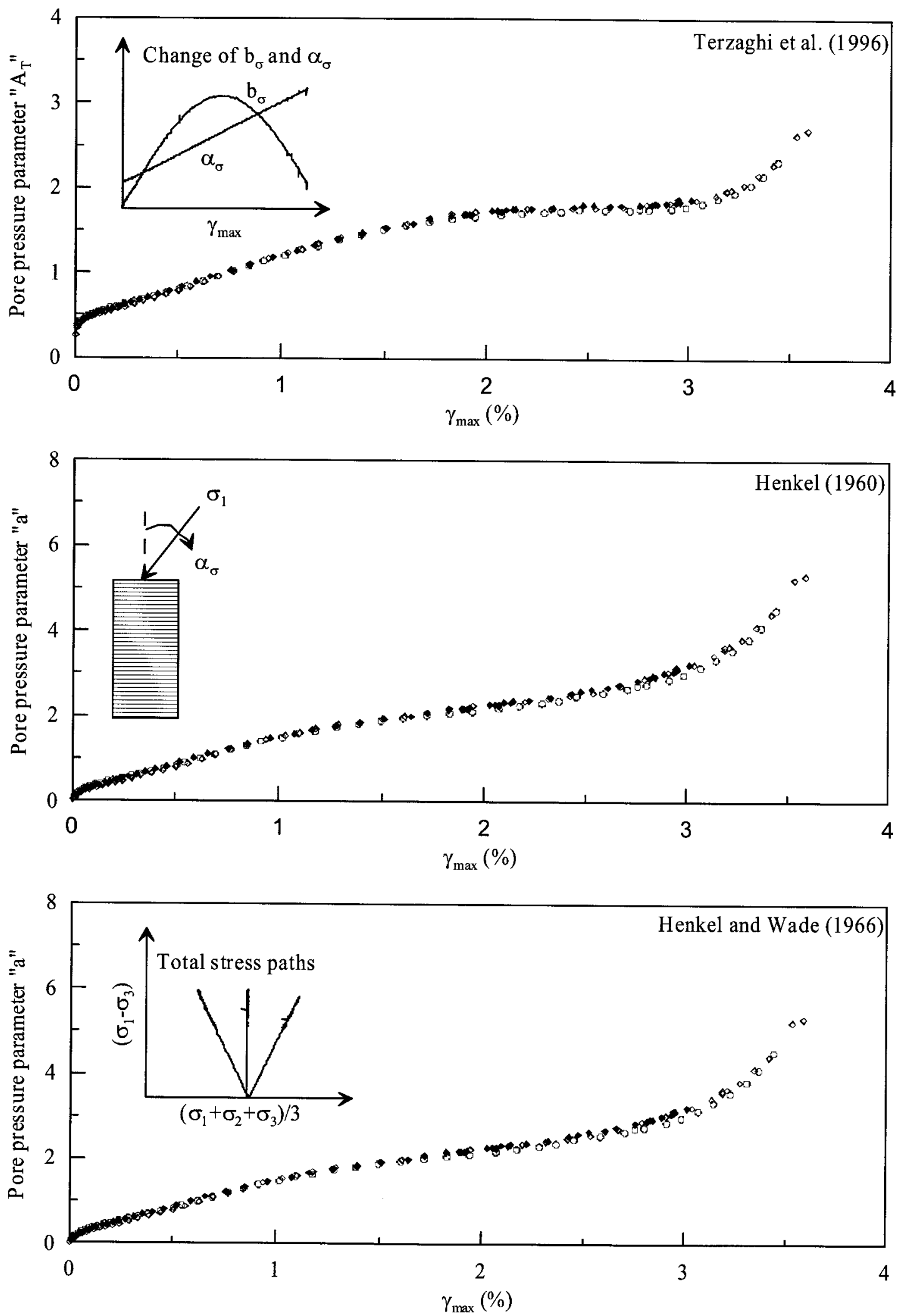

Fig. 7.34 Variation of pore pressure parameters during change of $b_{\sigma}$ and rotation of $\alpha_{\sigma}$ 


\subsection{Summary}

Experiments were carried out to explore the effect of change in the intermediate principal stress parameter $b_{\sigma}$ and the principal stress direction $\alpha_{\sigma}$ on soil behaviour during the application of different total stress paths. Based on the experimental work on Fraser River sand using the automated HCT device, the following conclusions are drawn.

In practice, two different approaches are used to divide the total stress increment tensor as hydrostatic stress increment and deviatoric stress increment tensors. In the first approach, the hydrostatic stress tensor is formed based on minor increment of principal stress $\left(\Delta \sigma_{3}\right)$ and the deviatoric stress tensor is formed in terms of incremental deviatoric stress $\left(\Delta \sigma_{1}-\Delta \sigma_{3}\right)$ that is estimated from principal components of stress increments. In the second approach, based on mechanics, the hydrostatic stress tensor is formed by the increment of total stress $\Delta \sigma_{m}$ and $\left(\Delta \sigma_{1}-\Delta \sigma_{3}\right) . \Delta \sigma_{3}$ can be expressed in terms of $b_{\Delta \sigma}$ and $\Delta \sigma_{m}$ and $\Delta \sigma_{m}$ is only dependent on total stress paths imposed. The first approach can only be used when $b_{\sigma}$ is constant during shearing. When $b_{\Delta \sigma}$ is changed during shearing, $\Delta \sigma_{3}$ may result in an incorrect response of excess pore pressure induced by deviatoric stress and that response fails to reflect the actual behaviour of sands (contractive/dilative).

The experimental evidence shows that the first approach does not display the correct trends in excess pore pressure generation during change of $b_{\sigma}$ along different total stress paths. In addition, predication of soil behaviour based on trend of excess pore pressure development (contractive and dilative) is misleading. However, consideration of either $\Delta \sigma_{m}$ or $\Delta \sigma_{3}$ results in identical excess pore pressure responses by deviatoric stress for each total stress path. 
Different formulations (excess pore pressure equations) have been proposed to quantify the excess pore pressure generated by hydrostatic and deviatoric stress increments during application of total stress increment. However, in practice, these formulations are not widely used because the parameter to quantify the deviatoric stress induced excess pore pressure ( $A_{T}$ and $a$ ) is dependent on several parameters. In this study, three different formulations (Terzaghi et al., 1996; Henkel, 1960; Henkel and Wade, 1966) were analyzed. Each formulation seems to work out for three-dimensional loading problems and $A_{T}$ and $a$ are independent on total stress paths imposed. For the formulation suggested by Terzaghi et al. (1996), the principal components of the stress increments should be considered. The formulations suggested by Henkel (1960) and Henkel and Wade (1966) do not necessarily require the principal components of the stress increments and can be formulated correctly from both principal components of stress increments and increments of principal stresses. The pore pressure parameters are independent of the total stress paths, even though principal stress direction is rotated with a change of $b_{\sigma}$.

The undrained response of soil is independent of total stress paths at constant $b_{\sigma}$ and fixed $\alpha_{\sigma}$ as noted in the previous research work and experiments conducted in this study. However, the undrained response is dependent on $b_{\sigma}$ and $\alpha_{\sigma}$. This study shows that the undrained response of soil is independent of total stress paths even $b_{\sigma}$ and/or $\alpha_{\sigma}$ are changing independently or simultaneously. The research reported herein suggests that the undrained stress path, and stress-strain curves are independent of total stress paths under all loadings. 


\section{SUMMARY AND CONCLUSIONS}

A new Hollow Cylinder Torsional shear (HCT) device was set up in the geotechnical laboratory at Carleton University. The HCT device is operated using advanced control techniques to permit testing along different stress/strain paths (including the ability to conduct partially drained tests). In addition, it is cable of applying cyclic loading under generalized stress states with controlled stress rotation. The required data acquisition and control programs were developed in-house to suit the research needs.

At the beginning of the research, undrained anisotropy of Fraser River sand was investigated. The anisotropy was studied by maintaining a fixed principal stress direction with respect to the bedding plane. In addition, the effect of intermediate principal stress on the sand behaviour was also studied. The tests on Fraser River sand revealed the directional dependency behaviour due to anisotropy formed by deposition process (water pluviation).

The strain path controlled loading that imposed volume-pressure coupled deformation in the specimen was applied in a systematic manner under three-dimensional loading. It is recognized that the strain paths imposed in this study are not realistic field loading scenarios. However, these experiments would provide an insight into the volumepressure coupled deformation problems that may occur in the field.

Cyclic behaviour of the sand was investigated under three-dimensional loading with rotation of principal stress directions. Two types of stress rotation (smooth and jump) were assessed. During smooth rotation that is very common in in-situ loading, principal stress direction was smoothly rotated on either side of the specimen deposition 
direction. During jump rotation, the principal direction was changed suddenly on either side of the deposition for positive and negative cycle of loading.

Undrained behaviour of the sand was investigated along different total stress paths with the rotation of principal stress direction and change of the intermediate principal stress parameter. This study further expanded the understanding of uniqueness of undrained response in terms of total stress paths, principal stress direction and the intermediate principal stress.

Based on the experimental evidence the following conclusions are drawn.

Undrained response of Fraser River sand is highly dependent on loading direction. The sand is completely dilative, when major principal stress direction is along the deposition direction. It behaves more contractively and shows limited liquefaction type of response when principal stress direction is closer to the bedding plane. In addition, the undrained strength of the sand is influenced by intermediate principal stress parameter. The sand is stronger at $b_{\sigma}=0$ and weaker at $b_{\sigma}=1.0$.

The undrained tests on Fraser River sand clearly show that the minimum undrained strength from triaxial tests is only applicable to specific loading scenarios $\left(b_{\sigma}=\right.$ $0, \alpha_{\sigma}=0^{\circ}$ and $\left.b_{\sigma}=1.0, \alpha_{\sigma}=90^{\circ}\right)$ and it would not yield correct solutions to the practical problems. The mobilized friction angle at Phase Transformation (PT) state is unique for particular sand regardless of $\alpha_{\sigma}$ and $b_{\sigma}$. Strength at PT state is dependent on not only void ratio and effective confining stress but also on $\alpha_{\sigma}$ and $b_{\sigma}$.

Volume-pressure coupled deformation leading to expansive volumetric strains clearly indicates that strain softening could be triggered in sands that are dilative and stable under undrained conditions. This kind of in-situ problem represents a critical risk, 
when the strength of such materials is evaluated using traditional undrained considerations.

During application of expansive volumetric strain, strength of the sand at maximum pore pressure state reduces systematically with increasing $b_{\sigma}$ at $\alpha_{\sigma}=0^{\circ}$. However, the reduction of the strength comparing with the corresponding undrained strength is not affected much by change of $b_{\sigma}$. Peak strength of the sand systematically reduces and friction angle mobilized at this state does not vary much with increasing $b_{\sigma}$.

A small amount of expansive volumetric strain transforms the sand into complete strain softening at fixed $\alpha_{\sigma}$ of $45^{\circ}, 60^{\circ}$ and $90^{\circ}$. The strength of the sand at peak pore pressure state during the application of expansive volumetric strain systematically reduces with increasing $\alpha_{\sigma}$. As noted in undrained tests, reduction in strength at peak pore pressure state is significant when $\alpha_{\sigma}$ changes from $0^{\circ}$ to $45^{\circ}$ and it is relatively minor when $\alpha_{\sigma}$ exceeds $45^{\circ}$. At a given $\alpha_{\sigma}$, reduction of strength compared to the corresponding undrained strength is almost the same regardless of $\alpha_{\sigma}$.

Increased rate of expansive volumetric strain significantly affects the strength of the sand. During the application of higher rate of expansive volumetric strain, the sand become softer and experiences a much lower strength than that of undrained strength. A systematic reduction of strength is noted with increasing rate of expansive volumetric strain. On the other hand, contractive volumetric strain results in a stronger response than undrained.

The peak strength and friction angle mobilized at this state reduces significantly with increasing rate of expansive volumetric strain. Friction angle mobilized at peak pore pressure state can be treated as a unique material property regardless of $b_{\sigma}, \alpha_{\sigma}, K_{c}$ and 
rate of expansive volumetric strain, and it is equal to the friction angle noted at PT state of undrained loading.

Cyclic resistance of soils in the laboratory is commonly assessed using triaxial and simple shear devices. The reliability of these results is questionable, because the nature of principal stress rotation during loading is different compared to the field problem. In addition, assessment of cyclic resistance by these devices differs from each other. Test data reported herein, and the literature show that cyclic resistance of soils based on triaxial mode of cyclic loading is higher than that of simple shear mode. Noted difference in liquefaction resistance at the same cyclic stress ratio in these devices can be attributed to the nature of principal stress rotation and the stress states.

Number of cycles required to liquefy Fraser River sand decreases with increasing cyclic stress ratio during smooth and jump rotations of principal stress direction between $-45^{\circ}$ and $+45^{\circ}$. The nature of rotation of principal stress directions does not influence much the cyclic resistance of sand (rotation between $-45^{\circ}$ and $+45^{\circ}$ ). Essentially, similar variation in cyclic resistance is noted with increasing cyclic stress ratio for both types of rotation.

A particular sand exhibits different cyclic resistance at a fixed cyclic stress ratio depending on the magnitude of maximum principal stress rotation $\alpha_{\sigma, \max }$. The sand exhibits higher cyclic resistance when principal stress rotation is much closer to deposition direction. The cyclic resistance reduces when the maximum inclination of principal stress $\alpha_{\sigma, \max }$ increases to $45^{\circ}\left(\alpha_{\sigma, \max }<45^{\circ}\right)$. The influence of the nature of principal stress rotation is insignificant within that range of $\alpha_{\sigma, \max }$. Essentially, a similar variation of cyclic resistance is noted for both types of rotation. When $\alpha_{\sigma, \max }$ is more than 
$60^{\circ}$, the nature of principal stress rotation influences the cyclic resistance of the sand. The sand exhibits higher cyclic resistance during jump rotation than smooth rotation of principal stress. This influence can be justified by the nature of shear stress experienced by the sand. However, further detail study is required to make a firm conclusion on this observation. The friction angle mobilized at PT state during the cyclic loading is equal to the friction angle mobilized at PT state under static loading.

During increment of principal stresses, hydrostatic stress components are commonly expressed in terms of either total mean normal stress $\Delta \sigma_{m}$ or minor increment of principal stress $\Delta \sigma_{3}$. Since $\Delta \sigma_{m}$ is mean of increments of three principal stresses and $\Delta \sigma_{3}$ is the minor increment of principal stresses, both $\Delta \sigma_{m}$ and $\Delta \sigma_{3}$, except some special loading conditions (e.g. equal increments of principal stresses) manipulate unequal amount for excess pore pressure induced by deviatoric stress. When $b_{\sigma}$ is fixed during shearing, consideration of hydrostatic increment of $\Delta \sigma_{m}$ or $\Delta \sigma_{3}$ would exhibit an actual trend of excess pore pressure induced by deviatoric stress, even though the magnitude is different. When $b_{\sigma}$ is changed during shearing, consideration of $\Delta \sigma_{3}$ may result in misleading interpretations of soil behaviour and consideration of $\Delta \sigma_{m}$ would enable proper assessment of dilation vs. contraction based on the excess pore pressure.

The undrained response of sand has been reported to be independent of the total stress path at fixed $\alpha_{\sigma}$ and $b_{\sigma}$. The experiments carried out for this research study confirm the above findings. In addition, it expand the above concepts that undrained effective stress paths, and stress-strain relations are independent of total stress paths even under generalized loading conditions. 
Excess pore pressure generated in a soil is expressed as a function in terms of hydrostatic stress increment and deviatoric stress increment. Different types of formulations (Terzaghi et al., 1996; Henkel, 1960; Henkel and Wade, 1966) have been introduced in the theory of soil mechanics. In these formulations, the excess pore pressure induced by deviatoric stress is obtained by multiplying the deviatoric stress component by pore pressure parameter that is estimated experimentally. The pore pressure parameters are independent of total stress paths, in which $b_{\sigma}$ and $\alpha_{\sigma}$ are controlled same manner. Even though, the formulations of excess pore pressure are quite different from each other, all formulations can be applicable to three-dimensional loading on saturated soils regardless of total stress paths.

\subsection{Recommendations for future research}

The research study explored different topics that have important theoretical and practical implications. The scope was limited to the assessment of specific issues, and many other aspects of these problems are still unknown. The followings are recommended for further work.

- Extensive studies on effect of $b_{\sigma}, \alpha_{\sigma}, K_{c}$ and $\varsigma$ can be performed to confidently interpret the soil behaviour under pressure-volume coupled deformation. The strain paths imposed on soils to study the pressure-volume coupled deformation can be more realistic considering the field deformation.

- Cyclic behaviour of sand under generalized loading can be studied to further improve the understanding on this area. In addition, an extensive investigation can be performed to clearly understand the effect of nature of principal stress rotation on soil behaviour. 


\section{REFERENCES}

Arthur, J.R.F., Chua, K.S., and Dunstan, T., (1977), "Induced Anisotropy in a Sand", Geotechnique, Vol. 27(1), pp. 13-30.

Arthur, J.R.F., Chua, K.S., Dunstan, T., and del Camino, J.R., (1980), "Principal Stress Rotation: A Missing Parameter", Journal of the Geotechnical Engineering Division, ASCE, Vol. 106(4), pp. 419-433.

Arthur, J.R.F., and Menzies, B.K., (1972), Correspondence on "A New Soil Testing Apparatus", Geotechnique, Vol. 18(2), pp. 271-272.

Arthur, J.R.F., and Menzies, B.K., (1972), "Inherent Anisotropy in Sand", Geotechnique, Vol. 22 (1), pp. 115-128.

ASTM. 2001a. Standard Test Methods for Maximum Index Density and Unit Weight of Soils Using a Vibratory Table (D-4253-00). Annual Book of ASTM Standards, American Society for Testing and Materials, Philadelphia, PA. Vol. 04.08.

ASTM. 2001b. Standard Test Methods for Minimum Index Density and Unit Weight of Soils and Calculation of Relative Density (D-4254-00). Annual Book of ASTM Standards, American Society for Testing and Materials, Philadelphia, PA. Vol. 04.08 .

Atkinson, J.H., and Evans, J.S., (1985), Discussion on "The Measurement of Soil Stiffness in the Triaxial Apparatus", Geotechnique, Vol. 35(3), pp. 378-380.

Barden, L., and Khayatt, A.J., (1966), "Incremental Strain Rate Ratios and Strength of Sand in the Triaxial Test", Geotechnique, Vol. 16(4), pp. 338-357.

Been, K., Jefferies, M.G., and Hachey, J., (1991), "The Critical State of Sands", Geotechnique, Vol. 41(3), pp. 365-381.

Bishop, A.W., (1966), "Strength of Soils as Engineering Materials", $6{ }^{\text {th }}$ Rankine Lecture, Geotechnique, Vol. 16(2), pp. 91-128.

Bishop, A.W., (1971), "Shear Strength Parameters for Undisturbed and Remolded Soil Specimens", Proceedings of Roscoe Memorial Symposium on Stress-Strain Behaviour of Soils" Cambridge University, pp. 3-58.

Bishop, A.W., and Green, G.E., (1965), "The Influence of End Restraint on the Compression Strength of a Cohesionless Soil", Geotechnique, Vol. 15(3), pp. 243-266. 
Bishop, A. W., and Wesley, L. D., (1975), “A Hydraulic Triaxial Apparatus for Controlled Stress Path Testing", Geotechnique, Vol. 25(4), pp. 657-670.

Bjerrum, L., and Landva, A., (1966), "Direct Simple-Shear Tests on a Norwegian Quick Clay", Geotechnique, Vol. 16(1), pp. 1-20.

Boulanger, R. W., Chan, C.K., Seed, H.B., and Seed, R.B., (1993), “A Low-Compliance Bi-Directional Cyclic Simple Shear Apparatus", Geotechnical Testing Journal, Vol. 16(1), pp. 36-45.

Broms, B.B., and Casbarian, A.O., (1965), "Effects of Rotation of the Principal Stress Axes and of the Intermediate Principal Stress on the Shear Strength", Proceedings of $6^{\text {th }}$ International Conference on Soil Mechanics and Foundation Engineering, Vol. 1, pp. 179-183.

Budhu, M., (1984), "Nonuniformities Imposed by Simple Shear Apparatus", Canadian Geotechnical Journal, Vol. 21(2), pp. 125-137.

Chaney, R., and Mulilis, J. P., (1978), "Suggested Method for Soil Specimen Remolding by Wet-Raining", Geotechnical Testing Journal, ASTM, Vol. 1(2), pp. 107-108.

Casabarian, A.O.P., and Jamal, A.K., (1963), "Discussion on Study of Failure Envelope of Soils", Journal of Soil Mechanics and Foundation Engineering, ASCE, Vol. 89(4), pp. 243-245.

Casagrande, A., (1975), "Liquefaction and Cyclic Deformation of Sands: a Critical Review", Proceedings of $5^{\text {th }}$ Pan-American Conference on Soil Mechanics and Foundation Engineering, pp. 79-133.

Casagrande, A., and Carrillo, N., (1944), "Shear Failure of Anisotropic Materials", Journal of the Boston Society of Civil Engineers, Vol. 31(4), pp. 122-135.

Castro, G., (1969), "Liquefaction of Sands", PhD Thesis, Harvard University, Cambridge, Massachusetts, 231p.

Castro, G., (1987), "On the Behaviour of Soils during the Earthquakes-Liquefaction", Proceedings of Soil Dynamics and Liquefaction, No. 42. Edited by A.S. Cakmak. Elsevier, pp. 169-204.

Castro, G., and Poulos, S.J., (1977), "Factors Affecting Liquefaction and Cyclic Mobility", Journal of Geotechnical Engineering Division, ASCE, Vol. 103(6), pp. 501-516. 
Castro, G., Poulos, S.J., France, J.W., and Enos, J.L., (1982), "Liquefaction Induced by Cyclic Loading", Report submitted to the National Science Foundation, March 1982.

Chern, J.C., (1985), "Undrained Response of Sands with Emphasis on Liquefaction and Cyclic Mobility", PhD Thesis, University of British Columbia, Vancouver, Canada, 213p.

Chu, J., Lo, S. C. R., and Lee, I. K., (1992), "Strain Softening Behaviour of Granular Sands under Strain Path Testing", Journal of Geotechnical Engineering, ASCE, Vol. 118(2), pp. 191-208.

Costa Filho, L.M., and Kupper, A.M.G., (1983), "A Study of Load Cell Deformability in Triaxial Tests", (in Portuguese), Solose Rochas, Brazilian Association of Soil Mechanics, Vol. 6(1), pp. 29-36.

Duncan, J.M., and Dunlop, P, (1969), "Behaviour of Soils in Simple Shear Tests", Proceedings of $7^{\text {th }}$ International Conference on Soil Mechanics and Foundation Engineering, Vol. 1, pp. 101-108.

Duncan, J.M., and Seed, H.B., (1966), "Anisotropy and Stress Reorientation in Clay", Journal of Soil Mechanics and Foundations Division, ASCE, Vol. 92(5), pp. 2150.

Dyvik, R., Berre, T., lacasse, S., and Raadim, B., (1987), "Comparison of Truly Undrained and Constant Volume Direct Simple Shear Tests", Geotechnique, Vol. 37(1), pp. 3-10.

Eliadorani, A., (2000), "The Response of Sands under Partially Drained States with Emphasis on Liquefaction", $P h D$ Thesis, University of British Columbia, Vancouver, Canada.

El-Ruwayih, A.A., (1975), "Stress-Strain Characteristics of Rockfill and Clay under High Porewater Tension", PhD Thesis, University of London, England.

El-Sohby, M.A., and Andrawes, K.Z., (1972), "Deformation Characteristics of Granular Materials under Hydrostatic Compression", Canadian Geotechnical Journal, Vol. 9, pp. 338-350. 
Ergun, M.U., (1981), "Evaluation of Three-Dimensional Shear Testing", Proceedings of $10^{\text {th }}$ International Conference on Soil Mechanics and Foundation Engineering, Vol. 1, pp. 593-596.

Field, W.G., (1963), "Towards the Statistical Definition of Granular Mass", in Proceedings of $4^{\text {th }}$ American and New Zealand Conference on Soil Mechanics, Vol. 1, pp. 143-148.

Finn, W.D.L., Pickering, J., and Bransby, P.L., (1971), "Sand Liquefaction in Triaxial and Simple Shear Test", Journal of Soil Mechanics and Foundations Division, ASCE, Vol. 97(4), pp. 839-851.

Finn, W. D. L., and Vaid, Y.P., (1977), "Liquefaction Potential from Drained Constant Volume Simple Shear Tests", Proceedings of $6^{\text {th }}$ World Conference on Earthquake Engineering, Vol. 1, pp. 2157-2162.

Finn, W. D. L., Vaid, Y.P., and Bhatia, K.K., (1978), "Constant Volume Simple Shear Testing", Proceedings of $2^{\text {nd }}$ International Conference on Microzonation for Safer Construction, Vol. 2, pp. 839-851.

Franke, E., Kiekbusch, M., and Schuppener, B., (1979), "A New Direct Simple Shear Device", Geotechnical Testing Journal, Vol. 2(4), pp. 190-199.

Frydman, S., Zeitlen, J.G., and Alpan, I., (1971), “The Membrane Effect in Triaxial Testing of Granular Soils", Journal of Testing and Evaluation, Vol. 1(1), pp. 3741.

Gananathan, N., (2002). "Partially Drained Response of Sands", M.A.Sc. Thesis, The University of British Columbia, Vancouver, Canada.

Green, G.E., (1971), "Strength and Deformation of Sand Measured in an Independent Stress Control Cell", Proceedings of Roscoe Memorial Symposium on StressStrain Behaviour of Soils, Cambridge University, pp. 285-323

Ha, D., (2003), "Effect of Initial Stress State on the Undrained Cyclic Behaviour of Sands", M.A.Sc. Thesis, Carleton University, Ottawa, Canada.

Habib, P., (1953), "Influence of the Variation of the Average Principal Stress upon the Shearing Strength of Soils", (in French), Proceedings of $3^{\text {rd }}$ International Conference on Soil Mechanics and Foundation Engineering, Vol. 1, pp. 131-136. 
Hansen, J.B., and Gibson, R.E., (1948), "Undrained Shear Strength of Anisotropically Consolidated Clays", Geotechnique, Vol. 1(3), pp. 189-204.

Haruyama, M., (1981), "Anisotropic Deformation-Strength Characteristics of an Assembly of Spherical Particles under 3-D Stresses", Soils and Foundations, Vol. 21(4), pp. 41-55.

Haruyama, M., (1985), "Drained Deformation - Strength Characteristics of Loose Shirasum (Volcanic Sandy Soil) Under Three Dimensional Stresses", Soils and Foundations, Vol. 25(1), pp. 65-76.

Hatanaka, M., Sugimoto, M., and Suzuki, Y., (1985), "Liquefaction Resistance of Two Alluvial Volcanic Soils Sampled by In-situ Freezing", Soils and Foundations, Vol. 25(3), pp. 49-63.

Henkel, D. J., (1960), "The Shear Strength of Saturated Remoulded Clays", Proceedings of ASCE Research Conference on Shear Strength of Cohesive Soils, Boulder, Colo., New York, pp. 533-554.

Henkel, D.J., and Wade, N.H., (1966). "Plane Strain Tests on Remoulded Clay", Journal of the Soil Mechanics and Foundation Division, ASCE, Vol. 92(6), pp. 67-80.

Hight, D.W., Gens, A., and Symes, M.J., (1983), “The Development of a New Hollow Cylinder Apparatus for Investigating the Effects of Principal Stress Rotation in Soils", Geotechnique, Vol. 33(4), pp. 355-383.

Hight, D.W., Shibuya, S., and Symes, M.J., (1988), Discussion on "The Engineering Application of Direct and Simple Shear Testing", Geotechnique, Vol. 38(1), pp. 139-140.

Hosseini, S.M.M., and Cousens, T.W., (1988), "A Simple Cubic Triaxial Test Apparatus", Symposium on New Concepts in Field and Laboratory Tests, Rio de Janeiro, Vol. 1, pp. 85-96.

Ishihara, K., (1993), "Liquefaction and Flow Failure during Earthquakes", Geotechnique, Vol. 43(3), pp. 351-415.

Ishihara, K., Tatsuoka, F., and Yasuda, S., (1975), "Undrained Deformation and Liquefaction of Sands under Cyclic Stresses", Soils and Foundations, Vol. 15(1), pp. 29-44. 
Ishihara, K., and Yamazaki, F., (1980), "Cyclic Simple Shear Tests on Saturated Sand in Multi-Directional Loading", Soils and Foundations, Vol. 20(1), pp. 45-59.

Jardine, R.J., Symes, M.J., and Burland, J.B., (1985), Reply on "The Measurement of Soil Stiffness in the Triaxial Apparatus", Geotechnique, Vol. 35, (3), pp. 380-382.

Kallstenius, T., and Bergau, W., (1961), "Research on the Texture of Granular Masses" Proceedings of $5^{\text {th }}$ international conference on Soil Mechanics and Foundation Engineering, Vol. 1, pp. 165-170.

Kammerer, A.M., (2002), "Undrained Response of Monterey 0/30 sand under Multidirectional Cyclic Simple Shear Loading Conditions", PhD Thesis, University of California, Berkeley, U.S.

Kjellman, W., (1936), "Report on an Apparatus for Consummate Investigation of the Mechanical Properties of Soils", Proceedings of $1^{\text {st }}$ International Conference on Soil Mechanics and Foundation Engineering, Vol. 2, pp. 16-20.

Kokusho, T., (2003), "Current State of Research on Flow Failure Considering Void Redistribution in Liquefied Deposits", Soil Dynamics and Earthquake Engineering, Elsevier, Vol. 23, pp. 585- 603.

Kolbuszewski, J.J., (1948), "An Experimental Study of the Maximum and Minimum Porosities of Sands", Proceedings of $2^{\text {nd }}$ International Conference on Soil Mechanics and Foundation Engineering, Vol. 1, pp. 158-165.

Ko, H.Y., and Scott, R.F., (1967), “A New Soil Testing Apparatus”, Geotechnique, Vol. $17(1)$, pp. 40-57.

Kramer, S.L., and Seed, H.B., (1988), "Initiation of Soil Liquefaction under Static Loading Conditions", Journal of Geotechnical Engineering, ASCE, Vol. 114(4), pp. $412-430$.

Kuerbis, R. H., (1989), "Effect of Gradation and Fines Content on the Undrained Response of Sand", MASc Thesis, University of British Columbia, Vancouver, Canada, 292p.

Kuerbis, R., Negussey, D., and Vaid, Y.P., (1988), "Effect of Gradation and Fines Content on the Undrained Response of Sand", Proceedings of Hydraulic Fill Structures, Fort Collins, Colo., 15-18 August 1988. Geotechnical Special 
Publication No. 21. Edited by D.J.A. Van Zyl and S.G. Vick. American Society of Civil Engineers, New York. pp. 330-345.

Lacasse, S., and Vucetic, M., (1981), Discussion of "State of the Art: Laboratory Strength Testing of Soils", Symposium on Laboratory Shear Strength of Soil, ASTM, STP 740, pp. 633-637.

Ladd, R.S., (1978), “Preparing Test Specimens Using Undercompaction”, Geotechnical Testing Journal, ASTM, Vol. 1(1), pp. 16-23.

Ladd, C.C., Foot, R., Ishihara, K., Schlosser, F., and Poulos, H.G., (1977), "StressDeformation and Strength Characteristics", State of the Art Report, $9^{\text {th }}$ International Conference on Soil Mechanics and Foundation Engineering, Vol. 2, pp. 421-494.

Ladd, P.V., and Lambe, T.W., (1963), "The Strength of Undisturbed Clay Determined from Undrained Tests", Proceedings of Symposium on Laboratory Shear Testing of Soils, ASTM, Philadelphia, PA, STP 361, pp. 342-371.

Lade, P.V., (1978), "Cubical Triaxial Apparatus for Soil Testing”, Geotechnical Testing Journal, ASTM, Vol. 1(2), pp. 93-101.

Lade, P.V., (1982), "Localization Effects in Triaxial Tests on Sand", Proceedings of IUTAM Symposium on Deformation and Failure of Granular Materials, Edited by P.A. Vermeer and H.J. Luger, Balkema, Rotterdam, pp. 461-471.

Lade, P.V., (1992), "Static Instability and Liquefaction of Loose Fine Sandy Slopes", Journal of Geotechnical Engineering, ASCE, Vol. 118(1), pp. 51-71.

Lade, P.V., and Duncan, J.M., (1973), "Cubical Triaxial Tests on Cohesionless Soil”, Journal of the Soil Mechanics and Foundation Division, ASCE, Vol. 99(10), pp. 793-811.

Lade, P.V., and Duncan, J.M., (1975), "Elasto-Plastic Stress Strain Theory for Cohesionless Soil", Journal of the Soil Mechanics and Foundation Division, ASCE, Vol. 101(10), pp. 1037-1053.

Lade, P. V., Nelson, R. B., and Ito, Y. M., (1988), "Instability of Granular Materials with Non-associated Flow", Journal of Engineering Mechanics, ASCE, Vol. 114(12), pp. 2173-2191. 
Lade, P.V., and Wasif, U., (1988), "Effect of Height- to- Diameter ratio in Triaxial Specimens on the Cross Anisotropic Sand", Advanced Triaxial Testing of Soil and Rock, ASTM Special Technical Publication 977, Edited by R.T. Donaghe, R.C. Chaney, and M.L. Silver, American Society for Testing and Materials, Philadelphia, ASCE, pp. 706-714.

Lam, W.K., and Tatsuoka, F., (1988), "Effects of Initial Anisotropic Fabric and $\sigma_{2}$ on Strength and Deformation Characteristics of Sand", Soils and Foundations, Vol. 28(1), pp. 89-106.

Lambe, T. W., (1951), "Soil Testing for Engineers", John Wiley \& Sons Inc., New York, $165 \mathrm{p}$.

Law, K. T., and Holtz, R. D., (1978), “A Note on Skempton's A Parameter with Rotation of Principal Stresses", Geotechnique, Vol. 28(1), pp. 57-64.

Lee, K.L., and Seed, H.B., (1967), "Cyclic Stress Conditions Causing Liquefaction of Sand", Journal of Geotechnical Engineering Division, ASCE, 93(5), pp. 47-70.

Logeswaran, P., (2005), "Behaviour of Sands under Simultaneous Changes of Volume and Pore Pressure", MASc Thesis, Carleton University, Ottawa, Canada, 177p.

Love, A. E. H., (1927), "The Mathematical Theory of Elasticity", Cambridge University Press, Cambridge.

Menzies, B.K., (1987), “A Computer Controlled Hydraulic Testing System”, Proceedings of Symposium on Advanced Triaxial Testing, ASTM STP 977, Philadelphia, PA, Vol. 1, pp. 82-94.

Miura, K., Miura, S., and Toki, S., (1986), "Deformation Behaviour of Anisotropic Dense Sand under Principal Stress Axes Rotation", Soils and Foundations, Vol. 26(1), pp. $36-52$.

Miura, S., and Toki, S., (1982), "A Sample Preparation Method and Its Effect on Static and Cyclic Deformation - Strength Properties of Sand", Soils and Foundations, Vol. 22(1), pp. 61-77.

Miura, S., and Toki, S., (1984), “Anisotropy in Mechanical Properties and Its Simulation of Sands from Natural Deposits", Soils and Foundations, Vol. 24(3), pp. 69-84. 
Mohamad, R., and Dobry, R., (1986), "Undrained Monotonic and Cyclic Triaxial Strength of Sand", Journal of Geotechnical Engineering, ASCE, Vol. 112(10), pp. 941-958.

Mould, J.C., Sture, S., and Ko, H.Y., (1982), "Modeling of Elastic-Plastic Anisotropic Hardening and Rotating Principal Stress Directions in Sand", Proceedings of IUTAM Conference on Deformation and Failure of Granular Materials, Delft, pp. 431-439.

Nakata, Y., Hyodo, M., Murata. H., and Yasufuku, N., (1998), "Flow Deformation of Sands Subjected to Principal Stress Rotation", Soils and Foundations, Vol. 38(2), pp. 115-128.

Negussey, D., (1984), "An Experimental Study of the Small Strain Response of Sand", Ph.D. Thesis, Department of Civil Engineering, University of British Columbia, Vancouver, Canada.

Negussey, D., Wijewickreme, W.K.D., and Vaid, Y.P., (1988), "Constant Volume Friction Angle of Granular Materials", Canadian Geotechnical Journal, Vol. 25, pp. 50-55.

Noorany, I., and Seed, H.B., (1965), "In-situ Strength Characteristics of Soft Clays", Journal of Soil Mechanics and Foundation Engineering Division, ASCE, Vol. 91(2), pp. 49-80.

Oda, M., (1972a), "Initial Fabrics and Their Relations to Mechanical Properties of Granular Material", Soils and Foundations, Vol. 12(1), pp. 17 -36.

Oda, M., (1972b), “The Mechanism Fabric Changes during Compressional Deformation of Sand", Soils and Foundations, Vol. 12(2), pp. 1-18.

Oda, M., (1981). "Anisotropic Strength of Cohesionless Sands", Journal of the Geotechnical Engineering Division, ASCE, Vol. 107(9), pp.1219-1231.

Oda, M., Koishikawa, I., and Higuchi, T., (1978), "Experimental Study of Anisotropic Shear Strength of Sand by Plane Strain Test", Soils and Foundations, Vol. 18(1), pp. 25-38.

Oda, M., Nasser, S.N., and Konishi, J. (1985). "Stress-Induced Anisotropy in Granular Masses", Soils and Foundations, Vol. 25(3), pp. 85-97. 
Parkin, A.K., Gerrard, C.M., and Willoughby, D.R., (1968), "Discussion on Deformation of Sand in Shear", Journal of Soil Mechanics and Foundations Division, ASCE, Vol. 94(1), pp. 336-340.

Pearce, J.A., (1971), “A New Triaxial Apparatus", Proceedings of Roscoe Memorial Symposium on Stress-Strain Behaviour of Soils, Cambridge University, pp. 330339.

Phillips, A.B., and May, P.H., (1967), "A Form of Anisotropy in Granular Media", Special Task Report, Dept. of Civil and Municipal Engineering, University College, London.

Poulos, S.J., (1981), "The Steady State of Deformation", Journal of Geotechnical Engineering Division, ASCE, Vol. 107(5), pp. 553-562.

Poulos, S.J., Castro, G., and France, J.W., (1985), "Liquefaction Evaluation Procedure" Journal of Geotechnical Engineering, ASCE, Vol. 111(6), pp. 772-792.

Ramamurthy, T. and Rawat, P.C., (1973), "Shear Strength of Sand under General Stress System", Proceedings of $8^{\text {th }}$ International Conference on Soil Mechanics and Foundation Engineering, Vol. 1.2, pp. 339-342.

Reades, D.W., and Green, G.E., (1976), "Independent Stress Control and Triaxial Extension Tests on Sand", Geotechnique, Vol. 26(4), pp. 551-576.

Rendulic, L., (1936), "Relation between Void Ratio and Effective Principals Stress for a Remoulded Silty Clay, Proceedings of $1^{\text {st }}$ International Conference on Soil Mechanics, Boston, Mass., 22-26 June 1936, Delft Geotechnics, Delft, the Netherlands, pp. 48-51.

Riemer, M.F., and Seed, R.B., (1997), "Factors Affecting Apparent Position of Steady State Line", Journal of Geotechnical Engineering, ASCE, Vol. 123(3), pp. 271288.

Roscoe, K.H., (1953), “An Apparatus for Application of Simple Shear to Soil Samples”, Proceedings of $3^{\text {rd }}$ International Conference on Soil Mechanics and Foundation Engineering, Vol. 1, pp. 186-191.

Roscoe, K.H., Schofield, A.N. and Worth, C.P., (1958), "On the Yielding of soil" Geotechnique, Vol. 8(1), 27-53. 
Rowe, P.W., and Barden, L., (1964), "The Importance of Free Ends in the Triaxial Test", Journal of Soil Mechanics and Foundation Engineering Division", ASCE, 90(1), pp. 1-27.

Saada, A.S., (1981), Discussion on "Undrained Deformation Characteristics of Loose Sand under 3-D Stress Conditions", Soils and Foundations, Vol. 21(4), pp. 121.

Saada, A.S., and Baah, A.K., (1967), "Deformation and Failure of a Cross Anisotropic Clay under Combined Stress", Proceedings of $3^{\text {rd }}$ Pan-American Conference on Soil Mechanics and Foundation Engineering, Vol. 1, pp. 67-88.

Saada, A.S., and Bianchini, G.F., (1978), Discussion on "Three-Dimensional Behaviour of Remolded Clay", Journal of the Geotechnical Engineering Division, ASCE, Vol. 104(12), pp. 1537-1540.

Saada, A.S., and Puccini, P., (1985), Discussion on "The Development of a New Hollow Cylinder Apparatus for Investigating the Effects of Principal Stress Rotation in Soils", Geotechnique, Vol. 35(1), pp. 78-85.

Saada, A.S., and Townsend, F.C., (1981), "State of the Art: Laboratory Strength Testing of Soils", Symposium on Laboratory Shear Strength of Soil, ASTM Special Technical Publication 740, pp. 7-77.

Sarsby, R.W., Kalteziotis, N., and Haddad, E.H., (1980), "Bedding Error in Triaxial Tests on Granular Media", Geotechnique, Vol. 30(3), pp. 302-309.

Sayao, A.S.F., (1989), "Behaviour of Sand under General Stress Paths in the Hollow Cylinder Torsional Device", PhD Thesis, University of British Columbia, Vancouver, Canada, 196p.

Sayao, A.S.F., and Vaid, Y.P., (1991), "A Critical Assessment of Stress Nonuniformities in Hollow Cylinder Test Specimens", Soil and Foundations, Vol. 31(1), pp. 6072.

Seed, H.B., (1979), "Soil Liquefaction and Cyclic Mobility Evaluation for Level Ground during Earthquakes", Journal of Geotechnical Engineering Division, ASCE, Vol. 105(2), pp. 201-225.

Seed, H.B., Mori, K., and Chan, C.K., (1977), "Influence of Seismic History on Liquefaction of Sands", Journal of Geotechnical Engineering Division, Vol. 103(4), pp. 257-269. 
Seed, H.B., Singh, S., Chan, C.K., and Vilela, T.F., (1982), "Consideration in Undisturbed Sampling of Sands", Journal of the Geotechnical Engineering Division, ASCE, Vol. 108(2), pp. 265-283.

Shankariah, B., and Ramamurthy, T., (1980), "Strength and Deformation Behaviour of Sand under General Stress System", Proceedings of $3^{\text {rd }}$ Australia-New Zealand Conference on Geomechanics, Wellington, Vol. 1, pp. 207-212.

Shibuya, S., (1988), “A Servo System for Hollow Cylinder Torsional Testing of Soils", Geotechnical Testing Journal, ASTM, Vol. 11(2), pp. 109-118.

Shibuya, S., and Hight, D.W., (1987), "A Bounding Surface for Granular Materials", Soils and Foundations, Vol. 27(4), pp. 123-136.

Sivakugan, N., Chameau, J.L., Holtz, R.D., and Altschaeffl, A.G., (1988), "ServoControlled Cubical Shear Device”, Geotechnical Testing Journal, ASTM, Vol. 11(2), pp. 119-124.

Sivathayalan, S., (2000), "Fabric Initial State and Stress Path Effects on Liquefaction Susceptibility of Sands, PhD Thesis, University of British Columbia, Vancouver, Canada, 307p.

Sivathayalan, S., and Logeswaran, P., (2007), "Behaviour of Sands under Generalized Drainage Boundary Conditions", Canadian Geotechnical Journal, Vol. 44(2), pp. 138-150.

Sivathayalan, S., and Logeswaran, P., (2008), "Experimental Assessment of the Response of Sands under Shear-Volume Coupled Deformation", Canadian Geotechnical Journal, Vol. 45(9), pp.1310-1323.

Sivathayalan, S., and Vaid, Y.P., (2002), "Influence of Generalized Initial State and Principal Stress Rotation on the Undrained Response of Sands", Canadian Geotechnical Journal, Vol. 39(1), pp. 63-76.

Skempton, A.W., (1954), "The pore Pressure Coefficients A and B", Geotechnique, Vol. 4, pp. 143.

Skempton, A. W., (1961), "Horizontal Stresses in Overconsolidated London Clay", Proceedings of $5^{\text {th }}$ International Conference on Soil Mechanics and Foundation Engineering, Paris, Vol. 1, pp. 351-357. 
Sladen, J.A., D’Hollander, R.D., and Krahn, J., (1985), "The Liquefaction of Sands- A Collapse Surface Approach", Canadian Geotechnical Journal, Vol. 22 (4), pp. 564-578.

Smith, W.O., (1932), "Capillary Flow through an Ideal Uniform Soil”, Journal of Applied Physics, Vol. 3 (3), pp. 139-146.

Smith, P. R., Jardine, R. J., and Hight, D. W., (1992), “The Yielding of Bothkennar Clay", Geotechnique, Vol. 42(2), pp. 257-274.

Stedman, D., (1997), "Effects of Confining Pressure and Static Shear on Liquefaction Resistance of Fraser River Sand", MASc Thesis, University of British Columbia, Vancouver, Canada, 93p.

Stokoe, II, K.H., and Nazarian S., (1985), "Use of Rayleigh Wave in Liquefaction Studies, Proceedings of Measurement and Use of Shear Wave Velocity for Evaluating Dynamic Soil Properties", Edited by R. D. Woods, ASCE, New York, 1-17.

Sture, S., and Desai, C.S., (1979), "Fluid Cushion Truly Triaxial or Multiaxial Testing Devices", Geotechnical Testing Journal, Vol. 2(1), pp 20-33.

Sukumaran, B., Leonards, G.A., and Fox, P.J., (1996), Discussion on "Liquefaction and Post Liquefaction Behaviour of Sand", Journal of Geotechnical Engineering, Vol. 122(2), pp. 502-503.

Sutherland, H.B., and Mesdary, M.S., (1969), "The Influence of the Intermediate Principal Stress on the Strength of Sand", Proceedings of $7^{\text {th }}$ International Conference on Soil Mechanics and Foundation Engineering, Vol. 1, pp. 391-399.

Symes, M.J., Gens, A. and Hight, D.W. (1984), "Undrained Anisotropy and Principal Stress Rotation in Saturated Sand", Geotechnique, Vol. 34(1), pp. 11-27.

Symes, M.J., Gens, A., and Hight, D.W., (1988), "Drained Principal Stress Rotation in Saturated Sand", Geotechnique, Vol. 38(1), pp. 59-81.

Symes, M.J., Hight, D.W., and Gens, A., (1982), "Investigating Anisotropy and the Effects of Principal Stress Rotation and of the Intermediate Principal Stress Using a Hollow Cylinder Apparatus", Proceedings of IUTAM Conference on Deformation and Failure of Granular Materials, Delft, pp. 441-449. 
Symes, M.J., Shibuya, S., Hight, D.W. and Gens, A. (1985), "Liquefaction with Cyclic Principal Stress Rotation", Proceedings of $11^{\text {th }}$ International Conference on Soil Mechanics and Foundation Engineering, San Francisco, Vol. 4, pp. 1919-1922.

Taylor, D.W., (1948), "Fundamental of Soils mechanics" John Wiley \& Sons, New York, $700 \mathrm{p}$.

Terzaghi, K. and Peck, R.B., (1948), "Soil Mechanics in Engineering Practice", $2^{\text {nd }}$ edition, John Wiley and sons, New York.

Terzaghi, K, Peck, R.B., and Mesri, G. (1996). "Soil Mechanics in Engineering Practice", $3^{\text {rd }}$ Edition, New York, John Wiley \& Sons Inc., 504p.

Thomas, J., (1992), "Static, Cyclic and Post Liquefaction Behaviour of Fraser River Sand", M.A.Sc. Thesis, University of British Columbia, Vancouver, Canada, 117p.

Uthayakumar, M., (1995), "Liquefaction of Sands under Multi-axial Loading", $P h D$ Thesis, University of British Columbia, Vancouver, Canada, 185p.

Uthayakumar, M., and Vaid, Y.P. (1998), "Static Liquefaction of Sands under Multiaxial Loading”, Canadian Geotechnical Journal, Vol. 35(2), pp. 273-283.

Vaid, Y.P., and Campanella, R.G., (1974), “Time Dependent Behaviour of Undisturbed Clay", Journal of Geotechnical Engineering Division, ASCE, Vol. 103(7), pp. 693-709.

Vaid, Y.P., and Finn, W.D.L., (1978), "Static Shear and Liquefaction Potential", Journal of Geotechnical Engineering Division, ASCE, Vol. 105(10), pp1233-1246.

Vaid, Y.P., and Chern, J.C., (1983), "Mechanism of Deformation during Undrained Loading of Saturated Sand", International Journal of Soil Dynamics and Earthquake Engineering, Vol. 2(3), pp. 171-177.

Vaid, Y.P., and Chern, J.C., (1985), "Cyclic and Monotonic Undrained Response of Sands", Proceedings of Advances in the Art of Testing Soils under Cyclic Loading Conditions, Detroit, pp. 120-147.

Vaid, Y.P., Chung, E.K.F., and Kuerbis, R.H., (1989), "Preshearing and Undrained Response of sands", Soils and Foundations, Vol. 29(4), pp. 49-61.

Vaid, Y.P., Chung, E.K.F., and Kuerbis, R.H., (1990a), "Stress Path and Steady State", Canadian Geotechnical Journal, Vol. 27(1), pp. 1-7. 
Vaid, Y.P., and Eliadorani, A., (1998), "Instability and Liquefaction of Granular Soils under Undrained and Partially Drained States", Canadian Geotechnical Journal, Vol. 35(6), pp. 1053-1062.

Vaid, Y.P., and Eliadorani, A., (2000), "Undrained and Drained (?) Stress-Strain Response", Canadian Geotechnical Journal, Vol. 37(5), pp. 1126-1130.

Vaid, Y.P., Sayao, A., Enhuang, H. and Negussey, D., (1990b), “Generalized Stress-Path Dependent Soil Behaviour with a New Hollow Cylinder Torsional Apparatus", Canadian Geotechnical Journal, Vol. 27(5), pp. 601-616.

Vaid, Y.P., and Negussey, D., (1988), "Preparation of Reconstituted Sand Specimens", Proceedings of Symposium on Advanced Triaxial Testing of Soil and Rock, ASTM Special Technical Publication 977, Philadelphia, pp. 405-417.

Vaid, Y.P., and Sivathayalan, S., (1996), "Static and Cyclic Liquefaction Potential of Fraser Delta Sand in Simple Shear and Triaxial Tests", Canadian Geotechnical Journal, Vol. 33(2), pp. 281-289.

Vaid, Y.P., Sivathayalan, S., and Stedman, D., (1999), "Influence of Specimen Reconstitution Method on Undrained Response of Sand", Geotechnical Testing Journal, ASTM, Vol. 22(3), pp. 187-195.

Vaid, Y.P., and Sivathayalan, S., (2000), "Fundamental Factors Affecting Liquefaction Susceptibility of Sands", Canadian Geotechnical Journal, Vol. 37, pp. 592-606.

Vaid, Y.P., Stedman, J.D., and Sivathayalan, S. (2001), "Confining Stress and Static Shear Effects in Cyclic Liquefaction", Canadian Geotechnical Journal, Vol. 38(3), pp. 580-591.

Vaid, Y.P., and Thomas, J., (1994), "Post Liquefaction Behaviour of Sand", Proceedings of $13^{\text {th }}$ International Conference on Soil Mechanics and Foundation Engineering, New Delhi, India, pp. 1305-1310.

Vaid, Y.P., and Thomas, J., (1995), "Liquefaction and Post Liquefaction of Sand", Journal of Geotechnical Engineering", Vol. 121(2), pp. 163-173.

Vaid, Y.P., and Thomas, J., (1996), Closure on "Liquefaction and Post Liquefaction of Sand", Journal of Geotechnical Engineering, Vol. 122(2), pp. 503-504.

Vaughan, P. R., Davachi, M. M., El-Ghamrawy, M. K., Hamza, M. M., and Hight, D.W., (1976), "Stability Analysis of Large Gravity structures", Proceedings of $l^{s t}$ 
International Conference on Behaviour of Off-Shore Structures, Trondheim, Norway, Vol. 1, pp. 467-487.

Wijewickreme, D., (1990), "Behaviour of Sand under Simultaneous Increase in Stress Ratio and Principal Stress rotation", Ph.D. Thesis, University of British Columbia, Canada, 225p

Wijewickreme, D., and Vaid, Y.P., (1991), "Stress Nonuniformities in Hollow Cylinder Torsional Specimen", Geotechnical Testing Journal, Vol. 14(4), pp. 349-362.

Wong, R.K.S., and Arthur, J.R.F., (1985), "Induced and Inherent Anisotropy in Sand", Geotechnique, Vol. 35, (4), pp. 471-481.

Yamada, Y. and Ishihara, K., (1979), "Anisotropic Deformation Characteristics of Sand under Three Dimensional Stress Conditions", Soils and Foundations, Vol. 19(2), pp. 79-94.

Yoshimi, Y., Hatanaka, M., and Oh-Oka, H., (1977), “A Simple Method for Undisturbed Sampling by Freezing", International Conference on Soil Mechanics and Foundation Engineering, Vol. 9(1), pp. 23-28.

Yoshimi, Y., Hatanaka, M., and Oh-Oka, H., (1978), "Undisturbed Sampling of Saturated Sands by Freezing", Soil and Foundations, Vol. 18(3), pp. 59-73.

Yoshimi, Y., Tokimatsu, K., and Hosaka, Y., (1989), "Evaluation of Liquefaction Resistance of Clean Sands Based on High Quality Undisturbed Samples", Soils and Foundations, Vol. 29(1), pp. 93-104.

Yoshimine, M., Ishihara, K., and Vargas, W., (1998), "Effects of Principal Stress Direction and Intermediate Principal Stress on Undrained Shear Behaviour of Sand", Soils and Foundations, Vol. 38(3), pp. 179-188. 


\section{Appendix (Model view of HCT DAQ Program)}




\begin{tabular}{|c|c|c|c|c|}
\hline \multirow{3}{*}{\multicolumn{2}{|c|}{ 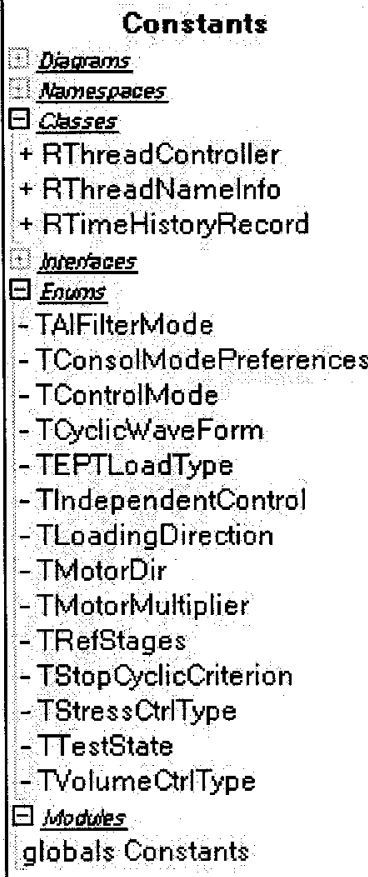 }} & \multirow{3}{*}{\multicolumn{2}{|c|}{ 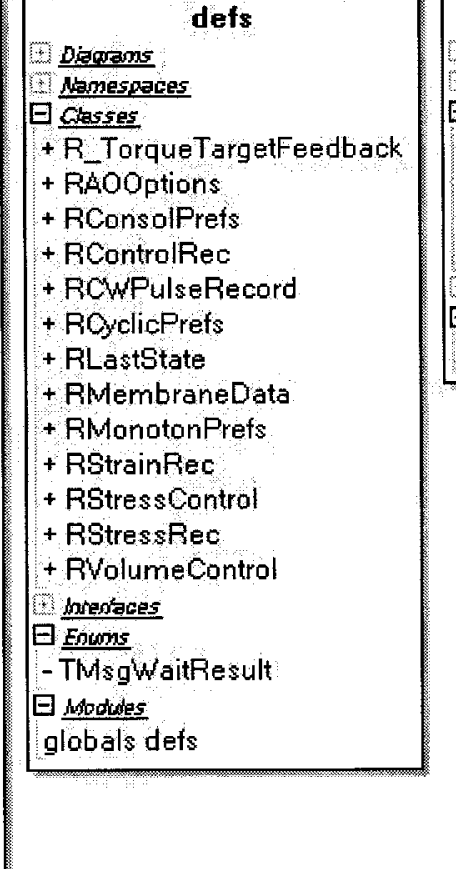 }} & \multirow{3}{*}{ 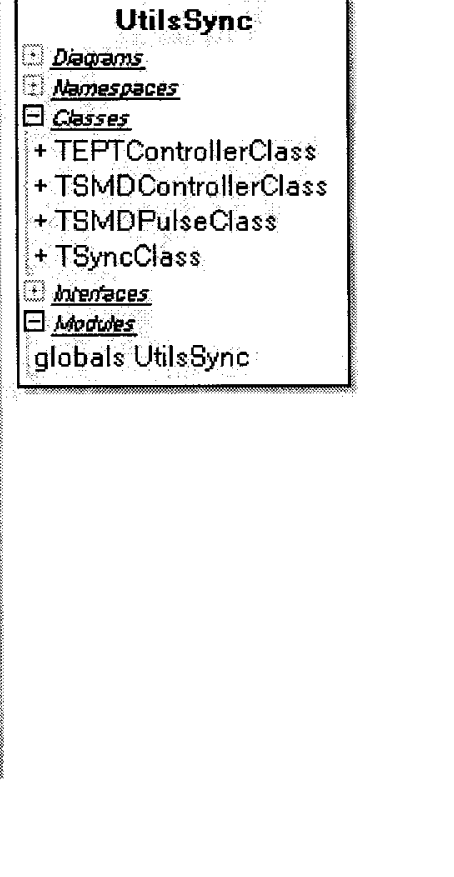 } \\
\hline & & & & \\
\hline & & & & \\
\hline & & & & \\
\hline \multicolumn{2}{|c|}{ 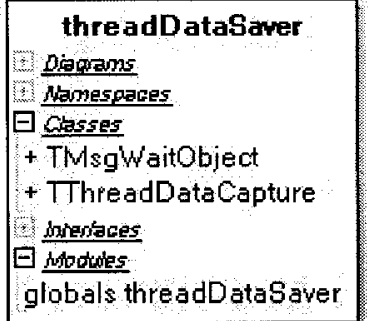 } & 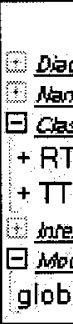 & $\begin{array}{l}\text { UtilsStack } \\
\text { sps } \\
\text { saces } \\
\text { nerRecoid } \\
\text { eadSafeTimerRecordStack } \\
\frac{\text { ges }}{\text { s UtilsStack }}\end{array}$ & 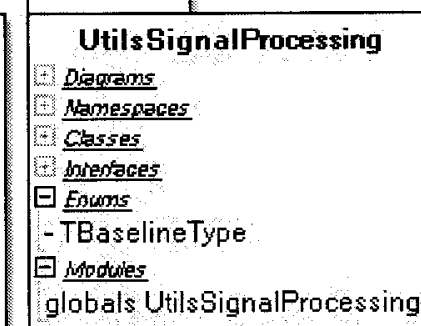 \\
\hline 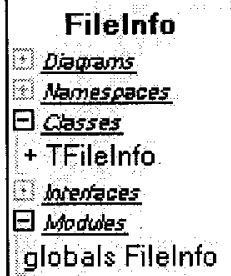 & \multicolumn{2}{|c|}{ 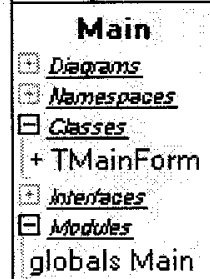 } & 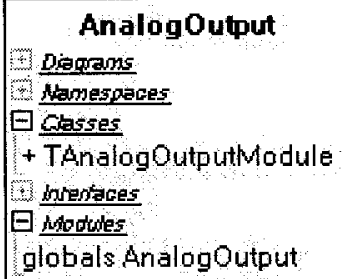 & 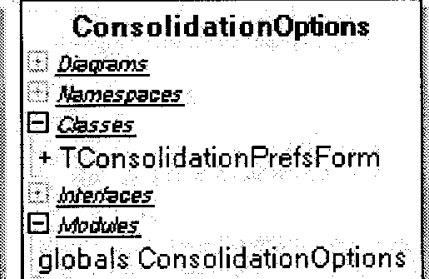 \\
\hline
\end{tabular}

Figure 1 Modules of Data Acquisition program of the HCT device - 1 


\begin{tabular}{|c|}
\hline 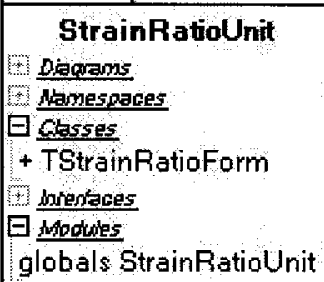 \\
\hline
\end{tabular}

\begin{tabular}{|c|}
\hline 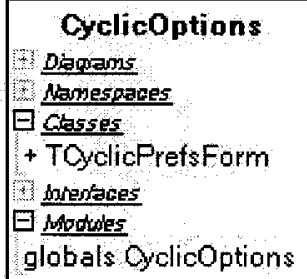 \\
\hline
\end{tabular}

\begin{tabular}{|c|}
\hline 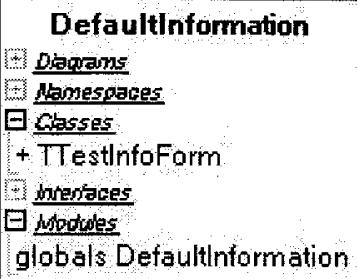 \\
\hline
\end{tabular}

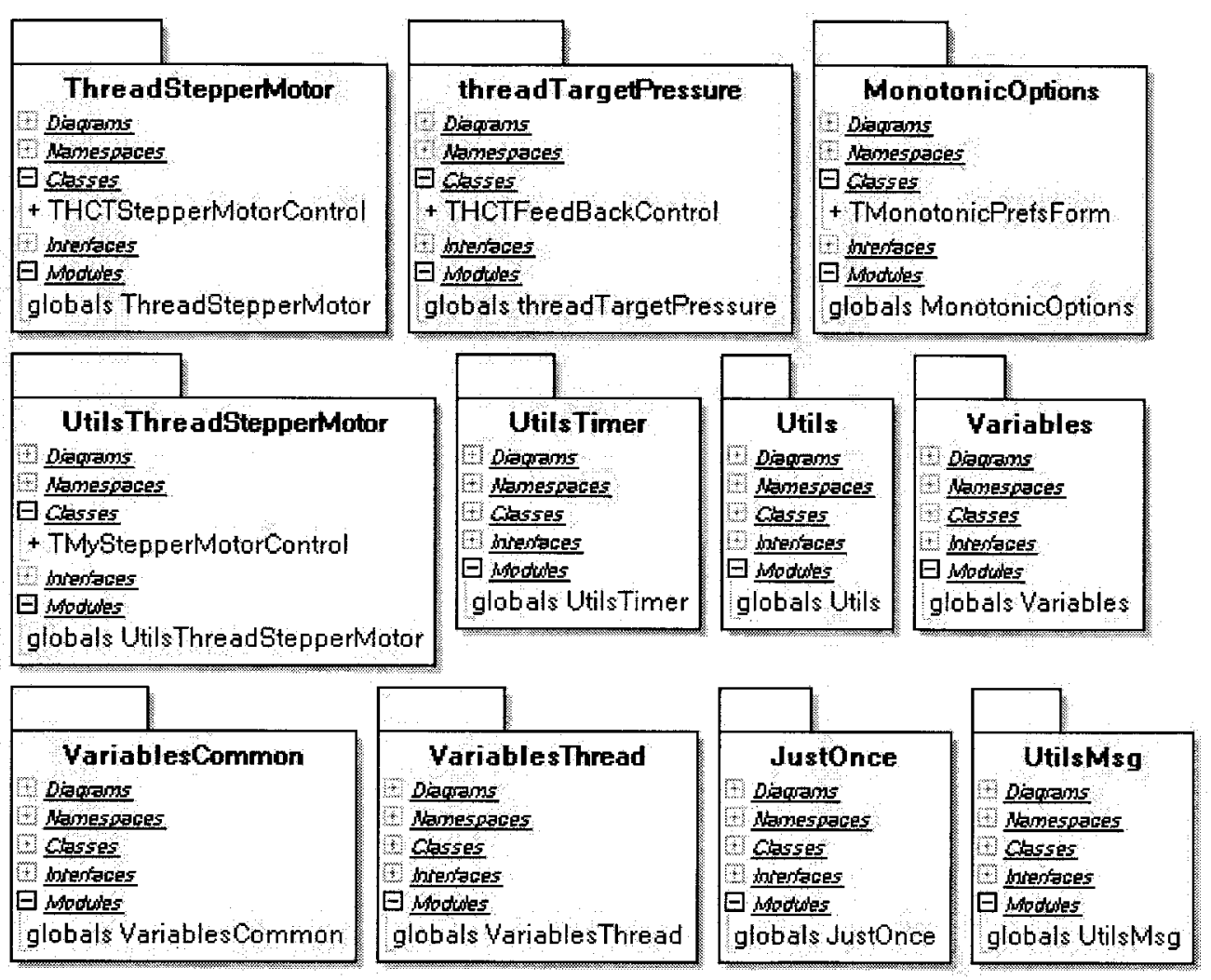

Figure 2 Modules of Data Acquisition program of the HCT device - 2 


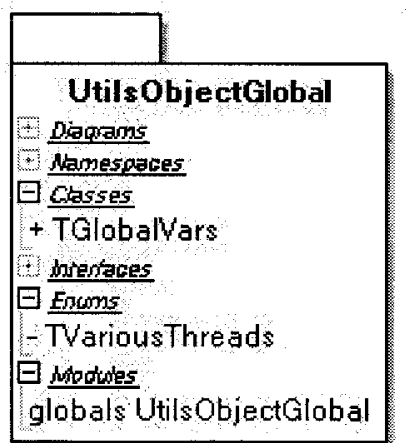

\begin{tabular}{|c|}
\hline 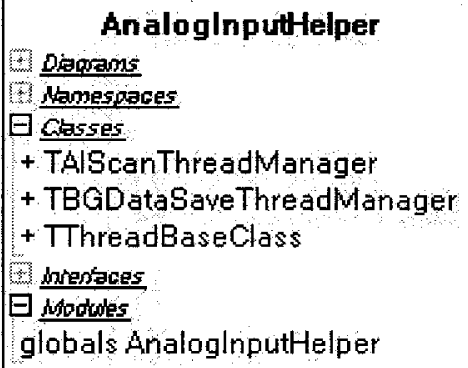 \\
\hline
\end{tabular}

\begin{tabular}{|c|}
\hline 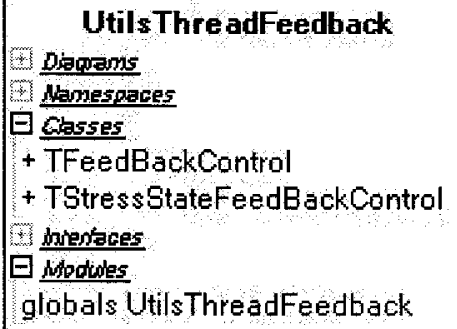 \\
\hline
\end{tabular}

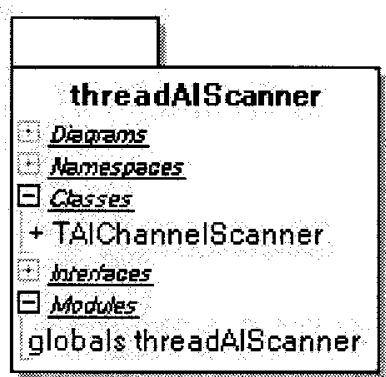

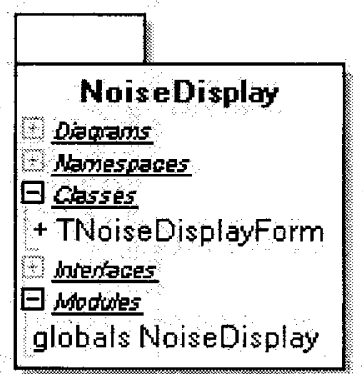

\begin{tabular}{|c|}
\hline 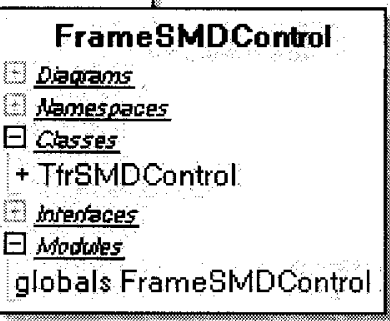 \\
\hline
\end{tabular}

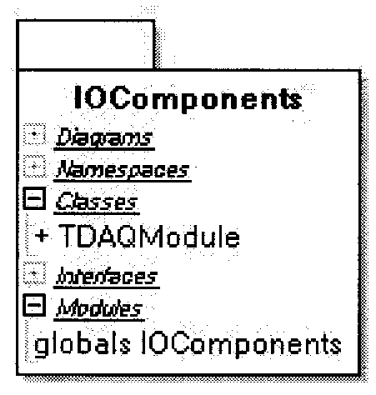

\begin{tabular}{|c|}
\hline 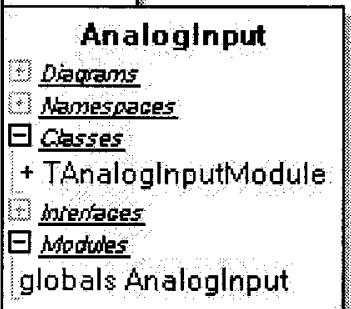 \\
\hline
\end{tabular}

\begin{tabular}{|c|}
\hline 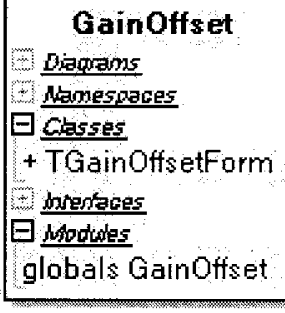 \\
\hline
\end{tabular}

Figure 3 Modules of Data Acquisition program of the HCT device - 3 


\begin{tabular}{|c|}
\hline 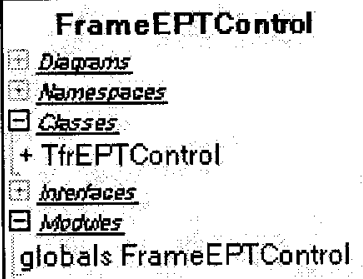 \\
\hline
\end{tabular}

\begin{tabular}{|c|}
\hline 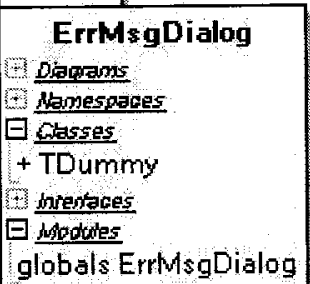 \\
\hline
\end{tabular}

\begin{tabular}{|c|}
\hline 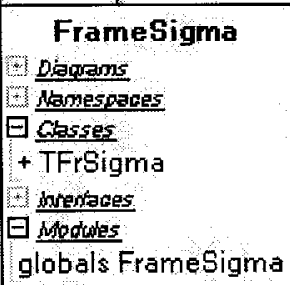 \\
\hline
\end{tabular}

\begin{tabular}{|c|}
\hline 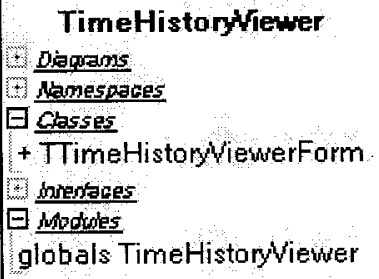 \\
\hline
\end{tabular}

\begin{tabular}{|c|c|}
\hline \multicolumn{2}{|c|}{ 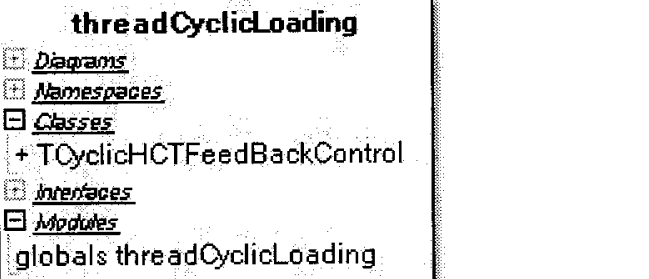 } \\
\hline 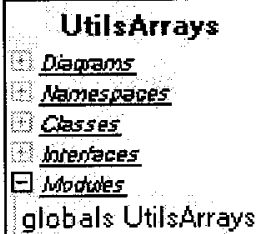 & 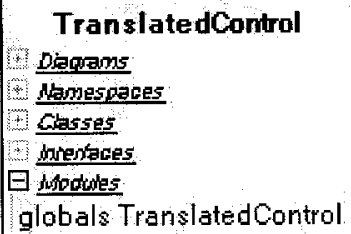 \\
\hline
\end{tabular}

\begin{tabular}{|c|}
\hline 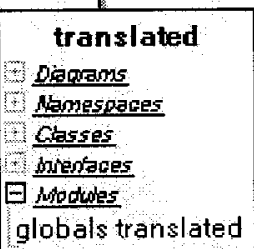 \\
\hline
\end{tabular}

Figure 4 Modules of Data Acquisition program of the HCT device - 4 


\begin{tabular}{|c|}
\hline 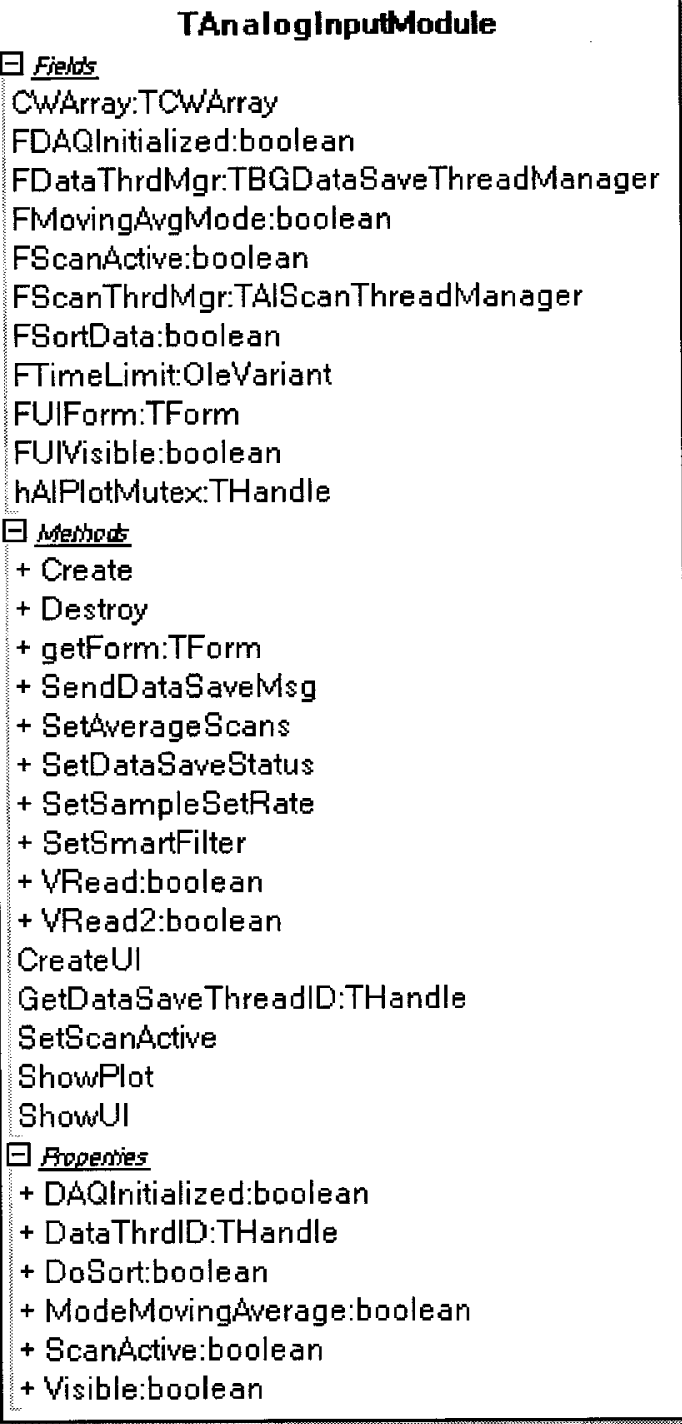 \\
\hline
\end{tabular}

Figure 5 Fields, methods and properties of TAnalogInputModule class 


\begin{tabular}{|c|c|c|}
\hline 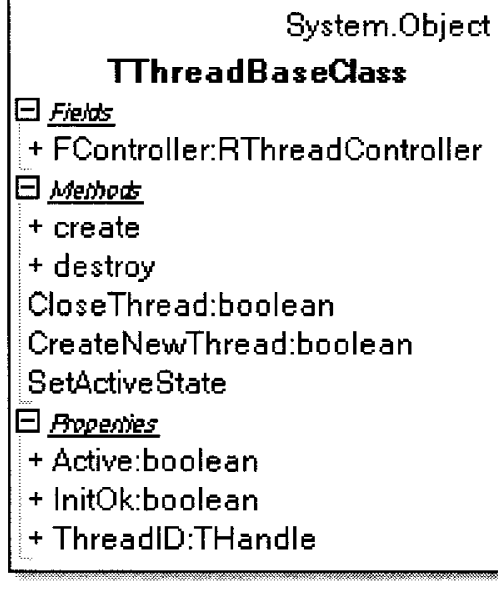 & 5 & 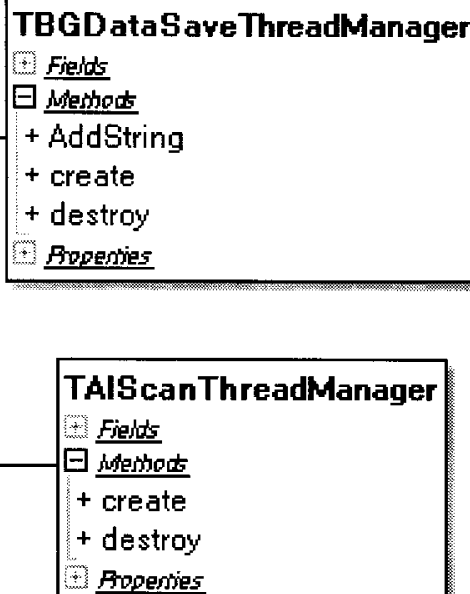 \\
\hline
\end{tabular}

Figure 6 Fields, methods and properties of classes (TThreadBaseClass, TBGDataSaveThreadManager and TAIScanThreadManager) in AnalogInputHelper module

\begin{tabular}{|c|}
\hline 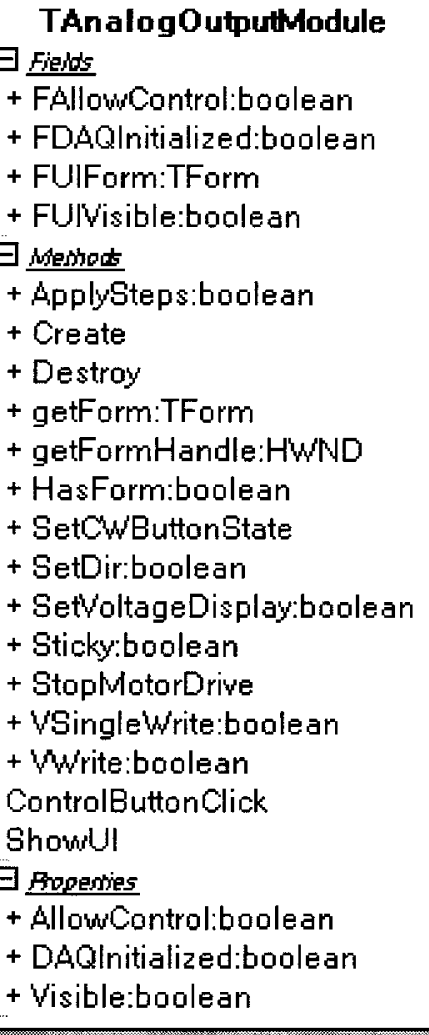 \\
\hline
\end{tabular}

Figure 7 Fields, methods and properties of TAnalogOutputModule class 


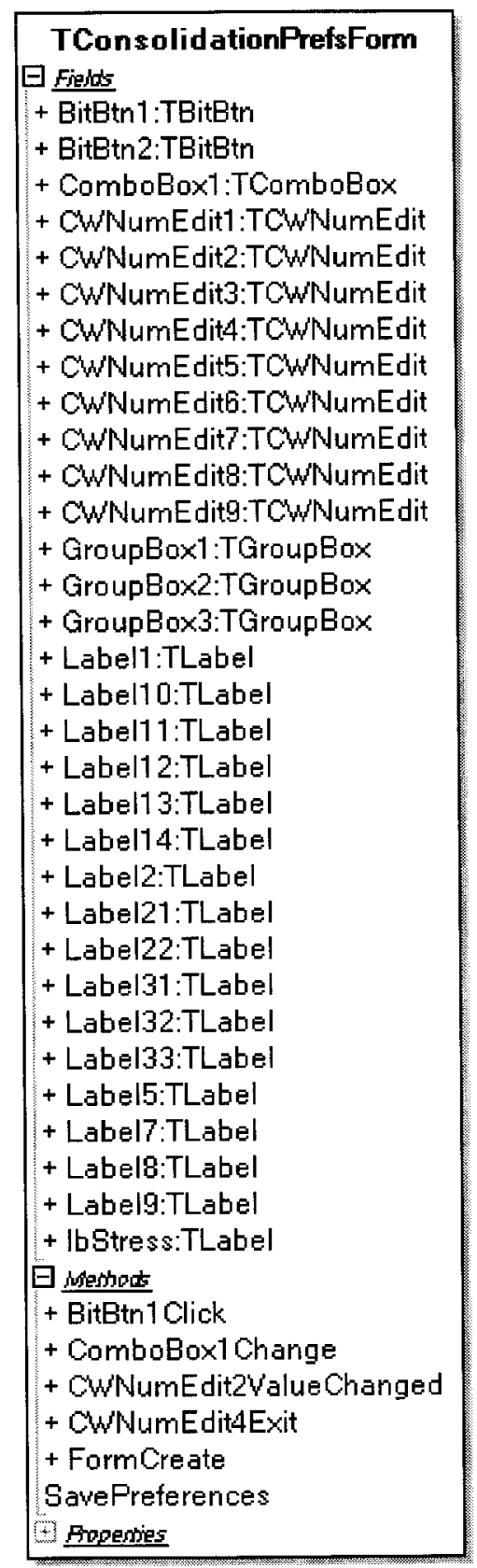

Figure 8 Fields, methods and properties of class of TConsolidationPrefsForm class in ConsolidationOptions module 


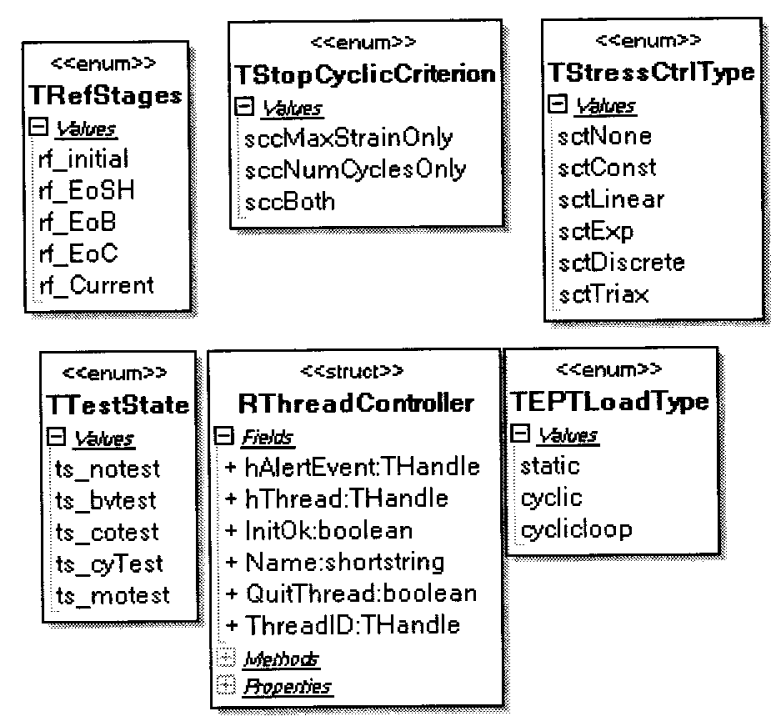

\begin{tabular}{l|} 
cenumss \\
TIndependentControl \\
E values \\
ic_Vertical \\
ic_Torsional
\end{tabular}

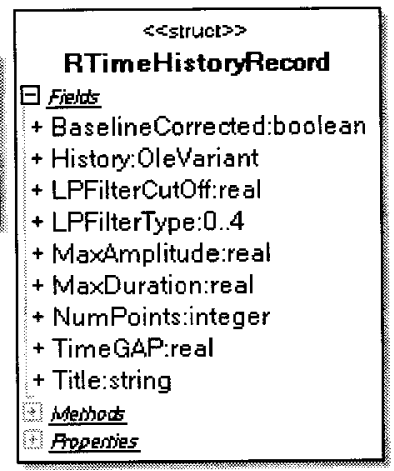

esenumss

TMotorMultiplier

$\Xi$ values

Speed25x

SpeedRegular

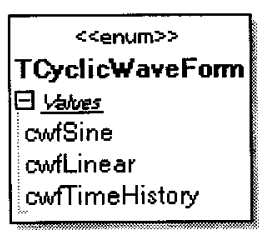

csenums?

TVolumeCtrIType

$\checkmark$ vatues

vothone

vetConst

votLinear

wotExp

vetDiscrete

\begin{tabular}{|c|}
\hline $\begin{array}{l}\text { \&senumss } \\
\text { TPrincipalRotation } \\
\square \text { Loutes } \\
\text { smooth } \\
\text { jump }\end{array}$ \\
\hline $\begin{array}{l}\text { ccenumbs } \\
\text { TControlMode } \\
\square \text { Eadors } \\
\text { cm_Stress } \\
\text { cm_Strain } \\
\text { cm_None }\end{array}$ \\
\hline
\end{tabular}

Esenums?

TCons olModePreferences

曰 heluses

cmp_MleanNormal

cmp_Triax

cmp_Simpleshear

\begin{tabular}{|} 
\&senumps \\
TAlFilterMode \\
$\square$ valugs \\
fm_None \\
fm_DropExtreme \\
fm_Stat
\end{tabular}

\begin{tabular}{|c|}
\hline cenumps \\
ThotorDir \\
$\square$ vatus \\
Comp_OutFlow \\
Ext_InFlow
\end{tabular}

estructss

RThreadNamelnfo

曰 Fiejds

+ FFlags:Longword

+ FName:PChar

+ FThreadID:Longword

+ FType:LongWord

Mptempot

Fopenies

cenum>:

TLoadingDirection

$\square$ values

Id_Pasitive

Id_Negative

Figure 9 Structures and enumerations in Constants module

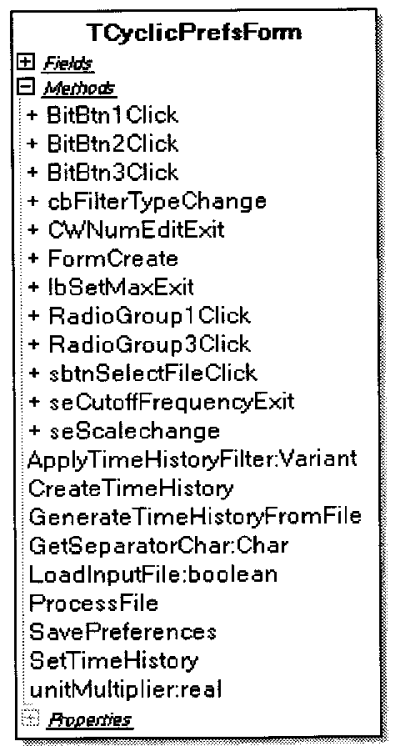

Figure 10 Fields, methods and properties of TCyclicPrefsForm class in CyclicOptions module 


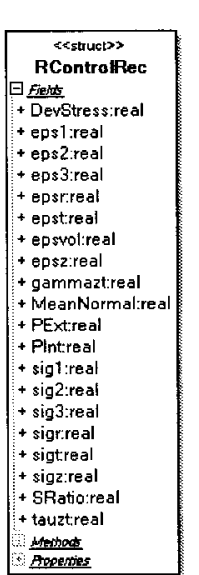

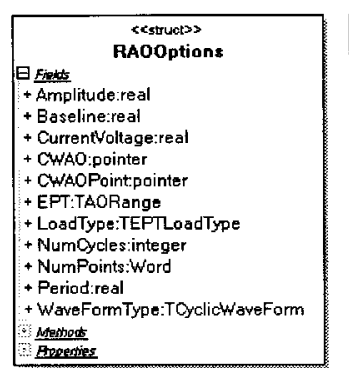

Epponips

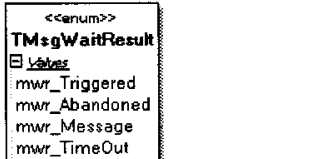

mour_Failed

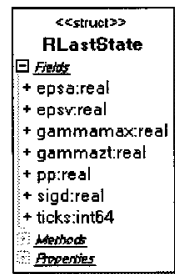

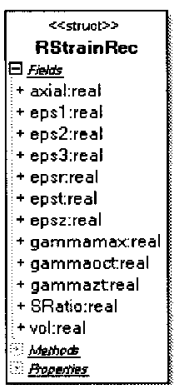

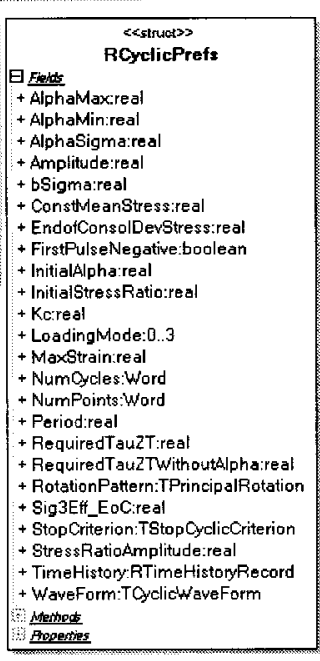

[Cstucts

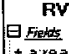

+ aryeal

+ FileNamesstring

+ InitialSlope Factorr

+ Mode:TVolumeCrrType

+ NumPoints integer

+ PPControl:boolean

+ required boolean

- ScaleFactor:real

+ smooth boolean

+ SplineSlopes:Variant

+ xarray of real

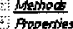
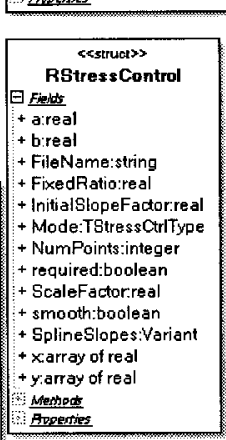

Eestruet>>
RStressRee

$$
\text { RSt }
$$

P Fiekt

+ DevStress:real
+ EPExtreal

+ EPExtreal

+ MeanNormalireal

+ PP:real

+ sig1:real

$+\operatorname{sig} 2$ rea

+ sig3real

+ sigrreal

+ sigt:real

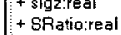

+ SRatio:real

intempoce

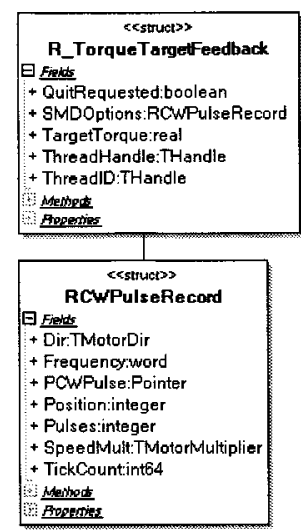

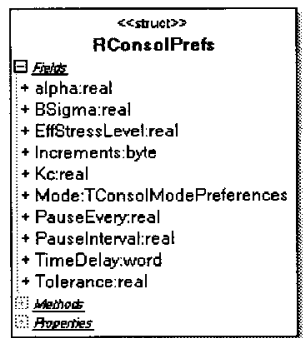

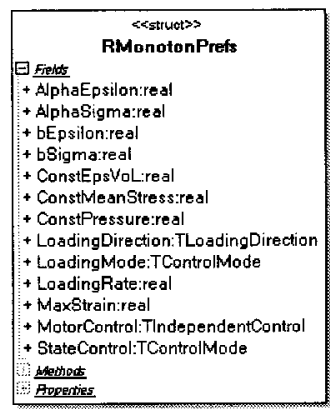

Figure 11 Structures and enumerations of Defs module

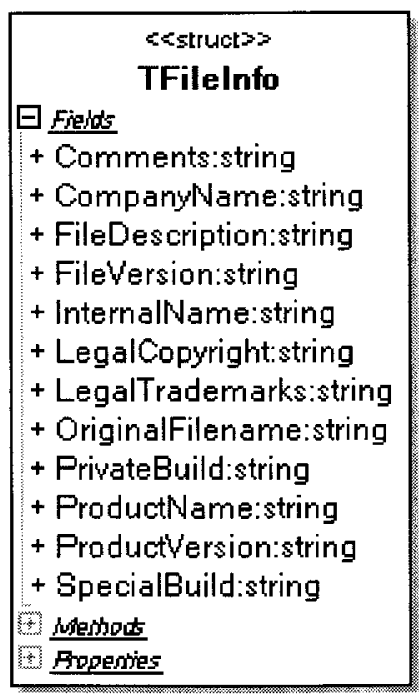

Figure 12 Structures in TFileInfo class in FileInfo Module 


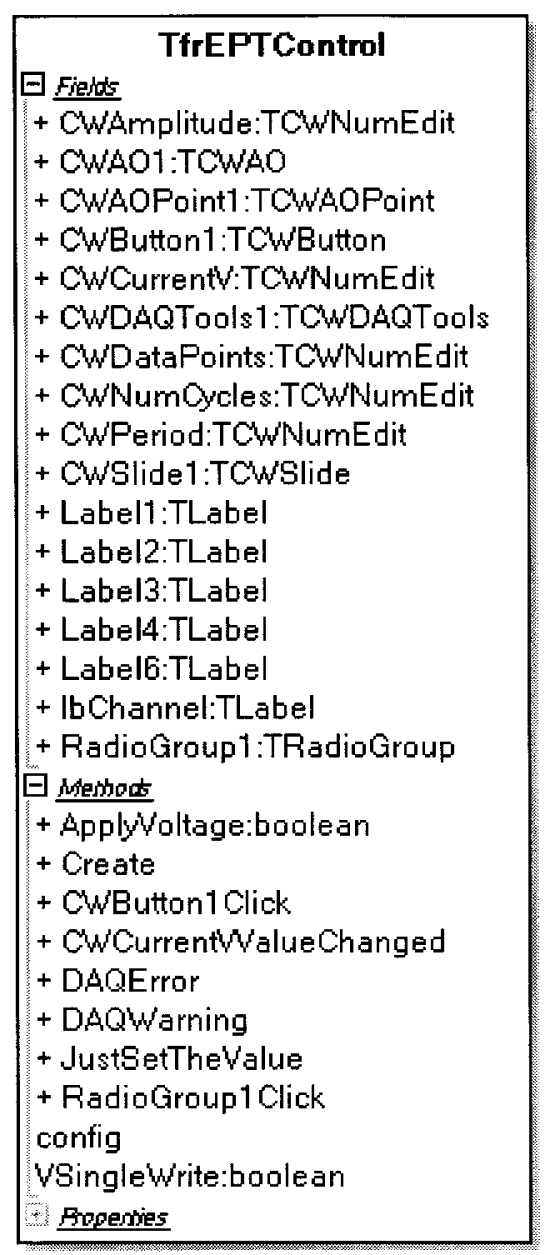

Figure 13 Fields, methods and properties of TfrEPTControl class in FrameEPTControl module

\begin{tabular}{|c|}
\hline 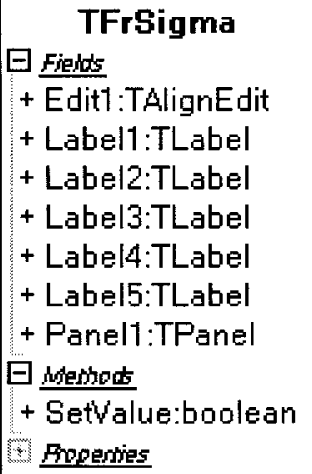 \\
\hline
\end{tabular}

Figure 14 Fields, methods and properties of TFrSigma class in FrameSigma module 


\begin{tabular}{|c|}
\hline 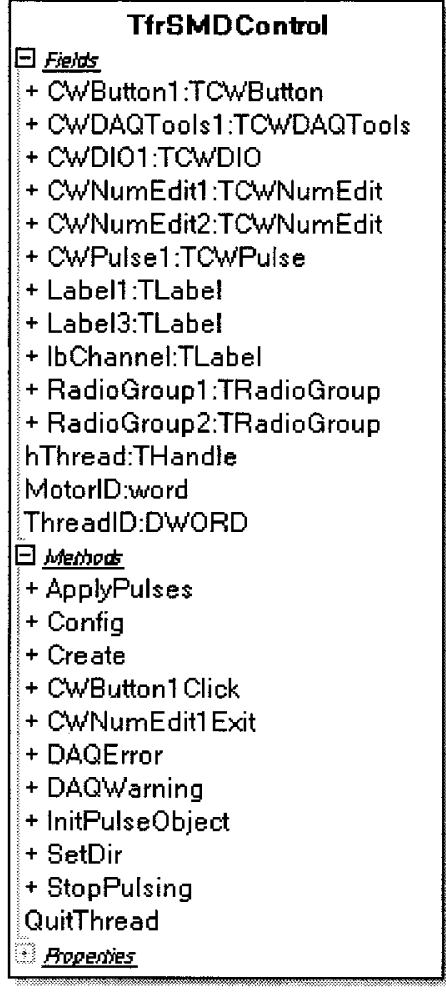 \\
\hline
\end{tabular}

Figure 15 Fields, methods and properties of TfrSMDControl class in FrameSMDControl module

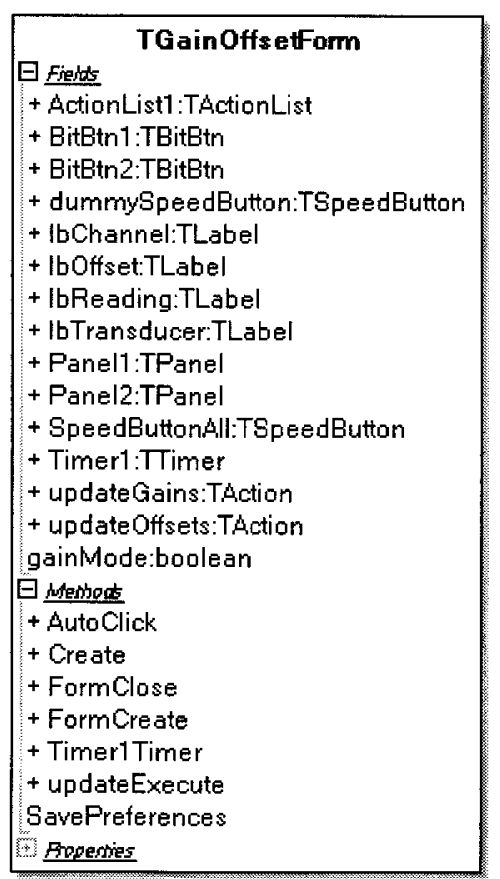

Figure 16 Fields, methods and properties of TGainOffsetForm class in GainOffset module 


\begin{tabular}{|c|}
\hline 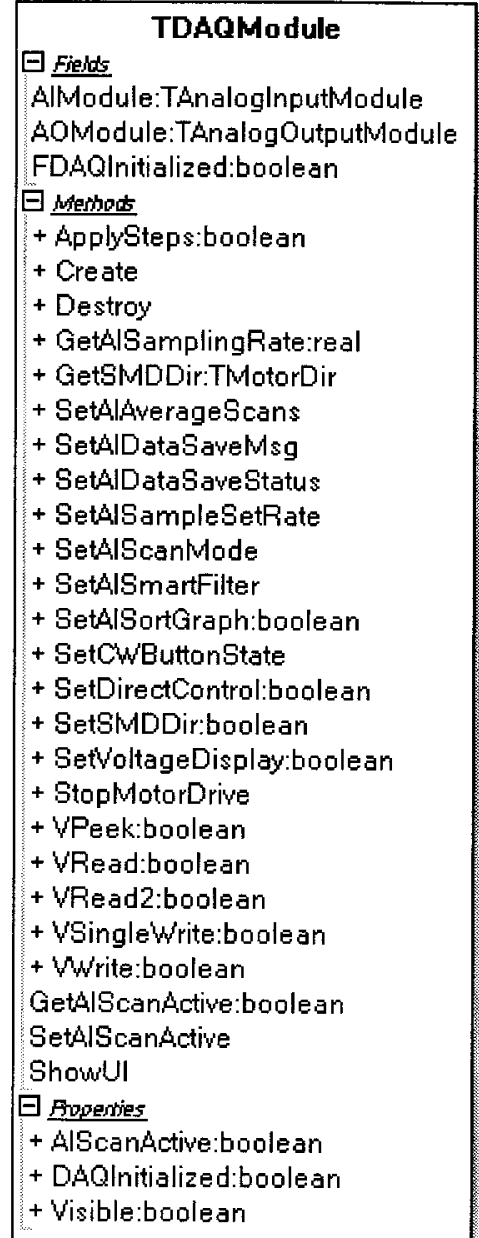 \\
\hline
\end{tabular}

Figure 17 Fields, methods and properties of TDAQModule class in IOComponents module

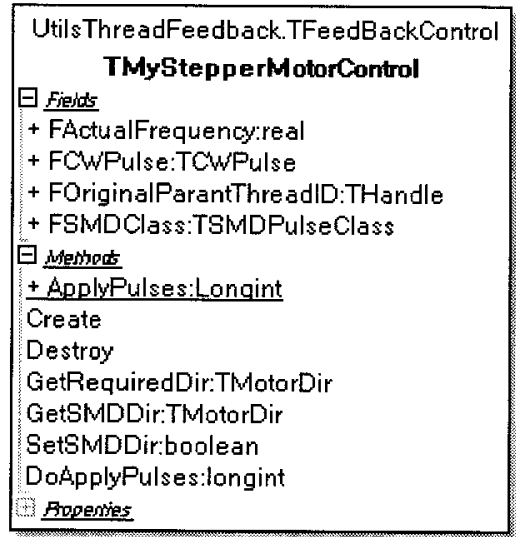

Figure 18 Fields, methods and properties of TMyStepperMotorControl class in UtilsThreadStepperMotor module 


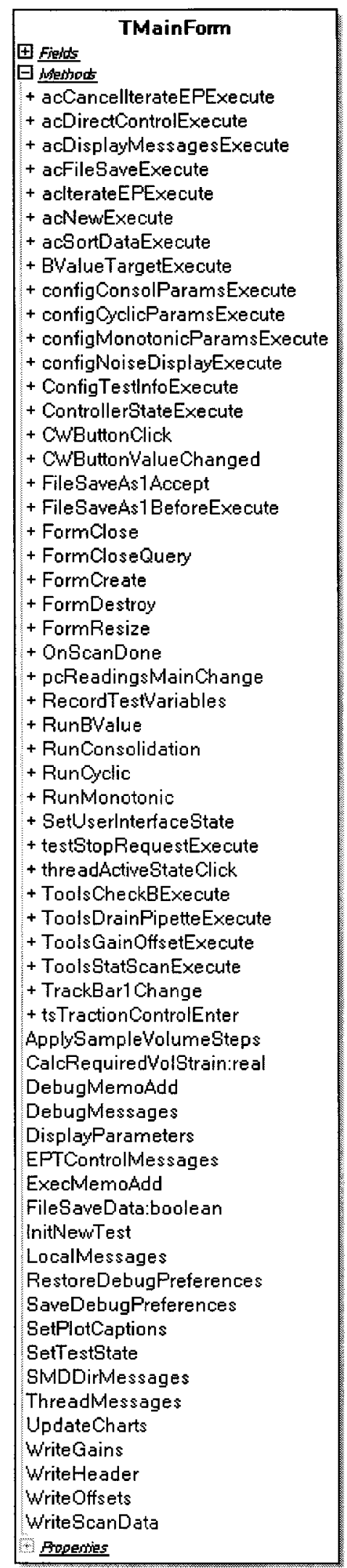

Figure 19 Fields, methods and properties of TMainForm class in Main module 


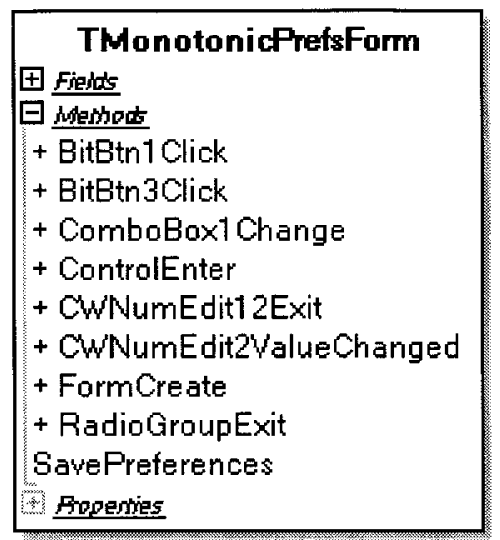

Figure 20 Fields, methods and properties of TMonotonicPrefsForm class in MonotonicOptions module

\begin{tabular}{|c|}
\hline 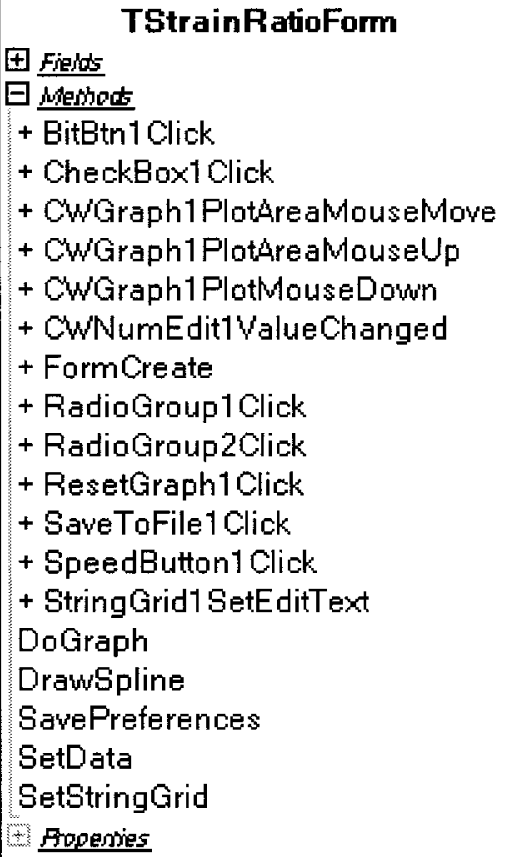 \\
\hline
\end{tabular}

Figure 21 Fields, methods and properties of TStrainRatioForm class in StrainRatioUnit module 


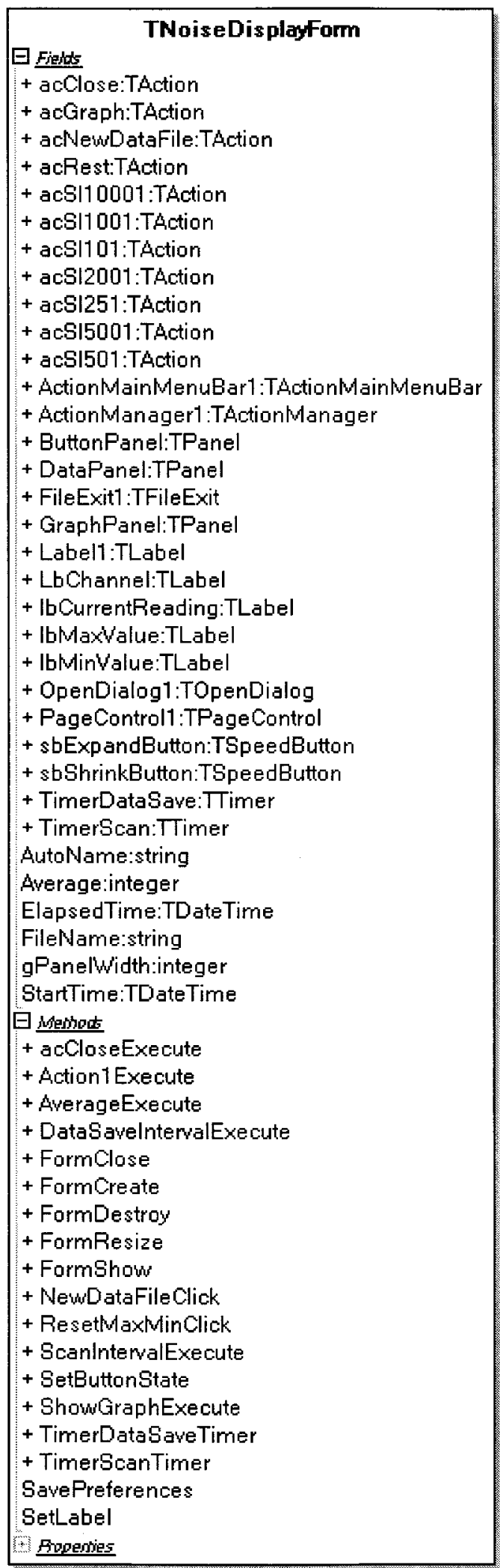

Figure 22 Fields, methods and properties of TNoiseDisplayForm class in NoiseDisplay module 


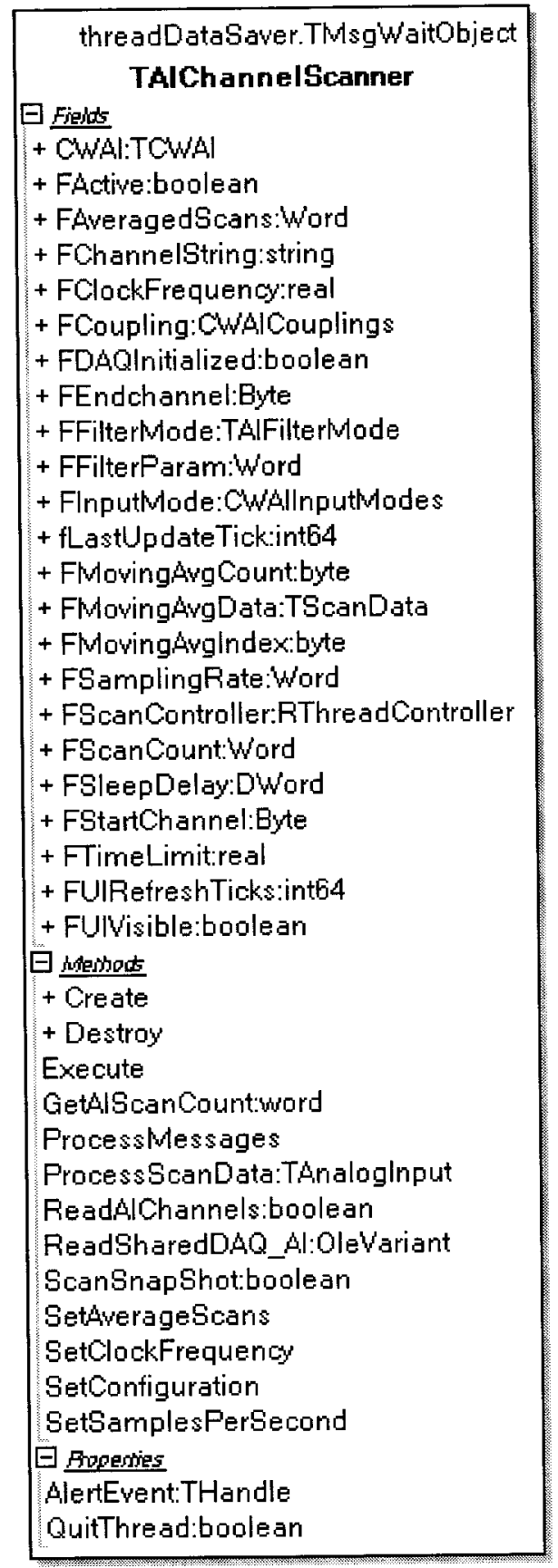

Figure 23 Fields, methods and properties of TAIChannelScanner class in ThreadAIScanner module 


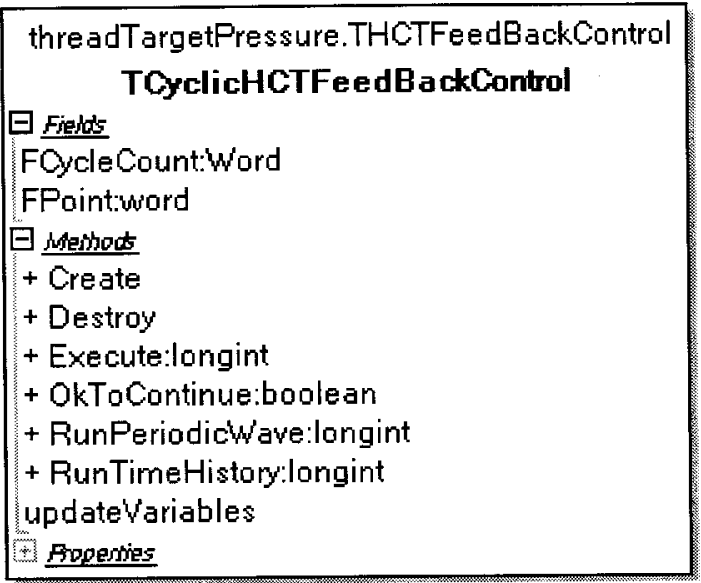

Figure 24 Fields, methods and properties of TCyclicHCTFeedBackControl class in ThreadCyclicLoading module

\begin{tabular}{|c|c|}
\hline 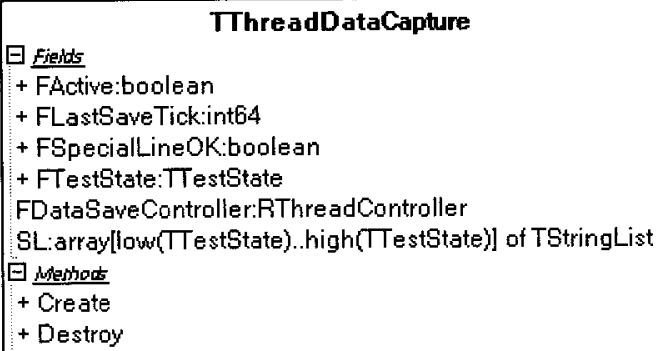 & 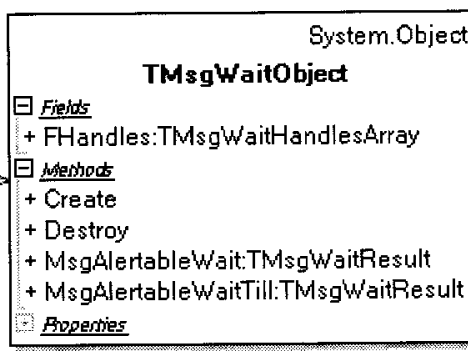 \\
\hline $\begin{array}{l}+ \text { Run:Longint } \\
\text { Execute } \\
\text { Add } \\
\text { AddDataLine } \\
\text { AddDataLineSpecial } \\
\text { Processhessages } \\
\text { Bopenies } \\
\text { AlertEvent:THandle } \\
\text { QuitThread:boolean }\end{array}$ & \\
\hline
\end{tabular}

Figure 25 Fields, methods and properties of classes (TSThreadDataCapture and TMsgWaitObject) in ThreadDataSaver module

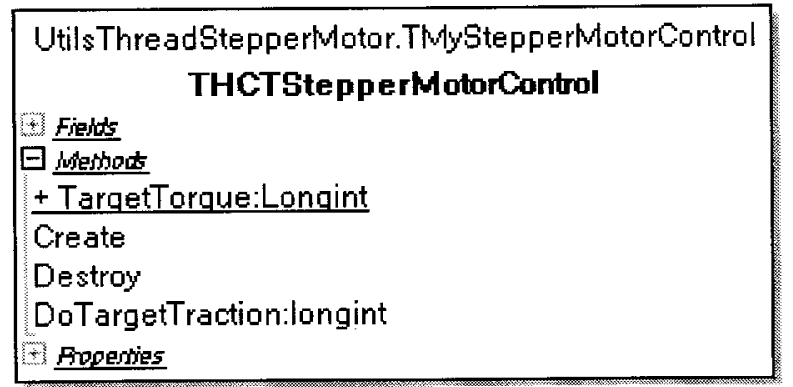

Figure 26 Fields, methods and properties of THCTStepperMotorControl class in ThreadStepperMotor module 


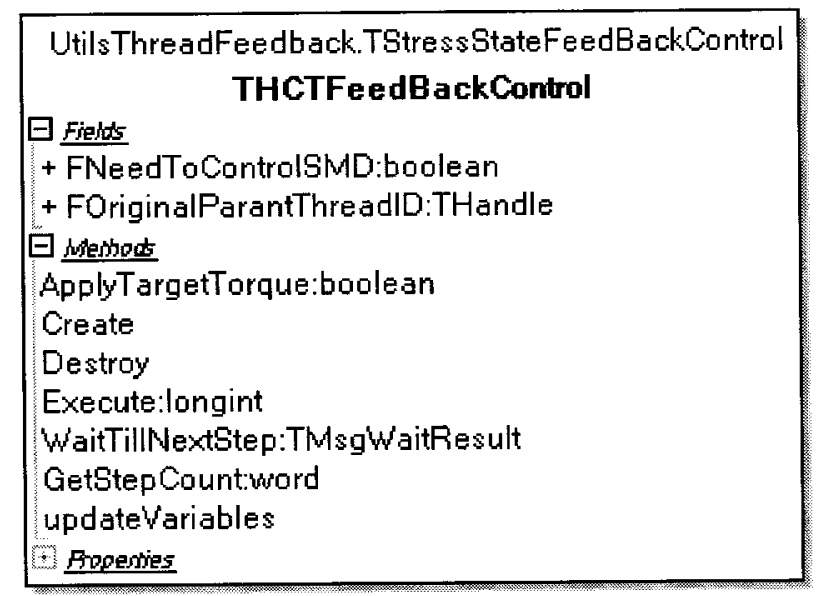

Figure 27 Fields, methods and properties of THCTFeedBackControl class in threadTargetPressure module

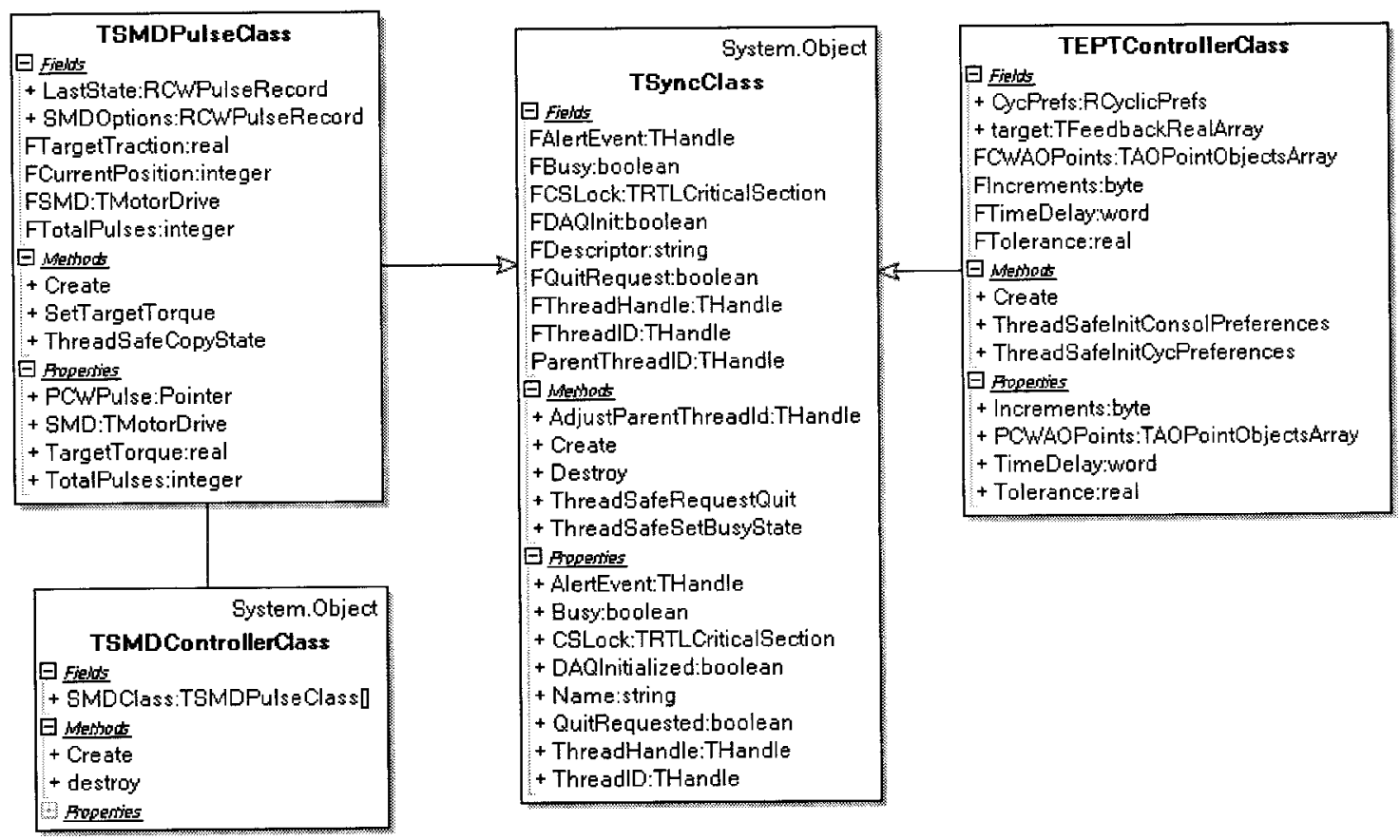

Figure 28 Fields, methods and properties of classes (TSMDPulseClass, TEPTControllerClass, TSMDControllerClass and TSyncClass) in UtilsSync module 


\begin{tabular}{|c|}
\hline 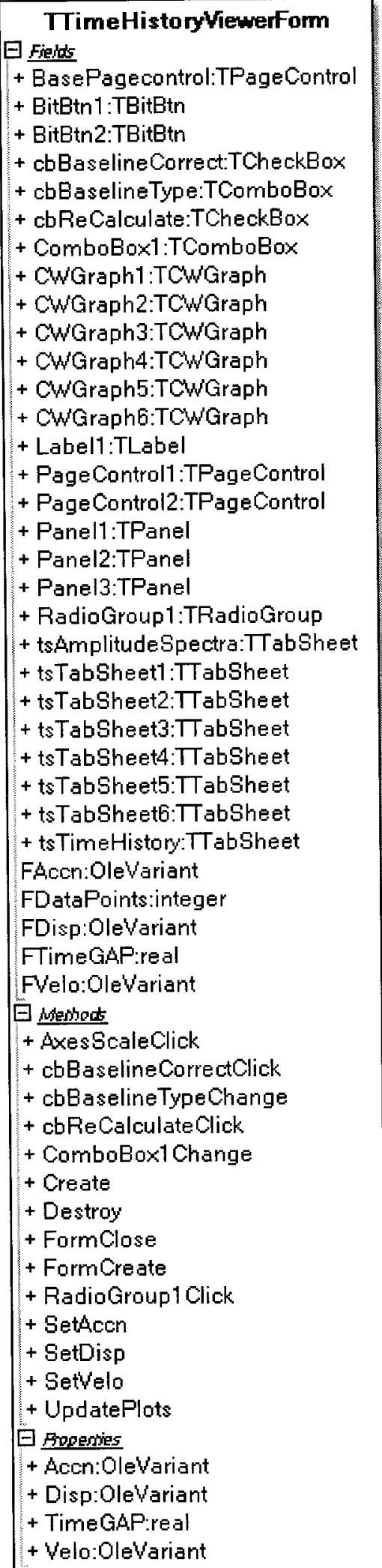 \\
\hline
\end{tabular}

Figure 29 Fields, methods and properties of TTimeHistoryViewerForm class in TimeHistoryViewer 


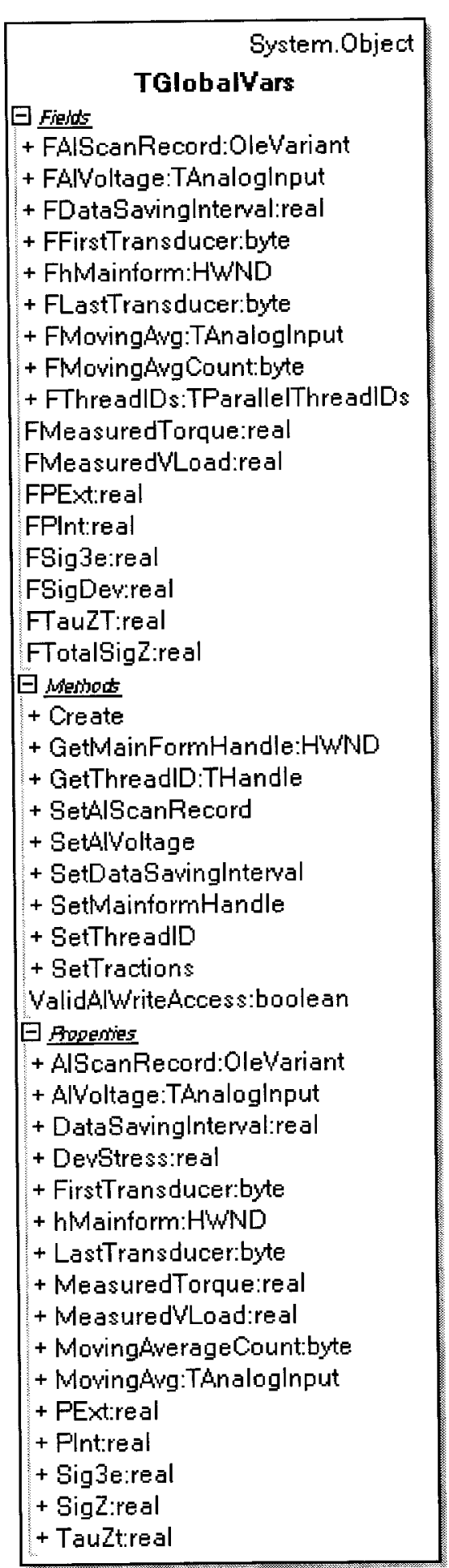

\begin{tabular}{|l|} 
ceenumps \\
TVariousThreads \\
$\square$ Uabes \\
th_Main \\
th_AlScan \\
th_DTSave
\end{tabular}

Figure $30 \quad$ Fields, methods, enumerations and properties of TGlobalVars class in UtilsObjectGlobal module 


\begin{tabular}{|c|}
\hline 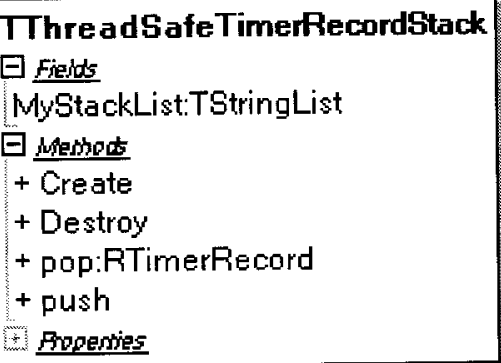 \\
\hline
\end{tabular}

\begin{tabular}{|c|}
\hline $\begin{array}{l}\text { Cestructs } \\
\text { RTimerRecord } \\
\theta \text { fields } \\
+ \text { StrID:TMaxStackStrSize } \\
+ \text { Ticks:int64 } \\
+\frac{\text { dudechot }}{\text { Bropenties }}\end{array}$ \\
\hline
\end{tabular}

Figure 31 Fields, methods and properties of TThreadSafeTimerRecordStack class and structures of RTimerRecord in UtilsStack module

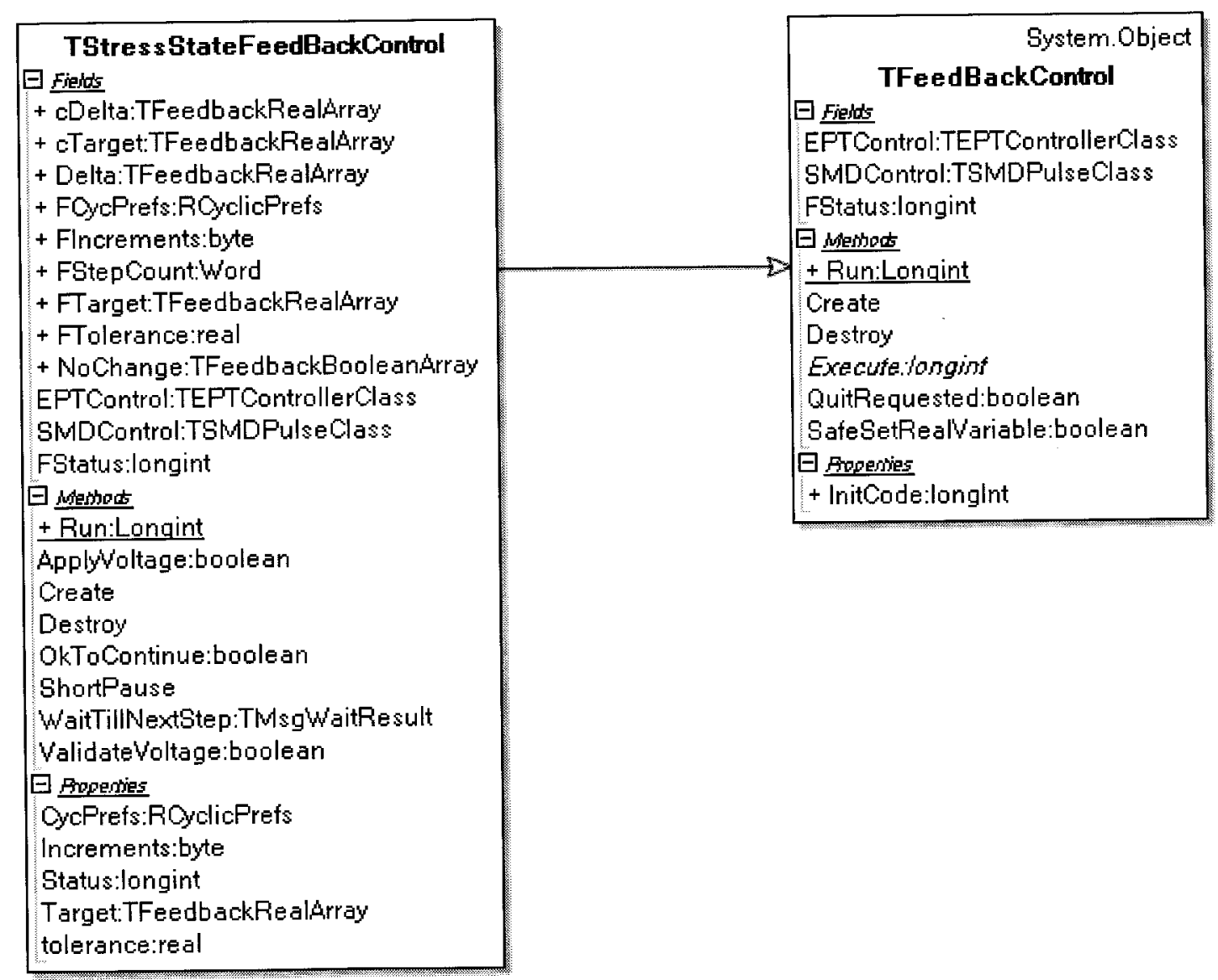

Figure 32 Fields, methods and properties of TStressStateFeedBackControl and TFeedBackControl classes in UtilsThreadFeedback module 